\title{
Tandem Iridium Catalysis as a General Strategy for Atroposelective Construction of Axially Chiral Styrenes
}

Jie Wang, ${ }^{\mathrm{a}}$ Xiaotian Qi, ${ }^{\mathrm{b}}$ Xiao-Long Min, ${ }^{\mathrm{a}}$ Wenbin Yi, ${ }^{\mathrm{a}}$ Peng Liu*b and Ying $\mathrm{He}^{* \mathrm{a}}$

${ }^{a}$ School of Chemistry and Chemical Engineering, Nanjing University of Science \& Technology, Nanjing 210094, China

${ }^{\mathrm{b}}$ Department of Chemistry, University of Pittsburgh, Pittsburgh, Pennsylvania 15260, United States

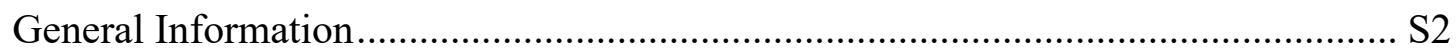

General procedure $\mathbf{A}$ : The synthesis of cinnamyl carbonates............................... S2

General procedure B: The synthesis of naphthol derivatives ................................ S3

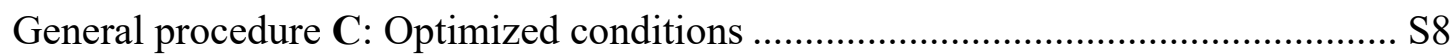

General procedure D: Synthesis of axially chiral product based on 2-naphthyl sulfonate...... S8

General procedure E: Synthesis of axially chiral product based on 2-naphthol....... S23

General procedure F: Synthesis of axially chiral products based on 1-naphthols.... S30

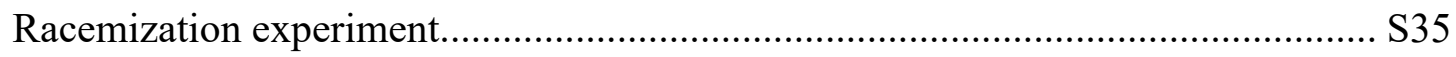

General procedure G: Large scale experiment ….............................................. S38

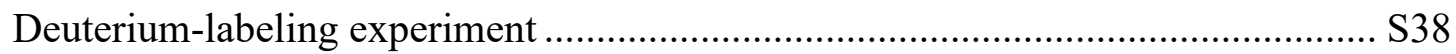

General procedure $\mathbf{H}$ : Synthetic transformations ................................................ S39

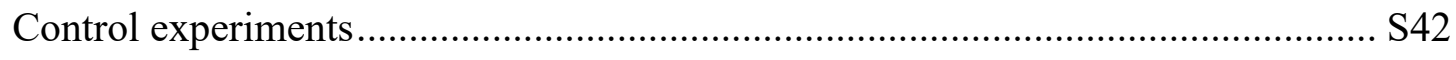

Stereospecific 1,3-H transfer of enantioenriched 1-(1-phenylallyl)naphthalen-2-ol.S43

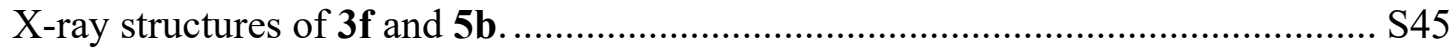

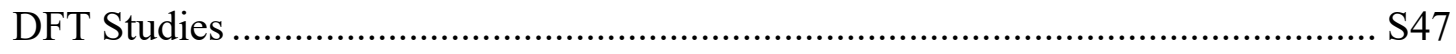

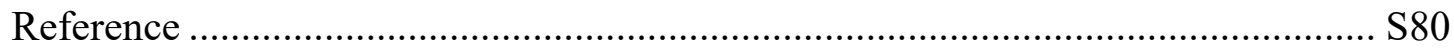

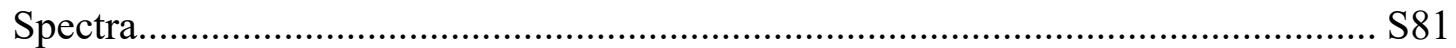




\section{General Information}

Unless otherwise noted, all starting materials were purchased from commercial sources and used without any further purification. The reactions were carried out in the glovebox unless otherwise stated. Toluene, 1, 4-dioxane, DCM, and DME were obtained from commercial sources and anhydrous THF is gained from distilling apparatus. Chemicals were used as obtained from the suppliers. The analytical data for the known compounds were found to match with the literature data and stored at $-20{ }^{\circ} \mathrm{C}$ under an inert atmosphere. Room temperature $=23-25{ }^{\circ} \mathrm{C}$. TLC plates were visualized under UV light $(254 \mathrm{~nm})$ or by staining with phosphomolybdic acid or $\mathrm{KMnO}_{4}$ followed by heating. Abbreviations are reported as following: EA = ethyl acetate, DCM $=$ dichloromethane, $\mathrm{THF}=$ tetrahydrofuran, $\mathrm{PE}=$ petroleum ether. ${ }^{1} \mathrm{H} \mathrm{NMR},{ }^{19} \mathrm{~F}$ NMR and ${ }^{13} \mathrm{C}$ NMR spectra were recorded on Bruker-AVANCE 500, and chemical shifts are reported in ppm. Multiplicities are reported using the following abbreviations: $\mathrm{s}=$ singlet, $\mathrm{d}=$ doublet, $\mathrm{t}=$ triplet, $\mathrm{q}=$ quartet, $\mathrm{m}=$ multiplet. The impurities of grease were found in some cases of NMR, however, not affect the yields of the products. High resolution mass spectral data were acquired on Agilent Technologies AccurateMass Q-TQF LC/MS 6520 operated by China Pharmaceutical University. Enantiomeric excess was determined on a Thermo Fisher UltiMate 3000 Chiral HPLC using $\mathrm{AD}$, $\mathrm{OD}$ and IA column.

\section{General procedure A: The synthesis of cinnamyl carbonates}

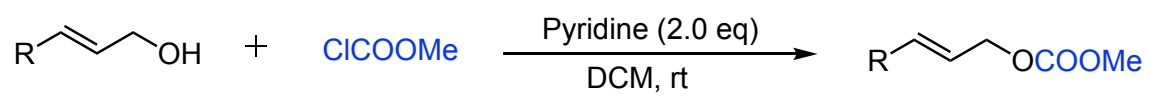

Under an argon atmosphere, cinnamyl alcohol (10 mmol, $1.0 \mathrm{eq})$, pyridine (20 mmol, 2.0 eq), was dissolved in DCM (25 mL). Then methyl chloroformate (15 mmol, 1.5 eq) was added dropwise under ice bath. The mixture was warmed to room temperature and stirred for 12 hours, then quenched by $\mathrm{HCl}$ aq $(1 \mathrm{M}, 30 \mathrm{~mL})$. The layers were separated and the aqueous phase was extracted by DCM $(20 \mathrm{~mL}$ x 3$)$. The combined organic layers were dried over anhydrous $\mathrm{Na}_{2} \mathrm{SO}_{4}$, filtered and concentrated 
in vacuo. The crude product was purified by column chromatography over silica gel (PE: $E A=20: 1)$ to get the cinnamyl carbonates.

\section{General procedure B: The synthesis of naphthol derivatives}

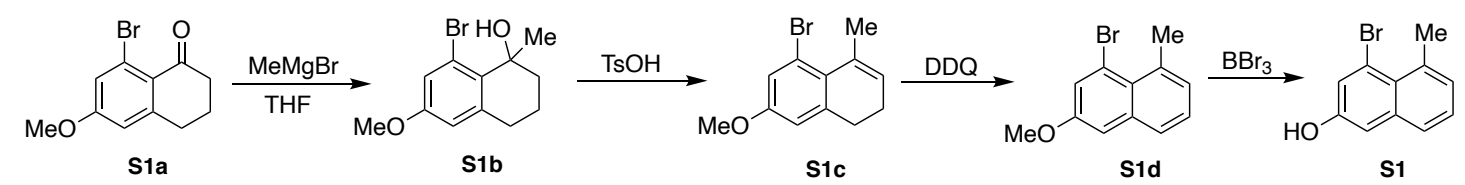

To a $500 \mathrm{~mL}$ round bottom flask filled with argon was added a magnetic bar. S1a (12.7 g, $50 \mathrm{mmol}, 1.0 \mathrm{eq})$ was dissolved in THF (150 mL), and then $\operatorname{MeMgBr}(20 \mathrm{~mL}$, $3.0 \mathrm{M}, 1.2 \mathrm{eq})$ was added at $0{ }^{0} \mathrm{C}$. The mixture was allowed to warm to room temperature and stirred for 4 hours. Then the mixture was quenched by $\mathrm{NH}_{4} \mathrm{Cl}$ aqueous $(20 \mathrm{~mL})$ and extracted with EA (40 mL x 3). The combined organic layers were washed with saturated $\mathrm{NaCl}$ aqueous $(50 \mathrm{~mL})$, dried over anhydrous $\mathrm{Na}_{2} \mathrm{SO}_{4}$, filtered and concentrated in vacuo. The crude product $\mathbf{S 1 b}$ was used for the next step without further purification.

To a $500 \mathrm{~mL}$ round bottom flask was added the crude product $\mathbf{S 1 b}$ and DCM (100 $\mathrm{mL})$, then $p$-toluenesulfonic acid hydrate $(0.475 \mathrm{~g}, 2.5 \mathrm{mmol}, 0.05 \mathrm{eq})$ was added and the mixture was stirred at room temperature for 1 hour. $\mathrm{H}_{2} \mathrm{O}$ was then added; after that the solution was separated and extracted with DCM (40 mL x 3). The combined organic layers were washed with saturated $\mathrm{NaCl}$ aqueous $(50 \mathrm{~mL})$, dried over anhydrous $\mathrm{Na}_{2} \mathrm{SO}_{4}$, filtered and concentrated in vacuo. The crude product S1c was used for the next step without further purification.

To a $500 \mathrm{~mL}$ round bottom flask under ice bath was added the crude product S1c and DCM (100 mL); and then DDQ (17.025 g, $75 \mathrm{mmol}, 1.5 \mathrm{eq})$ was added. The mixture was stirred at room temperature for 2 hours. After the starting material was completely consumed, saturated $\mathrm{NaHCO}_{3}$ aqueous $(100 \mathrm{~mL})$ was added dropwise into the flask. The mixture was filtered and the filtrate was separated and extracted with DCM (40 mL x 3). The combined organic layers were washed with saturated $\mathrm{NaCl}$ aqueous $(50 \mathrm{~mL})$, dried over anhydrous $\mathrm{Na}_{2} \mathrm{SO}_{4}$, filtered and concentrated in vacuo. 
The crude product was purified by column chromatography over silica gel (PE: EA= 50:1) to afford the product S1d as white solid (9.00 g, 72\% yield in three steps).

Under an argon atmosphere, S1d $(9.00 \mathrm{~g}, 36 \mathrm{mmol}, 1.0 \mathrm{eq})$ and DCM (50 mL) was added to a $250 \mathrm{~mL}$ round bottom flask. Then the mixture was cooled down to $-78{ }^{\circ} \mathrm{C}$ before $\mathrm{BBr}_{3}(3.22 \mathrm{~mL}, 43.2 \mathrm{mmol}, 1.2 \mathrm{eq})$ was added. The mixture was warmed to room temperature and reacted for 4 hours. After starting material was consumed completely, $\mathrm{H}_{2} \mathrm{O}$ was added to the mixture. The solution was separated and extracted with DCM (40 mL x 3). The combined organic layers were washed with saturated $\mathrm{NaCl}$ aqueous $(50 \mathrm{~mL})$, dried over anhydrous $\mathrm{Na}_{2} \mathrm{SO}_{4}$, filtered and concentrated in vacuo. The crude product was purified by column chromatography over silica gel (PE: EA= 20:1) to afford the product $\mathbf{S 1}$ as white solid (7.65 g, 90\% yield). ${ }^{1} \mathrm{H}$ NMR (500 MHz, Chloroform- $d$ ) $\delta 7.59-7.46(\mathrm{~m}, 2 \mathrm{H}), 7.41-7.23(\mathrm{~m}, 1 \mathrm{H}), 7.18(\mathrm{~d}, J=6.8 \mathrm{~Hz}, 1 \mathrm{H})$, 7.11 (q, $J=2.6 \mathrm{~Hz}, 1 \mathrm{H}), 4.96$ (s, 1H), 3.07 (s, 3H). ${ }^{13} \mathrm{C}$ NMR (126 MHz, Chloroformd) $\delta 150.91,136.12,134.47,127.79,125.81,125.64,125.62,123.78,119.74,110.16$, 25.09. HRMS(ESI) m/z: calculated for $\left[\mathrm{C}_{11} \mathrm{H}_{19} \mathrm{BrO}+\mathrm{H}\right]^{+} 236.9915$, found 236.9908 .

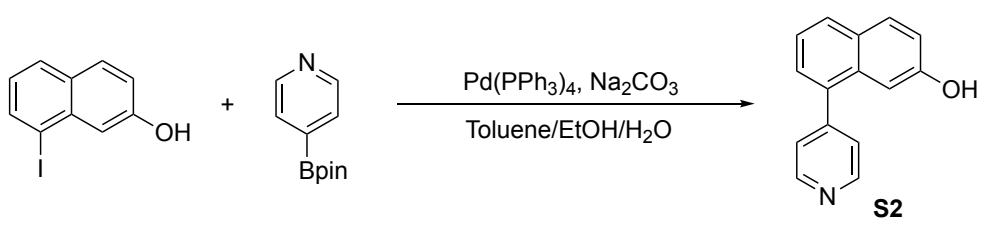

To a flame-dried round-bottom flask filled with argon were added 8iodonaphthalen-2-ol (2.7 g, $10 \mathrm{mmol}, 1.0 \mathrm{eq})$, 4-pyridine boronic acid pinacol ester (4.1 g, $20 \mathrm{mmol}, 2.0 \mathrm{eq}), \mathrm{Na}_{2} \mathrm{CO}_{3}(2.12 \mathrm{~g}, 20 \mathrm{mmol}, 2.0 \mathrm{eq})$ and $\mathrm{Pd}\left(\mathrm{PPh}_{3}\right)_{4}(1.155 \mathrm{~g}, 1 \mathrm{mmol}$, $0.1 \mathrm{eq})$. Toluene/EtOH/ $\mathrm{H}_{2} \mathrm{O}(10: 1.2: 1) 30 \mathrm{~mL}$ were added before the mixture was heated to $110^{\circ} \mathrm{C}$, and then the mixture was stirred for 12 hours. After cooling down to room temperature, the mixture was filtered through celite and treated with water. Then the solution was separated and extracted with EA $(20 \mathrm{~mL}$ x 3$)$. The combined organic layers were washed with saturated $\mathrm{NaCl}$ aqueous $(30 \mathrm{~mL})$, dried over anhydrous $\mathrm{Na}_{2} \mathrm{SO}_{4}$, filtered and concentrated in vacuo. The crude product was purified by column chromatography over silica gel (DCM: $\mathrm{MeOH}=50: 1$ to $20: 1$ ) to afford the product $\mathbf{S 2}$ 
as white solid (1.61g, 73\% yield). ${ }^{1} \mathrm{H}$ NMR $\left(500 \mathrm{MHz}, \mathrm{DMSO}-d_{6}\right) \delta 9.80(\mathrm{~s}, 1 \mathrm{H}), 8.96$ $-8.54(\mathrm{~m}, 2 \mathrm{H}), 8.07-7.79(\mathrm{~m}, 2 \mathrm{H}), 7.61-7.43(\mathrm{~m}, 2 \mathrm{H}), 7.48-7.26(\mathrm{~m}, 2 \mathrm{H}), 7.13$ (dd, $J=8.8,2.4 \mathrm{~Hz}, 1 \mathrm{H}), 7.07$ (d, $J=2.3 \mathrm{~Hz}, 1 \mathrm{H}) .{ }^{13} \mathrm{C}$ NMR $\left(126 \mathrm{MHz}, \mathrm{DMSO}-d_{6}\right) \delta 155.53$, $149.18,147.63$, 134.32, 131.15, 129.62, 127.98, 127.46, 126.57, 124.17, 121.71, 118.22, 105.35, 78.59. HRMS(ESI) m/z: calculated for $\left[\mathrm{C}_{15} \mathrm{H}_{11} \mathrm{NO}+\mathrm{H}\right]^{+} 222.0919$, found 222.0917 .

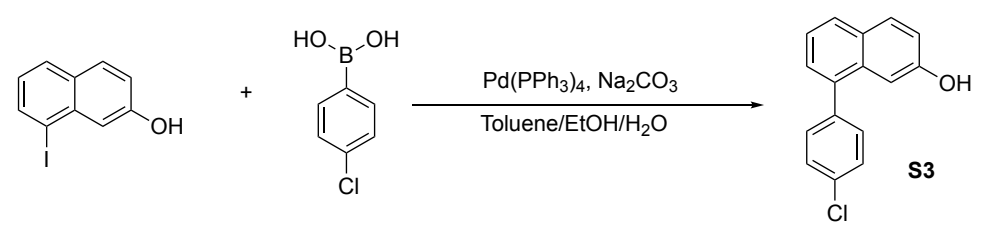

To a flame-dried round-bottom flask filled with argon were added 8iodonaphthalen-2-ol (2.7 g, $10 \mathrm{mmol}, 1.0 \mathrm{eq})$, boronic acid (3.12 g, $20 \mathrm{mmol}, 2.0 \mathrm{eq})$, $\mathrm{Na}_{2} \mathrm{CO}_{3}(2.12 \mathrm{~g}, 20 \mathrm{mmol}, 2.0 \mathrm{eq})$ and $\mathrm{Pd}\left(\mathrm{PPh}_{3}\right)_{4}(1.155 \mathrm{~g}, 1 \mathrm{mmol}, 0.1 \mathrm{eq})$. Toluene/EtOH/ $\mathrm{H}_{2} \mathrm{O}(10: 1.2: 1) 30 \mathrm{~mL}$ were added before the mixture was heated to 110 ${ }^{\circ} \mathrm{C}$, and then the mixture was stirred for 12 hours. After cooling down to room temperature, the mixture was filtered through celite and treated with water. Then the solution was separated and extracted with EA $(20 \mathrm{~mL} \times 3)$. The combined organic layers were washed with saturated $\mathrm{NaCl}$ aqueous $(30 \mathrm{~mL})$, dried over anhydrous $\mathrm{Na}_{2} \mathrm{SO}_{4}$, filtered and concentrated in vacuo. The crude product was purified by column chromatography over silica gel (PE: $\mathrm{EA}=50: 1$ to $20: 1)$ to afford the product $\mathbf{S 3}$ as yellow oil (1.85g, 73\% yield). ${ }^{1} \mathrm{H}$ NMR (500 MHz, Chloroform-d) $\delta 7.80$ (ddd, J = 10.9, 8.2, $1.9 \mathrm{~Hz}, 2 \mathrm{H}), 7.44(\mathrm{~d}, \mathrm{~J}=8.5 \mathrm{~Hz}, 2 \mathrm{H}), 7.41-7.27(\mathrm{~m}, 4 \mathrm{H}), 7.11(\mathrm{~d}, \mathrm{~J}=7.5 \mathrm{~Hz}, 2 \mathrm{H})$, 5.10 (s, 1H). 13C NMR (126 MHz, Chloroform-d) $\delta 152.80,138.28,136.39,132.26$, $131.69,130.23,129.39,128.25,127.54,126.82,126.52,122.14,116.71,106.60$. HRMS (ESI) m/z: calculated for $\left[\mathrm{C}_{16} \mathrm{H}_{11} \mathrm{ClO}+\mathrm{Na}\right]^{+} 277.0396$, found 277.0389 .

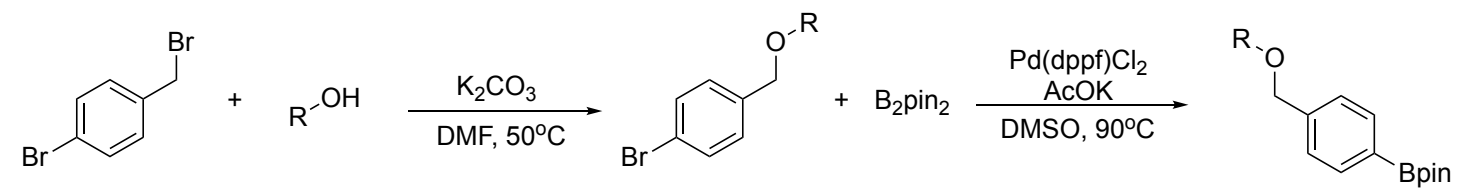

To a $250 \mathrm{~mL}$ round-bottom flask was added to 4-bromobenzyl bromide (6.0 g, 24 mmol, 1.2 eq), alcohol (20 mmol, 1.0 eq) and $\mathrm{K}_{2} \mathrm{CO}_{3}$ (5.52 g, $\left.40 \mathrm{mmol}, 2.0 \mathrm{eq}\right)$. Then 
the flask was charged with DMF $(80 \mathrm{~mL})$ and stirred at $50{ }^{\circ} \mathrm{C}$ for 6 hours. After cooling down to room temperature, the reaction was quenched by water. The layers were separated and extracted with EA $(30 \mathrm{~mL}$ x 3). The combined organic layers were washed with saturated $\mathrm{NaCl}$ aqueous $(30 \mathrm{~mL})$, dried over anhydrous $\mathrm{Na}_{2} \mathrm{SO}_{4}$, filtered and concentrated in vacuo. The crude product was purified by column chromatography over silica gel (PE: $\mathrm{EA}=50: 1)$ to afford the ether.

Under an argon atmosphere, an $100 \mathrm{~mL}$ round-bottom flask was charged with ether (10 mmol, 1.0 eq), B2pin (5.08 g, 20 mmol, 2.0 eq), AcOK (2.94 g, 30 mmol, 3.0 eq), and $\mathrm{Pd}(\mathrm{dppf}) \mathrm{Cl}_{2}(220 \mathrm{mg}, 0.3 \mathrm{mmol}, 0.03 \mathrm{eq})$, and DMSO (30 mL) was added. Then, the mixture was heated to $90{ }^{\circ} \mathrm{C}$ and stirred for 12 hours. After cooling down to room temperature, the mixture was filtered through celite and treated with water. Then the solution was separated and extracted with EA $(20 \mathrm{~mL}$ x 3). The combined organic layers were washed with saturated $\mathrm{NaCl}$ aqueous $(30 \mathrm{~mL})$, dried over anhydrous $\mathrm{Na}_{2} \mathrm{SO}_{4}$, filtered and concentrated in vacuo. The crude product was purified by column chromatography over silica gel (PE: EA= 50:1 to 20:1) to afford the boronated ester.

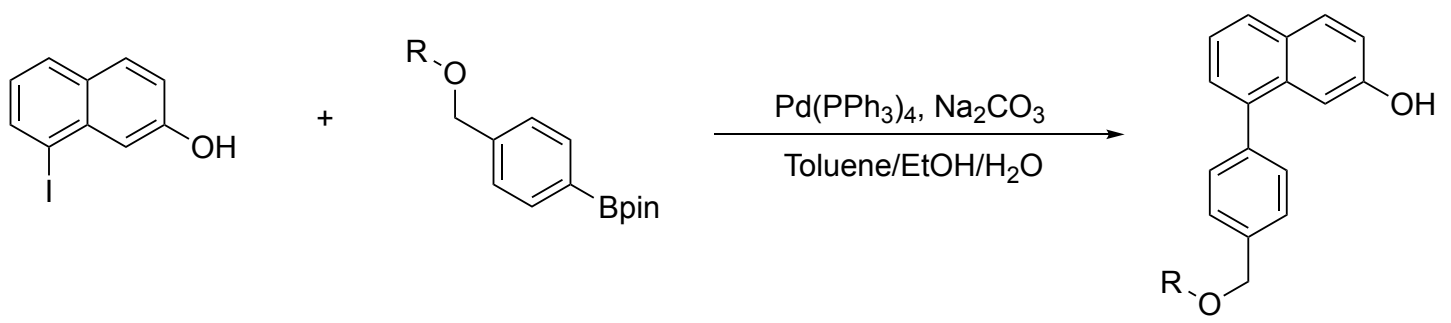

To a flame-dried round-bottom flask filled with argon were added 8iodonaphthalen-2-ol (2.7 g, $10 \mathrm{mmol}, 1.0 \mathrm{eq})$, the boronated ester (20 mmol, $2.0 \mathrm{eq})$, $\mathrm{Na}_{2} \mathrm{CO}_{3}(2.12 \mathrm{~g}, 20 \mathrm{mmol}, 2.0 \mathrm{eq})$ and $\mathrm{Pd}\left(\mathrm{PPh}_{3}\right)_{4}(1.155 \mathrm{~g}, 1.0 \mathrm{mmol}, 0.1 \mathrm{eq})$. Toluene/EtOH/ $\mathrm{H}_{2} \mathrm{O}(10: 1.2: 1) 30 \mathrm{~mL}$ were added before the mixture was heated to 110 ${ }^{\circ} \mathrm{C}$ and stirred for 12 hours. After cooling down to room temperature, the mixture was filtered through celite and treated with water. Then the solution was separated and extracted with EA $(20 \mathrm{~mL} \times 3)$, the combined organic layers were washed with saturated $\mathrm{NaCl}$ aqueous $(30 \mathrm{~mL})$, dried over anhydrous $\mathrm{Na}_{2} \mathrm{SO}_{4}$, filtered and concentrated in vacuo. The crude product was purified by column chromatography over silica gel (PE: $\mathrm{EA}=50: 1$ to $20: 1)$ to afford the final product. 


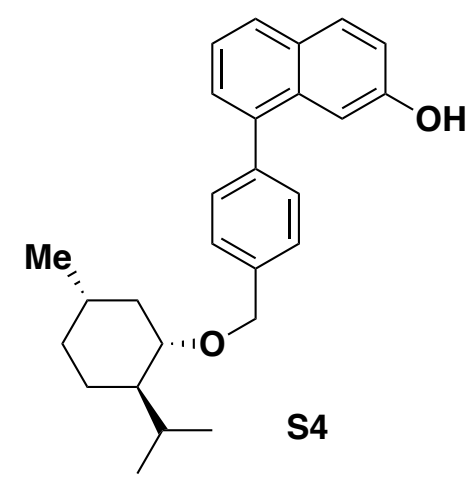

Following the general procedure, $\mathbf{S} 4$ was obtained as orange oil (2.95 g, 76\% yield). ${ }^{1} \mathrm{H}$ NMR (500 MHz, Chloroform-d) $\delta 7.74(\mathrm{t}, J=7.4 \mathrm{~Hz}, 2 \mathrm{H}), 7.39(\mathrm{~d}, J=1.7 \mathrm{~Hz}, 4 \mathrm{H})$, $7.36-7.26(\mathrm{~m}, 2 \mathrm{H}), 7.11-7.01(\mathrm{~m}, 2 \mathrm{H}), 5.83(\mathrm{~s}, 1 \mathrm{H}), 4.71(\mathrm{~d}, J=11.2 \mathrm{~Hz}, 1 \mathrm{H}), 4.45$ (d, $J=11.1 \mathrm{~Hz}, 1 \mathrm{H}), 3.26(\mathrm{td}, J=10.6,4.1 \mathrm{~Hz}, 1 \mathrm{H}), 2.34(\mathrm{pd}, J=7.0,2.6 \mathrm{~Hz}, 1 \mathrm{H}), 2.27$ $-2.18(\mathrm{~m}, 1 \mathrm{H}), 1.64$ (tq, $J=9.9,3.1 \mathrm{~Hz}, 2 \mathrm{H}), 1.47-1.29(\mathrm{~m}, 2 \mathrm{H}), 1.07-0.96(\mathrm{~m}, 2 \mathrm{H})$, $0.95(\mathrm{~d}, J=6.5 \mathrm{~Hz}, 3 \mathrm{H}), 0.88(\mathrm{~d}, J=7.1 \mathrm{~Hz}, 4 \mathrm{H}), 0.75(\mathrm{~d}, J=6.9 \mathrm{~Hz}, 3 \mathrm{H}) .{ }^{13} \mathrm{C} \mathrm{NMR}$ (126 MHz, Chloroform-d) $\delta$ 153.01, 139.29, 137.41, 136.52, 131.87, 129.14, 128.98, $128.09,127.14,126.46,126.41,122.00,116.80,106.78,78.24,69.45,47.20,39.31$, $33.51,30.67,24.57,22.25,21.41,20.09,15.10$. HRMS(ESI) $\mathrm{m} / \mathrm{z}$ : calculated for $\left[\mathrm{C}_{27} \mathrm{H}_{32} \mathrm{O}_{2}+\mathrm{K}\right]^{+}$427.2039, found 427.2065.

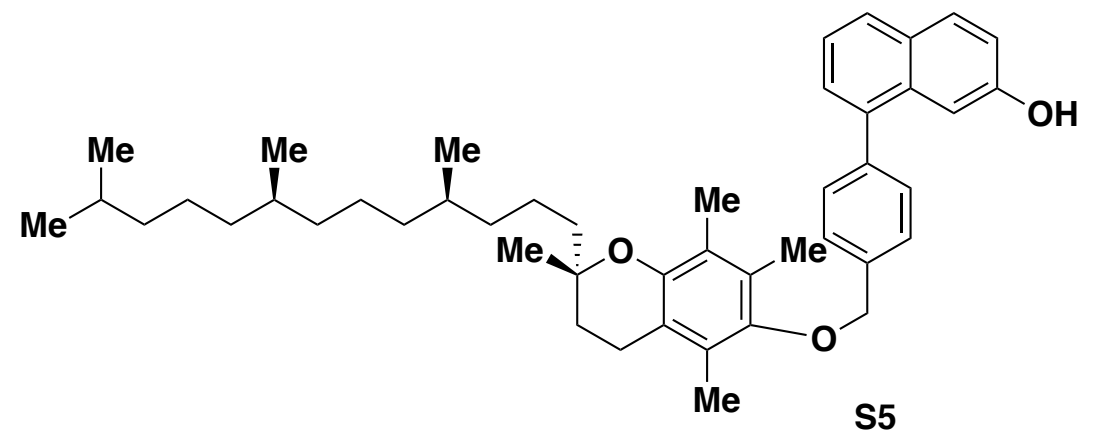

Following the general procedure, $\mathbf{S 5}$ was obtained as white solid $\left(4.3 \mathrm{~g}, 65 \%\right.$ yield) ${ }^{1} \mathrm{H}$ NMR (500 MHz, Chloroform-d) $\delta 7.76$ (dd, $J=9.1,5.2 \mathrm{~Hz}, 2 \mathrm{H}), 7.58$ (d, $J=7.8 \mathrm{~Hz}$, 2H), $7.46(\mathrm{~d}, J=7.9 \mathrm{~Hz}, 2 \mathrm{H}), 7.37-7.31(\mathrm{~m}, 2 \mathrm{H}), 7.12(\mathrm{~d}, J=2.5 \mathrm{~Hz}, 1 \mathrm{H}), 7.05$ (dd, $J=8.9,2.5 \mathrm{~Hz}, 1 \mathrm{H}), 5.40(\mathrm{~s}, 1 \mathrm{H}), 4.77(\mathrm{~s}, 2 \mathrm{H}), 2.61(\mathrm{t}, J=6.8 \mathrm{~Hz}, 2 \mathrm{H}), 2.28(\mathrm{~s}, 3 \mathrm{H})$, $2.23(\mathrm{~s}, 3 \mathrm{H}), 2.13(\mathrm{~s}, 3 \mathrm{H}), 1.88-1.72(\mathrm{~m}, 2 \mathrm{H}), 1.61-1.49(\mathrm{~m}, 4 \mathrm{H}), 1.46-1.33(\mathrm{~m}$, $5 \mathrm{H}), 1.33(\mathrm{~s}, 3 \mathrm{H}), 1.19-1.02(\mathrm{~m}, 8 \mathrm{H}), 0.86(\mathrm{dd}, J=8.7,6.6 \mathrm{~Hz}, 15 \mathrm{H}) .{ }^{13} \mathrm{C}$ NMR $(126$ MHz, Chloroform-d) $\delta 152.84,147.04,139.52,137.42,135.82,133.97,131.90,129.24$, 
$129.09,128.21,127.33,126.96,126.89,126.52,126.45,125.77,124.99,122.11$, $122.04,116.71,116.68,106.85,73.93,73.62,39.08,38.40,36.52,36.49,36.46,36.32$, $31.83,31.74,27.01,23.86,23.83,23.48,22.92,21.75,21.66,20.08,19.74,18.79,18.70$, 11.98, 11.11, 10.88. HRMS(EI) $\mathrm{m} / \mathrm{z}$ : calculated for $\left[\mathrm{C}_{46} \mathrm{H}_{62} \mathrm{O}_{3}+\mathrm{Na}\right]^{+} 685.4597$, found 685.4592 .

\section{General procedure C: Optimized conditions}
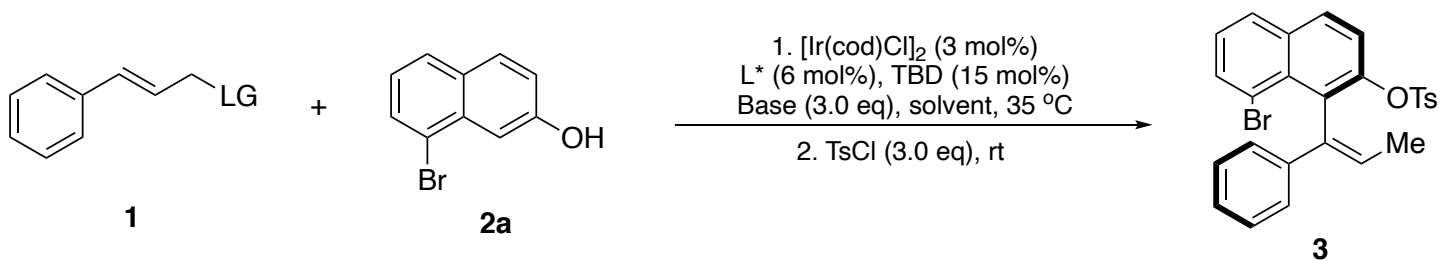

The reaction was proceeded in the glovebox under argon atmosphere. $[\operatorname{Ir}(\operatorname{cod}) \mathrm{Cl}]_{2}$ (2.1mg, $0.003 \mathrm{mmol}, 0.03 \mathrm{eq}), \mathbf{L}^{*}(0.006 \mathrm{mmol}, 0.06 \mathrm{eq})$, TBD (2.1mg, $0.015 \mathrm{mmol}$, 0.15 eq) were added to a 2 dram scintillation vial (vial A) equipped with a magnetic stirring bar. The vial was then charged with solvent $(0.5 \mathrm{~mL})$ and stirred at $25^{\circ} \mathrm{C}$ for 30 min, generating an orange solution. To another 2 dram scintillation vial (vial B) was added 1 ( $0.1 \mathrm{mmol}, 1.0 \mathrm{eq}), \mathbf{2 a}(0.15 \mathrm{mmol}, 1.5 \mathrm{eq})$, base $(0.3 \mathrm{mmol}, 3.0 \mathrm{eq})$ and solvent $(0.5 \mathrm{~mL})$. The preformed catalyst solution (vial A) was then transferred to vial $\mathbf{B}$. The mixture was stirred at $35{ }^{\circ} \mathrm{C}$ with stirring 12 hours. Then the mixture was taken out of the glovebox, and $\mathrm{TsCl}(0.3 \mathrm{mmol}, 3.0 \mathrm{eq})$ was added. After stirring at room temperature for 1 hour, the residue was purified directly by column chromatography over silica gel (PE: EA $=20: 1$ to 10:1) to afford the desired product.

General procedure D: Synthesis of axially chiral product based on 2-naphthyl sulfonate

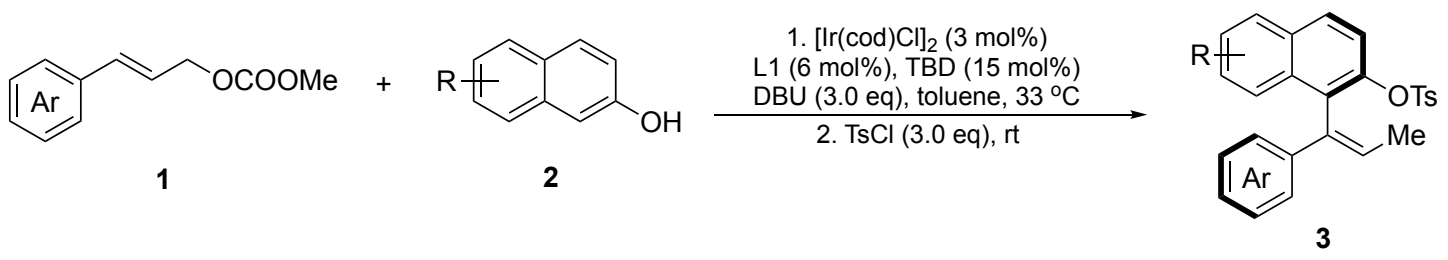

The reaction was proceeded in the glovebox under argon atmosphere. $[\operatorname{Ir}(\operatorname{cod}) \mathrm{Cl}]_{2}$ (2.1 mg, $0.003 \mathrm{mmol}, 0.03 \mathrm{eq}), \mathbf{L 1}$ (32.4mg, $0.006 \mathrm{mmol}, 0.06 \mathrm{eq})$, TBD (2.1 mg, $0.015 \mathrm{mmol}, 0.15 \mathrm{eq}$ ) were added to a 2 dram scintillation vial (vial A) equipped with a 
magnetic stirring bar. The vial was then charged with toluene $(0.5 \mathrm{~mL})$ and stirred at $25{ }^{\circ} \mathrm{C}$ for $30 \mathrm{~min}$, generating an orange solution. To another 2 dram scintillation vial (vial B) was added 1 (0.1 mmol, $1.0 \mathrm{eq}), 2$ (0.15 mmol, $1.5 \mathrm{eq})$, DBU (0.3 mmol, 3.0 eq) and toluene $(0.5 \mathrm{~mL})$. The preformed catalyst solution (vial A) was then transferred to vial $\mathbf{B}$. The mixture was stirred at $33^{\circ} \mathrm{C}$ with stirring 12 hours. Then the mixture was taken out of the glovebox, and $\mathrm{TsCl}(0.3 \mathrm{mmol}, 3.0 \mathrm{eq})$ was added. After stirring at room temperature for 1 hour, the residue was purified directly by column chromatography over silica gel (PE: EA $=20: 1$ to 10:1) to afford the desired product. (PS: The small difference of reaction temperature from 35 to $33{ }^{\circ} \mathrm{C}$ was due to the slight lower ee of 3a (1-2\%) was obtained when the reaction was carried out in $35{ }^{\circ} \mathrm{C}$. We supposed that the slow racemization of $\mathbf{3 a}$ may be occurred at $35^{\circ} \mathrm{C}$ during the reaction process in the system.)<smiles>C/C=C(\c1ccccc1)c1c(OC)ccc2cccc(Br)c12</smiles>

Following the general procedure D, 3a was obtained as white solid (41.3 $\mathrm{mg}, 84 \%$ yield). ${ }^{1} \mathrm{H}$ NMR (500 MHz, Chloroform-d) $\delta 7.88$ (dd, J = 10.9, 8.5 Hz, 2H), 7.85 $7.80(\mathrm{~m}, 1 \mathrm{H}), 7.73(\mathrm{~d}, \mathrm{~J}=9.0 \mathrm{~Hz}, 1 \mathrm{H}), 7.53$ (d, J = 8.2 Hz, 2H), 7.29 (t, J=7.8 Hz, 1H), $7.19-7.13(\mathrm{~m}, 5 \mathrm{H}), 7.04(\mathrm{dd}, \mathrm{J}=6.8,3.0 \mathrm{~Hz}, 2 \mathrm{H}), 6.33$ (q, J = 7.0 Hz, 1H), $2.41(\mathrm{~s}$, $3 \mathrm{H}), 1.36(\mathrm{~d}, \mathrm{~J}=7.0 \mathrm{~Hz}, 3 \mathrm{H}) .{ }^{13} \mathrm{C} \mathrm{NMR}(126 \mathrm{MHz}$, Chloroform- $d$ ) $\delta 146.17,143.84$, $140.36,134.17,133.88,133.08,132.78,130.20,129.45,128.41,128.20,127.69$, $127.52,126.96,126.90,125.30,125.22,125.17,119.97,118.93,20.67,14.92$. HRMS(ESI) m/z: calculated for $\left[\mathrm{C}_{26} \mathrm{H}_{21} \mathrm{BrO}_{3} \mathrm{~S}+\mathrm{Na}\right]^{+}$515.0292, found 515.0284. Isotopic MS, calculated 517.0272, found 550.517.0302. HPLC data (Chiralpak IA column, hexane: isopropanol $=98: 2,1.0 \mathrm{~mL} / \mathrm{min}), \operatorname{tr}=18.7 \mathrm{~min}$ (major), $\operatorname{tr}=17.6 \mathrm{~min}$ (minor), ee $=95 \%$. 
<smiles>C/C=C(\c1ccc(Cl)cc1)c1c(OC(F)(F)F)ccc2cccc(Br)c12</smiles>

3b

Following the general procedure D, 3b was obtained as colorless oil (40.0 mg, 76\% yield). ${ }^{1} \mathrm{H}$ NMR (500 MHz, Chloroform- $d$ ) $\delta 7.90(\mathrm{~d}, J=9.0 \mathrm{~Hz}, 1 \mathrm{H}), 7.87$ (dd, $J=8.2$, $1.3 \mathrm{~Hz}, 1 \mathrm{H}), 7.82(\mathrm{dd}, J=7.4,1.3 \mathrm{~Hz}, 1 \mathrm{H}), 7.74$ (d, $J=9.0 \mathrm{~Hz}, 1 \mathrm{H}), 7.54$ (d, $J=8.4$ $\mathrm{Hz}, 2 \mathrm{H}), 7.29$ (t, $J=7.8 \mathrm{~Hz}, 1 \mathrm{H}), 7.18(\mathrm{~d}, J=8.0 \mathrm{~Hz}, 2 \mathrm{H}), 7.08(\mathrm{~d}, J=8.6 \mathrm{~Hz}, 2 \mathrm{H})$, $6.93(\mathrm{~d}, J=8.6 \mathrm{~Hz}, 2 \mathrm{H}), 6.33(\mathrm{q}, J=7.0 \mathrm{~Hz}, 1 \mathrm{H}), 2.42(\mathrm{~s}, 3 \mathrm{H}), 1.37$ (d, $J=7.0 \mathrm{~Hz}, 3 \mathrm{H})$. ${ }^{13} \mathrm{C}$ NMR (126 MHz, Chloroform- $d$ ) $\delta$ 146.21, 144.07, 138.95, 133.96, 133.16, 133.08, $132.60,130.99$, 130.03, 129.69, 128.84, 128.44, 128.26, 127.64, 127.02, 126.87, 126.32, 125.35, 119.94, 118.72, 20.69, 14.97. HRMS(ESI) m/z: calculated for $\left[\mathrm{C}_{26} \mathrm{H}_{20} \mathrm{BrClO} 3 \mathrm{~S}+\mathrm{Na}\right]^{+}$548.9903, found 548.9904. Isotopic MS, calculated 550.9882, found 550.9894. HPLC data (Chiralpak IC column, hexane: isopropanol = 98: 2, 1.0 $\mathrm{mL} / \mathrm{min}$ ), $\operatorname{tr}=18.2 \mathrm{~min}$ (major), $\operatorname{tr}=19.7 \mathrm{~min}$ (minor), ee $=90 \%$.<smiles>C/C=C(\c1ccc(Br)cc1)c1c(OC(F)(F)F)ccc2cccc(Br)c12</smiles>

Following the general procedure D, 3c was obtained as colorless oil (42.4 mg, 74\% yield). ${ }^{1} \mathrm{H}$ NMR (500 MHz, Chloroform- $d$ ) $\delta 7.91$ (d, $\left.J=9.0 \mathrm{~Hz}, 1 \mathrm{H}\right), 7.88$ (dd, $J=8.2$, $1.3 \mathrm{~Hz}, 1 \mathrm{H}), 7.82(\mathrm{dd}, J=7.5,1.3 \mathrm{~Hz}, 1 \mathrm{H}), 7.74(\mathrm{~d}, J=9.0 \mathrm{~Hz}, 1 \mathrm{H}), 7.53$ (d, $J=8.3$ $\mathrm{Hz}, 1 \mathrm{H}), 7.30$ (t, $J=7.8 \mathrm{~Hz}, 1 \mathrm{H}), 7.23$ (d, $J=8.6 \mathrm{~Hz}, 2 \mathrm{H}), 7.18(\mathrm{~d}, J=8.0 \mathrm{~Hz}, 2 \mathrm{H})$, $6.87(\mathrm{~d}, J=8.7 \mathrm{~Hz}, 1 \mathrm{H}), 6.34$ (q, $J=7.0 \mathrm{~Hz}, 1 \mathrm{H}), 2.43$ (s, 3H), 1.36 (d, $J=7.0 \mathrm{~Hz}$, $3 \mathrm{H}) .{ }^{13} \mathrm{C}$ NMR (126 MHz, Chloroform- $d$ ) $\delta$ 146.23, 144.10, 139.42, 133.98, 133.23, $133.08,130.04,129.97,129.72,128.46,128.27,127.77,126.88,126.78,126.70$, 125.73, 125.36, 119.93, 119.22, 118.72, 20.72, 14.99. HRMS(ESI) m/z: calculated for $\left[\mathrm{C}_{26} \mathrm{H}_{20} \mathrm{Br}_{2} \mathrm{O}_{3} \mathrm{~S}+\mathrm{Na}\right]^{+}$592.9398, found 592.9408. Isotopic MS, calculated 594.9377, 
found 594.9373, calculated 596.9357, found 596.9348. HPLC data (Chiralpak IC column, hexane: isopropanol $=98: 2,1.0 \mathrm{~mL} / \mathrm{min}$ ), $\operatorname{tr}=19.7 \mathrm{~min}$ (major), $\operatorname{tr}=21.4 \mathrm{~min}$ (minor), ee $=92 \%$.

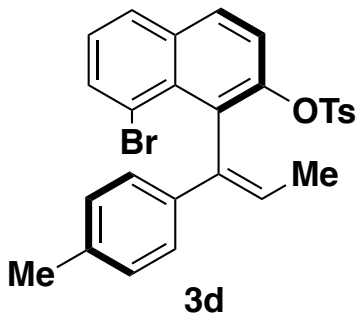

Following the general procedure D, 3d was obtained as colorless oil $(40.8 \mathrm{mg}, 81 \%$ yield). ${ }^{1} \mathrm{H}$ NMR (500 MHz, Chloroform- $d$ ) $\delta 7.95-7.84(\mathrm{~m}, 2 \mathrm{H}), 7.82$ (dd, $J=7.5,1.3$ $\mathrm{Hz}, 1 \mathrm{H}), 7.72(\mathrm{~d}, J=8.9 \mathrm{~Hz}, 1 \mathrm{H}), 7.55(\mathrm{~d}, J=8.3 \mathrm{~Hz}, 2 \mathrm{H}), 7.27(\mathrm{t}, J=7.8 \mathrm{~Hz}, 1 \mathrm{H})$, $7.17(\mathrm{~d}, J=8.0 \mathrm{~Hz}, 2 \mathrm{H}), 7.00-6.90(\mathrm{~m}, 5 \mathrm{H}), 6.27$ (q, $J=6.9 \mathrm{~Hz}, 1 \mathrm{H}), 2.41(\mathrm{~s}, 3 \mathrm{H})$, $2.30(\mathrm{~s}, 3 \mathrm{H}), 1.34$ (d, $J=7.0 \mathrm{~Hz}, 3 \mathrm{H}) .{ }^{13} \mathrm{C}$ NMR (126 MHz, Chloroform- $d$ ) $\delta$ 146.12, $143.83,137.73,134.88,133.99,133.86,133.07,132.78,130.18,129.36,128.37$, $128.19,127.76,127.64,127.01,126.09,125.20,125.06,119.97,118.96,20.70,20.13$, 14.88. HRMS(ESI) m/z: calculated for $\left[\mathrm{C}_{27} \mathrm{H}_{23} \mathrm{BrO}_{3} \mathrm{~S}+\mathrm{H}\right]^{+}$507.0630, found 507.0621. Isotopic MS, calculated 509.0609, found 509.0605. HPLC data (Chiralpak AD column, hexane: isopropanol $=98: 2,1.0 \mathrm{~mL} / \mathrm{min}$ ), $\operatorname{tr}=22.1 \mathrm{~min}$ (major), $\operatorname{tr}=19.1 \mathrm{~min}$ (minor), ee $=92 \%$.<smiles>C/C=C(\c1ccc(-c2ccccc2)cc1)c1c(OC(F)(F)F)ccc2cccc(Br)c12</smiles>

Following the general procedure D, 3e was obtained as white solid (45.0 mg, 79\% yield). ${ }^{1} \mathrm{H}$ NMR (500 MHz, Chloroform- $d$ ) $\delta 7.91$ (d, $\left.J=9.1 \mathrm{~Hz}, 1 \mathrm{H}\right), 7.88$ (dd, $J=8.1$, $1.3 \mathrm{~Hz}, 1 \mathrm{H}), 7.83(\mathrm{dd}, J=7.5,1.3 \mathrm{~Hz}, 1 \mathrm{H}), 7.76$ (d, $J=9.0 \mathrm{~Hz}, 1 \mathrm{H}), 7.58$ (dd, $J=8.4$, $1.3 \mathrm{~Hz}, 2 \mathrm{H}), 7.55$ (d, $J=8.3 \mathrm{~Hz}, 2 \mathrm{H}), 7.45-7.36$ (m, 4H), $7.35-7.26(\mathrm{~m}, 2 \mathrm{H}), 7.14$ $(\mathrm{d}, J=8.1 \mathrm{~Hz}, 2 \mathrm{H}), 7.10(\mathrm{~d}, J=8.4 \mathrm{~Hz}, 2 \mathrm{H}), 6.38(\mathrm{q}, J=6.9 \mathrm{~Hz}, 1 \mathrm{H}), 2.35(\mathrm{~s}, 3 \mathrm{H}), 1.38$ (d, $J=7.0 \mathrm{~Hz}, 3 \mathrm{H}) .{ }^{13} \mathrm{C}$ NMR (126 MHz, Chloroform-d) $\delta 146.21,143.90,139.76$, $139.48,137.82,133.92,133.84,133.10,132.73,130.19,129.53,128.40,128.24$, 
$127.73,127.41,127.12,126.95,126.08,125.78,125.53,125.51,125.28,120.02$, 118.93, 20.67, 15.01. HRMS(ESI) m/z: calculated for $\left[\mathrm{C}_{32} \mathrm{H}_{25} \mathrm{BrO}_{3} \mathrm{~S}+\mathrm{H}\right]^{+}$569.0786, found 569.0774. Isotopic MS, calculated 571.0766, found 571.0767. HPLC data (Chiralpak IA column, hexane: isopropanol =98:2,1.0 mL/min), $\operatorname{tr}=30.2 \mathrm{~min}$ (major), $\operatorname{tr}=36.2 \min ($ minor $)$, ee $=95 \%$.<smiles>C/C=C(\c1ccc(OC)cc1)c1c(OC)ccc2cccc(Br)c12</smiles>

Following the general procedure $\mathbf{D}, \mathbf{3 f}$ was obtained as white solid (45.1 $\mathrm{mg}, 86 \%$ yield). ${ }^{1} \mathrm{H}$ NMR (500 MHz, Chloroform- $d$ ) $\delta 7.96-7.84(\mathrm{~m}, 2 \mathrm{H}), 7.82$ (dd, J=7.5, $1.3 \mathrm{~Hz}$, 1H), $7.72(\mathrm{~d}, J=9.0 \mathrm{~Hz}, 1 \mathrm{H}), 7.56(\mathrm{~d}, J=8.0 \mathrm{~Hz}, 2 \mathrm{H}), 7.28(\mathrm{t}, J=7.8 \mathrm{~Hz}, 1 \mathrm{H}), 7.18$ $(\mathrm{d}, J=8.0 \mathrm{~Hz}, 2 \mathrm{H}), 6.95(\mathrm{~d}, J=8.8 \mathrm{~Hz}, 2 \mathrm{H}), 6.69(\mathrm{~d}, J=8.8 \mathrm{~Hz}, 2 \mathrm{H}), 6.19$ (q, $J=7.0$ $\mathrm{Hz}, 1 \mathrm{H}), 3.77$ (s, 3H), 2.41 (s, 3H), 1.33 (d, $J=7.0 \mathrm{~Hz}, 3 \mathrm{H}) .{ }^{13} \mathrm{C}$ NMR (126 MHz, Chloroform-d) $\delta 157.21,146.10,143.84,133.85,133.53,133.41,133.09,132.78$, $130.15,129.35,128.38,128.18,127.77,126.97,126.22,125.20,125.09,120.01$, 118.93, 112.25, 54.15, 20.68, 14.83. HRMS(ESI) m/z: calculated for $\left[\mathrm{C}_{27} \mathrm{H}_{23} \mathrm{BrO}_{4} \mathrm{~S}+\right.$ $\mathrm{H}]^{+}$523.0579, found 523.0576. Isotopic MS, calculated 525.0558, found 525.0559. HPLC data (Chiralpak IA column, hexane: isopropanol = 98: 2, $1.0 \mathrm{~mL} / \mathrm{min}$ ), $\operatorname{tr}=28.9$ $\min$ (major), $\operatorname{tr}=25.8 \min$ (minor), ee $=94 \%$.<smiles>C/C=C(\c1ccc(C(F)(F)F)cc1)c1c(O)ccc2cccc(Br)c12</smiles>

Following the general procedure D, 3g was obtained as white solid without Ts protection (17.0 mg, 42\% yield). ${ }^{1} \mathrm{H}$ NMR (500 MHz, Chloroform-d) $\delta 7.78$ (d, $J=8.9$ Hz, 1H), 7.72 (d, $J=8.0 \mathrm{~Hz}, 1 \mathrm{H}), 7.63$ (d, $J=7.5 \mathrm{~Hz}, 1 \mathrm{H}), 7.43$ (d, $J=8.2 \mathrm{~Hz}, 2 \mathrm{H})$, $7.26(\mathrm{dd}, J=10.9,8.6 \mathrm{~Hz}, 3 \mathrm{H}), 7.07$ (t, $J=7.8 \mathrm{~Hz}, 1 \mathrm{H}), 6.80(\mathrm{q}, J=6.9 \mathrm{~Hz}, 1 \mathrm{H}), 5.76$ 
(s, 1H), 1.65 (d, $J=6.9 \mathrm{~Hz}, 3 \mathrm{H}) .{ }^{13} \mathrm{C}$ NMR (126 MHz, Chloroform- $d$ ) $\delta 150.81,142.68$, $134.27,133.12,130.86,130.47,130.26,129.67,128.35,128.22,128.09$, 125.39, 124.48 (q, $J=3.9 \mathrm{~Hz}), 122.99,117.20,116.80,115.24,15.11 .{ }^{19} \mathrm{~F}$ NMR (470 MHz, Chloroform-d) $\delta-62.45$. HRMS(ESI) m/z: calculated for $\left[\mathrm{C}_{20} \mathrm{H}_{14} \mathrm{BrF}_{3} \mathrm{O}+\mathrm{H}\right]^{+}$407.0258, found 407.0253. HPLC data (Chiralpak AD column, hexane: isopropanol = 98: 2, 1.0 $\mathrm{mL} / \mathrm{min}$ ), $\operatorname{tr}=18.6 \mathrm{~min}$ (major), $\operatorname{tr}=12.2 \mathrm{~min}$ (minor), ee $=92 \%$.<smiles>C/C=C(\c1cccc(C)c1)c1c(OC)ccc2cccc(Br)c12</smiles>

Following the general procedure D, $\mathbf{3 h}$ was obtained as colorless oil (44.3 $\mathrm{mg}, 88 \%$ yield). ${ }^{1} \mathrm{H}$ NMR $(500 \mathrm{MHz}$, Chloroform- $d$ ) $\delta 7.89(\mathrm{t}, J=9.1 \mathrm{~Hz}, 2 \mathrm{H}), 7.85$ (d, $J=7.4$ $\mathrm{Hz}, 1 \mathrm{H}), 7.75$ (d, $J=8.9 \mathrm{~Hz}, 1 \mathrm{H}), 7.56(\mathrm{~d}, J=7.9 \mathrm{~Hz}, 2 \mathrm{H}), 7.30$ (t, $J=7.8 \mathrm{~Hz}, 1 \mathrm{H})$, $7.20(\mathrm{~d}, J=7.9 \mathrm{~Hz}, 2 \mathrm{H}), 7.05$ (t, $J=7.6 \mathrm{~Hz}, 1 \mathrm{H}), 6.97$ (d, $J=7.6 \mathrm{~Hz}, 1 \mathrm{H}), 6.86(\mathrm{~s}, 1 \mathrm{H})$, $6.82(\mathrm{~d}, J=7.8 \mathrm{~Hz}, 1 \mathrm{H}), 6.31$ (q, $J=7.0 \mathrm{~Hz}, 1 \mathrm{H}), 2.43$ (s, 3H), 2.23 (s, 3H), 1.35 (d, $J$ $=6.9 \mathrm{~Hz}, 3 \mathrm{H}) .{ }^{13} \mathrm{C}$ NMR (126 MHz, Chloroform- $d$ ) $\delta 146.13,143.77,140.37,136.12$, $134.28,133.86,133.04,132.77,130.21,129.38,128.37,128.21,127.72,127.00$, 126.80, 126.75, 126.14, 125.76, 125.18, 122.63, 120.01, 118.95, 20.68, 20.55, 14.87 . HRMS(ESI) $\mathrm{m} / \mathrm{z}$ : calculated for $\left[\mathrm{C}_{27} \mathrm{H}_{23} \mathrm{BrO}_{3} \mathrm{~S}+\mathrm{Na}\right]^{+}$529.0449, found 529.0453. Isotopic MS, calculated 531.0429, found 531.0431. HPLC data (Chiralpak IA column, hexane: isopropanol $=98: 2,1.0 \mathrm{~mL} / \mathrm{min}$ ), $\operatorname{tr}=15.2 \mathrm{~min}$ (major), $\operatorname{tr}=16.5 \mathrm{~min}$ (minor), ee $=95 \%$.<smiles>C/C=C(\c1cccc(OC)c1)c1c(OC)ccc2cccc(Br)c12</smiles>

Following the general procedure D, 3i was obtained as white solid ( $45.6 \mathrm{mg}, 87 \%$ yield). ${ }^{1} \mathrm{H}$ NMR (500 MHz, Chloroform- $d$ ) $\delta 7.98-7.85$ (m, 2H), 7.83 (dd, $J=7.5,1.4 \mathrm{~Hz}$, 1H), $7.73(\mathrm{~d}, J=9.0 \mathrm{~Hz}, 1 \mathrm{H}), 7.54(\mathrm{~d}, J=8.3 \mathrm{~Hz}, 2 \mathrm{H}), 7.29$ (t, $J=7.8 \mathrm{~Hz}, 1 \mathrm{H}), 7.18$ 
(d, $J=8.1 \mathrm{~Hz}, 2 \mathrm{H}), 7.08$ (t, $J=8.0 \mathrm{~Hz}, 1 \mathrm{H}), 6.71(\mathrm{dd}, J=8.1,2.6 \mathrm{~Hz}, 1 \mathrm{H}), 6.66$ (dd, $J$ $=7.7,1.7 \mathrm{~Hz}, 1 \mathrm{H}), 6.55(\mathrm{t}, J=2.1 \mathrm{~Hz}, 1 \mathrm{H}), 6.34(\mathrm{q}, J=7.0 \mathrm{~Hz}, 1 \mathrm{H}), 3.69(\mathrm{~s}, 3 \mathrm{H}), 2.41$ (s, 3H), 1.35 (d, $J=7.0 \mathrm{~Hz}, 3 \mathrm{H}) .{ }^{13} \mathrm{C}$ NMR (126 MHz, Chloroform-d) $\delta$ 158.21, 146.15, $143.85,141.89$, 133.96, 133.89, 133.04, 132.65, 130.13, 129.48, 128.39, 128.19, $127.74,127.37,127.29,126.96,125.21,119.92,118.91,118.00,111.06,110.53,54.00$, 20.67, 14.93. HRMS(ESI) m/z: calculated for $\left[\mathrm{C}_{27} \mathrm{H}_{23} \mathrm{BrO}_{4} \mathrm{~S}+\mathrm{H}\right]^{+}$523.0579, found 523.0571. Isotopic MS, calculated 525.0558, found 525.0558. HPLC data (Chiralpak OD column, hexane: isopropanol =98: $2,1.0 \mathrm{~mL} / \mathrm{min}$ ), $\operatorname{tr}=21.1 \mathrm{~min}$ (major), $\operatorname{tr}=17.6$ $\min ($ minor $)$, ee $=92 \%$.<smiles>C/C=C(\c1cccc(Cl)c1)c1c(OC)ccc2cccc(Br)c12</smiles>

Following the general procedure D, 3j was obtained as colorless oil $(38.2 \mathrm{mg}, 73 \%$ yield). ${ }^{1} \mathrm{H}$ NMR (500 MHz, Chloroform- $d$ ) $\delta 7.91$ (d, $\left.J=9.0 \mathrm{~Hz}, 1 \mathrm{H}\right), 7.88$ (dd, $J=8.2$, $1.3 \mathrm{~Hz}, 1 \mathrm{H}), 7.83(\mathrm{dd}, J=7.5,1.3 \mathrm{~Hz}, 1 \mathrm{H}), 7.74(\mathrm{~d}, J=8.9 \mathrm{~Hz}, 1 \mathrm{H}), 7.55$ (d, $J=7.9$ $\mathrm{Hz}, 2 \mathrm{H}), 7.30(\mathrm{t}, J=7.8 \mathrm{~Hz}, 1 \mathrm{H}), 7.21(\mathrm{~d}, J=8.0 \mathrm{~Hz}, 2 \mathrm{H}), 7.10(\mathrm{dt}, J=8.1,1.5 \mathrm{~Hz}$, $1 \mathrm{H}), 7.06(\mathrm{t}, J=7.8 \mathrm{~Hz}, 1 \mathrm{H}), 6.93(\mathrm{t}, J=2.0 \mathrm{~Hz}, 1 \mathrm{H}), 6.87(\mathrm{dt}, J=7.7,1.5 \mathrm{~Hz}, 1 \mathrm{H})$, $6.33(\mathrm{q}, J=7.0 \mathrm{~Hz}, 1 \mathrm{H}), 2.43(\mathrm{~s}, 3 \mathrm{H}), 1.37$ (d, $J=7.0 \mathrm{~Hz}, 3 \mathrm{H}) .{ }^{113} \mathrm{C} \mathrm{NMR}(126 \mathrm{MHz}$, Chloroform- $d$ ) $\delta 146.25,144.15,142.25,133.97,133.19,133.09,132.86,132.55$, $130.05,129.79,128.52,128.41,128.30,128.13,126.80,126.70,125.36,125.26$, $124.73,123.73,120.12,118.69,20.74,14.94$. HRMS(ESI) $\mathrm{m} / \mathrm{z}$ : calculated for $\left[\mathrm{C}_{26} \mathrm{H}_{20} \mathrm{BrClO} 3 \mathrm{~S}+\mathrm{H}\right]^{+}$527.0083, found 527.0085. HPLC data (Chiralpak ID column, hexane: isopropanol = 98:2, $1.0 \mathrm{~mL} / \mathrm{min}$ ), $\operatorname{tr}=18.9 \mathrm{~min}$ (major), $\operatorname{tr}=21.4 \mathrm{~min}$ (minor), ee $>99 \%$. 


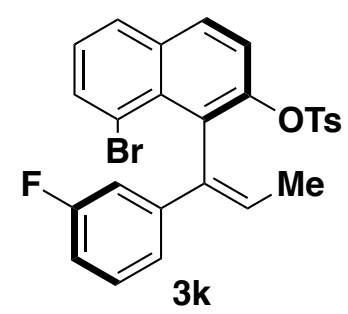

Following the general procedure D, 3k was obtained as colorless oil $(29.4 \mathrm{mg}, 58 \%$ yield). ${ }^{1} \mathrm{H}$ NMR (500 MHz, Chloroform- $d$ ) $\delta 7.91$ (d, $\left.J=9.0 \mathrm{~Hz}, 1 \mathrm{H}\right), 7.88$ (dd, $J=8.2$, $1.3 \mathrm{~Hz}, 1 \mathrm{H}), 7.83(\mathrm{dd}, J=7.5,1.3 \mathrm{~Hz}, 1 \mathrm{H}), 7.74(\mathrm{~d}, J=9.0 \mathrm{~Hz}, 1 \mathrm{H}), 7.55(\mathrm{~d}, J=8.2$ Hz, 2H), 7.30 (t, $J=7.8 \mathrm{~Hz}, 1 \mathrm{H}), 7.19$ (d, $J=8.1 \mathrm{~Hz}, 2 \mathrm{H}), 7.10$ (td, $J=8.1,6.2 \mathrm{~Hz}$, 1H), $6.86-6.75(\mathrm{~m}, 2 \mathrm{H}), 6.62(\mathrm{dt}, J=11.1,2.2 \mathrm{~Hz}, 1 \mathrm{H}), 6.35$ (q, $J=7.0 \mathrm{~Hz}, 1 \mathrm{H}), 2.42$ (s, 3H), $1.38(\mathrm{~d}, J=7.0 \mathrm{~Hz}, 3 \mathrm{H}) .{ }^{13} \mathrm{C}$ NMR $(126 \mathrm{MHz}$, Chloroform-d) $\delta 160.78,146.25$, $145.08,144.09,142.76,133.96,133.28,133.10,132.61,130.04,129.74,128.45$, $128.25(\mathrm{~d}, J=3.2 \mathrm{~Hz}), 128.14,126.85,126.79,125.33,120.93$ (d, $J=2.6 \mathrm{~Hz}), 120.02$, $118.73,112.00(\mathrm{~d}, J=21.5 \mathrm{~Hz}), 111.65(\mathrm{~d}, J=22.4 \mathrm{~Hz}), 20.65,14.95 .{ }^{19} \mathrm{~F}$ NMR $(470$ MHz, Chloroform- $d$ ) $\delta-114.16$ - -114.26 (m). HRMS(ESI) m/z: calculated for $\left[\mathrm{C}_{26} \mathrm{H}_{20} \mathrm{BrFO}_{3} \mathrm{~S}+\mathrm{Na}\right]^{+}$533.0198, found 533.0191. Isotopic MS, calculated 535.0178, found 535.0168. HPLC data (Chiralpak ID column, hexane: isopropanol = 98: 2, 1.0 $\mathrm{mL} / \mathrm{min}$ ), $\operatorname{tr}=22.1 \mathrm{~min}$ (major), $\operatorname{tr}=18.6 \mathrm{~min}$ (minor), ee $=96 \%$.

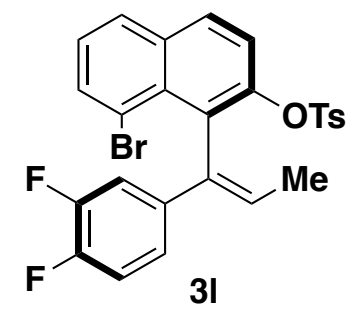

Following the general procedure D, 31 was obtained as colorless oil (36.1 mg, 68\% yield). ${ }^{1} \mathrm{H}$ NMR (500 MHz, Chloroform- $d$ ) $\delta 7.92$ (d, $\left.J=9.0 \mathrm{~Hz}, 1 \mathrm{H}\right), 7.88$ (dd, $J=8.2$, $1.4 \mathrm{~Hz}, 1 \mathrm{H}), 7.83(\mathrm{dd}, J=7.4,1.3 \mathrm{~Hz}, 1 \mathrm{H}), 7.73$ (d, $J=9.0 \mathrm{~Hz}, 1 \mathrm{H}), 7.57$ (d, $J=8.4$ $\mathrm{Hz}, 2 \mathrm{H}), 7.30$ (t, $J=7.8 \mathrm{~Hz}, 1 \mathrm{H}), 7.21(\mathrm{~d}, J=8.1 \mathrm{~Hz}, 2 \mathrm{H}), 6.91(\mathrm{dt}, J=10.3,8.7 \mathrm{~Hz}$, $1 \mathrm{H}), 6.78-6.65(\mathrm{~m}, 2 \mathrm{H}), 6.28(\mathrm{q}, J=7.0 \mathrm{~Hz}, 1 \mathrm{H}), 2.43(\mathrm{~s}, 3 \mathrm{H}), 1.37$ (d, $J=7.0 \mathrm{~Hz}$, 3H). ${ }^{13} \mathrm{C}$ NMR (126 MHz, Chloroform- $d$ ) $\delta$ 146.28, 144.29, 137.69 (d, $J=5.5 \mathrm{~Hz}$ ), $134.03,133.12$, 132.62, 132.47, 129.93, 129.89, 128.48, 128.32, 128.10, 128.09, 126.81, 126.51, 125.43, 121.10 (d, $J=3.2 \mathrm{~Hz}), 121.05$ (d, $J=3.3 \mathrm{~Hz}), 120.00,118.57$, 
$115.45(\mathrm{~d}, J=17.2 \mathrm{~Hz}), 113.58(\mathrm{~d}, J=18.2 \mathrm{~Hz}), 20.63,14.95 .{ }^{19} \mathrm{~F}$ NMR (470 MHz, Chloroform- $d$ ) $\delta$-138.69 (ddd, $J=20.9,12.1,8.3 \mathrm{~Hz}),-141.29(\mathrm{dtd}, J=17.9,10.5,9.1$, $5.0 \mathrm{~Hz}$ ). HRMS(ESI) $\mathrm{m} / \mathrm{z}$ : calculated for $\left[\mathrm{C}_{26} \mathrm{H}_{19} \mathrm{BrF}_{2} \mathrm{O}_{3} \mathrm{~S}+\mathrm{Na}\right]^{+}$551.0104, found 551.0108. Isotopic MS, calculated 553.0084, found 553.0090. HPLC data (Chiralpak IA column, hexane: isopropanol =98: 2, $1.0 \mathrm{~mL} / \mathrm{min}), \operatorname{tr}=17.7 \mathrm{~min}$ (major), $\operatorname{tr}=16.2$ $\min$ (minor), ee $=92 \%$.

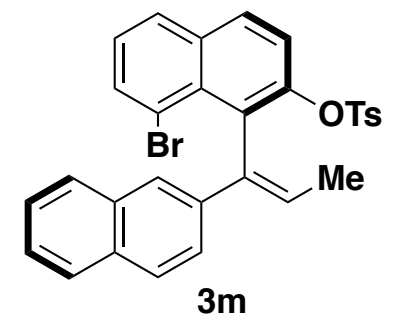

Following the general procedure D, 3m was obtained as white solid (37.2 mg, 69\% yield). ${ }^{1} \mathrm{H}$ NMR (500 MHz, Chloroform- $d$ ) $\delta 7.94$ (d, $\left.J=9.0 \mathrm{~Hz}, 1 \mathrm{H}\right), 7.90$ (dd, $J=8.2$, $1.3 \mathrm{~Hz}, 1 \mathrm{H}), 7.85-7.79(\mathrm{~m}, 2 \mathrm{H}), 7.78-7.73(\mathrm{~m}, 1 \mathrm{H}), 7.66(\mathrm{~d}, J=8.7 \mathrm{~Hz}, 1 \mathrm{H}), 7.53-$ $7.46(\mathrm{~m}, 1 \mathrm{H}), 7.44(\mathrm{dd}, J=8.8,1.9 \mathrm{~Hz}, 1 \mathrm{H}), 7.41-7.33(\mathrm{~m}, 4 \mathrm{H}), 7.29(\mathrm{t}, J=7.8 \mathrm{~Hz}$, 1H), $7.08(\mathrm{~d}, J=1.9 \mathrm{~Hz}, 1 \mathrm{H}), 6.88(\mathrm{~d}, J=8.0 \mathrm{~Hz}, 2 \mathrm{H}), 6.49$ (q, $J=7.0 \mathrm{~Hz}, 1 \mathrm{H}), 2.22$ (s, 3H), 1.43 (d, J=7.0 Hz, 3H). ${ }^{13} \mathrm{C}$ NMR (126 MHz, Chloroform- $d$ ) $\delta 146.29,143.76$, 137.89 , 134.15, 133.91, 133.10, 132.40, 132.36, 131.29, 130.22, 129.58, 128.23, $128.21,127.54,127.25,127.22,126.77,126.38,126.31,125.27,124.72,124.37$, 124.28, 123.32, 120.03, 118.96, 20.51, 15.06. HRMS(ESI) m/z: calculated for $\left[\mathrm{C}_{30} \mathrm{H}_{23} \mathrm{BrO}_{3} \mathrm{~S}+\mathrm{Na}\right]^{+}$565.0449, found 565.0445. Isotopic MS, calculated 567.0429, found 567.0428. HPLC data (Chiralpak IA column, hexane: isopropanol = 98: 2, 1.0 $\mathrm{mL} / \mathrm{min}$ ), $\operatorname{tr}=25.5 \min$ (major), $\operatorname{tr}=22.7 \mathrm{~min}$ (minor), ee $=96 \%$.<smiles>C/C=C(\c1ccc2c(c1)OCO2)c1c(OC)ccc2cccc(Br)c12</smiles>

Following the general procedure D, 3n was obtained as white solid (45.9 mg, 86\% yield). ${ }^{1} \mathrm{H}$ NMR (500 MHz, Chloroform- $d$ ) $\delta 7.93-7.85(\mathrm{~m}, 2 \mathrm{H}), 7.83$ (dd, $J=7.4,1.3$ Hz, 1H), 7.72 (d, $J=9.0 \mathrm{~Hz}, 1 \mathrm{H}), 7.62-7.57$ (m, 2H), 7.29 (t, $J=7.8 \mathrm{~Hz}, 1 \mathrm{H}), 7.21$ 
(d, $J=8.1 \mathrm{~Hz}, 2 \mathrm{H}), 6.59-6.53(\mathrm{~m}, 2 \mathrm{H}), 6.39(\mathrm{dd}, J=8.1,1.8 \mathrm{~Hz}, 1 \mathrm{H}), 6.17$ (q, $J=7.0$ $\mathrm{Hz}, 1 \mathrm{H}), 5.97-5.87(\mathrm{~m}, 2 \mathrm{H}), 2.43(\mathrm{~s}, 3 \mathrm{H}), 1.33$ (d, $J=7.0 \mathrm{~Hz}, 3 \mathrm{H}) .{ }^{13} \mathrm{C}$ NMR $(126$ MHz, Chloroform-d) $\delta 146.44,146.16,145.11,143.93$, 135.17, 133.91, 133.60, 133.09, $132.84,130.06,129.45,128.36,128.21,127.57,126.95,125.70,125.23,120.06$, 119.23, 118.86, 106.66, 105.32, 99.81, 20.66, 14.84. HRMS(ESI) m/z: calculated for $\left[\mathrm{C}_{27} \mathrm{H}_{21} \mathrm{BrO}_{5} \mathrm{~S}+\mathrm{Na}\right]^{+}$559.0191, found 559.0183. Isotopic MS, calculated 561.0170, found 561.0164. HPLC data (Chiralpak AD column, hexane: isopropanol = 95: 5, 1.0 $\mathrm{mL} / \mathrm{min}$ ), $\operatorname{tr}=24.7 \mathrm{~min}$ (major), $\operatorname{tr}=22.5 \min ($ minor), ee $=93 \%$.

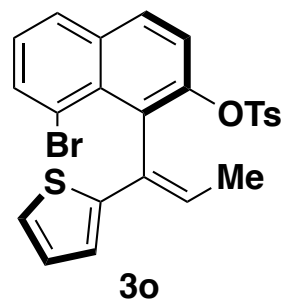

Following the general procedure D, 3o was obtained as white solid (42.0 mg, 84\% yield). ${ }^{1} \mathrm{H}$ NMR (500 MHz, Chloroform-d) $\delta 7.93$ (d, J = 9.0 Hz, 1H), 7.87 (dd, J = 8.1, $1.3 \mathrm{~Hz}, 1 \mathrm{H}), 7.82$ (d, J = 1.7 Hz, 1H), $7.82-7.79$ (m, 1H), 7.59 (d, J = 8.3 Hz, 2H), $7.28(\mathrm{t}, \mathrm{J}=7.9 \mathrm{~Hz}, 1 \mathrm{H}), 7.18(\mathrm{~d}, \mathrm{~J}=8.1 \mathrm{~Hz}, 2 \mathrm{H}), 7.06(\mathrm{~d}, \mathrm{~J}=5.1 \mathrm{~Hz}, 1 \mathrm{H}), 6.74(\mathrm{dd}, \mathrm{J}=$ 5.1, 3.6 Hz, 1H), 6.19 (q, J = 7.0 Hz, 1H), $6.11(\mathrm{~d}, \mathrm{~J}=3.5 \mathrm{~Hz}, 1 \mathrm{H}), 2.41(\mathrm{~s}, 3 \mathrm{H}), 1.38$ $(\mathrm{d}, \mathrm{J}=7.0 \mathrm{~Hz}, 3 \mathrm{H}) .{ }^{13} \mathrm{C}$ NMR (126 MHz, Chloroform- $d$ ) $\delta 146.08,146.05,143.86$, $133.90,133.15,132.59,129.81,129.74,129.13,128.42,128.11,126.91,126.43$, 126.17, 126.04, 125.27, 123.33, 121.75, 120.17, 118.88, 20.71, 14.57. HRMS(ESI) m/z: calculated for $\left[\mathrm{C}_{24} \mathrm{H}_{19} \mathrm{BrO}_{3} \mathrm{~S}_{2}+\mathrm{H}\right]^{+} 499.0037$, found 499.0027. Isotopic MS, calculated 501.0017, found 501.0012. HPLC data (Chiralpak AD column, hexane: isopropanol = 98: $2,1.0 \mathrm{~mL} / \mathrm{min}$ ), $\operatorname{tr}=26.8 \mathrm{~min}$ (major), $\operatorname{tr}=22.3 \mathrm{~min}$ (minor), ee $=92 \%$.

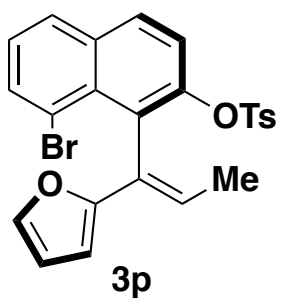

Following the general procedure D, 3p was obtained as orange solid (34.6 mg, 72\% yield). ${ }^{1} \mathrm{H}$ NMR (500 MHz, Chloroform- $d$ ) $\delta 7.91(\mathrm{~d}, J=9.0 \mathrm{~Hz}, 1 \mathrm{H}), 7.85$ (dd, $J=8.2$, 
$1.3 \mathrm{~Hz}, 1 \mathrm{H}), 7.80(\mathrm{dd}, J=7.4,1.3 \mathrm{~Hz}, 1 \mathrm{H}), 7.77$ (d, $J=9.0 \mathrm{~Hz}, 1 \mathrm{H}), 7.59$ (d, $J=8.4$ $\mathrm{Hz}, 2 \mathrm{H}), 7.31-7.27(\mathrm{~m}, 1 \mathrm{H}), 7.27-7.24(\mathrm{~m}, 1 \mathrm{H}), 7.19$ (d, $J=8.0 \mathrm{~Hz}, 2 \mathrm{H}), 6.30$ (q, $J$ $=7.2 \mathrm{~Hz}, 1 \mathrm{H}), 6.20(\mathrm{dd}, J=3.3,1.8 \mathrm{~Hz}, 1 \mathrm{H}), 5.38(\mathrm{~d}, J=3.3 \mathrm{~Hz}, 1 \mathrm{H}), 2.41(\mathrm{~s}, 3 \mathrm{H})$, $1.40(\mathrm{~d}, J=7.3 \mathrm{~Hz}, 3 \mathrm{H}) .{ }^{13} \mathrm{C}$ NMR $(126 \mathrm{MHz}$, Chloroform- $d) \delta 154.17,146.30,143.84$, $139.71,133.86,133.21,132.68,129.99,129.85,128.39,128.06,126.93,125.32$, 125.22, 124.88, 124.84, 120.27, 119.01, 110.23, 105.49, 20.69, 14.12. HRMS(ESI) m/z: calculated for $\left[\mathrm{C}_{24} \mathrm{H}_{19} \mathrm{BrO}_{4} \mathrm{~S}+\mathrm{H}\right]^{+}$483.0266, found 483.0263. Isotopic MS, calculated 485.0245, found 485.0249. HPLC data (Chiralpak AD column, hexane: isopropanol = 98: $2,1.0 \mathrm{~mL} / \mathrm{min}$ ), $\operatorname{tr}=25.8 \mathrm{~min}$ (major), $\operatorname{tr}=22.1 \mathrm{~min}($ minor), $\mathrm{ee}=92 \%$.

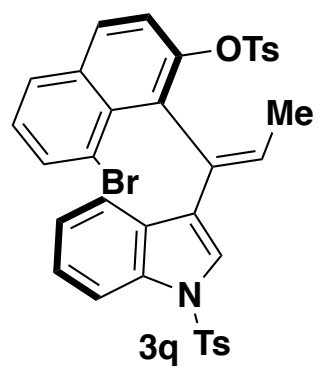

Following the general procedure D, 3q was obtained as white solid $(51.2 \mathrm{mg}, 75 \%$ yield). ${ }^{1} \mathrm{H}$ NMR (500 MHz, Chloroform- $d$ ) $\delta 7.95$ (dd, $\left.J=8.8,2.4 \mathrm{~Hz}, 2 \mathrm{H}\right), 7.90(\mathrm{~d}, J$ $=8.1 \mathrm{~Hz}, 1 \mathrm{H}), 7.84(\mathrm{dd}, J=9.1,2.7 \mathrm{~Hz}, 1 \mathrm{H}), 7.78(\mathrm{dd}, J=7.2,1.3 \mathrm{~Hz}, 1 \mathrm{H}), 7.66(\mathrm{~d}, J$ $=8.1 \mathrm{~Hz}, 1 \mathrm{H}), 7.53(\mathrm{~d}, J=8.7 \mathrm{~Hz}, 2 \mathrm{H}), 7.34(\mathrm{dd}, J=8.6,2.2 \mathrm{~Hz}, 2 \mathrm{H}), 7.30(\mathrm{td}, J=7.8$, $3.7 \mathrm{~Hz}, 2 \mathrm{H}), 7.20$ (t, $J=7.6 \mathrm{~Hz}, 1 \mathrm{H}), 7.10$ (d, $J=8.5 \mathrm{~Hz}, 2 \mathrm{H}), 6.84$ (d, $J=8.0 \mathrm{~Hz}, 2 \mathrm{H})$, $6.56(\mathrm{~s}, 1 \mathrm{H}), 6.44(\mathrm{q}, J=6.9 \mathrm{~Hz}, 1 \mathrm{H}), 2.26(\mathrm{~s}, 3 \mathrm{H}), 2.25-2.22(\mathrm{~s}, 3 \mathrm{H}), 1.45(\mathrm{~d}, J=7.1$ $\mathrm{Hz}, 3 \mathrm{H}) .{ }^{13} \mathrm{C}$ NMR (126 MHz, Chloroform- $d$ ) $\delta$ 146.15, 143.97, 143.63, 134.68, 133.83, $133.31,132.22,129.89,129.59,128.68,128.39,128.23,127.92,127.78,127.62$, $126.71,126.56,125.76,125.23,124.52,123.99,123.42,122.46,120.42,120.25$, 119.00, 112.85, 98.94, 20.57, 20.52, 14.77. HRMS(ESI) m/z: calculated for $\left[\mathrm{C}_{35} \mathrm{H}_{28} \mathrm{BrNO}_{5} \mathrm{~S}_{2}+\mathrm{H}\right]^{+}$686.0671, found 686.0665. Isotopic MS, calculated 688.0650, found 688.0651. HPLC data (Chiralpak IA column, hexane: isopropanol = 95: 5, 1.0 $\mathrm{mL} / \mathrm{min}), \operatorname{tr}=24.0 \mathrm{~min}$ (major), $\operatorname{tr}=28.1 \mathrm{~min}(\operatorname{minor})$, ee $=92 \%$. 


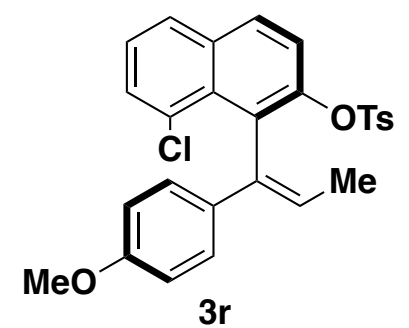

Following the general procedure D, 3r was obtained as colorless oil $(29.5 \mathrm{mg}, 62 \%$ yield). ${ }^{1} \mathrm{H}$ NMR (500 MHz, Chloroform- $d$ ) $\delta 7.89(\mathrm{~d}, J=9.0 \mathrm{~Hz}, 1 \mathrm{H}), 7.81$ (dd, $J=8.2$, $1.3 \mathrm{~Hz}, 1 \mathrm{H}), 7.73$ (d, $J=9.0 \mathrm{~Hz}, 1 \mathrm{H}), 7.56(\mathrm{~d}, J=8.3 \mathrm{~Hz}, 2 \mathrm{H}), 7.53$ (dd, $J=7.5,1.3$ $\mathrm{Hz}, 1 \mathrm{H}), 7.37$ (t, $J=7.8 \mathrm{~Hz}, 1 \mathrm{H}), 7.17(\mathrm{~d}, J=8.1 \mathrm{~Hz}, 2 \mathrm{H}), 6.95(\mathrm{~d}, J=8.9 \mathrm{~Hz}, 2 \mathrm{H})$, $6.69(\mathrm{~d}, J=8.8 \mathrm{~Hz}, 2 \mathrm{H}), 6.16(\mathrm{q}, J=7.0 \mathrm{~Hz}, 1 \mathrm{H}), 3.76(\mathrm{~s}, 3 \mathrm{H}), 2.41(\mathrm{~s}, 3 \mathrm{H}), 1.37$ (d, $J$ $=7.0 \mathrm{~Hz}, 3 \mathrm{H}) .{ }^{13} \mathrm{C}$ NMR $(126 \mathrm{MHz}$, Chloroform- $d$ ) $\delta 157.22,146.01,143.83,134.13$, $133.28,133.09,132.80,130.53,129.36,129.36,129.21,128.38,127.32,126.96$, 126.93, 126.07, 124.81, 123.95, 120.07, 112.29, 54.15, 20.65, 14.66. HRMS(ESI) m/z: calculated for $\left[\mathrm{C}_{27} \mathrm{H}_{23} \mathrm{ClO}_{4} \mathrm{~S}+\mathrm{H}\right]^{+}$479.1084, found 479.1088. HPLC data (Chiralpak IA column, hexane: isopropanol $=98: 2,1.0 \mathrm{~mL} / \mathrm{min}$ ), $\operatorname{tr}=28.1 \mathrm{~min}$ (major), $\operatorname{tr}=25.5$ $\min ($ minor), ee $=94 \%$.<smiles>C/C=c1/c2ccc(OC)cc2c2cccc3ccc(O)c1c32</smiles>

Following the general procedure D, 3s was obtained as white solid ( $31.8 \mathrm{mg}, 56 \%$ yield). ${ }^{1} \mathrm{H}$ NMR (500 MHz, Chloroform- $d$ ) $\delta 7.89$ (d, $\left.J=9.1 \mathrm{~Hz}, 1 \mathrm{H}\right), 7.81(\mathrm{dd}, J=8.2,1.3$ $\mathrm{Hz}, 1 \mathrm{H}), 7.73(\mathrm{~d}, J=9.0 \mathrm{~Hz}, 1 \mathrm{H}), 7.56(\mathrm{~d}, J=8.3 \mathrm{~Hz}, 2 \mathrm{H}), 7.53(\mathrm{dd}, J=7.5,1.3 \mathrm{~Hz}$, 1H), $7.37(\mathrm{t}, J=7.8 \mathrm{~Hz}, 1 \mathrm{H}), 7.17(\mathrm{~d}, J=8.1 \mathrm{~Hz}, 2 \mathrm{H}), 6.95(\mathrm{~d}, J=8.8 \mathrm{~Hz}, 2 \mathrm{H}), 6.69$ $(\mathrm{d}, J=8.8 \mathrm{~Hz}, 2 \mathrm{H}), 6.16(\mathrm{q}, J=7.0 \mathrm{~Hz}, 1 \mathrm{H}), 3.76(\mathrm{~s}, 3 \mathrm{H}), 2.41$ (s, 3H), 1.37 (d, $J=7.0$ $\mathrm{Hz}, 3 \mathrm{H}) .{ }^{13} \mathrm{C}$ NMR (126 MHz, Chloroform- $d$ ) $\delta 157.21,146.00,143.87,134.12,133.28$, $133.10,132.75,130.53,129.39,129.34,129.24,128.41,127.35,126.98,126.95$, 126.08, 124.84, 123.99, 120.08, 112.29, 54.16, 20.69, 14.69. HRMS(ESI) m/z: calculated for $\left[\mathrm{C}_{27} \mathrm{H}_{23} \mathrm{IO}_{4} \mathrm{~S}+\mathrm{H}\right]^{+}$571.0440, found 571.0436. HPLC data (Chiralpak ID 
column, hexane: isopropanol $=98: 2,1.0 \mathrm{~mL} / \mathrm{min}$ ), $\operatorname{tr}=49.4 \mathrm{~min}$ (major), $\operatorname{tr}=43.8 \mathrm{~min}$ (minor), ee $=94 \%$.<smiles>C/C=C(\c1ccc(OC)cc1)c1c(OC)ccc2ccccc12</smiles>

Following the general procedure $\mathbf{D}, 3 \mathbf{3 t}$ was obtained as white solid ( $32.0 \mathrm{mg}, 72 \%$ yield). ${ }^{1} \mathrm{H}$ NMR (500 MHz, Chloroform- $d$ ) $\delta 7.87(\mathrm{~d}, J=8.1 \mathrm{~Hz}, 1 \mathrm{H}), 7.85(\mathrm{~d}, J=9.1 \mathrm{~Hz}, 1 \mathrm{H})$, $7.74(\mathrm{~d}, J=8.5 \mathrm{~Hz}, 1 \mathrm{H}), 7.62(\mathrm{~d}, J=8.2 \mathrm{~Hz}, 2 \mathrm{H}), 7.58$ (d, $J=9.0 \mathrm{~Hz}, 1 \mathrm{H}), 7.47$ (ddd, $J=8.1,6.8,1.3 \mathrm{~Hz}, 1 \mathrm{H}), 7.41(\mathrm{ddd}, J=8.3,6.8,1.4 \mathrm{~Hz}, 1 \mathrm{H}), 7.16(\mathrm{~d}, J=8.0 \mathrm{~Hz}, 2 \mathrm{H})$, $6.93(\mathrm{~d}, J=8.8 \mathrm{~Hz}, 2 \mathrm{H}), 6.67(\mathrm{~d}, J=8.8 \mathrm{~Hz}, 2 \mathrm{H}), 6.36$ (q, $J=6.9 \mathrm{~Hz}, 1 \mathrm{H}), 3.75$ (s, 3H), $2.39(\mathrm{~s}, 3 \mathrm{H}), 1.39$ (d, $J=7.0 \mathrm{~Hz}, 3 \mathrm{H}) .{ }^{13} \mathrm{C}$ NMR (126 MHz, Chloroform- $d$ ) $\delta$ 157.49, $144.11,143.84,132.72,132.59,132.55,131.78,130.94,128.90,128.45,128.13$, $128.11,127.15,127.12,126.06,125.96,125.41,125.06,119.69,112.50,54.19,20.66$, 14.74. HRMS(ESI) $\mathrm{m} / \mathrm{z}$ : calculated for $\left[\mathrm{C}_{27} \mathrm{H}_{24} \mathrm{O}_{4} \mathrm{~S}+\mathrm{H}\right]^{+} 445.1474$, found 445.1470 . HPLC data (Chiralpak AD column, hexane: isopropanol = 97: 3, $1.0 \mathrm{~mL} / \mathrm{min}$ ), $\operatorname{tr}=27.7$ $\min ($ major $), \operatorname{tr}=40.1 \mathrm{~min}$ (minor), ee $=83 \%$.<smiles>C/C=C(\c1ccc(OC)cc1)c1c(OC)ccc2ccc(C)cc12</smiles>

Following the general procedure D, 3u was obtained as white solid (37.2 $\mathrm{mg}, 81 \%$ yield). ${ }^{1} \mathrm{H}$ NMR (500 MHz, Chloroform- $d$ ) $\delta 7.78(\mathrm{dd}, J=10.5,8.7 \mathrm{~Hz}, 2 \mathrm{H}), 7.61(\mathrm{~d}, J$ $=8.4 \mathrm{~Hz}, 2 \mathrm{H}), 7.49(\mathrm{~d}, J=9.1 \mathrm{~Hz}, 2 \mathrm{H}), 7.30(\mathrm{dd}, J=8.4,1.7 \mathrm{~Hz}, 1 \mathrm{H}), 7.16(\mathrm{~d}, J=8.0$ $\mathrm{Hz}, 2 \mathrm{H}), 6.95$ (d, $J=8.8 \mathrm{~Hz}, 2 \mathrm{H}), 6.68$ (d, $J=8.8 \mathrm{~Hz}, 2 \mathrm{H}), 6.35$ (q, $J=7.0 \mathrm{~Hz}, 1 \mathrm{H})$, $3.76(\mathrm{~s}, 3 \mathrm{H}), 2.39$ (s, 6H), 1.39 (d, J=7.0 Hz, 3H). ${ }^{13} \mathrm{C}$ NMR (126 MHz, Chloroformd) $\delta 157.47,144.23,143.72,135.92,132.84,132.69,132.64,131.97,129.15,128.40$, $127.77,127.43,127.33,127.09,126.95,125.94,124.87,124.19,118.61,112.50,54.18$, 20.99, 20.64, 14.73. HRMS(ESI) $\mathrm{m} / \mathrm{z}$ : calculated for $\left[\mathrm{C}_{28} \mathrm{H}_{26} \mathrm{O}_{4} \mathrm{~S}+\mathrm{H}\right]^{+} 459.1630$, found 
459.1635. HPLC data (Chiralpak OD column, hexane: isopropanol $=98: 2,1.0$ $\mathrm{mL} / \mathrm{min}$ ), $\operatorname{tr}=18.1 \mathrm{~min}$ (major), $\operatorname{tr}=16.5 \mathrm{~min}$ (minor), ee $\approx 82 \%$.

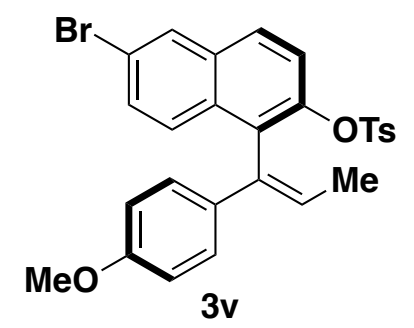

Following the general procedure D, 3v was obtained as colorless oil (38.6 mg, 74\% yield). ${ }^{1} \mathrm{H}$ NMR (500 MHz, Chloroform- $\left.d\right) \delta 8.03(\mathrm{~d}, J=1.9 \mathrm{~Hz}, 1 \mathrm{H}), 7.76(\mathrm{~d}, J=9.0$ $\mathrm{Hz}, 1 \mathrm{H}), 7.66-7.57(\mathrm{~m}, 4 \mathrm{H}), 7.47(\mathrm{dd}, J=9.0,2.0 \mathrm{~Hz}, 1 \mathrm{H}), 7.16(\mathrm{~d}, J=8.1 \mathrm{~Hz}, 2 \mathrm{H})$, $6.90(\mathrm{~d}, J=8.8 \mathrm{~Hz}, 2 \mathrm{H}), 6.67(\mathrm{~d}, J=8.9 \mathrm{~Hz}, 2 \mathrm{H}), 6.36(\mathrm{q}, J=6.9 \mathrm{~Hz}, 1 \mathrm{H}), 3.75(\mathrm{~s}, 3 \mathrm{H})$, 2.39 (s, 3H), 1.39 (d, $J=7.0 \mathrm{~Hz}, 3 \mathrm{H}) .{ }^{13} \mathrm{C}$ NMR (126 MHz, Chloroform- $d$ ) $\delta 157.62$, $144.30,143.98,132.59,132.26,132.18,132.02,130.35,129.44,129.13,128.50$, $128.46,127.27,127.16,127.10,125.90,125.32$, 120.95, 119.30, 112.58, 54.20, 20.66, 14.71. HRMS(ESI) m/z: calculated for $\left[\mathrm{C}_{27} \mathrm{H}_{23} \mathrm{BrO}_{4} \mathrm{~S}+\mathrm{H}\right]^{+}$523.0579, found 523.0574. Isotopic MS, calculated 525.0558, found 525.0558. HPLC data (Chiralpak IA column, hexane: isopropanol $=98: 2,1.0 \mathrm{~mL} / \mathrm{min}), \operatorname{tr}=35.3 \mathrm{~min}$ (major), $\operatorname{tr}=39.1 \mathrm{~min}($ minor $)$, ee $=78 \%$.

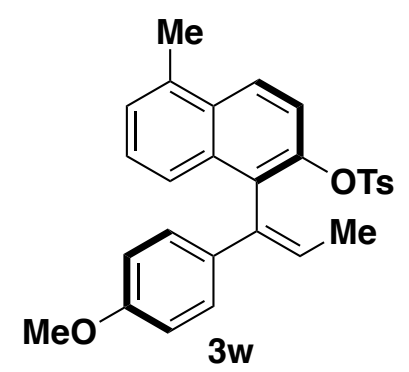

Following the general procedure $\mathbf{D}, \mathbf{3 w}$ was obtained as white solid $(35.6 \mathrm{mg}, 78 \%$ yield). ${ }^{1} \mathrm{H}$ NMR (500 MHz, Chloroform- $d$ ) $\delta 8.02(\mathrm{dd}, J=9.3,0.8 \mathrm{~Hz}, 1 \mathrm{H}), 7.73-7.52$ (m, 4H), 7.29 (d, $J=6.5 \mathrm{~Hz}, 2 \mathrm{H}), 7.15(\mathrm{~d}, J=8.1 \mathrm{~Hz}, 2 \mathrm{H}), 6.93(\mathrm{~d}, J=8.9 \mathrm{~Hz}, 2 \mathrm{H})$, $6.66(\mathrm{~d}, J=8.8 \mathrm{~Hz}, 2 \mathrm{H}), 6.35(\mathrm{q}, J=6.9 \mathrm{~Hz}, 1 \mathrm{H}), 3.74(\mathrm{~s}, 3 \mathrm{H}), 2.72(\mathrm{~s}, 3 \mathrm{H}), 2.38(\mathrm{~s}$, 3H), $1.38(\mathrm{~d}, J=6.9 \mathrm{~Hz}, 3 \mathrm{H}) .{ }^{13} \mathrm{C}$ NMR $(126 \mathrm{MHz}$, Chloroform-d) $\delta$ 157.45, 144.00, $143.79,133.64,132.87,132.76,132.66,132.07,130.13,128.43,128.42,127.10$, $125.94,125.92,125.88,124.90,124.36,123.83,119.19,112.48,54.18,20.65,18.69$, 
14.71. HRMS(ESI) $\mathrm{m} / \mathrm{z}$ : calculated for $\left[\mathrm{C}_{28} \mathrm{H}_{26} \mathrm{O}_{4} \mathrm{~S}+\mathrm{H}\right]^{+} 459.1630$, found 459.1635 . HPLC data (Chiralpak AD column, hexane: isopropanol = 95: 5, $1.0 \mathrm{~mL} / \mathrm{min}$ ), $\operatorname{tr}=18.1$ $\min$ (major), $\operatorname{tr}=22.7 \min ($ minor), $\mathrm{ee}=84 \%$.

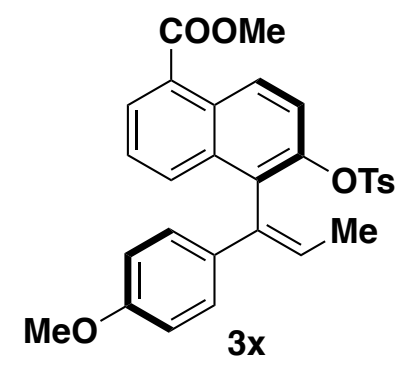

Following the general procedure $\mathbf{D}, \mathbf{3 x}$ was obtained as white solid (37.1 $\mathrm{mg}, 74 \%$ yield). ${ }^{1} \mathrm{H}$ NMR (500 MHz, Chloroform- $d$ ) $\delta 8.93(\mathrm{~d}, J=9.4 \mathrm{~Hz}, 1 \mathrm{H}), 8.14$ (dd, $J=7.2$, $1.3 \mathrm{~Hz}, 1 \mathrm{H}), 7.97(\mathrm{dd}, J=8.5,1.2 \mathrm{~Hz}, 1 \mathrm{H}), 7.67$ (d, $J=9.5 \mathrm{~Hz}, 1 \mathrm{H}), 7.64-7.56(\mathrm{~m}$, 2H), $7.42(\mathrm{dd}, J=8.5,7.2 \mathrm{~Hz}, 1 \mathrm{H}), 7.16(\mathrm{~d}, J=8.1 \mathrm{~Hz}, 2 \mathrm{H}), 6.92(\mathrm{~d}, J=8.8 \mathrm{~Hz}, 2 \mathrm{H})$, $6.67(\mathrm{~d}, J=8.9 \mathrm{~Hz}, 2 \mathrm{H}), 6.41$ (q, $J=6.9 \mathrm{~Hz}, 1 \mathrm{H}), 4.01(\mathrm{~s}, 3 \mathrm{H}), 3.75$ (s, 3H), 2.39 (s, 3H), $1.39(\mathrm{~d}, J=6.9 \mathrm{~Hz}, 3 \mathrm{H}) .{ }^{13} \mathrm{C}$ NMR $(126 \mathrm{MHz}$, Chloroform- $d$ ) $\delta$ 166.87, 157.59, $144.36,143.93,132.64,132.49,132.36,130.43,129.08,128.86,128.50,128.42$, $127.12,126.65,126.16,126.04,125.93,125.30,124.85,121.13,112.57,54.18,51.35$, 20.64, 14.69. HRMS(ESI) $\mathrm{m} / \mathrm{z}$ : calculated for $\left[\mathrm{C}_{29} \mathrm{H}_{26} \mathrm{O}_{6} \mathrm{~S}+\mathrm{H}\right]^{+} 503.1528$ found 503.1531. HPLC data (Chiralpak AD column, hexane: isopropanol $=90: 10,1.0$ $\mathrm{mL} / \mathrm{min}$ ), $\operatorname{tr}=16.0 \min$ (major), $\operatorname{tr}=38.1 \mathrm{~min}$ (minor), ee $=89 \%$.<smiles>C/C=C(\c1ccc(OC)cc1)c1c(OC)cc(Br)c2c(C)cccc12</smiles>

Following the general procedure D, $\mathbf{3 y}$ was obtained as white solid $(38.8 \mathrm{mg}, 72 \%$ yield). ${ }^{1} \mathrm{H}$ NMR (500 MHz, Chloroform-d) $\delta 7.96$ (s, 1H), 7.72 (dd, $J=8.6,1.4 \mathrm{~Hz}$, 1H), $7.61(\mathrm{~d}, J=8.3 \mathrm{~Hz}, 2 \mathrm{H}), 7.33(\mathrm{dt}, J=7.0,1.2 \mathrm{~Hz}, 1 \mathrm{H}), 7.29-7.22(\mathrm{~m}, 1 \mathrm{H}), 7.17$ $(\mathrm{d}, J=8.1 \mathrm{~Hz}, 2 \mathrm{H}), 6.91(\mathrm{~d}, J=8.9 \mathrm{~Hz}, 2 \mathrm{H}), 6.66(\mathrm{~d}, J=8.9 \mathrm{~Hz}, 2 \mathrm{H}), 6.36(\mathrm{q}, J=7.0$ $\mathrm{Hz}, 1 \mathrm{H}), 3.75$ (s, 3H), $3.14(\mathrm{~s}, 3 \mathrm{H}), 2.40(\mathrm{~s}, 3 \mathrm{H}), 1.38$ (d, $J=6.9 \mathrm{~Hz}, 3 \mathrm{H}) .{ }^{13} \mathrm{C}$ NMR (126 MHz, Chloroform-d) $\delta$ 157.61, 144.08, 142.68, 134.84, 134.14, 132.77, 132.45, 
$132.04,130.19,129.05,128.59,128.53,127.12,126.49,126.16,125.88,125.12$, 125.11, 118.69, 112.57, 54.19, 25.38, 20.65, 14.65. HRMS(ESI) m/z: calculated for $\left[\mathrm{C}_{28} \mathrm{H}_{25} \mathrm{BrO}_{4} \mathrm{~S}+\mathrm{H}\right]^{+}$537.0735, found 537.0729. Isotopic MS, calculated 539.0715, found 539.0723. HPLC data (Chiralpak IA column, hexane: isopropanol = 98: 2, 1.0 $\mathrm{mL} / \mathrm{min}), \operatorname{tr}=15.5 \min ($ major $), \operatorname{tr}=17.7 \min (\operatorname{minor})$, ee $=85 \%$.<smiles>C/C=C(\c1ccc(OC)cc1)c1c(O)ccc2ccc3ccccc3c12</smiles>

$3 z$

Following the general procedure $\mathbf{D}, \mathbf{3 z}$ was obtained as white solid without Ts protection (17.7 mg, 52\% yield). ${ }^{1} \mathrm{H}$ NMR $(500 \mathrm{MHz}$, Chloroform- $d$ ) $\delta 9.09(\mathrm{~d}, J=8.7$ $\mathrm{Hz}, 1 \mathrm{H}), 7.87$ (d, $J=8.6 \mathrm{~Hz}, 1 \mathrm{H}), 7.79(\mathrm{dd}, J=7.9,1.6 \mathrm{~Hz}, 1 \mathrm{H}), 7.71(\mathrm{~d}, J=8.7 \mathrm{~Hz}$, 1H), $7.60(\mathrm{~d}, J=8.7 \mathrm{~Hz}, 1 \mathrm{H}), 7.48-7.42(\mathrm{~m}, 1 \mathrm{H}), 7.38(\mathrm{~d}, J=8.6 \mathrm{~Hz}, 1 \mathrm{H}), 7.32$ (ddd, $J=8.6,6.9,1.6 \mathrm{~Hz}, 1 \mathrm{H}), 7.26(\mathrm{~d}, J=8.7 \mathrm{~Hz}, 2 \mathrm{H}), 6.78(\mathrm{q}, J=6.9 \mathrm{~Hz}, 1 \mathrm{H}), 6.73(\mathrm{~d}, J$ $=8.9 \mathrm{~Hz}, 2 \mathrm{H}), 5.93(\mathrm{~s}, 1 \mathrm{H}), 3.71(\mathrm{~s}, 3 \mathrm{H}), 1.67(\mathrm{~d}, J=6.9 \mathrm{~Hz}, 3 \mathrm{H}) \cdot{ }^{13} \mathrm{C} \mathrm{NMR}(126 \mathrm{MHz}$, Chloroform- $d$ ) $\delta 158.46,150.42,137.12,132.47,129.87,129.48,129.35,127.40$, $126.98,126.67,126.35,125.55,125.15,125.11,124.53,124.05,118.67,114.94$, 113.13, 54.18, 14.31. HRMS(NSI) m/z: calculated for $\left[\mathrm{C}_{24} \mathrm{H}_{20} \mathrm{O}_{2}+\mathrm{H}\right]^{+} 341.1542$, found 341.1531. HPLC data (Chiralpak IA column, hexane: isopropanol = 98: 2, 1.0 $\mathrm{mL} / \mathrm{min}), \operatorname{tr}=26.2 \mathrm{~min}$ (major), $\operatorname{tr}=18.1 \mathrm{~min}($ minor $)$, ee $=92 \%$.

General procedure E: Synthesis of axially chiral product based on 2-naphthol<smiles>[R]c1cccc2ccc(O)cc12</smiles><smiles>COc1ccc(/C(=C/N)c2c(O)ccc3ccccc23)cc1</smiles>

3

The reaction was proceeded in the glovebox under argon atmosphere. $[\operatorname{Ir}(\operatorname{cod}) \mathrm{Cl}]_{2}$ (2.1 mg, $0.003 \mathrm{mmol}, 0.03 \mathrm{eq}), \mathbf{L 1}$ (32.4mg, $0.006 \mathrm{mmol}, 0.06 \mathrm{eq})$, TBD (2.1mg, $0.015 \mathrm{mmol}, 0.15 \mathrm{eq}$ ) were added to a 2 dram scintillation vial (vial A) equipped with a magnetic stirring bar. The vial was then charged with toluene $(0.5 \mathrm{~mL})$ and stirred at 
$25{ }^{\circ} \mathrm{C}$ for $30 \mathrm{~min}$, generating an orange solution. To another 2 dram scintillation vial (vial B) was added $1 \mathbf{f}(0.1 \mathrm{mmol}, 1.0 \mathrm{eq}), 2$ (0.15 mmol, $1.5 \mathrm{eq})$, DBU (0.3 mmol, 3.0 eq) and toluene $(0.5 \mathrm{~mL})$. The preformed catalyst solution (vial A) was then transferred to vial $\mathbf{B}$. The mixture was stirred at $35^{\circ} \mathrm{C}$ with stirring 12 hours. Then the residue was purified directly by column chromatography over silica gel (PE: EA $=20: 1$ to $10: 1)$ to afford the desired product.<smiles>C/C=C(\c1ccc(OC)cc1)c1c(OC)ccc2cccc(O)c12</smiles>

Following the general procedure E, 3a' was obtained as white solid with Ts protection (34.9 mg, 76\% yield) $(Z / E=13: 1) .{ }^{1} \mathrm{H}$ NMR (500 MHz, Chloroform- $\left.d\right) \delta 7.86(\mathrm{~d}, J=$ $9.0 \mathrm{~Hz}, 1 \mathrm{H}), 7.75(\mathrm{~d}, J=8.1 \mathrm{~Hz}, 1 \mathrm{H}), 7.63(\mathrm{~d}, J=9.0 \mathrm{~Hz}, 1 \mathrm{H}), 7.56(\mathrm{~d}, J=8.3 \mathrm{~Hz}, 2 \mathrm{H})$, $7.35(\mathrm{t}, J=7.6 \mathrm{~Hz}, 1 \mathrm{H}), 7.25(\mathrm{~d}, J=8.7 \mathrm{~Hz}, 1 \mathrm{H}), 7.16(\mathrm{~d}, J=8.0 \mathrm{~Hz}, 2 \mathrm{H}), 6.97(\mathrm{~d}, J=$ $8.8 \mathrm{~Hz}, 2 \mathrm{H}), 6.69(\mathrm{~d}, J=8.8 \mathrm{~Hz}, 2 \mathrm{H}), 6.24(\mathrm{t}, J=7.0 \mathrm{~Hz}, 1 \mathrm{H}), 3.76(\mathrm{~s}, 3 \mathrm{H}), 2.51(\mathrm{~s}$, 3H), $2.40(\mathrm{~s}, 3 \mathrm{H}), 1.40(\mathrm{~d}, J=6.9 \mathrm{~Hz}, 3 \mathrm{H}) \cdot{ }^{13} \mathrm{C}$ NMR (126 MHz, Chloroform- $\left.d\right) \delta$ $157.42,145.18,143.65,135.59,134.97,133.03,132.82,132.31,131.75,129.57$, $129.39,128.36,127.41,126.92,126.65,125.80,124.66,123.18,118.75,112.54,54.19$, 22.32, 20.67, 14.74. HRMS(ESI) m/z: calculated for $\left[\mathrm{C}_{28} \mathrm{H}_{26} \mathrm{O}_{4} \mathrm{~S}+\mathrm{H}\right]^{+} 459.1630$, found 459.1631. HPLC data (Chiralpak IA column, hexane: isopropanol = 95: 5, $1.0 \mathrm{~mL} / \mathrm{min}$ ), $\operatorname{tr}=16.1 \min$ (major), $\operatorname{tr}=15.3 \min$ (minor), ee $=95 \%$.<smiles>C/C=C(\c1ccc(OC)cc1)c1c(O)ccc2cccc(O)c12</smiles>

$3 b^{\prime}$

Following the general procedure E, 3b' was obtained as colorless oil (26.1 mg, 78\% yield). ${ }^{1} \mathrm{H}$ NMR (500 MHz, Chloroform- $d$ ) $\delta 7.79(\mathrm{~d}, J=8.8 \mathrm{~Hz}, 1 \mathrm{H}), 7.55$ (d, $J=7.8$ $\mathrm{Hz}, 1 \mathrm{H}), 7.29$ (d, $J=8.8 \mathrm{~Hz}, 1 \mathrm{H}), 7.22(\mathrm{t}, J=7.7 \mathrm{~Hz}, 1 \mathrm{H}), 7.10(\mathrm{dd}, J=7.3,1.2 \mathrm{~Hz}$, 
1H), $6.94(\mathrm{~d}, J=8.8 \mathrm{~Hz}, 2 \mathrm{H}), 6.69(\mathrm{~d}, J=8.8 \mathrm{~Hz}, 2 \mathrm{H}), 6.34$ (q, $J=6.9 \mathrm{~Hz}, 1 \mathrm{H}), 6.20$ (s, 1H), $3.71(\mathrm{~s}, 3 \mathrm{H}), 2.33(\mathrm{~s}, 3 \mathrm{H}), 2.04(\mathrm{~s}, 3 \mathrm{H}), 1.78(\mathrm{~d}, J=6.9 \mathrm{~Hz}, 3 \mathrm{H}) .{ }^{13} \mathrm{C} \mathrm{NMR}(126$ MHz, Chloroform- $d$ ) $\delta 157.18,149.82,149.78,137.45,134.00,130.46,129.44,129.15$, $124.99,124.12,122.39,118.77,118.32,115.72,114.59,112.28,54.20,47.15,42.23$, 14.66. HRMS(ESI) m/z: calculated for $\left[\mathrm{C}_{22} \mathrm{H}_{23} \mathrm{NO}_{2}+\mathrm{Na}\right]^{+} 356.1626$, found 356.1604. HPLC data (Chiralpak IA column, hexane: isopropanol = 98: $2,1.0 \mathrm{~mL} / \mathrm{min}$ ), $\operatorname{tr}=10.6$ $\min$ (major), $\operatorname{tr}=9.5 \min$ (minor), ee $=93 \%$.<smiles>C/C=C(\c1ccc(OC)cc1)c1c(O)ccc2cccc(O)c12</smiles>

Following the general procedure E, 3c' was obtained as white solid (27.0 mg, 62\% yield). ${ }^{1} \mathrm{H}$ NMR (500 MHz, Chloroform- $d$ ) $\delta 7.96$ (dd, $\left.J=8.2,1.4 \mathrm{~Hz}, 1 \mathrm{H}\right), 7.95-7.92$ $(\mathrm{m}, 2 \mathrm{H}), 7.79(\mathrm{td}, J=7.5,1.1 \mathrm{~Hz}, 1 \mathrm{H}), 7.73(\mathrm{td}, J=7.5,1.1 \mathrm{~Hz}, 1 \mathrm{H}), 7.56-7.52(\mathrm{~m}$, $1 \mathrm{H}), 7.41(\mathrm{dd}, J=8.2,7.3 \mathrm{~Hz}, 1 \mathrm{H}), 7.35(\mathrm{~d}, J=8.9 \mathrm{~Hz}, 1 \mathrm{H}), 7.19(\mathrm{dd}, J=7.3,1.4 \mathrm{~Hz}$, $1 \mathrm{H}), 6.43(\mathrm{q}, J=9.1 \mathrm{~Hz}, 4 \mathrm{H}), 6.24(\mathrm{q}, J=6.9 \mathrm{~Hz}, 1 \mathrm{H}), 5.81(\mathrm{~s}, 1 \mathrm{H}), 3.71(\mathrm{~s}, 3 \mathrm{H}), 1.62$ $(\mathrm{d}, J=6.9 \mathrm{~Hz}, 3 \mathrm{H}) .{ }^{13} \mathrm{C} \mathrm{NMR}(126 \mathrm{MHz}$, Chloroform- $d) \delta 166.61,165.44,158.41$, 150.82 , 133.51, 132.75, 131.92, 130.90, 130.36, 130.15, 130.12, 130.03, 129.17, $129.01,125.56,125.43,125.41,124.55,122.37,122.27,122.09,116.57,113.21$, 112.65, 54.26, 14.55. HRMS(ESI) $\mathrm{m} / \mathrm{z}$ : calculated for $\left[\mathrm{C}_{22} \mathrm{H}_{20} \mathrm{O}_{2}+\mathrm{H}\right]^{+} 436.1549$, found 436.1550. HPLC data (Chiralpak AD column, hexane: isopropanol $=70: 30,1.0$ $\mathrm{mL} / \mathrm{min}$ ), $\operatorname{tr}=10.7 \mathrm{~min}$ (major), $\operatorname{tr}=14.7 \mathrm{~min}$ (minor), ee $=90 \%$.

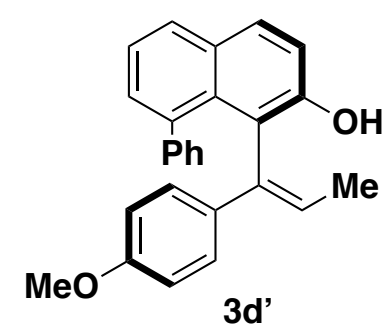

Following the general procedure E, 3d' was obtained as white solid (26.0 mg, 71\% yield). ${ }^{1} \mathrm{H}$ NMR $(500 \mathrm{MHz}$, Chloroform- $d) \delta 7.90$ (d, $\left.J=8.9 \mathrm{~Hz}, 1 \mathrm{H}\right), 7.82$ (d, $J=8.1$ 
Hz, 1H), 7.32 (d, $J=8.8 \mathrm{~Hz}, 1 \mathrm{H}), 7.28(\mathrm{~d}, J=7.6 \mathrm{~Hz}, 1 \mathrm{H}), 7.20(\mathrm{t}, J=7.1 \mathrm{~Hz}, 1 \mathrm{H})$, $7.17-7.10(\mathrm{~m}, 2 \mathrm{H}), 7.09$ (dd, $J=6.9,1.3 \mathrm{~Hz}, 1 \mathrm{H}), 6.89$ (t, $J=7.5 \mathrm{~Hz}, 1 \mathrm{H}), 6.54$ (d, $J$ $=8.7 \mathrm{~Hz}, 2 \mathrm{H}), 6.50(\mathrm{~d}, J=7.6 \mathrm{~Hz}, 1 \mathrm{H}), 6.43(\mathrm{~d}, J=8.4 \mathrm{~Hz}, 2 \mathrm{H}), 5.94(\mathrm{q}, J=6.9 \mathrm{~Hz}$, 1H), $5.81(\mathrm{~s}, 1 \mathrm{H}), 3.72(\mathrm{~s}, 3 \mathrm{H}), 1.64(\mathrm{~d}, J=6.9 \mathrm{~Hz}, 3 \mathrm{H}) .{ }^{13} \mathrm{C}$ NMR (126 MHz, Chloroform-d) $\delta 157.92,150.03,142.02$, 138.44, 134.68, 131.19, 129.94, 129.73, $129.68,129.39,129.02,128.58,127.58,125.94,125.89$, 125.25, 124.34, 124.25, 121.45, 115.87, 115.73, 112.11, 54.19, 15.13. HRMS(NSI) $\mathrm{m} / \mathrm{z}$ : calculated for $\left[\mathrm{C}_{26} \mathrm{H}_{22} \mathrm{O}_{2}+\mathrm{H}\right]^{+}$367.1698, found 367.1688. HPLC data (Chiralpak AD column, hexane: isopropanol $=98: 2,1.0 \mathrm{~mL} / \mathrm{min}), \operatorname{tr}=15.0 \mathrm{~min}$ (major), $\operatorname{tr}=29.3 \mathrm{~min}($ minor), ee $=$ $95 \%$.

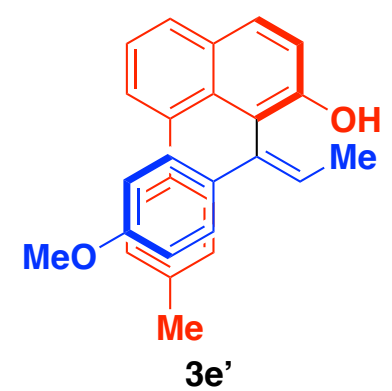

Following the general procedure E, 3e' was obtained as colorless oil $(21.9 \mathrm{mg}, 58 \%$ yield). ${ }^{1} \mathrm{H}$ NMR (500 MHz, Chloroform- $d$ ) $\delta 7.89$ (d, $\left.J=8.9 \mathrm{~Hz}, 1 \mathrm{H}\right), 7.80$ (dd, $J=8.1$, $1.5 \mathrm{~Hz}, 1 \mathrm{H}), 7.32$ (d, $J=8.9 \mathrm{~Hz}, 1 \mathrm{H}), 7.28$ (dd, $J=8.1,7.0 \mathrm{~Hz}, 1 \mathrm{H}), 7.08$ (dd, $J=7.0$, $1.5 \mathrm{~Hz}, 1 \mathrm{H}), 7.01(\mathrm{dd}, J=7.7,2.0 \mathrm{~Hz}, 1 \mathrm{H}), 6.97(\mathrm{dd}, J=7.7,1.9 \mathrm{~Hz}, 1 \mathrm{H}), 6.68(\mathrm{dd}, J$ $=7.7,1.9 \mathrm{~Hz}, 1 \mathrm{H}), 6.59-6.50(\mathrm{~m}, 2 \mathrm{H}), 6.45-6.41(\mathrm{~m}, 2 \mathrm{H}), 6.38(\mathrm{dd}, J=7.7,2.0 \mathrm{~Hz}$, 1H), $5.95(\mathrm{q}, J=6.9 \mathrm{~Hz}, 1 \mathrm{H}), 5.81(\mathrm{~s}, 1 \mathrm{H}), 3.72(\mathrm{~s}, 3 \mathrm{H}), 2.37(\mathrm{~s}, 3 \mathrm{H}), 1.64(\mathrm{~d}, J=7.0$ $\mathrm{Hz}, 3 \mathrm{H}) .{ }^{13} \mathrm{C}$ NMR (126 MHz, Chloroform- $d$ ) $\delta$ 157.91, 149.96, 139.04, 138.46, 134.73, $131.24,130.08,129.75,129.66,129.39,128.90,128.51,127.42,126.50,125.82$, 125.03, 124.24, 121.47, 115.92, 115.66, 111.99, 54.21, 20.24, 15.10. HRMS(NSI) m/z: calculated for $\left[\mathrm{C}_{27} \mathrm{H}_{24} \mathrm{O}_{2}+\mathrm{H}\right]^{+} 381.1855$, found 381.1844. HPLC data (Chiralpak IA column, hexane: isopropanol $=98: 2,1.0 \mathrm{~mL} / \mathrm{min}), \operatorname{tr}=12.5 \mathrm{~min}($ major $), \operatorname{tr}=19.2 \mathrm{~min}$ (minor), ee $=92 \%$. 


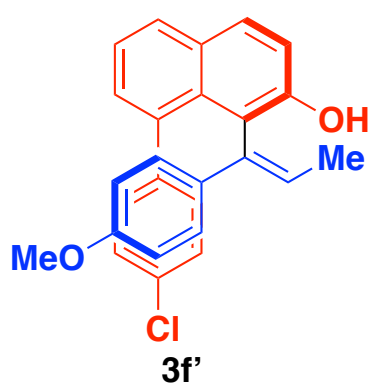

Following the general procedure E, 3f' was obtained as white solid (26.4 mg, $66 \%$ yield). ${ }^{1} \mathrm{H}$ NMR (500 MHz, Chloroform-d) $\delta 7.90$ (d, J = 8.9 Hz, 1H), 7.83 (dd, J = 8.2, $1.4 \mathrm{~Hz}, 1 \mathrm{H}), 7.33(\mathrm{~d}, \mathrm{~J}=8.8 \mathrm{~Hz}, 1 \mathrm{H}), 7.31-7.27(\mathrm{~m}, 1 \mathrm{H}), 7.14(\mathrm{dd}, \mathrm{J}=8.2,2.3 \mathrm{~Hz}$, 1H), 7.05 (ddd, J = 7.1, 5.9, $1.8 \mathrm{~Hz}, 2 \mathrm{H}), 6.82(\mathrm{dd}, \mathrm{J}=8.2,2.3 \mathrm{~Hz}, 1 \mathrm{H}), 6.63-6.54$ (m, 2H), $6.52-6.44(\mathrm{~m}, 2 \mathrm{H}), 6.40(\mathrm{dd}, \mathrm{J}=8.2,2.2 \mathrm{~Hz}, 1 \mathrm{H}), 6.04(\mathrm{q}, \mathrm{J}=6.9 \mathrm{~Hz}, 1 \mathrm{H}), 5.79$ (s, 1H), 3.74 (s, 3H), 1.64 (d, J = 7.0 Hz, 3H). ${ }^{13} \mathrm{C}$ NMR (126 MHz, Chloroform-d) $\delta$ 158.12 , 150.22, 140.34, 137.03, 134.66, 131.31, 130.87, 130.25, 129.89, 129.76, $129.67,129.36,127.96,125.81,125.73,124.47,124.44,121.42,115.94,112.23,54.23$, 15.01. HRMS(ESI) m/z: calculated for $\left[\mathrm{C}_{26} \mathrm{H}_{21} \mathrm{ClO}_{2}+\mathrm{H}\right]^{+} 401.1308$, found 401.1305. HPLC data (Chiralpak AD column, hexane: isopropanol = 98: 2, $1.0 \mathrm{~mL} / \mathrm{min}$ ), $\operatorname{tr}=19.6$ $\min$ (major), $\operatorname{tr}=37.1 \mathrm{~min}$ (minor), ee $=94 \%$.<smiles></smiles>

Following the general procedure E, 3g' was obtained as white solid (24.6 mg, 67\% yield). ${ }^{1} \mathrm{H}$ NMR (500 MHz, Chloroform- $d$ ) $\delta 8.41(\mathrm{~s}, 1 \mathrm{H}), 8.13(\mathrm{~s}, 1 \mathrm{H}), 7.93(\mathrm{~d}, J=8.9$ $\mathrm{Hz}, 1 \mathrm{H}), 7.88$ (dd, $J=8.1,1.4 \mathrm{~Hz}, 1 \mathrm{H}), 7.36$ (d, $J=8.9 \mathrm{~Hz}, 1 \mathrm{H}), 7.32$ (dd, $J=8.1,7.0$ $\mathrm{Hz}, 1 \mathrm{H}), 7.13$ (d, $J=5.1 \mathrm{~Hz}, 1 \mathrm{H}), 7.04$ (dd, $J=7.1,1.4 \mathrm{~Hz}, 1 \mathrm{H}), 6.59$ (d, $J=9.0 \mathrm{~Hz}$, 2H), $6.50-6.41(\mathrm{~m}, 3 \mathrm{H}), 6.04(\mathrm{q}, J=7.0 \mathrm{~Hz}, 1 \mathrm{H}), 5.83(\mathrm{~s}, 1 \mathrm{H}), 3.74(\mathrm{~s}, 3 \mathrm{H}), 1.67$ (d, $J=7.0 \mathrm{~Hz}, 3 \mathrm{H}) .{ }^{13} \mathrm{C}$ NMR $(126 \mathrm{MHz}$, Chloroform- $d$ ) $\delta 158.25,150.54,150.33,146.89$, $145.21,135.33,134.44,130.57,129.94,129.37,129.32,128.92,128.74,125.77$, $125.31,121.44,116.30,115.28,112.55,54.25,15.08$. HRMS(ESI) $\mathrm{m} / \mathrm{z}$ : calculated for 
$\left[\mathrm{C}_{25} \mathrm{H}_{21} \mathrm{NO}_{2}+\mathrm{H}\right]^{+}$368.3651, found 368.3654. HPLC data (Chiralpak IA column, hexane: isopropanol $=95: 5,1.0 \mathrm{~mL} / \mathrm{min}$ ), $\operatorname{tr}=34.5 \mathrm{~min}$ (major), $\operatorname{tr}=30.9 \mathrm{~min}$ (minor), ee $=95 \%$.

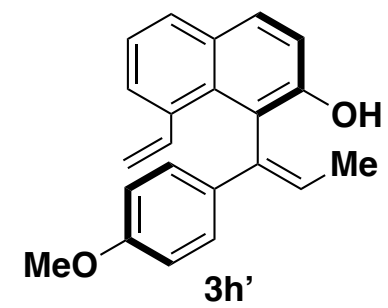

Following the general procedure E, 3h' was obtained as colorless oil (17.1 mg, 54\% yield). ${ }^{1} \mathrm{H}$ NMR (500 MHz, Chloroform- $d$ ) $\delta 7.81(\mathrm{~d}, J=8.8 \mathrm{~Hz}, 1 \mathrm{H}), 7.75$ (dd, $J=8.1$, $1.5 \mathrm{~Hz}, 1 \mathrm{H}), 7.33$ (t, J=7.0 Hz, 1H), $7.30-7.24(\mathrm{~m}, 3 \mathrm{H}), 7.21-7.15(\mathrm{~m}, 2 \mathrm{H}), 6.77$ (d, $J=8.9 \mathrm{~Hz}, 2 \mathrm{H}), 6.58(\mathrm{q}, J=6.9 \mathrm{~Hz}, 1 \mathrm{H}), 5.65(\mathrm{~s}, 1 \mathrm{H}), 5.22(\mathrm{dd}, J=16.9,1.9 \mathrm{~Hz}, 1 \mathrm{H})$, $4.90(\mathrm{dd}, J=10.7,1.9 \mathrm{~Hz}, 1 \mathrm{H}), 3.75(\mathrm{~s}, 3 \mathrm{H}), 1.56(\mathrm{~d}, J=6.9 \mathrm{~Hz}, 3 \mathrm{H}) .{ }^{13} \mathrm{C}$ NMR $(126$ MHz, Chloroform- $d$ ) $\delta 158.18,149.83,137.99,135.78,135.32,131.34,129.89,129.52$, $128.95,127.98,126.82,126.32,126.19,122.24,116.36,115.87,113.31,113.04,54.23$, 14.69. HRMS(ESI) m/z: calculated for $\left[\mathrm{C}_{22} \mathrm{H}_{20} \mathrm{O}_{2}+\mathrm{K}\right]^{+} 355.1100$, found 355.0958. HPLC data (Chiralpak AD column, hexane: isopropanol = 98: 2, $1.0 \mathrm{~mL} / \mathrm{min}$ ), $\operatorname{tr}=22.7$ $\min$ (major), $\operatorname{tr}=15.4 \min ($ minor), ee $=94 \%$.

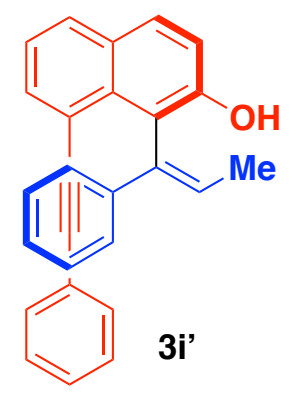

Following the general procedure E, 3i' was obtained as orange solid $(28.2 \mathrm{mg}, 72 \%$ yield). ${ }^{1} \mathrm{H}$ NMR (500 MHz, Chloroform- $d$ ) $\delta 7.87-7.78(\mathrm{~m}, 2 \mathrm{H}), 7.68(\mathrm{dd}, J=7.1,1.4$ $\mathrm{Hz}, 1 \mathrm{H}), 7.47(\mathrm{~d}, J=8.5 \mathrm{~Hz}, 1 \mathrm{H}), 7.33-7.29(\mathrm{~m}, 1 \mathrm{H}), 7.30-7.26(\mathrm{~m}, 3 \mathrm{H}), 7.17-7.13$ (m, 2H), 7.10 (d, $J=8.7 \mathrm{~Hz}, 2 \mathrm{H}), 6.70$ (d, $J=8.7 \mathrm{~Hz}, 2 \mathrm{H}), 6.60$ (q, $J=6.9 \mathrm{~Hz}, 1 \mathrm{H})$, $5.79(\mathrm{~s}, 1 \mathrm{H}), 3.72(\mathrm{~s}, 3 \mathrm{H}), 1.67$ (d, J=7.0 Hz, 3H). ${ }^{13} \mathrm{C}$ NMR (126 MHz, Chloroformd) $\delta 157.95,150.38,133.73,133.68,131.87,131.05,130.03,129.48,128.79,128.03$, 127.22, 127.18, 126.76, 126.23, 125.77, 121.79, 117.76, 117.12, 116.47, 112.71, 92.02, 
89.64, 54.22, 14.67. HRMS(ESI) m/z: calculated for $\left[\mathrm{C}_{28} \mathrm{H}_{22} \mathrm{O}_{2}+\mathrm{H}\right]^{+} 391.1698$, found 391.1693. HPLC data (Chiralpak AD column, hexane: isopropanol = 98: 2, 1.0 $\mathrm{mL} / \mathrm{min}$ ), $\operatorname{tr}=25.9 \min$ (major), $\operatorname{tr}=24.6 \mathrm{~min}$ (minor), ee $>99 \%$.

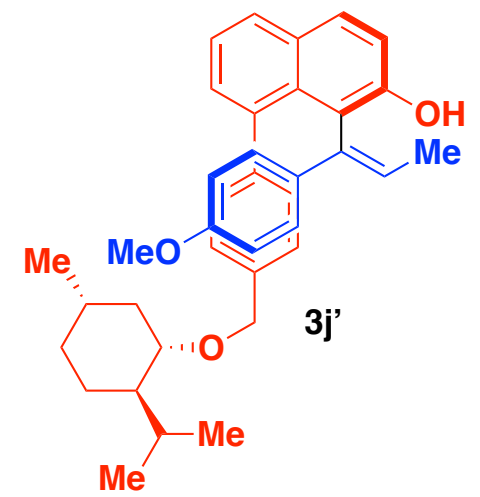

Following the general procedure E, 3j' was obtained as white solid (24.6mg, $46 \%$ yield). ${ }^{1} \mathrm{H}$ NMR $(500 \mathrm{MHz}$, Chloroform- $d$ ) $\delta 7.89(\mathrm{~d}, J=8.9 \mathrm{~Hz}, 1 \mathrm{H}), 7.81$ (dd, $J=8.2$, $1.4 \mathrm{~Hz}, 1 \mathrm{H}), 7.32(\mathrm{~d}, J=8.8 \mathrm{~Hz}, 1 \mathrm{H}), 7.28(\mathrm{t}, J=7.6 \mathrm{~Hz}, 1 \mathrm{H}), 7.18-7.14(\mathrm{~m}, 1 \mathrm{H})$, 7.08 (ddd, $J=12.9,7.5,1.7 \mathrm{~Hz}, 2 \mathrm{H}), 6.91-6.79(\mathrm{~m}, 1 \mathrm{H}), 6.53(\mathrm{~d}, J=8.9 \mathrm{~Hz}, 2 \mathrm{H})$, $6.48-6.41(\mathrm{~m}, 3 \mathrm{H}), 5.93(\mathrm{q}, J=6.9 \mathrm{~Hz}, 1 \mathrm{H}), 5.81(\mathrm{~s}, 1 \mathrm{H}), 4.68(\mathrm{~d}, J=11.4 \mathrm{~Hz}, 1 \mathrm{H})$, $4.42(\mathrm{~d}, J=11.4 \mathrm{~Hz}, 1 \mathrm{H}), 3.72(\mathrm{~s}, 3 \mathrm{H}), 3.24(\mathrm{td}, J=10.5,4.2 \mathrm{~Hz}, 1 \mathrm{H}), 2.59-2.29(\mathrm{~m}$, 1H), $2.24(\mathrm{~d}, J=13.2 \mathrm{~Hz}, 1 \mathrm{H}), 1.69(\mathrm{~d}, J=13.4 \mathrm{~Hz}, 2 \mathrm{H}), 1.64(\mathrm{~d}, J=6.9 \mathrm{~Hz}, 3 \mathrm{H}), 1.47$ - 1.39 (m, 2H), $1.08-1.00$ (m, 2H), 0.97 (d, J=6.5 Hz, 3H), 0.94 (d, J=7.1 Hz, 4H), $0.81(\mathrm{~d}, J=6.9 \mathrm{~Hz}, 3 \mathrm{H}) .{ }^{13} \mathrm{C} \mathrm{NMR}(126 \mathrm{MHz}$, Chloroform- $d) \delta 157.93,150.01,141.03$, 138.32 , 136.26, 134.74, 131.23, 129.96, 129.73, 129.67, 129.38, 129.01, 128.42, $127.52,125.89,125.30,124.40,123.80,121.45,115.88,115.70,112.07,78.02,69.60$, $54.18,47.51,39.64,33.63,30.69,24.76,22.49,21.42$, 20.03, 15.34, 15.09. HRMS(EI) $\mathrm{m} / \mathrm{z}$ : calculated for $\left[\mathrm{C}_{37} \mathrm{H}_{42} \mathrm{O}_{3}+\mathrm{K}\right]^{+}$573.2771, found 573.2786. $>20: 1 \mathrm{dr}$.

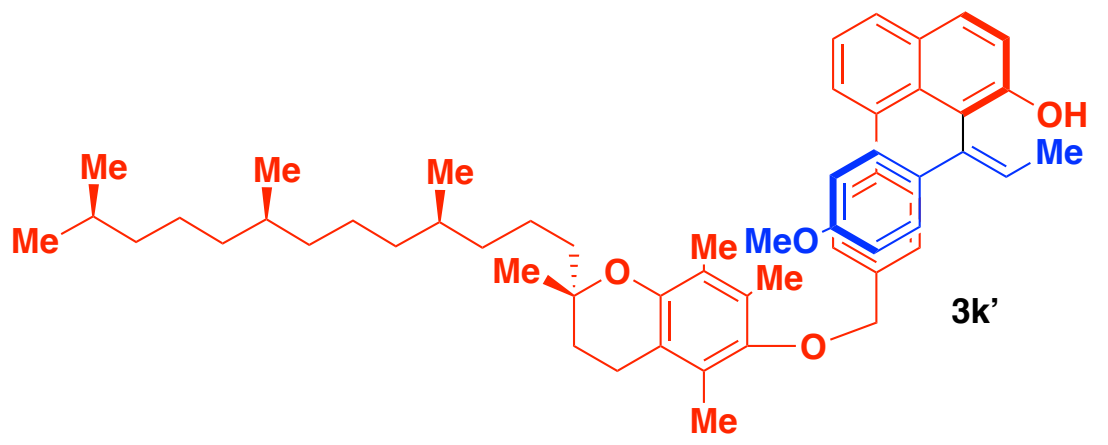


Following the general procedure E, 3l' was obtained as orange oil (29.9 mg, 37\% yield). ${ }^{1} \mathrm{H}$ NMR $(500 \mathrm{MHz}$, Chloroform- $d) \delta 7.91(\mathrm{~d}, J=8.9 \mathrm{~Hz}, 1 \mathrm{H}), 7.83(\mathrm{~d}, J=8.1 \mathrm{~Hz}, 1 \mathrm{H})$, $7.39-7.28(\mathrm{~m}, 3 \mathrm{H}), 7.18(\mathrm{dd}, J=7.7,1.9 \mathrm{~Hz}, 1 \mathrm{H}), 7.11$ (dd, $J=7.1,1.4 \mathrm{~Hz}, 1 \mathrm{H}), 6.99$ $(\mathrm{dd}, J=7.8,1.8 \mathrm{~Hz}, 1 \mathrm{H}), 6.62-6.37(\mathrm{~m}, 5 \mathrm{H}), 6.00(\mathrm{q}, J=6.9 \mathrm{~Hz}, 1 \mathrm{H}), 5.82(\mathrm{~s}, 1 \mathrm{H})$, $4.70(\mathrm{~d}, J=4.1 \mathrm{~Hz}, 2 \mathrm{H}), 3.72$ (s, 3H), 2.64 (t, $J=6.8 \mathrm{~Hz}, 2 \mathrm{H}), 2.32$ (s, 3H), 2.27 (s, $3 \mathrm{H}), 2.15(\mathrm{~s}, 3 \mathrm{H}), 1.82(\mathrm{~m}, 2 \mathrm{H}), 1.66(\mathrm{~d}, J=6.9 \mathrm{~Hz}, 3 \mathrm{H}), 1.62-1.48(\mathrm{~m}, 5 \mathrm{H}), 1.26(\mathrm{~d}$, $J=6.3 \mathrm{~Hz}, 16 \mathrm{H}), 1.11(\mathrm{~m}, 7 \mathrm{H}), 0.86(\mathrm{dd}, J=8.7,6.5 \mathrm{~Hz}, 14 \mathrm{H}) .{ }^{13} \mathrm{C} \mathrm{NMR}(126 \mathrm{MHz}$, Chloroform-d) $\delta 157.93,150.06,147.29,146.92,141.46,138.16,135.02,134.64$, $131.12,129.90,129.70,129.37,129.04,128.70,127.65,126.92,125.82,125.50$, $124.94,124.50,123.94,122.01,121.45,116.67,115.76,112.12,73.86,73.81,54.19$, $39.03,38.38,36.50,36.46,36.44,36.29,31.81,31.73,26.99,23.82,23.46,22.91,21.74$, 21.64, 20.06, 19.75, 18.77, 18.68, 15.11, 11.98, 11.11, 10.89. HRMS(EI) m/z: calculated for $\left[\mathrm{C}_{56} \mathrm{H}_{72} \mathrm{O}_{4}+\mathrm{Na}\right]^{+}$831.5328, found 831.5283. $>20: 1 \mathrm{dr}$.

General procedure F: Synthesis of axially chiral products based on 1-naphthols
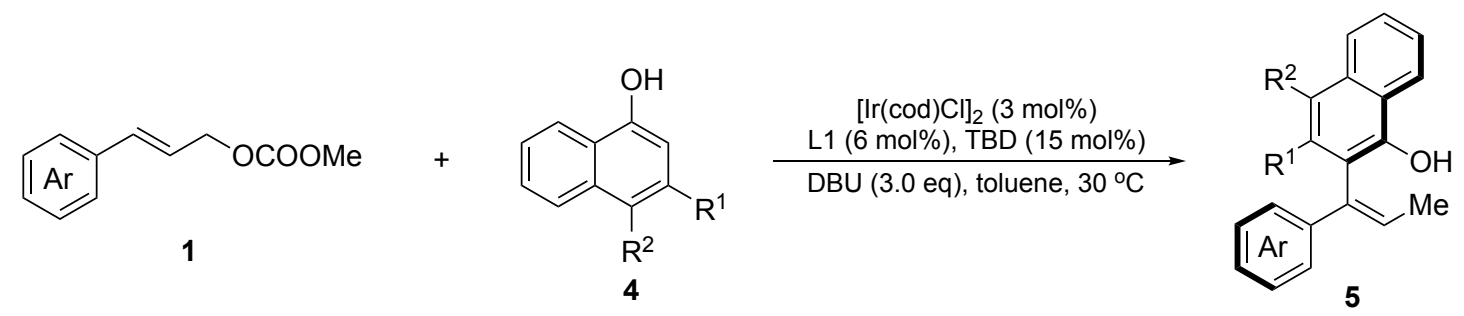

The reaction was proceeded in the glovebox under argon atmosphere. $[\operatorname{Ir}(\operatorname{cod}) \mathrm{Cl}]_{2}$ (2.1mg, $0.003 \mathrm{mmol}, 0.03 \mathrm{eq}), \mathbf{L 1}$ (32.4mg, $0.006 \mathrm{mmol}, 0.06 \mathrm{eq})$, TBD (2.1mg, $0.015 \mathrm{mmol}, 0.15 \mathrm{eq}$ ) were added to a 2 dram scintillation vial (vial $\mathbf{A}$ ) equipped with a magnetic stirring bar. The vial was then charged with toluene $(0.5 \mathrm{~mL})$ and stirred at $25{ }^{\circ} \mathrm{C}$ for $30 \mathrm{~min}$, generating an orange solution. To another 2 dram scintillation vial (vial B) was added 1 (0.1 mmol, 1.0 eq), 2 (0.15 mmol, 1.5 eq), DBU (0.3 mmol, 3.0 eq) and toluene $(0.5 \mathrm{~mL})$. The preformed catalyst solution (vial A) was then transferred to vial $\mathbf{B}$. The mixture was stirred at $30{ }^{\circ} \mathrm{C}$ with stirring 12 hours. Then the residue was purified directly by column chromatography over silica gel (PE: EA = 50:1) to afford the desired product. 


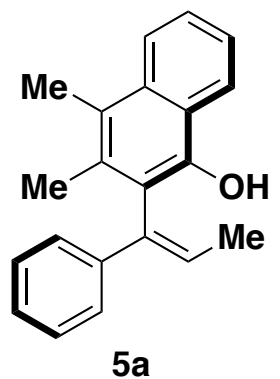

Following the general procedure F, 5a was obtained as colorless oil $(21.6 \mathrm{mg}, 75 \%$ yield). ${ }^{1} \mathrm{H}$ NMR (500 MHz, DMSO- $\left.d_{6}\right) \delta 8.45(\mathrm{~s}, 1 \mathrm{H}), 8.20(\mathrm{~d}, J=8.3 \mathrm{~Hz}, 1 \mathrm{H}), 8.01(\mathrm{~d}$, $J=8.6 \mathrm{~Hz}, 1 \mathrm{H}), 7.52(\mathrm{ddd}, J=8.4,6.7,1.4 \mathrm{~Hz}, 1 \mathrm{H}), 7.43$ (t, $J=7.5 \mathrm{~Hz}, 1 \mathrm{H}), 7.31-$ $7.16(\mathrm{~m}, 5 \mathrm{H}), 6.56(\mathrm{q}, J=6.7 \mathrm{~Hz}, 1 \mathrm{H}), 2.49(\mathrm{~s}, 3 \mathrm{H}), 2.10(\mathrm{~s}, 3 \mathrm{H}), 1.54(\mathrm{~d}, J=6.8 \mathrm{~Hz}$, $3 \mathrm{H}) .{ }^{13} \mathrm{C}$ NMR $\left(126 \mathrm{MHz}, \mathrm{DMSO}-d_{6}\right) \delta 146.52,140.03,136.02,131.99,131.84,127.70$, $126.04,125.58,125.26,125.01,123.05,122.94,122.02,121.42,119.94,16.47,14.71$, 13.80. HRMS(ESI) $\mathrm{m} / \mathrm{z}$ : calculated for $\left[\mathrm{C}_{21} \mathrm{H}_{20} \mathrm{O}+\mathrm{H}\right]^{+}$289.1592, found 289.1581 . HPLC data (Chiralpak IA column, hexane: isopropanol $=98: 2,1.0 \mathrm{~mL} / \mathrm{min}$ ), $\operatorname{tr}=9.8$ $\min$ (major), $\operatorname{tr}=8.4 \min$ (minor), ee $=85 \%$.<smiles>C/C=C(\c1ccc(OC)cc1)c1c(C)c(C)c2ccccc2c1OC</smiles>

Following the general procedure $\mathbf{F}, \mathbf{5 b}$ was isolated with Ts protection as white solid (38.3 mg, 81\% yield). ${ }^{1} \mathrm{H}$ NMR (500 MHz, Chloroform- $d$ ) $\delta 8.32-8.18(\mathrm{~m}, 1 \mathrm{H}), 8.08$ $(\mathrm{d}, J=8.4 \mathrm{~Hz}, 1 \mathrm{H}), 7.65-7.47$ (m, 4H), $7.15(\mathrm{~d}, J=8.0 \mathrm{~Hz}, 2 \mathrm{H}), 6.93-6.79(\mathrm{~m}, 2 \mathrm{H})$, $6.74-6.62(\mathrm{~m}, 2 \mathrm{H}), 5.87(\mathrm{q}, J=6.9 \mathrm{~Hz}, 1 \mathrm{H}), 3.77(\mathrm{~s}, 3 \mathrm{H}), 2.64(\mathrm{~s}, 3 \mathrm{H}), 2.44(\mathrm{~s}, 3 \mathrm{H})$, $2.18(\mathrm{~s}, 3 \mathrm{H}), 1.52$ (d, $J=7.0 \mathrm{~Hz}, 3 \mathrm{H}) .{ }^{13} \mathrm{C}$ NMR (126 MHz, Chloroform- $d$ ) $\delta$ 157.54, $143.14,142.15,134.79,134.34,132.42,132.20,131.98,130.38,129.89$, 127.93, $126.52,126.09,125.82,125.45,124.82,123.74,122.75,122.56,112.27,54.21,20.66$, 16.43, 14.81, 14.38. HRMS(ESI) m/z: calculated for $\left[\mathrm{C}_{29} \mathrm{H}_{28} \mathrm{O}_{4} \mathrm{~S}+\mathrm{H}\right]^{+} 473.1787$, found 
473.1793. HPLC data (Chiralpak IA column, hexane: isopropanol = 98:2, $1.0 \mathrm{~mL} / \mathrm{min}$ ), $\operatorname{tr}=23.6 \min$ (major), $\operatorname{tr}=18.2 \min$ (minor), ee $=91 \%$.<smiles>C/C=C(\c1ccc(-c2ccccc2)cc1)c1c(C)c(C)c2ccccc2c1O</smiles>

Following the general procedure F, 5c was obtained as white solid $(25.2 \mathrm{mg}, 69 \%$ yield). ${ }^{1} \mathrm{H}$ NMR $(500 \mathrm{MHz}$, Chloroform- $d$ ) $\delta 8.29(\mathrm{dd}, J=8.3,1.4 \mathrm{~Hz}, 1 \mathrm{H}), 8.04(\mathrm{~d}, J=$ $8.5 \mathrm{~Hz}, 1 \mathrm{H}), 7.60-7.54$ (m, 3H), 7.49 (d, $J=8.4 \mathrm{~Hz}, 2 \mathrm{H}), 7.41$ (t, $J=7.6 \mathrm{~Hz}, 3 \mathrm{H})$, $7.33(\mathrm{dd}, J=7.7,5.3 \mathrm{~Hz}, 3 \mathrm{H}), 6.77$ (q, $J=6.9 \mathrm{~Hz}, 1 \mathrm{H}), 5.56(\mathrm{~s}, 1 \mathrm{H}), 2.57$ (s, 3H), 2.18 (s, 3H), $1.70(\mathrm{~d}, J=6.9 \mathrm{~Hz}, 3 \mathrm{H}) .{ }^{13} \mathrm{C}$ NMR $(126 \mathrm{MHz}$, Chloroform- $d) \delta 144.95,139.59$, $139.27,137.56,135.23,132.14,131.26,127.77,127.32$, 126.31, 125.93, 125.33, 125.25, 124.73, 123.04, 122.67, 122.54, 121.82, 121.42, 118.20, 16.45, 14.46, 13.61. HRMS(ESI) m/z: calculated for $\left[\mathrm{C}_{27} \mathrm{H}_{24} \mathrm{O}+\mathrm{H}\right]^{+}$365.1905, found 365.1924. HPLC data (Chiralpak IA column, hexane: isopropanol = 98: 2, $1.0 \mathrm{~mL} / \mathrm{min}$ ), $\operatorname{tr}=15.7 \mathrm{~min}$ (major), $\operatorname{tr}=11.2 \min ($ minor $)$, ee $=85 \%$.<smiles>C/C=C(\c1cccc(C)c1)c1c(C)c(C)c2ccccc2c1O</smiles>

Following the general procedure F, 5d was obtained as colorless oil $(21.8 \mathrm{mg}, 72 \%$ yield). ${ }^{1} \mathrm{H}$ NMR (500 MHz, Chloroform- $d$ ) $\delta 8.28$ (dd, $\left.J=8.4,1.4 \mathrm{~Hz}, 1 \mathrm{H}\right), 8.03$ (d, $J$ $=8.5 \mathrm{~Hz}, 1 \mathrm{H}), 7.53(\mathrm{ddd}, J=8.4,6.8,1.4 \mathrm{~Hz}, 1 \mathrm{H}), 7.46(\mathrm{ddd}, J=8.0,6.8,1.1 \mathrm{~Hz}, 1 \mathrm{H})$, $7.14(\mathrm{t}, J=7.6 \mathrm{~Hz}, 1 \mathrm{H}), 7.09(\mathrm{~s}, 1 \mathrm{H}), 7.05(\mathrm{t}, J=7.9 \mathrm{~Hz}, 2 \mathrm{H}), 6.68(\mathrm{q}, J=6.9 \mathrm{~Hz}, 1 \mathrm{H})$, $5.55(\mathrm{~s}, 1 \mathrm{H}), 2.55(\mathrm{~s}, 3 \mathrm{H}), 2.27(\mathrm{~s}, 3 \mathrm{H}), 2.14(\mathrm{~s}, 3 \mathrm{H}), 1.66(\mathrm{~d}, J=6.9 \mathrm{~Hz}, 3 \mathrm{H}) .{ }^{13} \mathrm{C} \mathrm{NMR}$ (126 MHz, Chloroform-d) $\delta$ 144.91, 138.74, 137.19, 135.78, 132.09, 131.32, 127.51, $127.32,127.20,125.55,125.15,122.94,122.63,122.40,122.21,121.83,121.42$, 
118.46, 20.50, 16.40, 14.36, 13.56. HRMS(NSI) m/z: calculated for $\left[\mathrm{C}_{22} \mathrm{H}_{22} \mathrm{O}+\mathrm{H}\right]^{+}$ 303.1749, found 303.1740. HPLC data (Chiralpak IA column, hexane: isopropanol = 98: $2,1.0 \mathrm{~mL} / \mathrm{min}$ ), $\operatorname{tr}=9.6 \mathrm{~min}$ (major), $\operatorname{tr}=6.4 \mathrm{~min}$ (minor), ee $=90 \%$.

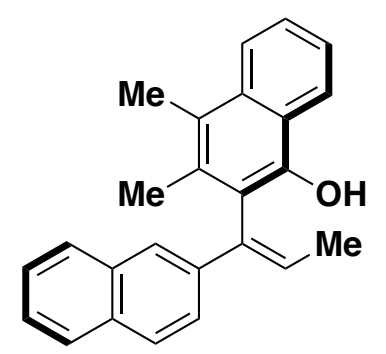

$5 e$

Following the general procedure F, 5e was obtained as white solid $(23.7 \mathrm{mg}, 70 \%$ yield). ${ }^{1} \mathrm{H}$ NMR $(500 \mathrm{MHz}$, Chloroform- $d) \delta 8.31(\mathrm{dd}, J=8.3,1.5 \mathrm{~Hz}, 1 \mathrm{H}), 8.06(\mathrm{~d}, J=$ $8.6 \mathrm{~Hz}, 1 \mathrm{H}), 7.77(\mathrm{dd}, J=8.9,2.4 \mathrm{~Hz}, 2 \mathrm{H}), 7.63(\mathrm{ddd}, J=16.2,7.8,2.1 \mathrm{~Hz}, 2 \mathrm{H}), 7.56$ (ddd, $J=8.4,6.7,1.5 \mathrm{~Hz}, 1 \mathrm{H}), 7.52-7.46(\mathrm{~m}, 2 \mathrm{H}), 7.39$ (tt, $J=6.9,5.2 \mathrm{~Hz}, 2 \mathrm{H}), 6.85$ (q, $J=6.9 \mathrm{~Hz}, 1 \mathrm{H}), 5.59$ (s, 1H), 2.57 (s, 3H), 2.15 (s, 3H), $1.72(\mathrm{~d}, J=6.9 \mathrm{~Hz}, 3 \mathrm{H})$. ${ }^{13} \mathrm{C}$ NMR (126 MHz, Chloroform- $d$ ) $\delta$ 145.06, 135.92, 135.68, 132.55, 132.20, 131.84, $131.35,127.82,127.27,127.27,126.47,125.24,125.16,124.90,124.17,123.01$, 122.76, 122.67, 122.56, 121.89, 121.49, 118.28, 16.41, 14.50, 13.58. HRMS(NSI) m/z: calculated for $\left[\mathrm{C}_{25} \mathrm{H}_{22} \mathrm{O}+\mathrm{H}\right]^{+}$339.1740, found 339.1749. HPLC data (Chiralpak IA column, hexane: isopropanol =98: $2,1.0 \mathrm{~mL} / \mathrm{min}$ ), $\operatorname{tr}=20.8 \mathrm{~min}$ (major), $\operatorname{tr}=10.2 \mathrm{~min}$ (minor), ee $=92 \%$.<smiles>C/C=C(\c1ccc2c(c1)OCO2)c1c(C)c(C)c2ccccc2c1O</smiles>

$5 f$

Following the general procedure F, $5 \mathbf{f}$ was obtained as white solid (24.9 $\mathrm{mg}, 75 \%$ yield). ${ }^{1} \mathrm{H}$ NMR (500 MHz, Chloroform- $d$ ) $\delta 8.26(\mathrm{dd}, J=8.4,1.4 \mathrm{~Hz}, 1 \mathrm{H}), 8.02(\mathrm{~d}, J=$ $8.5 \mathrm{~Hz}, 1 \mathrm{H}), 7.53(\mathrm{ddd}, J=8.4,6.7,1.4 \mathrm{~Hz}, 1 \mathrm{H}), 7.45(\mathrm{ddd}, J=8.1,6.7,1.2 \mathrm{~Hz}, 1 \mathrm{H})$, $6.83(\mathrm{~d}, J=1.1 \mathrm{~Hz}, 1 \mathrm{H}), 6.66(\mathrm{~d}, J=1.1 \mathrm{~Hz}, 2 \mathrm{H}), 6.56(\mathrm{q}, J=6.9 \mathrm{~Hz}, 1 \mathrm{H}), 5.92(\mathrm{~d}, J=$ 
$1.3 \mathrm{~Hz}, 2 \mathrm{H}), 5.54(\mathrm{~s}, 1 \mathrm{H}), 2.55(\mathrm{~s}, 3 \mathrm{H}), 2.14(\mathrm{~s}, 3 \mathrm{H}), 1.64(\mathrm{~d}, J=6.9 \mathrm{~Hz}, 3 \mathrm{H}) .{ }^{13} \mathrm{C} \mathrm{NMR}$ (126 MHz, Chloroform-d) $\delta$ 147.07, 146.12, 144.85, 135.16, 133.15, 132.09, 131.18, $125.72,125.20,122.99,122.62,122.49,121.77,121.39,118.88,118.40,107.25$, 105.18, 100.09, 16.31, 14.29, 13.55. HRMS(ESI) m/z: calculated for $\left[\mathrm{C}_{22} \mathrm{H}_{20} \mathrm{O}_{3}+\mathrm{H}\right]^{+}$ 333.1491, found 333.1482. HPLC data (Chiralpak IA column, hexane: isopropanol = 98: $2,1.0 \mathrm{~mL} / \mathrm{min}), \operatorname{tr}=19.6 \mathrm{~min}$ (major), $\operatorname{tr}=15.3 \mathrm{~min}($ minor $)$, ee $=91 \%$.

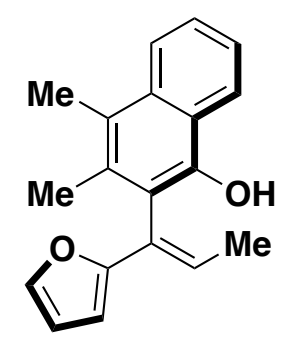

$5 g$

Following the general procedure F, $\mathbf{5 g}$ was obtained as orange oil (15.3 $\mathrm{mg}, 55 \%$ yield). ${ }^{1} \mathrm{H}$ NMR (500 MHz, Chloroform-d) $\delta 8.25$ (d, $\left.J=8.3 \mathrm{~Hz}, 1 \mathrm{H}\right), 8.02$ (d, $J=8.6$ $\mathrm{Hz}, 1 \mathrm{H}), 7.53$ (ddd, $J=8.4,6.7,1.4 \mathrm{~Hz}, 1 \mathrm{H}), 7.44$ (t, $J=7.5 \mathrm{~Hz}, 1 \mathrm{H}), 7.39$ (d, $J=1.8$ $\mathrm{Hz}, 1 \mathrm{H}), 6.76(\mathrm{q}, J=7.1 \mathrm{~Hz}, 1 \mathrm{H}), 6.27$ (dd, $J=3.2,1.8 \mathrm{~Hz}, 1 \mathrm{H}), 5.70$ (d, $J=3.4 \mathrm{~Hz}$, 1H), $5.46(\mathrm{~s}, 1 \mathrm{H}), 2.57(\mathrm{~s}, 3 \mathrm{H}), 2.23(\mathrm{~s}, 3 \mathrm{H}), 1.63(\mathrm{~d}, J=7.1 \mathrm{~Hz}, 3 \mathrm{H}) .{ }^{13} \mathrm{C}$ NMR $(126$ MHz, Chloroform-d) $\delta 152.43,145.40,141.18,132.21,131.30,125.88,125.33,124.81$, $122.96,122.58,122.32,121.80,121.46,115.88,110.40,106.55,16.16,13.57,13.41$. HRMS(NSI) $\mathrm{m} / \mathrm{z}$ : calculated for $\left[\mathrm{C}_{19} \mathrm{H}_{18} \mathrm{O}_{2}+\mathrm{H}\right]^{+}$279.1385, found 279.1376. HPLC data (Chiralpak IA column, hexane: isopropanol $=98: 2,1.0 \mathrm{~mL} / \mathrm{min}$ ), $\operatorname{tr}=8.0 \mathrm{~min}$ (major), $\operatorname{tr}=6.9 \min$ (minor), ee $=90 \%$.

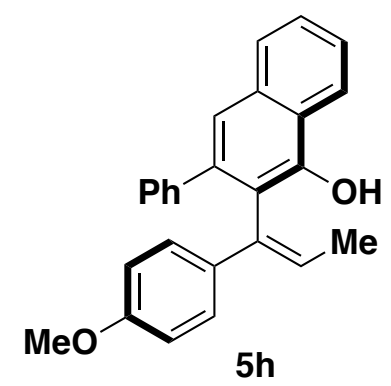

Following the general procedure $\mathbf{F}, \mathbf{5 h}$ was obtained as colorless oil $(22.6 \mathrm{mg}, 62 \%$ yield). ${ }^{1} \mathrm{H}$ NMR (500 MHz, Chloroform- $d$ ) $\delta 8.37-8.23(\mathrm{~m}, 1 \mathrm{H}), 7.89-7.78(\mathrm{~m}, 1 \mathrm{H})$, $7.59-7.46(\mathrm{~m}, 2 \mathrm{H}), 7.43(\mathrm{~s}, 1 \mathrm{H}), 7.19-7.09(\mathrm{~m}, 5 \mathrm{H}), 6.99-6.88(\mathrm{~m}, 2 \mathrm{H}), 6.76-6.51$ 
$(\mathrm{m}, 2 \mathrm{H}), 6.34(\mathrm{q}, J=6.9 \mathrm{~Hz}, 1 \mathrm{H}), 5.90(\mathrm{~s}, 1 \mathrm{H}), 3.72(\mathrm{~s}, 3 \mathrm{H}), 1.70(\mathrm{~d}, J=6.8 \mathrm{~Hz}, 3 \mathrm{H})$. ${ }^{13} \mathrm{C}$ NMR (126 MHz, Chloroform- $d$ ) $\delta$ 157.86, 147.09, 140.42, 139.43, 134.81, 132.82, $132.02,127.74,126.54,126.43,126.40,125.86,125.75,125.63,124.26,121.91$, 121.62, 119.87, 117.27, 112.55, 54.19, 14.72. HRMS(NSI) $\mathrm{m} / \mathrm{z}$ : calculated for $\left[\mathrm{C}_{26} \mathrm{H}_{22} \mathrm{O}+\mathrm{H}\right]^{+}$367.1698, found 367.1688. HPLC data (Chiralpak AD column, hexane: isopropanol $=98: 2,1.0 \mathrm{~mL} / \mathrm{min}$ ), $\operatorname{tr}=26.4 \mathrm{~min}$ (major), $\operatorname{tr}=21.7 \mathrm{~min}$ (minor), ee $=$ $84 \%$.

As to alkyl substituted allylic carbonate with 8-bromonaphthalen-2-ol for our reaction system. The allylic etherification product was obtained instead of axially chiral styrenes.

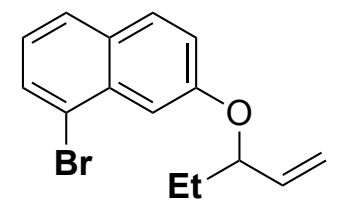

$5 \mathbf{i}$

Following the general procedure D, 5i was obtained as colorless oil $(11.9 \mathrm{mg}, 41 \%$ yield). ${ }^{1} \mathrm{H}$ NMR (500 MHz, Chloroform- $d$ ) $\delta 7.87-7.70(\mathrm{~m}, 3 \mathrm{H}), 7.63(\mathrm{~d}, J=2.6 \mathrm{~Hz}$, 1H), $7.29-7.15(\mathrm{~m}, 2 \mathrm{H}), 5.94(\mathrm{ddd}, J=17.2,10.6,6.6 \mathrm{~Hz}, 1 \mathrm{H}), 5.46(\mathrm{dt}, J=17.4,1.3$ Hz, 1H), $5.36(\mathrm{dt}, J=10.6,1.2 \mathrm{~Hz}, 1 \mathrm{H}), 4.81(\mathrm{q}, J=6.4 \mathrm{~Hz}, 1 \mathrm{H}), 1.97(\mathrm{dp}, J=14.4$, $7.3 \mathrm{~Hz}, 1 \mathrm{H}), 1.92-1.80(\mathrm{~m}, 1 \mathrm{H}), 1.11(\mathrm{t}, J=7.4 \mathrm{~Hz}, 3 \mathrm{H}) \cdot{ }^{13} \mathrm{C}$ NMR (126 MHz, Chloroform-d) $\delta 156.51,136.27,132.18,129.18,128.93,128.82,126.51,122.75$, $120.48,119.34,116.35,107.46,79.53,27.45,8.66$.

\section{Racemization experiment}

Compound $\mathbf{A}, \mathbf{B}$, or $\mathbf{C}(0.1 \mathrm{mmol})$ was dissolved in toluene $(1.0 \mathrm{~mL})$ in a sealed tube, respectively. The tube was placed in metal heating plate at $50{ }^{\circ} \mathrm{C}$. At given interval of time, small samples $(5 \mu \mathrm{L})$ was removed via syringe and subjected into the HPLC to measure the enantiomeric excess (Figure S1). 
The enantiomerisation barrier, corresponding to barrier to rotation for $\mathbf{A}, \mathbf{B}$, or $\mathbf{C}$ atropisomers, was obtained by kinetic of racemization of an enantiomer. ${ }^{[1]}$ The slope of the first-order kinetic line gives the racemization constant $(\mathrm{k}$ racemization $=2 * \mathrm{k}$ enantiomersation). Eyring equation gives the enantiomerisation barrier from enantiomerisation constant ( $\mathrm{k}$ enantiomerisation), $\mathrm{R}=8.31451 \mathrm{~J} \cdot \mathrm{K}^{-1} \cdot \mathrm{mol}^{-1}, \mathrm{~h}=$ $6.62608^{*} 10^{-34} \mathrm{~J} \cdot \mathrm{s}$ and $\mathrm{k}_{\mathrm{B}}=1.3806610^{-23} \mathrm{~J} \cdot \mathrm{K}^{-1} \cdot(-\ln \mathrm{ee}=\ln /\{(1+\boldsymbol{M} / \boldsymbol{P}) /(1-\boldsymbol{M} / \boldsymbol{P})\})$

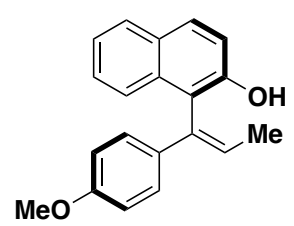

A

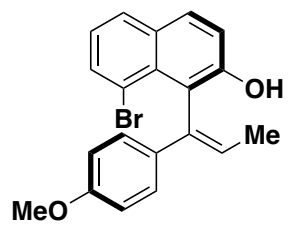

B

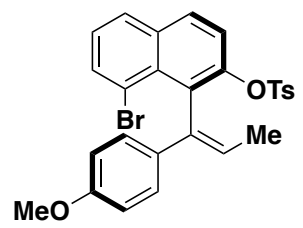

C

\begin{tabular}{|c|c|c|c|c|c|c|}
\hline Time (s) & A ee & A - In ee & B ee & B - In ee & C ee & C $\quad-$ In ee \\
\hline 0 & 77.8 & -4.354141431 & 91.74 & -4.518958489 & 92.66 & -4.52893688 \\
\hline 3600 & 68.72 & -4.230040278 & -- & -- & -- & -- \\
\hline 7200 & -- & -- & -- & -- & -- & -- \\
\hline 10800 & 61.8 & -4.123903364 & - & -- & 92.58 & -4.528073136 \\
\hline 14400 & 58.26 & -4.064915752 & -- & -- & -- & -- \\
\hline 18000 & 56.54 & -4.034948352 & -- & -- & -- & -- \\
\hline 25200 & 53 & -3.970291914 & -- & -- & -- & -- \\
\hline 28800 & 52.12 & -3.953548752 & -- & -- & -- & -- \\
\hline 32400 & 51.26 & -3.936910721 & -- & -- & -- & -- \\
\hline 39600 & -- & -- & -- & -- & 92.32 & -4.525260803 \\
\hline 57600 & -- & -- & 85.74 & -4.451319461 & -- & -- \\
\hline 61200 & -- & -- & -- & -- & -- & -- \\
\hline 79200 & 42.84 & -3.757472246 & -- & -- & -- & -- \\
\hline 82800 & -- & -- & -- & -- & -- & -- \\
\hline 86400 & -- & -- & -- & -- & 92.22 & -4.524177027 \\
\hline 93600 & 41.24 & -3.719408659 & -- & -- & -- & -- \\
\hline 100800 & 40.68 & -3.705736571 & -- & -- & -- & -- \\
\hline 108000 & 39.56 & -3.677818507 & -- & -- & -- & -- \\
\hline 115200 & 38.48 & -3.650138626 & -- & -- & -- & -- \\
\hline 162000 & 34 & -3.526360525 & -- & -- & -- & -- \\
\hline 169200 & -- & -- & 79.46 & -4.37525375 & 91.62 & -4.517649588 \\
\hline 183600 & 30.3 & -3.411147713 & -- & -- & -- & -- \\
\hline 244800 & 26.02 & -3.258865473 & -- & -- & -- & -- \\
\hline 259200 & 24.3 & -3.19047635 & -- & -- & -- & -- \\
\hline 342000 & -- & -- & 62.74 & -4.138999203 & -- & -- \\
\hline 345600 & -- & -- & -- & -- & 90.2 & -4.502029427 \\
\hline
\end{tabular}




\begin{tabular}{ccccccc}
\hline 356400 & 18.6 & -2.923161581 & -- & -- & -- & -- \\
518400 & -- & -- & -- & -- & 88.68 & -4.485034385 \\
666000 & -- & -- & 36.7 & -3.602776755 & -- & -- \\
691200 & -- & -- & -- & -- & 87.28 & -4.469121342 \\
774000 & -- & -- & 32.3 & -3.47506723 & -- & -- \\
885600 & -- & -- & -- & -- & 85.82 & -4.45225208 \\
1231200 & -- & -- & -- & -- & 84.2 & -4.433194921 \\
1612800 & -- & -- & -- & -- & 82.2 & -4.409155302 \\
2440800 & -- & -- & -- & -- & 76.26 & -4.334148554 \\
\hline
\end{tabular}

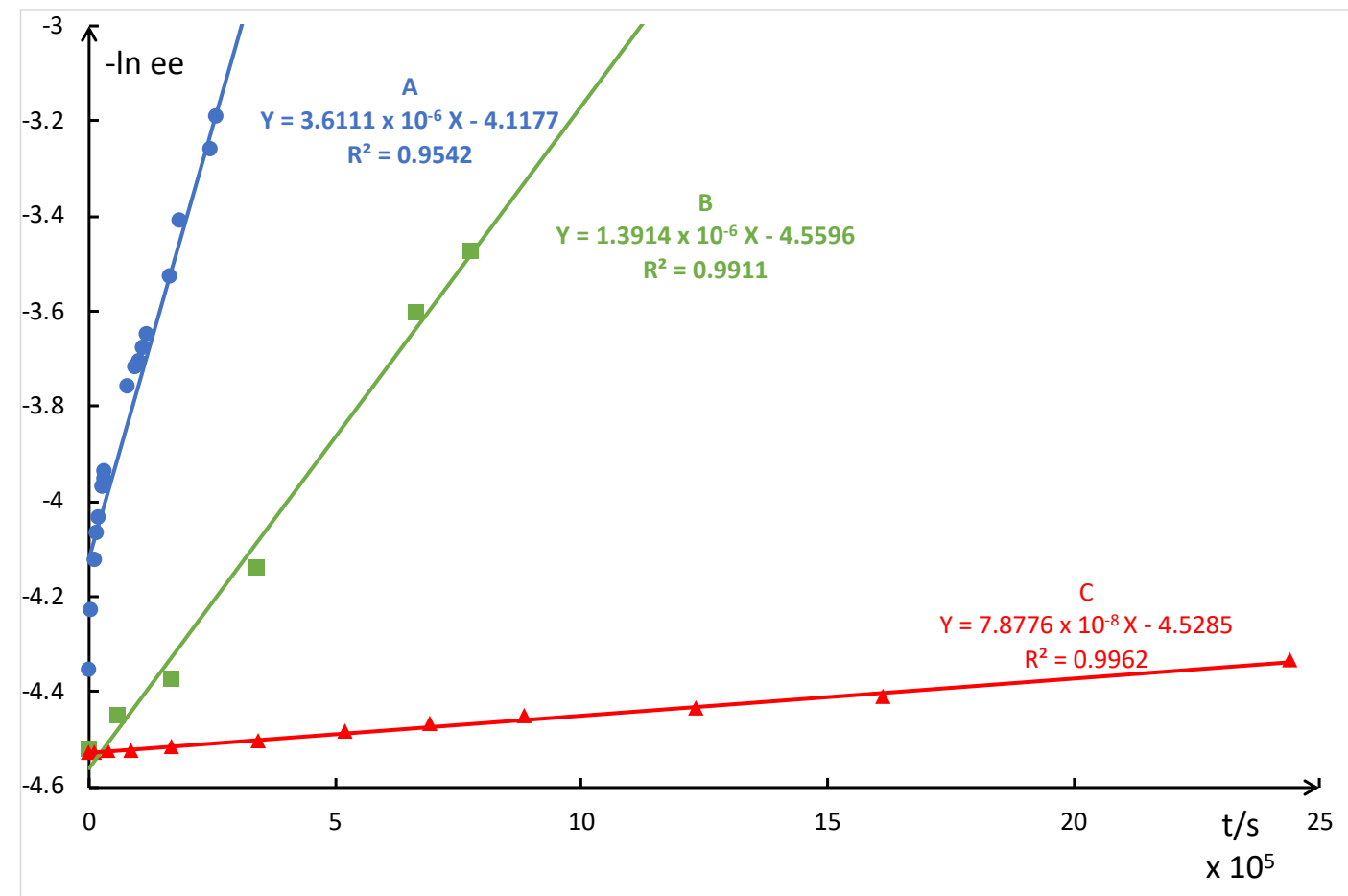

Figure S1 Racemization experiments.

$\mathbf{K}_{\mathbf{A}}$ racemization $=3.6111 \times 10^{-6} \mathrm{~S}^{-1}$

$\mathbf{K}_{\mathbf{A}}$ enantiomerization $=1.8056 \times 10^{-6} \mathrm{~S}^{-1}$

Half-life time $\mathbf{t}_{\mathbf{A} \mathbf{1 / 2}}{ }^{\mathbf{3 2 3 K}}=53.32 \mathrm{~h}$

$\Delta \mathbf{G}_{\mathbf{A}}{ }^{323 \mathrm{~K}}=26.996 \mathrm{Kcal} / \mathrm{mol}$

$\mathbf{K}_{\mathbf{B}}$ racemization $=1.3914 \times 10^{-6} \mathrm{~S}^{-1}$

$\mathbf{K}_{\mathbf{B}}$ enantiomerization $=6.957 \times 10^{-7} \mathrm{~S}^{-1}$

Half-life time $\mathbf{t}_{B} \mathbf{1 / 2}{ }^{\mathbf{3 2 3 K}}=138.38 \mathrm{~h}$

$\Delta \mathbf{G}_{\mathbf{B}}{ }^{323 \mathrm{~K}}=27.608 \mathrm{Kcal} / \mathrm{mol}$

$\mathbf{K}_{\mathbf{C}}$ racemization $=7.8776 \times 10^{-8} \mathrm{~S}^{-1}$ 
$\mathbf{K}_{\mathbf{C}}$ enantiomerization $=3.9388 \times 10^{-8} \mathrm{~S}^{-1}$

Half-life $\mathbf{t}_{\mathbf{C} \mathbf{1} / \mathbf{2}}{ }^{\mathbf{3 2 3 K}}=2444.15 \mathrm{~h}$

$\Delta \mathbf{G}_{\mathbf{C}}{ }^{323 K}=29.451 \mathrm{Kcal} / \mathrm{mol}$

General procedure G: Large scale experiment

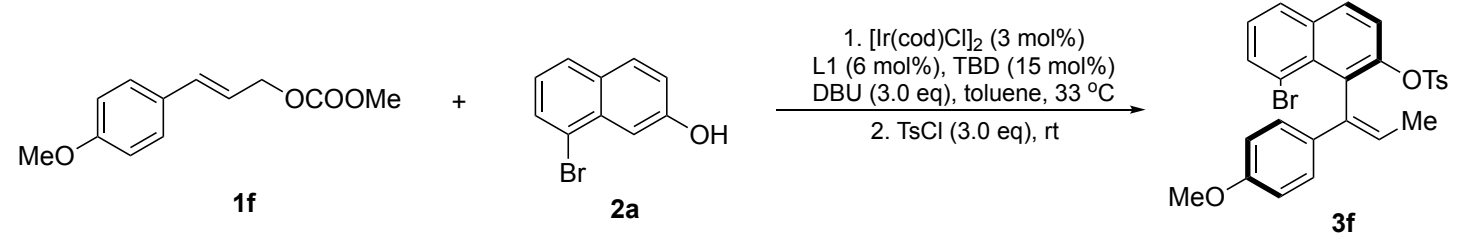

The reaction was proceeded in the glovebox under argon atmosphere. $[\operatorname{Ir}(\operatorname{cod}) \mathrm{Cl}]_{2}$ (21mg, $0.03 \mathrm{mmol}, 0.03 \mathrm{eq})$, L1 (33mg, $0.06 \mathrm{mmol}, 0.06 \mathrm{eq})$, TBD (21mg, 0.15 mmol, 0.15 eq) were added to a vial equipped with a magnetic stirring bar. The vial (vial A) was then charged with toluene $(5.0 \mathrm{~mL})$ and stirred at $25^{\circ} \mathrm{C}$ for $30 \mathrm{~min}$. To another vial (vial B) was added 1 (1.0 mmol, $1.0 \mathrm{eq}), 2$ (1.5 mmol, $1.5 \mathrm{eq})$, DBU (3.0 mmol, $3.0 \mathrm{eq})$ and toluene $(5.0 \mathrm{~mL})$. The preformed catalyst solution (vial $\mathbf{A})$ was then transferred to vial B. The mixture was stirred at $33{ }^{\circ} \mathrm{C}$ with stirring 12 hours. Then the mixture was taken out of the glovebox, and $\mathrm{TsCl}(3.0 \mathrm{mmol}, 3.0 \mathrm{eq})$ was added. After stirring at room temperature for 1 hour, the solution was concentrated in vacuo. The residue was purified directly by column chromatography over silica gel (PE: EA $=20: 1$ to $10: 1)$ to afford the desired product as white solid $(376 \mathrm{mg}, 72 \%$ yield, ee $=94 \%$.)

\section{Deuterium-labeling experiment}

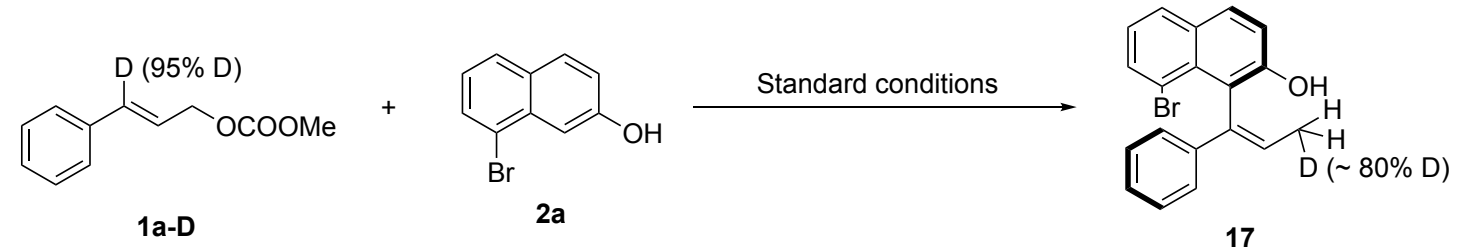

The substrates 1a-D was synthesized according to literature, ${ }^{[2]}$ the general procedure $\mathbf{E}$ was used to synthesize 17 using substrate 1a-D labeled with deuterium next to the aryl ring. As a result, product 17 with $\sim 80 \% \mathrm{D}$-incorporation at the methyl group was obtained. The incomplete D migration in the course of the transformation was attributed to $\mathrm{H} / \mathrm{D}$ exchange of iridium hydride with solvent or water during the reaction process. The high level central-to-axial chirality transfer maybe attributed to 
the coordination between allylic iridium hydride and hydroxyl group which restrict the rotation of reaction intermediate via chiral axis during the chiral transfer process.<smiles>[2H]C(C=C(c1ccccc1)c1c(O)ccc2ccccc12)C(=O)O</smiles>

Following the general procedure E, 17 was obtained as colorless oil (23.6 mg,70\% yield). ${ }^{1} \mathrm{H}$ NMR (500 MHz, Chloroform- $d$ ) $\delta 7.82(\mathrm{~d}, J=8.9 \mathrm{~Hz}, 1 \mathrm{H}), 7.77$ (dd, $J=8.1$, $1.3 \mathrm{~Hz}, 1 \mathrm{H}), 7.69$ (dd, $J=7.3,1.3 \mathrm{~Hz}, 1 \mathrm{H}), 7.33$ (d, $J=8.8 \mathrm{~Hz}, 1 \mathrm{H}), 7.28-7.18$ (m, $5 \mathrm{H}), 7.11(\mathrm{t}, J=7.7 \mathrm{~Hz}, 1 \mathrm{H}), 6.76(\mathrm{td}, J=6.9,1.8 \mathrm{~Hz}, 1 \mathrm{H}), 5.95(\mathrm{~s}, 1 \mathrm{H}), 2.01-1.61$ (m, 2.2 H).

\section{General procedure H: Synthetic transformations}
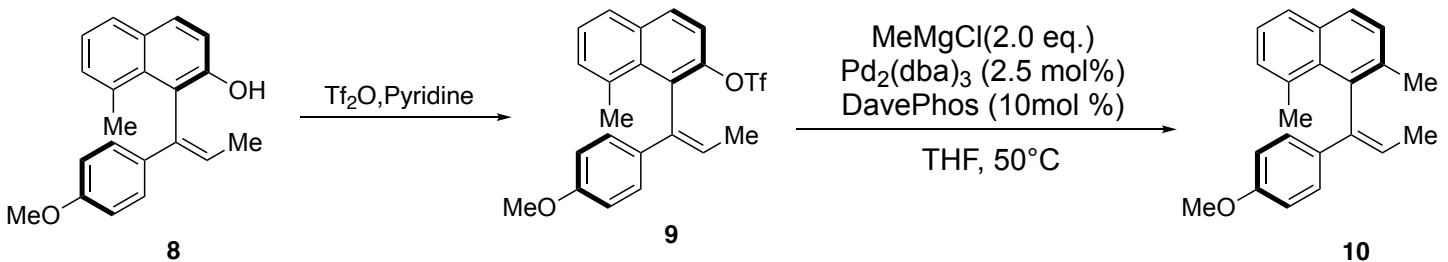

Following the general procedure E, 8 was obtained as colorless oil in $95 \%$ ee. Compound 8 (306 mg, 1.0 mmol, 1.0 eq) was dissolved in DCM (10 mL), Tf $2 \mathrm{O}(315$ $\mathrm{mg}, 1.5 \mathrm{mmol}, 1.5 \mathrm{eq})$ and pyridine (158 mg, $2.0 \mathrm{mmol}, 2.0 \mathrm{eq})$ was added dropwise. The mixture was stirred at room temperature for 6 hours, then water was added and the solution was extracted with DCM (10 mL x 3). The combined organic layers were washed with saturated $\mathrm{NaCl}$ aqueous $(30 \mathrm{~mL})$, dried over anhydrous $\mathrm{Na}_{2} \mathrm{SO}_{4}$, filtered and concentrated in vacuo. The crude product was purified by column chromatography over silica gel (PE: EA= 20:1 to 10:1) to afford 9 as white solid (359 mg, 82\% yield, 94\% ee). Then 9 (359 mg, $0.82 \mathrm{mmol}, 1.0 \mathrm{eq}$ ) was dissolved in anhydrous THF (10 $\mathrm{mL}), \mathrm{Pd}_{2}(\mathrm{dba})_{3}(18.8 \mathrm{mg}, 0.02 \mathrm{mmol}, 2.5 \mathrm{~mol} \%$ ), DavePhos (32.2 mg, $0.082 \mathrm{mmol}, 10$ $\mathrm{mol} \%)$ and $\mathrm{MeMgCl}(2.5 \mathrm{M}$ in $\mathrm{THF}, 0.66 \mathrm{ml}, 1.64 \mathrm{mmol}, 2.0 \mathrm{eq})$ was added. The mixture was heated to $50{ }^{\circ} \mathrm{C}$ and stirred for 5 hours, then the mixture was quenched by $\mathrm{NH}_{4} \mathrm{Cl}$ aqueous $(5 \mathrm{~mL})$ and extracted with EA $(10 \mathrm{~mL}$ x 3$)$, the combined organic layers were washed with saturated $\mathrm{NaCl}$ aqueous $(20 \mathrm{~mL})$, dried over anhydrous $\mathrm{Na}_{2} \mathrm{SO}_{4}$, 
filtered and concentrated in vacuo. The crude product was purified by column chromatography over silica gel $(\mathrm{PE}: \mathrm{EA}=50: 1)$ to afford $\mathbf{1 0}$ as white solid (187 mg, $76 \%$ yield) (Figure S2) $(Z / E=14: 1) .{ }^{1} \mathrm{H}$ NMR $(500 \mathrm{MHz}$, Chloroform- $d) \delta 7.75(\mathrm{~d}, J$ $=8.2 \mathrm{~Hz}, 1 \mathrm{H}), 7.70(\mathrm{~d}, J=8.1 \mathrm{~Hz}, 1 \mathrm{H}), 7.38(\mathrm{~d}, J=8.3 \mathrm{~Hz}, 1 \mathrm{H}), 7.29-7.23(\mathrm{~m}, 1 \mathrm{H})$, $7.18(\mathrm{~d}, J=7.0 \mathrm{~Hz}, 1 \mathrm{H}), 7.14(\mathrm{~d}, J=8.4 \mathrm{~Hz}, 2 \mathrm{H}), 6.76(\mathrm{~d}, J=9.0 \mathrm{~Hz}, 2 \mathrm{H}), 6.41$ (q, $J=$ $6.9 \mathrm{~Hz}, 1 \mathrm{H}), 3.75(\mathrm{~s}, 3 \mathrm{H}), 2.58(\mathrm{~s}, 3 \mathrm{H}), 2.22(\mathrm{~s}, 3 \mathrm{H}), 1.45(\mathrm{~d}, J=6.9 \mathrm{~Hz}, 3 \mathrm{H}) .{ }^{13} \mathrm{C} \mathrm{NMR}$ (126 MHz, Chloroform-d) $\delta 157.45,140.56,134.18,134.07,133.86,132.88,132.85$, $131.20,128.62,127.57,127.45,126.59,125.81,123.42,120.66,112.79,54.18,23.05$, 19.64, 14.29. HRMS(NSI) $\mathrm{m} / \mathrm{z}$ : calculated for $\left[\mathrm{C}_{22} \mathrm{H}_{22} \mathrm{O}_{2}+\mathrm{H}\right]^{+} 303.1749$, found 303.1743. HPLC data (Chiralpak IA column, hexane: isopropanol = 99.9: 0.1, 1.0 $\mathrm{mL} / \mathrm{min}$ ), $\operatorname{tr}=7.9 \min$ (major), $\operatorname{tr}=9.0 \mathrm{~min}$ (minor), ee $=93 \%$.

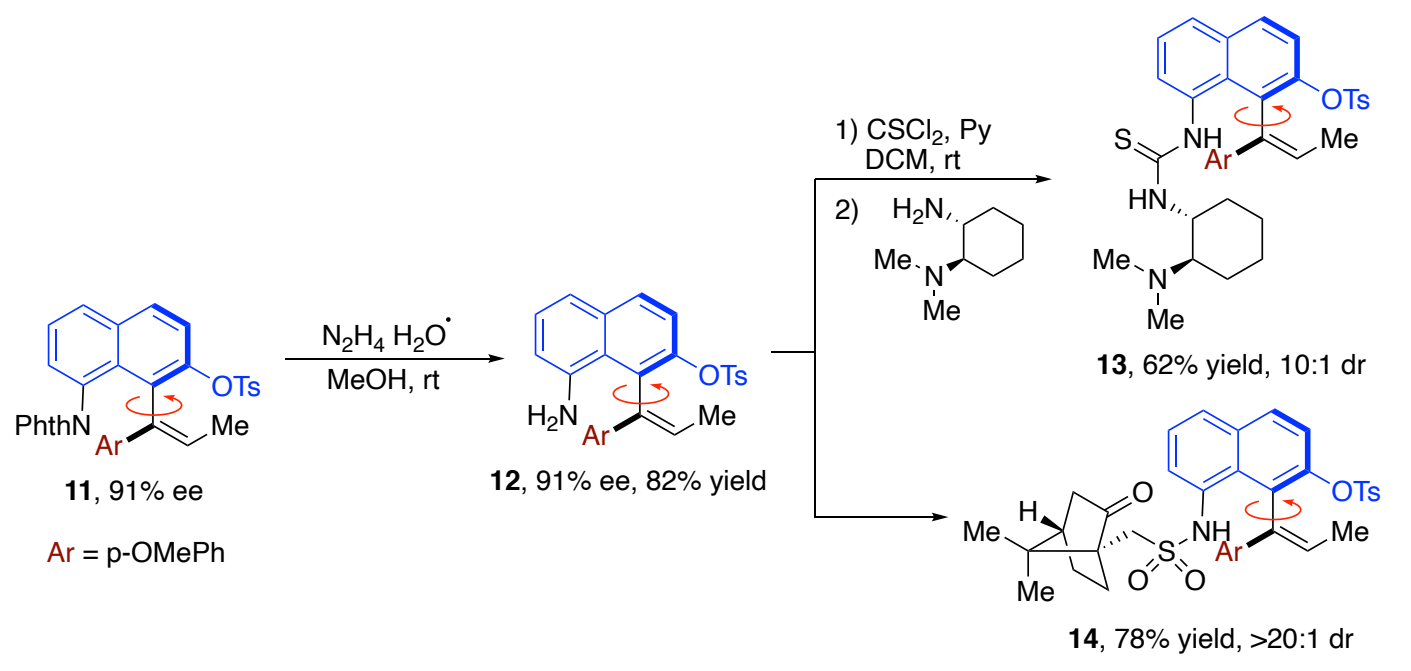

Figure S2 Synthetic transformations

Following the general procedure D, 11 was obtained in 91\% ee. Compound $\mathbf{1 1}$ (0.589 g, $1.0 \mathrm{mmol}, 1.0 \mathrm{eq})$ was dissolved in $\mathrm{MeOH}(10 \mathrm{~mL})$, and $80 \%$ hydrazine hydrate $(10 \mathrm{~mL})$ was added dropwise. The mixture was stirred at room temperature for 6 hours, then water was added and the solution was extracted with DCM (10 mL x 3), the combined organic layers were washed with saturated $\mathrm{NaCl}$ aqueous $(30 \mathrm{~mL})$, dried over anhydrous $\mathrm{Na}_{2} \mathrm{SO}_{4}$, filtered and concentrated in vacuo. The crude product was purified by column chromatography over silica gel (PE: EA=20:1 to 10:1) to afford 12 as white solid (0.376 g, 82\% yield, 91\% ee) (Figure S2). ${ }^{1} \mathrm{H}$ NMR (500 MHz, 
Chloroform-d) $\delta 7.75(\mathrm{~d}, J=9.0 \mathrm{~Hz}, 1 \mathrm{H}), 7.56(\mathrm{~d}, J=8.2 \mathrm{~Hz}, 2 \mathrm{H}), 7.49(\mathrm{~d}, J=9.1 \mathrm{~Hz}$, 1H), $7.31-7.23(\mathrm{~m}, 2 \mathrm{H}), 7.17(\mathrm{~d}, J=8.1 \mathrm{~Hz}, 2 \mathrm{H}), 7.09-6.99(\mathrm{~m}, 2 \mathrm{H}), 6.78-6.68(\mathrm{~m}$, 2H), $6.63(\mathrm{dd}, J=5.4,3.3 \mathrm{~Hz}, 1 \mathrm{H}), 6.35(\mathrm{q}, J=6.9 \mathrm{~Hz}, 1 \mathrm{H}), 4.73(\mathrm{~s}, 2 \mathrm{H}), 3.76(\mathrm{~s}, 3 \mathrm{H})$, $2.41(\mathrm{~s}, 3 \mathrm{H}), 1.52(\mathrm{~d}, J=6.9 \mathrm{~Hz}, 3 \mathrm{H}) .{ }^{13} \mathrm{C}$ NMR (126 MHz, Chloroform- $d$ ) $\delta$ 157.87, $143.67,143.66,136.02,133.11,132.98,131.77,129.12$, 128.39, 126.94, 126.11, $125.71,125.70,124.53,121.00,118.76,117.57,112.65,110.71,54.18,20.62,14.66$. HRMS(ESI) m/z: calculated for $\left[\mathrm{C}_{22} \mathrm{H}_{20} \mathrm{O}_{2}+\mathrm{H}\right]^{+}$460.1583, found 460.1580. HPLC data (Chiralpak AD column, hexane: isopropanol = 80:20,1.0 $\mathrm{mL} / \mathrm{min}$ ), $\operatorname{tr}=26.5 \mathrm{~min}$ (major), $\operatorname{tr}=22.2 \min$ (minor), ee $=96 \%$.

To a solution of 12 (45.9 mg, $0.1 \mathrm{mmol}, 1.0 \mathrm{eq})$ in DCM (1 mL) was added $\mathrm{CSCl}_{2}$ (13.8 mg, $0.12 \mathrm{mmol}, 1.2 \mathrm{eq})$ and pyridine (11.9 mg, $0.3 \mathrm{mmol}, 1.5 \mathrm{eq})$ dropwise. Then the mixture was stirred at room temperature for 1 hour. The solution was purified directly by a short column chromatography over silica gel (PE: EA $=10: 1$ to $5: 1)$ to afford the intermediate. Then the intermediate was dissolved in DCM (1 mL), amine (21.3 mg, $0.3 \mathrm{mmol}, 1.5 \mathrm{eq})$ and pyridine (15.8 mg, $0.4 \mathrm{mmol}, 2.0 \mathrm{eq})$ was added dropwise. The mixture was stirred at room temperature for 3 hours. The residue was purified directly by column chromatography over silica gel (DCM: $\mathrm{MeOH}=20: 1$ to 10:1) to afford 13 as yellow solid (39.9 mg, 62\% yield in two steps) (Figure S2). ${ }^{1} \mathrm{H}$ NMR (500 MHz, Chloroform-d) $\delta 7.94$ (d, $J=9.0 \mathrm{~Hz}, 1 \mathrm{H}), 7.87$ (d, $J=8.2 \mathrm{~Hz}, 1 \mathrm{H})$, $7.80(\mathrm{~d}, J=8.7 \mathrm{~Hz}, 1 \mathrm{H}), 7.53(\mathrm{~d}, J=8.0 \mathrm{~Hz}, 2 \mathrm{H}), 7.46(\mathrm{t}, J=7.7 \mathrm{~Hz}, 1 \mathrm{H}), 7.36(\mathrm{~s}, 1 \mathrm{H})$, $7.10(\mathrm{~d}, J=7.9 \mathrm{~Hz}, 2 \mathrm{H}), 7.02(\mathrm{~d}, J=8.9 \mathrm{~Hz}, 2 \mathrm{H}), 6.69(\mathrm{~d}, J=8.5 \mathrm{~Hz}, 2 \mathrm{H}), 6.46$ (q, $J=$ $6.9 \mathrm{~Hz}, 1 \mathrm{H}), 3.75(\mathrm{~s}, 3 \mathrm{H}), 2.56(\mathrm{~s}, 1 \mathrm{H}), 2.37(\mathrm{~s}, 3 \mathrm{H}), 2.21(\mathrm{~s}, 6 \mathrm{H}), 1.77$ (d, J=11.2 Hz, 2H), 1.59 (d, $J=13.6 \mathrm{~Hz}, 1 \mathrm{H}), 1.45(\mathrm{~d}, J=6.9 \mathrm{~Hz}, 3 \mathrm{H}), 1.34-1.21(\mathrm{~m}, 3 \mathrm{H}), 1.20-$ $1.01(\mathrm{~m}, 2 \mathrm{H}), 0.93-0.69(\mathrm{~m}, 2 \mathrm{H}) .{ }^{13} \mathrm{C}$ NMR (126 MHz, Chloroform-d) $\delta$ 179.14, $157.89,145.91,143.88,133.36,132.65,131.37,129.16,128.44,128.33,127.84$, 127.51, 126.86, 126.00, 125.40, 124.44, 123.45, 120.32, 112.95, 65.64, 54.18, 31.29, 28.68, 24.06, 24.04, 23.32, 20.62, 20.42, 14.96. HRMS(ESI) m/z: calculated for $\left[\mathrm{C}_{36} \mathrm{H}_{41} \mathrm{~N}_{3} \mathrm{O}_{4} \mathrm{~S}_{2}+\mathrm{H}\right]^{+}$644.2617, found 644.2627. 10:1 dr. 
To a solution of 12 (45.9 mg, $0.1 \mathrm{mmol}, 1.0 \mathrm{eq})$ and Camphor sulfonyl chloride (37.6 mg, $0.15 \mathrm{mmol}, 1.5 \mathrm{eq})$ in DCM (1 mL) was added Et $3 \mathrm{~N}$ (30.3 mg, 0.3 mmol, 3.0 eq). The mixture was stirred at room temperature for 4 hours, and concentrated in vacuo after 12 was consumed completely. The residue was purified directly by column chromatography over silica gel (DCM: $\mathrm{MeOH}=20: 1$ to $10: 1)$ to afford 14 as yellow solid (52.5 mg, 78\% yield) (Figure S2). ${ }^{1} \mathrm{H}$ NMR (500 MHz, Chloroform- $d$ ) $\delta 8.67$ (s, 1H), $7.91(\mathrm{~d}, J=9.0 \mathrm{~Hz}, 1 \mathrm{H}), 7.79(\mathrm{dd}, J=7.8,1.2 \mathrm{~Hz}, 1 \mathrm{H}), 7.70(\mathrm{~d}, J=9.1 \mathrm{~Hz}, 1 \mathrm{H})$, $7.68(\mathrm{dd}, J=8.2,1.2 \mathrm{~Hz}, 1 \mathrm{H}), 7.55(\mathrm{~d}, J=8.3 \mathrm{~Hz}, 2 \mathrm{H}), 7.47$ (t, $J=7.9 \mathrm{~Hz}, 1 \mathrm{H}), 7.14$ $(\mathrm{d}, J=8.0 \mathrm{~Hz}, 2 \mathrm{H}), 7.02(\mathrm{~d}, J=8.9 \mathrm{~Hz}, 2 \mathrm{H}), 6.73(\mathrm{~d}, J=9.0 \mathrm{~Hz}, 2 \mathrm{H}), 6.54(\mathrm{q}, J=7.0$ Hz, 1H), $3.76(\mathrm{~s}, 3 \mathrm{H}), 2.76(\mathrm{~d}, J=14.9 \mathrm{~Hz}, 1 \mathrm{H}), 2.57$ (d, $J=14.9 \mathrm{~Hz}, 1 \mathrm{H}), 2.39(\mathrm{~s}, 3 \mathrm{H})$, $2.34-2.22(\mathrm{~m}, 1 \mathrm{H}), 2.14(\mathrm{td}, J=12.5,11.7,3.2 \mathrm{~Hz}, 1 \mathrm{H}), 2.00(\mathrm{t}, J=4.5 \mathrm{~Hz}, 1 \mathrm{H}), 1.92$ (tt, $J=12.3,3.7 \mathrm{~Hz}, 1 \mathrm{H}), 1.80(\mathrm{~d}, J=18.5 \mathrm{~Hz}, 1 \mathrm{H}), 1.59(\mathrm{~d}, J=7.0 \mathrm{~Hz}, 3 \mathrm{H}), 1.33$ (d, $J$ $=4.4 \mathrm{~Hz}, 1 \mathrm{H}), 1.31-1.28(\mathrm{~m}, 1 \mathrm{H}), 1.25(\mathrm{~s}, 3 \mathrm{H}), 1.01(\mathrm{~s}, 3 \mathrm{H}), 0.79(\mathrm{~s}, 3 \mathrm{H}) .{ }^{13} \mathrm{C} \mathrm{NMR}$ (126 MHz, Chloroform-d) $\delta$ 213.27, 158.28, 145.13, 144.02, 134.52, 133.62, 132.91, $132.68,129.91,129.83,128.47,126.86,126.22,126.02,125.39,124.28,123.84$, $122.71,119.63,115.78,113.08,57.38,54.13,47.05,46.62,42.23,41.54,28.70,25.69$, 25.04, 20.66, 18.89, 15.05. HRMS(ESI) $\mathrm{m} / \mathrm{z}$ : calculated for $\left[\mathrm{C}_{37} \mathrm{H}_{39} \mathrm{NO}_{7} \mathrm{~S}_{2}+\mathrm{H}\right]^{+}$ 674.2246, found 674.2246. $>20: 1 \mathrm{dr}$.

\section{Control experiments}

Following the general procedure $\mathbf{E}$, the reaction was carried out under the optimized conditions using protected 2-naphthols or 2-naphthylamine analogues as the nucleophile. However, no desired products were obtained in all cases. These results indicated that unprotected 2-naphthols were crucial for the reaction occurring (Figure S3). 


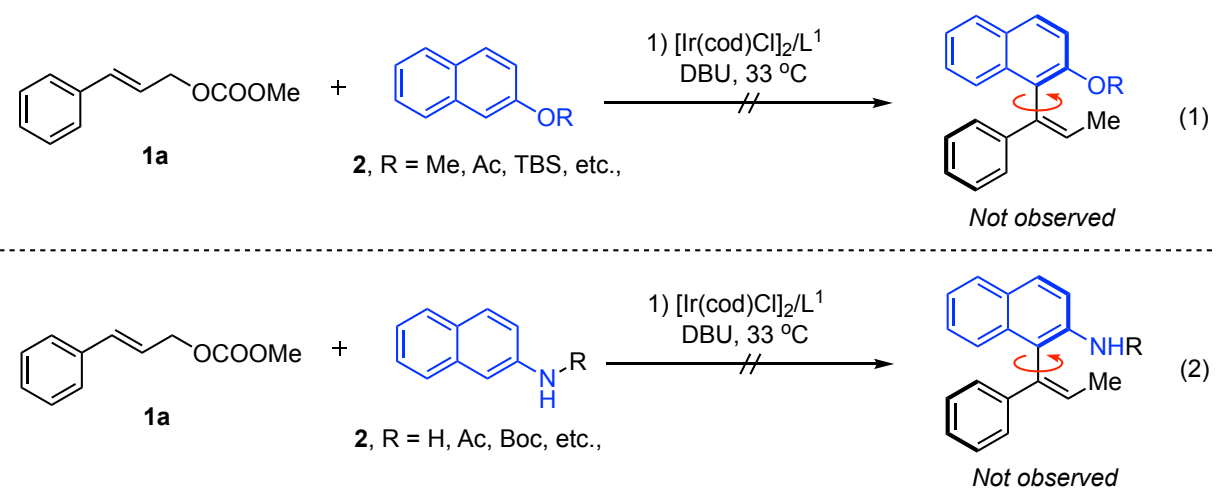

Figure S3 AASI conducted using protected 2-naphthols and 2-naphthylamines as substrates.

\section{Stereospecific 1,3-H transfer of enantioenriched 1-(1-phenylallyl)naphthalen-2-ol.}

Compound 15 was obtained according to the literature ${ }^{[3]}$. Compound $15(0.026 \mathrm{~g}$, $0.1 \mathrm{mmol})$ and toluene $(1.0 \mathrm{~mL})$ was subjected to a vial containing the stirring bar. DBU $(0.3 \mathrm{mmol})$ or TBD $(0.02 \mathrm{mmol})$ was then added to the mixture. The reaction was stirred at room temperature, $35{ }^{\circ} \mathrm{C}$ or $50{ }^{\circ} \mathrm{C}$ overnight and monitored by crude ${ }^{1} \mathrm{H}$ NMR. However, in all cases, only trace amount of product 16 was obtained ( $<5 \%$ yield). This result indicated that the base-promoted 1,3-proton transfer could not proceed the isomerization of allylic compound $\mathbf{1 5}$ (Figure S4).

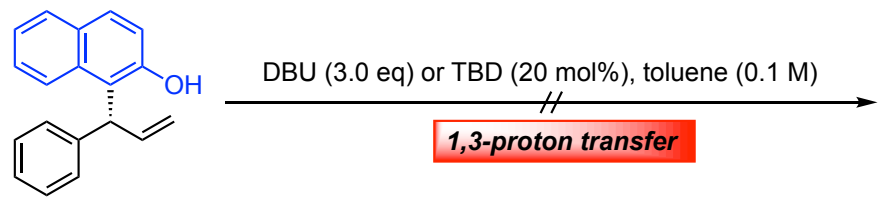

$15,70 \%$ ee

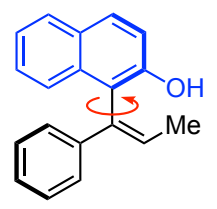

16, Trace product

Figure S4 Stereospecific 1,3-H transfer of 1-(1-phenylallyl)naphthalen-2-ol using base as

$$
\text { promotors }
$$

Compound $15(0.026 \mathrm{~g}, 0.1 \mathrm{mmol}),[\operatorname{Ir}(\operatorname{cod}) \mathrm{Cl}]_{2}(3.0 \mathrm{~mol} \%)$ and toluene $(1.0 \mathrm{~mL})$ was subjected to a vial containing the stirring bar. [For conditions 2, DBU (0.3 mmol) or $\mathrm{K}_{2} \mathrm{CO}_{3}(0.3 \mathrm{mmol})$ was then added to the mixture; For conditions 3 , DBU $(0.3 \mathrm{mmol})$ was then added to the mixture]. The reaction was stirred at room temperature or $33^{\circ} \mathrm{C}$ and monitored by TLC. In all cases, the reaction proceeded smoothly with full conversion of $\mathbf{1 5}$. The reaction was even faster if the iridium-catalyzed isomerization was conducted under condition 2. More importantly, product 16 could be readily 
isolated in 69\% ee (>98\% es) (PS: Compound 16 is easily racemizes at ambient temperature, so a quick test to confirm its optical purity is necessary.). These results indicated that the iridium catalyzed stereospecific 1,3-hydride transfer may occur for central-to-axial chirality transfer (Figure S5). After the reaction completed, compound $\mathbf{1 6}$ can be readily isolated beyond 90\% yield in all cases. Taking conditions 3 for example, $91 \%$ yield and $67 \%$ ee of $\mathbf{1 6}$ was obtained after the reaction completed $(96 \%$ es). This result further indicated that the reason of the relative lower ee of axially chiral styrenes bearing non-8-substituted naphthols may be attributed to their low configurational stability, that was consistent of our results illustrated in Table $\mathbf{3}, \mathbf{3 t}-\mathbf{3 y}$.

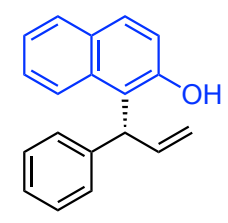

$15,70 \%$ ee
Condition 1: [lr] cat. (3.0 mol\%), rt, $12 \mathrm{~h}$

Condition 2: [Ir] cat. (3.0 mol\%), DBU or $\mathrm{K}_{2} \mathrm{CO}_{3}, \mathrm{rt}, 2 \mathrm{~h}$

Condition 3: [lr] cat. (3.0 mol\%), DBU, $33^{\circ} \mathrm{C}, 1 \mathrm{~h}$

\section{1,3-hydride transfer}

Full conversion

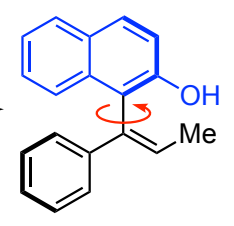

16

Figure S5 Stereospecific 1,3-H transfer of 1-(1-phenylallyl)naphthalen-2-ol by iridium

The isomerization was also conducted using the iridacycle catalyst. The experiment was performed in an argon-filled glove box. $[\operatorname{Ir}(\operatorname{cod}) \mathrm{Cl}]_{2}(2.1 \mathrm{mg}, 3 \mathrm{~mol} \%)$, L1 (6 mol\%), and TBD (15 mol\%) were added to a vial equipped with a magnetic stirring bar. The vial was then charged with toluene $(1.0 \mathrm{~mL})$ and stirred at $25{ }^{\circ} \mathrm{C}$ for $30 \mathrm{~min}$, generating an orange solution (Figure S6). The iridacycle catalyst containing the solvent was used directly for the catalysis system. As a result, the reaction also proceeded smoothly with full conversion in the presence of iridacycle catalyst at $33{ }^{\circ} \mathrm{C}$. Compound 16 could be readily isolated in $89 \%$ yield and $68 \%$ ee within $1.0 \mathrm{~h}(>97 \%$ es) (PS: Compound $\mathbf{1 6}$ is easily racemizes at ambient temperature, so a quick test to confirm its optical purity is necessary.). This result indicated that the ligand of iridium was not the key point for the stereospecific 1,3-hydride transfer process. 

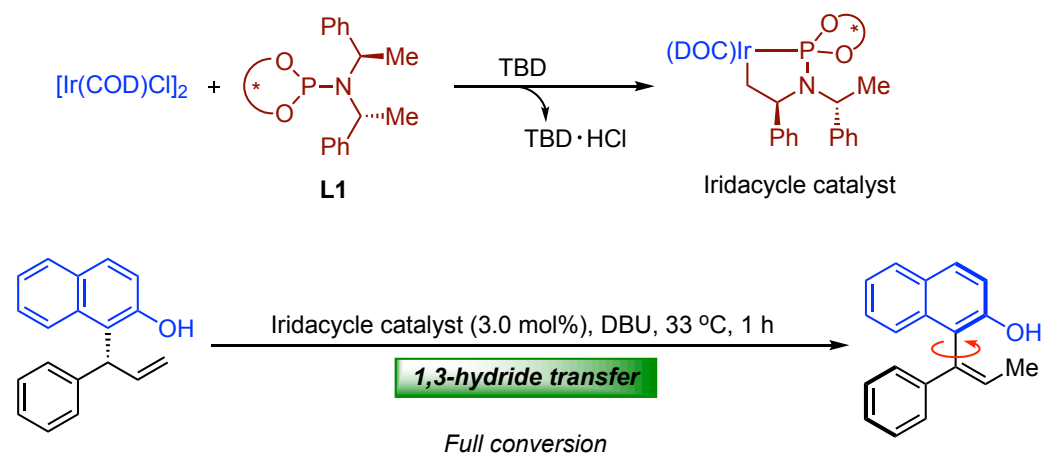

$15,70 \%$ ee

$16,68 \%$ ee

Figure S6 Stereospecific 1,3-H transfer catalyzed by iridacycle catalyst

X-ray structures of $3 f$ and $\mathbf{5 b}$.<smiles>C/C=C(\c1ccc(OC)cc1)c1c(OC)ccc2cccc(Br)c12</smiles>

$3 f$

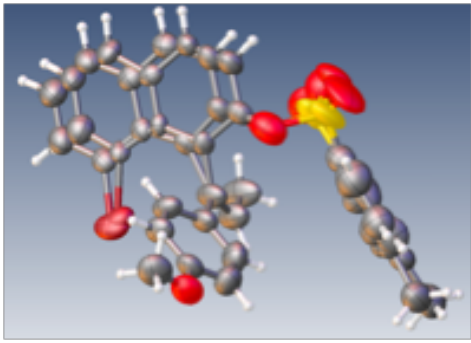

3f

Table 1 Crystal data and structure refinement for 2039913.

Identification code

2039913

Empirical formula

$\mathrm{C}_{27} \mathrm{H}_{23} \mathrm{BrNO}_{4} \mathrm{~S}$

Formula weight

523.42

Temperature/K

193(2)

Crystal system

orthorhombic

Space group

$\mathrm{P} 2{ }_{1} 2{ }_{1}{ }_{1}$

$\mathrm{a} / \AA$

7.2381(2)

$\mathrm{b} / \AA$

$10.1179(3)$

c/Å

32.6974(11)

$\alpha /^{\circ}$

90

$\beta /{ }^{\circ}$

90

$\gamma /{ }^{\circ}$

90

Volume $/ \AA^{3}$

2394.57(13)

$\mathrm{Z}$

4

$\rho_{\text {calc }} \mathrm{g} / \mathrm{cm}^{3}$

1.452

$\mu / \mathrm{mm}^{-1}$

2.249

$\mathrm{F}(000)$

1072.0

Crystal size $/ \mathrm{mm}^{3}$

$0.120 \times 0.110 \times 0.060$

Radiation

$\mathrm{GaK} \alpha(\lambda=1.34139)$

$2 \Theta$ range for data collection $/{ }^{\circ} 4.702$ to 107.862 
Index ranges

Reflections collected

Independent reflections

Data/restraints/parameter

Goodness-of-fit on $\mathrm{F}^{2}$

Final R indexes $[\mathrm{I}>=2 \sigma(\mathrm{I})] \quad \mathrm{R}_{1}=0.0534, \mathrm{wR}_{2}=0.1368$

Final R indexes [all data] $\quad \mathrm{R}_{1}=0.0610, \mathrm{wR}_{2}=0.1438$

Largest diff. peak/hole / e $\AA^{-3} 0.35 /-0.69$

Flack parameter

$0.008(9)$<smiles>C/C=C(\c1ccc(OC)cc1)c1c(C)c(C)c2ccccc2c1OC</smiles>

$5 b$

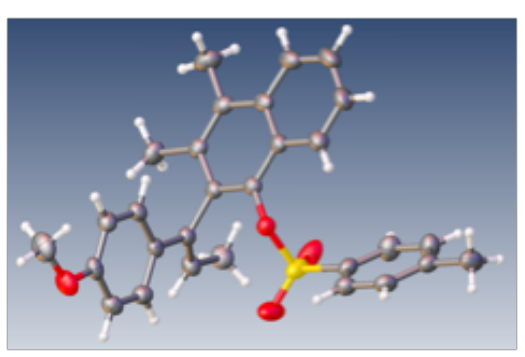

5b

Table 1 Crystal data and structure refinement for $\mathbf{2 0 3 9 9 2 0 .}$

Identification code

2039920

Empirical formula

$\mathrm{C}_{29} \mathrm{H}_{28} \mathrm{O}_{4} \mathrm{~S}$

Formula weight

472.57

Temperature/K

296(2)

Crystal system

orthorhombic

Space group

$\mathrm{P} 2{ }_{1} 2{ }_{1} 21$
a/Å
7.150(7)
$\mathrm{b} / \AA$
$12.868(13)$
c/Å
26.26(3)
$\alpha{ }^{\circ}$
90
$\beta /{ }^{\circ}$
90
$\gamma /{ }^{\circ}$
90
Volume $/ \AA^{3}$
$\mathrm{Z}$
2416(4)
$\rho_{\text {calc }} / \mathrm{cm}^{3}$
4
$\mu / \mathrm{mm}^{-1}$
1.299
$\mathrm{F}(000)$
1.459
Crystal size $/ \mathrm{mm}^{3}$
1000.0
Radiation
$0.200 \times 0.180 \times 0.160$
$\mathrm{CuK} \alpha(\lambda=1.54184)$

$2 \Theta$ range for data collection $/{ }^{\circ} 6.734$ to 136.662 
Index ranges

Reflections collected

Independent reflections

Data/restraints/parameters

Goodness-of-fit on $\mathrm{F}^{2}$

Final R indexes [I>=2 $\sigma(\mathrm{I})]$

Final R indexes [all data]

Largest diff. peak/hole / e $\AA^{-3}$

Flack parameter
$-7 \leq \mathrm{h} \leq 8,-15 \leq \mathrm{k} \leq 12,-31 \leq \mathrm{l} \leq 31$

8736

$4232\left[\mathrm{R}_{\text {int }}=0.0320, \mathrm{R}_{\text {sigma }}=0.0479\right]$

$4232 / 0 / 312$

1.018

$\mathrm{R}_{1}=0.0385, \mathrm{wR}_{2}=0.1013$

$\mathrm{R}_{1}=0.0414, \mathrm{wR}_{2}=0.1034$

\section{DFT Studies}

\section{Computational methods}

All density functional theory (DFT) calculations were performed using the Gaussian 16 software package. ${ }^{[4]}$ Geometries were optimized in toluene with the SMD solvation mode ${ }^{[5]}$ using the M06-2X functional ${ }^{[6]}$ with a mixed basis set of LANL2DZ for Ir and 6-31G(d) for other atoms. Vibrational frequency calculations were performed for all the stationary points to confirm if each optimized structure is a local minimum or a transition state structure. Single point energies were calculated using M06-2X with a mixed basis set of SDD for Ir and 6-311++G(d,p) for other atoms in toluene using the SMD solvation model. Intrinsic reaction coordinate (IRC) calculations have demonstrated that the transition state connects two corresponding intermediates along the reaction coordinate.

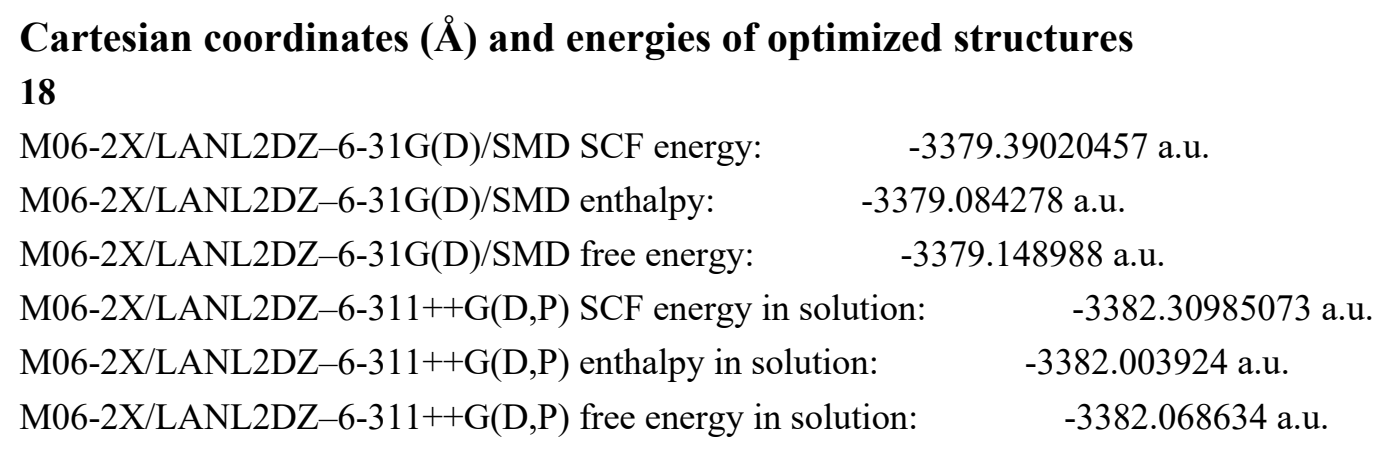

Cartesian coordinates

$\begin{array}{lcrr}\text { ATOM } & \text { X } & \text { Y } & \text { Z } \\ \text { C } & 2.504074 & -2.044861 & -0.370718 \\ \text { C } & 1.414451 & -1.239371 & -0.135690\end{array}$




\begin{tabular}{|c|c|c|c|}
\hline $\mathrm{C}$ & 1.511603 & 0.191852 & -0.097524 \\
\hline $\mathrm{C}$ & 2.794657 & 0.709095 & -0.485425 \\
\hline $\mathrm{C}$ & 3.899061 & -0.142642 & -0.735613 \\
\hline $\mathrm{C}$ & 3.773250 & -1.500530 & -0.643047 \\
\hline $\mathrm{H}$ & 2.367385 & -3.120059 & -0.384114 \\
\hline $\mathrm{C}$ & 2.976741 & 2.107372 & -0.642209 \\
\hline $\mathrm{H}$ & 4.847200 & 0.314218 & -1.004815 \\
\hline $\mathrm{H}$ & 4.615533 & -2.160255 & -0.821374 \\
\hline $\mathrm{C}$ & 1.950602 & 2.973562 & -0.420435 \\
\hline $\mathrm{C}$ & 0.714556 & 2.475138 & 0.054486 \\
\hline $\mathrm{H}$ & 3.950356 & 2.471367 & -0.956995 \\
\hline $\mathrm{H}$ & 2.078095 & 4.043882 & -0.563687 \\
\hline $\mathrm{C}$ & -0.809338 & 0.809913 & 1.032209 \\
\hline $\mathrm{H}$ & -1.069027 & 1.759941 & 1.515134 \\
\hline $\mathrm{C}$ & -0.610885 & -0.144200 & 2.201174 \\
\hline $\mathrm{H}$ & -1.427314 & -0.834717 & 2.400355 \\
\hline $\mathrm{C}$ & 0.450497 & -0.140942 & 3.002135 \\
\hline $\mathrm{H}$ & 1.295110 & 0.524462 & 2.838703 \\
\hline $\mathrm{H}$ & 0.515976 & -0.819642 & 3.846912 \\
\hline $\mathrm{C}$ & -2.031568 & 0.517377 & 0.165643 \\
\hline $\mathrm{C}$ & -3.262301 & 0.271445 & 0.782049 \\
\hline $\mathrm{C}$ & -1.986149 & 0.562564 & -1.228199 \\
\hline $\mathrm{C}$ & -4.410377 & 0.049574 & 0.026857 \\
\hline $\mathrm{C}$ & -3.133359 & 0.345705 & -1.987597 \\
\hline $\mathrm{H}$ & -1.040153 & 0.755368 & -1.726219 \\
\hline $\mathrm{C}$ & -4.350167 & 0.083155 & -1.364301 \\
\hline $\mathrm{H}$ & -3.072613 & 0.378303 & -3.071478 \\
\hline $\mathrm{H}$ & -5.244117 & -0.088824 & -1.955910 \\
\hline $\mathrm{H}$ & -3.328092 & 0.263175 & 1.867403 \\
\hline $\mathrm{H}$ & -5.354689 & -0.143148 & 0.527602 \\
\hline $\mathrm{O}$ & -0.283371 & 3.354122 & 0.341856 \\
\hline $\mathrm{H}$ & 0.038735 & 4.259066 & 0.213057 \\
\hline $\mathrm{C}$ & 0.480835 & 1.126258 & 0.278936 \\
\hline $\mathrm{Br}$ & -0.233215 & -2.185683 & -0.058738 \\
\hline
\end{tabular}

\section{$18 \mathbf{a}$}

M06-2X/LANL2DZ-6-31G(D)/SMD SCF energy: M06-2X/LANL2DZ-6-31G(D)/SMD enthalpy: -3379.39344980 a.u. M06-2X/LANL2DZ-6-31G(D)/SMD free energy: -3379.087364 a.u. -3379.153003 a.u. M06-2X/LANL2DZ-6-311++G(D,P) SCF energy in solution: -3382.31233367 a.u. M06-2X/LANL2DZ-6-311++G(D,P) enthalpy in solution: -3382.006248 a.u. 
Cartesian coordinates

$\begin{array}{lrrr}\text { ATOM } & \text { X } & \text { Y } & \text { Z } \\ \text { C } & 3.173963 & -1.511755 & -0.009120 \\ \text { C } & 1.885011 & -1.039772 & -0.058676 \\ \text { C } & 1.571323 & 0.356989 & 0.056904 \\ \text { C } & 2.685591 & 1.186346 & 0.424543 \\ \text { C } & 4.006959 & 0.675326 & 0.473999 \\ \text { C } & 4.260262 & -0.643123 & 0.218091 \\ \text { H } & 3.349252 & -2.577624 & -0.100383 \\ \text { C } & 2.466454 & 2.543664 & 0.768296 \\ \text { H } & 4.811501 & 1.359929 & 0.727445 \\ \text { H } & 5.269347 & -1.039861 & 0.244249 \\ \text { C } & 1.209719 & 3.072093 & 0.757368 \\ \text { C } & 0.129818 & 2.286436 & 0.295077 \\ \text { H } & 3.318910 & 3.149424 & 1.061164 \\ \text { H } & 1.032539 & 4.103417 & 1.052722 \\ \text { C } & -0.872849 & 0.350116 & -0.902928 \\ \text { H } & -0.537986 & -0.608531 & -1.296070 \\ \text { C } & -1.172333 & 1.184362 & -2.134363 \\ \text { H } & -1.595447 & 2.171387 & -1.969197 \\ \text { C } & -0.943228 & 0.750476 & -3.370773 \\ \text { H } & -0.526695 & -0.236506 & -3.562140 \\ \text { H } & -1.159397 & 1.367089 & -4.238727 \\ \text { C } & -2.116162 & 0.073146 & -0.060639 \\ \text { C } & -3.361410 & -0.075494 & -0.676850 \\ \text { C } & -2.031973 & -0.104413 & 1.322248 \\ \text { C } & -4.494818 & -0.386960 & 0.070354 \\ \text { C } & -3.162788 & -0.417450 & 2.071538 \\ \text { H } & -1.071164 & 0.004927 & 1.817675 \\ \text { C } & -4.400587 & -0.558594 & 1.448848 \\ \text { H } & -3.075130 & -0.550723 & 3.145891 \\ \text { H } & -5.283447 & -0.800537 & 2.032810 \\ \text { H } & -3.442895 & 0.052041 & -1.752692 \\ \text { H } & -5.453540 & -0.496189 & -0.428305 \\ \text { O } & -1.111686 & 2.833588 & 0.213550 \\ \text { H } & -1.075301 & 3.760383 & 0.495623 \\ & 0.287311 & 0.974880 & -0.127675 \\ \text { H } & 0.560324 & -2.417856 & -0.066741\end{array}$


19

M06-2X/LANL2DZ-6-31G(D)/SMD SCF energy:

M06-2X/LANL2DZ-6-31G(D)/SMD enthalpy:

-3841.25685339 a.u.

M06-2X/LANL2DZ-6-31G(D)/SMD free energy:

-3840.688672 a.u.

M06-2X/LANL2DZ-6-311++G(D,P) SCF energy in solution:

-3840.779126 a.u.

M06-2X/LANL2DZ-6-311++G(D,P) enthalpy in solution:

-3844.31253435 a.u.

M06-2X/LANL2DZ-6-311++G(D,P) free energy in solution:

-3843.744353 a.u.

-3843.834807 a.u.

Cartesian coordinates

$\begin{array}{lrrr}\text { ATOM } & \text { X } & \text { Y } & \text { Z } \\ \text { C } & 5.272734 & -0.258542 & -0.418741 \\ \text { C } & 3.946405 & 0.066213 & -0.270657 \\ \text { C } & 3.053049 & -0.719125 & 0.528066 \\ \text { C } & 3.683564 & -1.742137 & 1.313069 \\ \text { C } & 5.058729 & -2.054989 & 1.149508 \\ \text { C } & 5.834171 & -1.356913 & 0.266848 \\ \text { H } & 5.904516 & 0.372264 & -1.033652 \\ \text { C } & 2.926804 & -2.443140 & 2.282892 \\ \text { H } & 5.477819 & -2.860808 & 1.746498 \\ \text { H } & 6.883189 & -1.597140 & 0.127179 \\ \text { C } & 1.599074 & -2.162961 & 2.457162 \\ \text { C } & 0.959040 & -1.243458 & 1.602584 \\ \text { H } & 3.418431 & -3.200486 & 2.886537 \\ \text { H } & 1.014804 & -2.693192 & 3.207332 \\ \text { O } & -0.398148 & -1.072129 & 1.712233 \\ \text { C } & 0.839249 & 0.201514 & -0.412150 \\ \text { H } & -1.210429 & -0.637645 & -0.287801 \\ \text { C } & 1.018511 & -0.156865 & -1.796084 \\ \text { H } & 0.657464 & 0.580423 & -2.516279 \\ \text { C } & 1.595838 & -1.260635 & -2.329347 \\ \text { H } & 2.013601 & -2.054504 & -1.715134 \\ \text { H } & 1.709110 & -1.361824 & -3.404042 \\ \text { C } & 0.068505 & 1.352740 & -0.035311 \\ \text { C } & 0.130580 & 1.903432 & 1.277307 \\ \text { C } & -0.831373 & 2.015386 & -0.922310 \\ \text { C } & -0.635222 & 2.992707 & 1.662568 \\ \text { C } & -1.580694 & 3.116801 & -0.530652 \\ \text { H } & -0.959066 & 1.640518 & -1.933880 \\ \text { C } & -1.505753 & 3.624549 & 0.768104 \\ \text { H } & -2.245136 & 3.584010 & -1.255272 \\ \text { H } & -2.095796 & 4.484082 & 1.070195 \\ \text { H } & 0.817031 & 1.459486 & 1.992761 \\ \text { H } & -0.538563 & 3.369417 & 2.678713\end{array}$




$\begin{array}{lrrr}\mathrm{C} & -5.735995 & -0.673500 & -0.368184 \\ \mathrm{C} & -3.231597 & -0.640555 & -0.230455 \\ \mathrm{C} & -5.942082 & -0.532066 & 1.136836 \\ \mathrm{C} & -3.173210 & 0.239682 & 0.998852 \\ \mathrm{C} & -5.550830 & 0.845140 & 1.673441 \\ \mathrm{C} & -4.237809 & 1.337973 & 1.067417 \\ \mathrm{H} & -6.041810 & 0.242378 & -0.890990 \\ \mathrm{H} & -3.233684 & -0.409034 & 1.882556 \\ \mathrm{H} & -5.381525 & -1.327479 & 1.641147 \\ \mathrm{H} & -5.460180 & 0.792031 & 2.764257 \\ \mathrm{H} & -6.375837 & -1.479207 & -0.733903 \\ \mathrm{H} & -6.999088 & -0.724805 & 1.345748 \\ \mathrm{H} & -2.174867 & 0.683670 & 1.015913 \\ \mathrm{H} & -6.347159 & 1.566011 & 1.456981 \\ \mathrm{H} & -3.831774 & 2.163920 & 1.656621 \\ \mathrm{H} & -4.401435 & 1.743373 & 0.061818 \\ \mathrm{C} & -4.306449 & -1.773291 & -2.076534 \\ \mathrm{C} & -3.116037 & -2.718410 & -2.089235 \\ \mathrm{C} & -1.853958 & -1.913104 & -1.853187 \\ \mathrm{H} & -0.995561 & -2.540399 & -1.605458 \\ \mathrm{H} & -3.242047 & -3.471318 & -1.304384 \\ \mathrm{H} & -3.060905 & -3.234621 & -3.050152 \\ \mathrm{H} & -4.237609 & -1.050164 & -2.898830 \\ \mathrm{H} & -5.240286 & -2.325754 & -2.190953 \\ \mathrm{H} & -1.578244 & -1.327639 & -2.736080 \\ \mathrm{~N} & -2.067770 & -1.003076 & -0.730050 \\ \mathrm{~N} & -4.373532 & -1.045110 & -0.798590 \\ \mathrm{Br} & 3.466443 & 1.747911 & -1.030611 \\ \mathrm{H} & -0.720015 & -1.627387 & 2.438185 \\ \mathrm{C} & 1.618731 & -0.549900 & 0.591254\end{array}$

\section{(M)-20}

M06-2X/LANL2DZ-6-31G(D)/SMD SCF energy:

-3379.41280811 a.u.

M06-2X/LANL2DZ-6-31G(D)/SMD enthalpy:

-3379.106932 a.u.

M06-2X/LANL2DZ-6-31G(D)/SMD free energy:

-3379.173431 a.u.

M06-2X/LANL2DZ-6-311++G(D,P) SCF energy in solution:

-3382.32995253 a.u.

M06-2X/LANL2DZ-6-311++G(D,P) enthalpy in solution:

-3382.024076 a.u.

M06-2X/LANL2DZ-6-311++G(D,P) free energy in solution:

-3382.090575 a.u.

Cartesian coordinates

$\begin{array}{llll}\text { ATOM } & \mathrm{X} & \mathrm{Y} & \mathrm{Z}\end{array}$




$\begin{array}{lrrr}\mathrm{C} & -3.064528 & -1.411079 & -0.487224 \\ \mathrm{C} & -1.798494 & -0.968193 & -0.188631 \\ \mathrm{C} & -1.473170 & 0.427530 & -0.129625 \\ \mathrm{C} & -2.533954 & 1.315449 & -0.516699 \\ \mathrm{C} & -3.828197 & 0.830088 & -0.829950 \\ \mathrm{C} & -4.103165 & -0.508446 & -0.790871 \\ \mathrm{H} & -3.254703 & -2.477833 & -0.516392 \\ \mathrm{C} & -2.289631 & 2.709848 & -0.594715 \\ \mathrm{H} & -4.594824 & 1.550188 & -1.102110 \\ \mathrm{H} & -5.093121 & -0.887596 & -1.020557 \\ \mathrm{C} & -1.065190 & 3.229137 & -0.292094 \\ \mathrm{C} & -0.037663 & 2.366718 & 0.153232 \\ \mathrm{H} & -3.101991 & 3.360922 & -0.904233 \\ \mathrm{H} & -0.879341 & 4.298798 & -0.355166 \\ \mathrm{C} & 0.922969 & 0.257900 & 0.893915 \\ \mathrm{C} & 0.838468 & -0.201415 & 2.149649 \\ \mathrm{H} & 1.695109 & -0.742508 & 2.549615 \\ \mathrm{C} & -0.358061 & -0.118090 & 3.044274 \\ \mathrm{H} & -1.100556 & 0.595081 & 2.678124 \\ \mathrm{H} & -0.842356 & -1.100448 & 3.125523 \\ \mathrm{C} & 2.164196 & 0.106226 & 0.089895 \\ \mathrm{C} & 3.430186 & 0.149661 & 0.687143 \\ \mathrm{C} & 2.088091 & -0.086820 & -1.294545 \\ \mathrm{C} & 4.582488 & -0.023780 & -0.072156 \\ \mathrm{C} & 3.240389 & -0.258879 & -2.055756 \\ \mathrm{H} & 1.113584 & -0.113659 & -1.774589 \\ \mathrm{C} & 4.492940 & -0.232011 & -1.447319 \\ \mathrm{H} & 3.158353 & -0.416496 & -3.127134 \\ \mathrm{H} & 5.392886 & -0.362287 & -2.040601 \\ \mathrm{H} & -0.511004 & -2.358876 & 0.002990 \\ \mathrm{H} & -0.064941 & 0.176631 & 4.057897 \\ \mathrm{O} & & & \end{array}$

\section{(P)-20}

M06-2X/LANL2DZ-6-31G(D)/SMD SCF energy: -3379.41280811 a.u. M06-2X/LANL2DZ-6-31G(D)/SMD enthalpy: M06-2X/LANL2DZ-6-31G(D)/SMD free energy: -3379.106932 a.u. -3379.173431 a.u. 
M06-2X/LANL2DZ-6-311++G(D,P) SCF energy in solution: M06-2X/LANL2DZ-6-311++G(D,P) enthalpy in solution: M06-2X/LANL2DZ-6-311++G(D,P) free energy in solution:
-3382.32995253 a.u. -3382.024076 a.u. -3382.090575 a.u.

Cartesian coordinates

$\begin{array}{lrrr}\text { ATOM } & \text { X } & \text { Y } & \text { Z } \\ \text { C } & 3.064528 & -1.411079 & -0.487224 \\ \text { C } & 1.798494 & -0.968193 & -0.188631 \\ \text { C } & 1.473170 & 0.427530 & -0.129625 \\ \text { C } & 2.533954 & 1.315449 & -0.516699 \\ \text { C } & 3.828197 & 0.830088 & -0.829950 \\ \text { C } & 4.103165 & -0.508446 & -0.790871 \\ \text { H } & 3.254703 & -2.477833 & -0.516392 \\ \text { C } & 2.289631 & 2.709848 & -0.594715 \\ \text { H } & 4.594824 & 1.550188 & -1.102110 \\ \text { H } & 5.093121 & -0.887596 & -1.020557 \\ \text { C } & 1.065190 & 3.229137 & -0.292094 \\ \text { C } & 0.037663 & 2.366718 & 0.153232 \\ \text { H } & 3.101991 & 3.360922 & -0.904233 \\ \text { H } & 0.879341 & 4.298798 & -0.355166 \\ \text { C } & -0.922969 & 0.257900 & 0.893915 \\ \text { C } & -0.838468 & -0.201415 & 2.149649 \\ \text { H } & -1.695109 & -0.742508 & 2.549615 \\ \text { C } & 0.358061 & -0.118090 & 3.044274 \\ \text { H } & 1.100556 & 0.595081 & 2.678124 \\ \text { H } & 0.842356 & -1.100448 & 3.125523 \\ \text { C } & -2.164196 & 0.106226 & 0.089895 \\ \text { C } & -3.430186 & 0.149661 & 0.687143 \\ \text { C } & -2.088091 & -0.086820 & -1.294545 \\ \text { C } & -4.582488 & -0.023780 & -0.072156 \\ \text { C } & -3.240389 & -0.258879 & -2.055756 \\ \text { H } & -1.113584 & -0.113659 & -1.774589 \\ \text { C } & -4.492940 & -0.232011 & -1.447319 \\ \text { H } & -3.158353 & -0.416496 & -3.127134 \\ \text { H } & -5.392886 & -0.362287 & -2.040601 \\ \text { H } & -3.508825 & 0.348419 & 1.751969 \\ \text { H } & -5.554984 & 0.018530 & 0.409544 \\ \text { O } & -1.160227 & 2.875205 & 0.535278 \\ \text { H } & -1.165068 & 3.832689 & 0.384385 \\ \text { C } & 0.216618 & 0.995916 & 0.264285 \\ \text { H } & 0.511004 & -2.358876 & 0.002990 \\ & 0.064941 & 0.176631 & 4.057897\end{array}$


21

M06-2X/LANL2DZ-6-31G(D)/SMD SCF energy:

-4256.12030383 a.u.

M06-2X/LANL2DZ-6-31G(D)/SMD enthalpy:

-4255.614475 a.u.

M06-2X/LANL2DZ-6-31G(D)/SMD free energy:

-4255.705710 a.u.

M06-2X/LANL2DZ-6-311++G(D,P) SCF energy in solution:

-4258.84079015 a.u.

M06-2X/LANL2DZ-6-311++G(D,P) enthalpy in solution:

-4258.334961 a.u.

M06-2X/LANL2DZ-6-311++G(D,P) free energy in solution:

-4258.426196 a.u.

Cartesian coordinates

$\begin{array}{lccc}\text { ATOM } & \text { X } & \text { Y } & \text { Z } \\ \text { C } & 4.315553 & -0.998032 & -2.187541 \\ \text { C } & 3.424994 & -0.425279 & -1.308321 \\ \text { C } & 3.092495 & -1.020718 & -0.044824 \\ \text { C } & 3.870580 & -2.187193 & 0.276664 \\ \text { C } & 4.792678 & -2.748338 & -0.640449 \\ \text { C } & 4.993770 & -2.188008 & -1.870612 \\ \text { H } & 4.518839 & -0.498931 & -3.127832 \\ \text { C } & 3.739017 & -2.809773 & 1.545610 \\ \text { H } & 5.335114 & -3.638988 & -0.336837 \\ \text { H } & 5.688982 & -2.618981 & -2.582914 \\ \text { C } & 2.859337 & -2.331336 & 2.465832 \\ \text { C } & 2.022343 & -1.242382 & 2.130172 \\ \text { H } & 4.357571 & -3.676219 & 1.761162 \\ \text { H } & 2.741234 & -2.780765 & 3.445666 \\ \text { C } & 0.998635 & 0.450414 & 0.674407 \\ \text { H } & 0.259323 & 0.231407 & 1.452626 \\ \text { C } & 0.240658 & 0.264806 & -0.635395 \\ \text { H } & 0.015427 & 1.167655 & -1.196138 \\ \text { C } & -0.032931 & -0.942447 & -1.197915 \\ \text { H } & 0.300883 & -1.866926 & -0.734445 \\ \text { H } & -0.418732 & -1.009376 & -2.210226 \\ \text { C } & 1.382803 & 1.895620 & 0.975466 \\ \text { C } & 0.387131 & 2.875157 & 0.942971 \\ \text { C } & 2.671446 & 2.266107 & 1.358373 \\ \text { C } & 0.676960 & 4.201780 & 1.248432 \\ \text { C } & 2.964537 & 3.591364 & 1.670840 \\ \text { H } & 3.455520 & 1.515850 & 1.403105 \\ \text { C } & 1.971973 & 4.565788 & 1.609771 \\ \text { H } & 3.975374 & 3.861488 & 1.961202 \\ \text { H } & 2.202619 & 5.598615 & 1.851842 \\ \text { H } & -0.634057 & 2.589414 & 0.698040\end{array}$




$\begin{array}{lccc}\mathrm{H} & -0.111490 & 4.947862 & 1.215647 \\ \mathrm{O} & 1.133912 & -0.851483 & 3.095658 \\ \mathrm{H} & 0.256289 & -1.226276 & 2.866293 \\ \mathrm{C} & 2.088230 & -0.594651 & 0.903889 \\ \mathrm{Br} & 2.819808 & 1.289167 & -1.882191 \\ \mathrm{Ir} & -2.086349 & -0.437498 & -0.050354 \\ \mathrm{C} & -2.703621 & 1.413759 & -0.822627 \\ \mathrm{C} & -2.886919 & 0.410681 & -1.811296 \\ \mathrm{C} & -4.007464 & -0.329352 & 0.900939 \\ \mathrm{C} & -4.260879 & -0.128386 & -2.192413 \\ \mathrm{C} & -3.985394 & -1.399421 & -0.015956 \\ \mathrm{C} & -4.616160 & -1.389663 & -1.389547 \\ \mathrm{H} & -1.851551 & 2.078499 & -0.961285 \\ \mathrm{H} & -5.011446 & 0.654575 & -2.044453 \\ \mathrm{H} & -3.794442 & -2.390576 & 0.392609 \\ \mathrm{H} & -2.143909 & 0.375201 & -2.607946 \\ \mathrm{H} & -4.272860 & -0.363426 & -3.260352 \\ \mathrm{H} & -5.705015 & -1.508168 & -1.302549 \\ \mathrm{C} & -3.808139 & 2.028823 & 0.012681 \\ \mathrm{H} & -4.374034 & 2.760140 & -0.580967 \\ \mathrm{H} & -3.328391 & 2.589561 & 0.822763 \\ \mathrm{C} & -4.742242 & 0.973488 & 0.625103 \\ \mathrm{H} & -3.841898 & -0.578400 & 1.947437 \\ \mathrm{H} & -5.595908 & 0.778829 & -0.031691 \\ \mathrm{H} & -5.157677 & 1.354474 & 1.562071 \\ \mathrm{H} & -4.245730 & -2.271163 & -1.922177 \\ \mathrm{Cl} & -1.319535 & -2.112578 & 1.597794\end{array}$

22

M06-2X/LANL2DZ-6-31G(D)/SMD SCF energy:

M06-2X/LANL2DZ-6-31G(D)/SMD enthalpy:

M06-2X/LANL2DZ-6-31G(D)/SMD free energy:

-922.69166163 a.u.

-922.414750 a.u.

M06-2X/LANL2DZ-6-311++G(D,P) SCF energy in solution:

-922.467210 a.u.

M06-2X/LANL2DZ-6-311++G(D,P) enthalpy in solution:

-922.86538136 a.u.

M06-2X/LANL2DZ-6-311++G(D,P) free energy in solution:

-922.588470 a.u.

-922.640930 a.u.

Cartesian coordinates

$\begin{array}{lccc}\text { ATOM } & \mathrm{X} & \mathrm{Y} & \mathrm{Z} \\ \text { C } & -2.437216 & 0.595428 & 0.150109 \\ \text { C } & 0.014460 & 0.099429 & -0.013662 \\ \text { C } & -2.867403 & -0.597084 & -0.699641\end{array}$




$\begin{array}{lccc}\mathrm{C} & -0.157584 & -1.394872 & -0.175830 \\ \mathrm{C} & -2.634210 & -1.947744 & -0.020350 \\ \mathrm{C} & -1.273853 & -2.009175 & 0.674238 \\ \mathrm{H} & -2.715253 & 0.443946 & 1.201954 \\ \mathrm{H} & -0.319550 & -1.609055 & -1.239385 \\ \mathrm{H} & -2.347029 & -0.543472 & -1.662281 \\ \mathrm{H} & -2.702107 & -2.741371 & -0.772669 \\ \mathrm{H} & -2.972130 & 1.482239 & -0.196275 \\ \mathrm{H} & -3.932419 & -0.476931 & -0.921736 \\ \mathrm{H} & 0.811158 & -1.836569 & 0.074548 \\ \mathrm{H} & -3.427500 & -2.135818 & 0.711745 \\ \mathrm{H} & -1.012511 & -3.047998 & 0.892861 \\ \mathrm{H} & -1.316823 & -1.497034 & 1.642917 \\ \mathrm{C} & -0.723483 & 2.378157 & 0.341611 \\ \mathrm{C} & 0.571514 & 2.807921 & -0.330698 \\ \mathrm{C} & 1.692301 & 1.899759 & 0.138647 \\ \mathrm{H} & 2.591908 & 2.005252 & -0.472063 \\ \mathrm{H} & 0.459351 & 2.735147 & -1.417115 \\ \mathrm{H} & 0.789167 & 3.848540 & -0.079788 \\ \mathrm{H} & -0.670334 & 2.536813 & 1.426288 \\ \mathrm{H} & -1.564310 & 2.959906 & -0.041397 \\ \mathrm{H} & 1.965362 & 2.111625 & 1.178232 \\ \mathrm{~N} & 1.259158 & 0.513672 & 0.034708 \\ \mathrm{~N} & -1.009442 & 0.959308 & 0.073892 \\ \mathrm{H} & 2.026742 & -0.212505 & -0.022013 \\ \mathrm{Cl} & 3.498889 & -1.489676 & -0.085328\end{array}$

23

M06-2X/LANL2DZ-6-31G(D)/SMD SCF energy: M06-2X/LANL2DZ-6-31G(D)/SMD enthalpy: M06-2X/LANL2DZ-6-31G(D)/SMD free energy: -3795.32343253 a.u. -3794.833320 a.u. -3794.916316 a.u.

M06-2X/LANL2DZ-6-311++G(D,P) SCF energy in solution: -3797.99847186 a.u. M06-2X/LANL2DZ-6-311++G(D,P) enthalpy in solution: M06-2X/LANL2DZ-6-311++G(D,P) free energy in solution: -3797.508359 a.u. -3797.591355 a.u.

Cartesian coordinates

$\begin{array}{lcrr}\text { ATOM } & \text { X } & \text { Y } & \text { Z } \\ \text { C } & -4.790122 & -1.236484 & 0.923873 \\ \text { C } & -3.524858 & -0.729608 & 0.742643 \\ \text { C } & -2.545588 & -1.368158 & -0.081465 \\ \text { C } & -2.905972 & -2.685206 & -0.522064\end{array}$




\begin{tabular}{|c|c|c|c|}
\hline $\mathrm{C}$ & -4.210440 & -3.193785 & -0.322292 \\
\hline $\mathrm{C}$ & -5.160659 & -2.465143 & 0.345302 \\
\hline $\mathrm{H}$ & -5.486698 & -0.707235 & 1.564340 \\
\hline $\mathrm{C}$ & -1.920860 & -3.502502 & -1.145860 \\
\hline $\mathrm{H}$ & -4.438084 & -4.184881 & -0.705663 \\
\hline $\mathrm{H}$ & -6.165001 & -2.848253 & 0.490861 \\
\hline $\mathrm{C}$ & -0.643192 & -3.062767 & -1.300294 \\
\hline $\mathrm{C}$ & -0.290906 & -1.720372 & -0.954064 \\
\hline $\mathrm{H}$ & -2.208329 & -4.500894 & -1.465699 \\
\hline $\mathrm{H}$ & 0.131304 & -3.686762 & -1.735312 \\
\hline $\mathrm{C}$ & -0.861799 & 0.621250 & -0.588688 \\
\hline $\mathrm{H}$ & -0.244606 & 0.623635 & -1.501859 \\
\hline $\mathrm{C}$ & 0.105457 & 1.088298 & 0.475686 \\
\hline $\mathrm{H}$ & 0.464912 & 2.109733 & 0.368765 \\
\hline $\mathrm{C}$ & 0.444841 & 0.383853 & 1.599687 \\
\hline $\mathrm{H}$ & -0.031833 & -0.573133 & 1.806411 \\
\hline $\mathrm{H}$ & 0.965204 & 0.865788 & 2.422041 \\
\hline $\mathrm{C}$ & -1.966207 & 1.616713 & -0.947575 \\
\hline $\mathrm{C}$ & -2.031267 & 2.911176 & -0.426336 \\
\hline $\mathrm{C}$ & -2.898275 & 1.250499 & -1.924251 \\
\hline $\mathrm{C}$ & -3.004229 & 3.808948 & -0.858335 \\
\hline $\mathrm{C}$ & -3.876993 & 2.142559 & -2.351458 \\
\hline $\mathrm{H}$ & -2.857033 & 0.252098 & -2.351221 \\
\hline $\mathrm{C}$ & -3.934991 & 3.428668 & -1.820422 \\
\hline $\mathrm{H}$ & -4.594143 & 1.829944 & -3.104587 \\
\hline $\mathrm{H}$ & -4.697881 & 4.126136 & -2.152267 \\
\hline $\mathrm{H}$ & -1.342479 & 3.225391 & 0.351655 \\
\hline $\mathrm{H}$ & -3.038437 & 4.806636 & -0.430660 \\
\hline $\mathrm{O}$ & 0.948363 & -1.339006 & -1.192761 \\
\hline $\mathrm{C}$ & -1.266952 & -0.843192 & -0.463516 \\
\hline $\mathrm{Br}$ & -3.072658 & 0.732957 & 1.877525 \\
\hline Ir & 2.085899 & -0.134098 & 0.073896 \\
\hline $\mathrm{C}$ & 3.169766 & 1.511045 & 0.822633 \\
\hline $\mathrm{C}$ & 3.524641 & 0.342380 & 1.545990 \\
\hline $\mathrm{C}$ & 3.623583 & -0.032303 & -1.460251 \\
\hline $\mathrm{C}$ & 4.850493 & -0.389619 & 1.353700 \\
\hline $\mathrm{C}$ & 3.727681 & -1.244391 & -0.764459 \\
\hline $\mathrm{C}$ & 4.741691 & -1.528236 & 0.323636 \\
\hline $\mathrm{H}$ & 2.504081 & 2.213244 & 1.327048 \\
\hline $\mathrm{H}$ & 5.626045 & 0.326766 & 1.065422 \\
\hline $\mathrm{H}$ & 3.261185 & -2.115623 & -1.219263 \\
\hline $\mathrm{H}$ & 3.088615 & 0.227854 & 2.537329 \\
\hline $\mathrm{H}$ & 5.169678 & -0.806805 & 2.313174 \\
\hline
\end{tabular}




$\begin{array}{lrrc}\mathrm{H} & 5.723078 & -1.747438 & -0.119351 \\ \mathrm{C} & 4.008978 & 2.158853 & -0.260799 \\ \mathrm{H} & 4.821612 & 2.753811 & 0.178556 \\ \mathrm{H} & 3.359682 & 2.862265 & -0.794210 \\ \mathrm{C} & 4.567924 & 1.140560 & -1.269146 \\ \mathrm{H} & 3.050581 & -0.047672 & -2.385968 \\ \mathrm{H} & 5.547488 & 0.771245 & -0.952526 \\ \mathrm{H} & 4.721985 & 1.631580 & -2.234393 \\ \mathrm{H} & 4.420522 & -2.441533 & 0.834836\end{array}$

\section{$23 \mathbf{a}$}

M06-2X/LANL2DZ-6-31G(D)/SMD SCF energy: -3795.31158371 a.u. M06-2X/LANL2DZ-6-31G(D)/SMD enthalpy: -3794.821559 a.u. M06-2X/LANL2DZ-6-31G(D)/SMD free energy: -3794.904706 a.u.

M06-2X/LANL2DZ-6-311++G(D,P) SCF energy in solution: -3797.98644616 a.u. M06-2X/LANL2DZ-6-311++G(D,P) enthalpy in solution: -3797.496421 a.u. M06-2X/LANL2DZ-6-311++G(D,P) free energy in solution: -3797.579568 a.u.

Cartesian coordinates

$\begin{array}{lrrr}\text { ATOM } & \text { X } & \text { Y } & \text { Z } \\ \text { C } & 0.237160 & 3.955184 & -0.381709 \\ \text { C } & 0.365422 & 2.580725 & -0.536830 \\ \text { C } & 0.707253 & 1.710408 & 0.527435 \\ \text { C } & 0.799524 & 2.346020 & 1.799954 \\ \text { C } & 0.652146 & 3.731055 & 1.954667 \\ \text { C } & 0.398835 & 4.546115 & 0.868896 \\ \text { H } & -0.015551 & 4.561407 & -1.244186 \\ \text { C } & 1.024254 & 1.546201 & 2.989755 \\ \text { H } & 0.741746 & 4.152231 & 2.952262 \\ \text { H } & 0.294881 & 5.619612 & 0.982509 \\ \text { C } & 1.158810 & 0.211117 & 2.947687 \\ \text { C } & 1.205566 & -0.506460 & 1.668458 \\ \text { H } & 1.067172 & 2.076726 & 3.938685 \\ \text { H } & 1.335142 & -0.383317 & 3.838893 \\ \text { C } & 1.664197 & -0.325816 & -0.803495 \\ \text { H } & 1.791541 & 0.491499 & -1.513471 \\ \text { C } & 0.692037 & -1.304847 & -1.441745 \\ \text { H } & 0.307473 & -1.035140 & -2.424083 \\ \text { C } & 0.303903 & -2.505275 & -0.894918 \\ \text { H } & 0.797566 & -2.889973 & -0.007070 \\ \text { H } & -0.307971 & -3.190803 & -1.474208\end{array}$




\begin{tabular}{lccc}
$\mathrm{C}$ & 3.075055 & -0.831722 & -0.534070 \\
$\mathrm{C}$ & 3.580652 & -1.984667 & -1.132811 \\
$\mathrm{C}$ & 3.931313 & -0.060093 & 0.258014 \\
$\mathrm{C}$ & 4.908713 & -2.363610 & -0.939242 \\
$\mathrm{C}$ & 5.254909 & -0.435969 & 0.454702 \\
$\mathrm{H}$ & 3.550459 & 0.842873 & 0.731843 \\
$\mathrm{C}$ & 5.750817 & -1.593415 & -0.144277 \\
$\mathrm{H}$ & 5.901250 & 0.175079 & 1.077966 \\
$\mathrm{H}$ & 6.783672 & -1.890536 & 0.009474 \\
$\mathrm{H}$ & 2.935294 & -2.598708 & -1.754008 \\
$\mathrm{H}$ & 5.282091 & -3.267759 & -1.411320 \\
$\mathrm{O}$ & 1.522494 & -1.702803 & 1.661698 \\
$\mathrm{C}$ & 0.903458 & 0.245005 & 0.425923 \\
$\mathrm{Br}$ & -0.112337 & 1.965533 & -2.285842 \\
$\mathrm{Ir}$ & -0.999108 & -0.875477 & 0.040482 \\
$\mathrm{C}$ & -2.617110 & -1.668809 & -1.112947 \\
$\mathrm{C}$ & -2.616988 & -2.285463 & 0.159212 \\
$\mathrm{C}$ & -2.435930 & 0.737366 & 0.277270 \\
$\mathrm{C}$ & -3.606781 & -1.940244 & 1.264781 \\
$\mathrm{C}$ & -2.166140 & 0.149855 & 1.524744 \\
$\mathrm{C}$ & -3.063658 & -0.857179 & 2.215954 \\
$\mathrm{H}$ & -2.203571 & -2.244187 & -1.940769 \\
$\mathrm{H}$ & -4.559364 & -1.632061 & 0.823172 \\
$\mathrm{H}$ & -1.480641 & 0.678056 & 2.186560 \\
$\mathrm{H}$ & -2.181213 & -3.281612 & 0.222791 \\
$\mathrm{H}$ & -3.821047 & -2.844099 & 1.842559 \\
$\mathrm{H}$ & -3.889046 & -0.344061 & 2.729429 \\
$\mathrm{C}$ & -3.566261 & -0.578291 & -1.556443 \\
$\mathrm{H}$ & -4.545039 & -1.003484 & -1.819187 \\
$\mathrm{H}$ & -3.155098 & -0.144326 & -2.474163 \\
$\mathrm{C}$ & -3.719416 & 0.532484 & -0.507253 \\
$\mathrm{H}$ & -1.893939 & 1.649997 & 0.041172 \\
$\mathrm{H}$ & -4.541155 & 0.315071 & 0.181071 \\
-3.977442 & 1.470507 & -1.007808 \\
\hline & -2.464490 & -1.340527 & 2.995403 \\
\hline & & & \\
$\mathrm{H}$ & & & \\
$\mathrm{H}$ & & & \\
$\mathrm{H}$ & & & \\
$\mathrm{H}$ & & & \\
$\mathrm{H}$ & & &
\end{tabular}

24

M06-2X/LANL2DZ-6-31G(D)/SMD SCF energy: -3795.29894634 a.u. M06-2X/LANL2DZ-6-31G(D)/SMD enthalpy: -3794.811325 a.u.

M06-2X/LANL2DZ-6-31G(D)/SMD free energy: -3794.895664 a.u.

M06-2X/LANL2DZ-6-311++G(D,P) SCF energy in solution: -3797.97812014 a.u. 
M06-2X/LANL2DZ-6-311++G(D,P) enthalpy in solution:

M06-2X/LANL2DZ-6-311++G(D,P) free energy in solution:
-3797.490499 a.u.

-3797.574838 a.u.

Cartesian coordinates

$\begin{array}{lccc}\text { ATOM } & \text { X } & \text { Y } & \text { Z } \\ \text { C } & -5.096387 & -1.344210 & 0.662898 \\ \text { C } & -3.833050 & -0.797393 & 0.694573 \\ \text { C } & -2.747382 & -1.324798 & -0.073530 \\ \text { C } & -3.035053 & -2.555222 & -0.745457 \\ \text { C } & -4.334883 & -3.109185 & -0.757650 \\ \text { C } & -5.367073 & -2.496524 & -0.095745 \\ \text { H } & -5.879032 & -0.898385 & 1.266065 \\ \text { C } & -1.977629 & -3.239604 & -1.408708 \\ \text { H } & -4.493709 & -4.035305 & -1.303653 \\ \text { H } & -6.370103 & -2.909595 & -0.109382 \\ \text { C } & -0.714336 & -2.745144 & -1.393759 \\ \text { C } & -0.406770 & -1.481748 & -0.786887 \\ \text { H } & -2.202521 & -4.176142 & -1.912518 \\ \text { H } & 0.105567 & -3.264276 & -1.880250 \\ \text { C } & -1.219726 & 0.718488 & 0.046107 \\ \text { H } & 2.031267 & 1.365822 & -0.377474 \\ \text { C } & -0.228807 & 1.178268 & 0.832123 \\ \text { H } & -0.045242 & 2.252257 & 0.862191 \\ \text { C } & 0.741740 & 0.318855 & 1.551781 \\ \text { H } & 0.291108 & -0.642831 & 1.836714 \\ \text { H } & 1.141416 & 0.806062 & 2.448498 \\ \text { C } & -2.054389 & 1.660753 & -0.750392 \\ \text { C } & -2.366801 & 2.941794 & -0.271976 \\ \text { C } & -2.529687 & 1.299002 & -2.018266 \\ \text { C } & 3.631543 & 1.249256 & 1.109349 \\ \text { C } & -3.099145 & 3.837011 & -1.042716 \\ \text { C } & -3.266855 & 2.193480 & -2.789754 \\ \text { H } & -2.306748 & 0.309088 & -2.406648 \\ \text { C } & -3.552711 & 3.467954 & -2.308357 \\ \text { H } & -3.617721 & 1.890701 & -3.772039 \\ \text { H } & -4.131935 & 4.163953 & -2.907543 \\ \text { H } & -2.053697 & 3.223543 & 0.729282 \\ \text { H } & -3.332114 & 4.821335 & -0.646866 \\ \text { O } & 0.856374 & -1.119952 & -0.937957 \\ \text { C } & -1.435008 & -0.733689 & -0.193629 \\ \text { Br } & -3.614534 & 0.584161 & 1.991894 \\ \text { C } & 2.202307 & -0.066710 & 0.159163 \\ \text { C } & & & 1.539853\end{array}$




$\begin{array}{lrrr}\mathrm{C} & 3.886751 & 0.141431 & -1.592579 \\ \mathrm{C} & 5.003724 & -0.947678 & 1.092135 \\ \mathrm{C} & 3.823439 & -1.162637 & -1.188782 \\ \mathrm{C} & 4.703270 & -1.818930 & -0.145247 \\ \mathrm{H} & 3.085547 & 1.908820 & 1.781172 \\ \mathrm{H} & 5.877846 & -0.314421 & 0.917703 \\ \mathrm{H} & 3.165203 & -1.826664 & -1.744734 \\ \mathrm{H} & 3.403682 & -0.338956 & 2.504878 \\ \mathrm{H} & 5.274450 & -1.610724 & 1.918852 \\ \mathrm{H} & 5.645776 & -2.147311 & -0.603742 \\ \mathrm{C} & 4.496081 & 1.978123 & 0.099982 \\ \mathrm{H} & 5.384307 & 2.385314 & 0.601719 \\ \mathrm{H} & 3.920912 & 2.840478 & -0.254748 \\ \mathrm{C} & 4.928772 & 1.135037 & -1.117599 \\ \mathrm{H} & 3.272070 & 0.434430 & -2.440317 \\ \mathrm{H} & 5.855871 & 0.598276 & -0.901017 \\ \mathrm{H} & 5.159148 & 1.814848 & -1.943176 \\ \mathrm{H} & 4.192324 & -2.731038 & 0.182252\end{array}$

25

M06-2X/LANL2DZ-6-31G(D)/SMD SCF energy: -3795.32005408 a.u. M06-2X/LANL2DZ-6-31G(D)/SMD enthalpy: -3794.830429 a.u. M06-2X/LANL2DZ-6-31G(D)/SMD free energy: -3794.911515 a.u.

M06-2X/LANL2DZ-6-311++G(D,P) SCF energy in solution: -3797.99550174 a.u. M06-2X/LANL2DZ-6-311++G(D,P) enthalpy in solution: M06-2X/LANL2DZ-6-311++G(D,P) free energy in solution: -3797.505877 a.u. Imaginary frequency: $\quad-14.6606 \mathrm{~cm}-1$ -3797.586963 a.u.

Cartesian coordinates

$\begin{array}{lccr}\text { ATOM } & \text { X } & \text { Y } & \text { Z } \\ \text { C } & -4.585403 & 2.408572 & -0.448879 \\ \text { C } & -3.648007 & 1.403982 & -0.512536 \\ \text { C } & -2.418903 & 1.447967 & 0.223835 \\ \text { C } & -2.203025 & 2.667348 & 0.951471 \\ \text { C } & -3.178904 & 3.690765 & 0.995474 \\ \text { C } & -4.366026 & 3.561968 & 0.326645 \\ \text { H } & -5.497088 & 2.314227 & -1.027685 \\ \text { C } & -0.976400 & 2.862394 & 1.641983 \\ \text { H } & -2.958593 & 4.583308 & 1.574897 \\ \text { H } & -5.121816 & 4.339326 & 0.361732 \\ \text { C } & -0.005513 & 1.909512 & 1.630881\end{array}$




\begin{tabular}{|c|c|c|c|}
\hline $\mathrm{C}$ & -0.210229 & 0.662119 & 0.962697 \\
\hline $\mathrm{H}$ & -0.832645 & 3.796667 & 2.178770 \\
\hline $\mathrm{H}$ & 0.933761 & 2.050095 & 2.158633 \\
\hline $\mathrm{C}$ & -1.589449 & -0.970682 & -0.227882 \\
\hline $\mathrm{H}$ & 1.073807 & -1.091998 & -2.086452 \\
\hline $\mathrm{C}$ & -0.877513 & -1.448154 & -1.256391 \\
\hline $\mathrm{H}$ & -0.967977 & -2.502433 & -1.512116 \\
\hline $\mathrm{C}$ & 0.060573 & -0.654052 & -2.107582 \\
\hline $\mathrm{H}$ & 0.100532 & 0.399202 & -1.800017 \\
\hline $\mathrm{H}$ & -0.237502 & -0.669043 & -3.163855 \\
\hline $\mathrm{C}$ & -2.490888 & -1.859203 & 0.558229 \\
\hline $\mathrm{C}$ & -3.158513 & -2.935760 & -0.042462 \\
\hline $\mathrm{C}$ & -2.684689 & -1.642499 & 1.927675 \\
\hline $\mathrm{C}$ & -3.965681 & -3.784373 & 0.705521 \\
\hline $\mathrm{C}$ & -3.494284 & -2.491380 & 2.678090 \\
\hline $\mathrm{H}$ & -2.187760 & -0.805609 & 2.409640 \\
\hline $\mathrm{C}$ & -4.135353 & -3.568046 & 2.072417 \\
\hline $\mathrm{H}$ & -3.623481 & -2.307908 & 3.740678 \\
\hline $\mathrm{H}$ & -4.771350 & -4.226566 & 2.656190 \\
\hline $\mathrm{H}$ & -3.063889 & -3.089108 & -1.113719 \\
\hline $\mathrm{H}$ & -4.477271 & -4.608648 & 0.217229 \\
\hline $\mathrm{O}$ & 0.738976 & -0.264419 & 1.059853 \\
\hline $\mathrm{C}$ & -1.410020 & 0.425929 & 0.278990 \\
\hline $\mathrm{Br}$ & -4.088499 & 0.018790 & -1.747281 \\
\hline Ir & 2.355350 & 0.014498 & -0.191606 \\
\hline $\mathrm{C}$ & 3.843045 & -0.436037 & -1.618237 \\
\hline $\mathrm{C}$ & 4.021377 & 0.894204 & -1.165113 \\
\hline $\mathrm{C}$ & 3.564889 & -1.072435 & 1.067925 \\
\hline $\mathrm{C}$ & 5.136332 & 1.296026 & -0.206219 \\
\hline $\mathrm{C}$ & 3.522396 & 0.309306 & 1.468974 \\
\hline $\mathrm{C}$ & 4.640363 & 1.306531 & 1.249341 \\
\hline $\mathrm{H}$ & 3.385967 & -0.565530 & -2.600812 \\
\hline $\mathrm{H}$ & 5.983022 & 0.612599 & -0.325100 \\
\hline $\mathrm{H}$ & 2.868788 & 0.533700 & 2.311951 \\
\hline $\mathrm{H}$ & 3.657899 & 1.686954 & -1.820505 \\
\hline $\mathrm{H}$ & 5.507552 & 2.288716 & -0.476520 \\
\hline $\mathrm{H}$ & 5.464580 & 1.122446 & 1.951990 \\
\hline $\mathrm{C}$ & 4.667695 & -1.621306 & -1.148559 \\
\hline $\mathrm{H}$ & 5.664854 & -1.607167 & -1.608710 \\
\hline $\mathrm{H}$ & 4.169088 & -2.526356 & -1.511393 \\
\hline $\mathrm{C}$ & 4.778874 & -1.695011 & 0.386528 \\
\hline $\mathrm{H}$ & 2.936862 & -1.756708 & 1.638353 \\
\hline $\mathrm{H}$ & 5.686641 & -1.193213 & 0.737245 \\
\hline
\end{tabular}




$\begin{array}{llrl}\mathrm{H} & 4.867998 & -2.740175 & 0.694951 \\ \mathrm{H} & 4.241256 & 2.298520 & 1.487386\end{array}$

26

M06-2X/LANL2DZ-6-31G(D)/SMD SCF energy: -4256.14727212 a.u. M06-2X/LANL2DZ-6-31G(D)/SMD enthalpy: -4255.641270 a.u. M06-2X/LANL2DZ-6-31G(D)/SMD free energy: -4255.733509 a.u. M06-2X/LANL2DZ-6-311++G(D,P) SCF energy in solution: -4258.86496149 a.u. M06-2X/LANL2DZ-6-311++G(D,P) enthalpy in solution: -4258.358959 a.u.

M06-2X/LANL2DZ-6-311++G(D,P) free energy in solution: -4258.451198 a.u.

Cartesian coordinates

$\begin{array}{lrrr}\text { ATOM } & \text { X } & \text { Y } & \text { Z } \\ \text { C } & 4.604220 & -2.662291 & -0.310421 \\ \text { C } & 3.757094 & -1.581496 & -0.375112 \\ \text { C } & 2.575144 & -1.494465 & 0.430109 \\ \text { C } & 2.346565 & -2.605763 & 1.310404 \\ \text { C } & 3.239998 & -3.704697 & 1.357276 \\ \text { C } & 4.350477 & -3.739067 & 0.560683 \\ \text { H } & 5.484165 & -2.679049 & -0.943072 \\ \text { C } & 1.210720 & -2.619337 & 2.161919 \\ \text { H } & 3.017728 & -4.517631 & 2.042389 \\ \text { H } & 5.038010 & -4.577514 & 0.590183 \\ \text { C } & 0.310479 & -1.595104 & 2.152962 \\ \text { C } & 0.525148 & -0.520859 & 1.267577 \\ \text { H } & 1.076631 & -3.464991 & 2.829667 \\ \text { H } & -0.554933 & -1.577962 & 2.806810 \\ \text { C } & 1.664961 & 0.837582 & -0.387865 \\ \text { H } & -0.666487 & 0.102268 & -2.577625 \\ \text { C } & 1.036645 & 0.917700 & -1.569740 \\ \text { H } & 1.064250 & 1.867854 & -2.099830 \\ \text { C } & 0.343222 & -0.202478 & -2.275876 \\ \text { H } & 0.267641 & -1.099833 & -1.654709 \\ \text { H } & 0.888953 & -0.464869 & -3.191605 \\ \text { C } & 2.303619 & 2.020499 & 0.257642 \\ \text { C } & 1.834970 & 3.319573 & 0.013559 \\ \text { C } & 3.376116 & 1.854190 & 1.144611 \\ \text { C } & 2.442654 & 4.417762 & 0.614420 \\ \text { C } & 3.982916 & 2.953138 & 1.743179 \\ \text { H } & 3.746473 & 0.855587 & 1.359303 \\ \text { C } & 3.520791 & 4.240332 & 1.477998\end{array}$




$\begin{array}{lccc}\mathrm{H} & 4.818286 & 2.802046 & 2.420084 \\ \mathrm{H} & 3.989914 & 5.097822 & 1.950474 \\ \mathrm{H} & 0.968701 & 3.467745 & -0.624281 \\ \mathrm{H} & 2.058748 & 5.414168 & 0.418180 \\ \mathrm{O} & -0.455857 & 0.454520 & 1.277304 \\ \mathrm{H} & -0.130582 & 1.290217 & 0.873368 \\ \mathrm{C} & 1.616425 & -0.422737 & 0.427176 \\ \mathrm{Br} & 4.298121 & -0.237222 & -1.606340 \\ \mathrm{Ir} & -2.414903 & 0.290248 & 0.113615 \\ \mathrm{C} & -2.315725 & -1.782699 & -0.136768 \\ \mathrm{C} & -3.138306 & -1.485888 & 0.984144 \\ \mathrm{C} & -3.778179 & 0.137077 & -1.462586 \\ \mathrm{C} & -4.656645 & -1.613677 & 0.944718 \\ \mathrm{C} & -4.434755 & 0.589102 & -0.284041 \\ \mathrm{C} & -5.318097 & -0.270339 & 0.595905 \\ \mathrm{H} & -1.298127 & -2.118614 & 0.074154 \\ \mathrm{H} & -4.939328 & -2.388490 & 0.224960 \\ \mathrm{H} & -4.629944 & 1.658937 & -0.214462 \\ \mathrm{H} & -2.693234 & -1.604489 & 1.971587 \\ \mathrm{H} & -5.016887 & -1.957348 & 1.918528 \\ \mathrm{H} & -6.297638 & -0.421743 & 0.122035 \\ \mathrm{C} & -2.839183 & -2.212266 & -1.493591 \\ \mathrm{H} & -3.190713 & -3.252463 & -1.457930 \\ \mathrm{H} & -1.995038 & -2.192159 & -2.190985 \\ \mathrm{C} & -3.943064 & -1.275319 & -2.012483 \\ \mathrm{H} & -3.521505 & 0.900089 & -2.196353 \\ \mathrm{H} & -4.935676 & -1.654514 & -1.748742 \\ \mathrm{H} & -3.910723 & -1.240706 & -3.105118 \\ \mathrm{H} & -5.499133 & 0.290358 & 1.518796 \\ \mathrm{Cl} & -1.864038 & 2.634823 & -0.275027\end{array}$

\section{DBU}

M06-2X/LANL2DZ-6-31G(D)/SMD SCF energy:

-461.88251453 a.u.

M06-2X/LANL2DZ-6-31G(D)/SMD enthalpy:

-461.622170 a.u.

M06-2X/LANL2DZ-6-31G(D)/SMD free energy:

-461.667802 a.u.

M06-2X/LANL2DZ-6-311++G(D,P) SCF energy in solution:

-462.01364694 a.u.

M06-2X/LANL2DZ-6-311++G(D,P) enthalpy in solution:

-461.753302 a.u.

M06-2X/LANL2DZ-6-311++G(D,P) free energy in solution:

-461.798934 a.u.

Cartesian coordinates

$\begin{array}{llll}\text { ATOM } & \mathrm{X} & \mathrm{Y} & \mathrm{Z}\end{array}$




$\begin{array}{lrrc}\mathrm{C} & -0.848776 & 1.456765 & 0.272335 \\ \mathrm{C} & 0.369506 & -0.720483 & -0.137448 \\ \mathrm{C} & -2.110921 & 1.138205 & -0.528941 \\ \mathrm{C} & -0.923909 & -1.473865 & -0.402184 \\ \mathrm{C} & -2.946764 & 0.001962 & 0.066360 \\ \mathrm{C} & -2.078504 & -1.158102 & 0.557629 \\ \mathrm{H} & -1.064657 & 1.404408 & 1.352866 \\ \mathrm{H} & -1.244546 & -1.300266 & -1.437685 \\ \mathrm{H} & -1.812417 & 0.908770 & -1.557720 \\ \mathrm{H} & -3.650324 & -0.358882 & -0.693253 \\ \mathrm{H} & -0.568118 & 2.494390 & 0.067135 \\ \mathrm{H} & -2.721196 & 2.046142 & -0.580750 \\ \mathrm{H} & -0.641380 & -2.524892 & -0.331193 \\ \mathrm{H} & -3.551254 & 0.384501 & 0.897284 \\ \mathrm{H} & -2.699215 & -2.050767 & 0.684665 \\ \mathrm{H} & -1.670120 & -0.930428 & 1.549895 \\ \mathrm{C} & 1.581437 & 1.346485 & 0.298082 \\ \mathrm{C} & 2.775436 & 0.614521 & -0.288549 \\ \mathrm{C} & 2.719366 & -0.836097 & 0.168604 \\ \mathrm{H} & 3.473325 & -1.434427 & -0.355035 \\ \mathrm{H} & 2.722925 & 0.659937 & -1.382239 \\ \mathrm{H} & 3.705323 & 1.098966 & 0.024851 \\ \mathrm{H} & 1.680676 & 1.415531 & 1.393719 \\ \mathrm{H} & 1.528359 & 2.370161 & -0.088107 \\ \mathrm{H} & 2.964475 & -0.900811 & 1.238543 \\ \mathrm{~N} & 1.421747 & -1.453069 & -0.054796 \\ \mathrm{~N} & 0.339243 & 0.667544 & -0.062106\end{array}$

\section{TS-1}

M06-2X/LANL2DZ-6-31G(D)/SMD SCF energy:

-3841.24982979 a.u.

M06-2X/LANL2DZ-6-31G(D)/SMD enthalpy: -3840.687214 a.u.

M06-2X/LANL2DZ-6-31G(D)/SMD free energy: -3840.777041 a.u.

M06-2X/LANL2DZ-6-311++G(D,P) SCF energy in solution: -3844.30202507 a.u.

M06-2X/LANL2DZ-6-311++G(D,P) enthalpy in solution: -3843.739409 a.u. M06-2X/LANL2DZ-6-311++G(D,P) free energy in solution: -3843.829236 a.u.

Imaginary frequency: $\quad-1197.7876 \mathrm{~cm}-1$

Cartesian coordinates

$\begin{array}{lcrr}\text { ATOM } & \text { X } & \text { Y } & \text { Z } \\ \text { C } & -5.000241 & -0.452139 & 0.576694 \\ \text { C } & -3.709745 & -0.049661 & 0.337838\end{array}$




\begin{tabular}{|c|c|c|c|}
\hline $\mathrm{C}$ & -2.813621 & -0.801102 & -0.491293 \\
\hline $\mathrm{C}$ & -3.429181 & -1.869864 & -1.224170 \\
\hline $\mathrm{C}$ & -4.766575 & -2.268464 & -0.964551 \\
\hline $\mathrm{C}$ & -5.528113 & -1.605182 & -0.043339 \\
\hline $\mathrm{H}$ & -5.635645 & 0.158395 & 1.208282 \\
\hline $\mathrm{C}$ & -2.697639 & -2.521209 & -2.247312 \\
\hline $\mathrm{H}$ & -5.170824 & -3.108105 & -1.523789 \\
\hline $\mathrm{H}$ & -6.547795 & -1.909540 & 0.168552 \\
\hline $\mathrm{C}$ & -1.420358 & -2.131342 & -2.534583 \\
\hline $\mathrm{C}$ & -0.780637 & -1.166678 & -1.724368 \\
\hline $\mathrm{H}$ & -3.176581 & -3.316386 & -2.811344 \\
\hline $\mathrm{H}$ & -0.865419 & -2.598910 & -3.345531 \\
\hline $\mathrm{O}$ & 0.519852 & -0.842946 & -1.981086 \\
\hline $\mathrm{C}$ & -0.554006 & 0.182587 & 0.357898 \\
\hline $\mathrm{H}$ & 0.801741 & -0.516739 & 0.371092 \\
\hline $\mathrm{C}$ & -0.864296 & -0.069460 & 1.776860 \\
\hline $\mathrm{H}$ & -0.603575 & 0.740133 & 2.458998 \\
\hline $\mathrm{C}$ & -1.397242 & -1.168120 & 2.337149 \\
\hline $\mathrm{H}$ & -1.718167 & -2.026748 & 1.751689 \\
\hline $\mathrm{H}$ & -1.564020 & -1.218908 & 3.408661 \\
\hline $\mathrm{C}$ & -0.081531 & 1.535210 & 0.008284 \\
\hline $\mathrm{C}$ & -0.337345 & 2.119309 & -1.248798 \\
\hline $\mathrm{C}$ & 0.737634 & 2.277973 & 0.887838 \\
\hline $\mathrm{C}$ & 0.185187 & 3.358592 & -1.600720 \\
\hline $\mathrm{C}$ & 1.245179 & 3.525289 & 0.540383 \\
\hline $\mathrm{H}$ & 1.005314 & 1.857975 & 1.853888 \\
\hline $\mathrm{C}$ & 0.978855 & 4.081548 & -0.710285 \\
\hline $\mathrm{H}$ & 1.867450 & 4.062391 & 1.252395 \\
\hline $\mathrm{H}$ & 1.377872 & 5.053547 & -0.983322 \\
\hline $\mathrm{H}$ & -0.971293 & 1.588678 & -1.953393 \\
\hline $\mathrm{H}$ & -0.043840 & 3.771529 & -2.580018 \\
\hline $\mathrm{C}$ & 5.534732 & -0.807810 & 0.436436 \\
\hline $\mathrm{C}$ & 3.026565 & -0.743204 & 0.218373 \\
\hline $\mathrm{C}$ & 5.856703 & -0.199374 & -0.926426 \\
\hline $\mathrm{C}$ & 3.056712 & 0.391105 & -0.783441 \\
\hline $\mathrm{C}$ & 5.430427 & 1.264569 & -1.058650 \\
\hline $\mathrm{C}$ & 4.045864 & 1.517094 & -0.459077 \\
\hline $\mathrm{H}$ & 5.748047 & -0.084017 & 1.236814 \\
\hline $\mathrm{H}$ & 3.278126 & -0.034488 & -1.770329 \\
\hline $\mathrm{H}$ & 5.392609 & -0.818831 & -1.701647 \\
\hline $\mathrm{H}$ & 5.429549 & 1.540359 & -2.119441 \\
\hline $\mathrm{H}$ & 6.201277 & -1.658299 & 0.599137 \\
\hline $\mathrm{H}$ & 6.938085 & -0.279977 & -1.078240 \\
\hline
\end{tabular}




$\begin{array}{lccc}\mathrm{H} & 2.042406 & 0.777867 & -0.846416 \\ \mathrm{H} & 6.167553 & 1.909333 & -0.566505 \\ \mathrm{H} & 3.636696 & 2.457610 & -0.838549 \\ \mathrm{H} & 4.114920 & 1.638959 & 0.629013 \\ \mathrm{C} & 4.101511 & -2.419439 & 1.613194 \\ \mathrm{C} & 2.825373 & -3.221409 & 1.434495 \\ \mathrm{C} & 1.645069 & -2.271048 & 1.510452 \\ \mathrm{H} & 0.717809 & -2.755079 & 1.193339 \\ \mathrm{H} & 2.845283 & -3.722063 & 0.460460 \\ \mathrm{H} & 2.753717 & -3.989389 & 2.209016 \\ \mathrm{H} & 4.148602 & -1.988828 & 2.623449 \\ \mathrm{H} & 4.979246 & -3.057941 & 1.483011 \\ \mathrm{H} & 1.482766 & -1.921465 & 2.536147 \\ \mathrm{~N} & 1.850671 & -1.119831 & 0.636489 \\ \mathrm{~N} & 4.177063 & -1.351952 & 0.610684 \\ \mathrm{Br} & -3.290559 & 1.694449 & 0.977607 \\ \mathrm{H} & 0.836416 & -1.380289 & -2.722591 \\ \mathrm{C} & -1.397902 & -0.559507 & -0.635645\end{array}$

\section{TS-1a}

M06-2X/LANL2DZ-6-31G(D)/SMD SCF energy: M06-2X/LANL2DZ-6-31G(D)/SMD enthalpy: -3841.24288265 a.u. M06-2X/LANL2DZ-6-31G(D)/SMD free energy: -3840.679990 a.u. M06-2X/LANL2DZ-6-311++G(D,P) SCF energy in solution: -3840.770049 a.u. M06-2X/LANL2DZ-6-311++G(D,P) enthalpy in solution: M06-2X/LANL2DZ-6-311++G(D,P) free energy in solution: -3844.29609698 a.u. Imaginary frequency: $\quad-953.6242 \mathrm{~cm}-1$ -3843.733204 a.u. -3843.823263 a.u.

Cartesian coordinates

$\begin{array}{lccc}\text { ATOM } & \text { X } & \text { Y } & \text { Z } \\ \text { C } & 1.684035 & -3.161899 & 0.476548 \\ \text { C } & 1.587391 & -1.942271 & -0.149066 \\ \text { C } & 2.322057 & -0.785924 & 0.282989 \\ \text { C } & 3.316195 & -1.067599 & 1.285136 \\ \text { C } & 3.401021 & -2.335357 & 1.916010 \\ \text { C } & 2.569284 & -3.358547 & 1.556396 \\ \text { H } & 1.108032 & -3.997023 & 0.093739 \\ \text { C } & 4.244700 & -0.062884 & 1.650210 \\ \text { H } & 4.151893 & -2.472286 & 2.689604 \\ \text { H } & 2.623530 & -4.328084 & 2.040131 \\ \text { C } & 4.203167 & 1.164881 & 1.057899\end{array}$




\begin{tabular}{|c|c|c|c|}
\hline $\mathrm{C}$ & 3.159279 & 1.471992 & 0.154118 \\
\hline $\mathrm{H}$ & 4.999221 & -0.294567 & 2.396559 \\
\hline $\mathrm{H}$ & 4.932383 & 1.930870 & 1.313716 \\
\hline $\mathrm{O}$ & 3.072891 & 2.732156 & -0.340620 \\
\hline $\mathrm{C}$ & 0.949506 & 1.158921 & -0.880465 \\
\hline $\mathrm{H}$ & -0.426287 & 0.411671 & -0.915956 \\
\hline $\mathrm{C}$ & 1.139831 & 1.427412 & -2.312406 \\
\hline $\mathrm{H}$ & 0.425967 & 2.110946 & -2.772629 \\
\hline $\mathrm{C}$ & 2.054952 & 0.891395 & -3.135731 \\
\hline $\mathrm{H}$ & 2.810434 & 0.194788 & -2.781647 \\
\hline $\mathrm{H}$ & 2.070161 & 1.137421 & -4.193215 \\
\hline $\mathrm{C}$ & 0.295107 & 2.217017 & -0.067084 \\
\hline $\mathrm{C}$ & 0.384027 & 2.225423 & 1.345600 \\
\hline $\mathrm{C}$ & -0.584786 & 3.175480 & -0.619922 \\
\hline $\mathrm{C}$ & -0.344641 & 3.108527 & 2.134088 \\
\hline $\mathrm{C}$ & -1.314982 & 4.057575 & 0.170249 \\
\hline $\mathrm{H}$ & -0.701027 & 3.249919 & -1.696017 \\
\hline $\mathrm{C}$ & -1.210171 & 4.038018 & 1.559052 \\
\hline $\mathrm{H}$ & -1.970495 & 4.777852 & -0.313695 \\
\hline $\mathrm{H}$ & -1.777814 & 4.729876 & 2.173287 \\
\hline $\mathrm{H}$ & 1.044746 & 1.516279 & 1.838168 \\
\hline $\mathrm{H}$ & -0.230150 & 3.067958 & 3.214981 \\
\hline $\mathrm{C}$ & -4.444508 & -0.896737 & 1.089261 \\
\hline $\mathrm{C}$ & -2.256925 & -0.345460 & -0.015102 \\
\hline $\mathrm{C}$ & -3.869930 & -1.871457 & 2.115058 \\
\hline $\mathrm{C}$ & -1.426042 & -0.673790 & 1.206082 \\
\hline $\mathrm{C}$ & -2.967084 & -1.207109 & 3.157479 \\
\hline $\mathrm{C}$ & -2.016206 & -0.184419 & 2.532913 \\
\hline $\mathrm{H}$ & -4.745261 & 0.041300 & 1.578358 \\
\hline $\mathrm{H}$ & -1.255588 & -1.757734 & 1.231187 \\
\hline $\mathrm{H}$ & -3.336663 & -2.664109 & 1.578670 \\
\hline $\mathrm{H}$ & -2.389414 & -1.983791 & 3.671610 \\
\hline $\mathrm{H}$ & -5.351019 & -1.335108 & 0.664411 \\
\hline $\mathrm{H}$ & -4.711285 & -2.357089 & 2.620098 \\
\hline $\mathrm{H}$ & -0.449642 & -0.216237 & 1.035891 \\
\hline $\mathrm{H}$ & -3.582997 & -0.715410 & 3.919093 \\
\hline $\mathrm{H}$ & -1.196309 & 0.033245 & 3.223993 \\
\hline $\mathrm{H}$ & -2.528158 & 0.770910 & 2.367013 \\
\hline $\mathrm{C}$ & -4.324330 & -0.207456 & -1.275283 \\
\hline $\mathrm{C}$ & -3.449637 & -0.349757 & -2.511058 \\
\hline $\mathrm{C}$ & -2.185411 & 0.466020 & -2.305979 \\
\hline $\mathrm{H}$ & -1.430014 & 0.249162 & -3.068403 \\
\hline $\mathrm{H}$ & -3.191248 & -1.404562 & -2.654494 \\
\hline
\end{tabular}




$\begin{array}{llcl}\mathrm{H} & -3.996053 & -0.009672 & -3.394339 \\ \mathrm{H} & -4.681978 & 0.826491 & -1.172877 \\ \mathrm{H} & -5.200033 & -0.857381 & -1.350620 \\ \mathrm{H} & -2.400931 & 1.541389 & -2.349470 \\ \mathrm{~N} & -1.595792 & 0.153465 & -1.016589 \\ \mathrm{~N} & -3.586947 & -0.598768 & -0.068657 \\ \mathrm{C} & 2.158805 & 0.563517 & -0.190547 \\ \mathrm{H} & 3.771160 & 3.275517 & 0.053810 \\ \mathrm{Br} & 0.596076 & -1.980164 & -1.773656\end{array}$

\section{TS-2}

M06-2X/LANL2DZ-6-31G(D)/SMD SCF energy:

-3841.25622677 a.u.

M06-2X/LANL2DZ-6-31G(D)/SMD enthalpy: -3840.692950 a.u.

M06-2X/LANL2DZ-6-31G(D)/SMD free energy:

-3840.782104 a.u.

M06-2X/LANL2DZ-6-311++G(D,P) SCF energy in solution: -3844.30835336 a.u.

M06-2X/LANL2DZ-6-311++G(D,P) enthalpy in solution: -3843.745077 a.u. M06-2X/LANL2DZ-6-311++G(D,P) free energy in solution: -3843.834231 a.u.

Cartesian coordinates

$\begin{array}{lrrr}\text { ATOM } & \text { X } & \text { Y } & \text { Z } \\ \text { C } & 4.560210 & -1.237540 & -0.464066 \\ \text { C } & 3.429172 & -0.476316 & -0.295087 \\ \text { C } & 2.284180 & -0.963136 & 0.420011 \\ \text { C } & 2.477322 & -2.221340 & 1.085537 \\ \text { C } & 3.661593 & -2.982159 & 0.905154 \\ \text { C } & 4.673391 & -2.520476 & 0.111520 \\ \text { H } & 5.395380 & -0.821300 & -1.015683 \\ \text { C } & 1.475596 & -2.709569 & 1.959632 \\ \text { H } & 3.742480 & -3.937884 & 1.416029 \\ \text { H } & 5.577837 & -3.099579 & -0.042745 \\ \text { C } & 0.314145 & -2.013302 & 2.143707 \\ \text { C } & 0.071577 & -0.843529 & 1.389109 \\ \text { H } & 1.652606 & -3.641453 & 2.489112 \\ \text { H } & -0.445606 & -2.375129 & 2.834395 \\ \text { O } & -1.149264 & -0.233724 & 1.486572 \\ \text { C } & 0.585004 & 0.835518 & -0.341753 \\ \text { H } & -0.881929 & -1.128994 & -1.936064 \\ \text { C } & 0.482863 & 0.630443 & -1.702591 \\ \text { H } & 0.200777 & 1.507612 & -2.289205 \\ \text { C } & 0.566291 & -0.573344 & -2.436450\end{array}$




\begin{tabular}{|c|c|c|c|}
\hline $\mathrm{H}$ & 1.140632 & -1.399611 & -2.013986 \\
\hline $\mathrm{H}$ & 0.657197 & -0.486411 & -3.517416 \\
\hline $\mathrm{C}$ & 0.262957 & 2.114859 & 0.289477 \\
\hline $\mathrm{C}$ & 0.710348 & 2.408114 & 1.595421 \\
\hline $\mathrm{C}$ & -0.502244 & 3.119696 & -0.344855 \\
\hline $\mathrm{C}$ & 0.417697 & 3.613151 & 2.221162 \\
\hline $\mathrm{C}$ & -0.786036 & 4.329253 & 0.279004 \\
\hline $\mathrm{H}$ & -0.894646 & 2.948417 & -1.343706 \\
\hline $\mathrm{C}$ & -0.333793 & 4.591550 & 1.571319 \\
\hline $\mathrm{H}$ & -1.376988 & 5.072611 & -0.250584 \\
\hline $\mathrm{H}$ & -0.561026 & 5.534410 & 2.058940 \\
\hline $\mathrm{H}$ & 1.314032 & 1.671178 & 2.118135 \\
\hline $\mathrm{H}$ & 0.789859 & 3.793754 & 3.226554 \\
\hline $\mathrm{C}$ & -4.866837 & -0.748623 & 0.429619 \\
\hline $\mathrm{C}$ & -2.753319 & -0.923489 & -0.924419 \\
\hline $\mathrm{C}$ & -4.564896 & 0.680896 & 0.875734 \\
\hline $\mathrm{C}$ & -2.733193 & 0.536466 & -1.314371 \\
\hline $\mathrm{C}$ & -4.708499 & 1.707892 & -0.248024 \\
\hline $\mathrm{C}$ & -4.093693 & 1.194751 & -1.549102 \\
\hline $\mathrm{H}$ & -5.732563 & -0.769878 & -0.246769 \\
\hline $\mathrm{H}$ & -2.162908 & 1.066475 & -0.541325 \\
\hline $\mathrm{H}$ & -3.551411 & 0.711890 & 1.291789 \\
\hline $\mathrm{H}$ & -4.208659 & 2.635463 & 0.052673 \\
\hline $\mathrm{H}$ & -5.139494 & -1.338625 & 1.308621 \\
\hline $\mathrm{H}$ & -5.247382 & 0.929853 & 1.694553 \\
\hline $\mathrm{H}$ & -2.126274 & 0.586779 & -2.221112 \\
\hline $\mathrm{H}$ & -5.766635 & 1.945964 & -0.406209 \\
\hline $\mathrm{H}$ & -3.963571 & 2.025376 & -2.249329 \\
\hline $\mathrm{H}$ & -4.771120 & 0.485147 & -2.040203 \\
\hline $\mathrm{C}$ & -3.748556 & -2.928890 & -0.040200 \\
\hline $\mathrm{C}$ & -2.332344 & -3.436091 & 0.188412 \\
\hline $\mathrm{C}$ & -1.470451 & -3.012621 & -0.987268 \\
\hline $\mathrm{H}$ & -0.404095 & -3.073456 & -0.741315 \\
\hline $\mathrm{H}$ & -1.943893 & -2.997449 & 1.112673 \\
\hline $\mathrm{H}$ & -2.327117 & -4.523329 & 0.299216 \\
\hline $\mathrm{H}$ & -4.179394 & -3.382484 & -0.942462 \\
\hline $\mathrm{H}$ & -4.395663 & -3.181781 & 0.801576 \\
\hline $\mathrm{H}$ & -1.649356 & -3.660107 & -1.853694 \\
\hline $\mathrm{N}$ & -1.746649 & -1.634059 & -1.363144 \\
\hline $\mathrm{N}$ & -3.736291 & -1.468989 & -0.181184 \\
\hline $\mathrm{Br}$ & 3.599638 & 1.313252 & -0.922575 \\
\hline $\mathrm{H}$ & -1.661604 & -0.678085 & 2.179273 \\
\hline $\mathrm{C}$ & 1.006165 & -0.304178 & 0.512718 \\
\hline
\end{tabular}




\section{TS-3}

M06-2X/LANL2DZ-6-31G(D)/SMD SCF energy:

-3379.35478106 a.u.

M06-2X/LANL2DZ-6-31G(D)/SMD enthalpy:

-3379.050651 a.u.

M06-2X/LANL2DZ-6-31G(D)/SMD free energy:

-3379.113299 a.u.

M06-2X/LANL2DZ-6-311++G(D,P) SCF energy in solution:

-3382.27582647 a.u.

M06-2X/LANL2DZ-6-311++G(D,P) enthalpy in solution:

-3381.971696 a.u.

M06-2X/LANL2DZ-6-311++G(D,P) free energy in solution:

-3382.034344 a.u.

Imaginary frequency: $\quad-38.6668 \mathrm{~cm}-1$

Cartesian coordinates

$\begin{array}{lrrr}\text { ATOM } & \text { X } & \text { Y } & \text { Z } \\ \text { C } & 3.571751 & -0.145977 & 0.733377 \\ \text { C } & 2.319758 & -0.320369 & 0.188206 \\ \text { C } & 1.379944 & 0.752573 & 0.065622 \\ \text { C } & 1.960319 & 2.050663 & 0.225764 \\ \text { C } & 3.252737 & 2.218933 & 0.778667 \\ \text { C } & 4.022800 & 1.134065 & 1.105570 \\ \text { H } & 4.245356 & -0.993469 & 0.793724 \\ \text { C } & 1.241454 & 3.189098 & -0.232363 \\ \text { H } & 3.622522 & 3.230393 & 0.921920 \\ \text { H } & 5.007410 & 1.253248 & 1.544745 \\ \text { C } & 0.031538 & 3.028961 & -0.828143 \\ \text { C } & -0.622456 & 1.763200 & -0.799572 \\ \text { H } & 1.697951 & 4.170379 & -0.142551 \\ \text { H } & -0.499237 & 3.879318 & -1.250893 \\ \text { C } & -0.918148 & -0.394008 & 0.534653 \\ \text { H } & -0.730997 & -0.092922 & 1.575283 \\ \text { C } & -0.629043 & -1.877096 & 0.510781 \\ \text { H } & -0.722720 & -2.385724 & -0.444583 \\ \text { C } & -0.394586 & -2.575690 & 1.617009 \\ \text { H } & -0.307330 & -2.091572 & 2.587276 \\ \text { H } & -0.266750 & -3.653626 & 1.591848 \\ \text { C } & -2.429670 & -0.267032 & 0.349245 \\ \text { C } & -3.089449 & -0.858635 & -0.732789 \\ \text { C } & -3.188222 & 0.386754 & 1.318110 \\ \text { C } & -4.471356 & -0.782057 & -0.847859 \\ \text { C } & -4.576062 & 0.467069 & 1.206325 \\ \text { H } & -2.688005 & 0.841596 & 2.170015 \\ \text { C } & -5.222329 & -0.116476 & 0.122124 \\ \text { H } & -5.149648 & 0.982086 & 1.971521\end{array}$




$\begin{array}{lrrr}\mathrm{H} & -6.302972 & -0.059996 & 0.032921 \\ \mathrm{H} & -2.513347 & -1.361735 & -1.504707 \\ \mathrm{H} & -4.967219 & -1.241808 & -1.697775 \\ \mathrm{O} & -1.884012 & 1.705125 & -1.295149 \\ \mathrm{H} & -2.210807 & 2.603739 & -1.452967 \\ \mathrm{C} & -0.038569 & 0.639384 & -0.231123 \\ \mathrm{Br} & 2.104440 & -1.956603 & -0.766749\end{array}$

\section{TS-4}

M06-2X/LANL2DZ-6-31G(D)/SMD SCF energy: M06-2X/LANL2DZ-6-31G(D)/SMD enthalpy: -3379.36966430 a.u. M06-2X/LANL2DZ-6-31G(D)/SMD free energy: -3379.065175 a.u. M06-2X/LANL2DZ-6-311++G(D,P) SCF energy in solution: -3379.126677 a.u. M06-2X/LANL2DZ-6-311++G(D,P) enthalpy in solution: M06-2X/LANL2DZ-6-311++G(D,P) free energy in solution: -3382.28759590 a.u. Imaginary frequency: $\quad-53.3976 \mathrm{~cm}-1$ -3381.983107 a.u. -3382.044609 a.u.

Cartesian coordinates

$\begin{array}{lrrr}\text { ATOM } & \text { X } & \text { Y } & \text { Z } \\ \text { C } & -0.247587 & -2.859720 & -0.070849 \\ \text { C } & -0.063890 & -1.557147 & 0.328122 \\ \text { C } & 1.040468 & -0.770954 & -0.121056 \\ \text { C } & 2.092347 & -1.517932 & -0.735097 \\ \text { C } & 1.882289 & -2.852480 & -1.162786 \\ \text { C } & 0.703394 & -3.496775 & -0.893075 \\ \text { H } & -1.097952 & -3.416157 & 0.306829 \\ \text { C } & 3.385631 & -0.935179 & -0.818631 \\ \text { H } & 2.692299 & -3.361931 & -1.677164 \\ \text { H } & 0.536006 & -4.518458 & -1.216809 \\ \text { C } & 3.617220 & 0.266393 & -0.218350 \\ \text { C } & 2.535474 & 1.044489 & 0.272620 \\ \text { H } & 4.192027 & -1.496252 & -1.281016 \\ \text { H } & 4.623171 & 0.675665 & -0.156454 \\ \text { O } & 2.805374 & 2.194089 & 0.931292 \\ \text { C } & 0.089121 & 1.633949 & -0.086322 \\ \text { C } & 0.162462 & 2.984959 & -0.213536 \\ \text { H } & -0.798045 & 3.431491 & -0.458888 \\ \text { C } & 1.269150 & 4.000608 & -0.239990 \\ \text { H } & 1.668222 & 4.222630 & 0.753043 \\ \text { H } & 0.875353 & 4.928027 & -0.664392 \\ \text { C } & -1.265011 & 1.125723 & -0.519586\end{array}$




$\begin{array}{lccc}\mathrm{C} & -2.429918 & 1.581352 & 0.108754 \\ \mathrm{C} & -1.398928 & 0.304157 & -1.643292 \\ \mathrm{C} & -3.687334 & 1.208096 & -0.350737 \\ \mathrm{C} & -2.657726 & -0.078721 & -2.101343 \\ \mathrm{H} & -0.511992 & -0.036988 & -2.169665 \\ \mathrm{C} & -3.806913 & 0.366013 & -1.454765 \\ \mathrm{H} & -2.736551 & -0.719623 & -2.974582 \\ \mathrm{H} & -4.787428 & 0.065374 & -1.811267 \\ \mathrm{H} & -2.337887 & 2.217467 & 0.985050 \\ \mathrm{H} & -4.575531 & 1.565942 & 0.161637 \\ \mathrm{H} & 2.107153 & 3.676624 & -0.863050 \\ \mathrm{Br} & -1.160334 & -0.966104 & 1.764922 \\ \mathrm{C} & 1.207809 & 0.658210 & 0.084128 \\ \mathrm{H} & 3.751492 & 2.396878 & 0.858853\end{array}$

\section{TS-5}

M06-2X/LANL2DZ-6-31G(D)/SMD SCF energy:

-4256.06924012 a.u.

-4255.568492 a.u.

-4255.653927 a.u.

M06-2X/LANL2DZ-6-31G(D)/SMD free energy:

-4258.79184050 a.u.

M06-2X/LANL2DZ-6-311++G(D,P) SCF energy in solution: -4258.291092 a.u.

M06-2X/LANL2DZ-6-311++G(D,P) enthalpy in solution:

-4258.376527 a.u.

M06-2X/LANL2DZ-6-311++G(D,P) free energy in solution:

Imaginary frequency: $\quad-708.7114 \mathrm{~cm}-1$

Cartesian coordinates

$\begin{array}{lcrr}\text { ATOM } & \text { X } & \text { Y } & \text { Z } \\ \text { C } & 4.406382 & -0.978733 & -1.631086 \\ \text { C } & 3.387061 & -0.340110 & -0.964110 \\ \text { C } & 2.755094 & -0.898249 & 0.194733 \\ \text { C } & 3.401016 & -2.069383 & 0.712945 \\ \text { C } & 4.460040 & -2.703400 & 0.017760 \\ \text { C } & 4.929826 & -2.197539 & -1.162453 \\ \text { H } & 4.840786 & -0.507125 & -2.505018 \\ \text { C } & 3.003402 & -2.605237 & 1.966599 \\ \text { H } & 4.889527 & -3.602146 & 0.450687 \\ \text { H } & 5.729885 & -2.686042 & -1.708089 \\ \text { C } & 1.991397 & -2.034351 & 2.671230 \\ \text { C } & 1.257406 & -0.967299 & 2.103197 \\ \text { H } & 3.532468 & -3.472694 & 2.350214 \\ \text { H } & 1.674337 & -2.413496 & 3.637027 \\ \text { C } & 0.625661 & 0.570994 & 0.231290\end{array}$




\begin{tabular}{|c|c|c|c|}
\hline $\mathrm{H}$ & -0.987640 & 0.255164 & 1.097382 \\
\hline $\mathrm{C}$ & 0.235741 & 0.331297 & -1.141165 \\
\hline $\mathrm{H}$ & -0.050986 & 1.201784 & -1.721318 \\
\hline $\mathrm{C}$ & 0.070127 & -0.903897 & -1.776295 \\
\hline $\mathrm{H}$ & 0.521951 & -1.811020 & -1.390430 \\
\hline $\mathrm{H}$ & -0.201277 & -0.895643 & -2.826626 \\
\hline $\mathrm{C}$ & 0.699696 & 1.993676 & 0.733324 \\
\hline $\mathrm{C}$ & 0.477503 & 3.104299 & -0.097794 \\
\hline $\mathrm{C}$ & 1.051217 & 2.253372 & 2.066488 \\
\hline $\mathrm{C}$ & 0.567282 & 4.405580 & 0.387656 \\
\hline $\mathrm{C}$ & 1.138985 & 3.553381 & 2.552535 \\
\hline $\mathrm{H}$ & 1.251794 & 1.432878 & 2.742178 \\
\hline $\mathrm{C}$ & 0.892449 & 4.640561 & 1.719374 \\
\hline $\mathrm{H}$ & 1.407922 & 3.712438 & 3.592302 \\
\hline $\mathrm{H}$ & 0.965324 & 5.655024 & 2.098360 \\
\hline $\mathrm{H}$ & 0.257976 & 2.977682 & -1.152361 \\
\hline $\mathrm{H}$ & 0.393318 & 5.236874 & -0.288771 \\
\hline $\mathrm{O}$ & 0.161177 & -0.537717 & 2.805889 \\
\hline $\mathrm{H}$ & -0.528286 & -1.219664 & 2.667179 \\
\hline $\mathrm{C}$ & 1.569583 & -0.413863 & 0.874155 \\
\hline $\mathrm{Br}$ & 3.056807 & 1.428412 & -1.595549 \\
\hline Ir & -1.619572 & -0.516824 & -0.223426 \\
\hline $\mathrm{C}$ & -2.489095 & 1.453302 & -0.379458 \\
\hline $\mathrm{C}$ & -2.711109 & 0.778766 & -1.596825 \\
\hline $\mathrm{C}$ & -3.420829 & -0.874921 & 0.820132 \\
\hline $\mathrm{C}$ & -4.078341 & 0.249711 & -2.005180 \\
\hline $\mathrm{C}$ & -3.423257 & -1.602062 & -0.419721 \\
\hline $\mathrm{C}$ & -4.236924 & -1.227831 & -1.639421 \\
\hline $\mathrm{H}$ & -1.687205 & 2.184768 & -0.354082 \\
\hline $\mathrm{H}$ & -4.852105 & 0.861472 & -1.531171 \\
\hline $\mathrm{H}$ & -3.204313 & -2.663609 & -0.347085 \\
\hline $\mathrm{H}$ & -2.039455 & 1.009454 & -2.420630 \\
\hline $\mathrm{H}$ & -4.199644 & 0.384054 & -3.083900 \\
\hline $\mathrm{H}$ & -5.294396 & -1.488816 & -1.487881 \\
\hline $\mathrm{C}$ & -3.535726 & 1.653285 & 0.697176 \\
\hline $\mathrm{H}$ & -4.223911 & 2.456059 & 0.397206 \\
\hline $\mathrm{H}$ & -3.007670 & 2.007660 & 1.588353 \\
\hline $\mathrm{C}$ & -4.288626 & 0.361072 & 1.041315 \\
\hline $\mathrm{H}$ & -3.222242 & -1.467020 & 1.710030 \\
\hline $\mathrm{H}$ & -5.209829 & 0.272654 & 0.455341 \\
\hline $\mathrm{H}$ & -4.601072 & 0.396679 & 2.088682 \\
\hline $\mathrm{H}$ & -3.878313 & -1.842266 & -2.471529 \\
\hline $\mathrm{Cl}$ & -0.839017 & -2.650990 & 0.773909 \\
\hline
\end{tabular}




\section{TS-6}

M06-2X/LANL2DZ-6-31G(D)/SMD SCF energy: -3795.29491670 a.u. M06-2X/LANL2DZ-6-31G(D)/SMD enthalpy: -3794.809750 a.u.

M06-2X/LANL2DZ-6-31G(D)/SMD free energy: -3794.889703 a.u.

M06-2X/LANL2DZ-6-311++G(D,P) SCF energy in solution: -3797.97206480 a.u.

M06-2X/LANL2DZ-6-311++G(D,P) enthalpy in solution: -3797.486898 a.u. M06-2X/LANL2DZ-6-311++G(D,P) free energy in solution: -3797.566851 a.u. Imaginary frequency: $\quad-791.1638 \mathrm{~cm}-1$

Cartesian coordinates

$\begin{array}{lccc}\text { ATOM } & \text { X } & \text { Y } & \text { Z } \\ \text { C } & 4.967994 & -0.329861 & -0.176557 \\ \text { C } & 3.615771 & -0.231593 & -0.416031 \\ \text { C } & 2.664144 & -1.129134 & 0.158844 \\ \text { C } & 3.239916 & -2.271139 & 0.804481 \\ \text { C } & 4.630548 & -2.363771 & 1.032009 \\ \text { C } & 5.485900 & -1.382767 & 0.597435 \\ \text { H } & 5.640399 & 0.384331 & -0.638464 \\ \text { C } & 2.391677 & -3.352377 & 1.187219 \\ \text { H } & 5.007599 & -3.238932 & 1.554417 \\ \text { H } & 6.553156 & -1.443300 & 0.781152 \\ \text { C } & 1.057827 & -3.316528 & 0.942238 \\ \text { C } & 0.445623 & -2.130379 & 0.419117 \\ \text { H } & 2.846824 & -4.221168 & 1.655582 \\ \text { H } & 0.401824 & -4.142939 & 1.195583 \\ \text { C } & 0.519256 & 0.292438 & -0.156469 \\ \text { H } & -0.702150 & 0.198071 & 0.969288 \\ \text { C } & -0.162301 & 0.450923 & -1.433222 \\ \text { H } & -0.428343 & 1.456986 & -1.746655 \\ \text { C } & -0.659869 & -0.613115 & -2.185495 \\ \text { H } & -0.259783 & -1.615970 & -2.076571 \\ \text { H } & -1.186346 & -0.391422 & -3.107423 \\ \text { C } & 1.026586 & 1.518408 & 0.573408 \\ \text { C } & 0.939680 & 2.810591 & 0.043379 \\ \text { C } & 1.571638 & 1.367313 & 1.855062 \\ \text { C } & 1.372117 & 3.914165 & 0.774588 \\ \text { C } & 2.015000 & 2.466949 & 2.580468 \\ \text { H } & 1.637911 & 0.373384 & 2.288662 \\ \text { C } & 1.913531 & 3.749379 & 2.045513 \\ \text { C.436515 } & 2.319567 & 3.570042\end{array}$




$\begin{array}{lrrc}\mathrm{H} & 2.256579 & 4.609682 & 2.611559 \\ \mathrm{H} & 0.565985 & 2.972042 & -0.962136 \\ \mathrm{H} & 1.296573 & 4.905263 & 0.337675 \\ \mathrm{O} & -0.860772 & -2.138653 & 0.321972 \\ \mathrm{C} & 1.230473 & -1.012863 & 0.111598 \\ \mathrm{Br} & 3.140713 & 1.019048 & -1.777382 \\ \mathrm{Ir} & -1.786552 & -0.367838 & -0.164378 \\ \mathrm{C} & -2.577624 & 1.614810 & 0.142409 \\ \mathrm{C} & -3.145516 & 1.097057 & -1.037014 \\ \mathrm{C} & -3.238640 & -0.860742 & 1.266872 \\ \mathrm{C} & -4.587626 & 0.610167 & -1.119165 \\ \mathrm{C} & -3.587515 & -1.428703 & -0.008372 \\ \mathrm{C} & -4.690004 & -0.906271 & -0.907101 \\ \mathrm{H} & -1.757196 & 2.324208 & 0.035010 \\ \mathrm{H} & -5.190997 & 1.152647 & -0.384984 \\ \mathrm{H} & -3.370916 & -2.489131 & -0.128370 \\ \mathrm{H} & -2.709465 & 1.423966 & -1.978682 \\ \mathrm{H} & -4.993506 & 0.875646 & -2.099845 \\ \mathrm{H} & -5.678549 & -1.182000 & -0.512101 \\ \mathrm{C} & -3.286366 & 1.670476 & 1.483211 \\ \mathrm{H} & -4.001095 & 2.505358 & 1.492717 \\ \mathrm{H} & -2.524935 & 1.900007 & 2.235732 \\ \mathrm{C} & -3.969917 & 0.343708 & 1.855900 \\ \mathrm{H} & -2.810133 & -1.549973 & 1.990537 \\ \mathrm{H} & -5.015476 & 0.335527 & 1.530285 \\ \mathrm{H} & -3.994888 & 0.249988 & 2.945364 \\ \mathrm{H} & -4.587645 & -1.408640 & -1.874628\end{array}$

\section{TS-6a}

M06-2X/LANL2DZ-6-31G(D)/SMD SCF energy: -3795.24666422 a.u. M06-2X/LANL2DZ-6-31G(D)/SMD enthalpy: M06-2X/LANL2DZ-6-31G(D)/SMD free energy: -3794.762371 a.u. M06-2X/LANL2DZ-6-311++G(D,P) SCF energy in solution: -3794.844676 a.u. M06-2X/LANL2DZ-6-311++G(D,P) enthalpy in solution: -3797.92642825 a.u. M06-2X/LANL2DZ-6-311++G(D,P) free energy in solution: -3797.442135 a.u. Imaginary frequency: $\quad-829.1327 \mathrm{~cm}-1$ -3797.524440 a.u.

Cartesian coordinates

$\begin{array}{lcrr}\text { ATOM } & \text { X } & \text { Y } & \text { Z } \\ \text { C } & -1.271357 & -3.084568 & -1.705606 \\ \text { C } & -1.266827 & -1.891454 & -0.986406\end{array}$




\begin{tabular}{|c|c|c|c|}
\hline $\mathrm{C}$ & -0.728051 & -1.781592 & 0.320722 \\
\hline $\mathrm{C}$ & -0.192159 & -3.011107 & 0.828725 \\
\hline $\mathrm{C}$ & -0.183214 & -4.196092 & 0.084484 \\
\hline$C$ & -0.724224 & -4.248882 & -1.184327 \\
\hline $\mathrm{H}$ & -1.709601 & -3.088180 & -2.696777 \\
\hline $\mathrm{C}$ & 0.370061 & -3.087382 & 2.166611 \\
\hline $\mathrm{H}$ & 0.253518 & -5.082849 & 0.535056 \\
\hline $\mathrm{H}$ & -0.728446 & -5.165977 & -1.762897 \\
\hline $\mathrm{C}$ & 0.274408 & -2.078277 & 3.043076 \\
\hline $\mathrm{C}$ & -0.430393 & -0.855685 & 2.647243 \\
\hline $\mathrm{H}$ & 0.825986 & -4.032312 & 2.453171 \\
\hline $\mathrm{H}$ & 0.606428 & -2.156127 & 4.073554 \\
\hline $\mathrm{C}$ & -0.949892 & 0.828396 & 0.891369 \\
\hline $\mathrm{H}$ & -0.329548 & 0.783903 & -0.567183 \\
\hline $\mathrm{C}$ & -0.088875 & 1.844709 & 1.526285 \\
\hline $\mathrm{H}$ & -0.302934 & 2.869992 & 1.231811 \\
\hline $\mathrm{C}$ & 1.073872 & 1.593993 & 2.271567 \\
\hline $\mathrm{H}$ & 1.176269 & 0.778843 & 2.970276 \\
\hline $\mathrm{H}$ & 1.701575 & 2.456047 & 2.477295 \\
\hline $\mathrm{C}$ & -2.337525 & 1.279686 & 0.557985 \\
\hline $\mathrm{C}$ & -2.583047 & 2.409206 & -0.225912 \\
\hline $\mathrm{C}$ & -3.407718 & 0.584608 & 1.118402 \\
\hline $\mathrm{C}$ & -3.886288 & 2.829749 & -0.459054 \\
\hline $\mathrm{C}$ & -4.714212 & 1.008610 & 0.885488 \\
\hline $\mathrm{H}$ & -3.211553 & -0.284429 & 1.739770 \\
\hline $\mathrm{C}$ & -4.957326 & 2.127542 & 0.094993 \\
\hline $\mathrm{H}$ & -5.542354 & 0.459756 & 1.323129 \\
\hline $\mathrm{H}$ & -5.975955 & 2.453106 & -0.091481 \\
\hline $\mathrm{H}$ & -1.748091 & 2.934540 & -0.685661 \\
\hline $\mathrm{H}$ & -4.069619 & 3.700363 & -1.081402 \\
\hline $\mathrm{O}$ & -0.878718 & -0.090579 & 3.496290 \\
\hline $\mathrm{C}$ & -0.540335 & -0.582085 & 1.191041 \\
\hline $\mathrm{Br}$ & -2.046001 & -0.470792 & -1.998776 \\
\hline Ir & 1.171014 & 0.608562 & 0.223173 \\
\hline $\mathrm{C}$ & 1.778423 & 1.993253 & -1.324754 \\
\hline $\mathrm{C}$ & 2.765889 & 1.986527 & -0.316562 \\
\hline $\mathrm{C}$ & 1.973652 & -0.788565 & -1.133949 \\
\hline $\mathrm{C}$ & 4.130586 & 1.329275 & -0.478664 \\
\hline $\mathrm{C}$ & 2.788033 & -0.738035 & 0.051929 \\
\hline $\mathrm{C}$ & 4.151155 & -0.080355 & 0.126414 \\
\hline $\mathrm{H}$ & 1.086789 & 2.834380 & -1.333499 \\
\hline $\mathrm{H}$ & 4.397706 & 1.308017 & -1.539227 \\
\hline $\mathrm{H}$ & 2.649127 & -1.559809 & 0.756210 \\
\hline
\end{tabular}




$\begin{array}{lrrr}\mathrm{H} & 2.727443 & 2.805169 & 0.397097 \\ \mathrm{H} & 4.882175 & 1.955883 & 0.010893 \\ \mathrm{H} & 4.915402 & -0.704949 & -0.358595 \\ \mathrm{C} & 1.904744 & 1.253920 & -2.642684 \\ \mathrm{H} & 2.547344 & 1.824564 & -3.328466 \\ \mathrm{H} & 0.908227 & 1.226099 & -3.095017 \\ \mathrm{C} & 2.408767 & -0.188367 & -2.470591 \\ \mathrm{H} & 1.332623 & -1.661796 & -1.226267 \\ \mathrm{H} & 3.498256 & -0.240544 & -2.569013 \\ \mathrm{H} & 2.005804 & -0.802564 & -3.281448 \\ \mathrm{H} & 4.426197 & -0.010235 & 1.184225\end{array}$

\section{TS-7}

M06-2X/LANL2DZ-6-31G(D)/SMD SCF energy: -3795.27859709 a.u. M06-2X/LANL2DZ-6-31G(D)/SMD enthalpy: -3794.793611 a.u.

M06-2X/LANL2DZ-6-31G(D)/SMD free energy: -3794.875909 a.u. M06-2X/LANL2DZ-6-311++G(D,P) SCF energy in solution: -3797.95779950 a.u.

M06-2X/LANL2DZ-6-311++G(D,P) enthalpy in solution: -3797.472813 a.u. M06-2X/LANL2DZ-6-311++G(D,P) free energy in solution: -3797.555111 a.u. Imaginary frequency: $\quad-840.1120 \mathrm{~cm}-1$

Cartesian coordinates

$\begin{array}{lccc}\text { ATOM } & \text { X } & \text { Y } & \text { Z } \\ \text { C } & -4.111529 & 2.381485 & -0.992287 \\ \text { C } & -3.188591 & 1.373766 & -0.836618 \\ \text { C } & -2.159329 & 1.420750 & 0.155671 \\ \text { C } & -2.079937 & 2.650433 & 0.886845 \\ \text { C } & -3.033443 & 3.679257 & 0.705424 \\ \text { C } & -4.055442 & 3.541209 & -0.196621 \\ \text { H } & -4.867800 & 2.291216 & -1.763683 \\ \text { C } & -1.016712 & 2.837535 & 1.813179 \\ \text { H } & -2.932874 & 4.583965 & 1.298934 \\ \text { H } & -4.793929 & 4.323792 & -0.334650 \\ \text { C } & -0.060690 & 1.884448 & 1.984628 \\ \text { C } & -0.131817 & 0.628107 & 1.298751 \\ \text { H } & -0.980500 & 3.767982 & 2.374158 \\ \text { H } & 0.749252 & 2.019921 & 2.694706 \\ \text { C } & -1.470651 & -1.059483 & 0.120596 \\ \text { H } & 1.838243 & -1.875991 & -0.182781 \\ \text { C } & -0.579781 & -1.823906 & -0.535621 \\ \text { H } & -0.762063 & -2.895302 & -0.596001\end{array}$




$\begin{array}{lccc}\mathrm{C} & 0.637206 & -1.324930 & -1.204921 \\ \mathrm{H} & 0.459426 & -0.329977 & -1.658033 \\ \mathrm{H} & 0.984791 & -1.993268 & -1.994975 \\ \mathrm{C} & -2.704997 & -1.661430 & 0.697220 \\ \mathrm{C} & -3.352948 & -2.732334 & 0.065503 \\ \mathrm{C} & -3.245402 & -1.175251 & 1.894626 \\ \mathrm{C} & -4.483610 & -3.315507 & 0.625272 \\ \mathrm{C} & -4.380298 & -1.755726 & 2.453935 \\ \mathrm{H} & -2.761383 & -0.343189 & 2.398071 \\ \mathrm{C} & -5.003063 & -2.829915 & 1.824284 \\ \mathrm{H} & -4.777276 & -1.366310 & 3.386735 \\ \mathrm{H} & -5.891337 & -3.279390 & 2.257741 \\ \mathrm{H} & -2.979197 & -3.087552 & -0.890586 \\ \mathrm{H} & -4.971817 & -4.140620 & 0.114971 \\ \mathrm{O} & 0.798328 & -0.281293 & 1.568811 \\ \mathrm{C} & -1.220249 & 0.371700 & 0.447071 \\ \mathrm{Br} & -3.281183 & 0.002982 & -2.158187 \\ \mathrm{Ir} & 2.147367 & -0.306020 & -0.022206 \\ \mathrm{C} & 3.687660 & -1.031016 & -1.357786 \\ \mathrm{C} & 3.479067 & 0.331802 & -1.617011 \\ \mathrm{C} & 3.793780 & -0.237618 & 1.367370 \\ \mathrm{C} & 4.453667 & 1.419144 & -1.179180 \\ \mathrm{C} & 3.373749 & 1.081181 & 1.100634 \\ \mathrm{C} & 4.073089 & 2.051308 & 0.169946 \\ \mathrm{H} & 5.498952 & -1.526506 & 1.519263 \\ \mathrm{H} & 3.242435 & -1.741907 & -2.050831 \\ \mathrm{H} & 5.461875 & 0.995486 & -1.139307 \\ \mathrm{H} & 2.383630 & 2.883069 & -0.009850 \\ \mathrm{H} & 2.722827 & 1.535342 & 1.844029 \\ \mathrm{H} & 2.847552 & 0.575699 & -2.470419 \\ \mathrm{H} & 4.482732 & 2.195958 & -1.948670 \\ \mathrm{H} & 4.960141 & 2.484475 & 0.653290 \\ \mathrm{C} & 4.823862 & -1.595935 & -0.529651 \\ \mathrm{H} & 5.743127 & -1.629357 & -1.129994 \\ \mathrm{H} & 4.564414 & -2.633794 & -0.297065 \\ \mathrm{H} & 3.412220 & -0.697203 & 2.276497 \\ \mathrm{H} & 5.805418 & -0.040605 & 0.647335 \\ \mathrm{H} & & & \\ \mathrm{H} & & -836812 & 0.788255 \\ \mathrm{H} & & & \\ \mathrm{H} & & & \end{array}$




\section{Reference}

[1] S.-L. Li., C. Yang., Q. Wu., H.-L. Zheng., X. Li., J.-P. Cheng. Atroposelective catalytic asymmetric allylic alkylation reaction for axially chiral anilides with achiral Morita-Baylis-Hillman carbonates. J. Am. Chem. Soc. 2018, 140, 12836-12843.

[2] W.T. Hendrix., J. L. von Rosenberg. The mechanism of the rearrangement of the hydrocobalt carbonyl catalyzed isomerization of 3-phenylpropene. J. Am. Chem. Soc. 1976, $98,4850-4852$.

[3] C. Li, B. Breit, Rhodium-catalyzed dynamic kinetic asymmetric allylation of phenols and 2-hydroxypyridines. Chem. Eur. J. 2016, 22, 14655-14663.

[4] Frisch, M. J.; Trucks, G. W.; Schlegel, H. B.; Scuseria, G. E.; Robb, M. A.; Cheeseman, J. R.; Scalmani, G.; Barone, V.; Mennucci, B.; Petersson, G. A.; Nakatsuji, H.; Caricato, M.; Li, X.; Hratchian, H. P.; Izmaylov, A. F.; Bloino, J.; Zheng, G.; Sonnenberg, J. L.; Hada, M.; Ehara, M.; Toyota, K.; Fukuda, R.; Hasegawa, J.; Ishida, M.; Nakajima, T.; Honda, Y.; Kitao, O.; Nakai, H.; Vreven, T.; Montgomery, J. A., Jr.; ; Peralta, J. E.; Ogliaro, F.; Bearpark, M.; Heyd, J. J.; Brothers, E.; Kudin, K. N.; Staroverov, V. N.; Kobayashi, R.; Normand, J.; Raghavachari, K.; Rendell, A.; Burant, J. C.; Iyengar, S. S.; Tomasi, J.; Cossi, M.; Rega, N.; Millam, N. J.; Klene, M.; Knox, J. E.; Cross, J. B.; Bakken, V.; Adamo, C.; Jaramillo, J.; Gomperts, R.; Stratmann, R. E.; Yazyev, O.; Austin, A. J.; Cammi, R.; Pomelli, C.; Ochterski, J. W.; Martin, R. L.; Morokuma, K.; Zakrzewski, V. G.; Voth, G. A.; Salvador, P.; Dannenberg, J. J.; Dapprich, S.; Daniels, A. D.; Farkas, O.; Foresman, J. B.; Ortiz, J. V.; Cioslowski, J.; Fox, D. J. Gaussian 16, Revision A.03; Gaussian, Inc.: Wallingford, CT, 2016.

[5] Marenich, A. V.; Cramer, C. J.; Truhlar, D. G. Universal solvation model based on solute electron density and on a continuum model of the solvent defined by the bulk dielectric constant and atomic surface tensions. J. Phys. Chem. B 2009, 113, 6378-6396. [6] Zhao, Y.; Truhlar, D. G. The M06 suite of density functionals for main group thermochemistry, thermochemical kinetics, noncovalent interactions, excited states, and transition elements: two new functionals and systematic testing of four M06-class functionals and 12 other functionals. Theor. Chem. Acc. 2007, 120, 215-241. 


\section{Spectra}
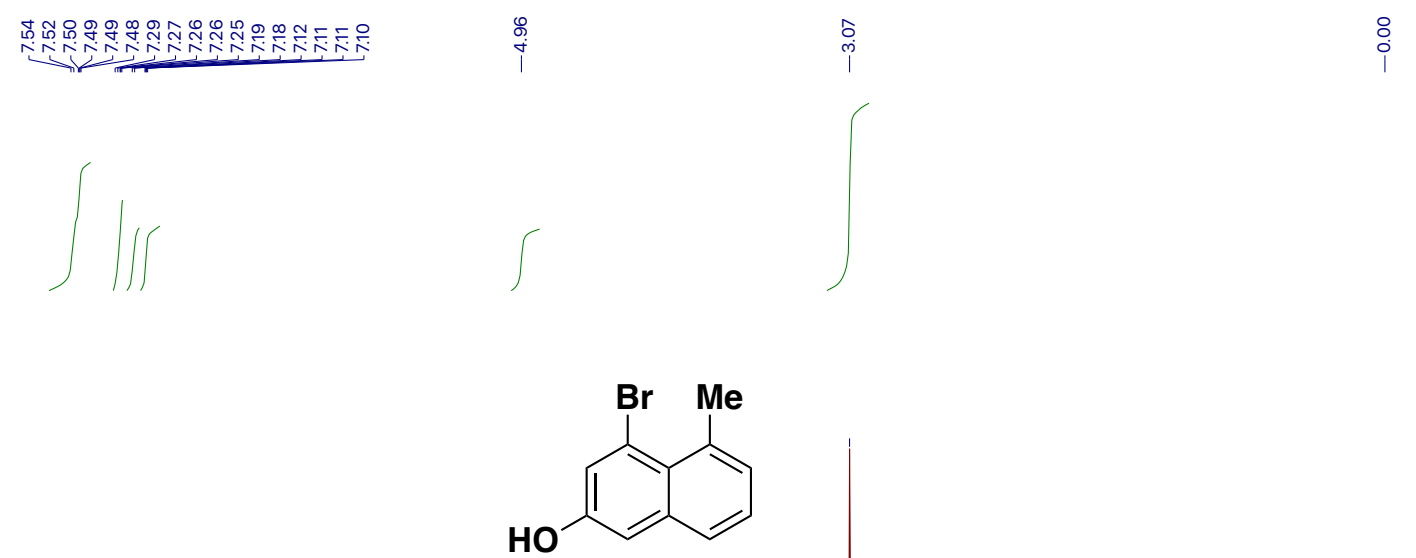

S1

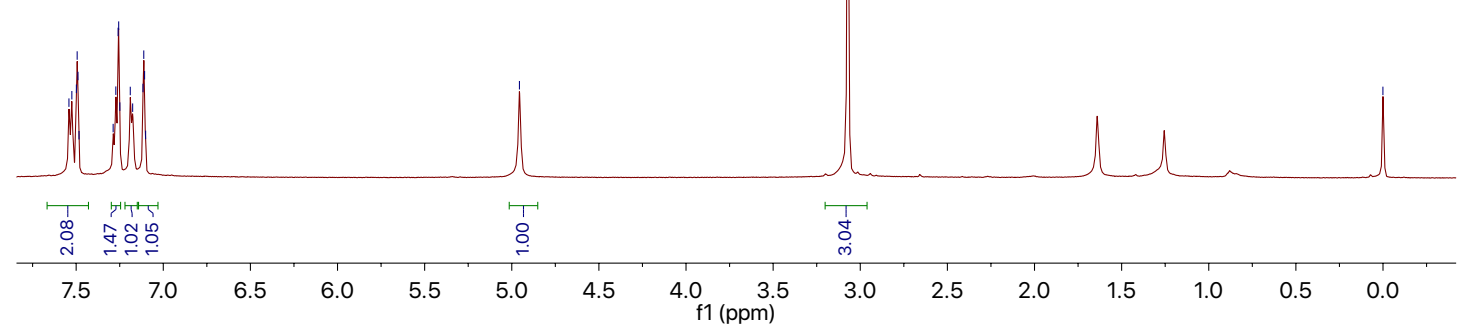

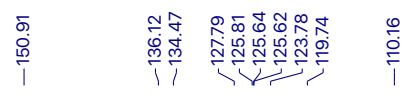<smiles>Cc1cccc2cc(O)cc(Br)c12</smiles>

S1

(10.t)

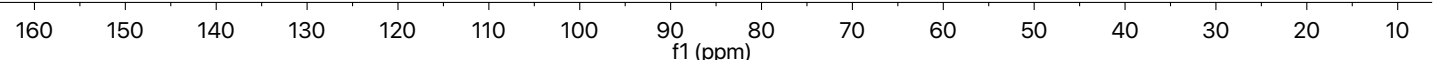




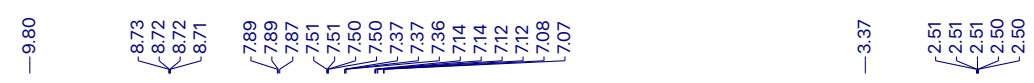

$\iiint||$<smiles>Oc1ccc2cccc(-c3ccncc3)c2c1</smiles>

S2

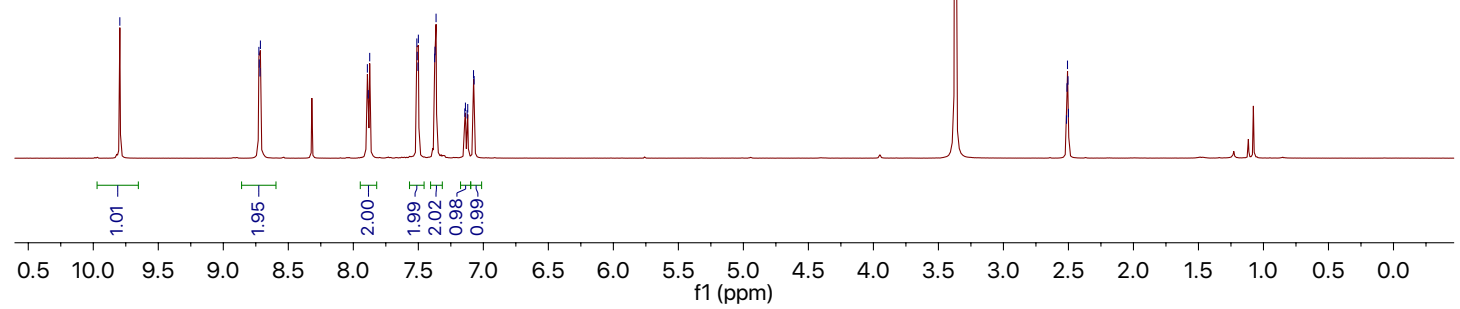

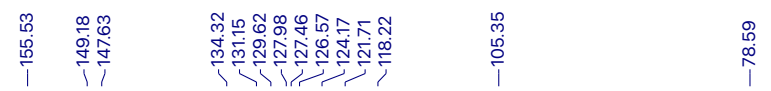<smiles>Oc1ccc2cccc(-c3ccncc3)c2c1</smiles>

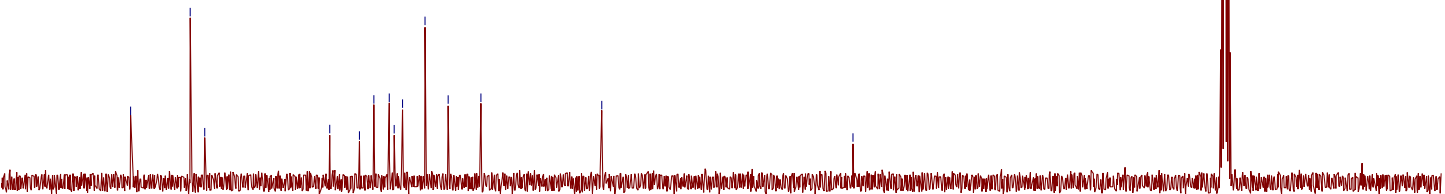

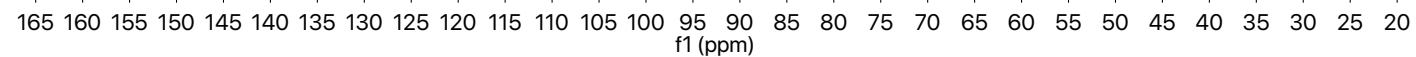



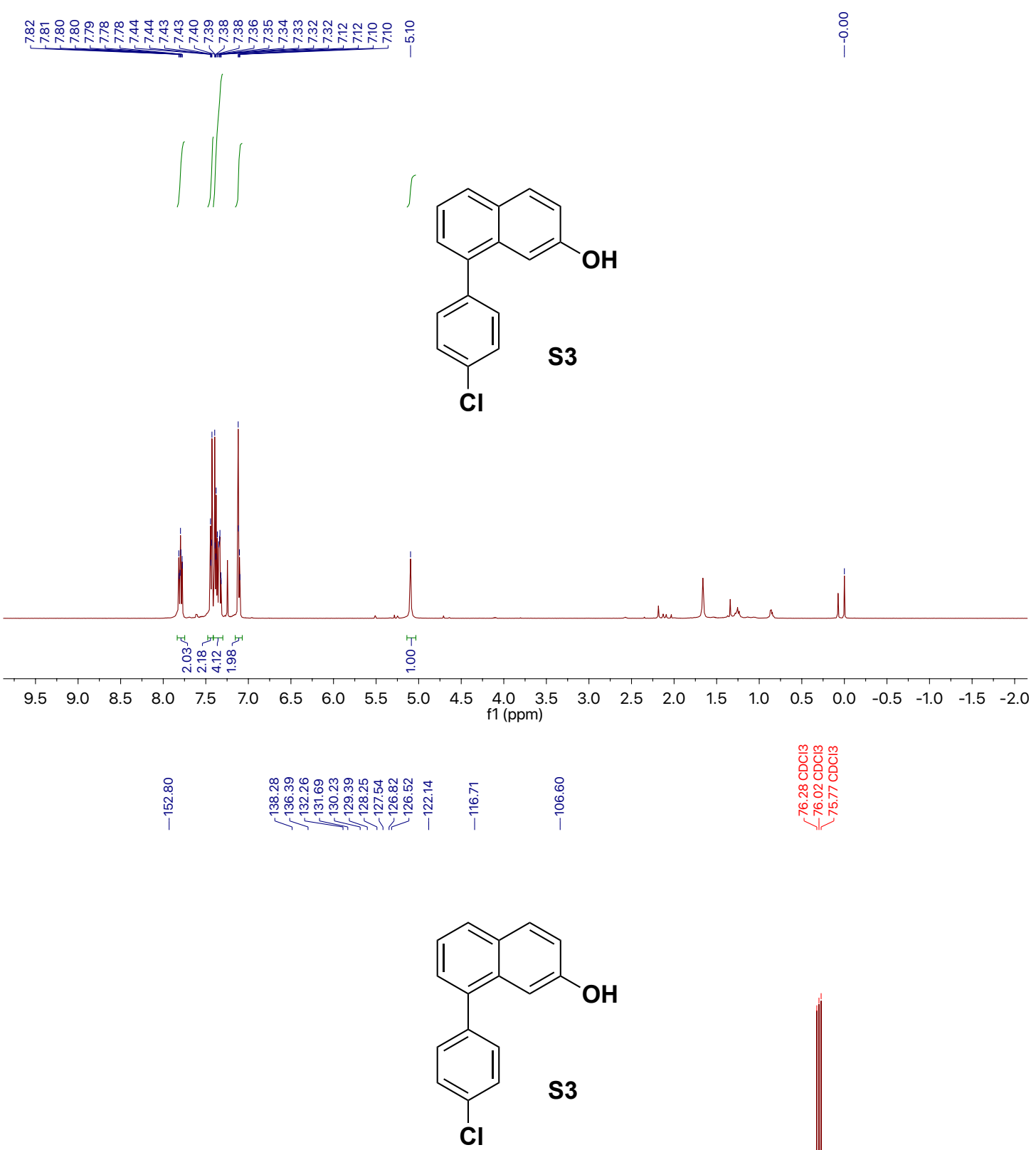

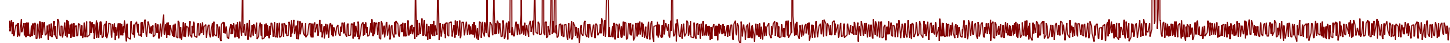

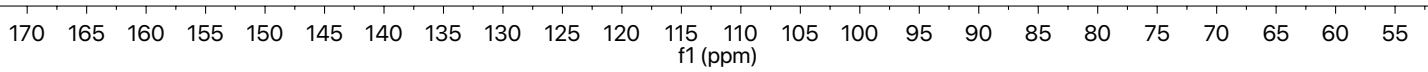


$\underbrace{.05}$
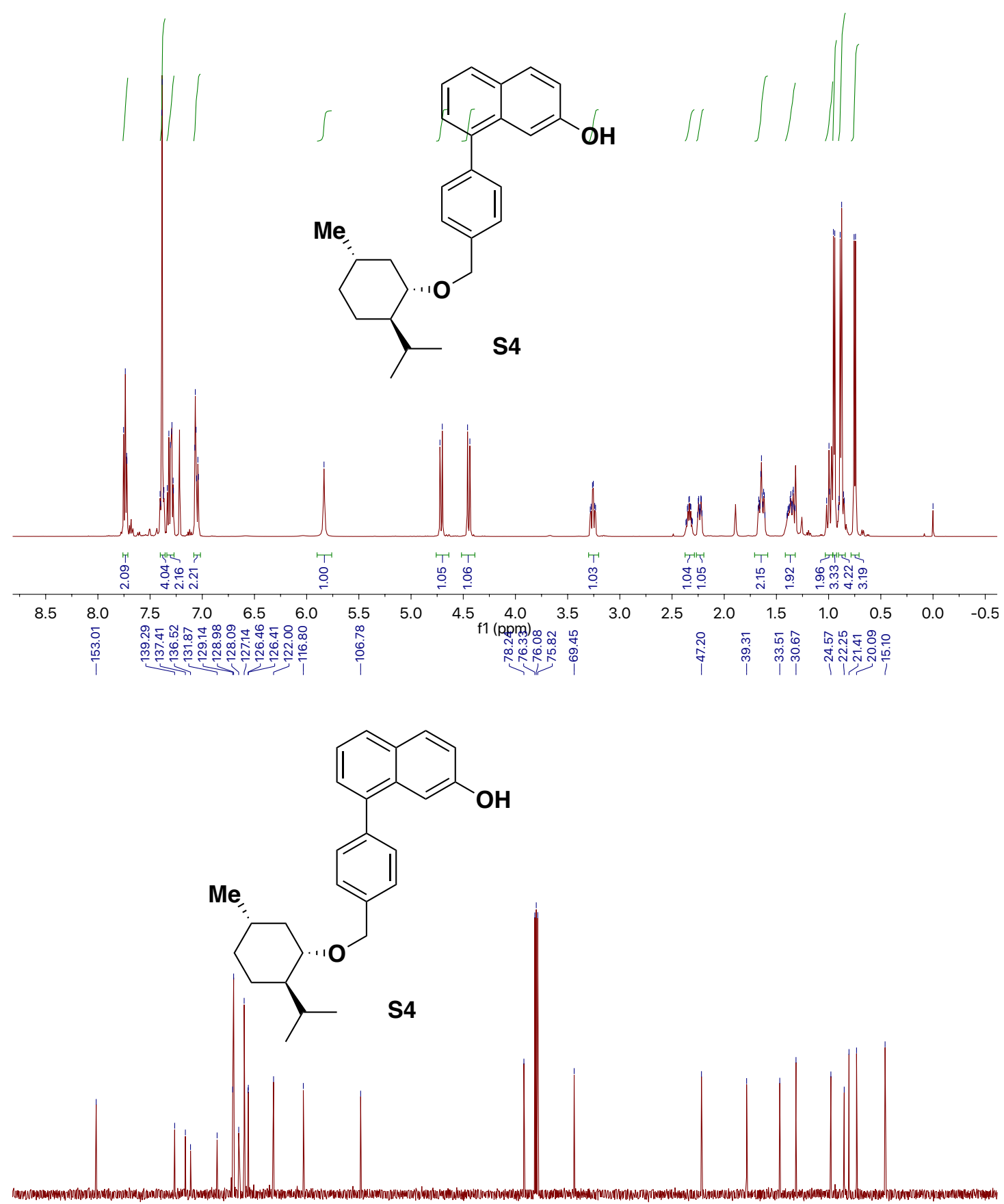

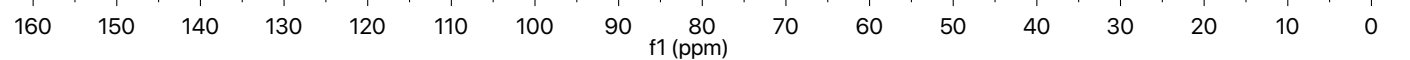




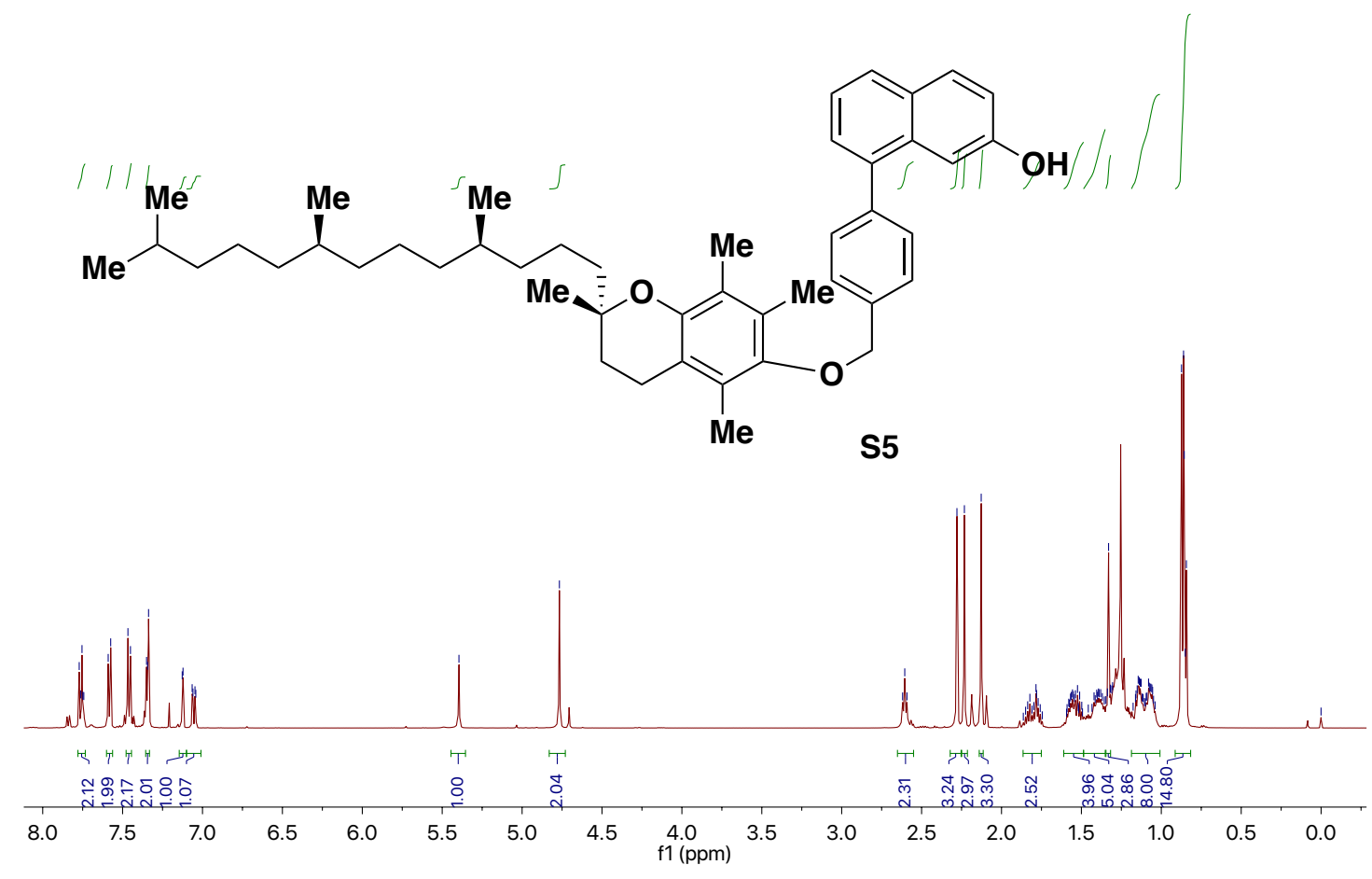

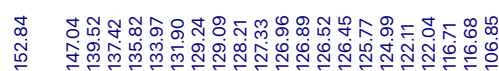

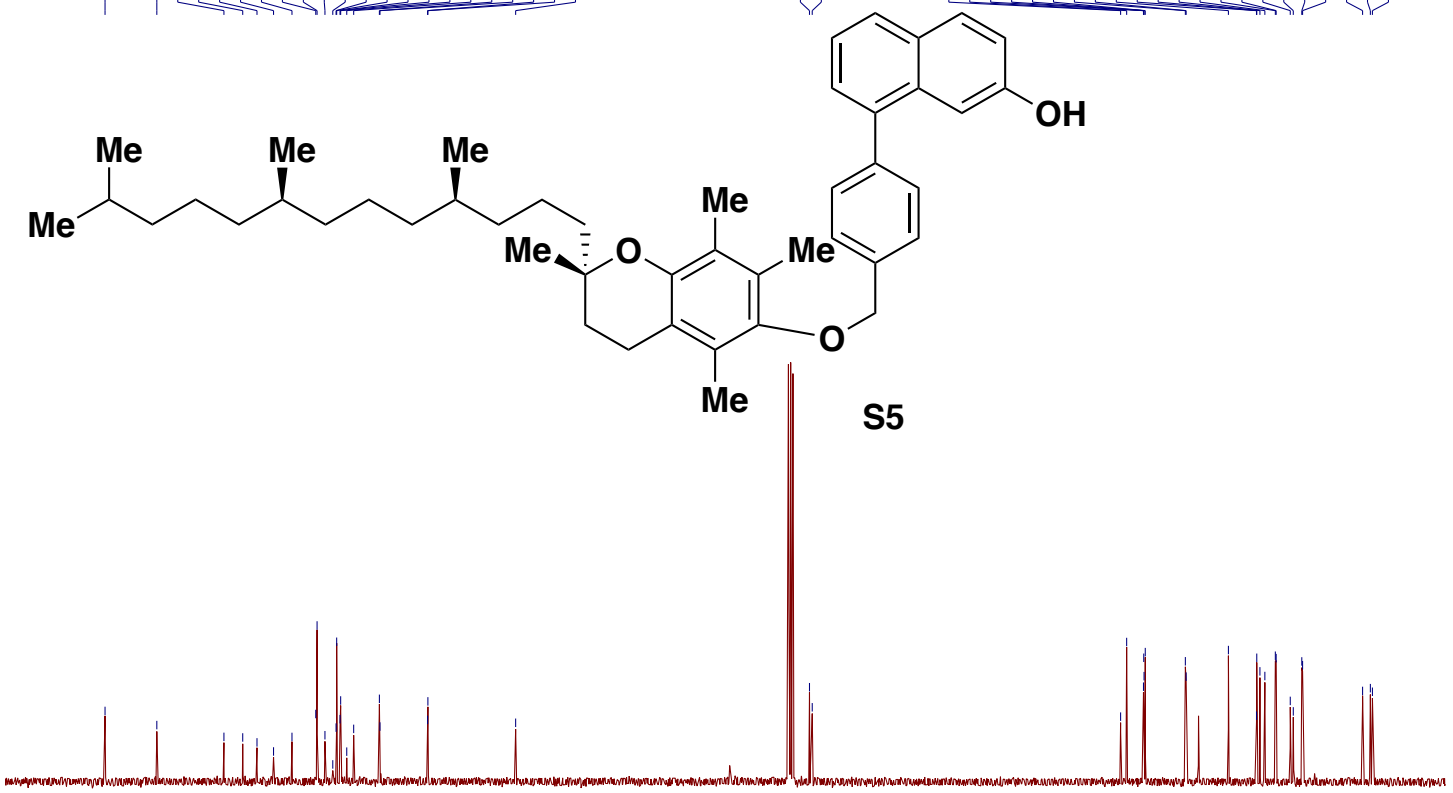

160

150140

130

120

$110 \quad 100$

$90 \begin{array}{r}80 \\ f 1(p p m)\end{array}$

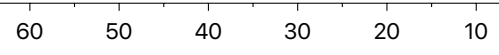



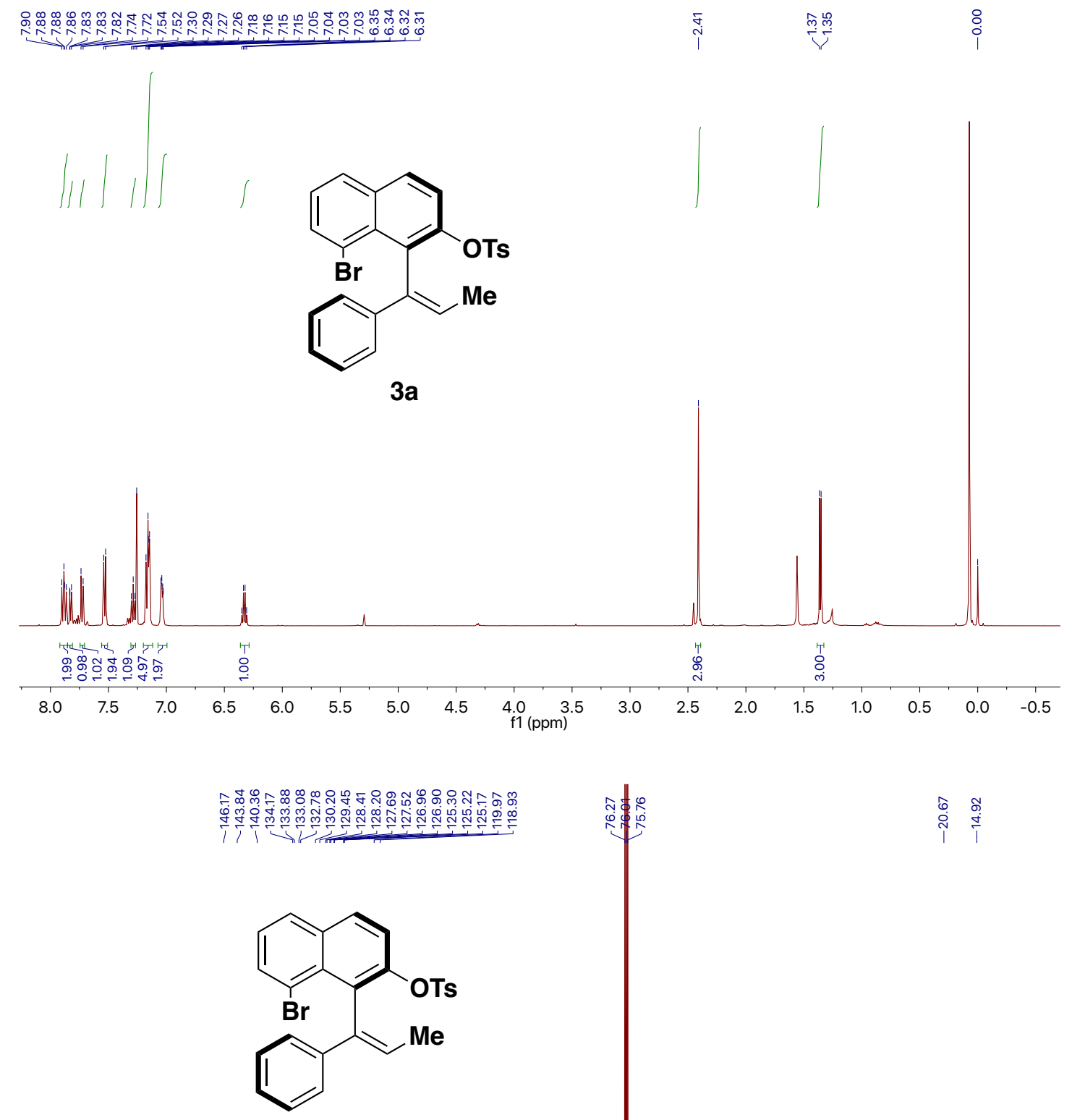

$3 a$

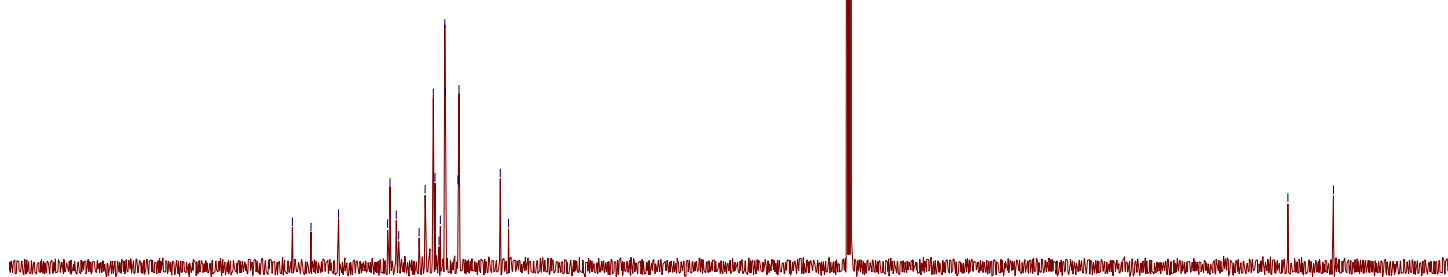

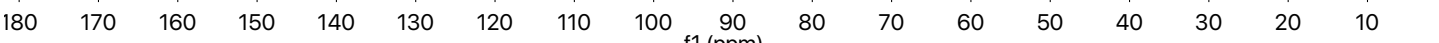



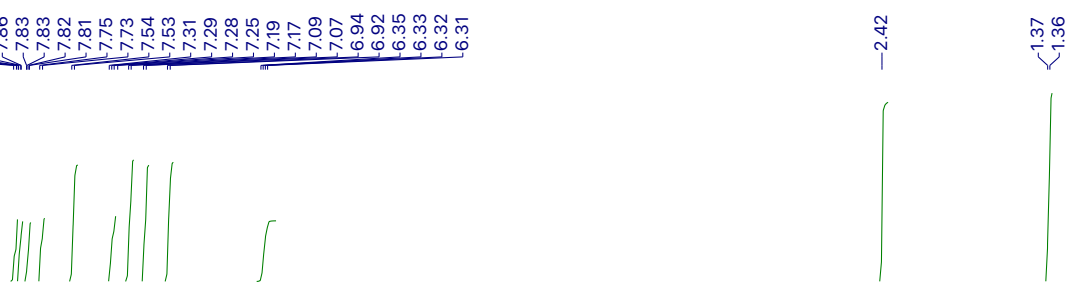<smiles>C/C=C(\c1ccc(Cl)cc1)c1c(O[Na])ccc2cccc(Br)c12</smiles>

3b

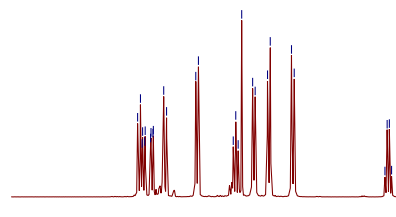

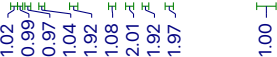

$\begin{array}{lllllllll}8.5 & 8.0 & 7.5 & 7.0 & 6.5 & 6.0 & 5.5 & 5.0 & 4.5 \\ \mathrm{f} 1(\mathrm{ppm})\end{array}$

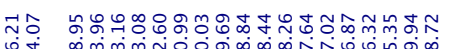

住<smiles>C/C=C(\c1ccc(Cl)cc1)c1c(OC)ccc2cccc(Br)c12</smiles>

$3 b$

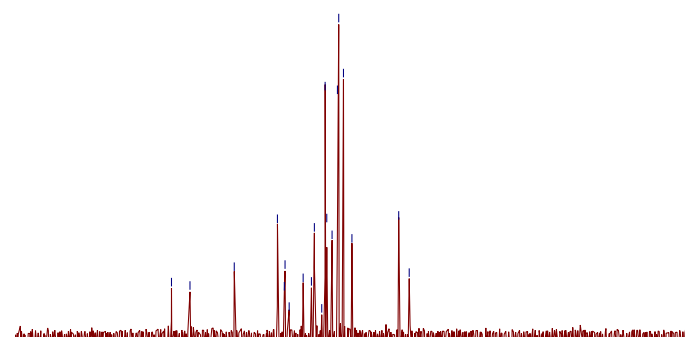

160

$150 \quad 140 \quad 130$

$120 \quad 110 \quad 100$

$90 \begin{gathered}80 \\ \mathrm{f} 1(\mathrm{ppm})\end{gathered}$

60

0

40

30

$\stackrel{T}{\stackrel{r}{m}}$

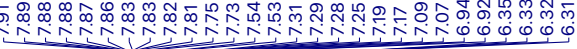

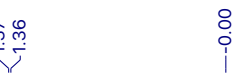




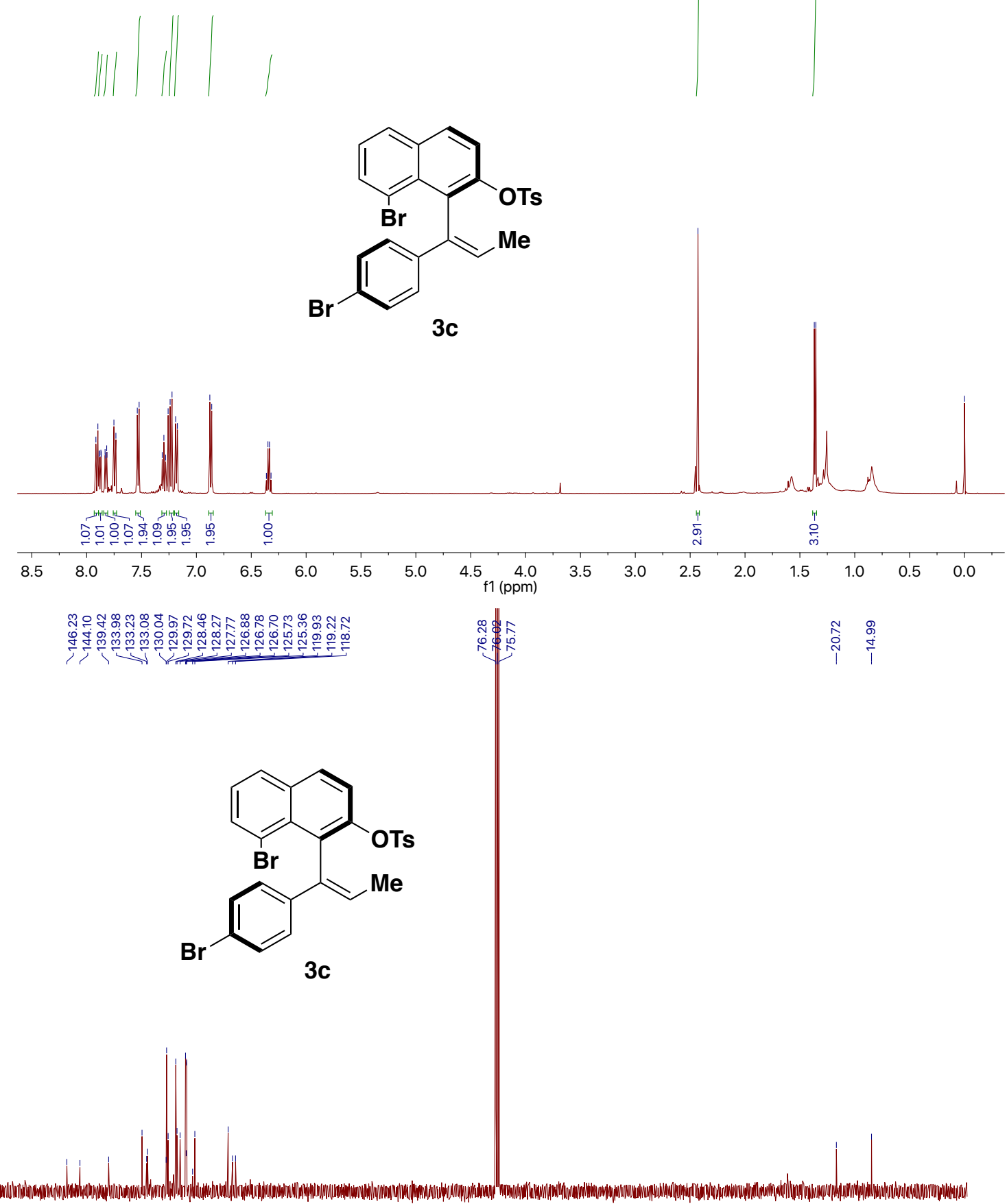

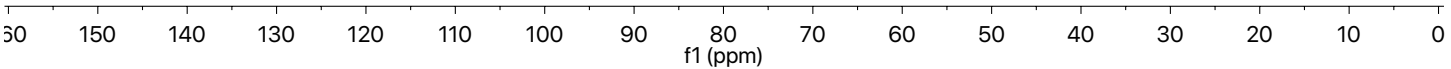



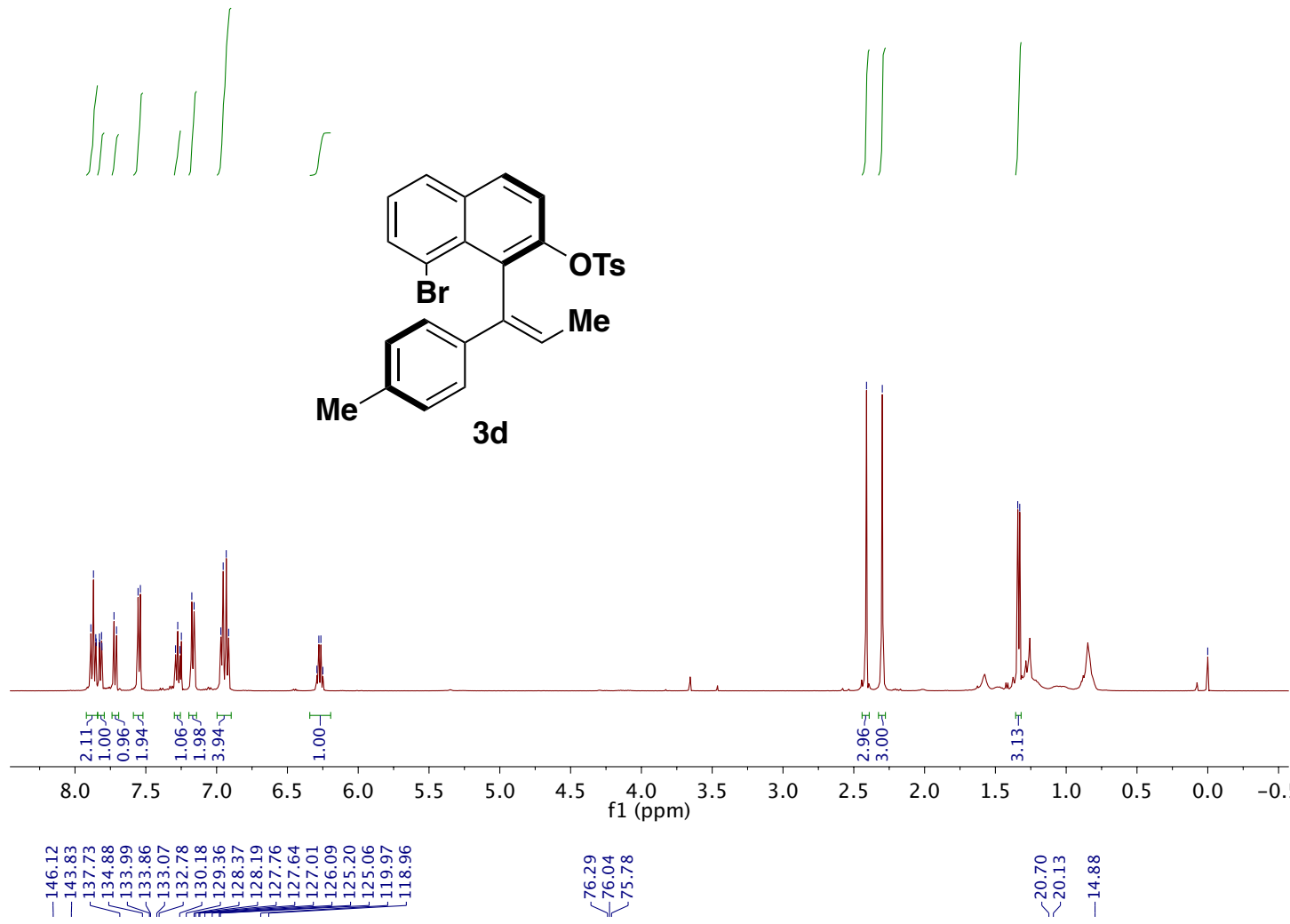<smiles>C/C=C(\c1ccc(C)cc1)c1c(OC)ccc2cccc(Br)c12</smiles>

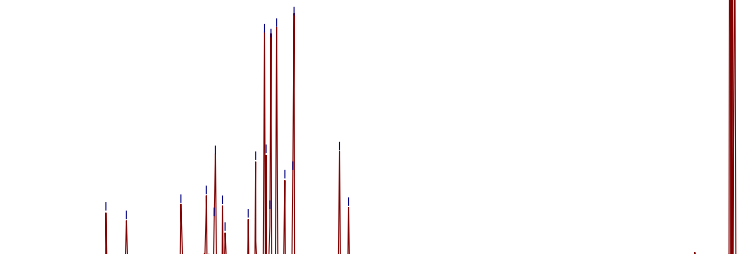

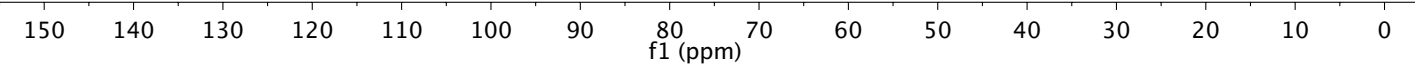




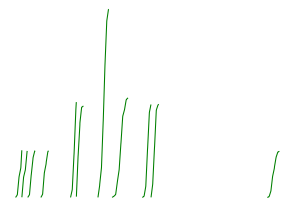<smiles>C/C=C(\c1ccc(-c2ccccc2)cc1)c1c(OC(F)(F)F)ccc2cccc(Br)c12</smiles>

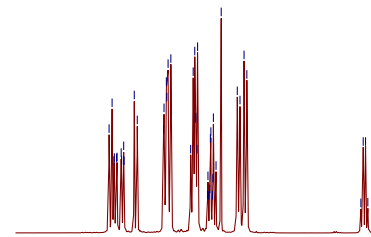

$3 e$

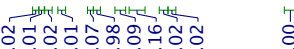

$\begin{array}{llll}8.0 & 7.5 & 7.0 & 6.5\end{array}$

6.

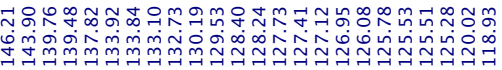

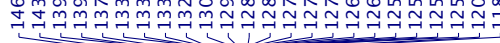
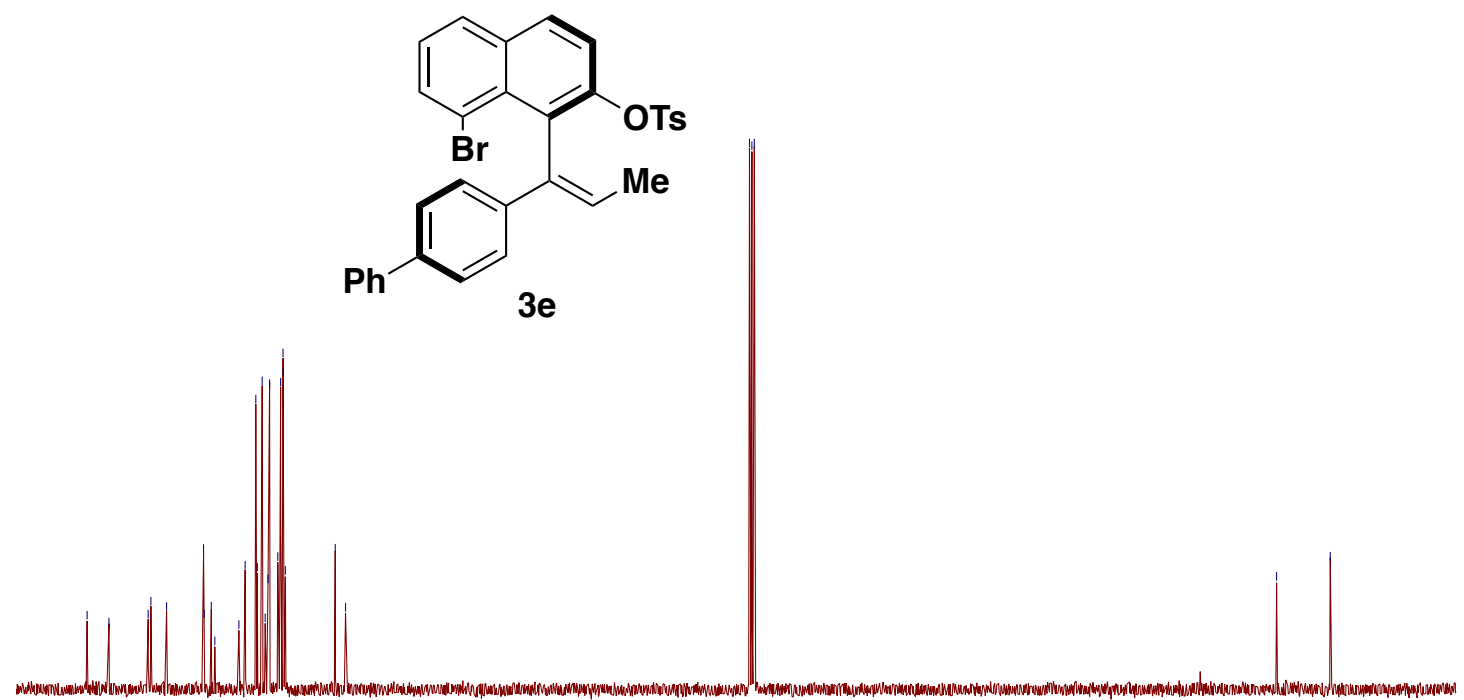

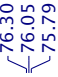

\begin{tabular}{ll}
$\hat{0}$ & \multicolumn{1}{c}{} \\
$\stackrel{0}{0}$ & ம் \\
1 & 1
\end{tabular}

$\begin{array}{lllllllllllllll}145 & 135 & 125 & 115 & 105 & 95 & 85 & \begin{array}{c}75 \\ \mathrm{f} 1(\mathrm{ppm})\end{array} & 65 & 55 & 45 & 35 & 25 & 15 & 5\end{array}$ 

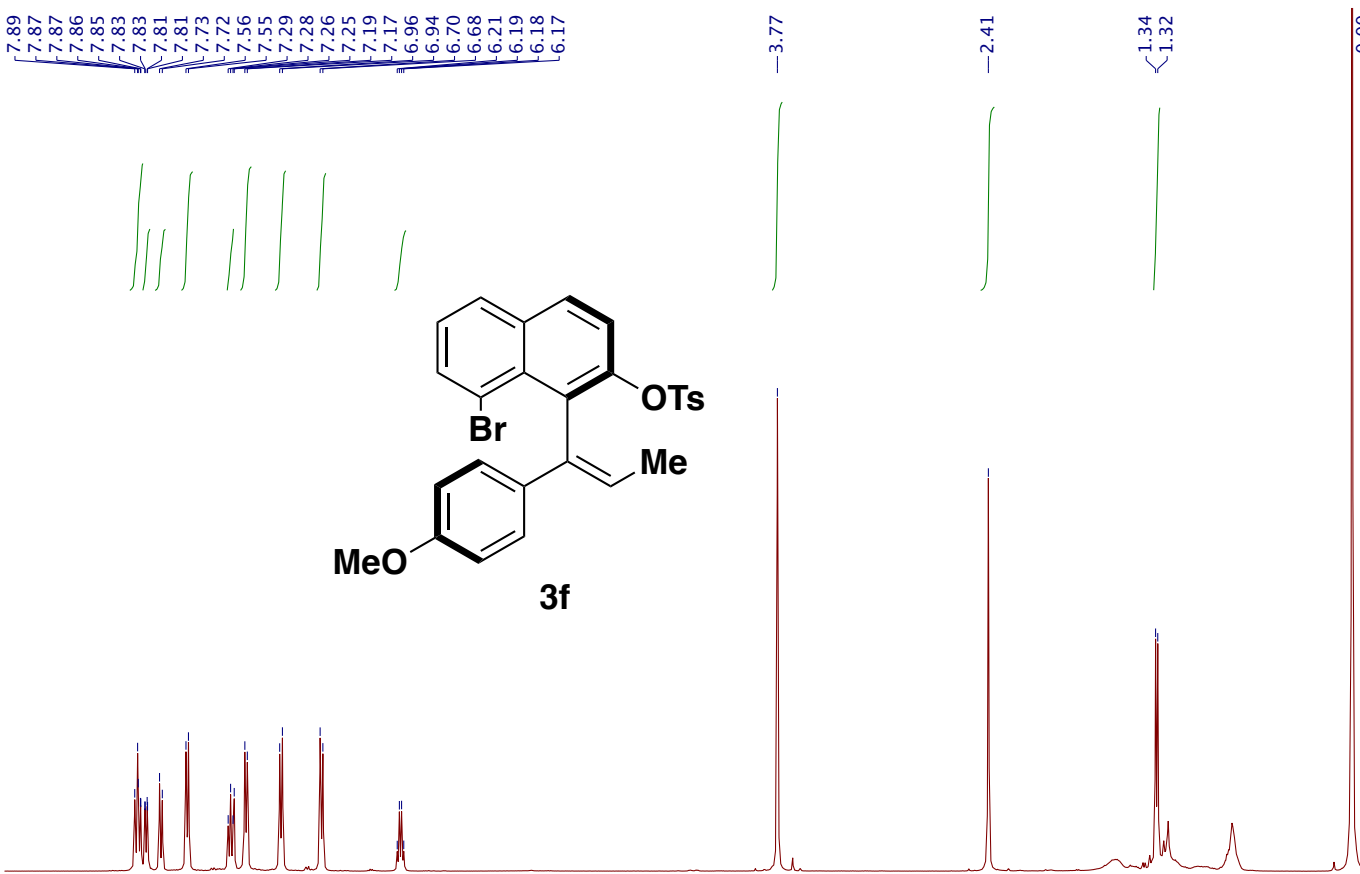

$3 f$

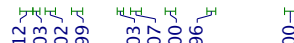

$\begin{array}{lllllllll}8.5 & 8.0 & 7.5 & 7.0 & 6.5 & 6.0 & 5.5 & 5.0 & 4.5 \\ \mathrm{f} 1\left(\begin{array}{c}4.0 \\ (\mathrm{ppm})\end{array}\right.\end{array}$

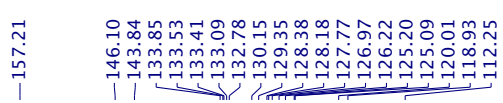

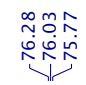

证

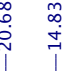<smiles>C/C=C(\c1ccc(OC)cc1)c1c(OC)ccc2cccc(Br)c12</smiles>

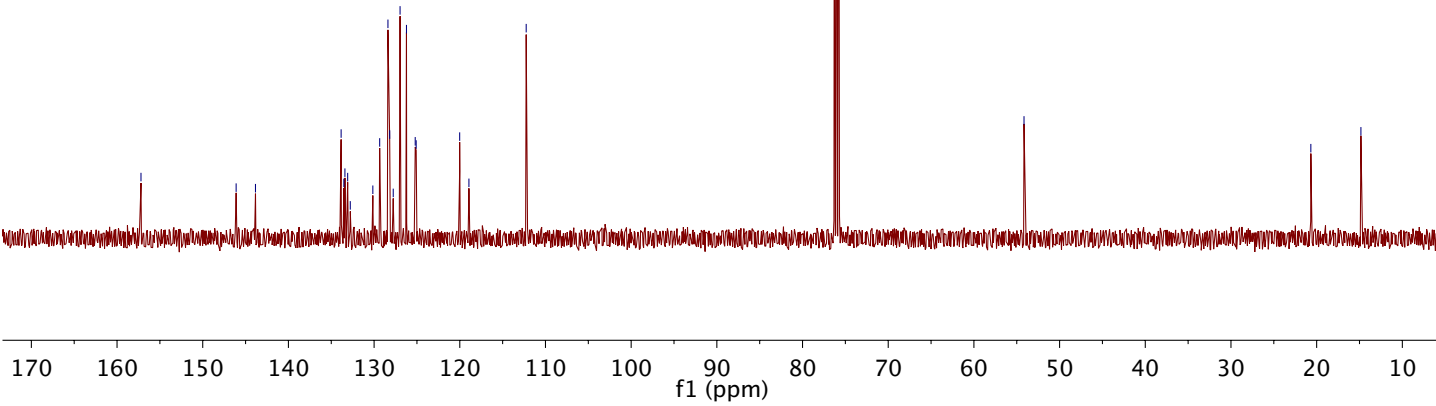




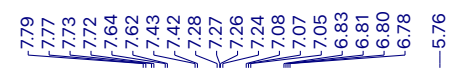

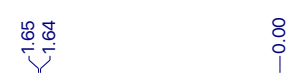

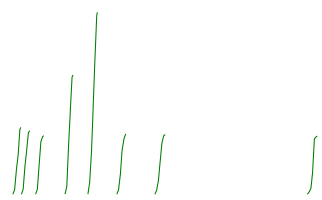

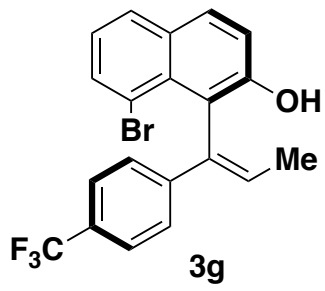

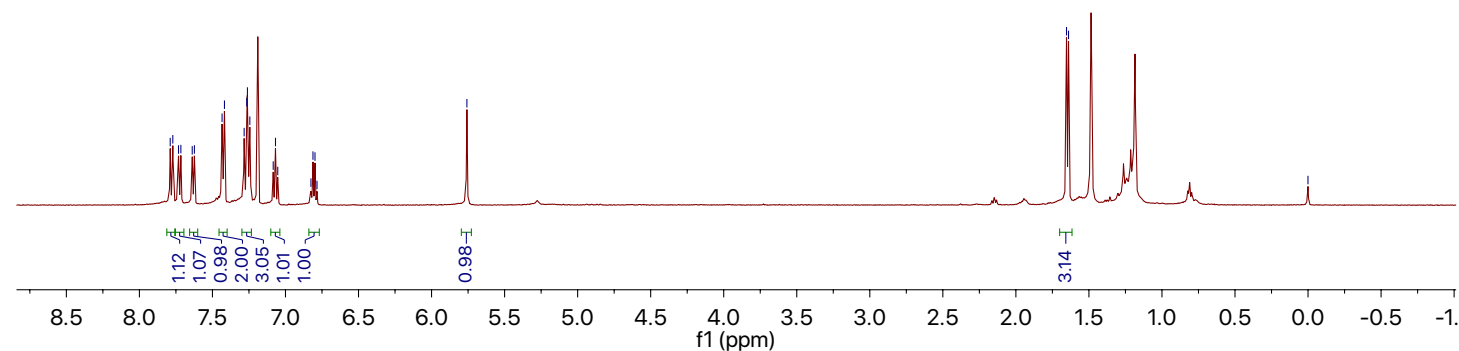

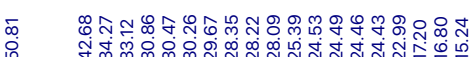

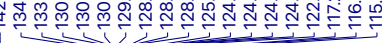
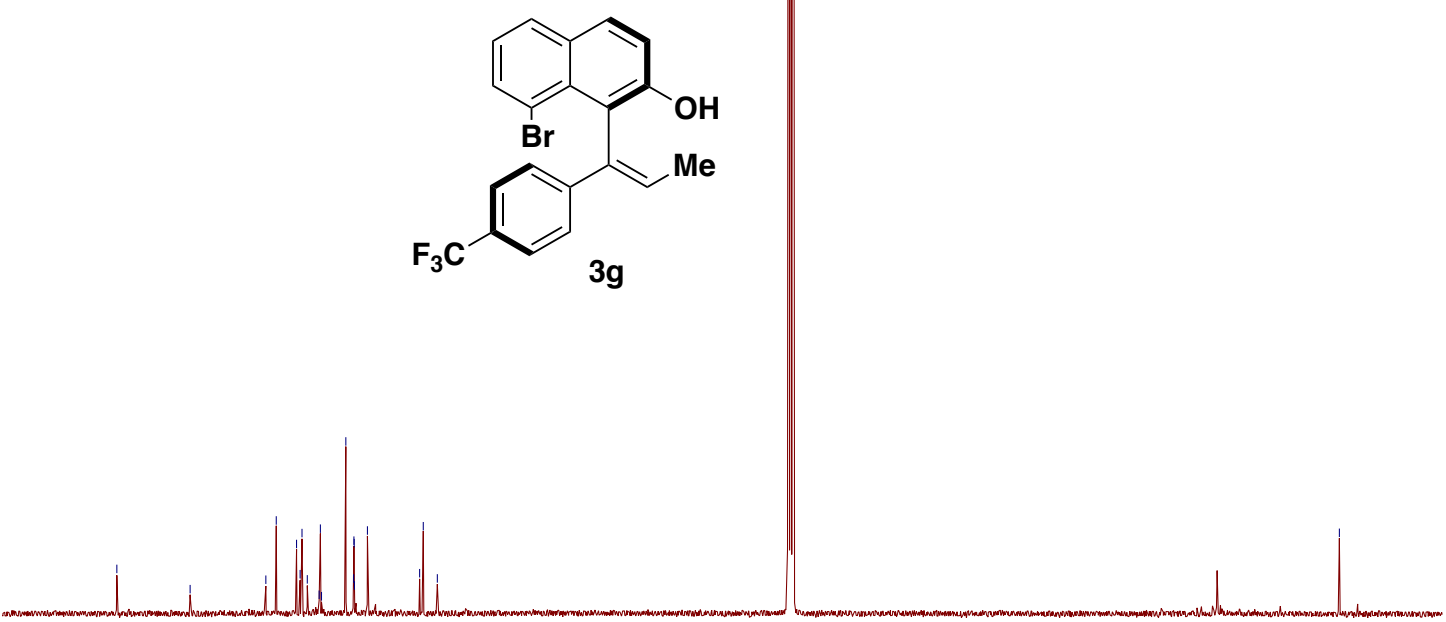

160

$\begin{array}{lllllll}150 & 140 & 130 & 120 & 110 & 100 & 90 \\ \mathrm{f} 1(\mathrm{ppm}) & 80\end{array}$ 

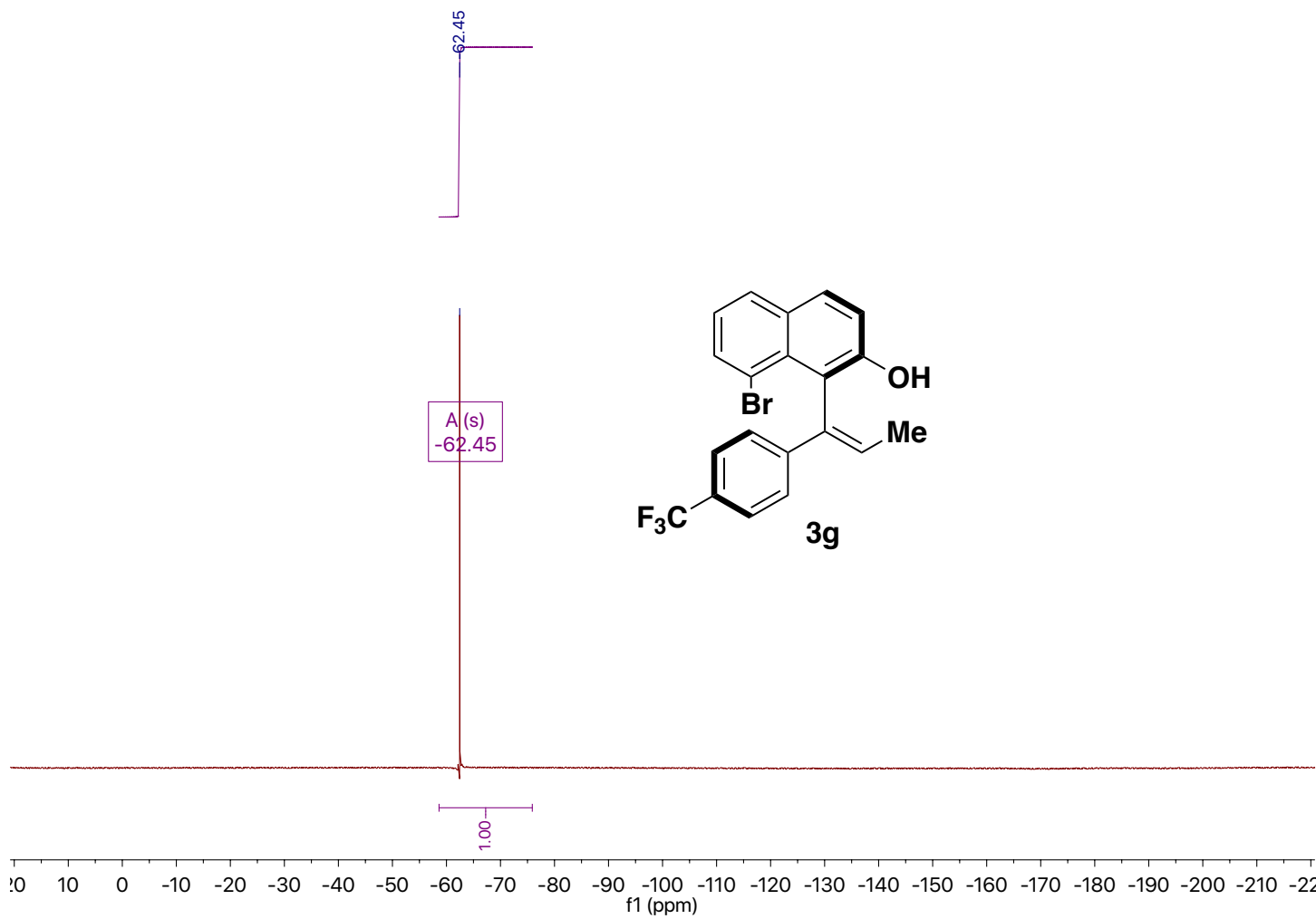
<smiles>C/C=C(\c1cccc(C)c1)c1c(OC)ccc2cccc(Br)c12</smiles>

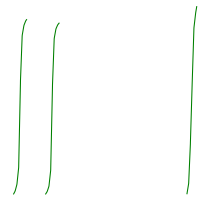

$3 h$

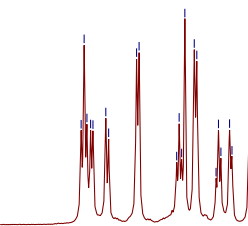

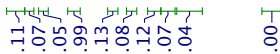

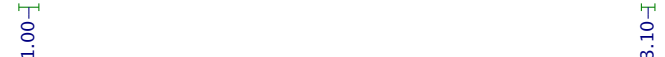

$\begin{array}{llllllllllllllllll}8.0 & 7.5 & 7.0 & 6.5 & 6.0 & 5.5 & 5.0 & 4.5 & \begin{array}{c}4.0 \\ \mathrm{f} 1(\mathrm{ppm})\end{array} 3.5 & 3.0 & 2.5 & 2.0 & 1.5 & 1.0 & 0.5 & 0.0 & -0.5\end{array}$<smiles>C/C=C(\c1cccc(C)c1)c1c(OC(F)(F)F)ccc2cccc(Br)c12</smiles>

$3 h$

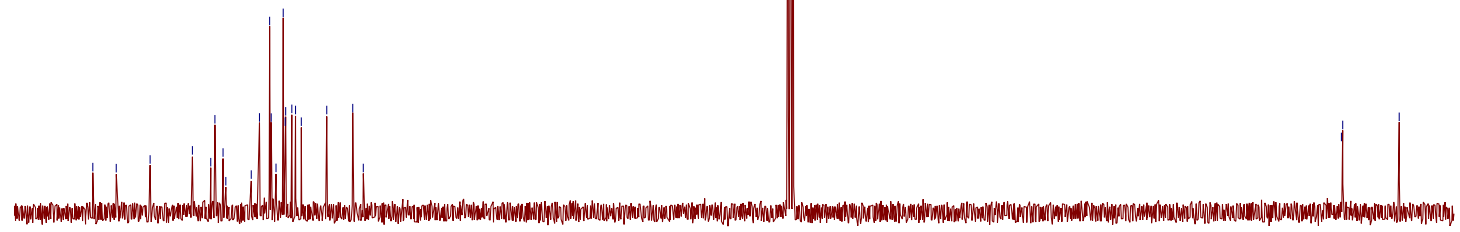

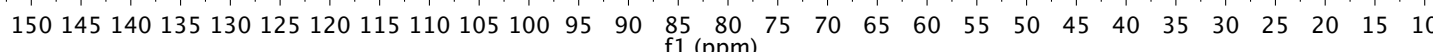




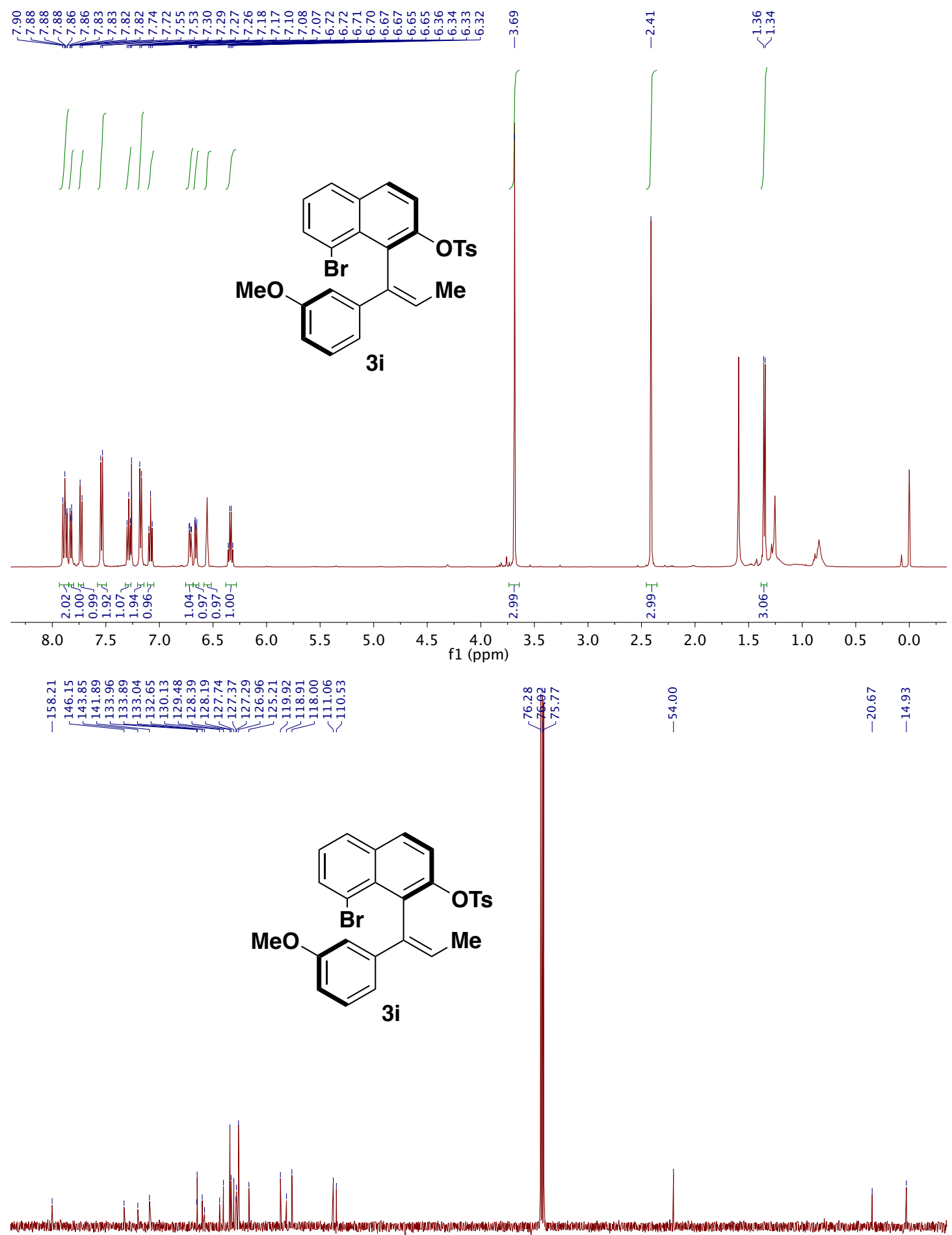

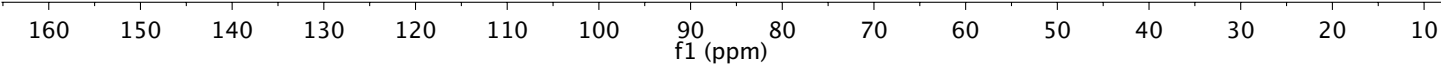



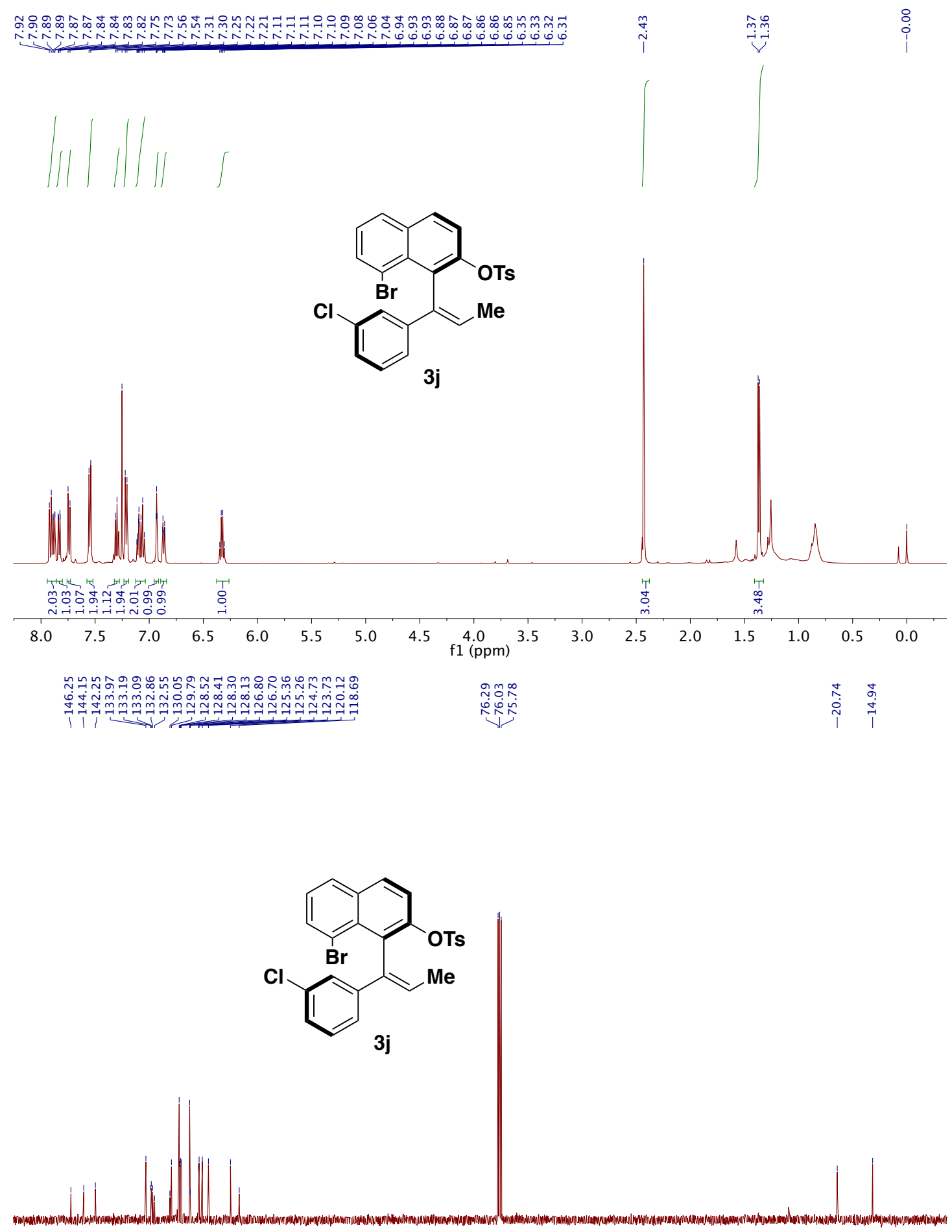

\begin{tabular}{|c|c|c|c|c|c|c|c|c|c|c|c|c|c|c|c|}
\hline 55 & 145 & 135 & 125 & 115 & 105 & 95 & ${ }^{85}$ f1 & $\begin{array}{l}75 \\
\text { pm) }\end{array}$ & 65 & 55 & 45 & 35 & 25 & 15 & 5 \\
\hline
\end{tabular}




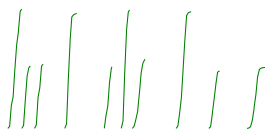<smiles>C/C=C(\c1cccc(F)c1)c1c(OC)ccc2cccc(Br)c12</smiles>

Hud dud

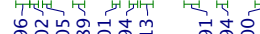

\begin{tabular}{|c|c|c|c|c|c|c|c|c|c|c|c|c|c|c|c|c|c|c|}
\hline & & & & 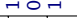 & & & & & & & & m & & $\sim$ & & & & \\
\hline 3.5 & 8.0 & 7.5 & 7.0 & 6.5 & 6.0 & 5.5 & 5.0 & 4.5 & $\begin{array}{c}4.0 \\
\mathrm{f} 1(\mathrm{ppm}\end{array}$ & 3.5 & 3.0 & 2.5 & 2.0 & 1.5 & 1.0 & 0.5 & 0.0 & -0.5 \\
\hline
\end{tabular}

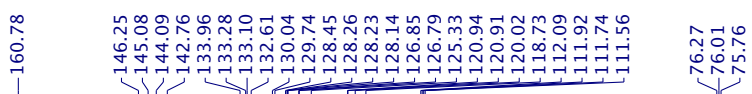

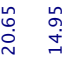<smiles>C/C=C(\c1cccc(F)c1)c1c(OC)ccc2cccc(Br)c12</smiles>

3k

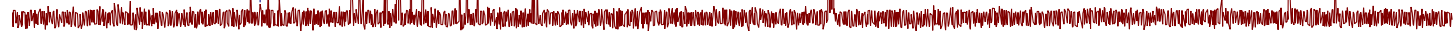

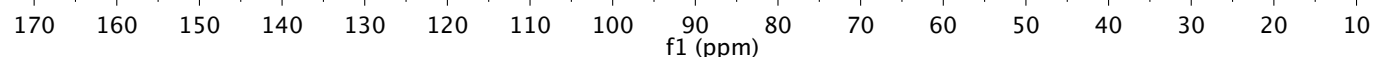


๓ำกสสำ

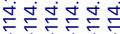<smiles>C/C=C(\c1cccc(F)c1)c1c(OC)ccc2cccc(Br)c12</smiles>

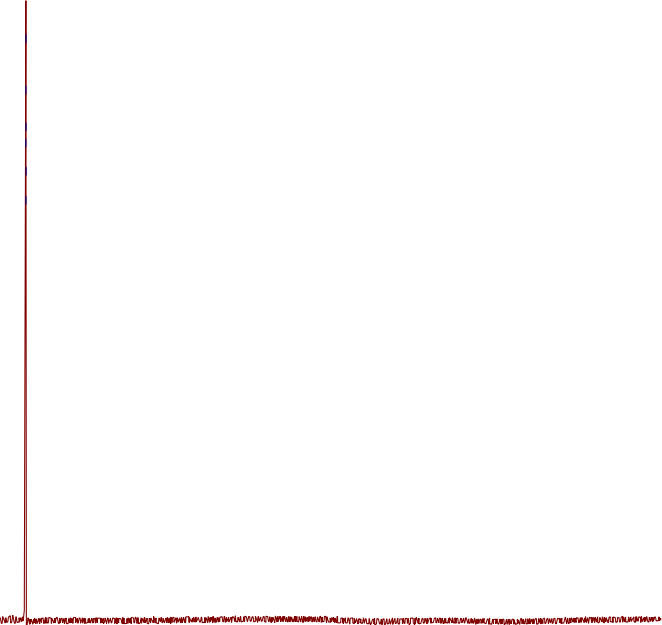

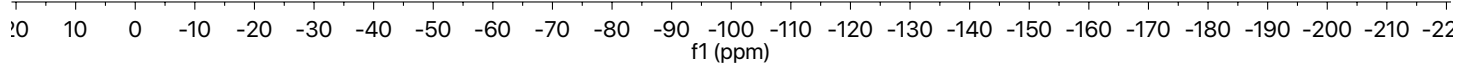

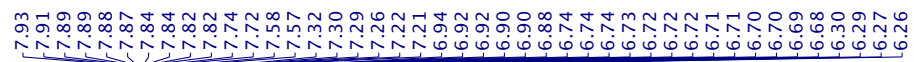

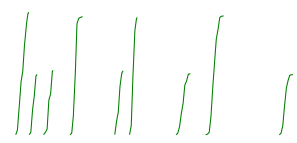<smiles>C/C=C(\c1ccc(F)c(F)c1)c1c(OC)ccc2cccc(Br)c12</smiles>

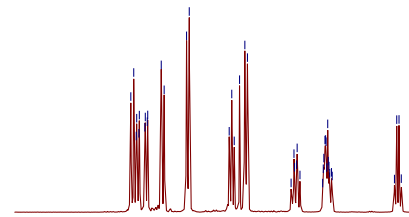

$\stackrel{\substack{\infty \\ i}}{i=1}$

$\stackrel{8}{\circ}$

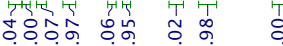

$\begin{array}{llllllllll}8.5 & 8.0 & 7.5 & 7.0 & 6.5 & 6.0 & 5.5 & 5.0 & 4.5 \\ \mathrm{f} 1(\mathrm{ppm})\end{array}$ 


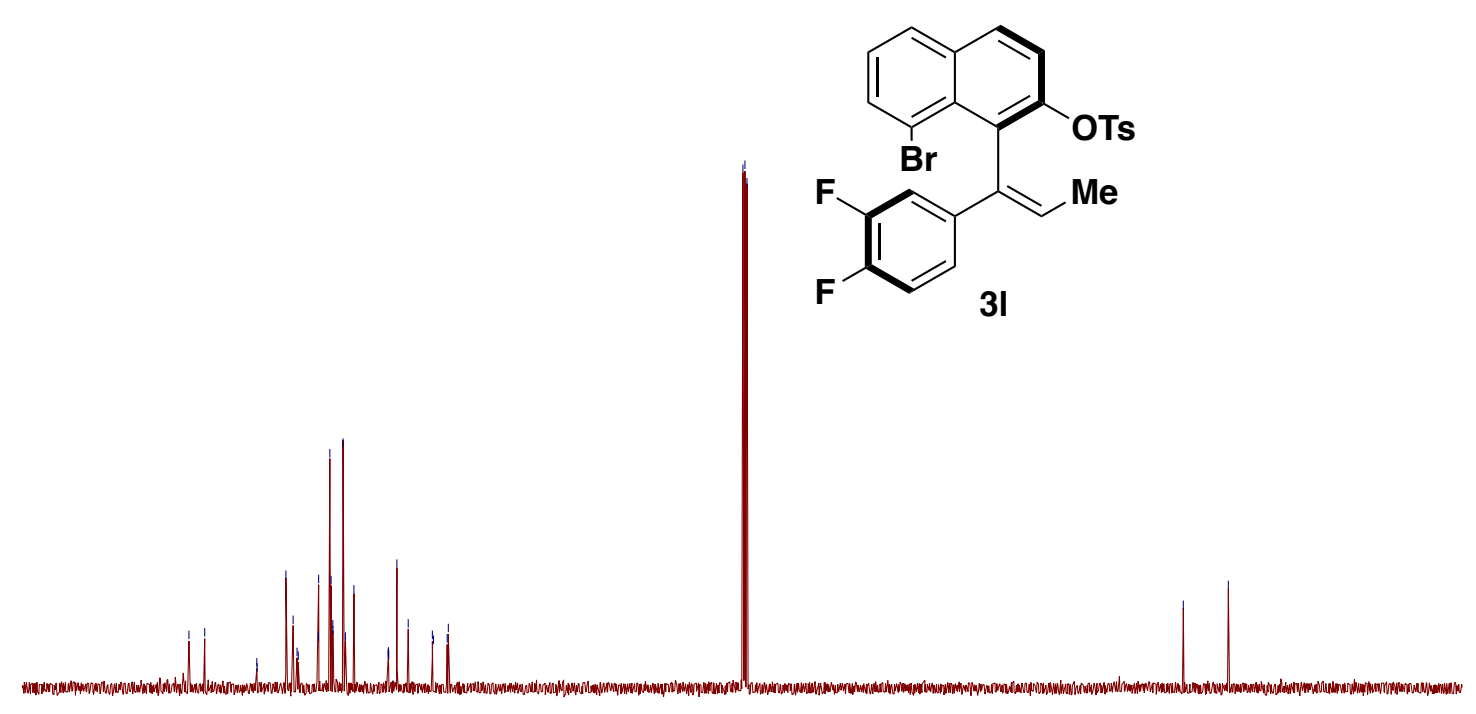

$\begin{array}{lllllllllllllllll}160 & 150 & 140 & 130 & 120 & 110 & 100 & 90 & \begin{array}{c}80 \\ \mathrm{f} 1(\mathrm{ppm})\end{array} & 60 & 50 & 40 & 30 & 20 & 10 & 0 & -10\end{array}$

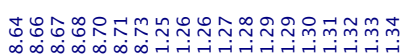

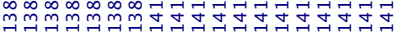

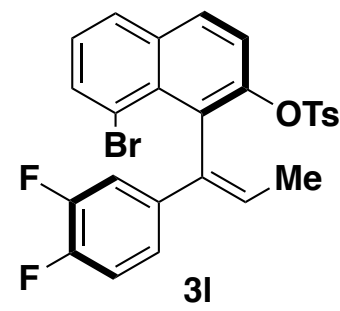

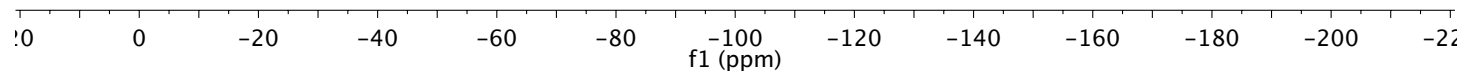




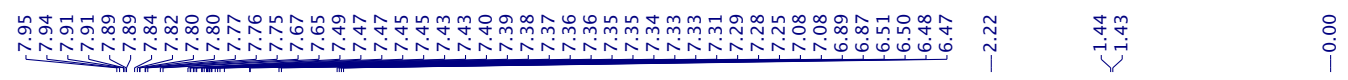

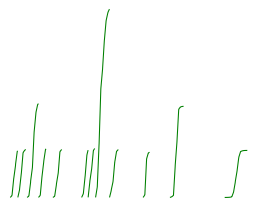<smiles>C/C=C(/c1ccc2ccccc2c1)c1c(OC)ccc2cccc(Br)c12</smiles>

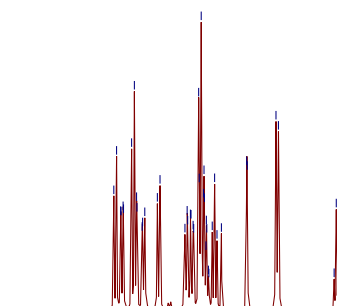

$3 \mathrm{~m}$

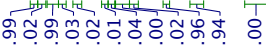

$\begin{array}{lllll}8.5 & 8.0 & 7.5 & 7.0 & 6.5\end{array}$

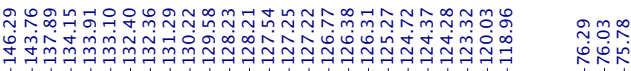

$\begin{array}{ll}\vec{n} & 0 \\ \stackrel{1}{0} & \dot{n} \\ & 1\end{array}$<smiles>C/C=C(\c1ccc2ccccc2c1)c1c(OC)ccc2cccc(Br)c12</smiles>

$3 \mathrm{~m}$

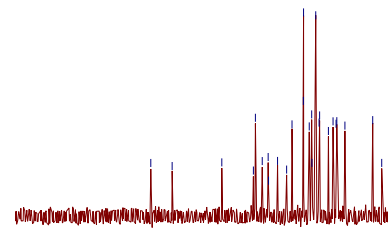

$160 \quad 150$

$\begin{array}{lll}140 & 130 \quad 120\end{array}$

$\begin{array}{llll}10 & 100 & 90 & 80\end{array}$ $\mathrm{f} 1(\mathrm{ppm})$ 
111

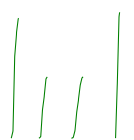<smiles>C/C=C(\c1ccc2c(c1)OCO2)c1c(OC)ccc2ccccc12</smiles>

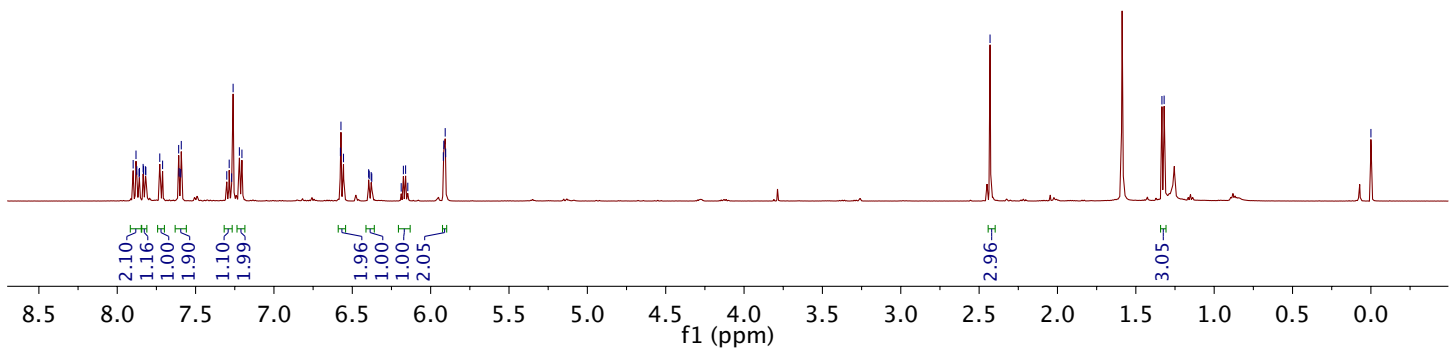

은

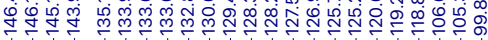

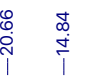<smiles>C/C=C(\c1ccc2c(c1)OCO2)c1c(OC)ccc2cccc(Br)c12</smiles>

3n

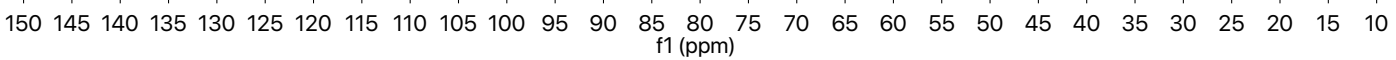




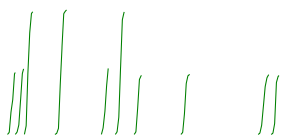<smiles>C/C=C(\c1cccs1)c1c(OCC)ccc2cccc(Br)c12</smiles>

30

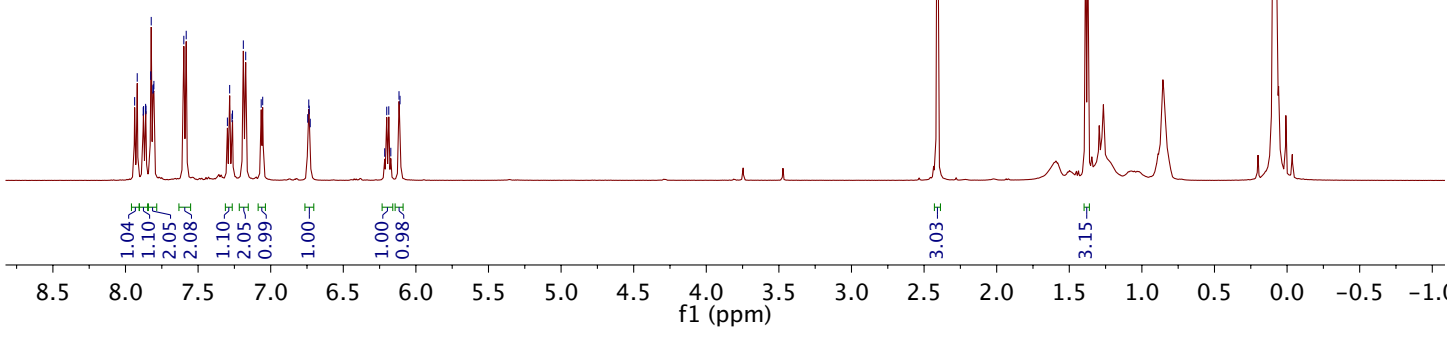

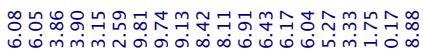

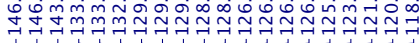
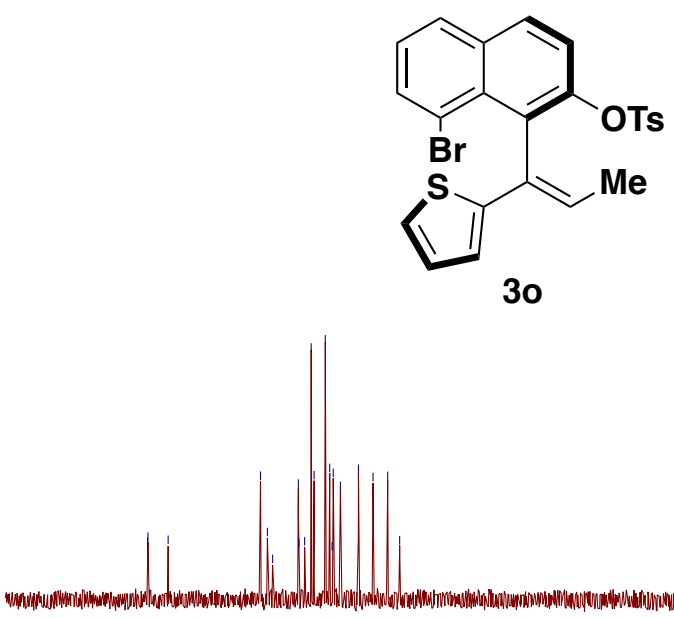

$\begin{array}{llllllll}60 & 150 & 140 & 130 & 120 & 110 & 100 & 90 \begin{array}{r}80 \\ \mathrm{f} 1(\mathrm{ppm})\end{array}\end{array}$

$\begin{array}{lllllll}70 & 60 & 50 & 40 & 30 & 20 & 10\end{array}$


$\| \int$<smiles>C1=CC=CC=1</smiles><smiles>C=CC</smiles>

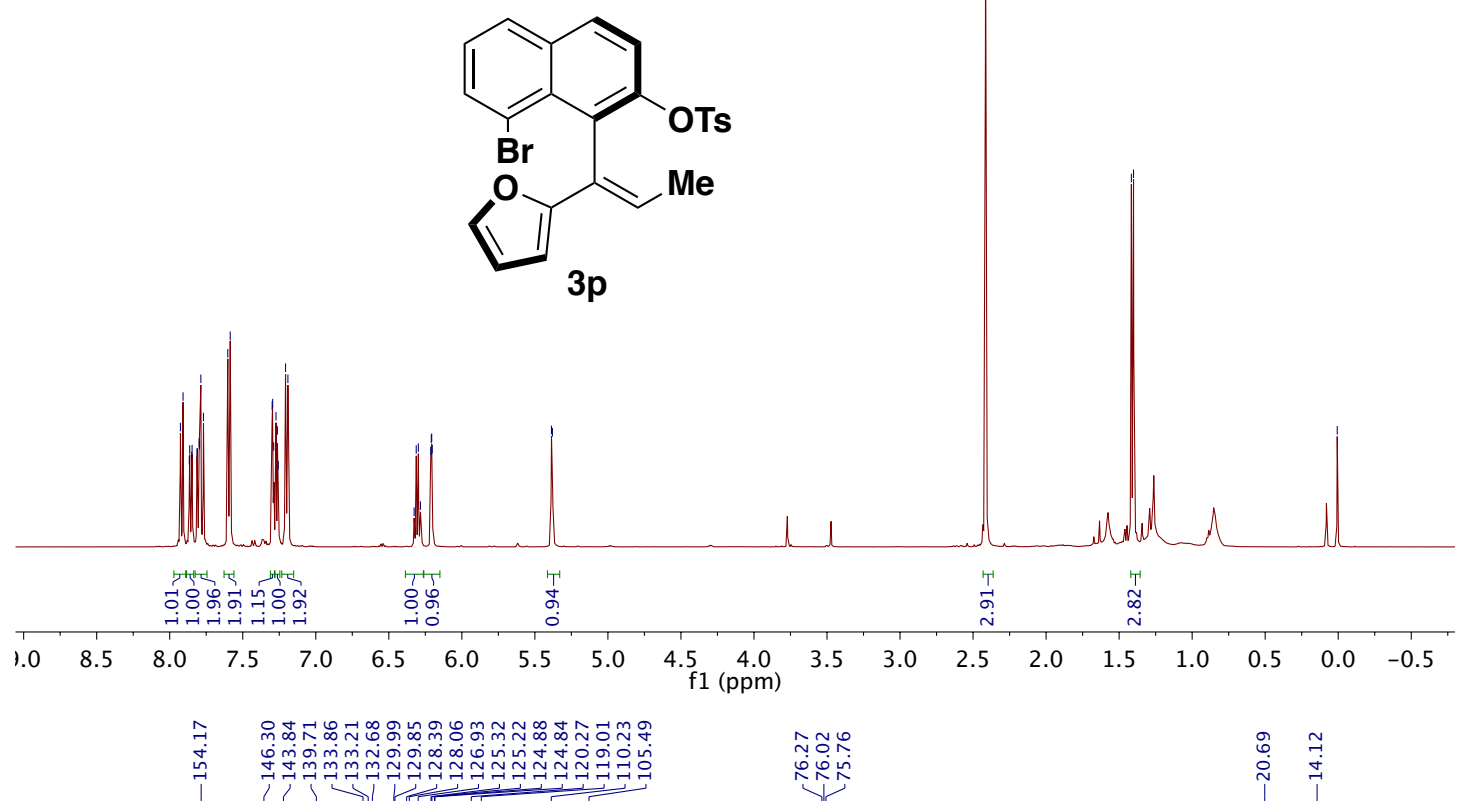<smiles>C/C=C(\c1ccco1)c1c(OC)ccc2cccc(Br)c12</smiles>

$3 p$

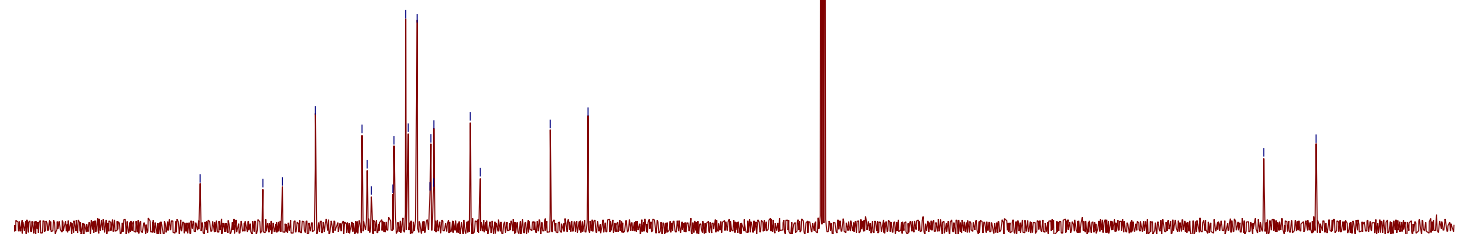

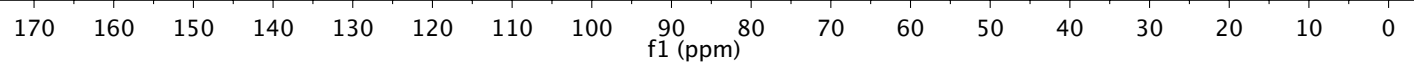




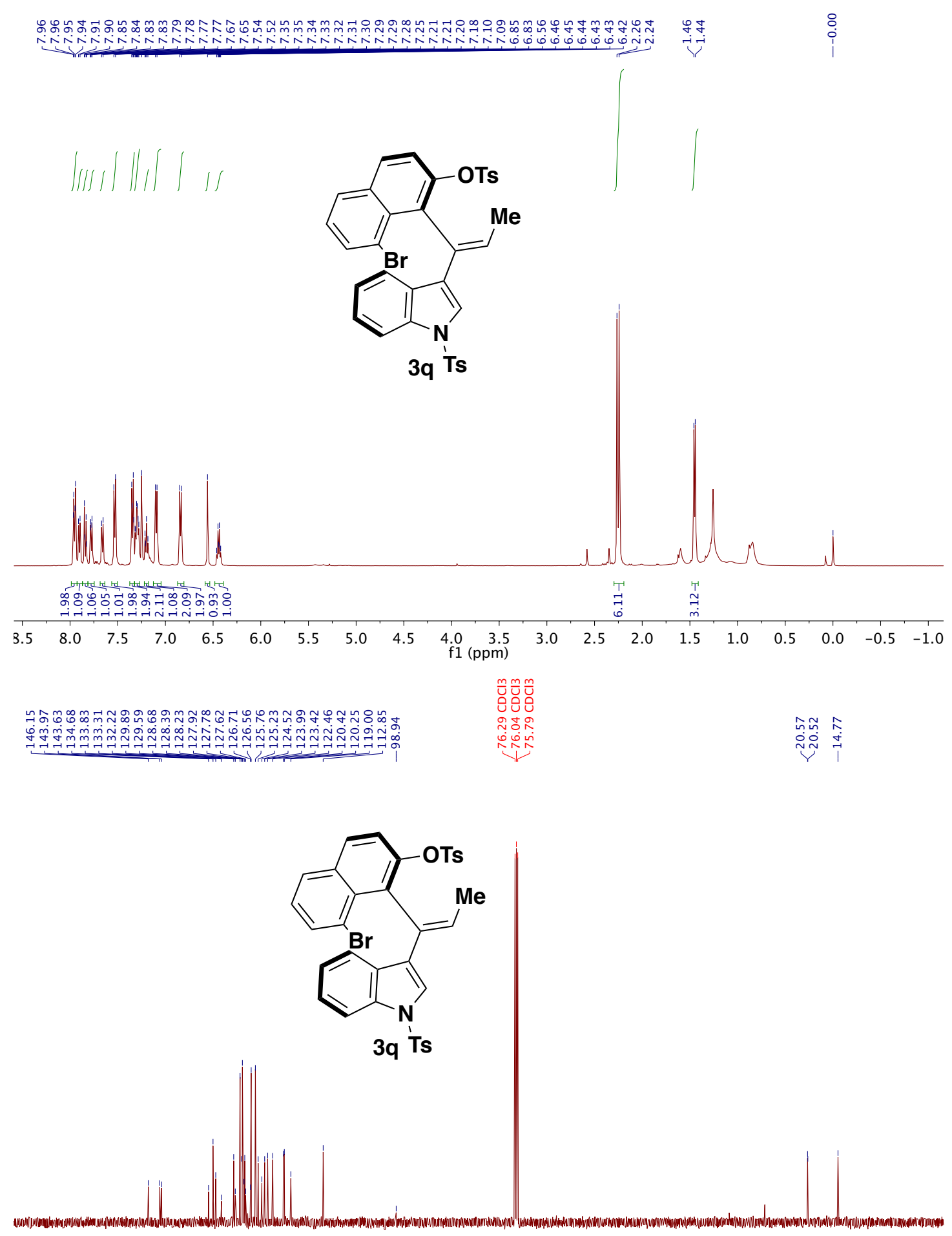

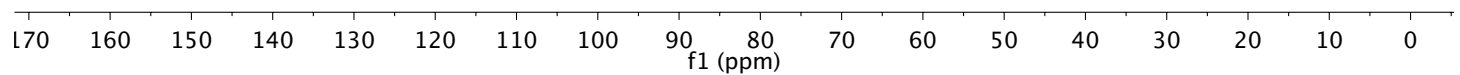




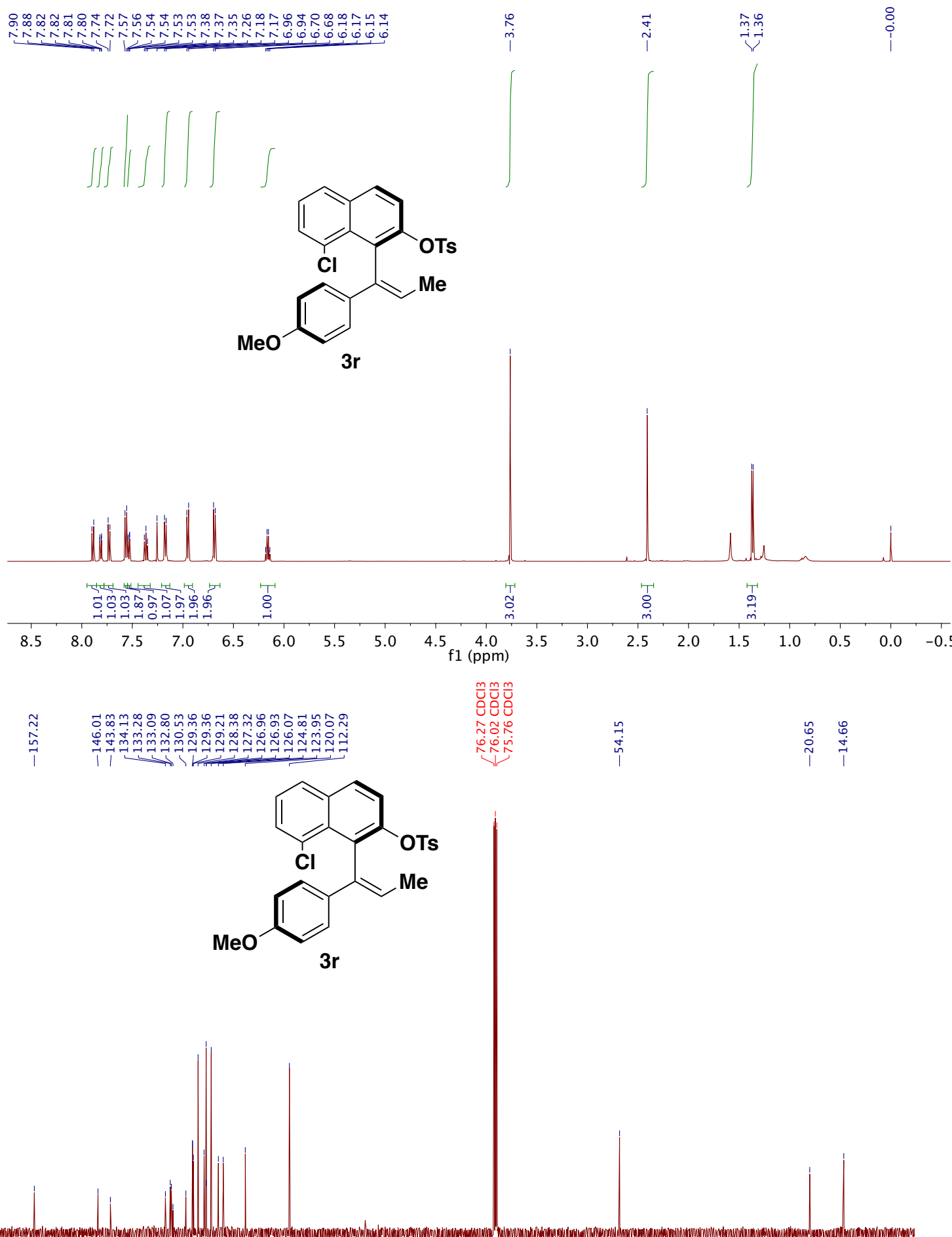

$\begin{array}{llllllllllllllll}160 & 150 & 140 & 130 & 120 & 110 & 100 & \underset{\mathrm{f} 1(\mathrm{ppm})}{80} & 70 & 60 & 50 & 40 & 30 & 20 & 10\end{array}$ 


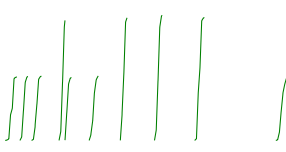<smiles></smiles>

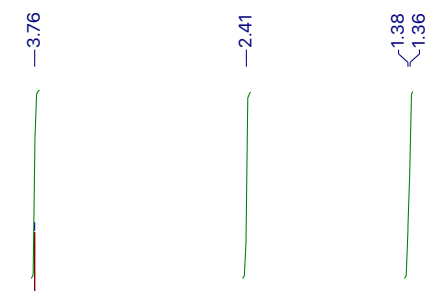

3 s

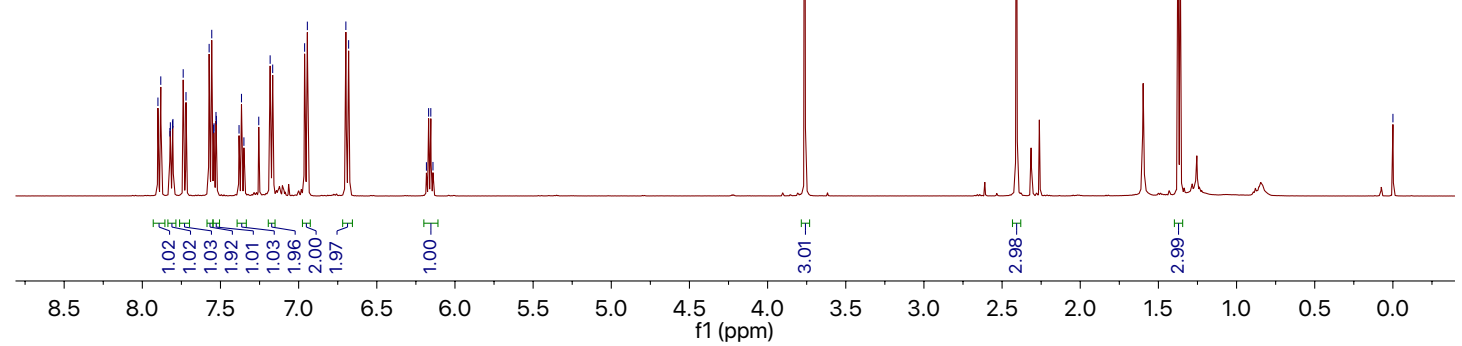<smiles>C/C=c1/c2ccc(OC)cc2c2cccc3ccc(O)c1c32</smiles>

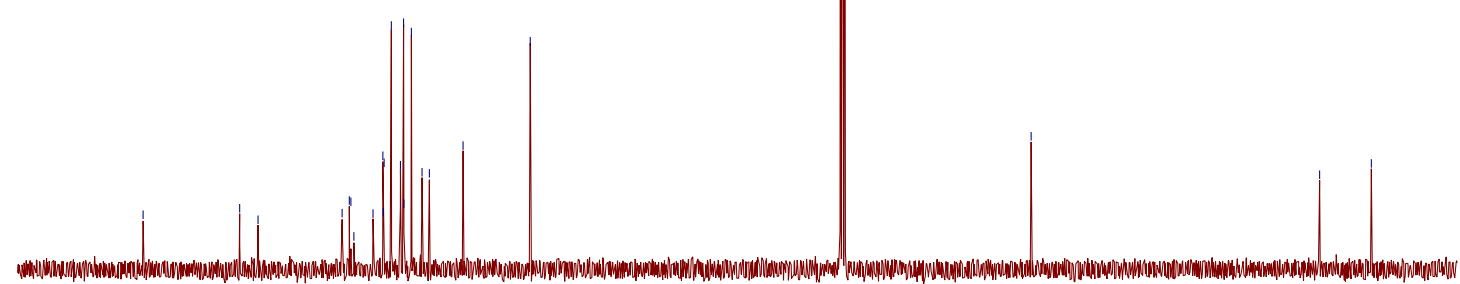

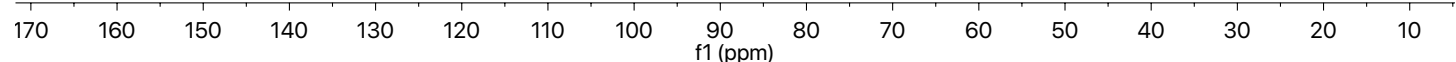



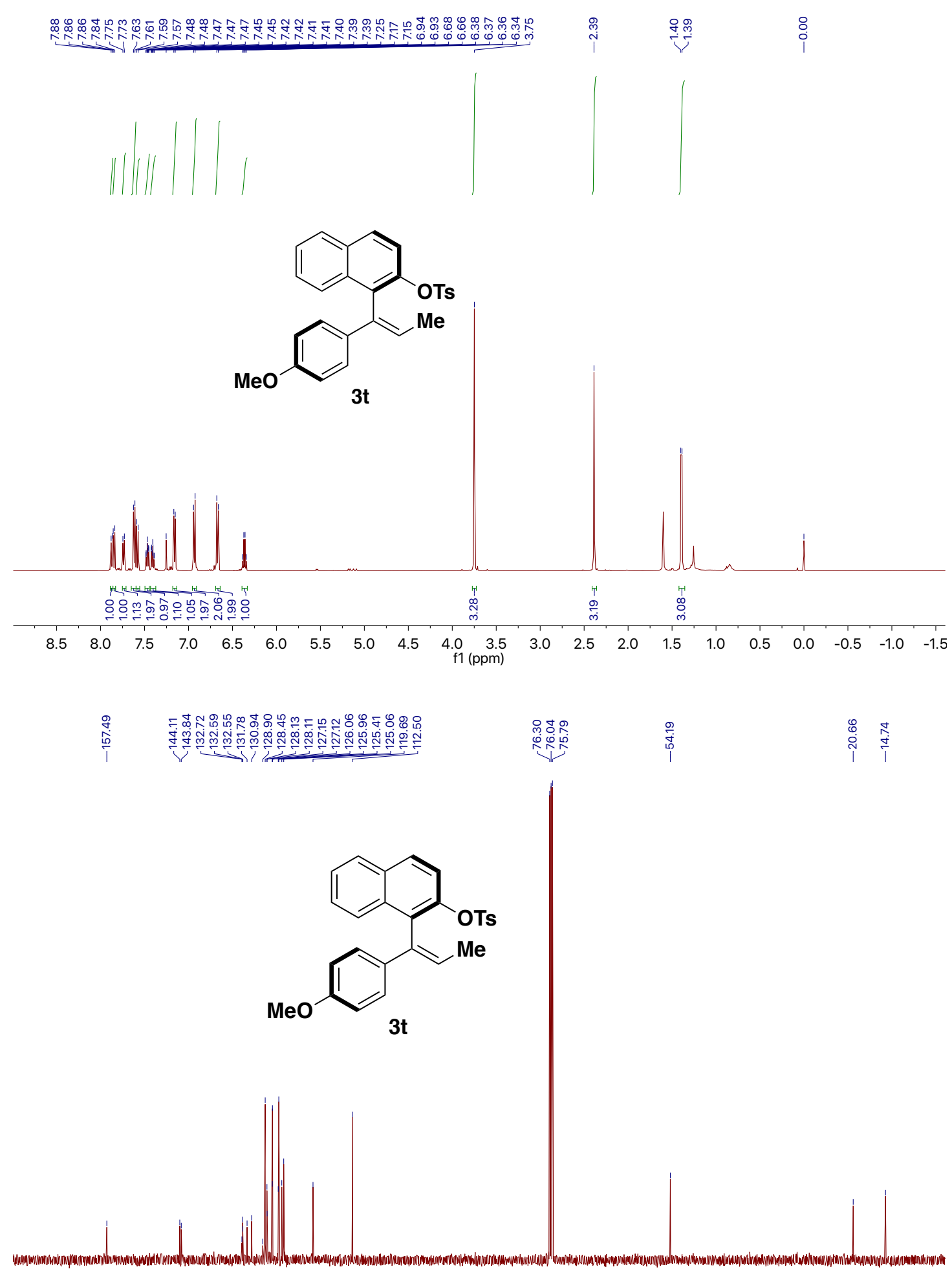

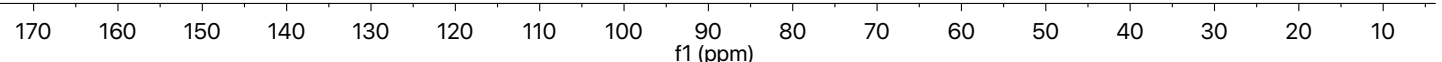


$\iiint \iiint$
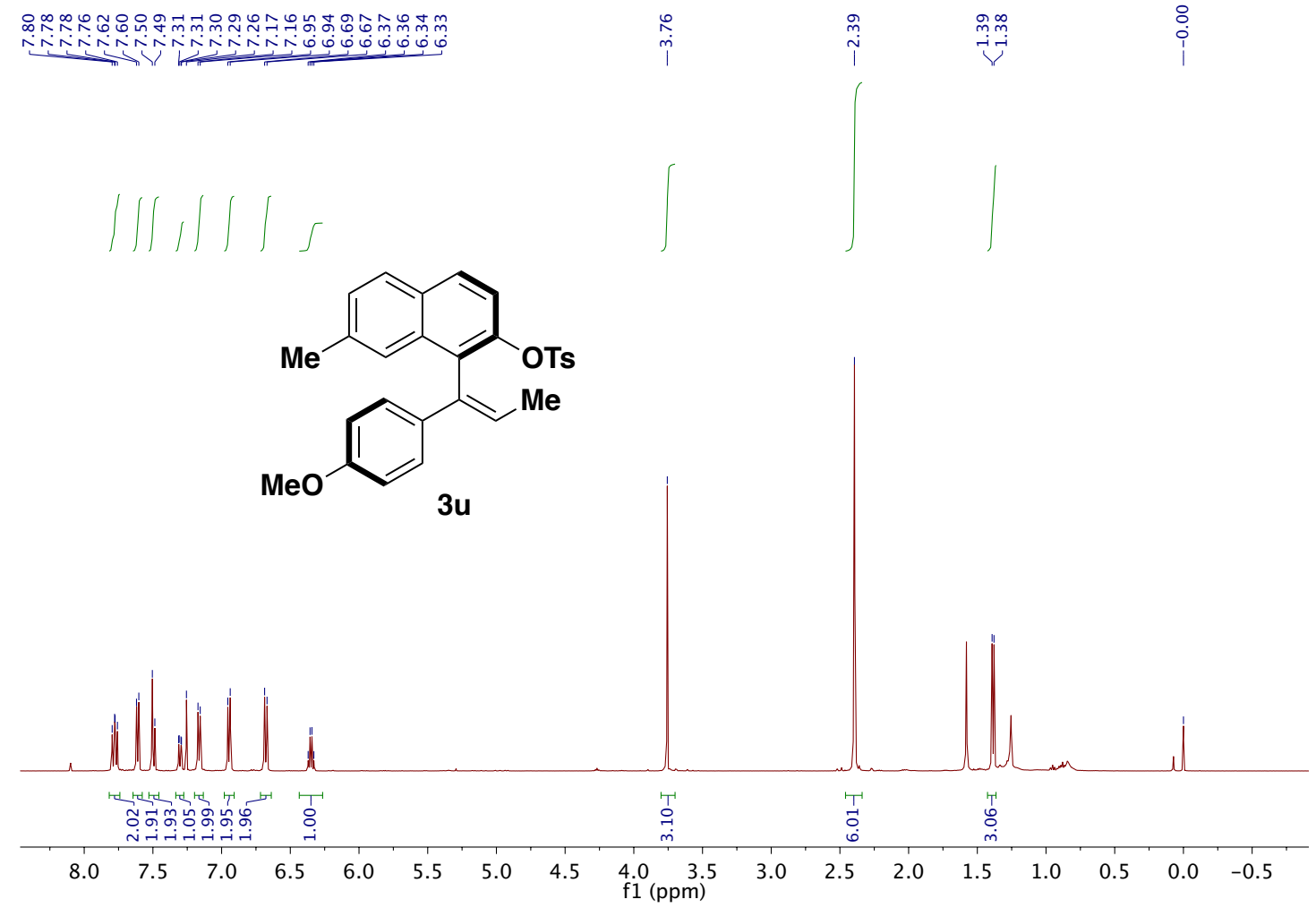

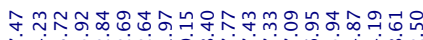

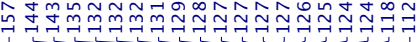

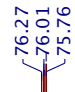

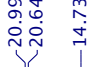

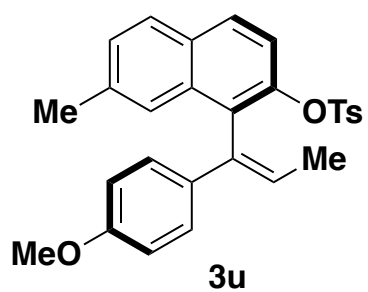

$70 \quad 160$

150

140

130

110

00

90
$\mathrm{f} 1(\mathrm{ppm})$

$80 \quad 70 \quad 60$

$\begin{array}{llllll}50 & 40 & 30 & 20 & 11\end{array}$ 

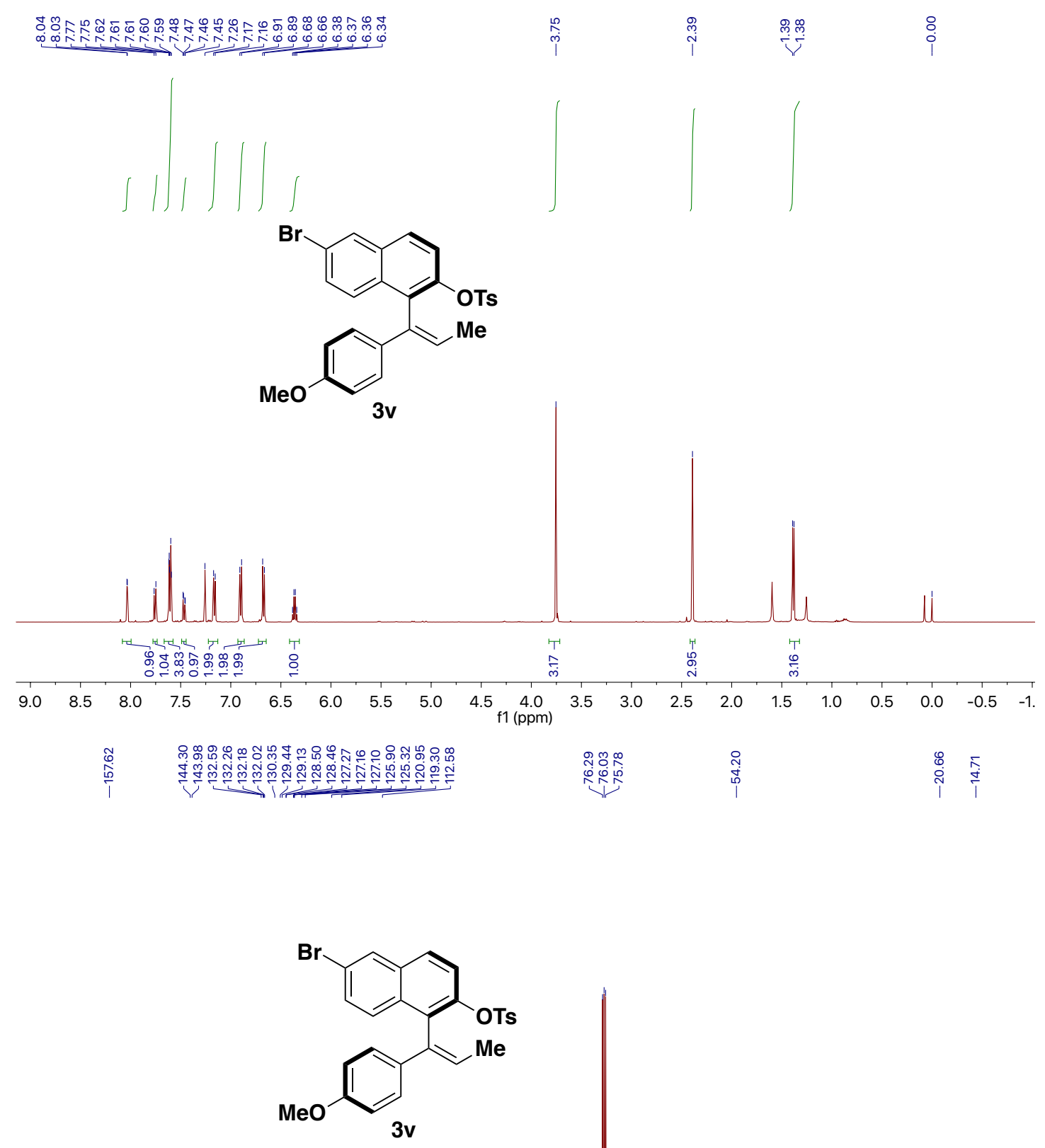

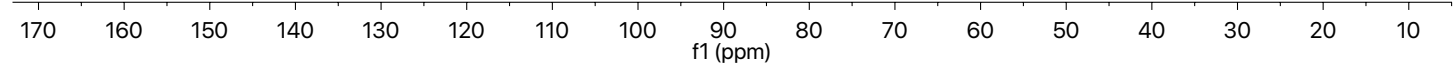




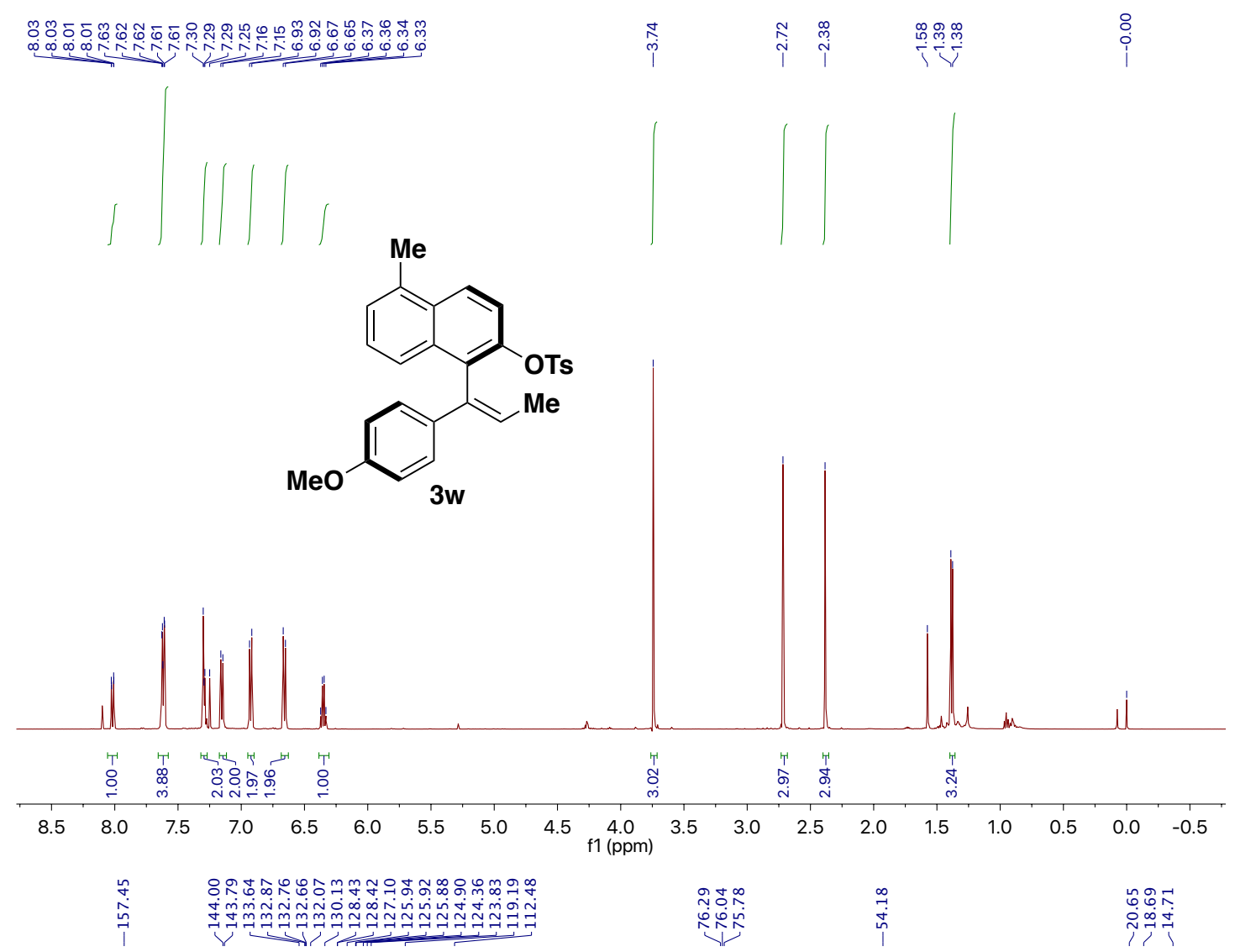<smiles>C/C=C(\c1ccc(OC)cc1)c1c(OC)ccc2c(C)cccc12</smiles>

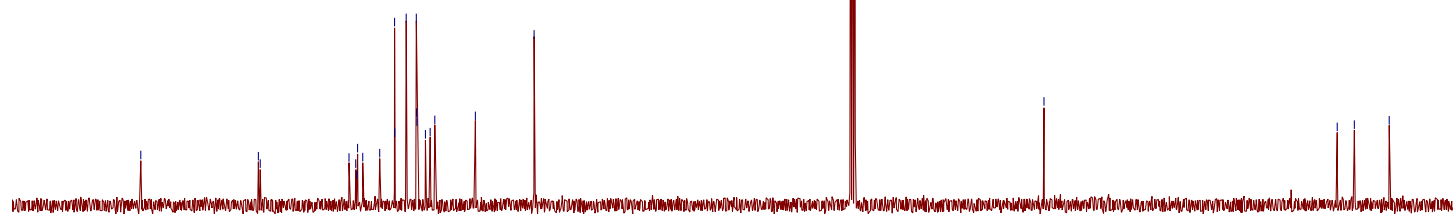

\begin{tabular}{lllllllllllllllll}
\hline 170 & 160 & 150 & 140 & 130 & 120 & 110 & 100 & $\begin{array}{c}90 \\
\mathrm{f} 1(\mathrm{ppm})\end{array}$ & 80 & 70 & 60 & 50 & 40 & 30 & 20 & 10
\end{tabular} 

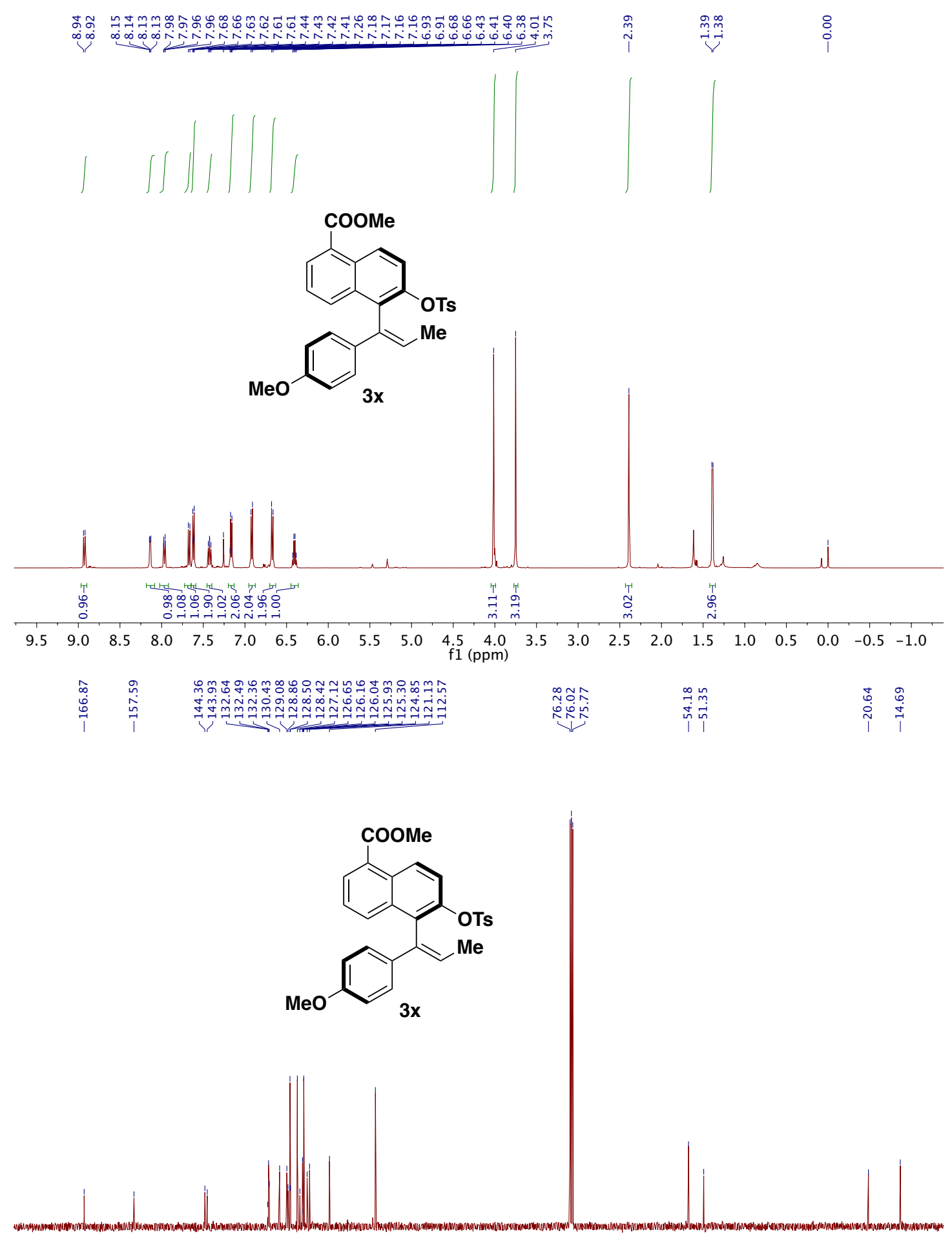

$\begin{array}{lllllllllllllllll}170 & 160 & 150 & 140 & 130 & 120 & 110 & \begin{array}{r}100 \\ \mathrm{f} 1(\mathrm{ppm})\end{array} & 80 & 70 & 60 & 50 & 40 & 30 & 20 & 10\end{array}$ 

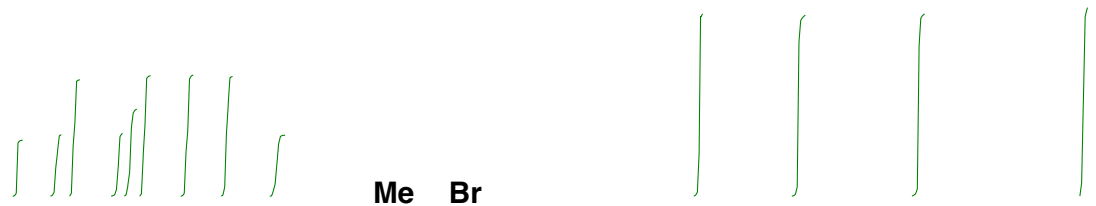<smiles>COc1ccc(/C(=C/[N+](=O)[O-])c2c(OC)cc(Br)c3c(C)cccc23)cc1</smiles>

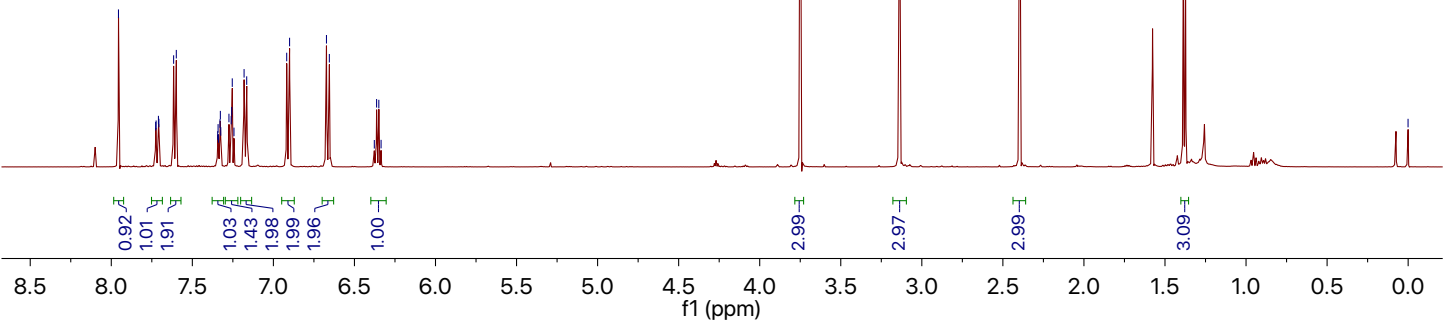<smiles>C/C=C(\c1ccc(OC)cc1)c1c(O[Na])cc(Br)c2c(C)cccc12</smiles>

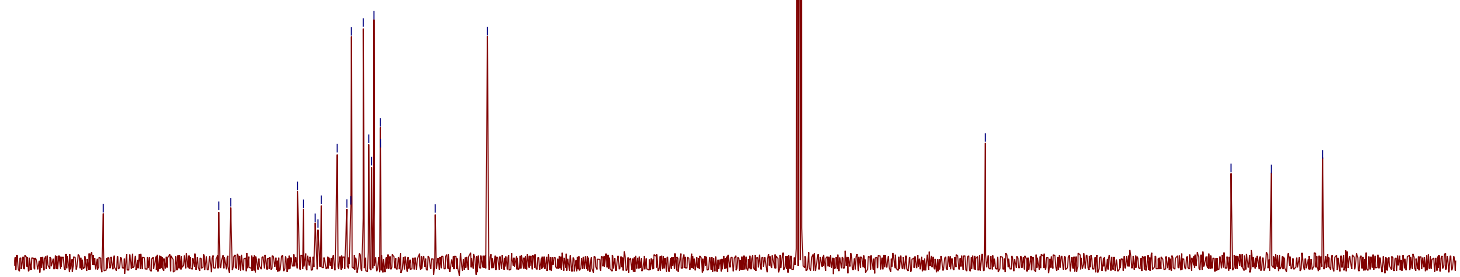

$\begin{array}{llllllll}160 & 150 & 140 & 130 & 120 & 110 & 100 & 90\end{array}$

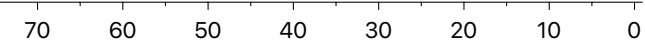




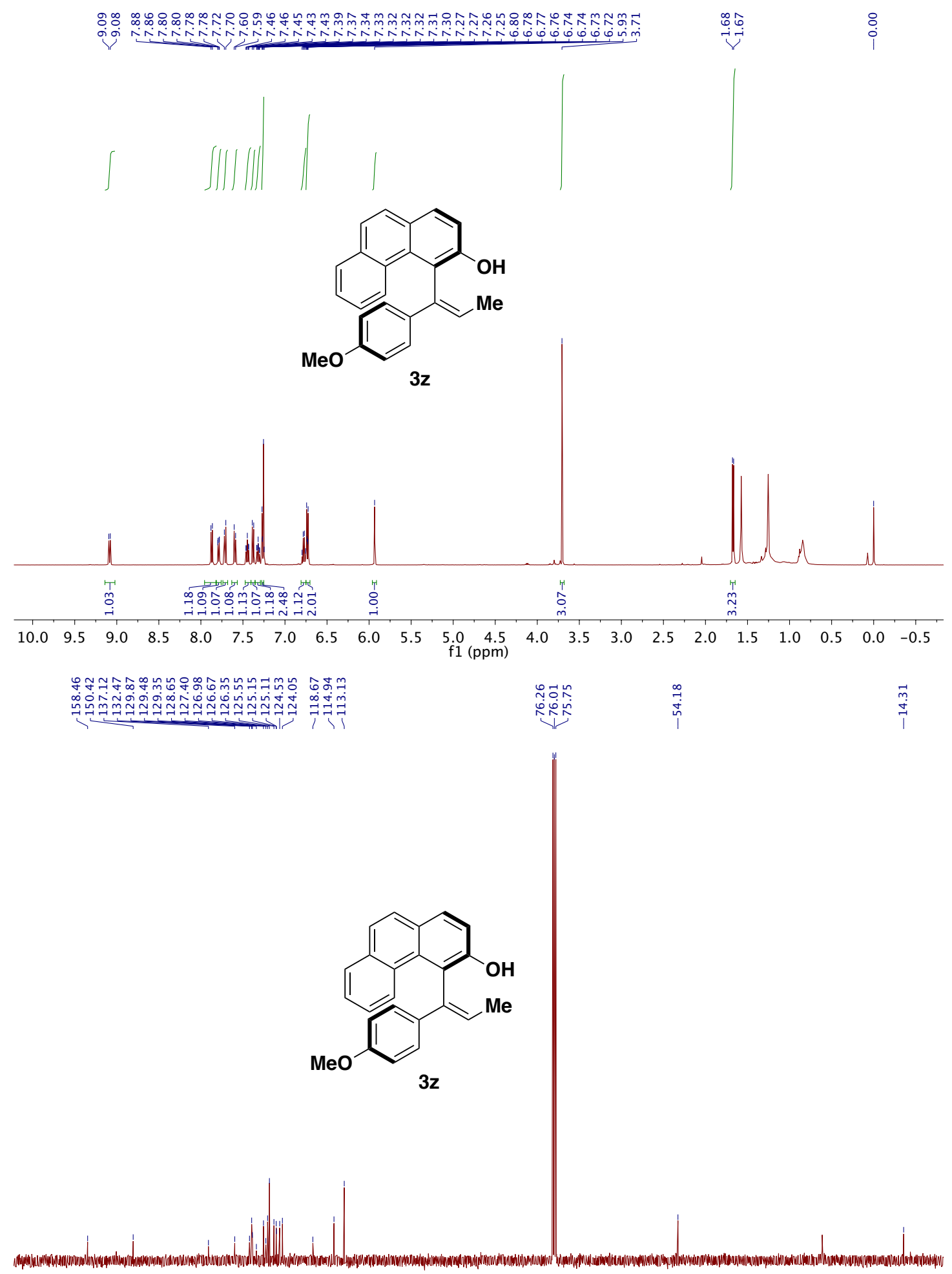




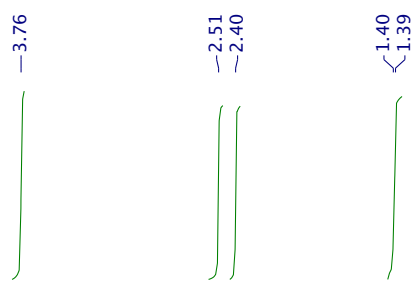<smiles>C/C=C(\c1ccc(OC)cc1)c1c(OC)ccc2ccccc12</smiles>

3a'

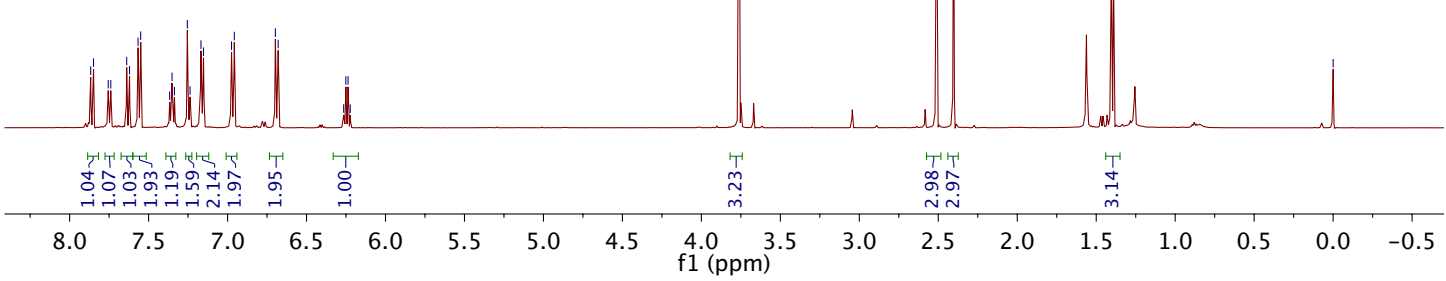

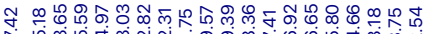

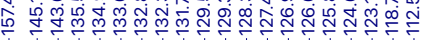

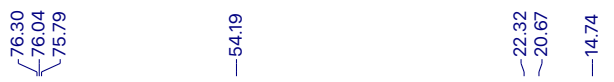
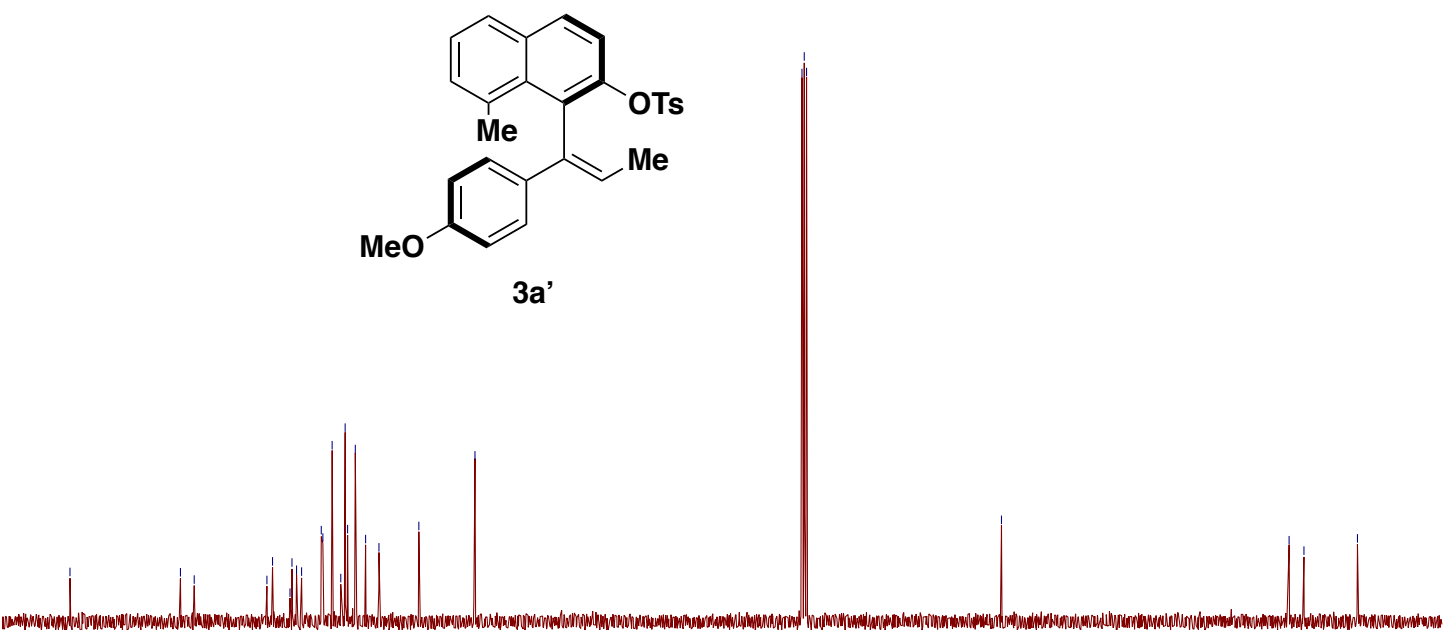

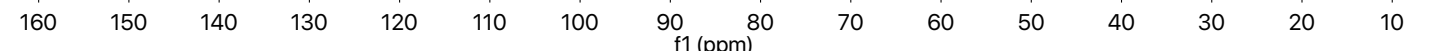




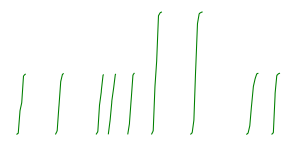<smiles>C/C=C(\c1ccc(OC)cc1)c1c(O)ccc2cccnc12</smiles>

3b'

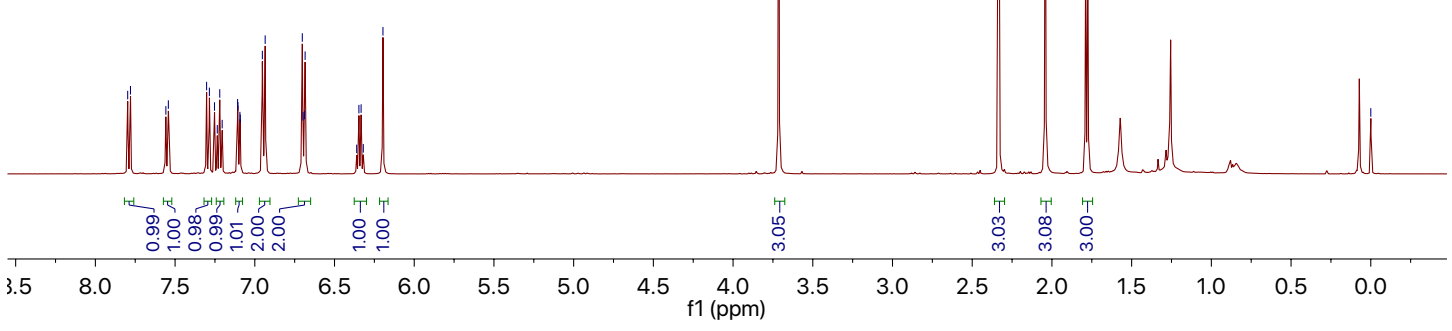

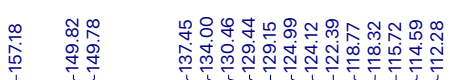

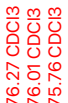

年

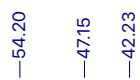

$\underset{\substack{0 \\ \hdashline}}{\stackrel{+}{+}}$<smiles>C/C=C(/c1ccc(OC)cc1)c1c(O)ccc2cccc(O)c12</smiles>

$3 b^{\prime}$ 


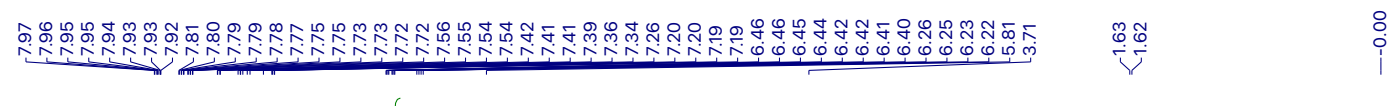
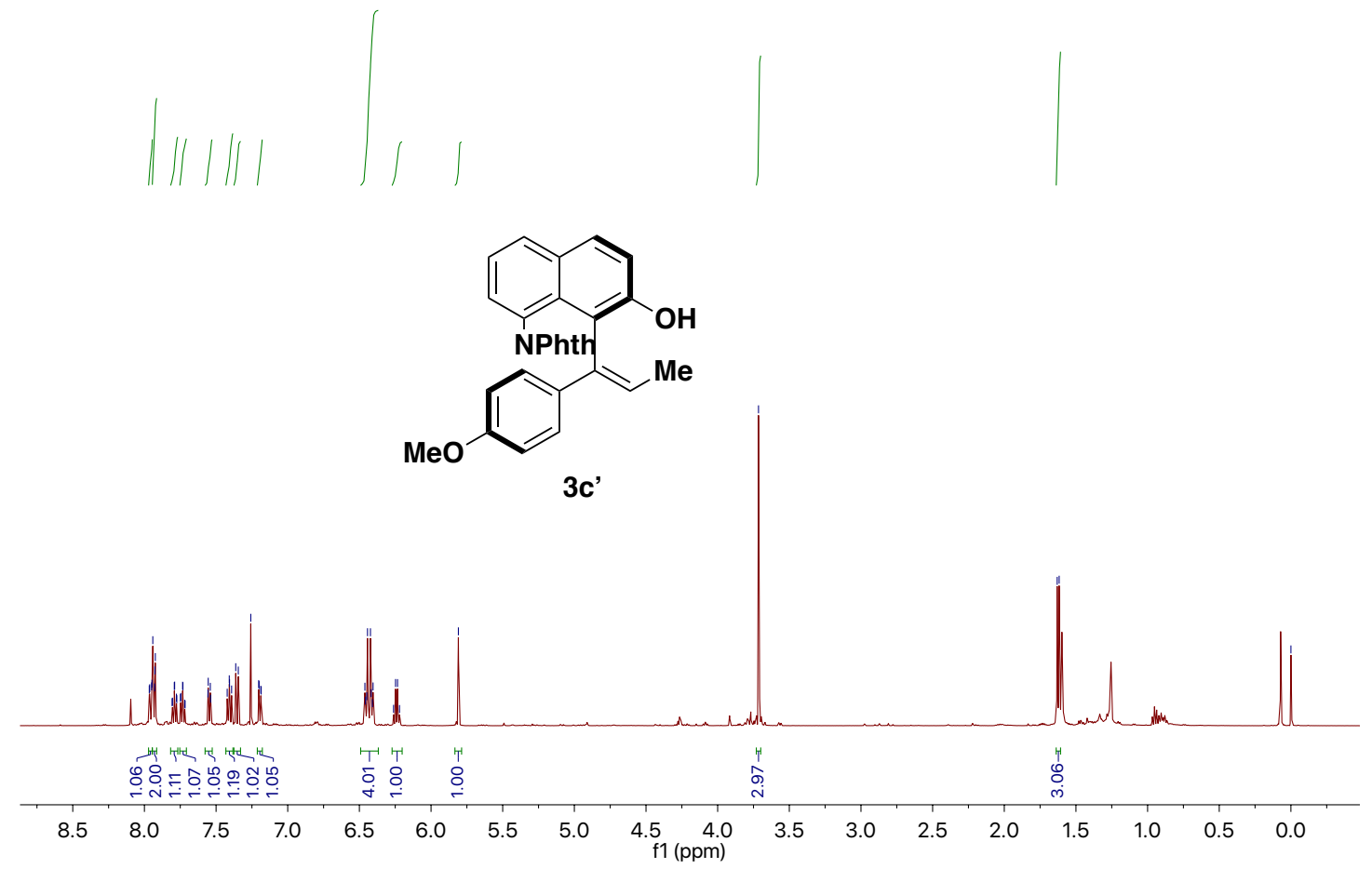

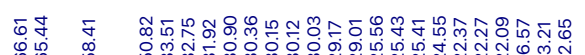

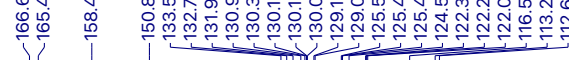

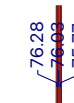

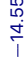

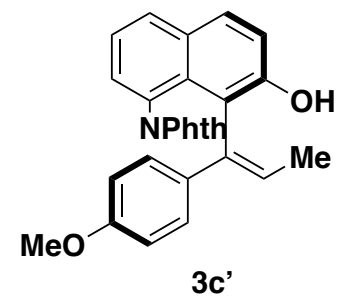

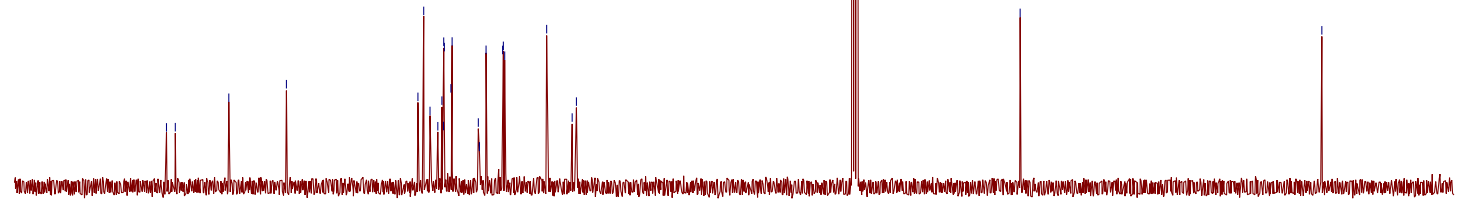

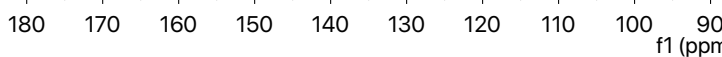
$\begin{array}{lllllllll}80 & 70 & 60 & 50 & 40 & 30 & 20 & 10 & 0\end{array}$ 


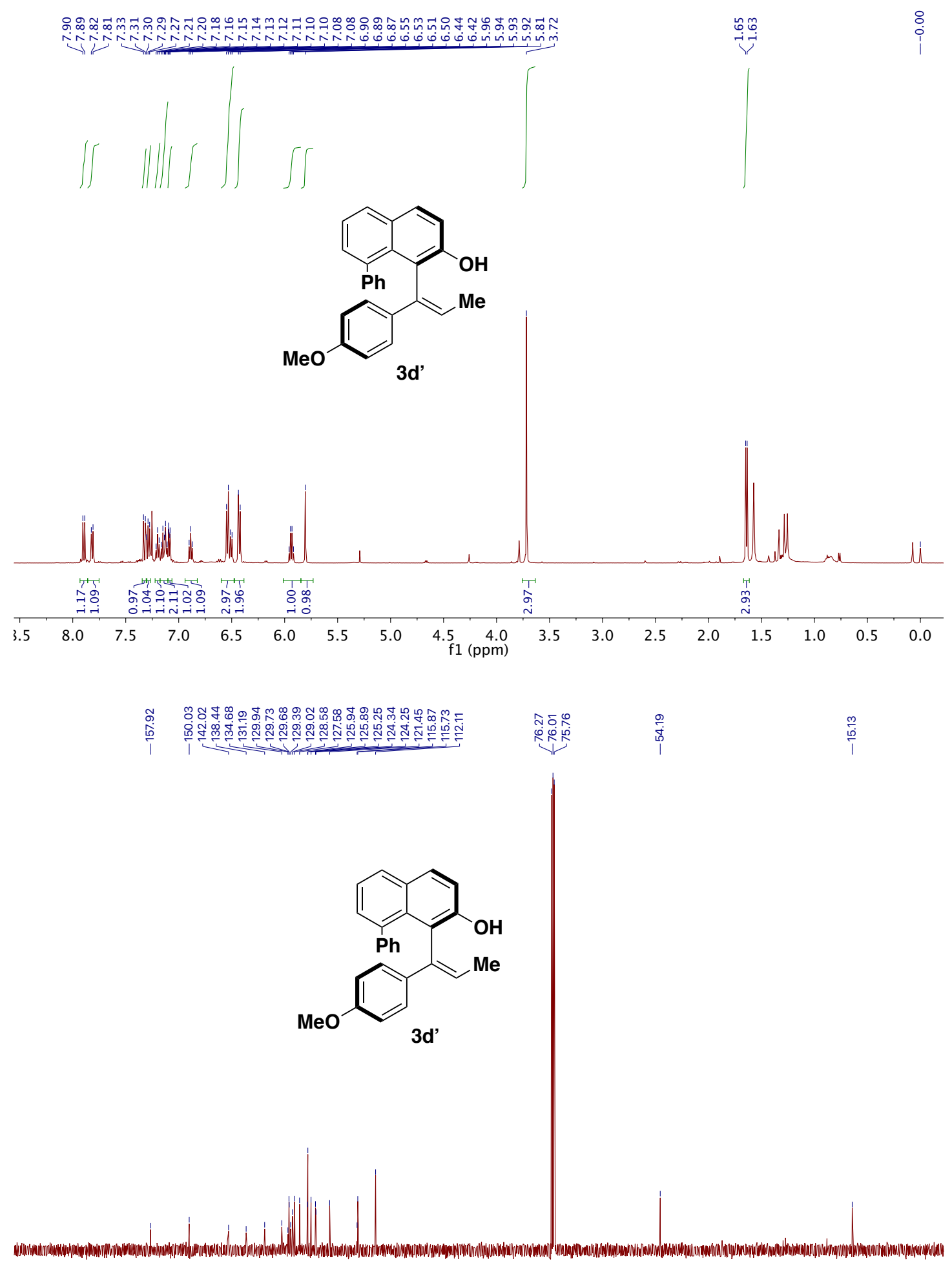

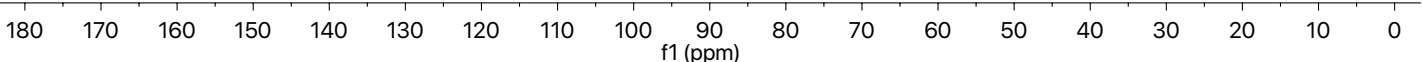




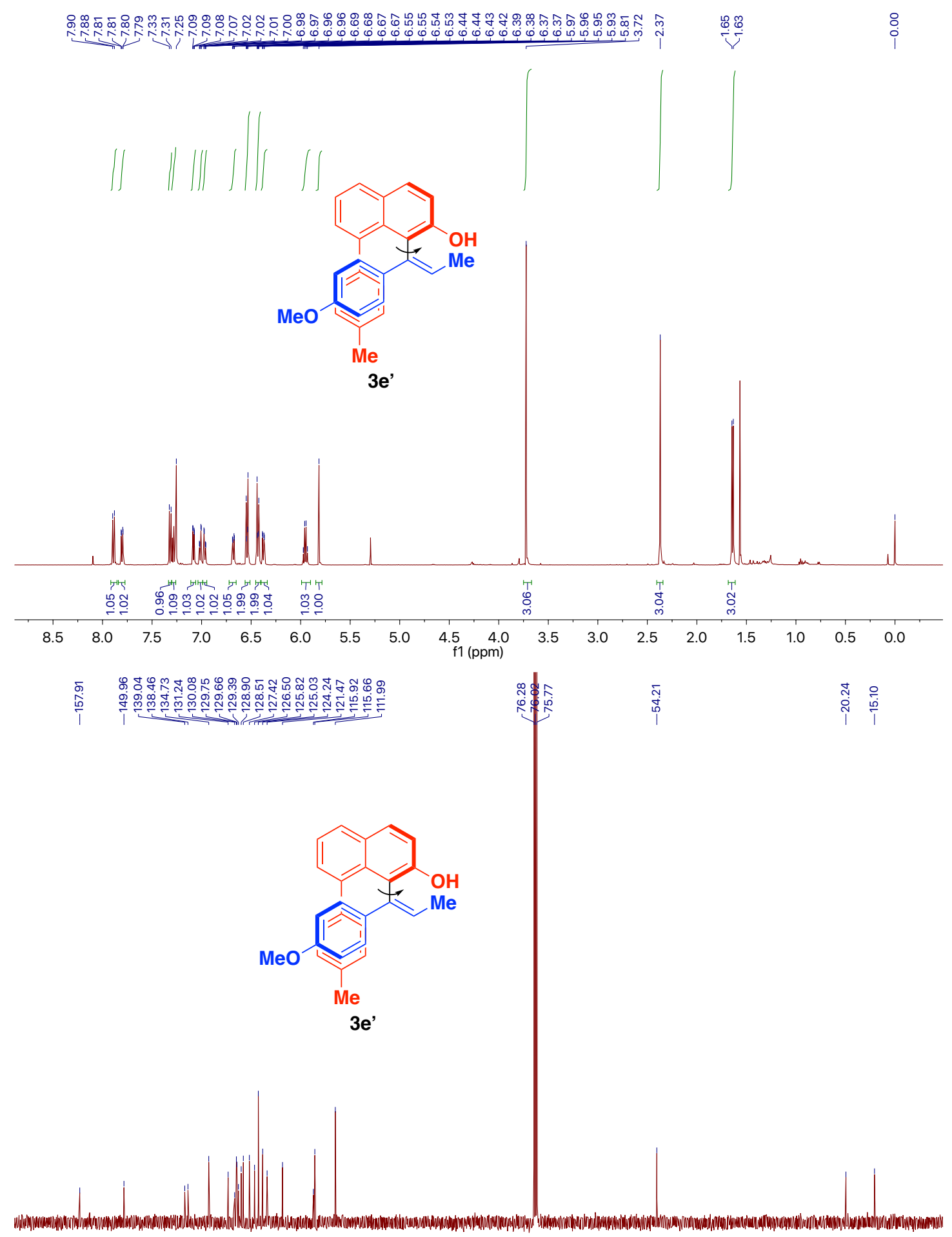

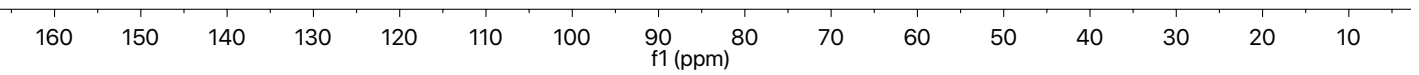



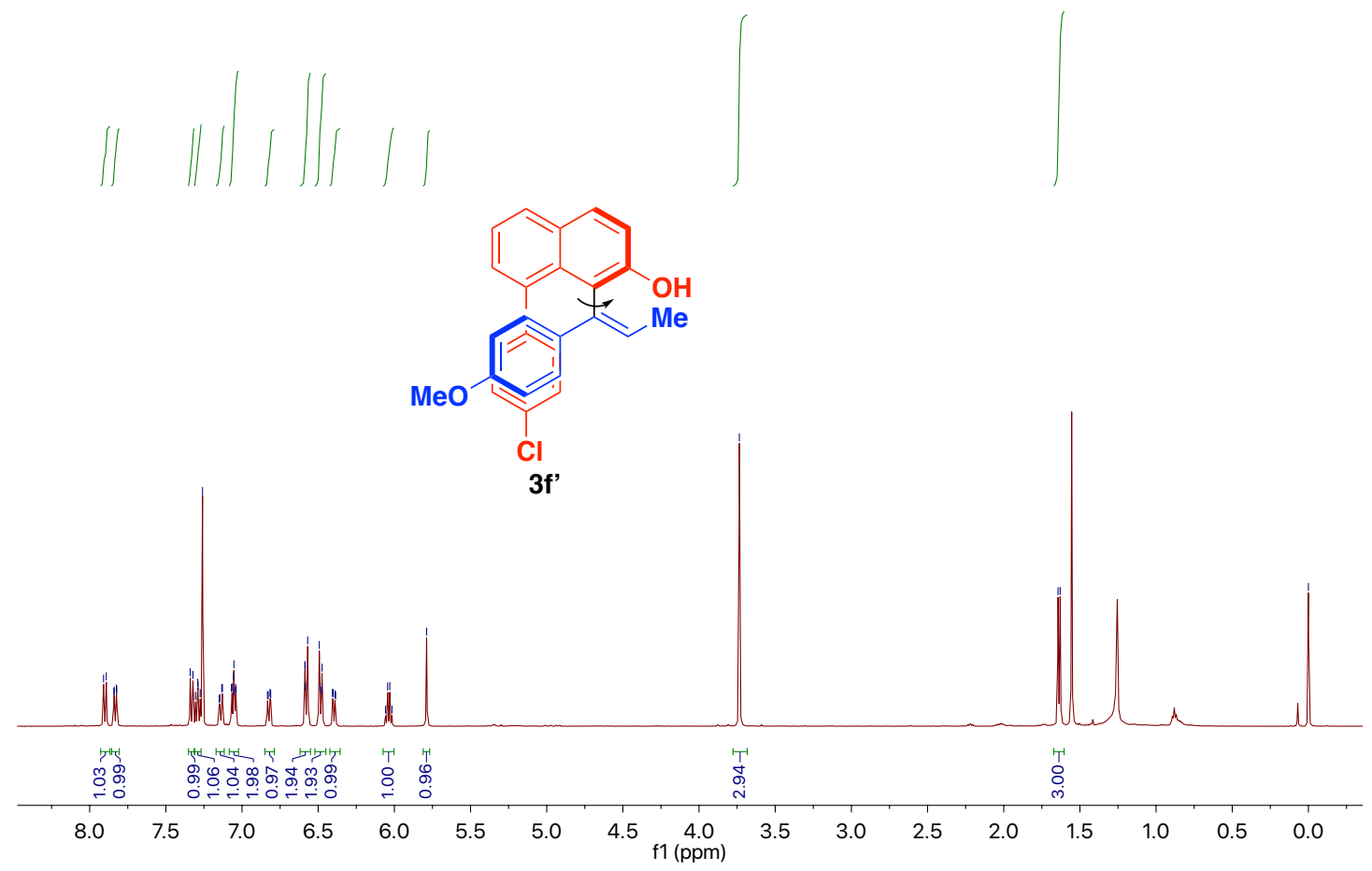

궁 สำ I

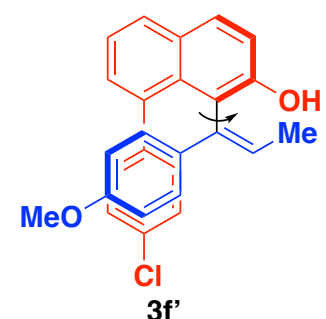

3f'

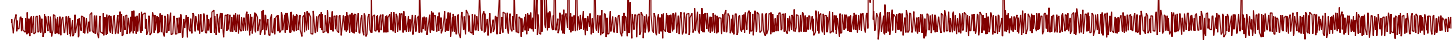

$\begin{array}{lllllllllllllllllllllll}210 & 200 & 190 & 180 & 170 & 160 & 150 & 140 & 130 & 120 & 110 & \begin{array}{c}100 \\ \mathrm{f} 1(\mathrm{ppm})\end{array} & 90 & 80 & 70 & 60 & 50 & 40 & 30 & 20 & 10 & 0 & -10\end{array}$ 


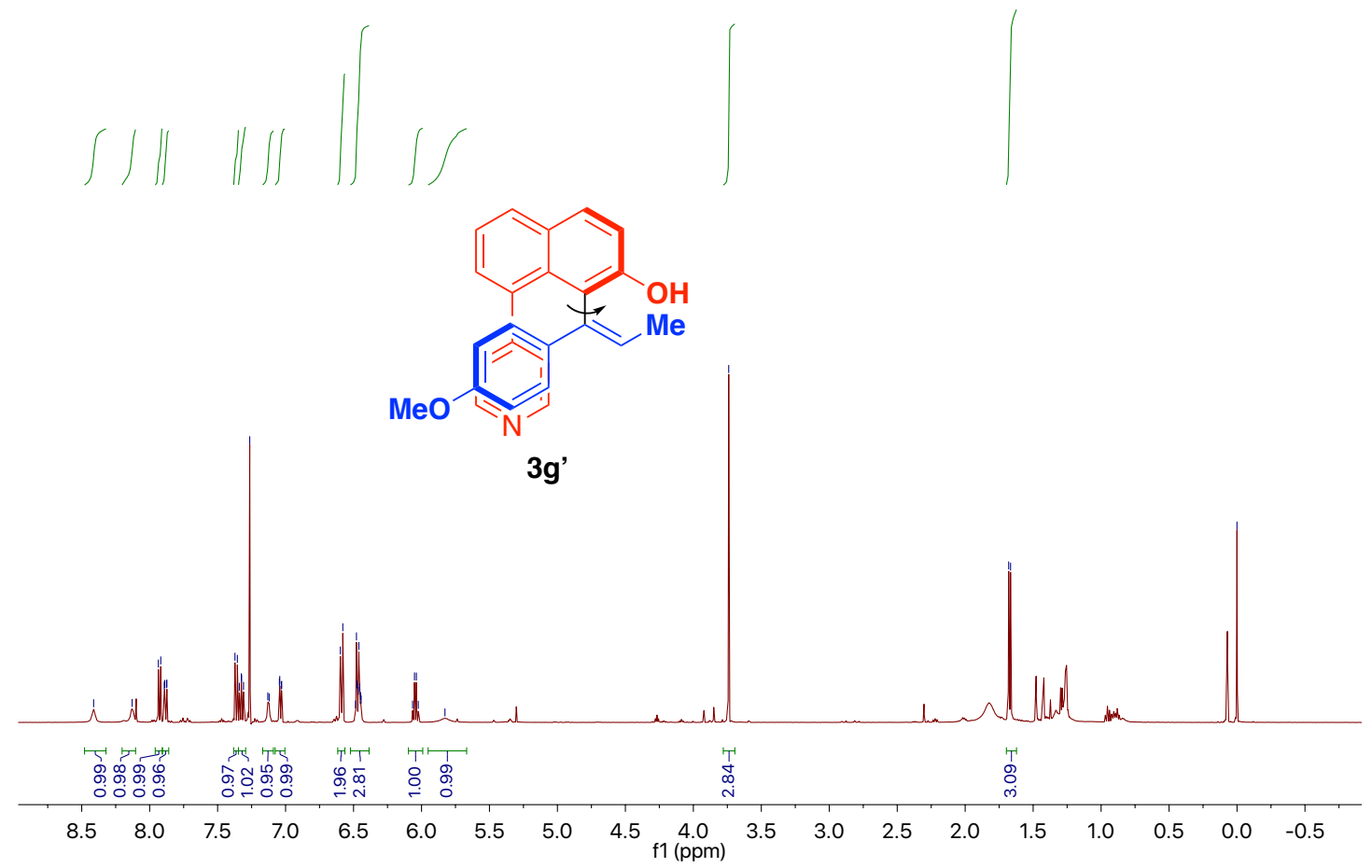

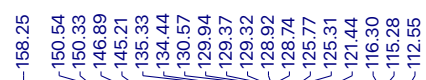<smiles>C/C=C(\C)c1c(O)ccc2cccc(-c3cc(OC)ncc3O)c12</smiles>

$3 g^{\prime}$

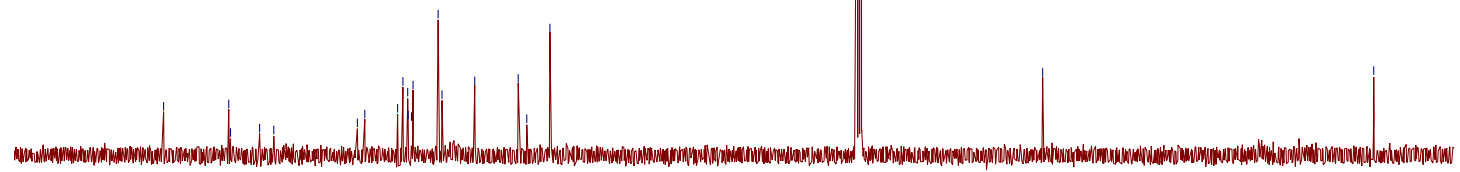

$\begin{array}{lllllllllllllllll}170 & 160 & 150 & 140 & 130 & 120 & 110 & 100 & \begin{array}{c}90 \\ \mathrm{f} 1(\mathrm{ppm})\end{array} & 80 & 70 & 60 & 50 & 40 & 30 & 20 & 10\end{array}$




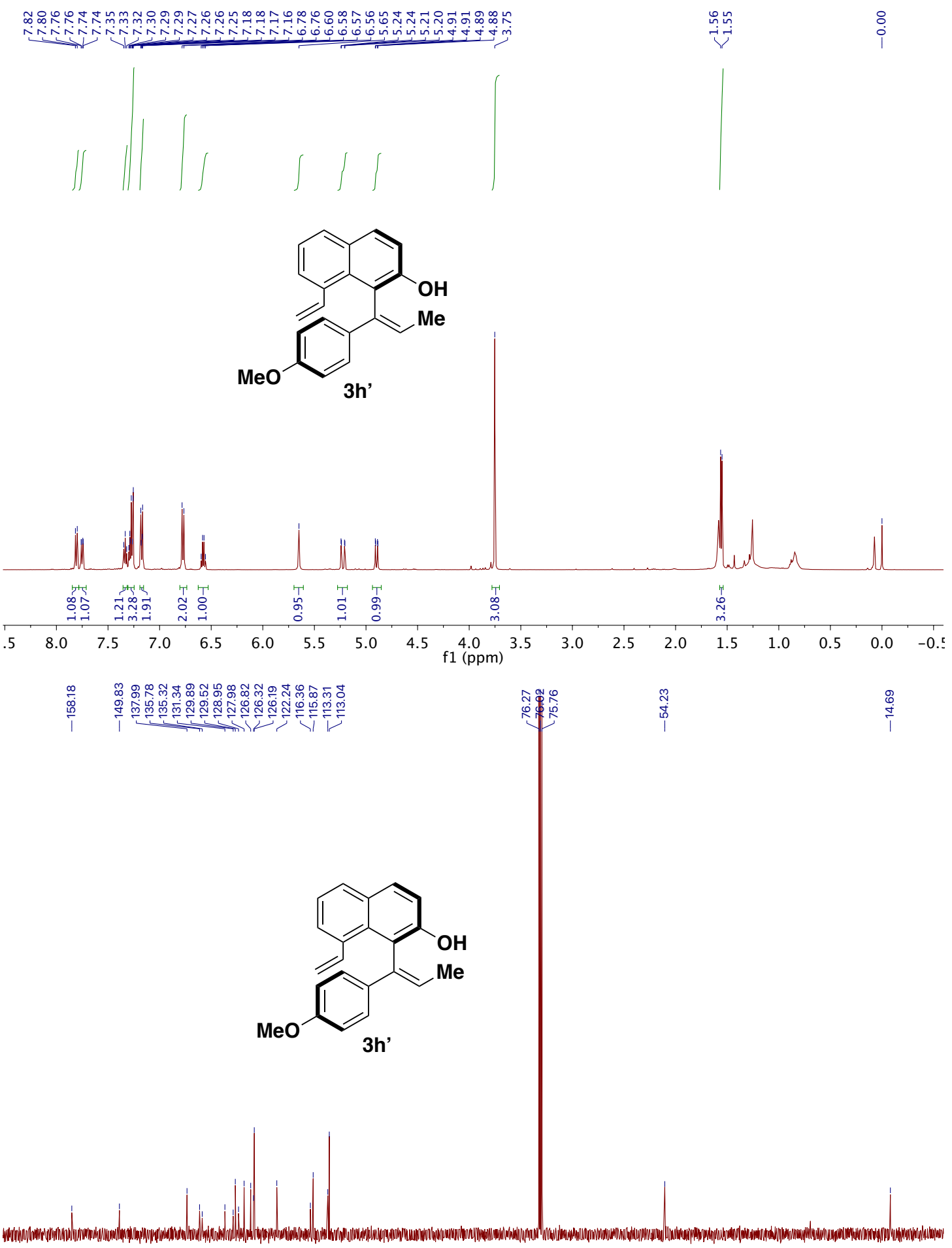

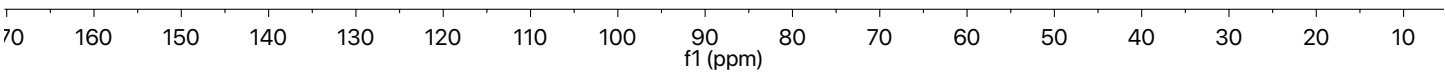




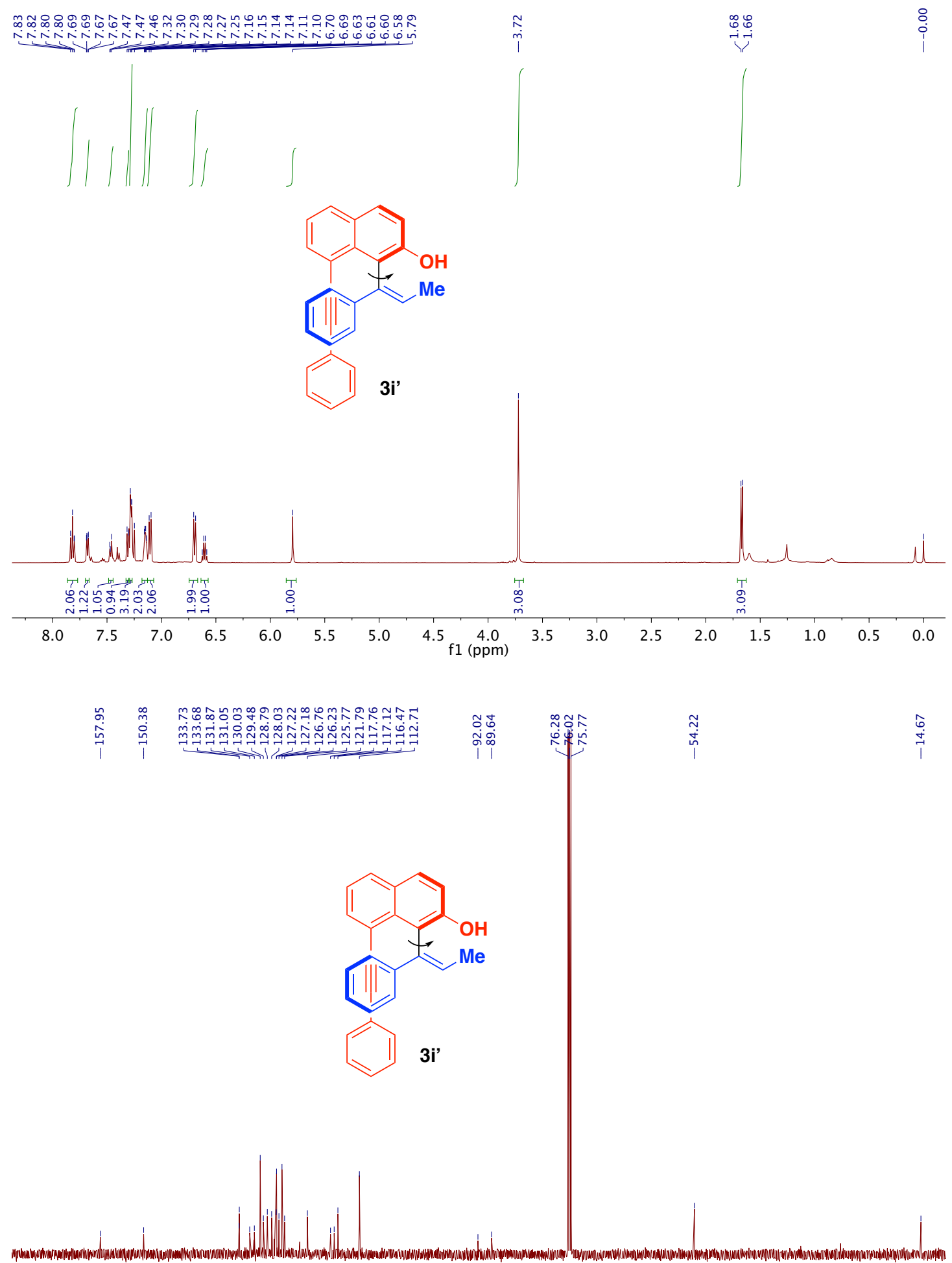

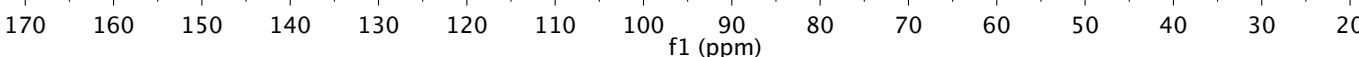




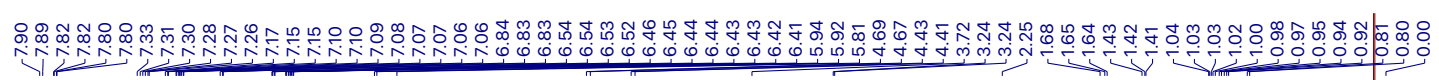

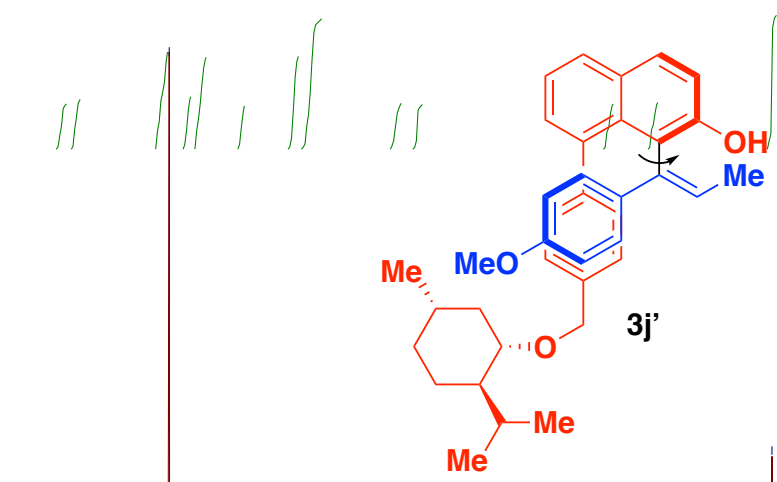
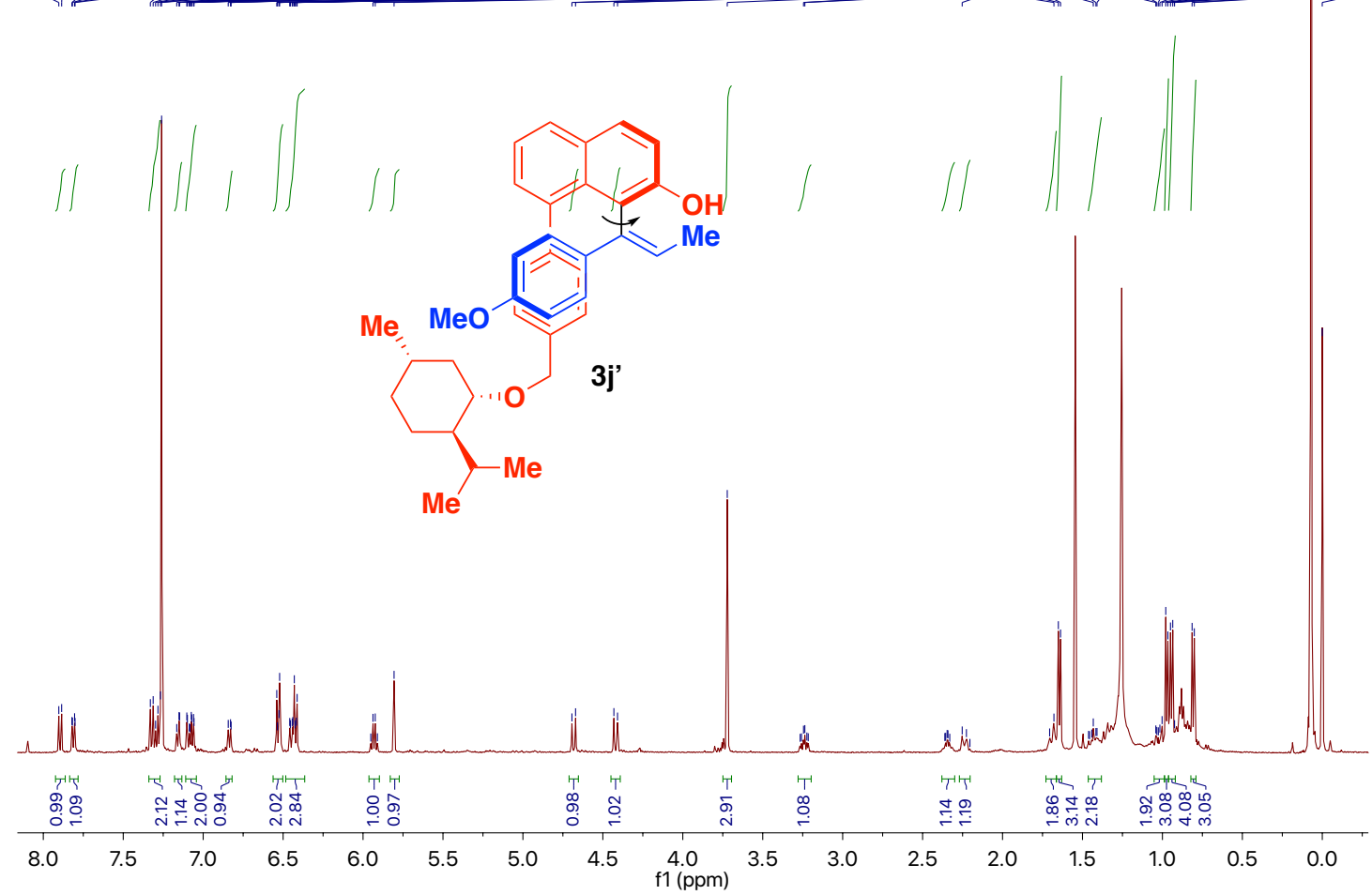

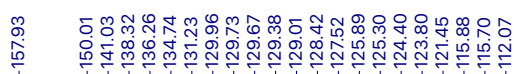

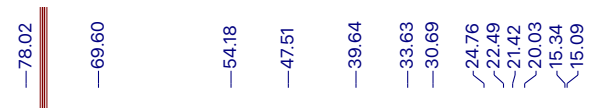
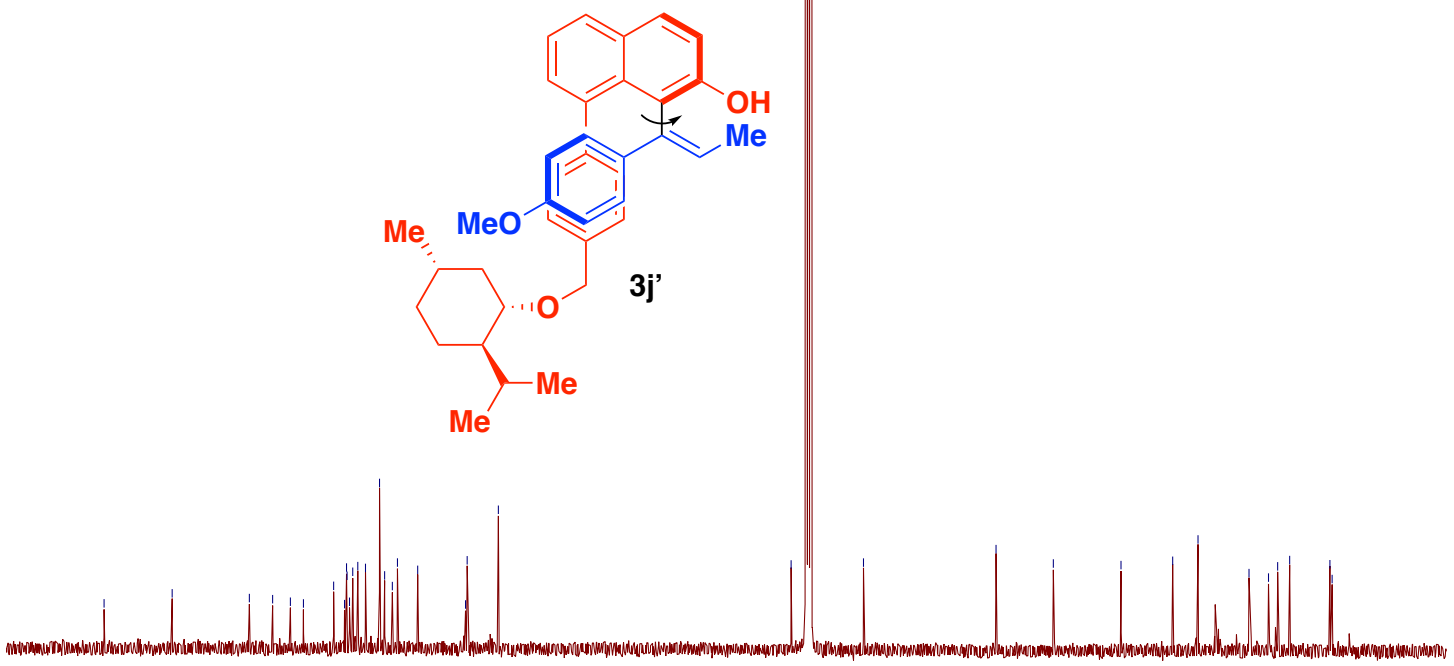

16

150

$130 \quad 120 \quad 110$

100

90
$\mathrm{f} 1(\mathrm{ppm})$ 


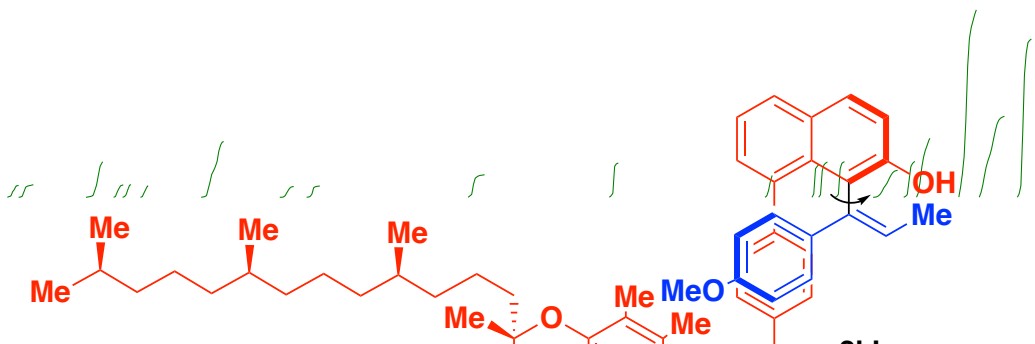

$3 k^{\prime}$

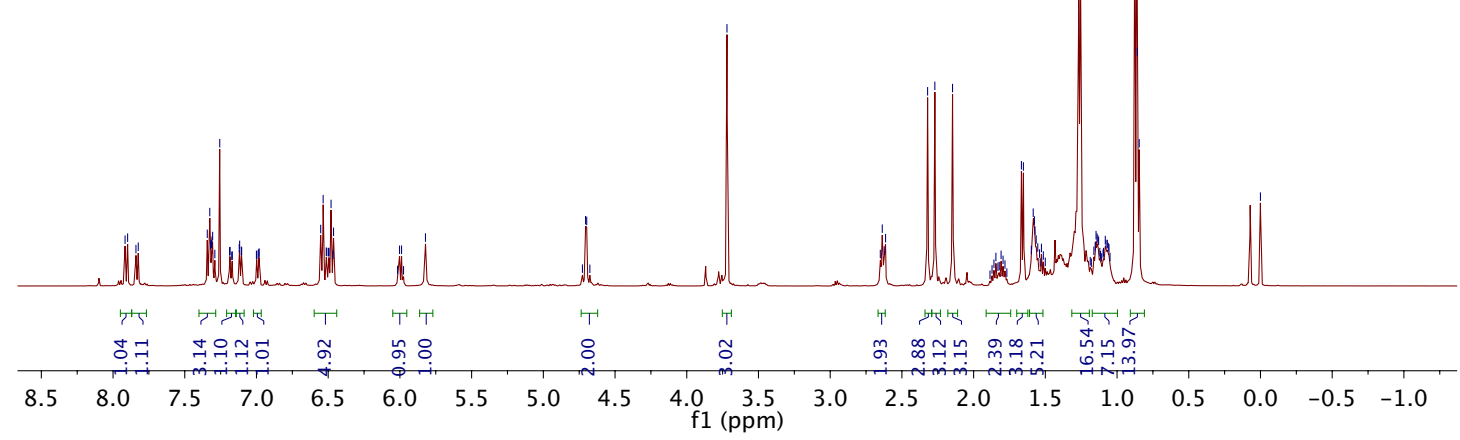

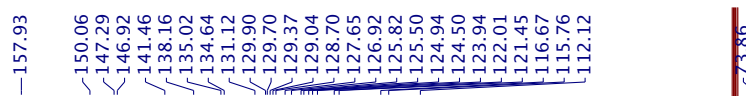

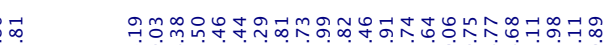

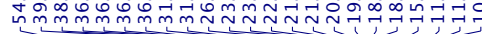

Me<smiles>CCCCOc1ccccc1OCc1c(C)c(C)c2c(c1C)CC[C@@](C)(CCC)O2</smiles>

3k'

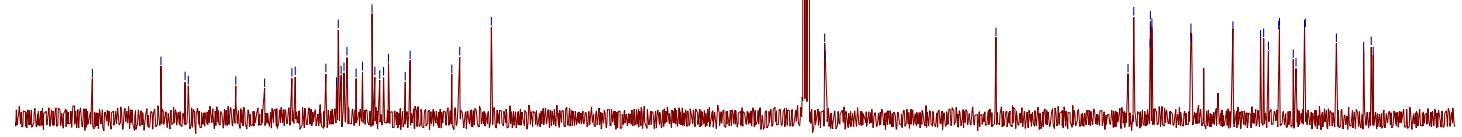




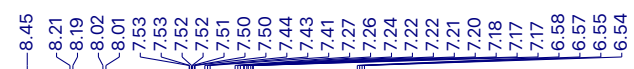

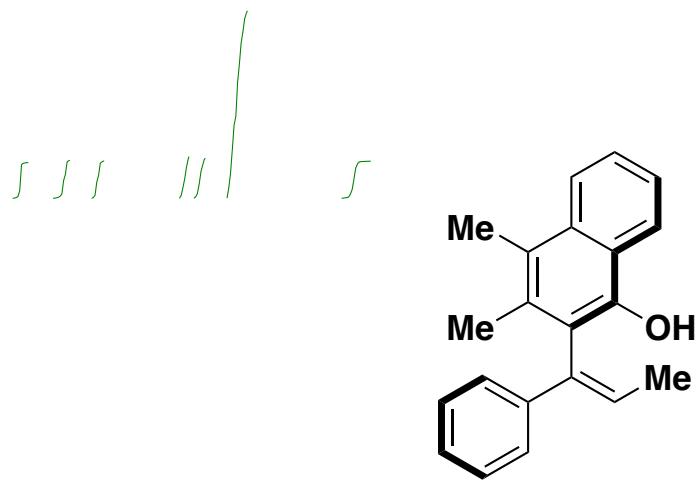

$5 a$

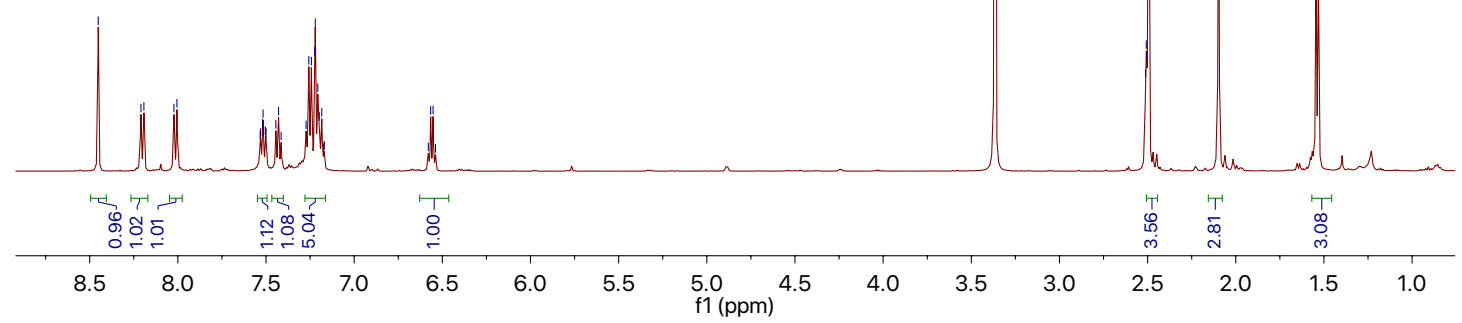

กำ

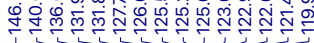<smiles>C/C=C(\c1ccccc1)c1c(C)c(C)c2ccccc2c1O</smiles>

$5 a$

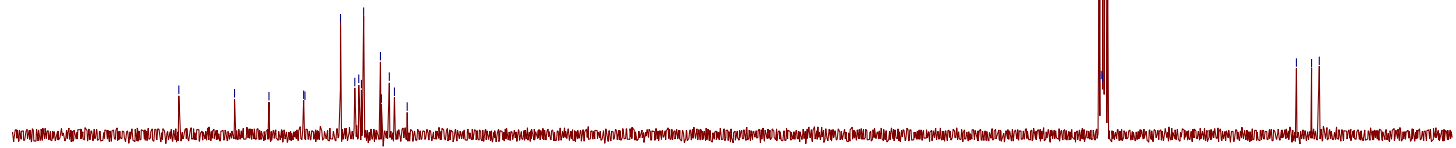

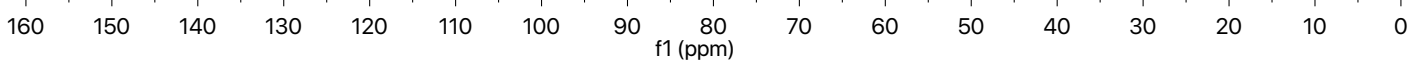




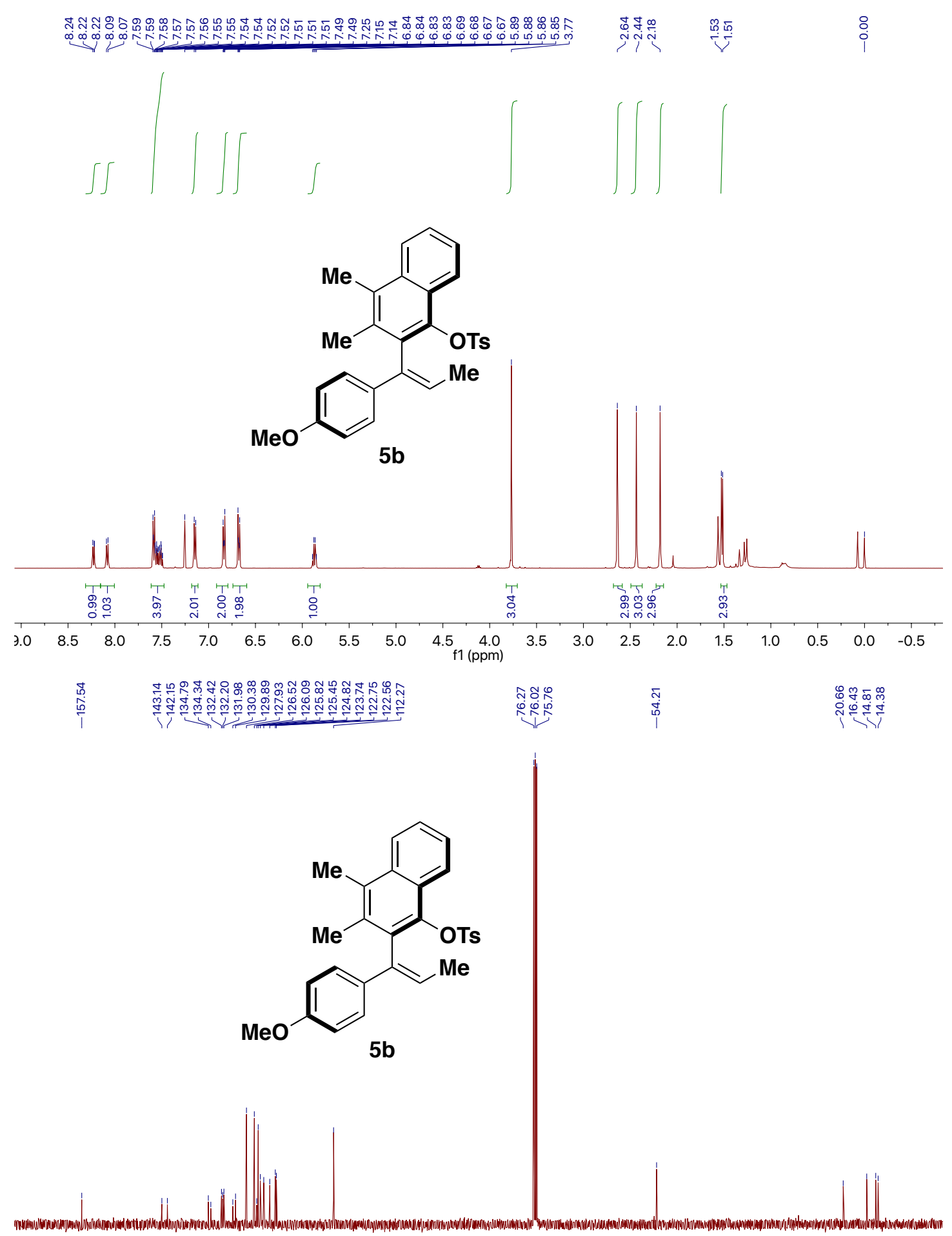

$\begin{array}{lllllllllllllll}160 & 150 & 140 & 130 & 120 & 110 & 100 & \underset{\mathrm{f} 1(\mathrm{ppm})}{90} & 70 & 60 & 50 & 40 & 30 & 20 & 10\end{array}$ 


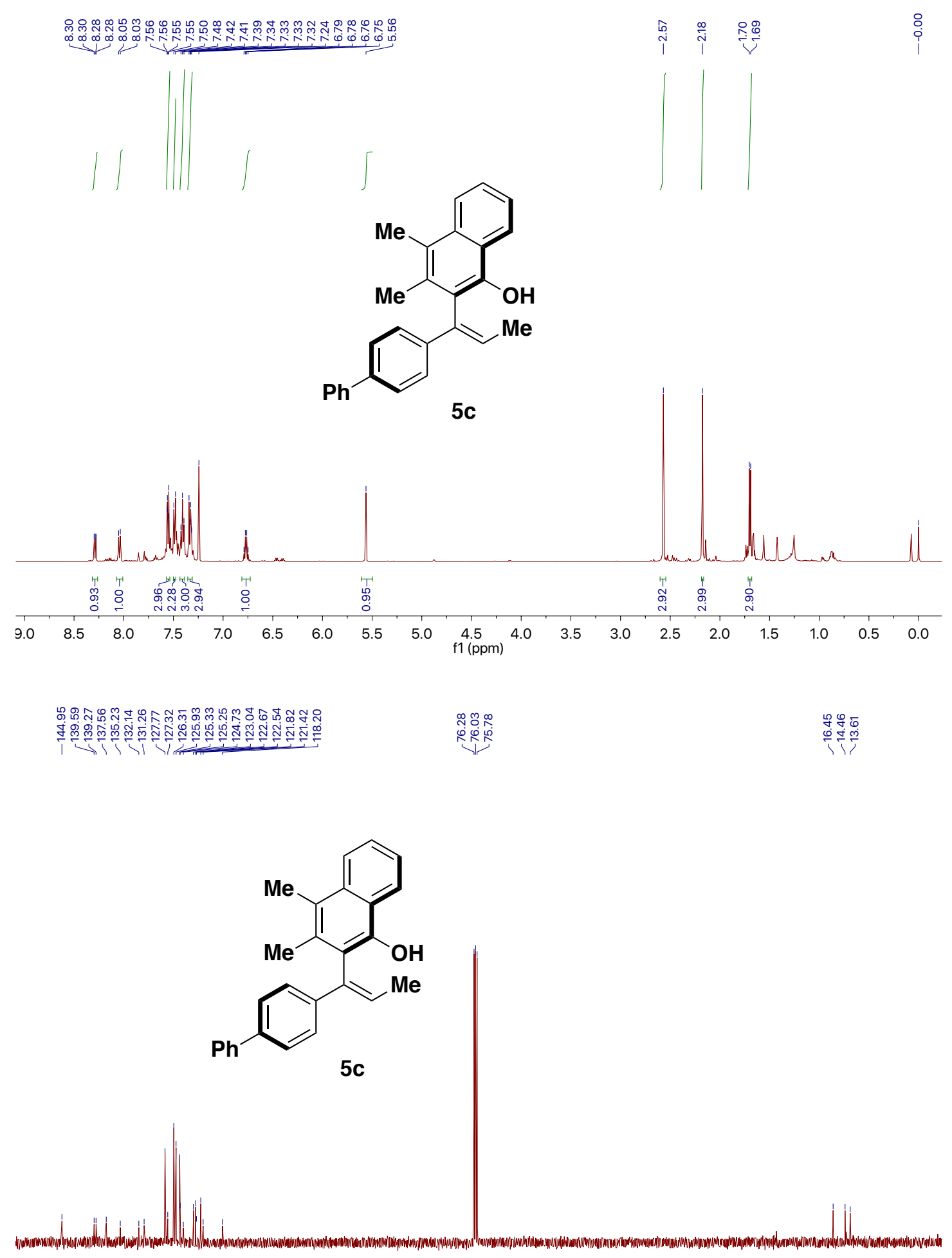

$\begin{array}{llllllllllllllllllllllllllllllll}150 & 145 & 140 & 135 & 130 & 125 & 120 & 115 & 110 & 105 & 100 & 95 & 90 & 85 & 80 & 75 & 70 & 65 & 60 & 55 & 50 & 45 & 40 & 35 & 30 & 25 & 20 & 15 & 10 & 5 & 0\end{array}$ 

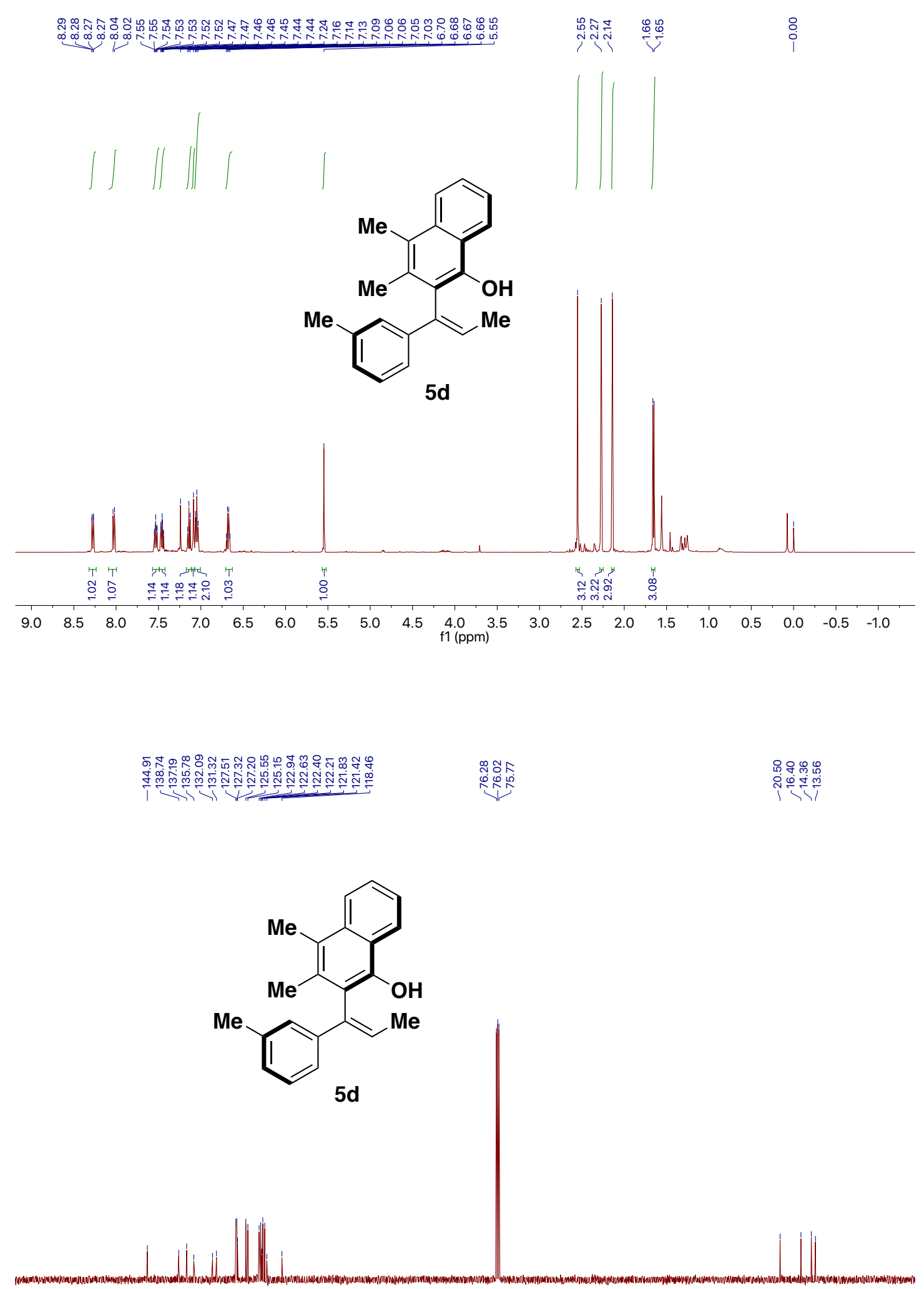

$\begin{array}{lllllllll}70 & 160 & 150 & 140 & 130 & 120 & 110 & 100 & 90\end{array}$ 


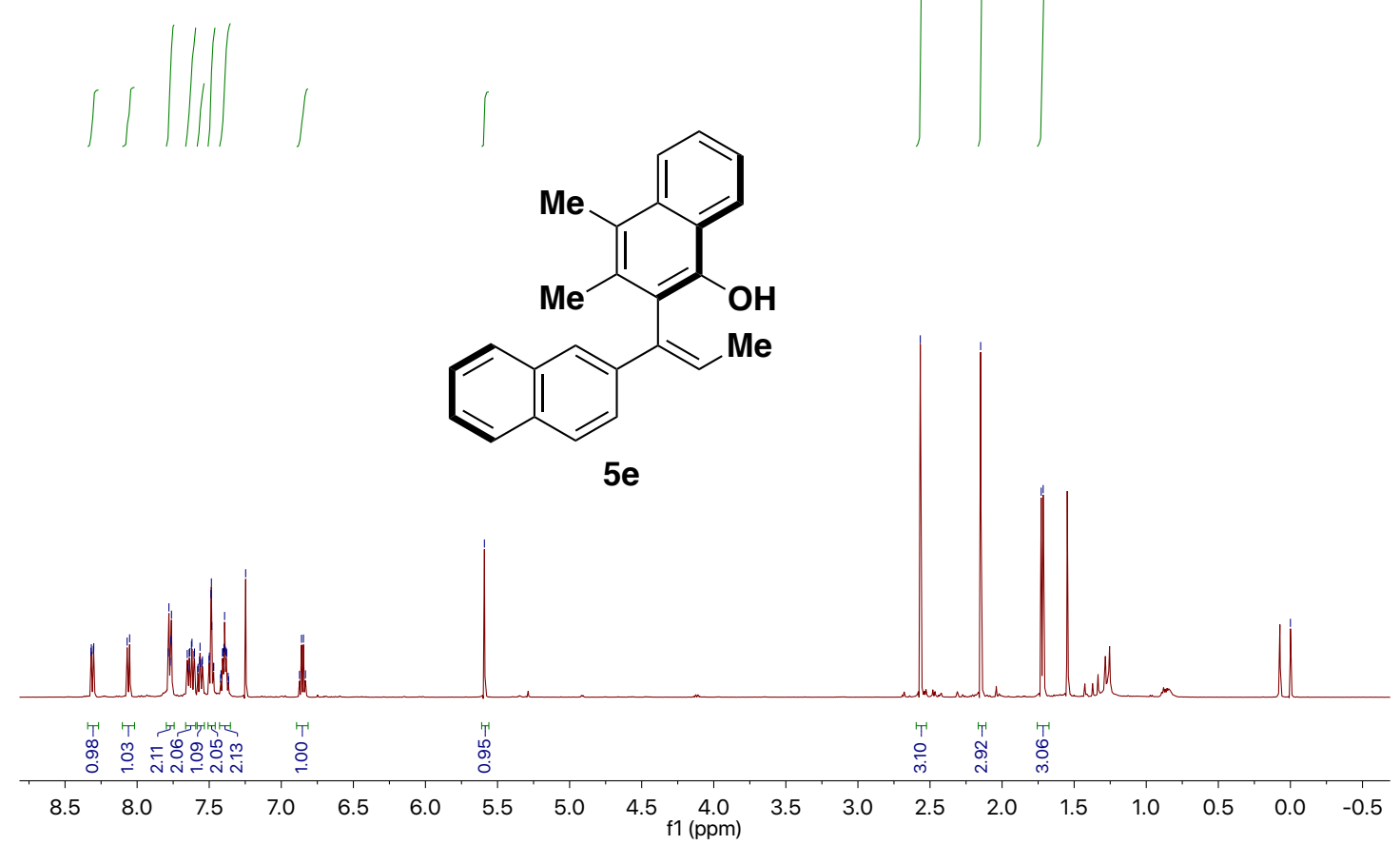

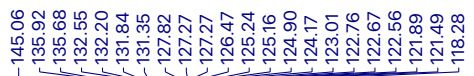<smiles>C/C=C(/c1ccc2ccccc2c1)c1c(C)c(C)c2ccccc2c1O</smiles>

$5 e$
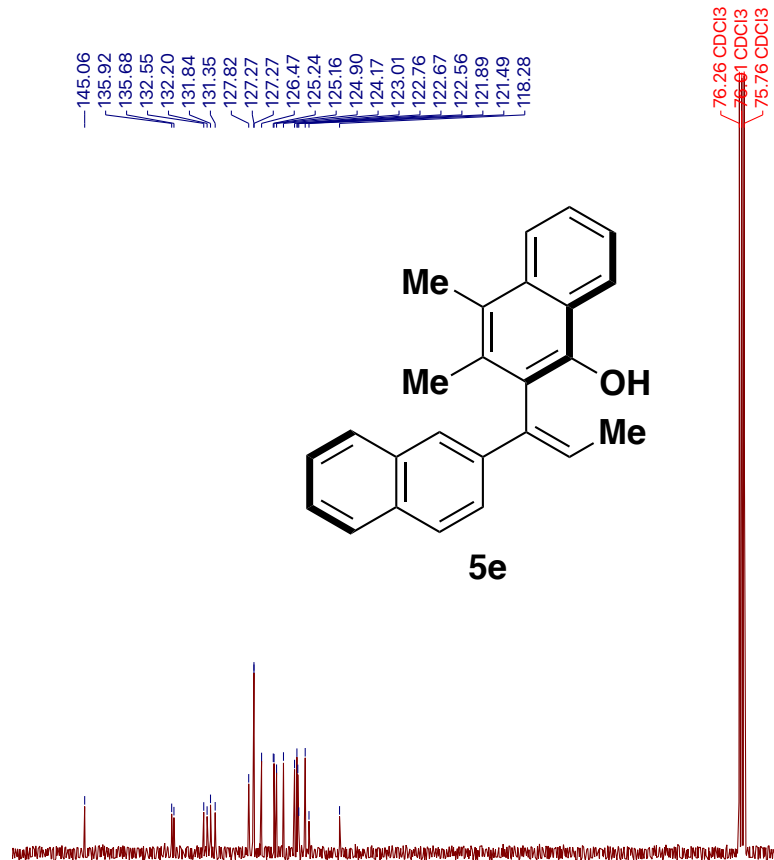

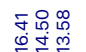

广传

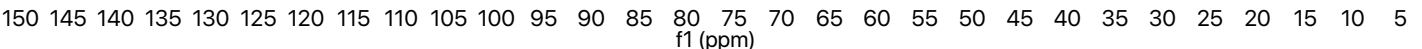




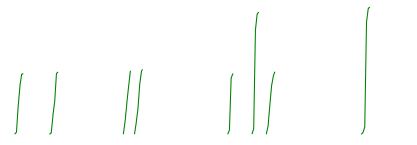<smiles>C/C=C(\c1ccc2c(c1)OCO2)c1c(C)c(C)c2ccccc2c1O</smiles>

$5 f$

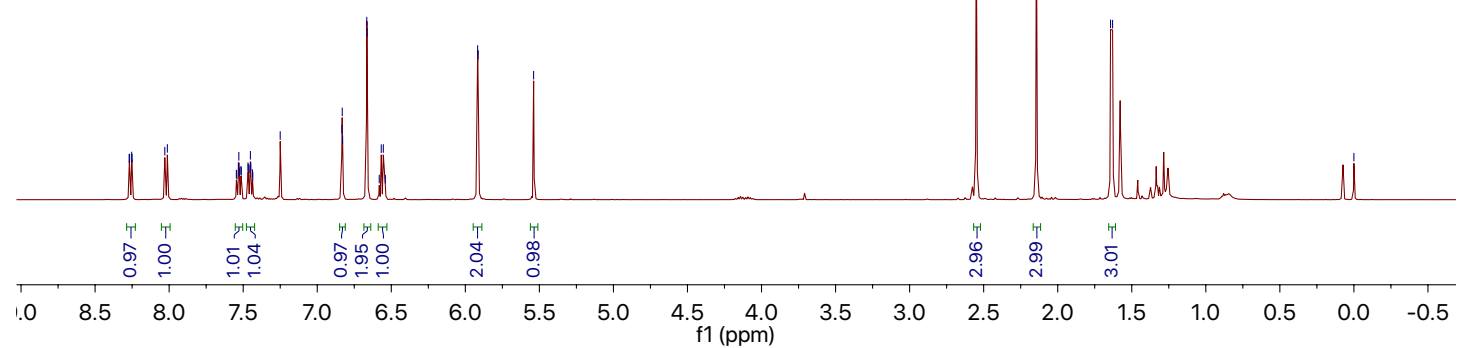

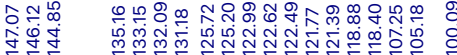

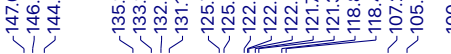

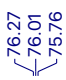

-

(r)<smiles>C/C=C(\c1ccc2c(c1)OCO2)c1c(C)c(C)c2ccccc2c1O</smiles>

$5 f$

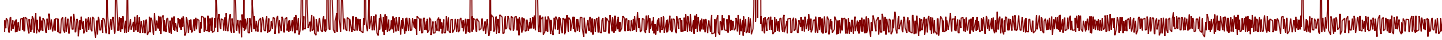

$\begin{array}{llllllllllllll}150 & 140 & 130 & 120 & 110 & 100 & 90 & 80 & 70 & 60 & 50 & 40 & 30 & 20\end{array}$ 

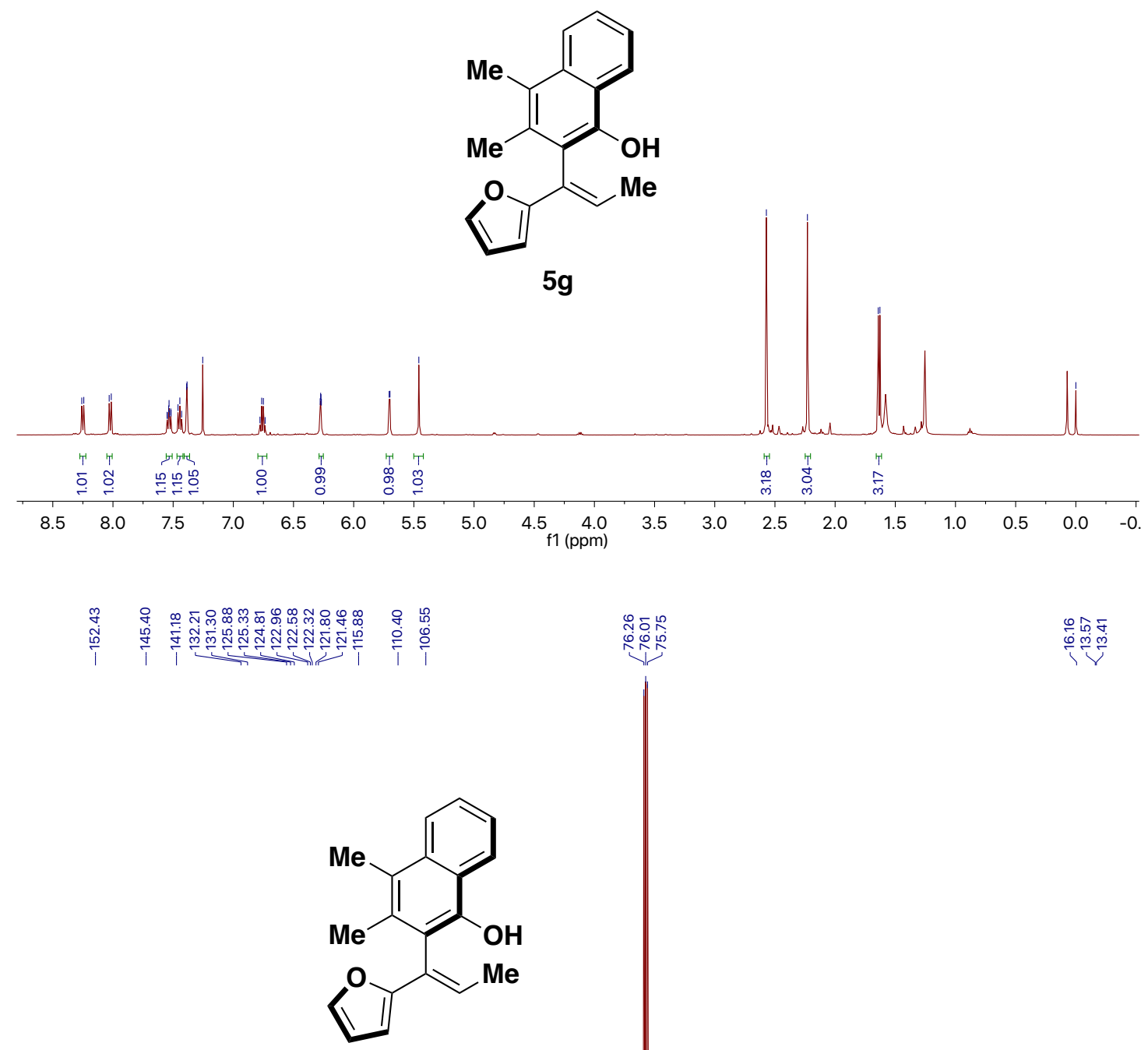

$5 g$

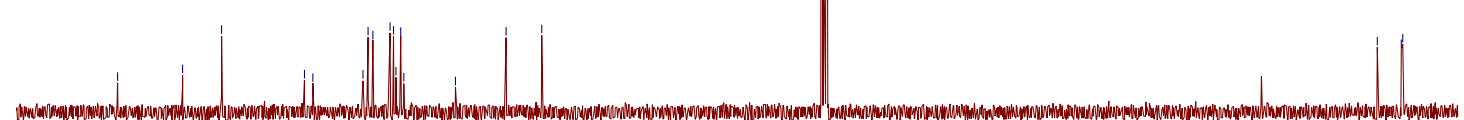

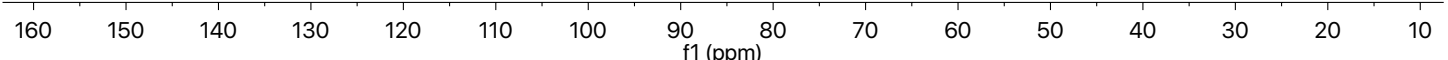




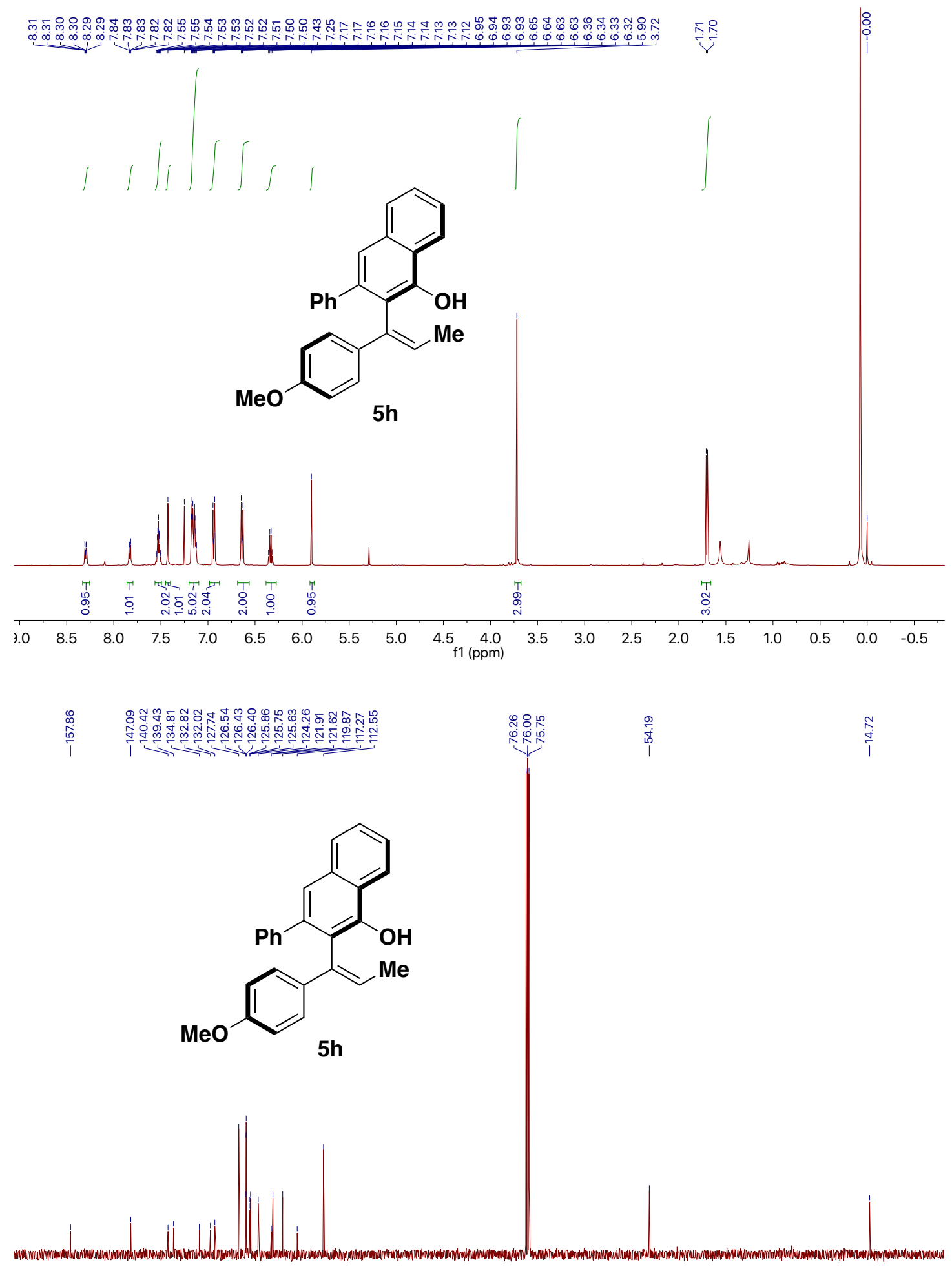

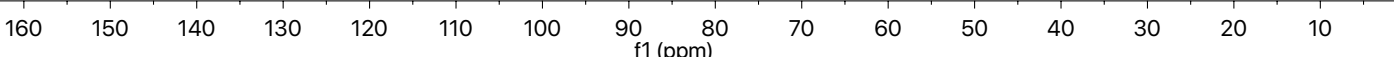




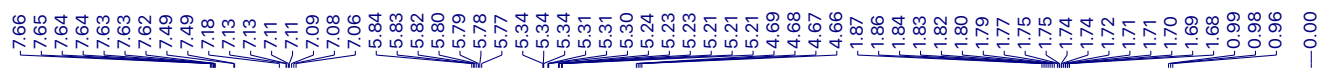
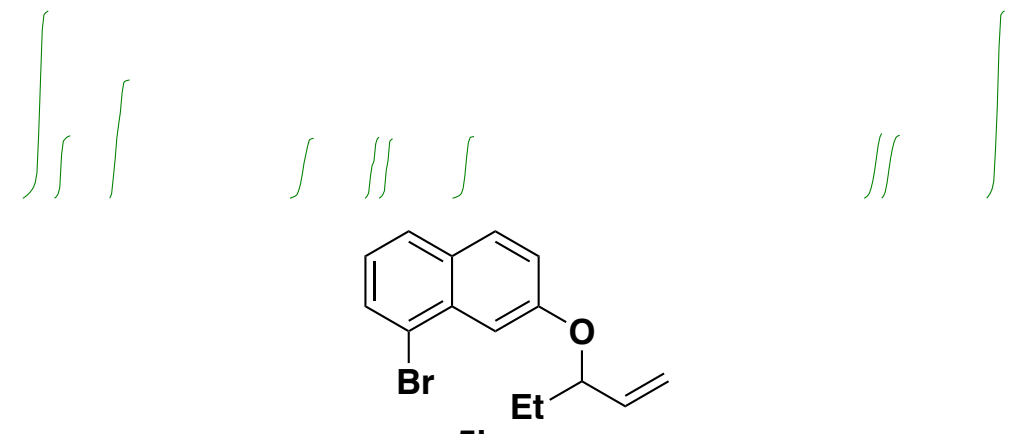

$5 i$
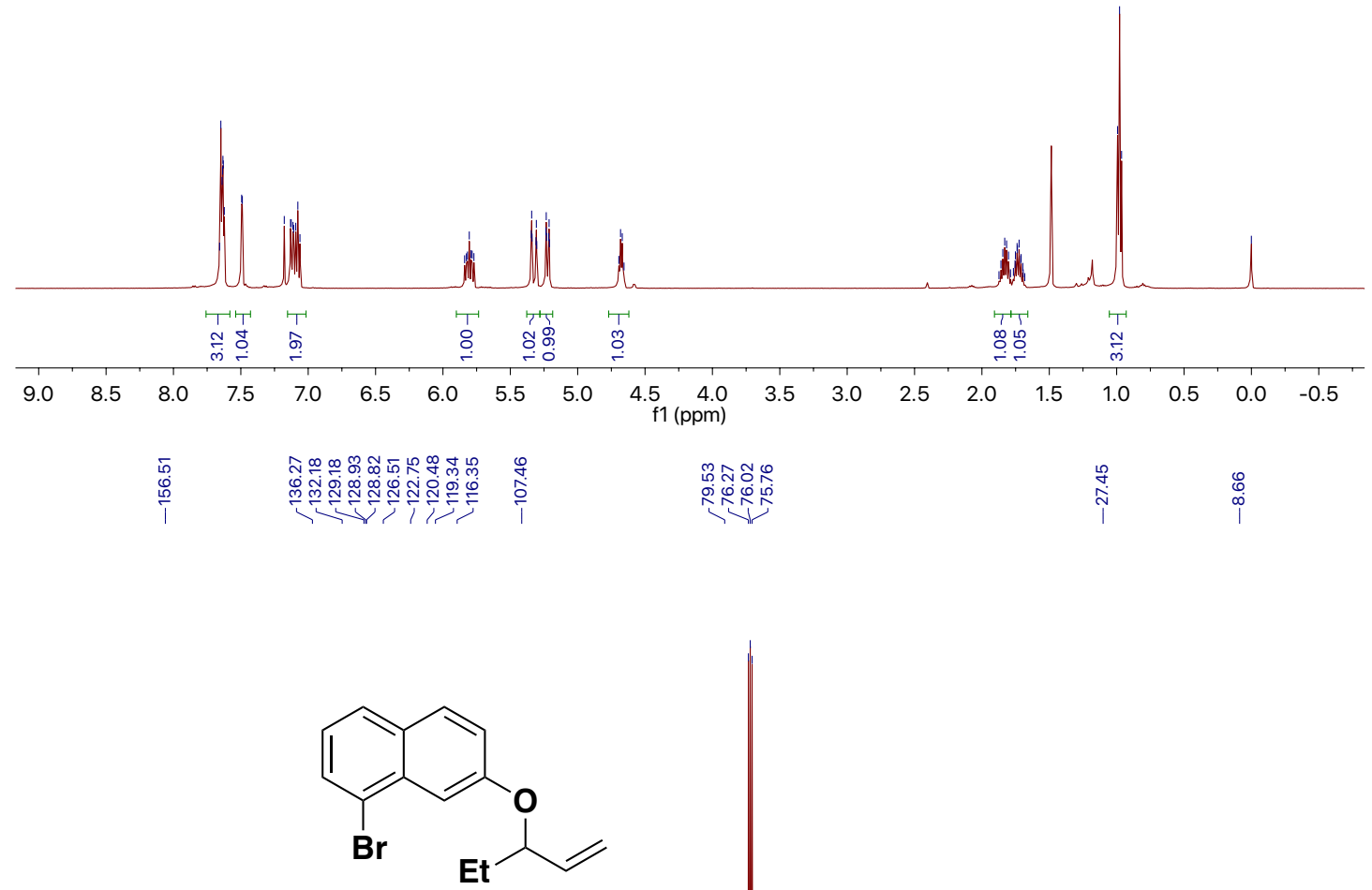

5

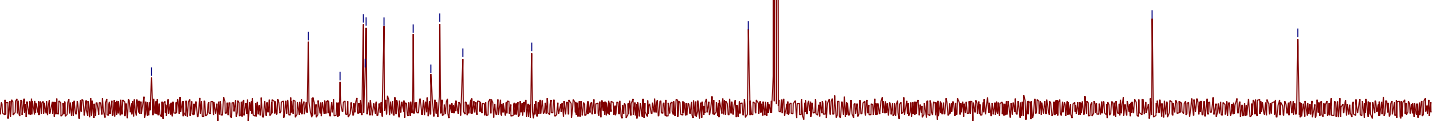

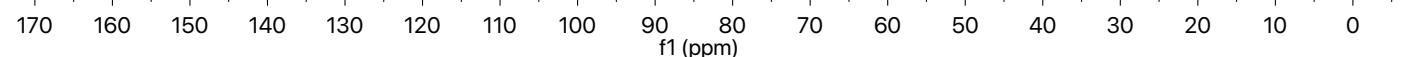




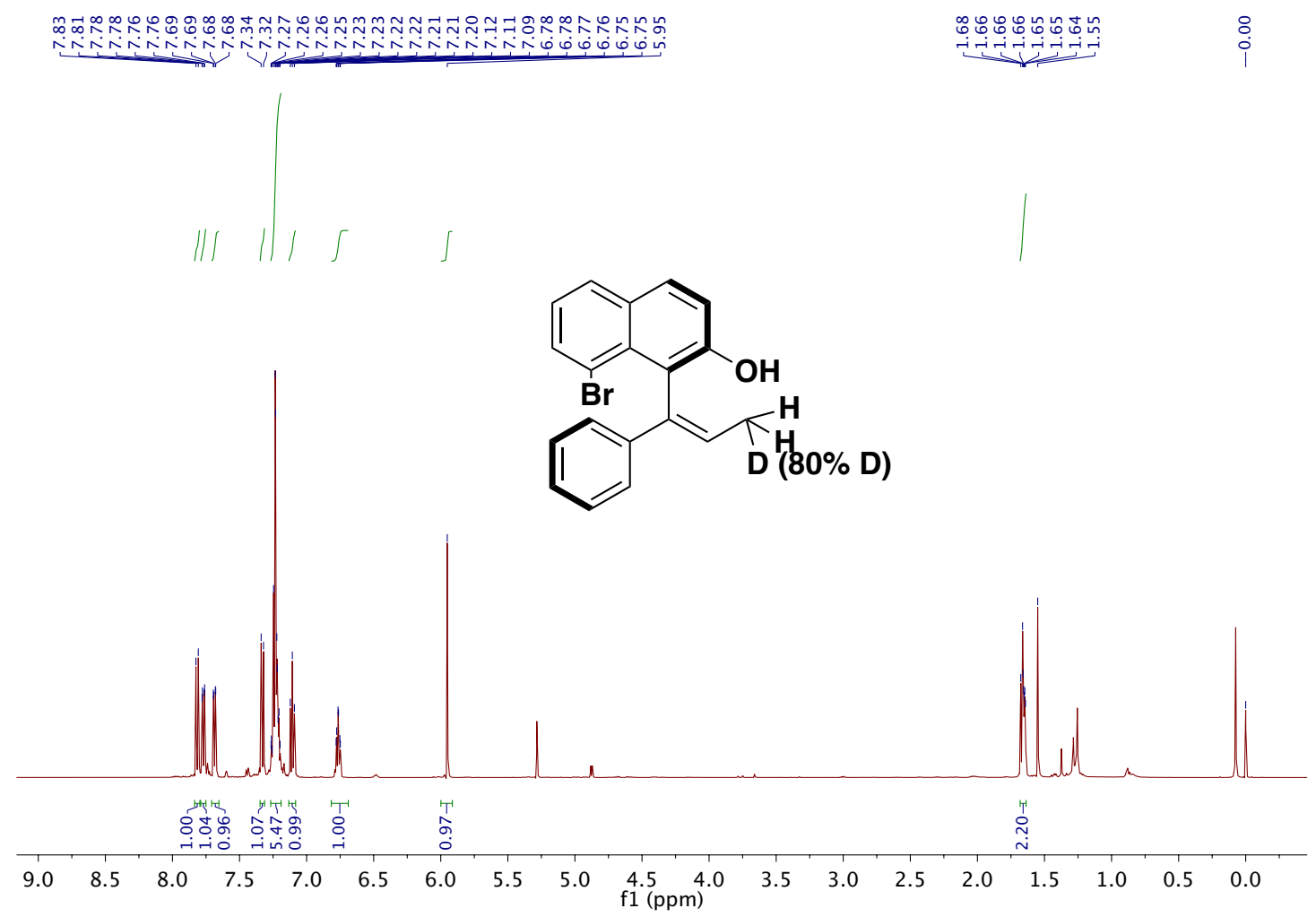



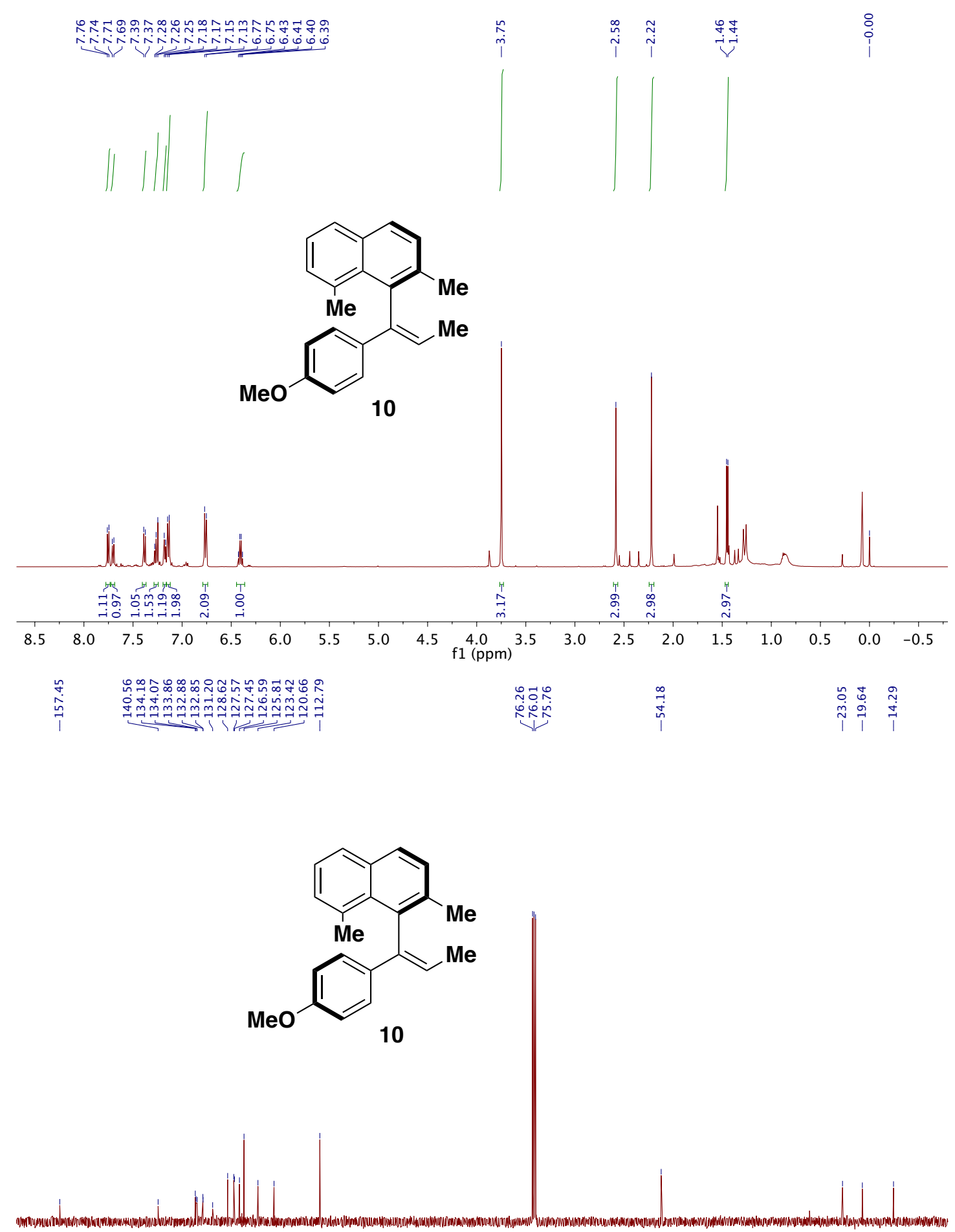

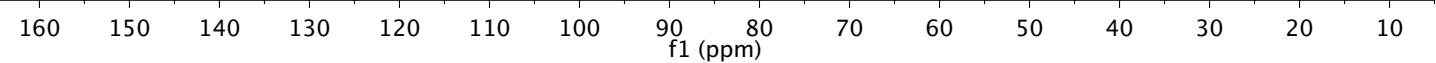



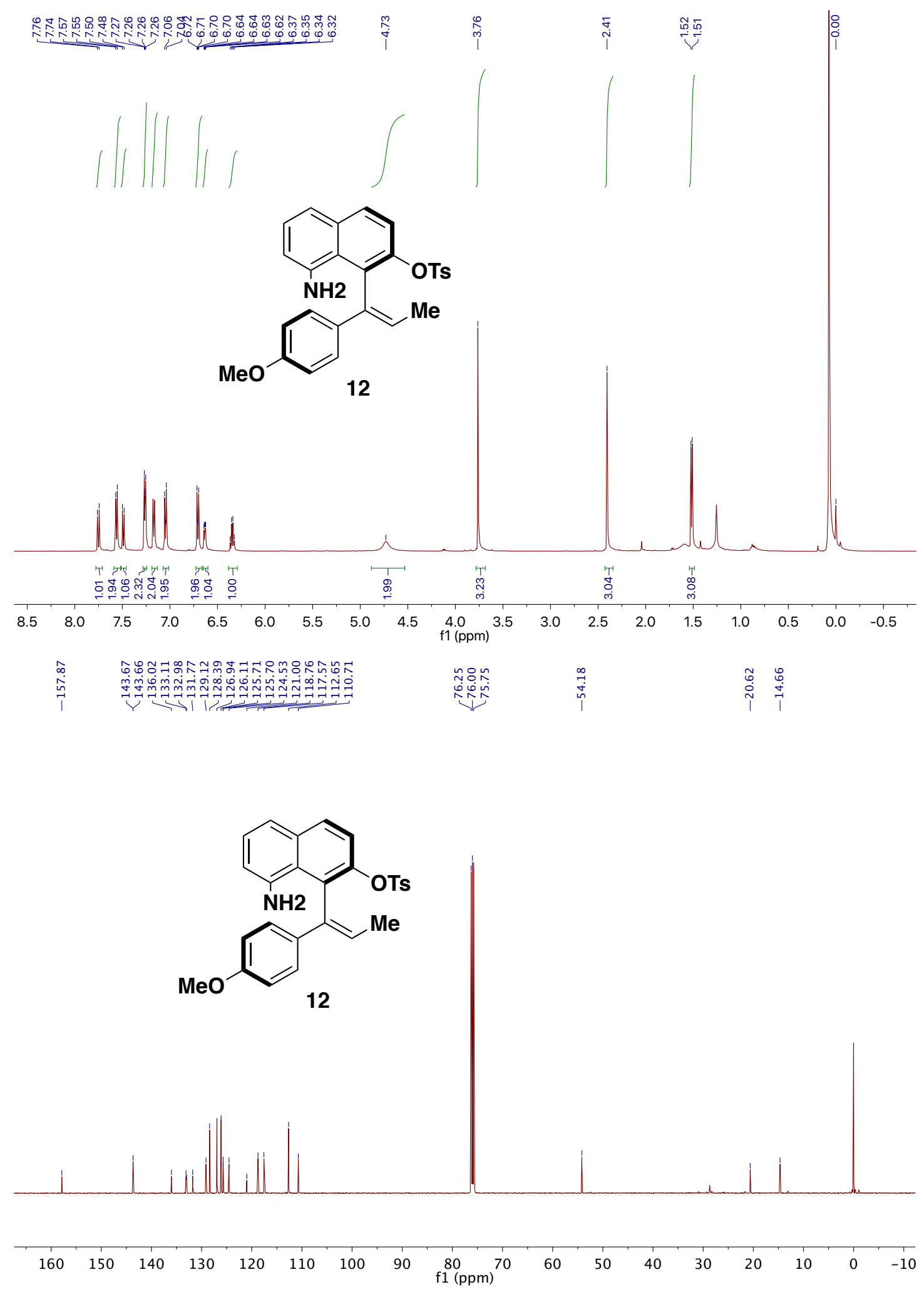


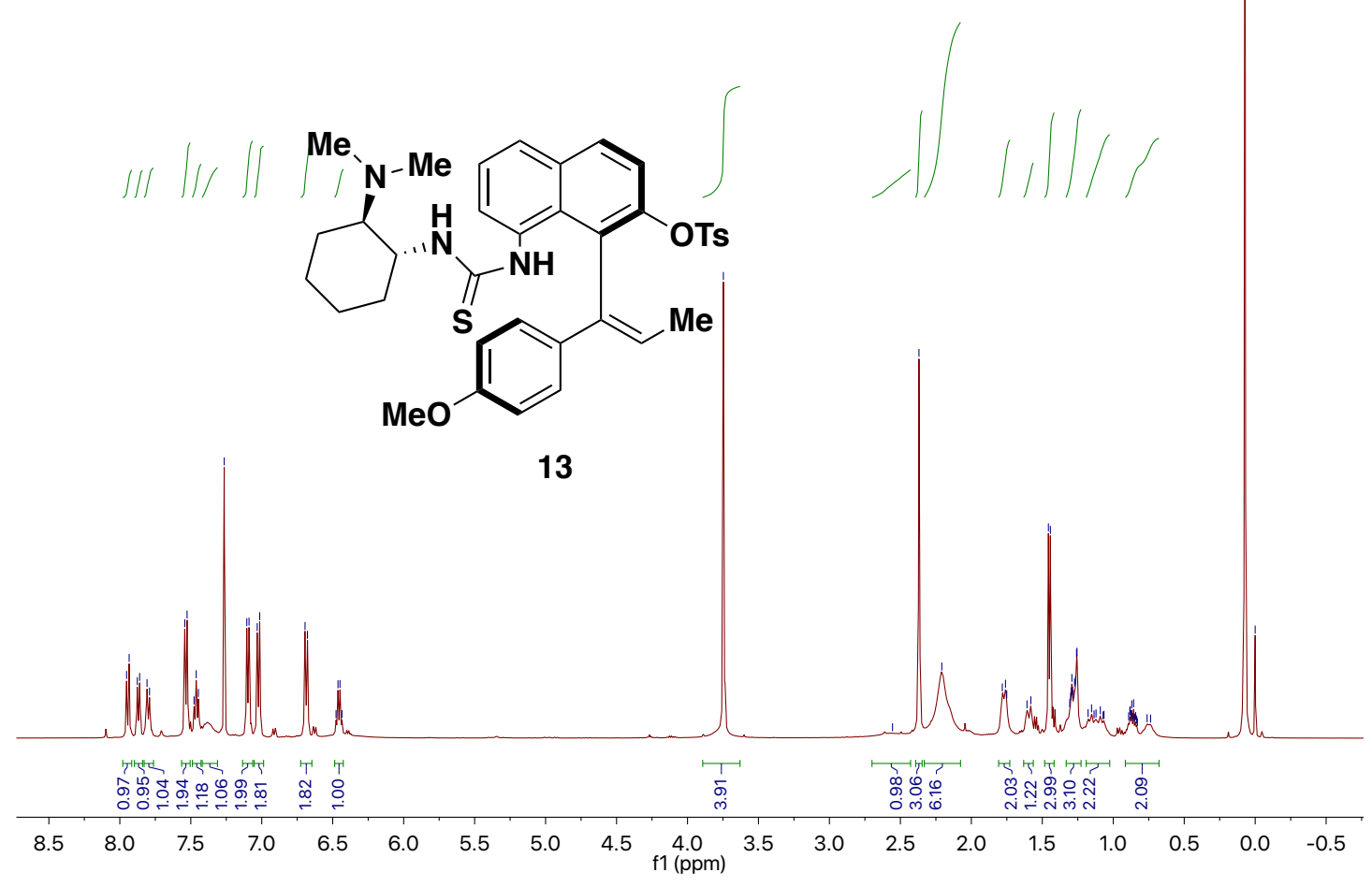

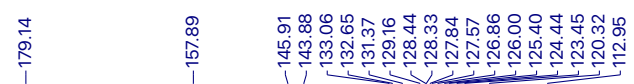

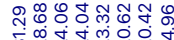

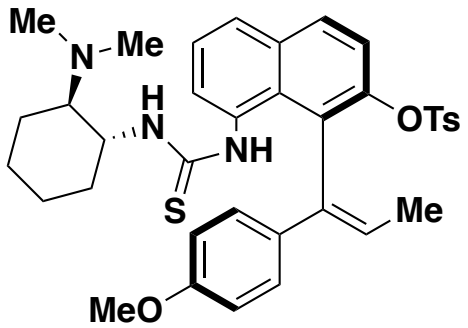

13

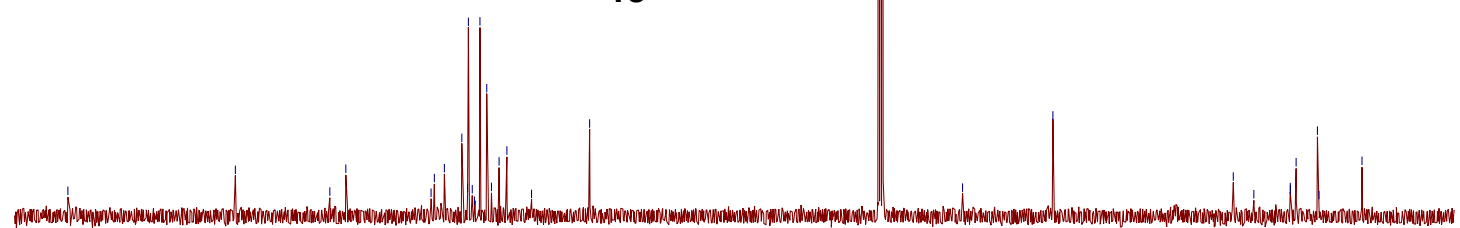

$\begin{array}{lllllllll}180 & 170 & 160 & 150 & 140 & 130 & 120 & 110 & 100 \\ \mathrm{f} 1(\mathrm{ppm})\end{array}$ 


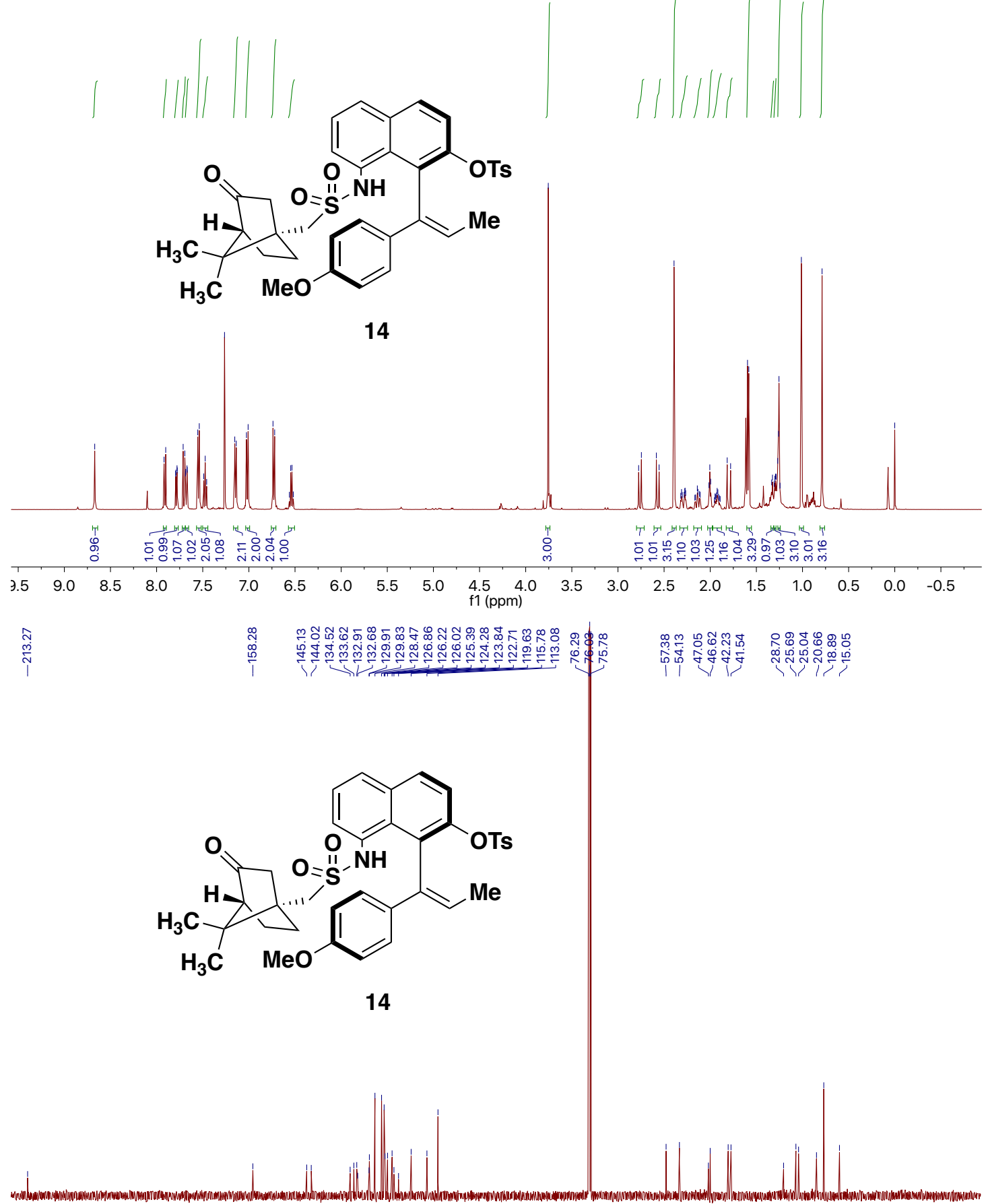

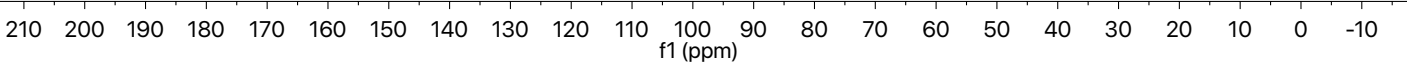




\section{HPLC data}
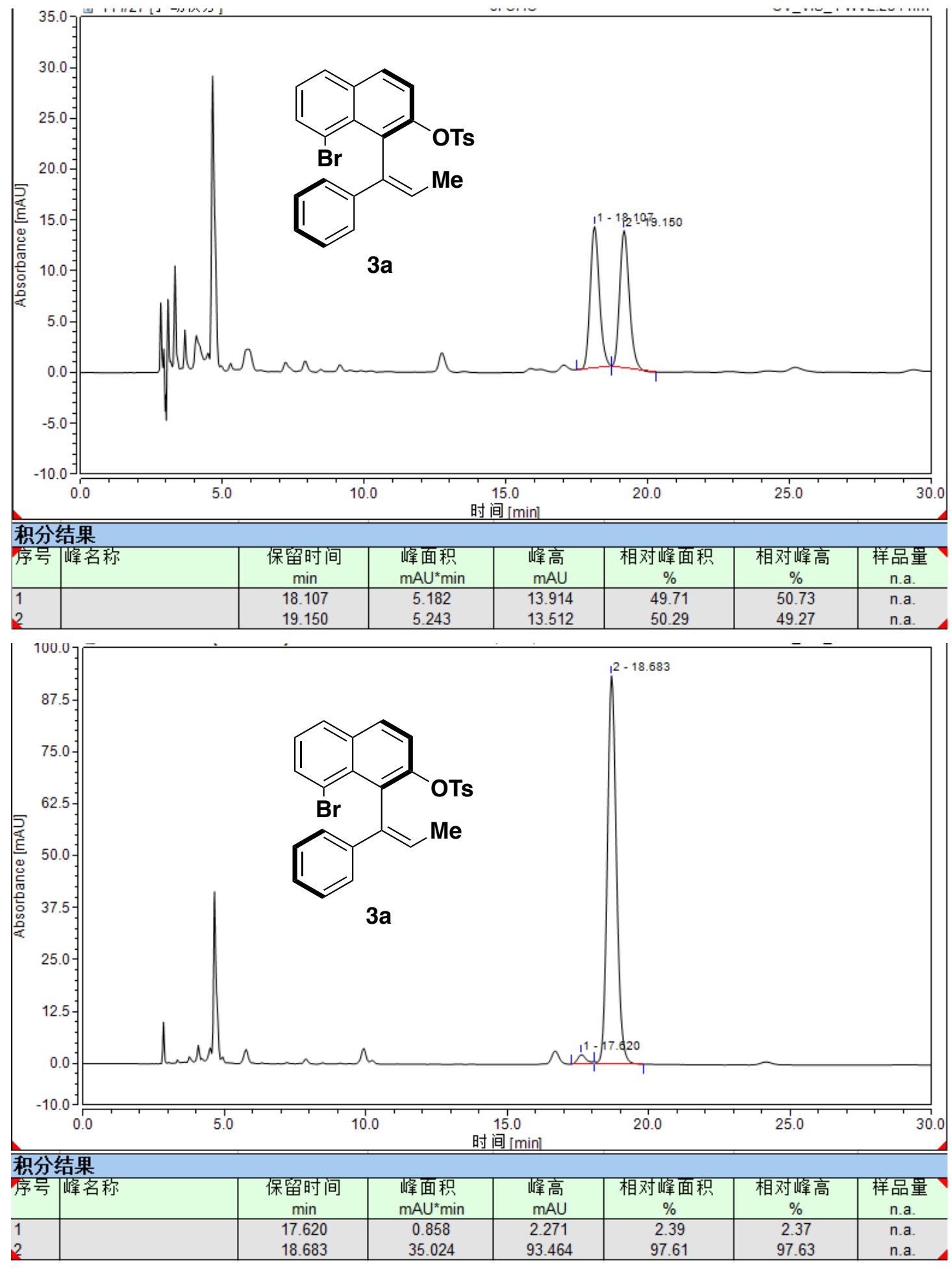

For comparison of Chinese and English of the HPLC data table.

\begin{tabular}{|l|l|l|l|l|l|l|l|}
\hline 序号 & $\begin{array}{l}\text { 峰名 } \\
\text { 称 }\end{array}$ & $\begin{array}{l}\text { 保留时间 } \\
(\mathrm{min})\end{array}$ & $\begin{array}{l}\text { 峰面积 } \\
(\mathrm{mAU} \text { *min) }\end{array}$ & $\begin{array}{l}\text { 峰高 } \\
(\mathrm{mAU})\end{array}$ & $\begin{array}{l}\text { 相对峰面 } \\
\text { 积 }(\%)\end{array}$ & $\begin{array}{l}\text { 相对峰高 } \\
(\%)\end{array}$ & $\begin{array}{l}\text { 样品量 } \\
\text { n.a. }\end{array}$ \\
\hline $\begin{array}{l}\text { Entr } \\
\mathrm{y}\end{array}$ & $\begin{array}{l}\text { Peak } \\
\text { name }\end{array}$ & $\begin{array}{l}\text { Retention } \\
\text { time (min) }\end{array}$ & $\begin{array}{l}\text { Peak area } \\
(\mathrm{mAU} * \mathrm{~min})\end{array}$ & $\begin{array}{l}\text { Peak } \\
\text { height } \\
(\mathrm{mAU})\end{array}$ & $\begin{array}{l}\text { Relative } \\
\text { peak area } \\
(\%)\end{array}$ & $\begin{array}{l}\text { Relative } \\
\text { Peak } \\
\text { height (\%) }\end{array}$ & $\begin{array}{l}\text { Sample } \\
\text { volume } \\
\text { n.a. }\end{array}$ \\
\hline
\end{tabular}



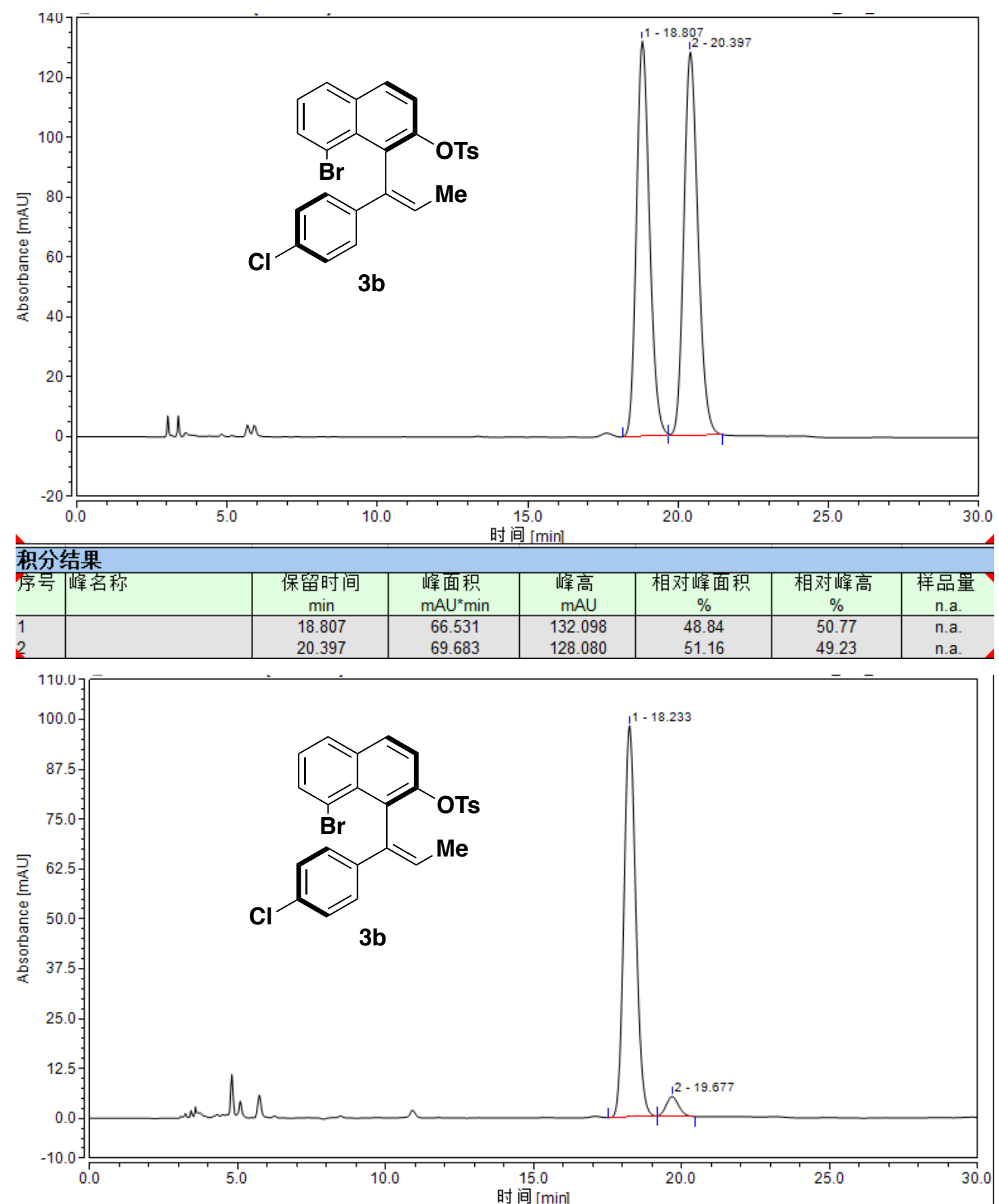

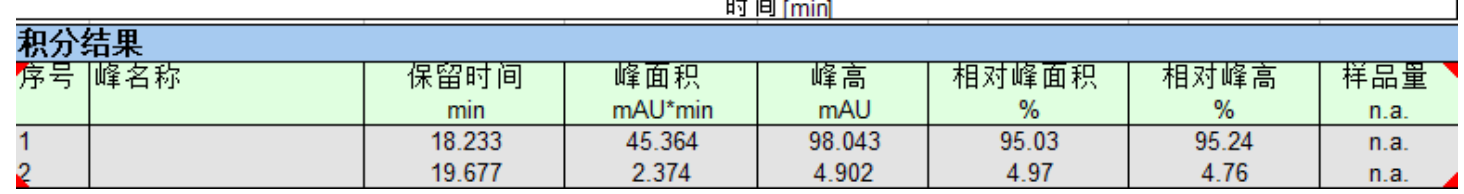

For comparison of Chinese and English of the HPLC data table.

\begin{tabular}{|l|l|l|l|l|l|l|l|}
\hline 序号 & $\begin{array}{l}\text { 峰名 } \\
\text { 称 }\end{array}$ & $\begin{array}{l}\text { 保留时间 } \\
(\mathrm{min})\end{array}$ & $\begin{array}{l}\text { 峰面积 } \\
(\mathrm{mAU} \text { *min) }\end{array}$ & $\begin{array}{l}\text { 峰高 } \\
(\mathrm{mAU})\end{array}$ & $\begin{array}{l}\text { 相对峰面 } \\
\text { 积 }(\%)\end{array}$ & $\begin{array}{l}\text { 相对峰高 } \\
(\%)\end{array}$ & $\begin{array}{l}\text { 样品量 } \\
\text { n.a. }\end{array}$ \\
\hline $\begin{array}{l}\text { Entr } \\
\mathrm{y}\end{array}$ & $\begin{array}{l}\text { Peak } \\
\text { name }\end{array}$ & $\begin{array}{l}\text { Retention } \\
\text { time (min) }\end{array}$ & $\begin{array}{l}\text { Peak area } \\
\left(\mathrm{mAU} \mathrm{U}^{*} \text { min }\right)\end{array}$ & $\begin{array}{l}\text { Peak } \\
\text { height } \\
(\mathrm{mAU})\end{array}$ & $\begin{array}{l}\text { Relative } \\
\text { peak area } \\
(\%)\end{array}$ & $\begin{array}{l}\text { Relative } \\
\text { Peak } \\
\text { height (\%) }\end{array}$ & $\begin{array}{l}\text { Sample } \\
\text { volume } \\
\text { n.a. }\end{array}$ \\
\hline
\end{tabular}



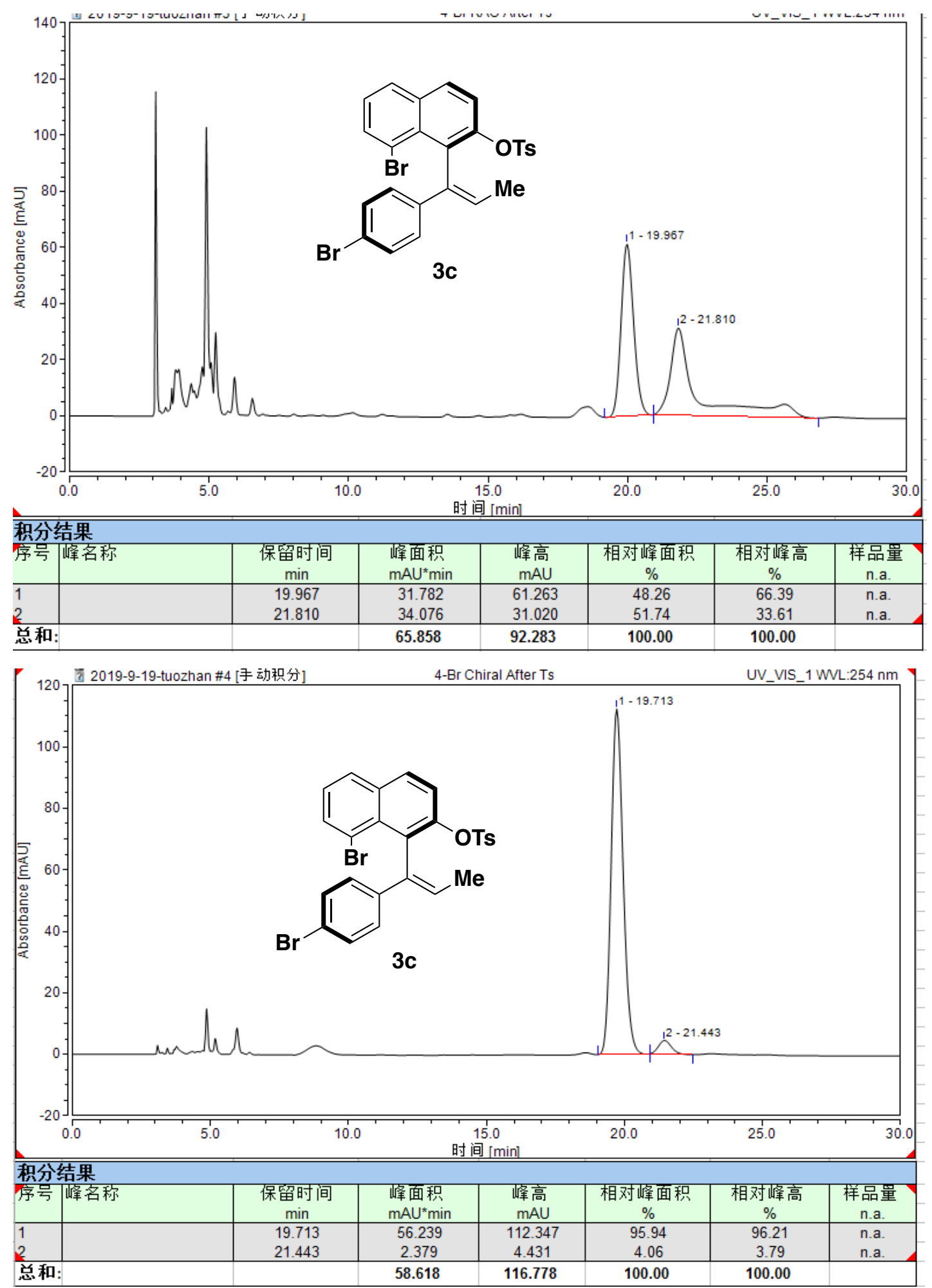

For comparison of Chinese and English of the HPLC data table.

\begin{tabular}{|c|c|c|c|c|c|c|c|}
\hline 序号 & $\begin{array}{l}\text { 峰名 } \\
\text { 称 }\end{array}$ & $\begin{array}{l}\text { 保留时间 } \\
\text { (min) }\end{array}$ & $\begin{array}{l}\text { 峰面积 } \\
\text { (mAU*min) }\end{array}$ & $\begin{array}{l}\text { 峰高 } \\
(\mathrm{mAU})\end{array}$ & $\begin{array}{l}\text { 相对峰面 } \\
\text { 积 }(\%)\end{array}$ & $\begin{array}{l}\text { 相对峰高 } \\
(\%)\end{array}$ & $\begin{array}{l}\text { 样品量 } \\
\text { n.a. }\end{array}$ \\
\hline $\begin{array}{l}\text { Entr } \\
\mathrm{y}\end{array}$ & $\begin{array}{l}\text { Peak } \\
\text { name }\end{array}$ & $\begin{array}{l}\text { Retention } \\
\text { time (min) }\end{array}$ & $\begin{array}{l}\text { Peak area } \\
\left(\mathrm{mAU}{ }^{*} \min \right)\end{array}$ & $\begin{array}{l}\text { Peak } \\
\text { height } \\
(\mathrm{mAU})\end{array}$ & $\begin{array}{l}\text { Relative } \\
\text { peak area } \\
(\%)\end{array}$ & $\begin{array}{l}\text { Relative } \\
\text { Peak } \\
\text { height (\%) }\end{array}$ & $\begin{array}{l}\text { Sample } \\
\text { volume } \\
\text { n.a. }\end{array}$ \\
\hline
\end{tabular}



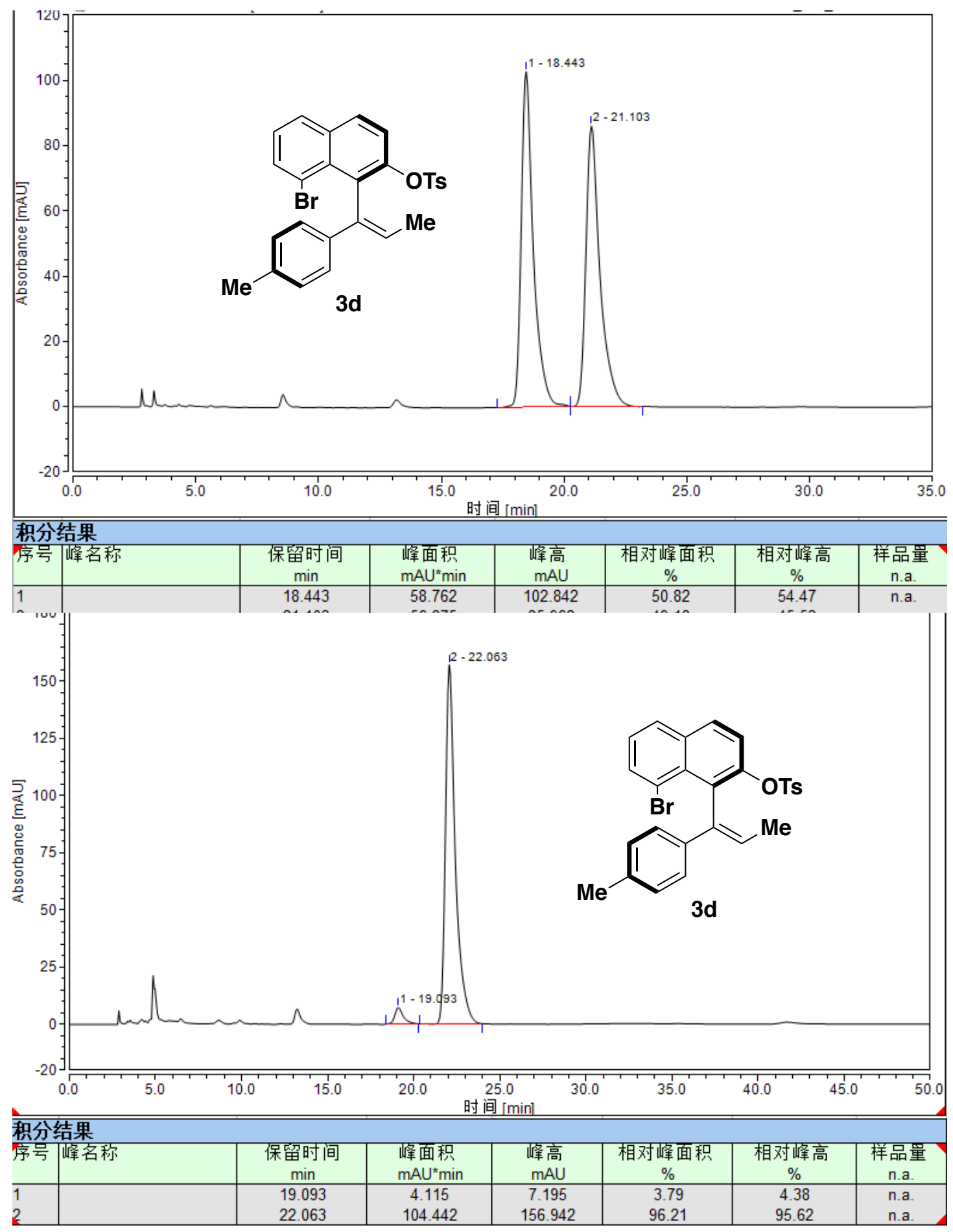

For comparison of Chinese and English of the HPLC data table.

\begin{tabular}{|c|c|c|c|c|c|c|c|}
\hline 序号 & $\begin{array}{l}\text { 峰名 } \\
\text { 称 }\end{array}$ & $\begin{array}{l}\text { 保留时间 } \\
\text { (min) }\end{array}$ & $\begin{array}{l}\text { 峰面积 } \\
\text { (mAU*min) }\end{array}$ & $\begin{array}{l}\text { 峰高 } \\
\text { (mAU) }\end{array}$ & $\begin{array}{l}\text { 相对峰面 } \\
\text { 积 (\%) }\end{array}$ & $\begin{array}{l}\text { 相对峰高 } \\
(\%)\end{array}$ & $\begin{array}{l}\text { 样品量 } \\
\text { n.a. }\end{array}$ \\
\hline $\begin{array}{l}\text { Entr } \\
\mathrm{y}\end{array}$ & $\begin{array}{l}\text { Peak } \\
\text { name }\end{array}$ & $\begin{array}{l}\text { Retention } \\
\text { time (min) }\end{array}$ & $\begin{array}{l}\text { Peak area } \\
\left(\mathrm{mAU}{ }^{*} \min \right)\end{array}$ & $\begin{array}{l}\text { Peak } \\
\text { height } \\
(\mathrm{mAU})\end{array}$ & $\begin{array}{l}\text { Relative } \\
\text { peak area } \\
(\%)\end{array}$ & $\begin{array}{l}\text { Relative } \\
\text { Peak } \\
\text { height (\%) }\end{array}$ & $\begin{array}{l}\text { Sample } \\
\text { volume } \\
\text { n.a. }\end{array}$ \\
\hline
\end{tabular}



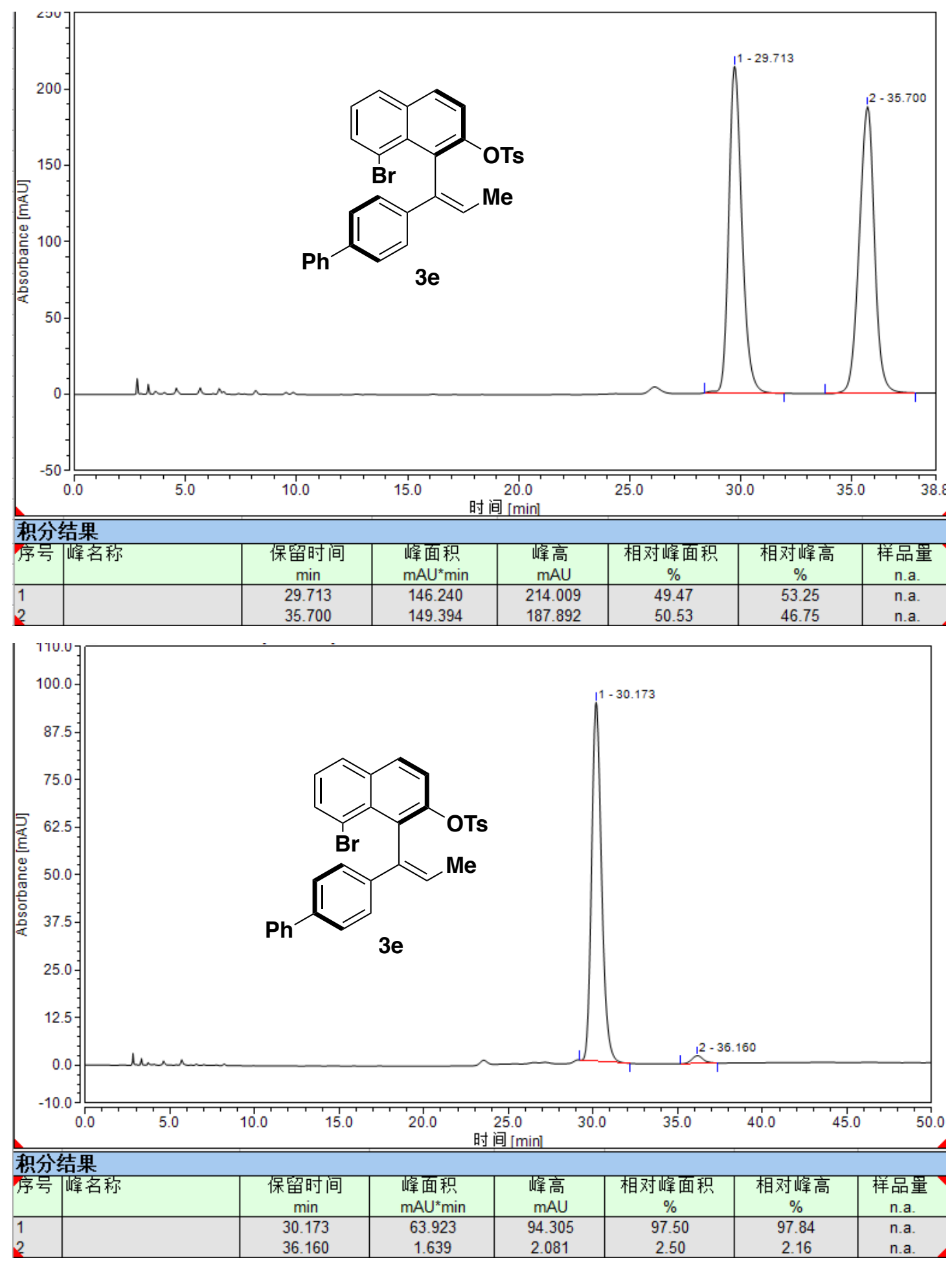

For comparison of Chinese and English of the HPLC data table.

\begin{tabular}{|l|l|l|l|l|l|l|l|}
\hline 序号 & $\begin{array}{l}\text { 峰名 } \\
\text { 称 }\end{array}$ & $\begin{array}{l}\text { 保留时间 } \\
(\mathrm{min})\end{array}$ & $\begin{array}{l}\text { 峰面积 } \\
(\mathrm{mAU} \text { *min) }\end{array}$ & $\begin{array}{l}\text { 峰高 } \\
(\mathrm{mAU})\end{array}$ & $\begin{array}{l}\text { 相对峰面 } \\
\text { 积 }(\%)\end{array}$ & $\begin{array}{l}\text { 相对峰高 } \\
(\%)\end{array}$ & $\begin{array}{l}\text { 样品量 } \\
\text { n.a. }\end{array}$ \\
\hline $\begin{array}{l}\text { Entr } \\
\mathrm{y}\end{array}$ & $\begin{array}{l}\text { Peak } \\
\text { name }\end{array}$ & $\begin{array}{l}\text { Retention } \\
\text { time (min) }\end{array}$ & $\begin{array}{l}\text { Peak area } \\
\left(\mathrm{mAU} \mathrm{U}^{*} \text { min }\right)\end{array}$ & $\begin{array}{l}\text { Peak } \\
\text { height } \\
(\mathrm{mAU})\end{array}$ & $\begin{array}{l}\text { Relative } \\
\text { peak area } \\
(\%)\end{array}$ & $\begin{array}{l}\text { Relative } \\
\text { Peak } \\
\text { height (\%) }\end{array}$ & $\begin{array}{l}\text { Sample } \\
\text { volume } \\
\text { n.a. }\end{array}$ \\
\hline
\end{tabular}



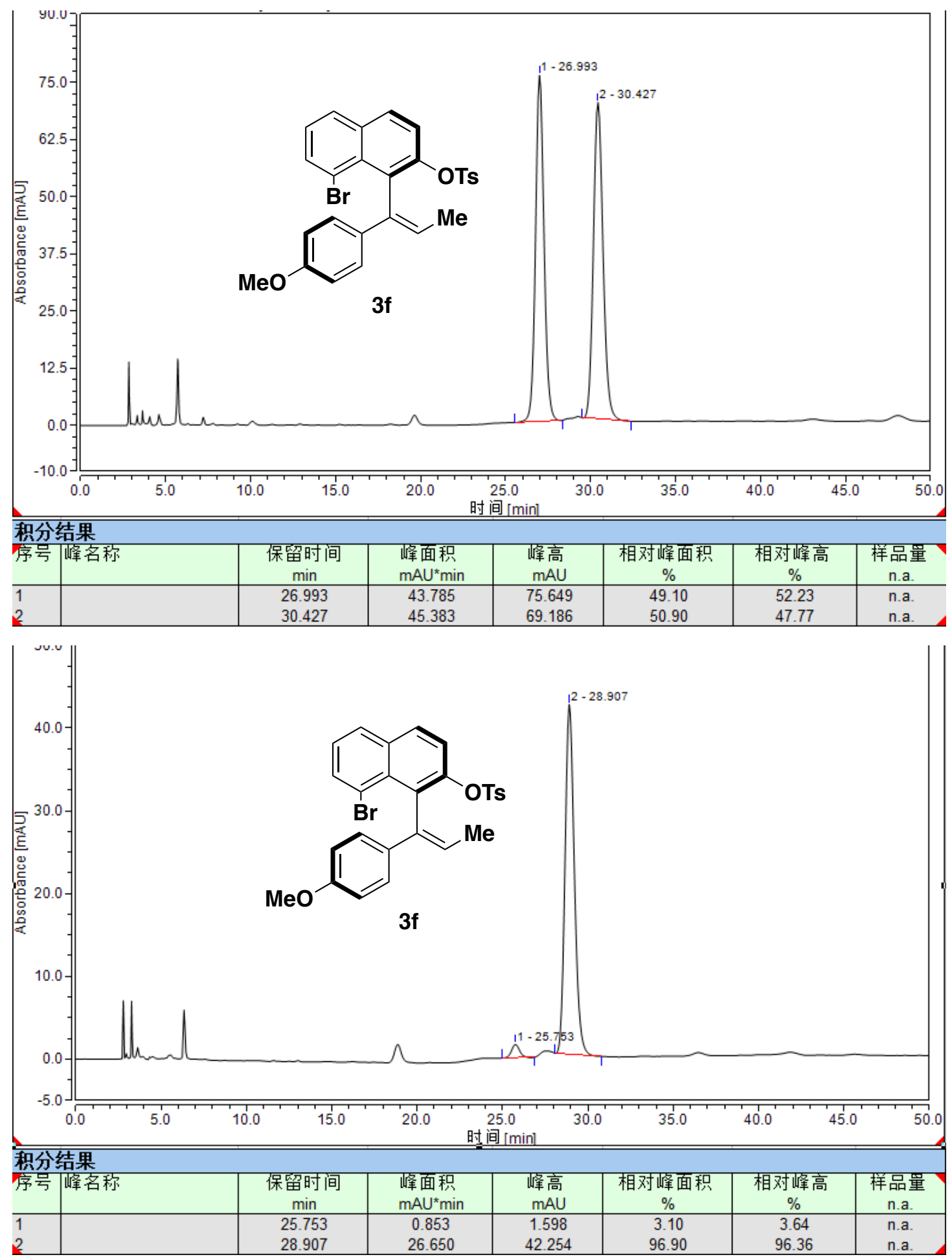

For comparison of Chinese and English of the HPLC data table.

\begin{tabular}{|l|l|l|l|l|l|l|l|}
\hline 序号 & $\begin{array}{l}\text { 峰名 } \\
\text { 称 }\end{array}$ & $\begin{array}{l}\text { 保留时间 } \\
(\mathrm{min})\end{array}$ & $\begin{array}{l}\text { 峰面积 } \\
(\mathrm{mAU} \text { *min) }\end{array}$ & $\begin{array}{l}\text { 峰高 } \\
(\mathrm{mAU})\end{array}$ & $\begin{array}{l}\text { 相对峰面 } \\
\text { 积 }(\%)\end{array}$ & $\begin{array}{l}\text { 相对峰高 } \\
(\%)\end{array}$ & $\begin{array}{l}\text { 样品量 } \\
\text { n.a. }\end{array}$ \\
\hline $\begin{array}{l}\text { Entr } \\
\mathrm{y}\end{array}$ & $\begin{array}{l}\text { Peak } \\
\text { name }\end{array}$ & $\begin{array}{l}\text { Retention } \\
\text { time (min) }\end{array}$ & $\begin{array}{l}\text { Peak area } \\
\left(\mathrm{mAU} \mathrm{U}^{*} \text { min }\right)\end{array}$ & $\begin{array}{l}\text { Peak } \\
\text { height } \\
(\mathrm{mAU})\end{array}$ & $\begin{array}{l}\text { Relative } \\
\text { peak area } \\
(\%)\end{array}$ & $\begin{array}{l}\text { Relative } \\
\text { Peak } \\
\text { height (\%) }\end{array}$ & $\begin{array}{l}\text { Sample } \\
\text { volume } \\
\text { n.a. }\end{array}$ \\
\hline
\end{tabular}



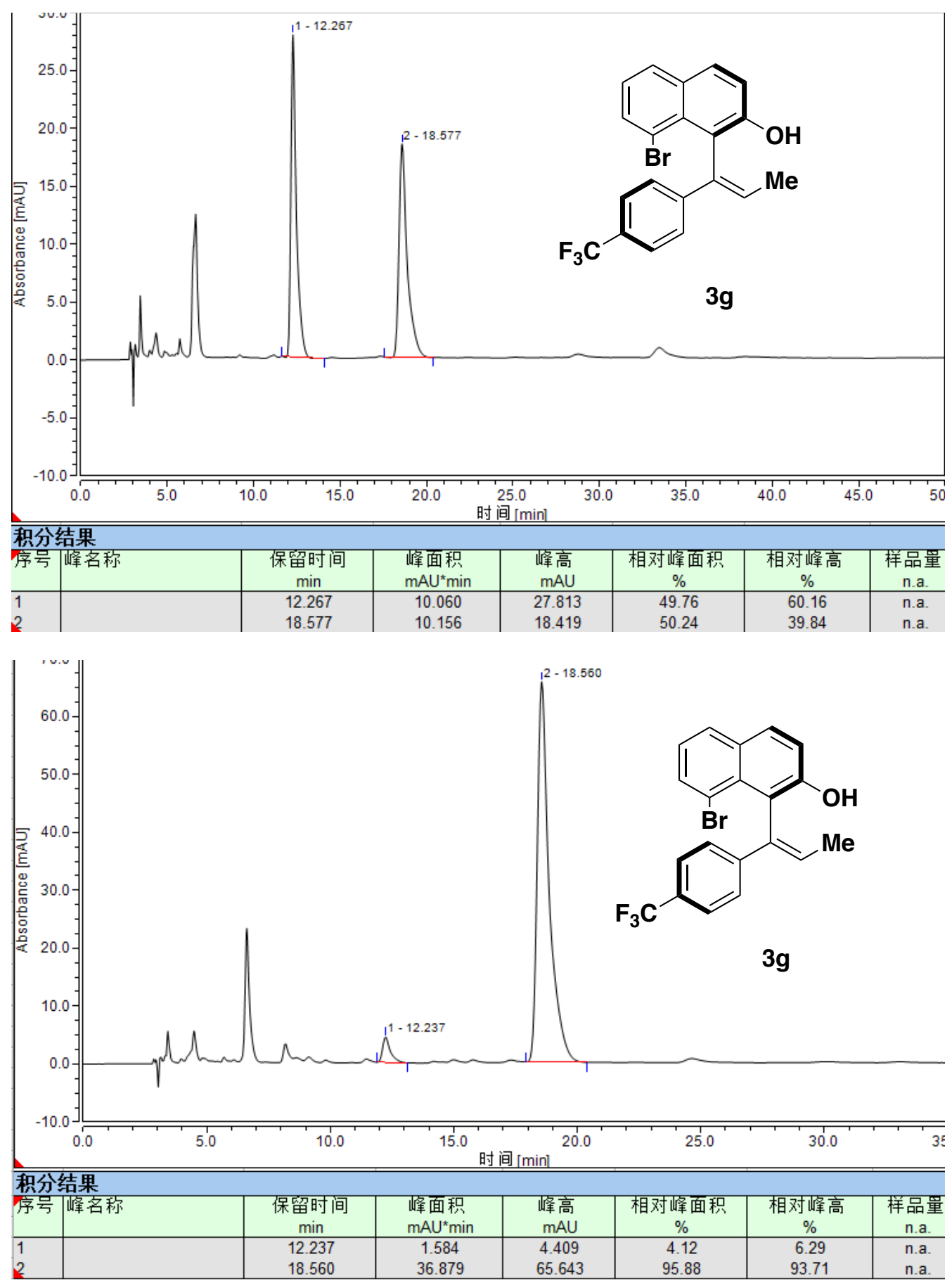

For comparison of Chinese and English of the HPLC data table.

\begin{tabular}{|l|l|l|l|l|l|l|l|}
\hline 序号 & $\begin{array}{l}\text { 峰名 } \\
\text { 称 }\end{array}$ & $\begin{array}{l}\text { 保留时间 } \\
(\mathrm{min})\end{array}$ & $\begin{array}{l}\text { 峰面积 } \\
(\mathrm{mAU} \text { *min) }\end{array}$ & $\begin{array}{l}\text { 峰高 } \\
(\mathrm{mAU})\end{array}$ & $\begin{array}{l}\text { 相对峰面 } \\
\text { 积 }(\%)\end{array}$ & $\begin{array}{l}\text { 相对峰高 } \\
(\%)\end{array}$ & $\begin{array}{l}\text { 样品量 } \\
\text { n.a. }\end{array}$ \\
\hline $\begin{array}{l}\text { Entr } \\
\mathrm{y}\end{array}$ & $\begin{array}{l}\text { Peak } \\
\text { name }\end{array}$ & $\begin{array}{l}\text { Retention } \\
\text { time (min) }\end{array}$ & $\begin{array}{l}\text { Peak area } \\
(\mathrm{mAU} * \mathrm{~min})\end{array}$ & $\begin{array}{l}\text { Peak } \\
\text { height } \\
(\mathrm{mAU})\end{array}$ & $\begin{array}{l}\text { Relative } \\
\text { peak area } \\
(\%)\end{array}$ & $\begin{array}{l}\text { Relative } \\
\text { Peak } \\
\text { height (\%) }\end{array}$ & $\begin{array}{l}\text { Sample } \\
\text { volume } \\
\text { n.a. }\end{array}$ \\
\hline
\end{tabular}



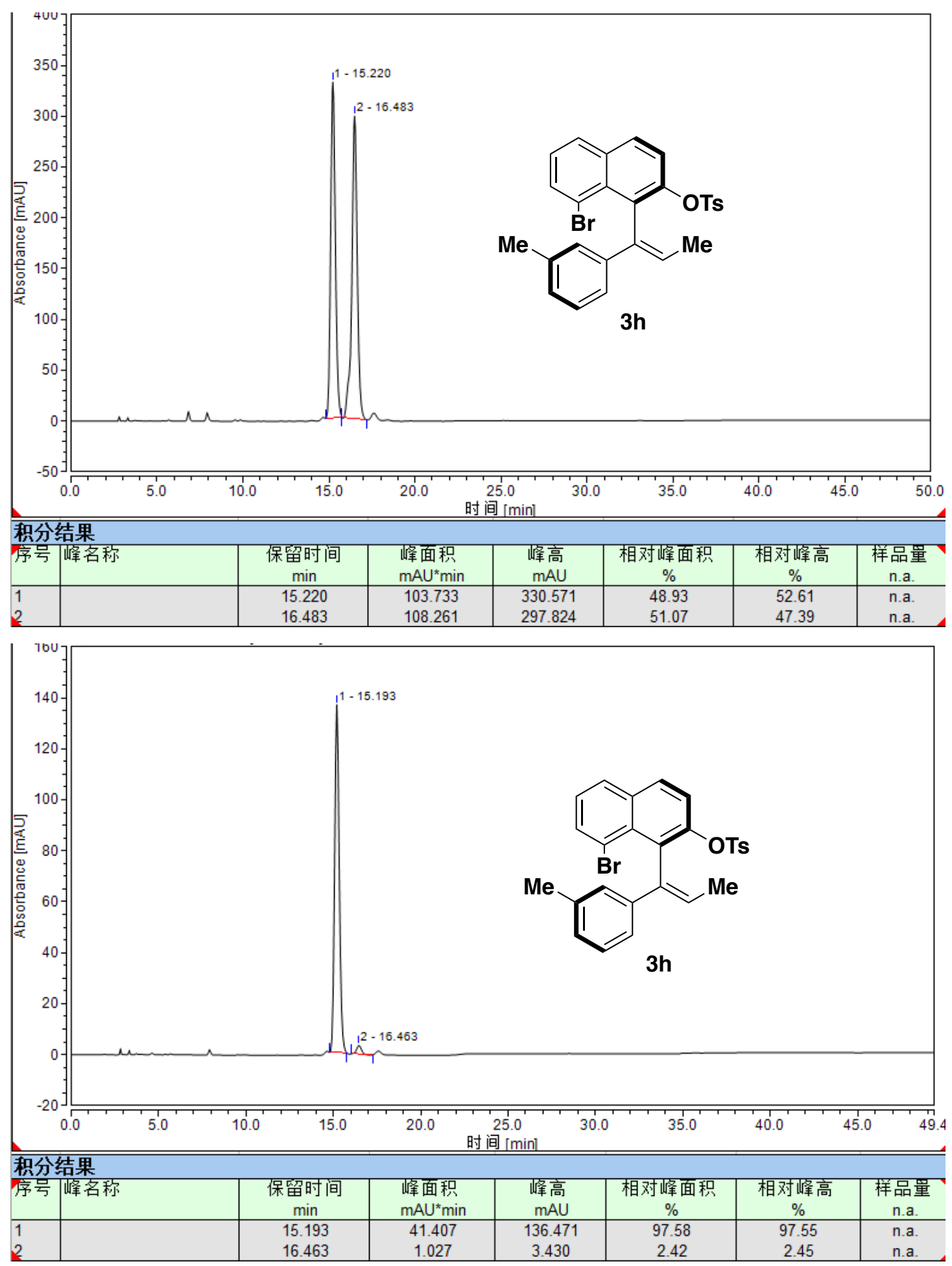

For comparison of Chinese and English of the HPLC data table.

\begin{tabular}{|l|l|l|l|l|l|l|l|}
\hline 序号 & $\begin{array}{l}\text { 峰名 } \\
\text { 称 }\end{array}$ & $\begin{array}{l}\text { 保留时间 } \\
(\mathrm{min})\end{array}$ & $\begin{array}{l}\text { 峰面积 } \\
(\mathrm{mAU} \text { *min) }\end{array}$ & $\begin{array}{l}\text { 峰高 } \\
(\mathrm{mAU})\end{array}$ & $\begin{array}{l}\text { 相对峰面 } \\
\text { 积 }(\%)\end{array}$ & $\begin{array}{l}\text { 相对峰高 } \\
(\%)\end{array}$ & $\begin{array}{l}\text { 样品量 } \\
\text { n.a. }\end{array}$ \\
\hline $\begin{array}{l}\text { Entr } \\
\mathrm{y}\end{array}$ & $\begin{array}{l}\text { Peak } \\
\text { name }\end{array}$ & $\begin{array}{l}\text { Retention } \\
\text { time (min) }\end{array}$ & $\begin{array}{l}\text { Peak area } \\
\left(\mathrm{mAU} \mathrm{U}^{*} \text { min }\right)\end{array}$ & $\begin{array}{l}\text { Peak } \\
\text { height } \\
(\mathrm{mAU})\end{array}$ & $\begin{array}{l}\text { Relative } \\
\text { peak area } \\
(\%)\end{array}$ & $\begin{array}{l}\text { Relative } \\
\text { Peak } \\
\text { height (\%) }\end{array}$ & $\begin{array}{l}\text { Sample } \\
\text { volume } \\
\text { n.a. }\end{array}$ \\
\hline
\end{tabular}



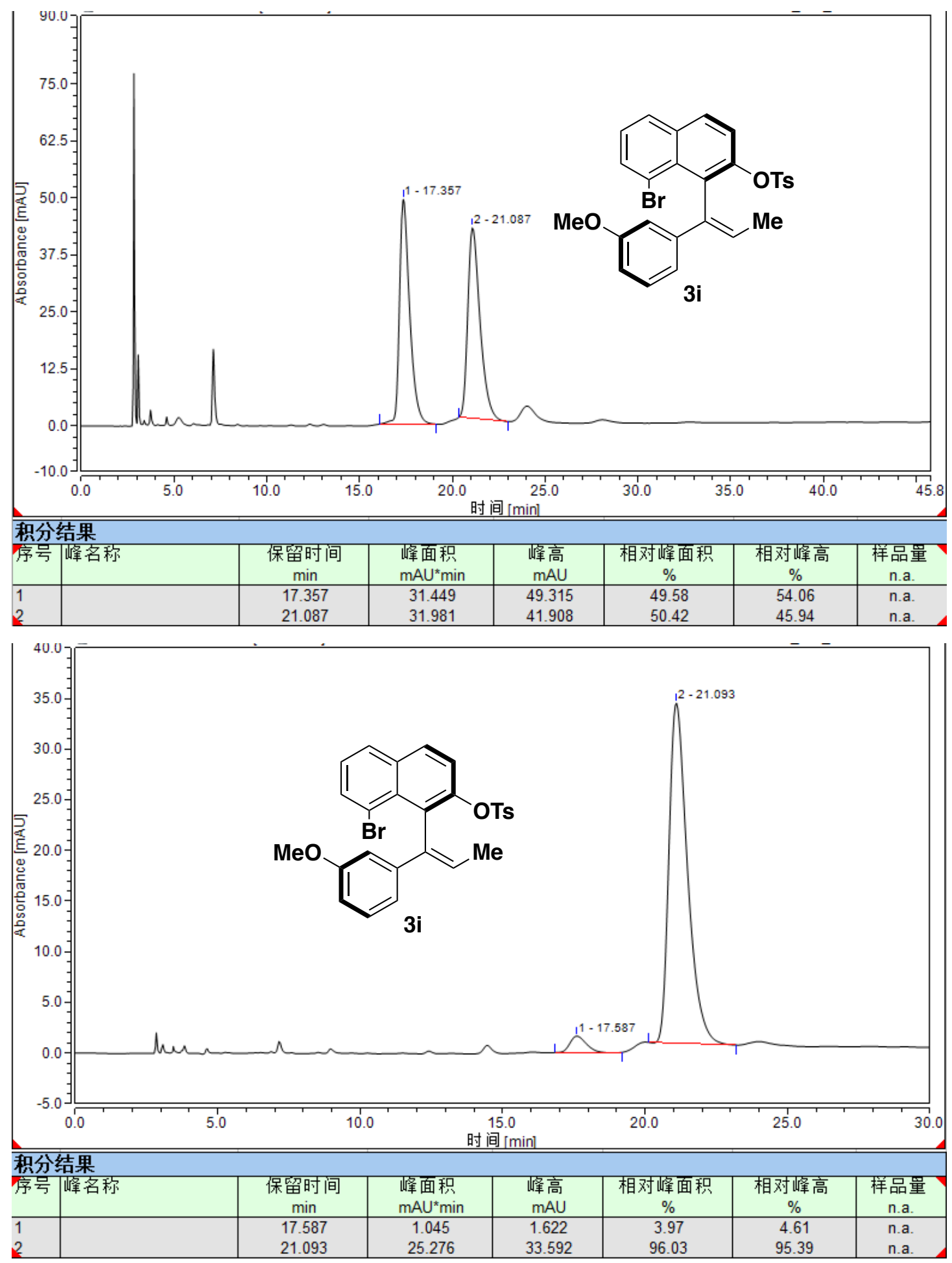

For comparison of Chinese and English of the HPLC data table.

\begin{tabular}{|l|l|l|l|l|l|l|l|}
\hline 序号 & $\begin{array}{l}\text { 峰名 } \\
\text { 称 }\end{array}$ & $\begin{array}{l}\text { 保留时间 } \\
(\mathrm{min})\end{array}$ & $\begin{array}{l}\text { 峰面积 } \\
(\mathrm{mAU} \text { *min) }\end{array}$ & $\begin{array}{l}\text { 峰高 } \\
(\mathrm{mAU})\end{array}$ & $\begin{array}{l}\text { 相对峰面 } \\
\text { 积 }(\%)\end{array}$ & $\begin{array}{l}\text { 相对峰高 } \\
(\%)\end{array}$ & $\begin{array}{l}\text { 样品量 } \\
\text { n.a. }\end{array}$ \\
\hline $\begin{array}{l}\text { Entr } \\
\mathrm{y}\end{array}$ & $\begin{array}{l}\text { Peak } \\
\text { name }\end{array}$ & $\begin{array}{l}\text { Retention } \\
\text { time (min) }\end{array}$ & $\begin{array}{l}\text { Peak area } \\
\left(\mathrm{mAU} \mathrm{U}^{*} \text { min }\right)\end{array}$ & $\begin{array}{l}\text { Peak } \\
\text { height } \\
(\mathrm{mAU})\end{array}$ & $\begin{array}{l}\text { Relative } \\
\text { peak area } \\
(\%)\end{array}$ & $\begin{array}{l}\text { Relative } \\
\text { Peak } \\
\text { height (\%) }\end{array}$ & $\begin{array}{l}\text { Sample } \\
\text { volume } \\
\text { n.a. }\end{array}$ \\
\hline
\end{tabular}




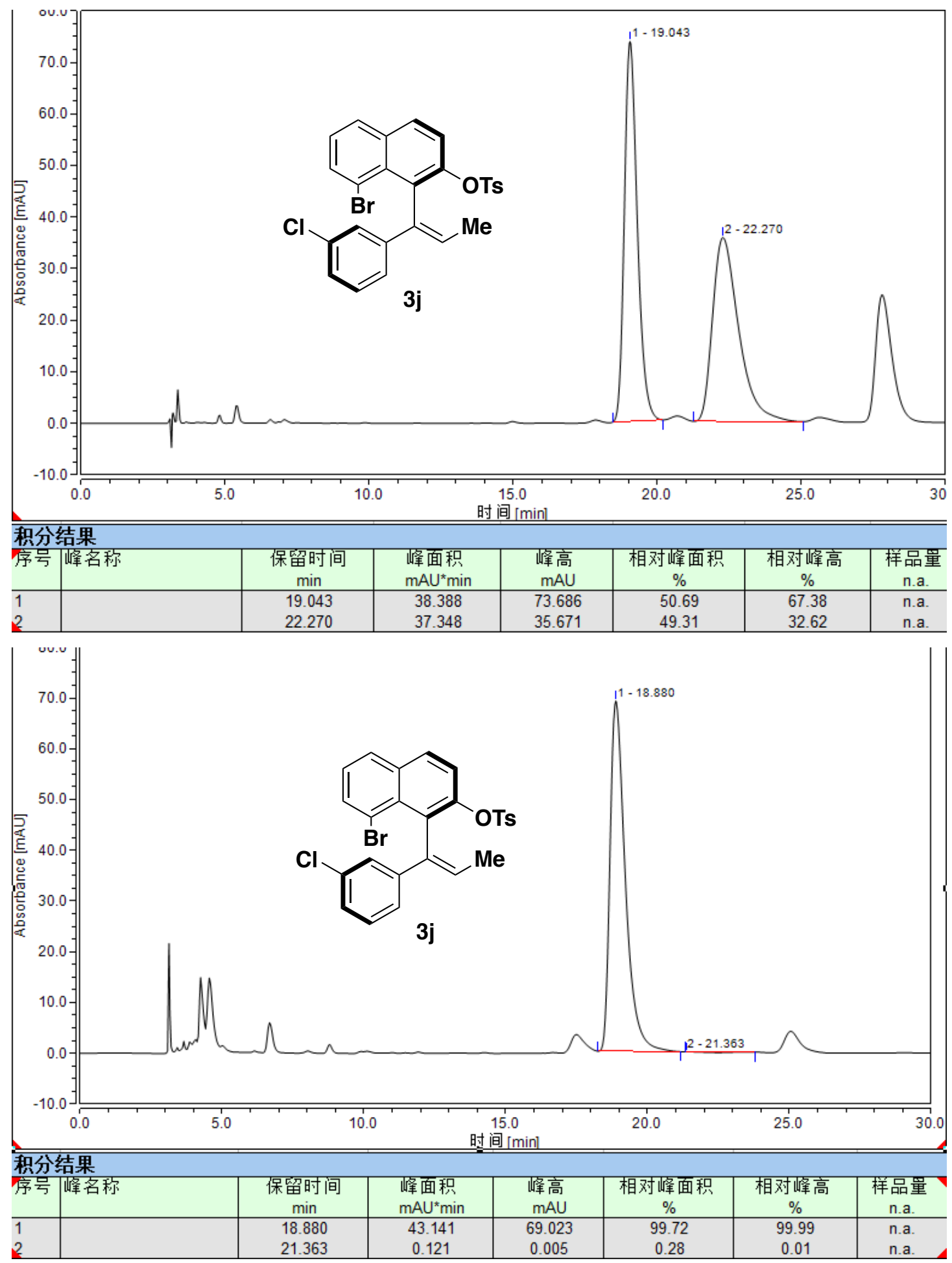

For comparison of Chinese and English of the HPLC data table.

\begin{tabular}{|l|l|l|l|l|l|l|l|}
\hline 序号 & $\begin{array}{l}\text { 峰名 } \\
\text { 称 }\end{array}$ & $\begin{array}{l}\text { 保留时间 } \\
(\mathrm{min})\end{array}$ & $\begin{array}{l}\text { 峰面积 } \\
(\mathrm{mAU} \text { *min) }\end{array}$ & $\begin{array}{l}\text { 峰高 } \\
(\mathrm{mAU})\end{array}$ & $\begin{array}{l}\text { 相对峰面 } \\
\text { 积 }(\%)\end{array}$ & $\begin{array}{l}\text { 相对峰高 } \\
(\%)\end{array}$ & $\begin{array}{l}\text { 样品量 } \\
\text { n.a. }\end{array}$ \\
\hline $\begin{array}{l}\text { Entr } \\
\mathrm{y}\end{array}$ & $\begin{array}{l}\text { Peak } \\
\text { name }\end{array}$ & $\begin{array}{l}\text { Retention } \\
\text { time (min) }\end{array}$ & $\begin{array}{l}\text { Peak area } \\
(\mathrm{mAU} \text { *min) }\end{array}$ & $\begin{array}{l}\text { Peak } \\
\text { height } \\
(\mathrm{mAU})\end{array}$ & $\begin{array}{l}\text { Relative } \\
\text { peak area } \\
(\%)\end{array}$ & $\begin{array}{l}\text { Relative } \\
\text { Peak } \\
\text { height (\%) }\end{array}$ & $\begin{array}{l}\text { Sample } \\
\text { volume } \\
\text { n.a. }\end{array}$ \\
\hline
\end{tabular}



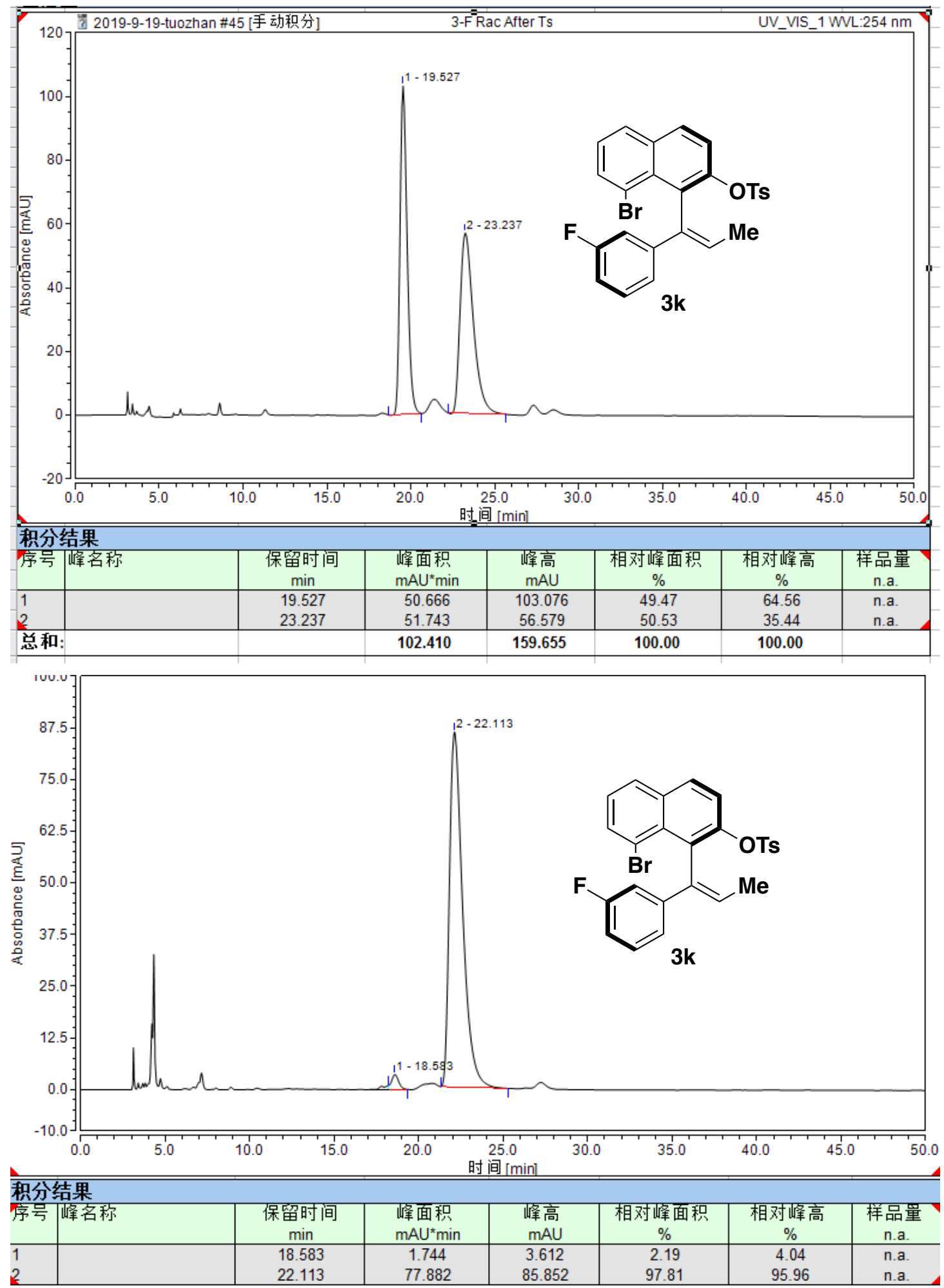

For comparison of Chinese and English of the HPLC data table.

\begin{tabular}{|l|l|l|l|l|l|l|l|}
\hline 序号 & $\begin{array}{l}\text { 峰名 } \\
\text { 称 }\end{array}$ & $\begin{array}{l}\text { 保留时间 } \\
(\mathrm{min})\end{array}$ & $\begin{array}{l}\text { 峰面积 } \\
(\mathrm{mAU} \text { *min) }\end{array}$ & $\begin{array}{l}\text { 峰高 } \\
(\mathrm{mAU})\end{array}$ & $\begin{array}{l}\text { 相对峰面 } \\
\text { 积 }(\%)\end{array}$ & $\begin{array}{l}\text { 相对峰高 } \\
(\%)\end{array}$ & $\begin{array}{l}\text { 样品量 } \\
\text { n.a. }\end{array}$ \\
\hline $\begin{array}{l}\text { Entr } \\
\mathrm{y}\end{array}$ & $\begin{array}{l}\text { Peak } \\
\text { name }\end{array}$ & $\begin{array}{l}\text { Retention } \\
\text { time (min) }\end{array}$ & $\begin{array}{l}\text { Peak area } \\
(\mathrm{mAU} * \mathrm{~min})\end{array}$ & $\begin{array}{l}\text { Peak } \\
\text { height } \\
(\mathrm{mAU})\end{array}$ & $\begin{array}{l}\text { Relative } \\
\text { peak area } \\
(\%)\end{array}$ & $\begin{array}{l}\text { Relative } \\
\text { Peak } \\
\text { height (\%) }\end{array}$ & $\begin{array}{l}\text { Sample } \\
\text { volume } \\
\text { n.a. }\end{array}$ \\
\hline
\end{tabular}




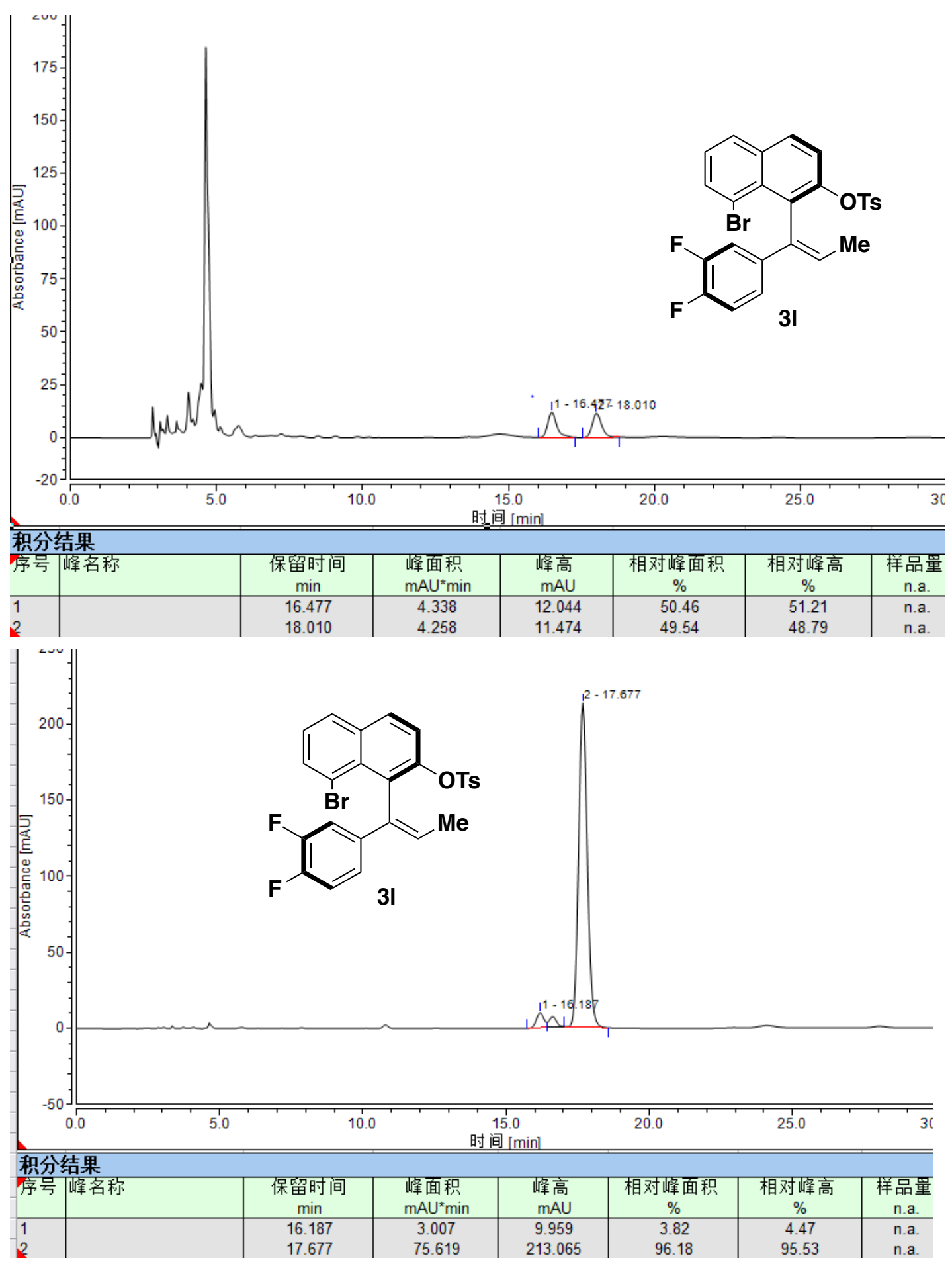

For comparison of Chinese and English of the HPLC data table.

\begin{tabular}{|c|c|c|c|c|c|c|c|}
\hline 序号 & $\begin{array}{l}\text { 峰名 } \\
\text { 称 } \\
\end{array}$ & $\begin{array}{l}\text { 保留时间 } \\
\text { (min) }\end{array}$ & $\begin{array}{l}\text { 峰面积 } \\
\left(\mathrm{mAU}{ }^{*} \min \right)\end{array}$ & $\begin{array}{l}\text { 峰高 } \\
(\mathrm{mAU})\end{array}$ & $\begin{array}{l}\text { 相对峰面 } \\
\text { 积 }(\%)\end{array}$ & $\begin{array}{l}\text { 相对峰高 } \\
(\%)\end{array}$ & $\begin{array}{l}\text { 样品量 } \\
\text { n.a. }\end{array}$ \\
\hline $\begin{array}{l}\text { Entr } \\
\mathrm{y}\end{array}$ & $\begin{array}{l}\text { Peak } \\
\text { name }\end{array}$ & $\begin{array}{l}\text { Retention } \\
\text { time (min) }\end{array}$ & $\begin{array}{l}\text { Peak area } \\
\left(\mathrm{mAU}{ }^{*} \min \right)\end{array}$ & $\begin{array}{l}\text { Peak } \\
\text { height } \\
(\mathrm{mAU})\end{array}$ & $\begin{array}{l}\text { Relative } \\
\text { peak area } \\
(\%)\end{array}$ & $\begin{array}{l}\text { Relative } \\
\text { Peak } \\
\text { height (\%) }\end{array}$ & $\begin{array}{l}\text { Sample } \\
\text { volume } \\
\text { n.a. }\end{array}$ \\
\hline
\end{tabular}



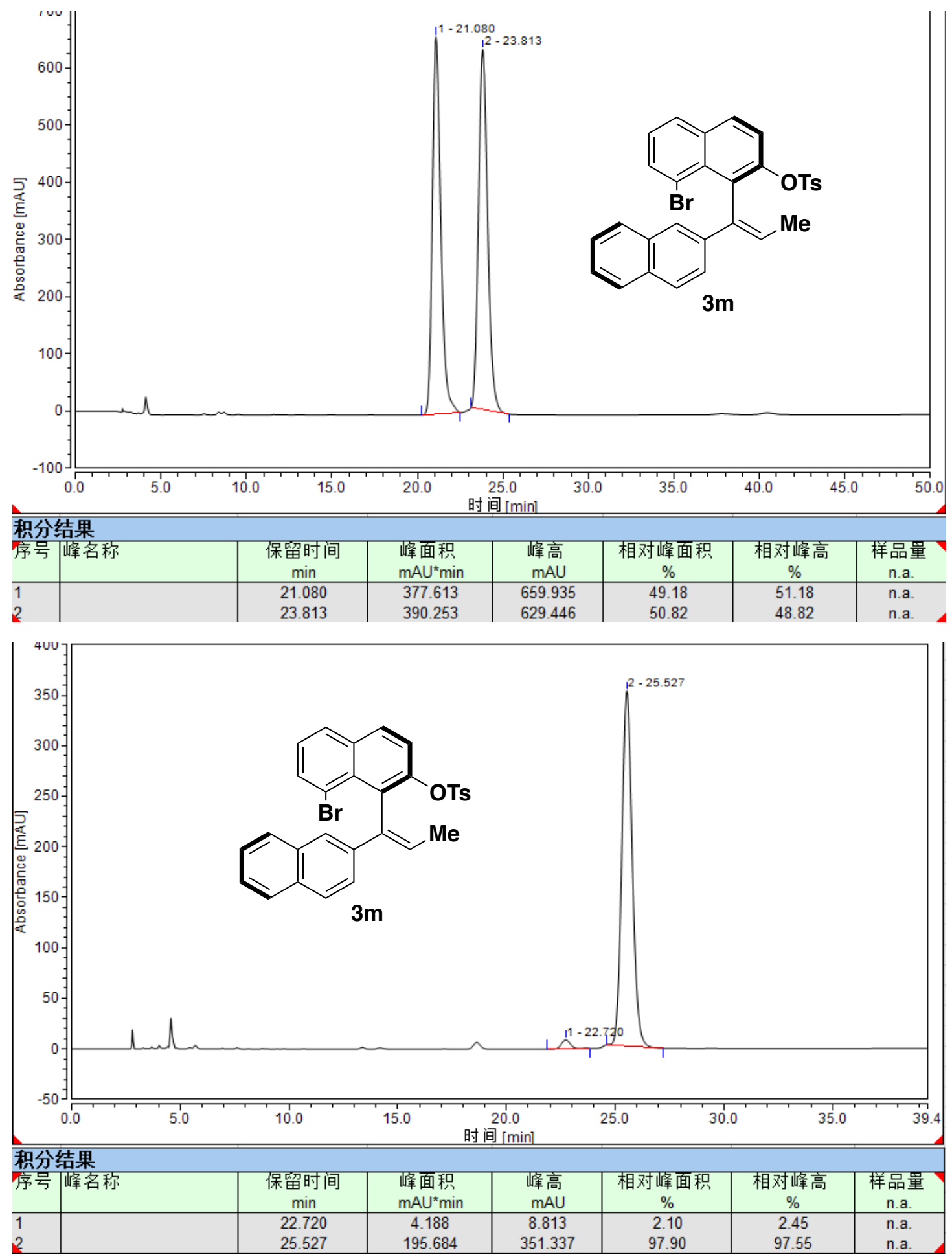

For comparison of Chinese and English of the HPLC data table.

\begin{tabular}{|l|l|l|l|l|l|l|l|}
\hline 序号 & $\begin{array}{l}\text { 峰名 } \\
\text { 称 }\end{array}$ & $\begin{array}{l}\text { 保留时间 } \\
(\mathrm{min})\end{array}$ & $\begin{array}{l}\text { 峰面积 } \\
(\mathrm{mAU} \text { *min) }\end{array}$ & $\begin{array}{l}\text { 峰高 } \\
(\mathrm{mAU})\end{array}$ & $\begin{array}{l}\text { 相对峰面 } \\
\text { 积 }(\%)\end{array}$ & $\begin{array}{l}\text { 相对峰高 } \\
(\%)\end{array}$ & $\begin{array}{l}\text { 样品量 } \\
\text { n.a. }\end{array}$ \\
\hline $\begin{array}{l}\text { Entr } \\
\mathrm{y}\end{array}$ & $\begin{array}{l}\text { Peak } \\
\text { name }\end{array}$ & $\begin{array}{l}\text { Retention } \\
\text { time (min) }\end{array}$ & $\begin{array}{l}\text { Peak area } \\
\left(\mathrm{mAU} \mathrm{U}^{*} \text { min }\right)\end{array}$ & $\begin{array}{l}\text { Peak } \\
\text { height } \\
(\mathrm{mAU})\end{array}$ & $\begin{array}{l}\text { Relative } \\
\text { peak area } \\
(\%)\end{array}$ & $\begin{array}{l}\text { Relative } \\
\text { Peak } \\
\text { height (\%) }\end{array}$ & $\begin{array}{l}\text { Sample } \\
\text { volume } \\
\text { n.a. }\end{array}$ \\
\hline
\end{tabular}



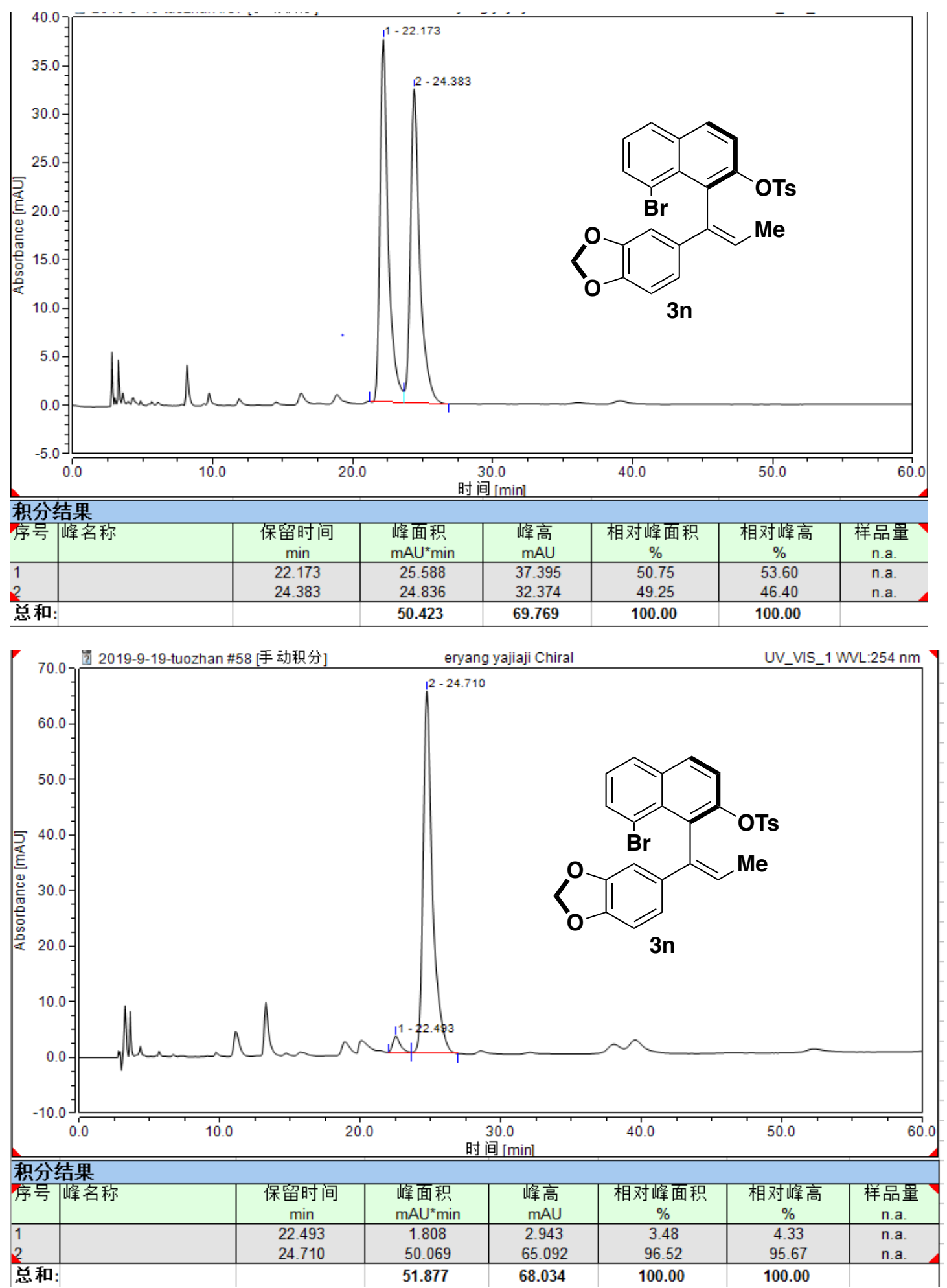

For comparison of Chinese and English of the HPLC data table.

\begin{tabular}{|l|l|l|l|l|l|l|l|}
\hline 序号 & $\begin{array}{l}\text { 峰名 } \\
\text { 称 }\end{array}$ & $\begin{array}{l}\text { 保留时间 } \\
(\mathrm{min})\end{array}$ & $\begin{array}{l}\text { 峰面积 } \\
(\mathrm{mAU} \text { *min) }\end{array}$ & $\begin{array}{l}\text { 峰高 } \\
(\mathrm{mAU})\end{array}$ & $\begin{array}{l}\text { 相对峰面 } \\
\text { 积 }\end{array}$ & $\begin{array}{l}\text { 相对 }) \\
(\%)\end{array}$ & $\begin{array}{l}\text { 峰品量 } \\
\text { n.a. }\end{array}$ \\
\hline $\begin{array}{l}\text { Entr } \\
\text { y }\end{array}$ & $\begin{array}{l}\text { Peak } \\
\text { name }\end{array}$ & $\begin{array}{l}\text { Retention } \\
\text { time (min) }\end{array}$ & $\begin{array}{l}\text { Peak area } \\
(\mathrm{mAU} \text { *min) }\end{array}$ & $\begin{array}{l}\text { Peak } \\
\text { height } \\
(\mathrm{mAU})\end{array}$ & $\begin{array}{l}\text { Relative } \\
\text { peak area } \\
(\%)\end{array}$ & $\begin{array}{l}\text { Relative } \\
\text { Peak } \\
\text { height (\%) }\end{array}$ & $\begin{array}{l}\text { Sample } \\
\text { volume } \\
\text { n.a. }\end{array}$ \\
\hline
\end{tabular}



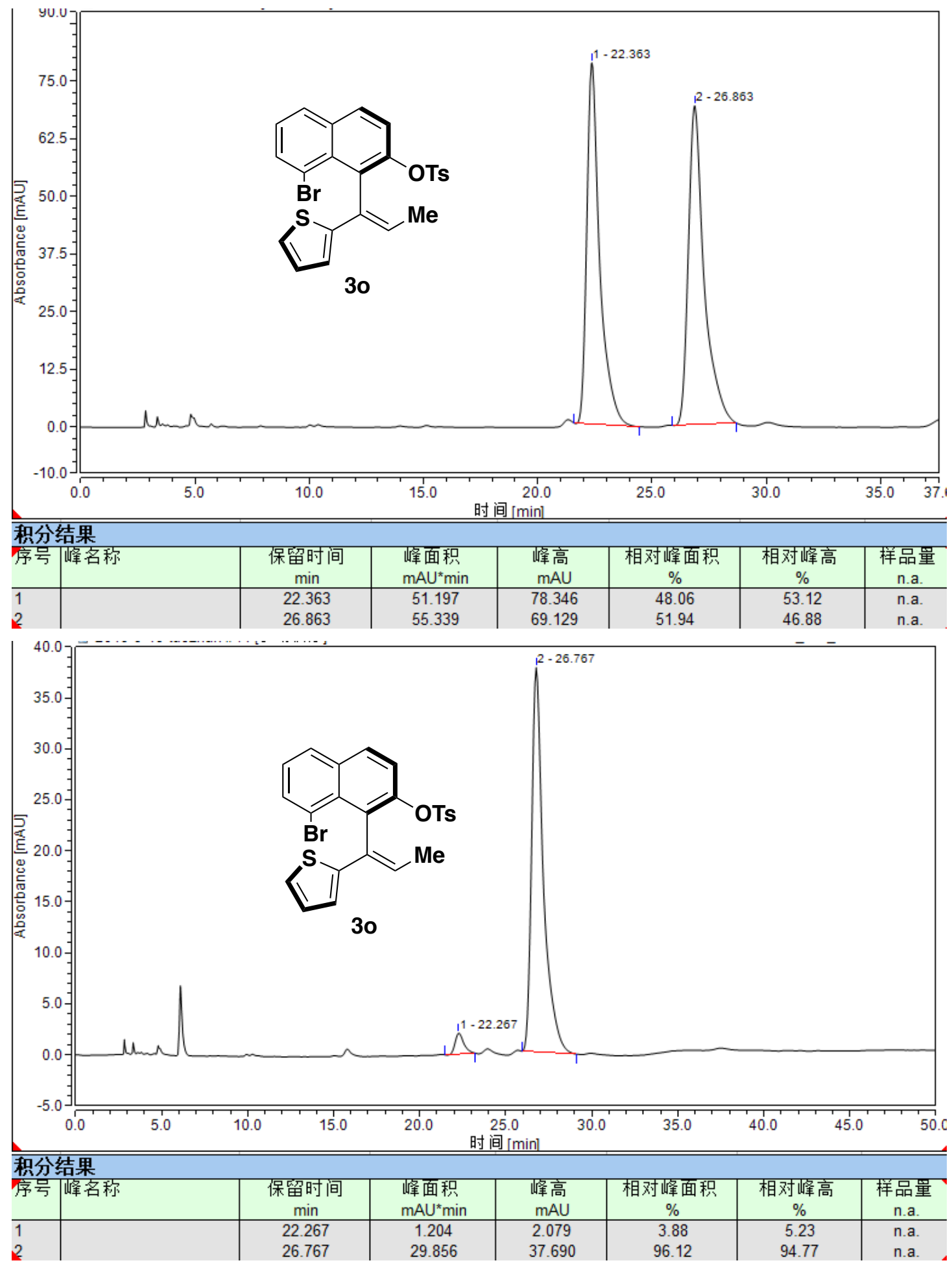

For comparison of Chinese and English of the HPLC data table.

\begin{tabular}{|l|l|l|l|l|l|l|l|}
\hline 序号 & $\begin{array}{l}\text { 峰名 } \\
\text { 称 }\end{array}$ & $\begin{array}{l}\text { 保留时间 } \\
(\mathrm{min})\end{array}$ & $\begin{array}{l}\text { 峰面积 } \\
(\mathrm{mAU} \text { *min) }\end{array}$ & $\begin{array}{l}\text { 峰高 } \\
(\mathrm{mAU})\end{array}$ & $\begin{array}{l}\text { 相对峰面 } \\
\text { 积 }(\%)\end{array}$ & $\begin{array}{l}\text { 相对峰高 } \\
(\%)\end{array}$ & $\begin{array}{l}\text { 样品量 } \\
\text { n.a. }\end{array}$ \\
\hline $\begin{array}{l}\text { Entr } \\
\mathrm{y}\end{array}$ & $\begin{array}{l}\text { Peak } \\
\text { name }\end{array}$ & $\begin{array}{l}\text { Retention } \\
\text { time (min) }\end{array}$ & $\begin{array}{l}\text { Peak area } \\
\left(\mathrm{mAU} \mathrm{U}^{*} \text { min }\right)\end{array}$ & $\begin{array}{l}\text { Peak } \\
\text { height } \\
(\mathrm{mAU})\end{array}$ & $\begin{array}{l}\text { Relative } \\
\text { peak area } \\
(\%)\end{array}$ & $\begin{array}{l}\text { Relative } \\
\text { Peak } \\
\text { height (\%) }\end{array}$ & $\begin{array}{l}\text { Sample } \\
\text { volume } \\
\text { n.a. }\end{array}$ \\
\hline
\end{tabular}



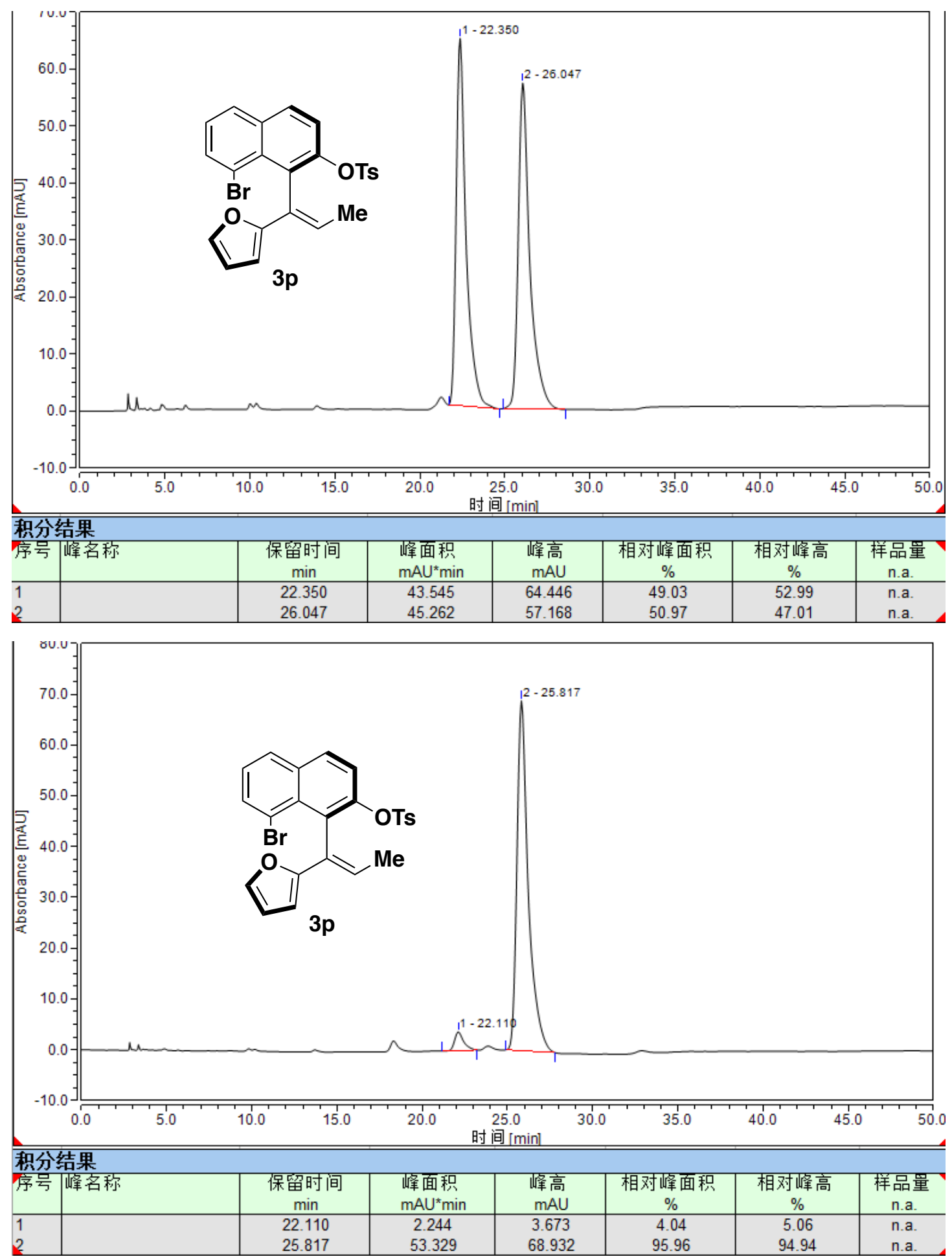

For comparison of Chinese and English of the HPLC data table.

\begin{tabular}{|l|l|l|l|l|l|l|l|}
\hline 序号 & $\begin{array}{l}\text { 峰名 } \\
\text { 称 }\end{array}$ & $\begin{array}{l}\text { 保留时间 } \\
(\mathrm{min})\end{array}$ & $\begin{array}{l}\text { 峰面积 } \\
(\mathrm{mAU} \text { *min) }\end{array}$ & $\begin{array}{l}\text { 峰高 } \\
(\mathrm{mAU})\end{array}$ & $\begin{array}{l}\text { 相对峰面 } \\
\text { 积 }(\%)\end{array}$ & $\begin{array}{l}\text { 相对峰高 } \\
(\%)\end{array}$ & $\begin{array}{l}\text { 样品量 } \\
\text { n.a. }\end{array}$ \\
\hline $\begin{array}{l}\text { Entr } \\
\mathrm{y}\end{array}$ & $\begin{array}{l}\text { Peak } \\
\text { name }\end{array}$ & $\begin{array}{l}\text { Retention } \\
\text { time (min) }\end{array}$ & $\begin{array}{l}\text { Peak area } \\
\left(\mathrm{mAU} \mathrm{U}^{*} \text { min }\right)\end{array}$ & $\begin{array}{l}\text { Peak } \\
\text { height } \\
(\mathrm{mAU})\end{array}$ & $\begin{array}{l}\text { Relative } \\
\text { peak area } \\
(\%)\end{array}$ & $\begin{array}{l}\text { Relative } \\
\text { Peak } \\
\text { height (\%) }\end{array}$ & $\begin{array}{l}\text { Sample } \\
\text { volume } \\
\text { n.a. }\end{array}$ \\
\hline
\end{tabular}



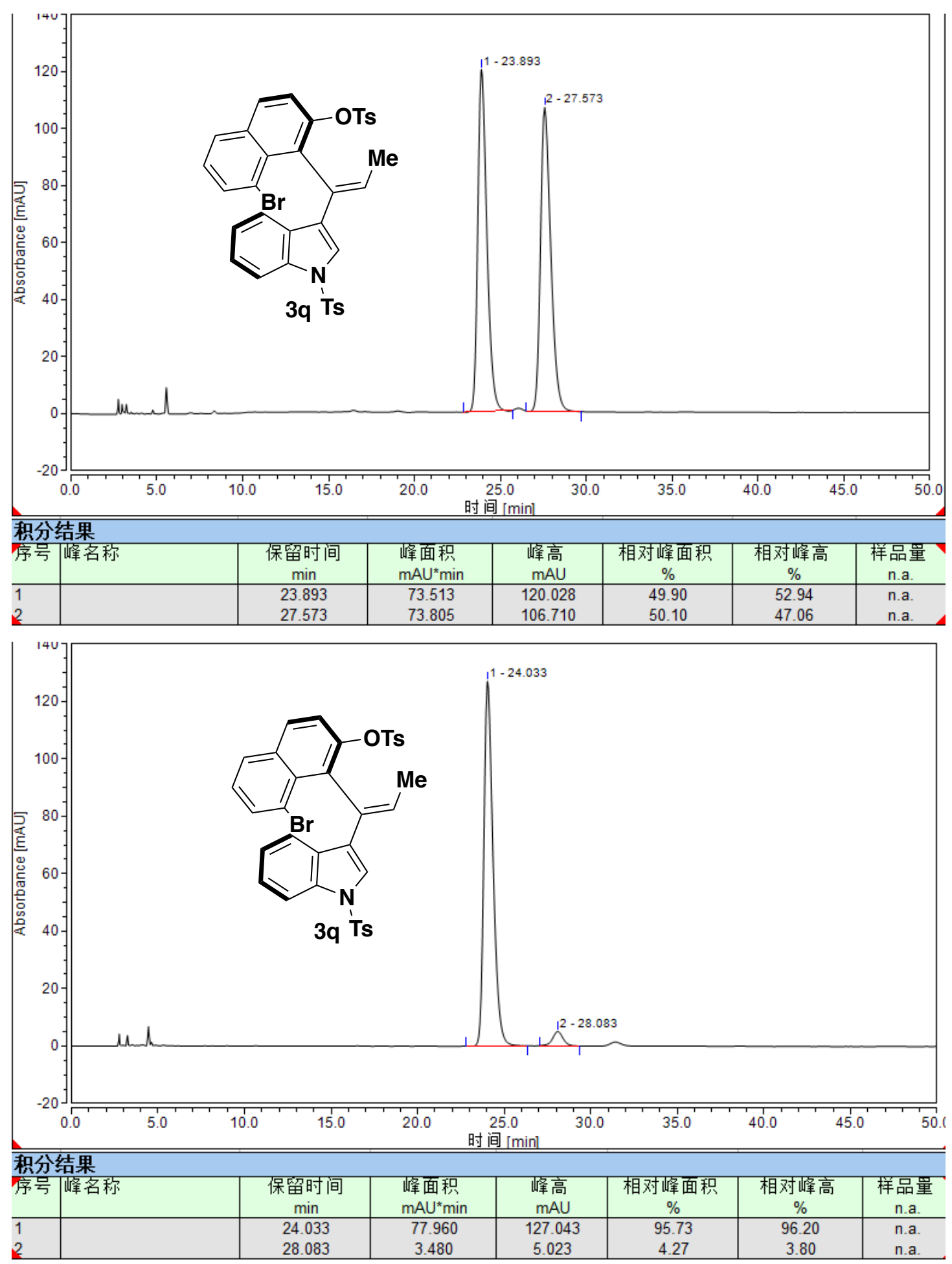

For comparison of Chinese and English of the HPLC data table.

\begin{tabular}{|l|l|l|l|l|l|l|l|}
\hline 序号 & $\begin{array}{l}\text { 峰名 } \\
\text { 称 }\end{array}$ & $\begin{array}{l}\text { 保留时间 } \\
(\mathrm{min})\end{array}$ & $\begin{array}{l}\text { 峰面积 } \\
(\mathrm{mAU} \text { *min) }\end{array}$ & $\begin{array}{l}\text { 峰高 } \\
(\mathrm{mAU})\end{array}$ & $\begin{array}{l}\text { 相对峰面 } \\
\text { 积 }(\%)\end{array}$ & $\begin{array}{l}\text { 相对峰高 } \\
(\%)\end{array}$ & $\begin{array}{l}\text { 样品量 } \\
\text { n.a. }\end{array}$ \\
\hline $\begin{array}{l}\text { Entr } \\
\mathrm{y}\end{array}$ & $\begin{array}{l}\text { Peak } \\
\text { name }\end{array}$ & $\begin{array}{l}\text { Retention } \\
\text { time (min) }\end{array}$ & $\begin{array}{l}\text { Peak area } \\
\left(\mathrm{mAU} \mathrm{U}^{*} \text { min }\right)\end{array}$ & $\begin{array}{l}\text { Peak } \\
\text { height } \\
(\mathrm{mAU})\end{array}$ & $\begin{array}{l}\text { Relative } \\
\text { peak area } \\
(\%)\end{array}$ & $\begin{array}{l}\text { Relative } \\
\text { Peak } \\
\text { height (\%) }\end{array}$ & $\begin{array}{l}\text { Sample } \\
\text { volume } \\
\text { n.a. }\end{array}$ \\
\hline
\end{tabular}



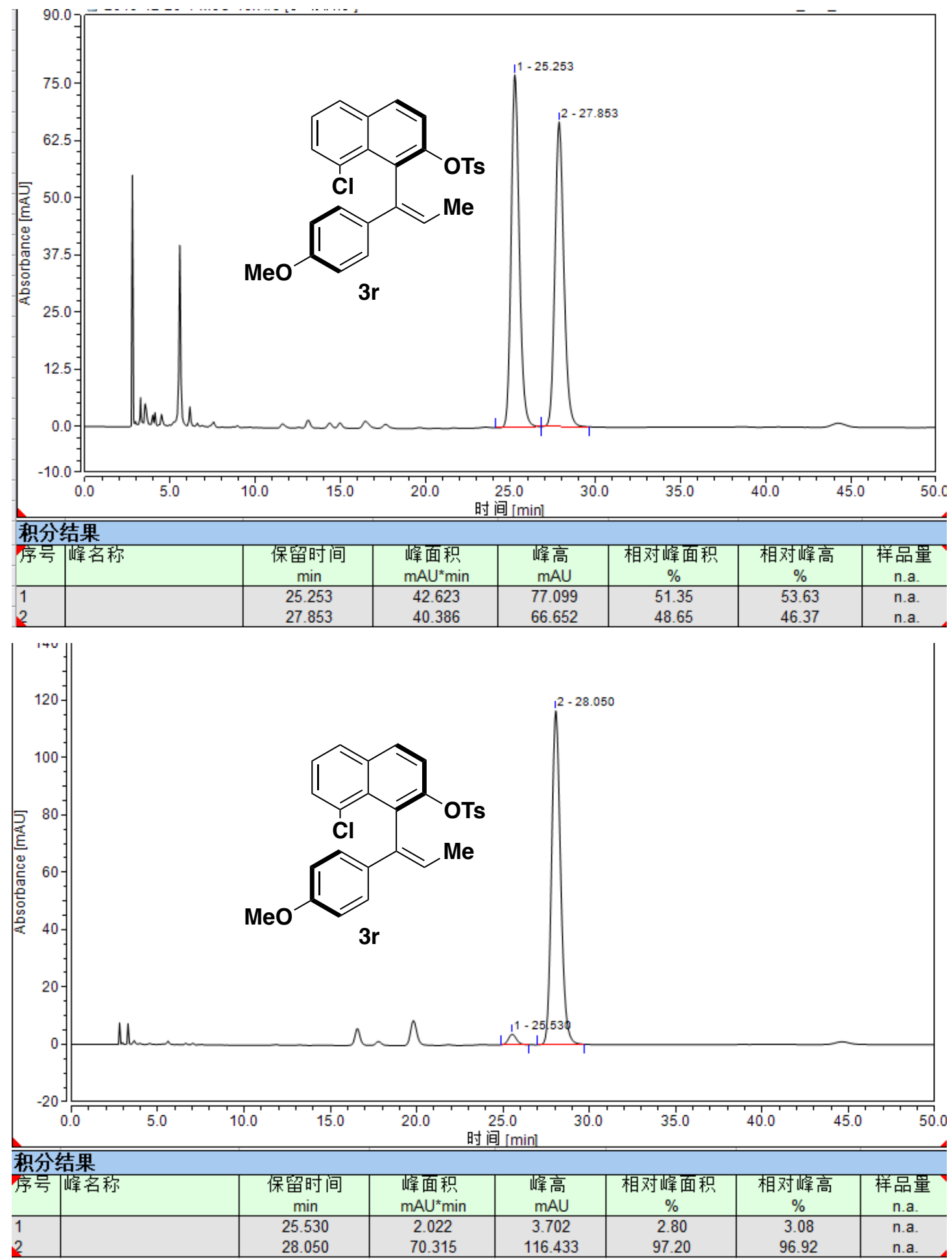

For comparison of Chinese and English of the HPLC data table.

\begin{tabular}{|l|l|l|l|l|l|l|l|}
\hline 序号 & $\begin{array}{l}\text { 峰名 } \\
\text { 称 }\end{array}$ & $\begin{array}{l}\text { 保留时间 } \\
(\mathrm{min})\end{array}$ & $\begin{array}{l}\text { 峰面积 } \\
(\mathrm{mAU} \text { *min) }\end{array}$ & $\begin{array}{l}\text { 峰高 } \\
(\mathrm{mAU})\end{array}$ & $\begin{array}{l}\text { 相对峰面 } \\
\text { 积 }(\%)\end{array}$ & $\begin{array}{l}\text { 相对峰高 } \\
(\%)\end{array}$ & $\begin{array}{l}\text { 样品量 } \\
\text { n.a. }\end{array}$ \\
\hline $\begin{array}{l}\text { Entr } \\
\mathrm{y}\end{array}$ & $\begin{array}{l}\text { Peak } \\
\text { name }\end{array}$ & $\begin{array}{l}\text { Retention } \\
\text { time (min) }\end{array}$ & $\begin{array}{l}\text { Peak area } \\
\left(\mathrm{mAU} \mathrm{U}^{*} \text { min }\right)\end{array}$ & $\begin{array}{l}\text { Peak } \\
\text { height } \\
(\mathrm{mAU})\end{array}$ & $\begin{array}{l}\text { Relative } \\
\text { peak area } \\
(\%)\end{array}$ & $\begin{array}{l}\text { Relative } \\
\text { Peak } \\
\text { height (\%) }\end{array}$ & $\begin{array}{l}\text { Sample } \\
\text { volume } \\
\text { n.a. }\end{array}$ \\
\hline
\end{tabular}



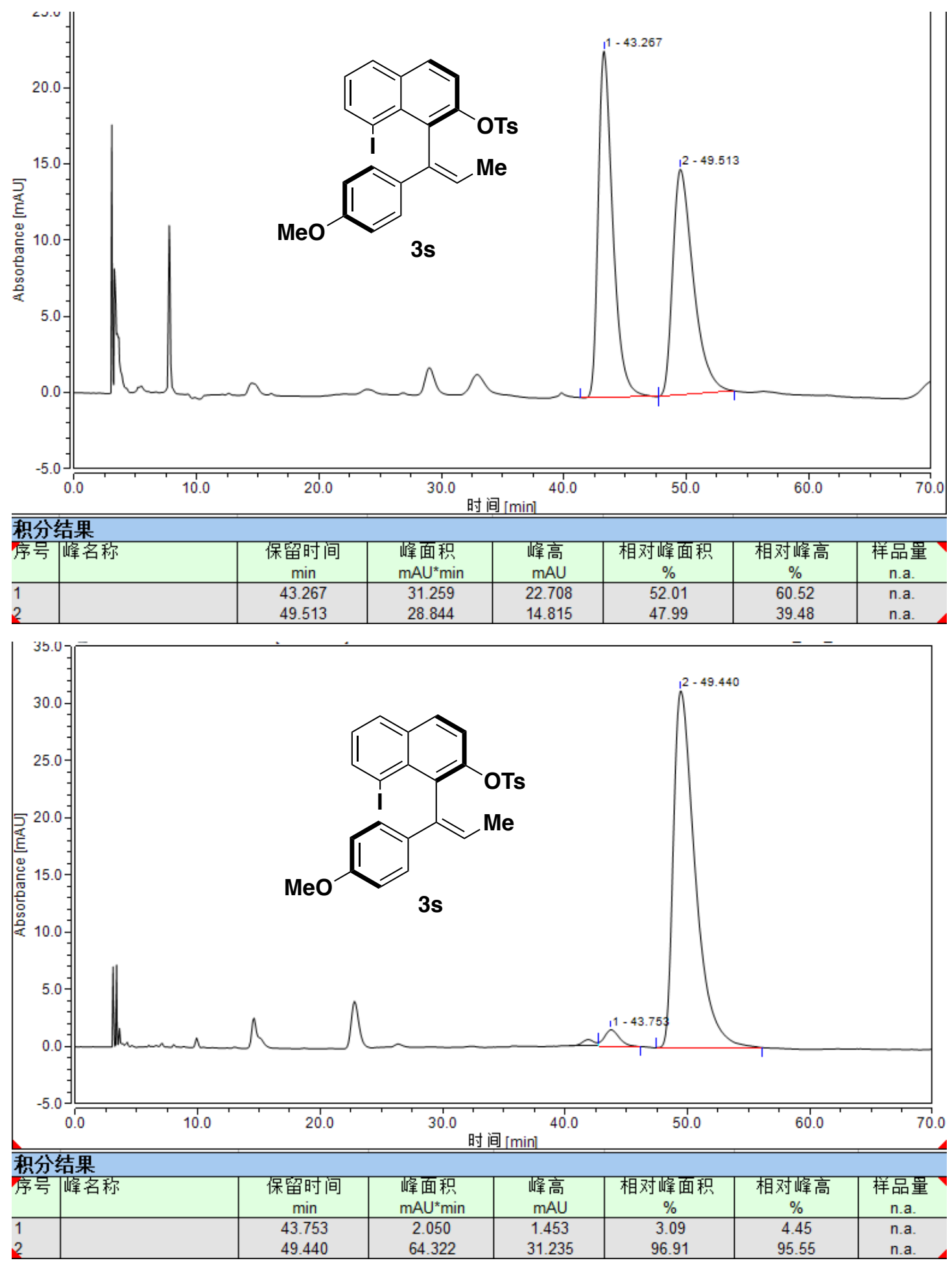

For comparison of Chinese and English of the HPLC data table.

\begin{tabular}{|l|l|l|l|l|l|l|l|}
\hline 序号 & $\begin{array}{l}\text { 峰名 } \\
\text { 称 }\end{array}$ & $\begin{array}{l}\text { 保留时间 } \\
(\mathrm{min})\end{array}$ & $\begin{array}{l}\text { 峰面积 } \\
(\mathrm{mAU} \text { *min) }\end{array}$ & $\begin{array}{l}\text { 峰高 } \\
(\mathrm{mAU})\end{array}$ & $\begin{array}{l}\text { 相对峰面 } \\
\text { 积 }(\%)\end{array}$ & $\begin{array}{l}\text { 相对峰高 } \\
(\%)\end{array}$ & $\begin{array}{l}\text { 样品量 } \\
\text { n.a. }\end{array}$ \\
\hline $\begin{array}{l}\text { Entr } \\
\mathrm{y}\end{array}$ & $\begin{array}{l}\text { Peak } \\
\text { name }\end{array}$ & $\begin{array}{l}\text { Retention } \\
\text { time (min) }\end{array}$ & $\begin{array}{l}\text { Peak area } \\
\left(\mathrm{mAU} \mathrm{U}^{*} \text { min }\right)\end{array}$ & $\begin{array}{l}\text { Peak } \\
\text { height } \\
(\mathrm{mAU})\end{array}$ & $\begin{array}{l}\text { Relative } \\
\text { peak area } \\
(\%)\end{array}$ & $\begin{array}{l}\text { Relative } \\
\text { Peak } \\
\text { height (\%) }\end{array}$ & $\begin{array}{l}\text { Sample } \\
\text { volume } \\
\text { n.a. }\end{array}$ \\
\hline
\end{tabular}



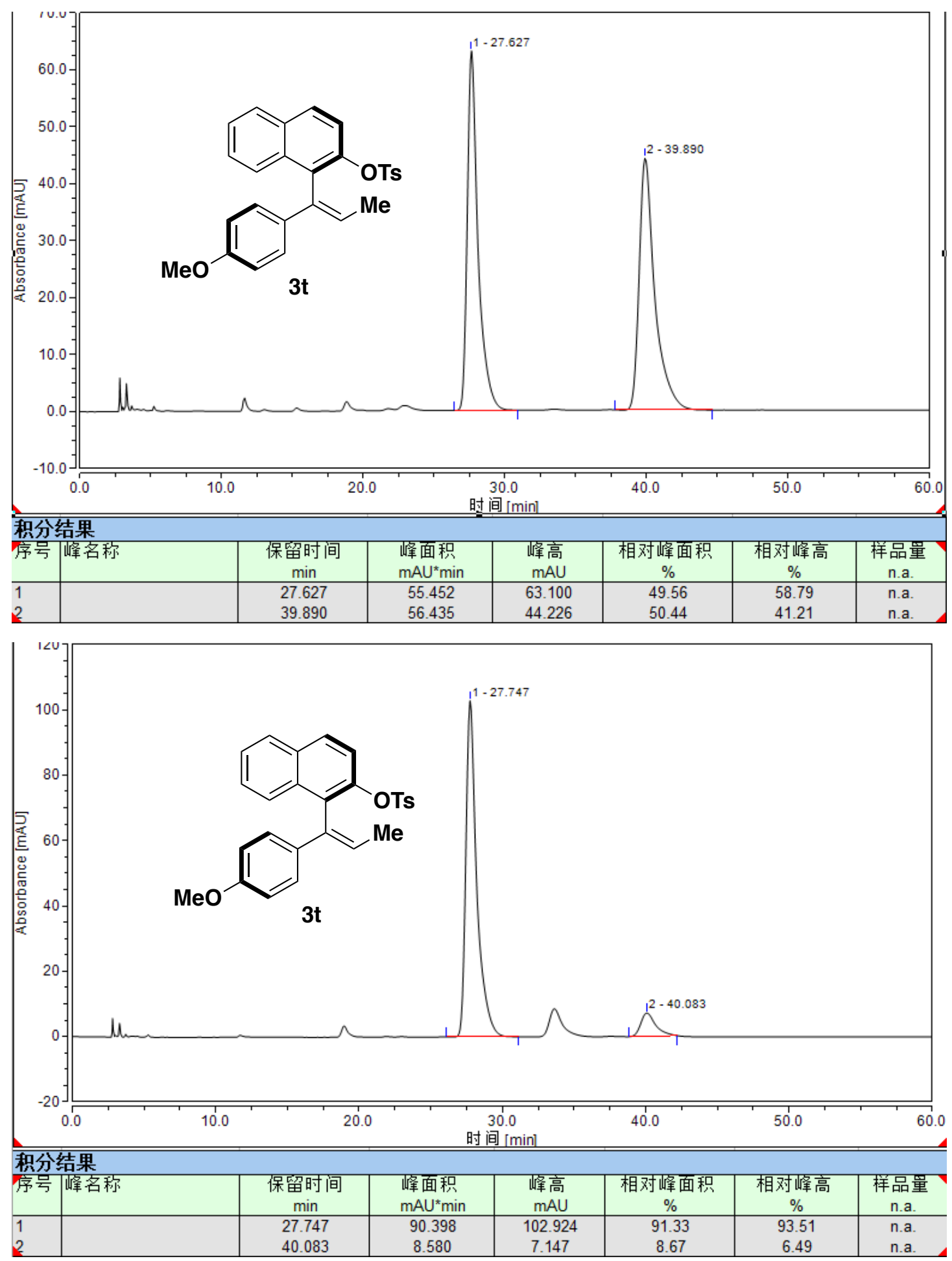

For comparison of Chinese and English of the HPLC data table.

\begin{tabular}{|l|l|l|l|l|l|l|l|}
\hline 序号 & $\begin{array}{l}\text { 峰名 } \\
\text { 称 }\end{array}$ & $\begin{array}{l}\text { 保留时间 } \\
(\mathrm{min})\end{array}$ & $\begin{array}{l}\text { 峰面积 } \\
(\mathrm{mAU} \text { *min) }\end{array}$ & $\begin{array}{l}\text { 峰高 } \\
(\mathrm{mAU})\end{array}$ & $\begin{array}{l}\text { 相对峰面 } \\
\text { 积 }(\%)\end{array}$ & $\begin{array}{l}\text { 相对峰高 } \\
(\%)\end{array}$ & $\begin{array}{l}\text { 样品量 } \\
\text { n.a. }\end{array}$ \\
\hline $\begin{array}{l}\text { Entr } \\
\mathrm{y}\end{array}$ & $\begin{array}{l}\text { Peak } \\
\text { name }\end{array}$ & $\begin{array}{l}\text { Retention } \\
\text { time (min) }\end{array}$ & $\begin{array}{l}\text { Peak area } \\
\left(\mathrm{mAU} \mathrm{U}^{*} \text { min }\right)\end{array}$ & $\begin{array}{l}\text { Peak } \\
\text { height } \\
(\mathrm{mAU})\end{array}$ & $\begin{array}{l}\text { Relative } \\
\text { peak area } \\
(\%)\end{array}$ & $\begin{array}{l}\text { Relative } \\
\text { Peak } \\
\text { height (\%) }\end{array}$ & $\begin{array}{l}\text { Sample } \\
\text { volume } \\
\text { n.a. }\end{array}$ \\
\hline
\end{tabular}




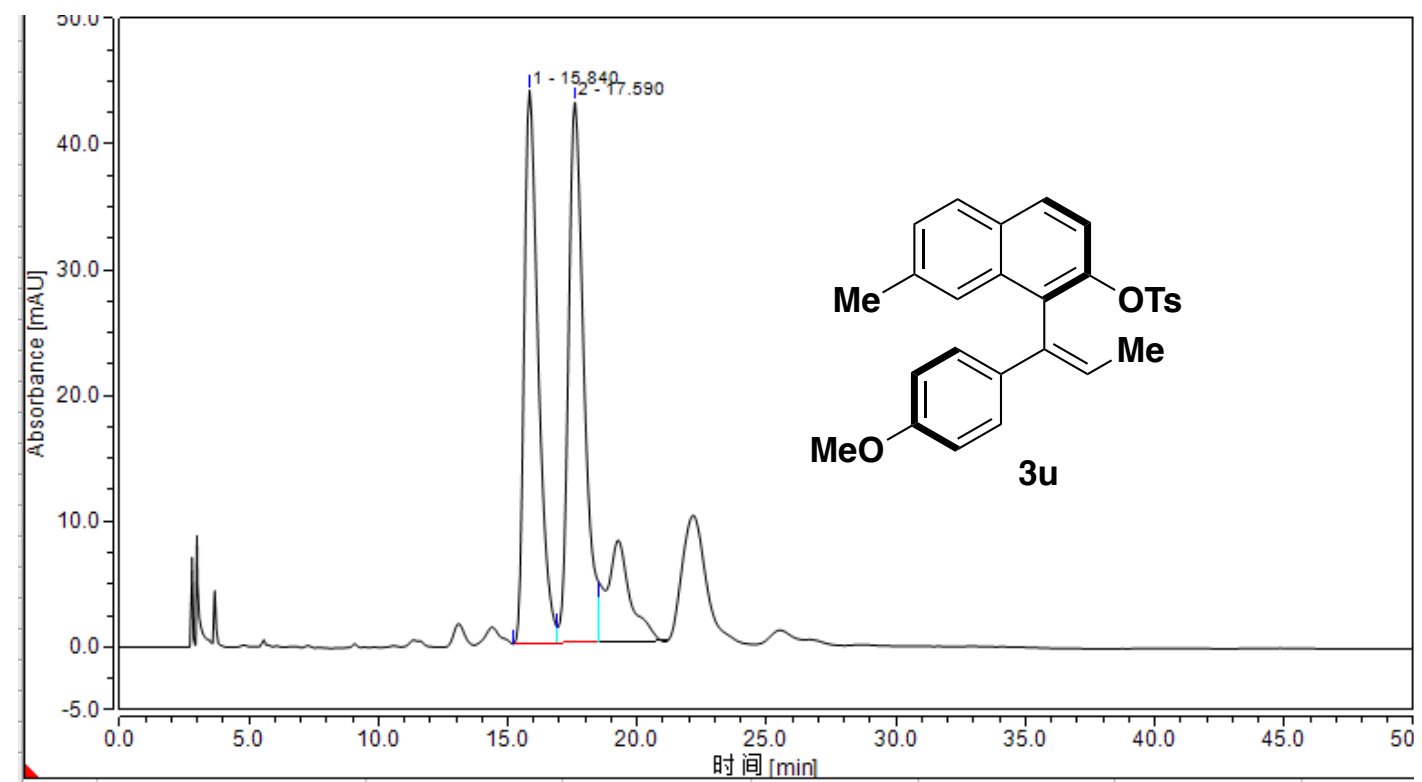

\begin{tabular}{|c|c|c|c|c|c|c|c|}
\hline \multicolumn{8}{|c|}{ 积分结果 } \\
\hline 序号 & 峰名称 & $\begin{array}{c}\text { 保留时间 } \\
\text { min }\end{array}$ & $\begin{array}{l}\text { 峰面积 } \\
m U^{*} \text { min }\end{array}$ & $\begin{array}{l}\text { 㷨高 } \\
\text { mAU }\end{array}$ & $\begin{array}{c}\text { 相对峰面积 } \\
\%\end{array}$ & $\begin{array}{c}\text { 相对峰高 } \\
\%\end{array}$ & $\begin{array}{c}\text { 样品墨 } \\
\text { n.a. }\end{array}$ \\
\hline $\begin{array}{l}1 \\
2\end{array}$ & & $\begin{array}{l}15.840 \\
17.590\end{array}$ & $\begin{array}{l}27.856 \\
29.742\end{array}$ & $\begin{array}{l}44.000 \\
42.964\end{array}$ & $\begin{array}{l}48.36 \\
51.64\end{array}$ & $\begin{array}{l}50.60 \\
49.40\end{array}$ & $\begin{array}{l}\text { n.a. } \\
\text { n.a. }\end{array}$ \\
\hline
\end{tabular}

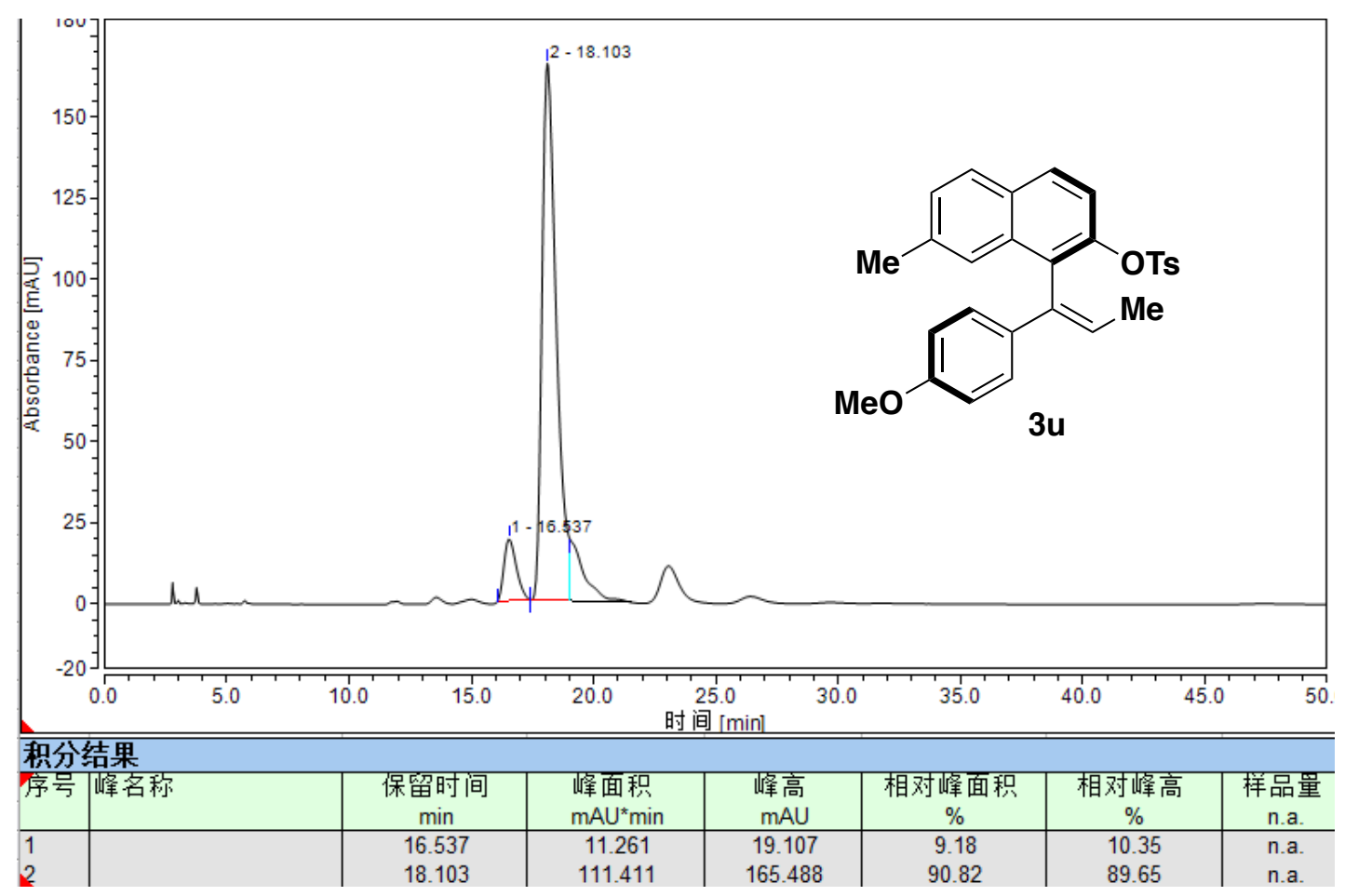

For comparison of Chinese and English of the HPLC data table.

\begin{tabular}{|c|c|c|c|c|c|c|c|}
\hline 序号 & $\begin{array}{l}\text { 峰名 } \\
\text { 称 }\end{array}$ & $\begin{array}{l}\text { 保留时间 } \\
\text { (min) }\end{array}$ & $\begin{array}{l}\text { 峰面积 } \\
\left(\mathrm{mAU} \mathrm{U}^{*} \mathrm{~min}\right)\end{array}$ & $\begin{array}{l}\text { 峰高 } \\
(\mathrm{mAU})\end{array}$ & $\begin{array}{l}\text { 相对峰面 } \\
\text { 积 }(\%)\end{array}$ & $\begin{array}{l}\text { 相对峰高 } \\
(\%)\end{array}$ & $\begin{array}{l}\text { 样品量 } \\
\text { n.a. }\end{array}$ \\
\hline $\begin{array}{l}\text { Entr } \\
\mathrm{y}\end{array}$ & $\begin{array}{l}\text { Peak } \\
\text { name }\end{array}$ & $\begin{array}{l}\text { Retention } \\
\text { time (min) }\end{array}$ & $\begin{array}{l}\text { Peak area } \\
\left(\mathrm{mAU} *^{*} \min \right)\end{array}$ & $\begin{array}{l}\text { Peak } \\
\text { height } \\
\text { (mAU) }\end{array}$ & $\begin{array}{l}\text { Relative } \\
\text { peak area } \\
(\%)\end{array}$ & $\begin{array}{l}\text { Relative } \\
\text { Peak } \\
\text { height }(\%)\end{array}$ & $\begin{array}{l}\text { Sample } \\
\text { volume } \\
\text { n.a. }\end{array}$ \\
\hline
\end{tabular}



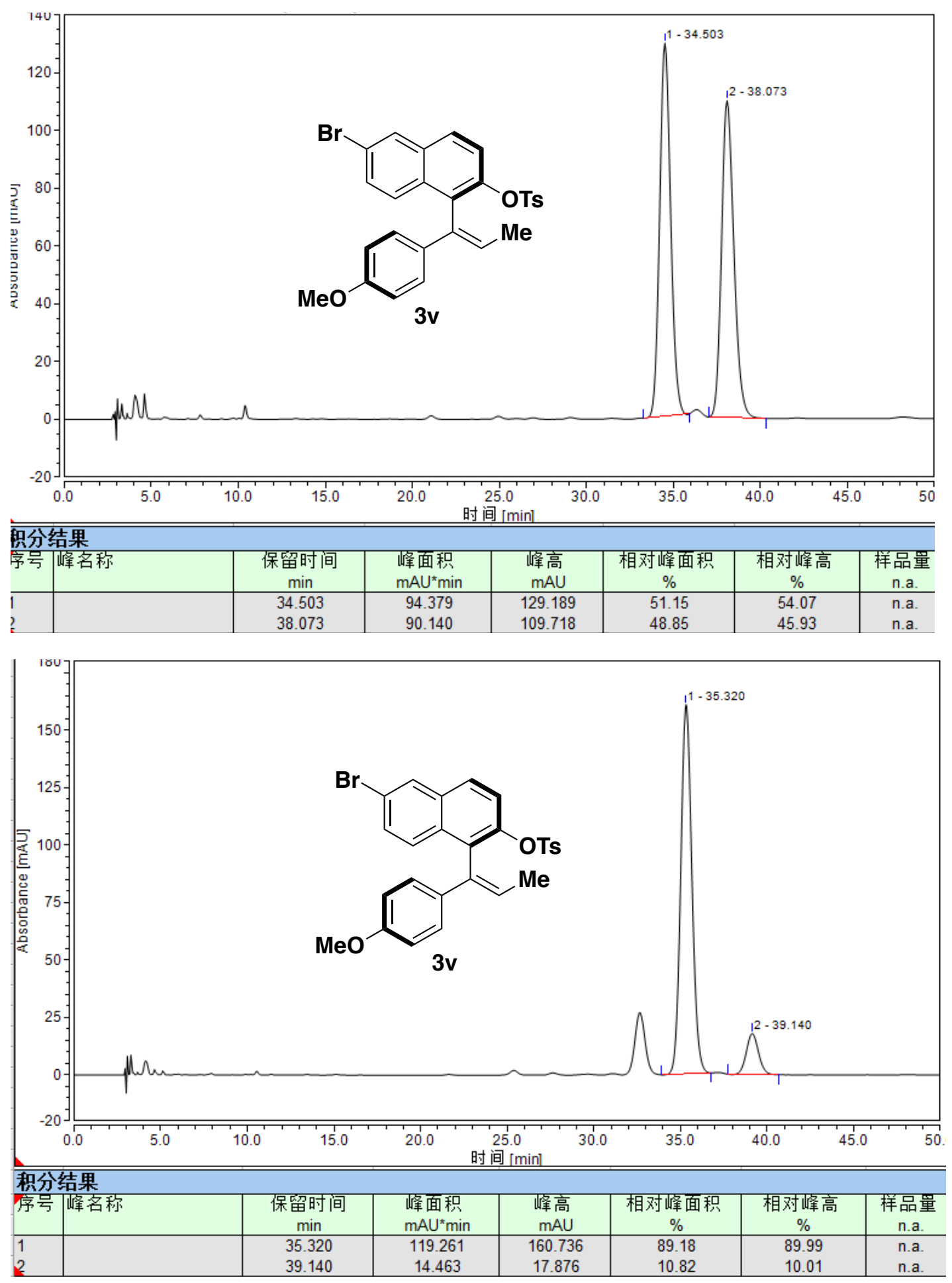

For comparison of Chinese and English of the HPLC data table.

\begin{tabular}{|l|l|l|l|l|l|l|l|}
\hline 序号 & $\begin{array}{l}\text { 峰名 } \\
\text { 称 }\end{array}$ & $\begin{array}{l}\text { 保留时间 } \\
(\mathrm{min})\end{array}$ & $\begin{array}{l}\text { 峰面积 } \\
(\mathrm{mAU} \text { *min) }\end{array}$ & $\begin{array}{l}\text { 峰高 } \\
(\mathrm{mAU})\end{array}$ & $\begin{array}{l}\text { 相对峰面 } \\
\text { 积 }(\%)\end{array}$ & $\begin{array}{l}\text { 相对峰高 } \\
(\%)\end{array}$ & $\begin{array}{l}\text { 样品量 } \\
\text { n.a. }\end{array}$ \\
\hline $\begin{array}{l}\text { Entr } \\
\mathrm{y}\end{array}$ & $\begin{array}{l}\text { Peak } \\
\text { name }\end{array}$ & $\begin{array}{l}\text { Retention } \\
\text { time (min) }\end{array}$ & $\begin{array}{l}\text { Peak area } \\
\left(\mathrm{mAU} \mathrm{U}^{*} \text { min }\right)\end{array}$ & $\begin{array}{l}\text { Peak } \\
\text { height } \\
(\mathrm{mAU})\end{array}$ & $\begin{array}{l}\text { Relative } \\
\text { peak area } \\
(\%)\end{array}$ & $\begin{array}{l}\text { Relative } \\
\text { Peak } \\
\text { height (\%) }\end{array}$ & $\begin{array}{l}\text { Sample } \\
\text { volume } \\
\text { n.a. }\end{array}$ \\
\hline
\end{tabular}



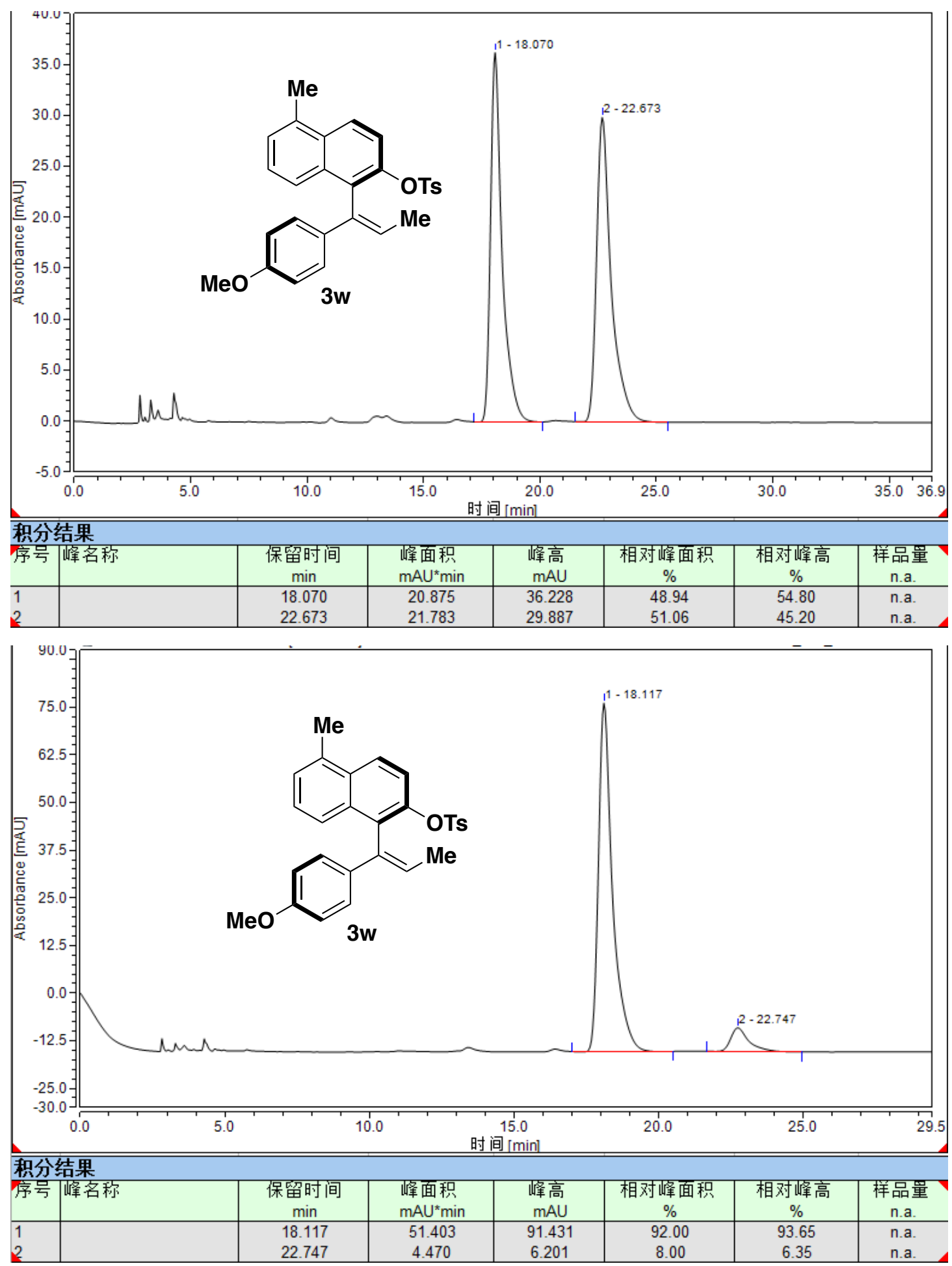

For comparison of Chinese and English of the HPLC data table.

\begin{tabular}{|l|l|l|l|l|l|l|l|}
\hline 序号 & $\begin{array}{l}\text { 峰名 } \\
\text { 称 }\end{array}$ & $\begin{array}{l}\text { 保留时间 } \\
(\mathrm{min})\end{array}$ & $\begin{array}{l}\text { 峰面积 } \\
(\mathrm{mAU} \text { *min) }\end{array}$ & $\begin{array}{l}\text { 峰高 } \\
(\mathrm{mAU})\end{array}$ & $\begin{array}{l}\text { 相对峰面 } \\
\text { 积 }(\%)\end{array}$ & $\begin{array}{l}\text { 相对峰高 } \\
(\%)\end{array}$ & $\begin{array}{l}\text { 样品量 } \\
\text { n.a. }\end{array}$ \\
\hline $\begin{array}{l}\text { Entr } \\
\mathrm{y}\end{array}$ & $\begin{array}{l}\text { Peak } \\
\text { name }\end{array}$ & $\begin{array}{l}\text { Retention } \\
\text { time (min) }\end{array}$ & $\begin{array}{l}\text { Peak area } \\
\left(\mathrm{mAU} \mathrm{U}^{*} \text { min }\right)\end{array}$ & $\begin{array}{l}\text { Peak } \\
\text { height } \\
(\mathrm{mAU})\end{array}$ & $\begin{array}{l}\text { Relative } \\
\text { peak area } \\
(\%)\end{array}$ & $\begin{array}{l}\text { Relative } \\
\text { Peak } \\
\text { height (\%) }\end{array}$ & $\begin{array}{l}\text { Sample } \\
\text { volume } \\
\text { n.a. }\end{array}$ \\
\hline
\end{tabular}



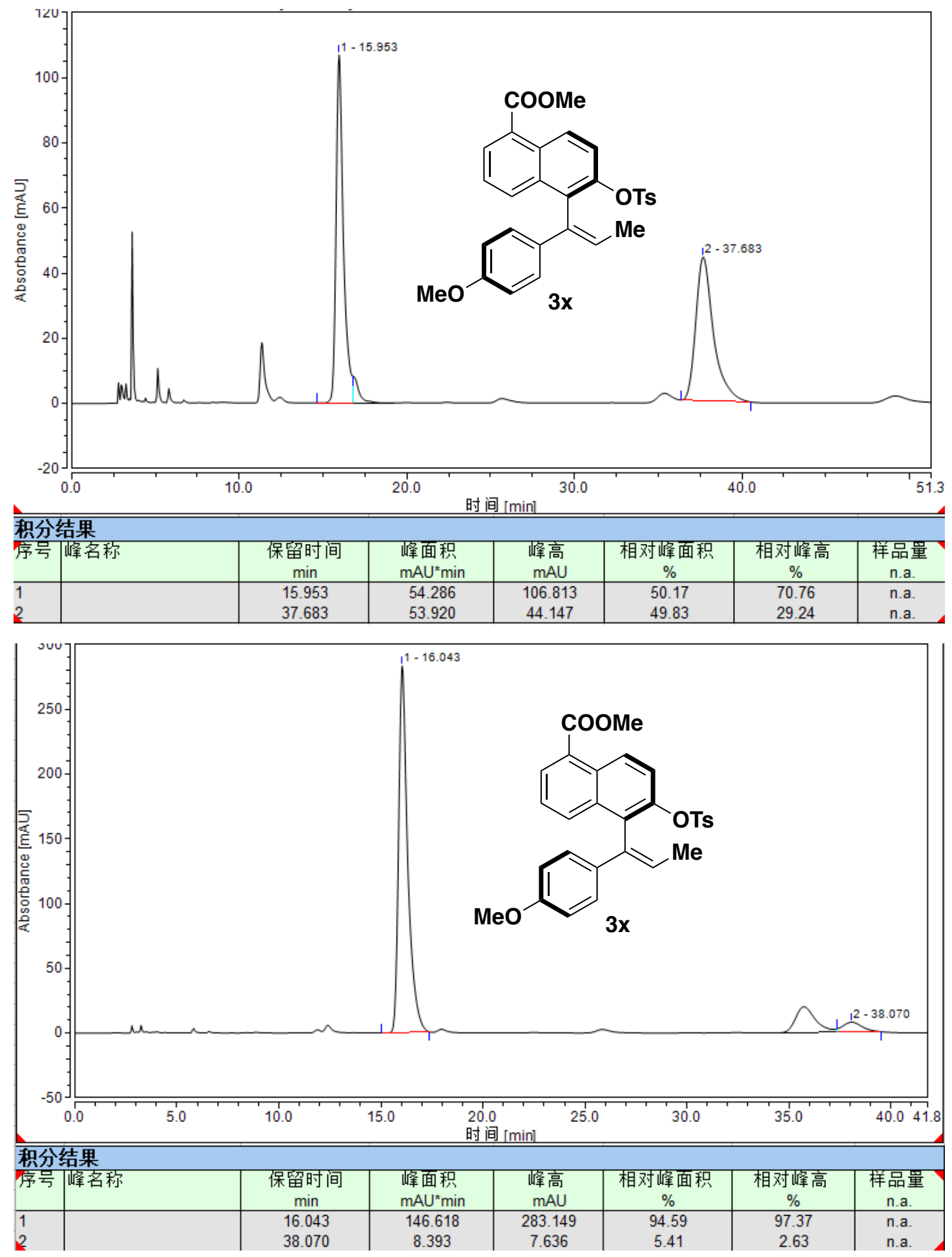

For comparison of Chinese and English of the HPLC data table.

\begin{tabular}{|l|l|l|l|l|l|l|l|}
\hline 序号 & $\begin{array}{l}\text { 峰名 } \\
\text { 称 }\end{array}$ & $\begin{array}{l}\text { 保留时间 } \\
(\mathrm{min})\end{array}$ & $\begin{array}{l}\text { 峰面积 } \\
(\mathrm{mAU} \text { *min) }\end{array}$ & $\begin{array}{l}\text { 峰高 } \\
(\mathrm{mAU})\end{array}$ & $\begin{array}{l}\text { 相对峰面 } \\
\text { 积 }(\%)\end{array}$ & $\begin{array}{l}\text { 相对峰高 } \\
(\%)\end{array}$ & $\begin{array}{l}\text { 样品量 } \\
\text { n.a. }\end{array}$ \\
\hline $\begin{array}{l}\text { Entr } \\
\mathrm{y}\end{array}$ & $\begin{array}{l}\text { Peak } \\
\text { name }\end{array}$ & $\begin{array}{l}\text { Retention } \\
\text { time (min) }\end{array}$ & $\begin{array}{l}\text { Peak area } \\
\left(\mathrm{mAU} \mathrm{U}^{*} \text { min }\right)\end{array}$ & $\begin{array}{l}\text { Peak } \\
\text { height } \\
(\mathrm{mAU})\end{array}$ & $\begin{array}{l}\text { Relative } \\
\text { peak area } \\
(\%)\end{array}$ & $\begin{array}{l}\text { Relative } \\
\text { Peak } \\
\text { height (\%) }\end{array}$ & $\begin{array}{l}\text { Sample } \\
\text { volume } \\
\text { n.a. }\end{array}$ \\
\hline
\end{tabular}



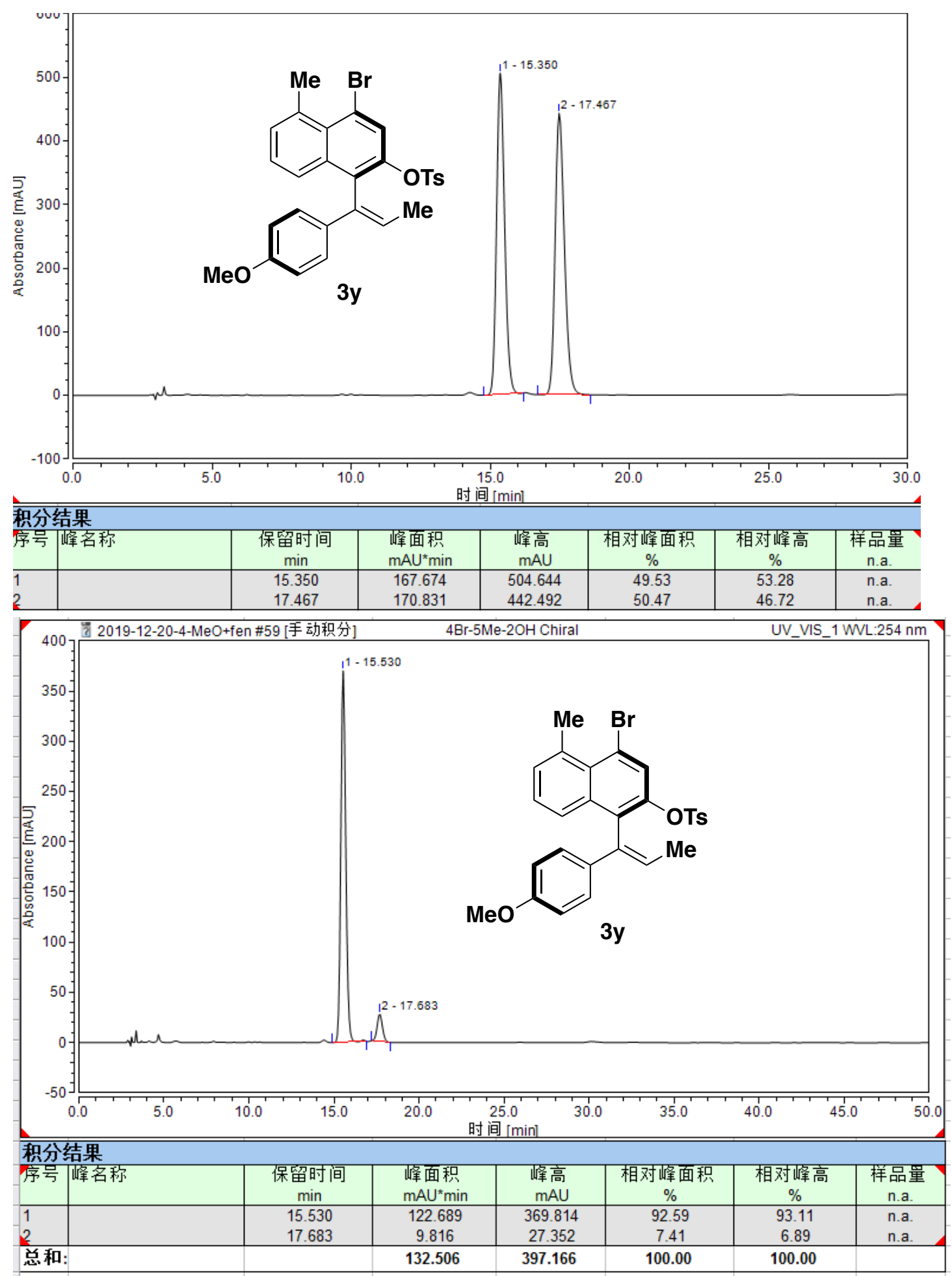

For comparison of Chinese and English of the HPLC data table.

\begin{tabular}{|l|l|l|l|l|l|l|l|}
\hline 序号 & $\begin{array}{l}\text { 峰名 } \\
\text { 称 }\end{array}$ & $\begin{array}{l}\text { 保留时间 } \\
(\mathrm{min})\end{array}$ & $\begin{array}{l}\text { 峰面积 } \\
(\mathrm{mAU} \text { *min) }\end{array}$ & $\begin{array}{l}\text { 峰高 } \\
(\mathrm{mAU})\end{array}$ & $\begin{array}{l}\text { 相对峰面 } \\
\text { 积 }(\%)\end{array}$ & $\begin{array}{l}\text { 相对峰高 } \\
(\%)\end{array}$ & $\begin{array}{l}\text { 样品量 } \\
\text { n.a. }\end{array}$ \\
\hline $\begin{array}{l}\text { Entr } \\
\mathrm{y}\end{array}$ & $\begin{array}{l}\text { Peak } \\
\text { name }\end{array}$ & $\begin{array}{l}\text { Retention } \\
\text { time (min) }\end{array}$ & $\begin{array}{l}\text { Peak area } \\
\left(\mathrm{mAU} \mathrm{U}^{*} \text { min }\right)\end{array}$ & $\begin{array}{l}\text { Peak } \\
\text { height } \\
(\mathrm{mAU})\end{array}$ & $\begin{array}{l}\text { Relative } \\
\text { peak area } \\
(\%)\end{array}$ & $\begin{array}{l}\text { Relative } \\
\text { Peak } \\
\text { height (\%) }\end{array}$ & $\begin{array}{l}\text { Sample } \\
\text { volume } \\
\text { n.a. }\end{array}$ \\
\hline
\end{tabular}



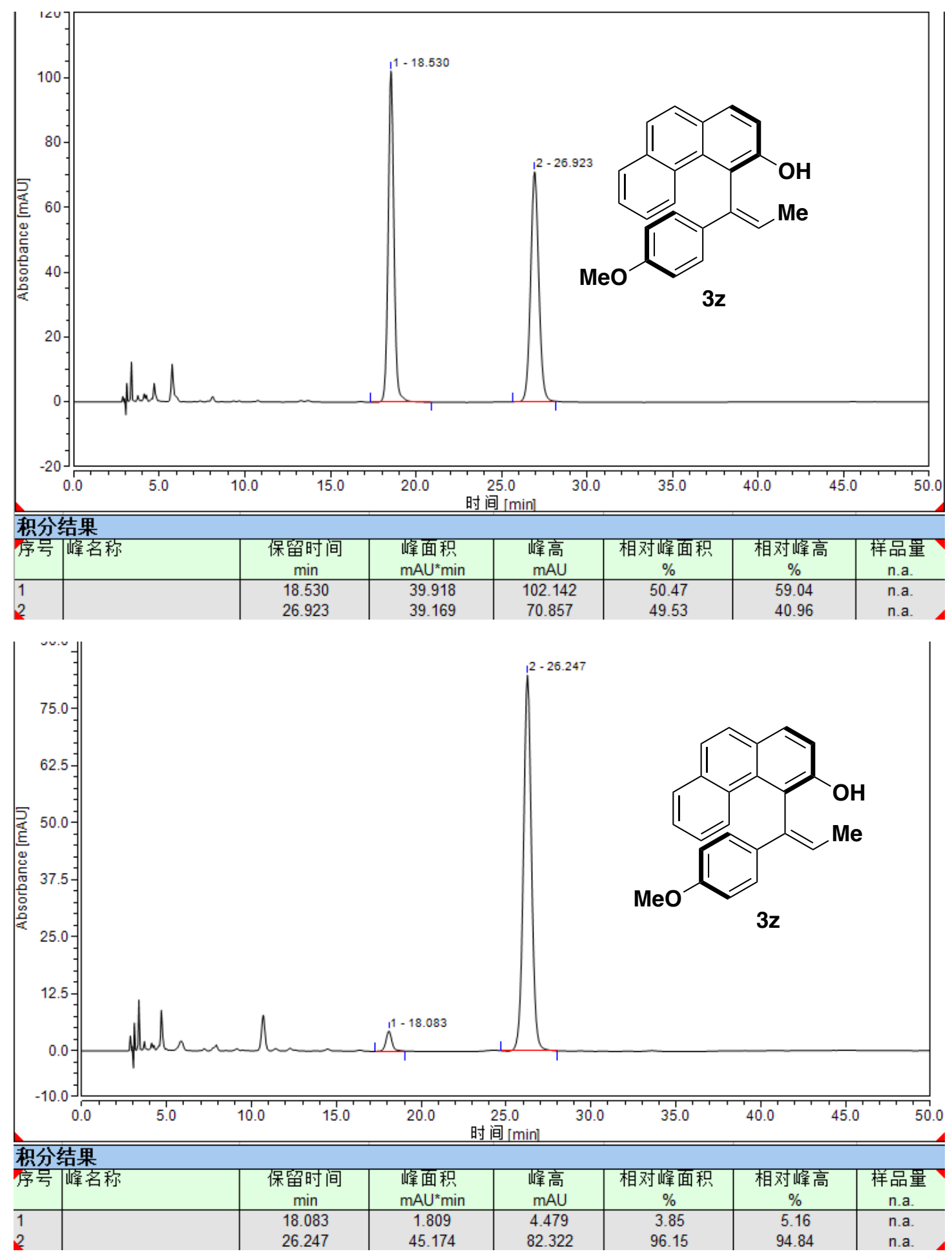

For comparison of Chinese and English of the HPLC data table.

\begin{tabular}{|l|l|l|l|l|l|l|l|}
\hline 序号 & $\begin{array}{l}\text { 峰名 } \\
\text { 称 }\end{array}$ & $\begin{array}{l}\text { 保留时间 } \\
(\mathrm{min})\end{array}$ & $\begin{array}{l}\text { 峰面积 } \\
(\mathrm{mAU} \text { *min) }\end{array}$ & $\begin{array}{l}\text { 峰高 } \\
(\mathrm{mAU})\end{array}$ & $\begin{array}{l}\text { 相对峰面 } \\
\text { 积 }(\%)\end{array}$ & $\begin{array}{l}\text { 相对峰高 } \\
(\%)\end{array}$ & $\begin{array}{l}\text { 样品量 } \\
\text { n.a. }\end{array}$ \\
\hline $\begin{array}{l}\text { Entr } \\
\mathrm{y}\end{array}$ & $\begin{array}{l}\text { Peak } \\
\text { name }\end{array}$ & $\begin{array}{l}\text { Retention } \\
\text { time (min) }\end{array}$ & $\begin{array}{l}\text { Peak area } \\
\left(\mathrm{mAU} \mathrm{U}^{*} \text { min }\right)\end{array}$ & $\begin{array}{l}\text { Peak } \\
\text { height } \\
(\mathrm{mAU})\end{array}$ & $\begin{array}{l}\text { Relative } \\
\text { peak area } \\
(\%)\end{array}$ & $\begin{array}{l}\text { Relative } \\
\text { Peak } \\
\text { height (\%) }\end{array}$ & $\begin{array}{l}\text { Sample } \\
\text { volume } \\
\text { n.a. }\end{array}$ \\
\hline
\end{tabular}



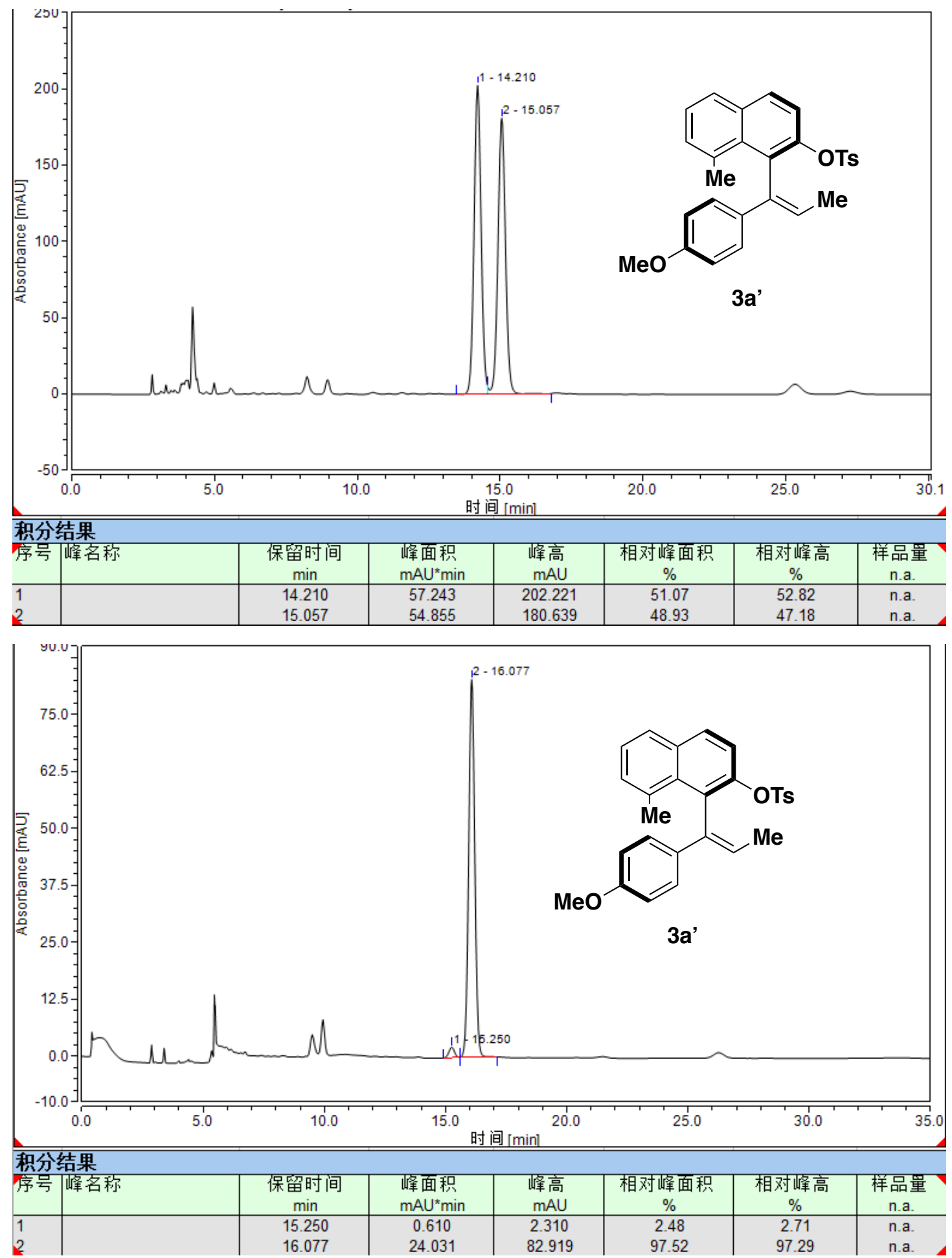

For comparison of Chinese and English of the HPLC data table.

\begin{tabular}{|l|l|l|l|l|l|l|l|}
\hline 序号 & $\begin{array}{l}\text { 峰名 } \\
\text { 称 }\end{array}$ & $\begin{array}{l}\text { 保留时间 } \\
(\mathrm{min})\end{array}$ & $\begin{array}{l}\text { 峰面积 } \\
(\mathrm{mAU} \text { *min) }\end{array}$ & $\begin{array}{l}\text { 峰高 } \\
(\mathrm{mAU})\end{array}$ & $\begin{array}{l}\text { 相对峰面 } \\
\text { 积 }(\%)\end{array}$ & $\begin{array}{l}\text { 相对峰高 } \\
(\%)\end{array}$ & $\begin{array}{l}\text { 样品量 } \\
\text { n.a. }\end{array}$ \\
\hline $\begin{array}{l}\text { Entr } \\
\mathrm{y}\end{array}$ & $\begin{array}{l}\text { Peak } \\
\text { name }\end{array}$ & $\begin{array}{l}\text { Retention } \\
\text { time (min) }\end{array}$ & $\begin{array}{l}\text { Peak area } \\
\left(\mathrm{mAU} \mathrm{U}^{*} \text { min }\right)\end{array}$ & $\begin{array}{l}\text { Peak } \\
\text { height } \\
(\mathrm{mAU})\end{array}$ & $\begin{array}{l}\text { Relative } \\
\text { peak area } \\
(\%)\end{array}$ & $\begin{array}{l}\text { Relative } \\
\text { Peak } \\
\text { height (\%) }\end{array}$ & $\begin{array}{l}\text { Sample } \\
\text { volume } \\
\text { n.a. }\end{array}$ \\
\hline
\end{tabular}



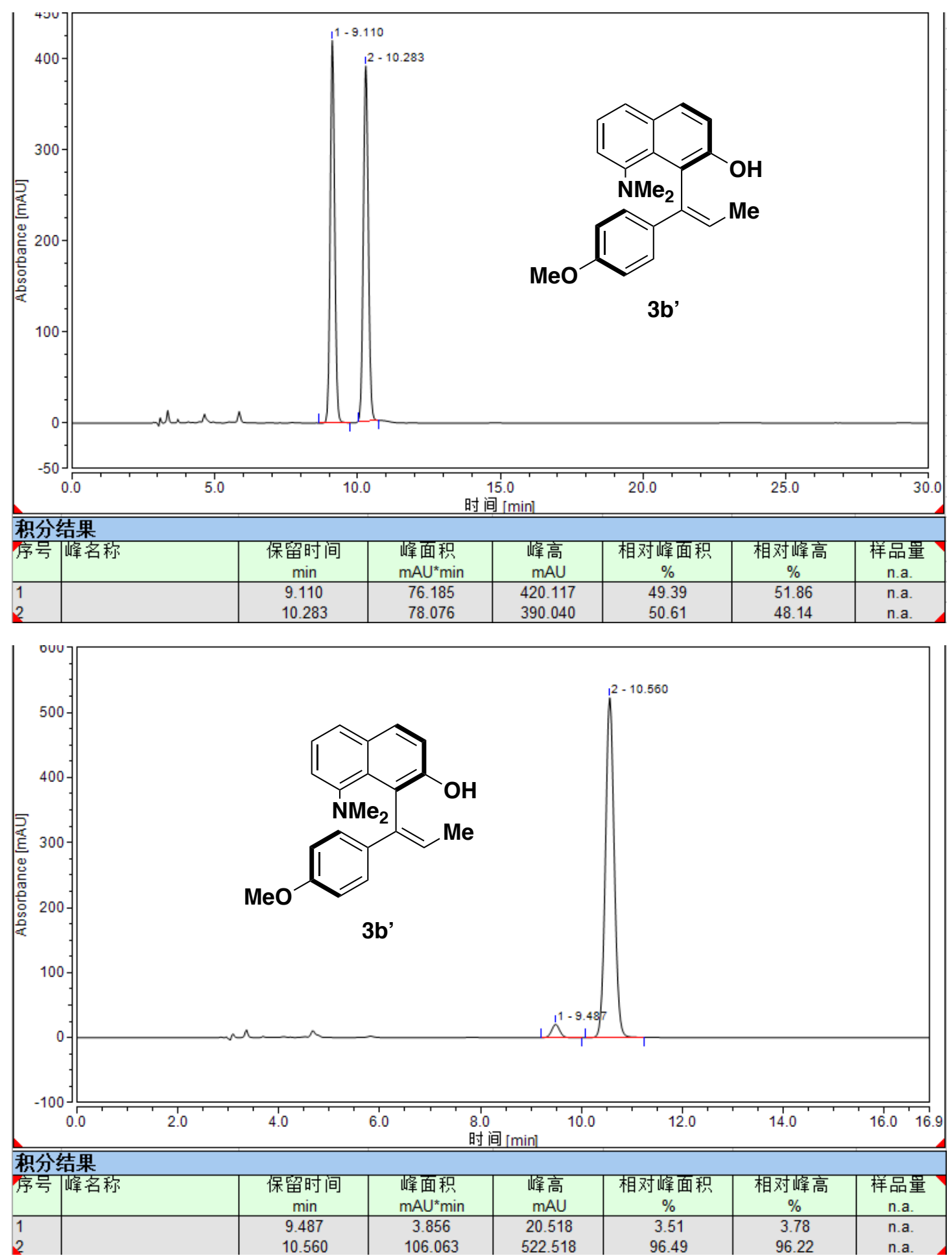

For comparison of Chinese and English of the HPLC data table.

\begin{tabular}{|l|l|l|l|l|l|l|l|}
\hline 序号 & $\begin{array}{l}\text { 峰名 } \\
\text { 称 }\end{array}$ & $\begin{array}{l}\text { 保留时间 } \\
(\mathrm{min})\end{array}$ & $\begin{array}{l}\text { 峰面积 } \\
(\mathrm{mAU} \text { *min) }\end{array}$ & $\begin{array}{l}\text { 峰高 } \\
(\mathrm{mAU})\end{array}$ & $\begin{array}{l}\text { 相对峰面 } \\
\text { 积 }(\%)\end{array}$ & $\begin{array}{l}\text { 相对峰高 } \\
(\%)\end{array}$ & $\begin{array}{l}\text { 样品量 } \\
\text { n.a. }\end{array}$ \\
\hline $\begin{array}{l}\text { Entr } \\
\mathrm{y}\end{array}$ & $\begin{array}{l}\text { Peak } \\
\text { name }\end{array}$ & $\begin{array}{l}\text { Retention } \\
\text { time (min) }\end{array}$ & $\begin{array}{l}\text { Peak area } \\
\left(\mathrm{mAU} \mathrm{U}^{*} \text { min }\right)\end{array}$ & $\begin{array}{l}\text { Peak } \\
\text { height } \\
(\mathrm{mAU})\end{array}$ & $\begin{array}{l}\text { Relative } \\
\text { peak area } \\
(\%)\end{array}$ & $\begin{array}{l}\text { Relative } \\
\text { Peak } \\
\text { height (\%) }\end{array}$ & $\begin{array}{l}\text { Sample } \\
\text { volume } \\
\text { n.a. }\end{array}$ \\
\hline
\end{tabular}



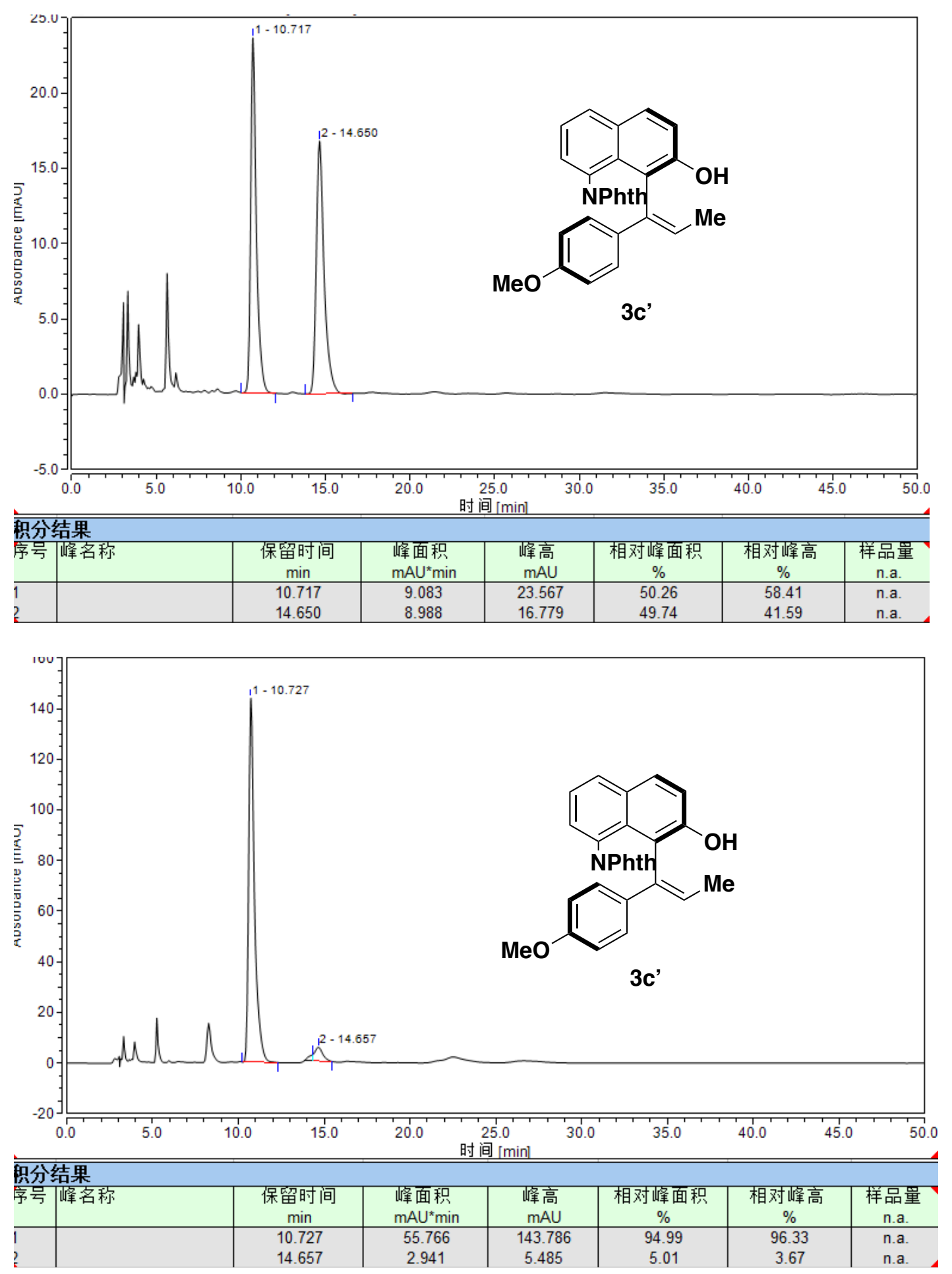

For comparison of Chinese and English of the HPLC data table.

\begin{tabular}{|l|l|l|l|l|l|l|l|}
\hline 序号 & $\begin{array}{l}\text { 峰名 } \\
\text { 称 }\end{array}$ & $\begin{array}{l}\text { 保留时间 } \\
(\mathrm{min})\end{array}$ & $\begin{array}{l}\text { 峰面积 } \\
(\mathrm{mAU} \text { *min) }\end{array}$ & $\begin{array}{l}\text { 峰高 } \\
(\mathrm{mAU})\end{array}$ & $\begin{array}{l}\text { 相对峰面 } \\
\text { 积 }(\%)\end{array}$ & $\begin{array}{l}\text { 相对峰高 } \\
(\%)\end{array}$ & $\begin{array}{l}\text { 样品量 } \\
\text { n.a. }\end{array}$ \\
\hline $\begin{array}{l}\text { Entr } \\
\mathrm{y}\end{array}$ & $\begin{array}{l}\text { Peak } \\
\text { name }\end{array}$ & $\begin{array}{l}\text { Retention } \\
\text { time (min) }\end{array}$ & $\begin{array}{l}\text { Peak area } \\
\left(\mathrm{mAU} \mathrm{U}^{*} \text { min }\right)\end{array}$ & $\begin{array}{l}\text { Peak } \\
\text { height } \\
(\mathrm{mAU})\end{array}$ & $\begin{array}{l}\text { Relative } \\
\text { peak area } \\
(\%)\end{array}$ & $\begin{array}{l}\text { Relative } \\
\text { Peak } \\
\text { height (\%) }\end{array}$ & $\begin{array}{l}\text { Sample } \\
\text { volume } \\
\text { n.a. }\end{array}$ \\
\hline
\end{tabular}



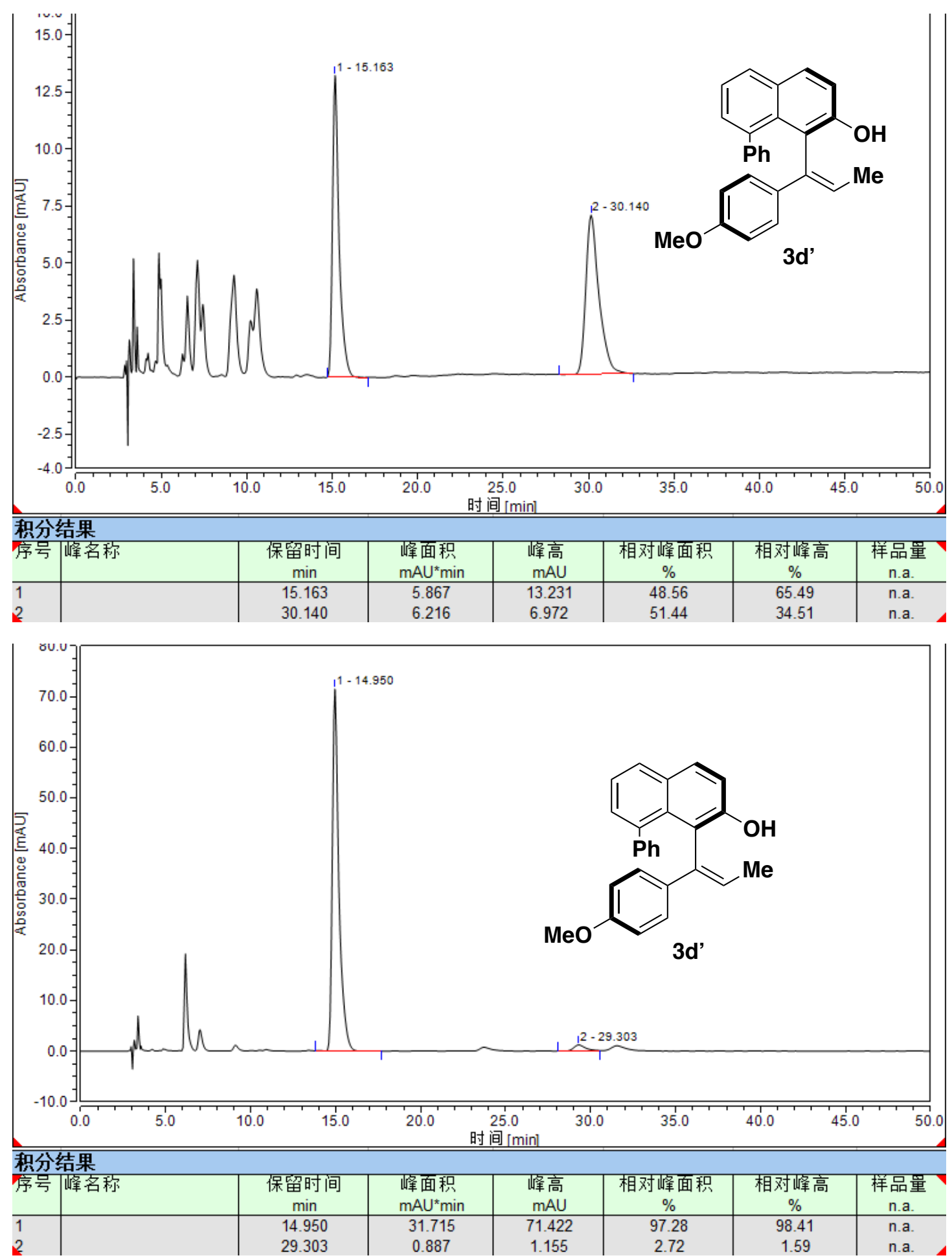

For comparison of Chinese and English of the HPLC data table.

\begin{tabular}{|l|l|l|l|l|l|l|l|}
\hline 序号 & $\begin{array}{l}\text { 峰名 } \\
\text { 称 }\end{array}$ & $\begin{array}{l}\text { 保留时间 } \\
(\mathrm{min})\end{array}$ & $\begin{array}{l}\text { 峰面积 } \\
(\mathrm{mAU} \text { *min) }\end{array}$ & $\begin{array}{l}\text { 峰高 } \\
(\mathrm{mAU})\end{array}$ & $\begin{array}{l}\text { 相对峰面 } \\
\text { 积 }(\%)\end{array}$ & $\begin{array}{l}\text { 相对峰高 } \\
(\%)\end{array}$ & $\begin{array}{l}\text { 样品量 } \\
\text { n.a. }\end{array}$ \\
\hline $\begin{array}{l}\text { Entr } \\
\mathrm{y}\end{array}$ & $\begin{array}{l}\text { Peak } \\
\text { name }\end{array}$ & $\begin{array}{l}\text { Retention } \\
\text { time (min) }\end{array}$ & $\begin{array}{l}\text { Peak area } \\
\left(\mathrm{mAU} \mathrm{U}^{*} \text { min }\right)\end{array}$ & $\begin{array}{l}\text { Peak } \\
\text { height } \\
(\mathrm{mAU})\end{array}$ & $\begin{array}{l}\text { Relative } \\
\text { peak area } \\
(\%)\end{array}$ & $\begin{array}{l}\text { Relative } \\
\text { Peak } \\
\text { height (\%) }\end{array}$ & $\begin{array}{l}\text { Sample } \\
\text { volume } \\
\text { n.a. }\end{array}$ \\
\hline
\end{tabular}



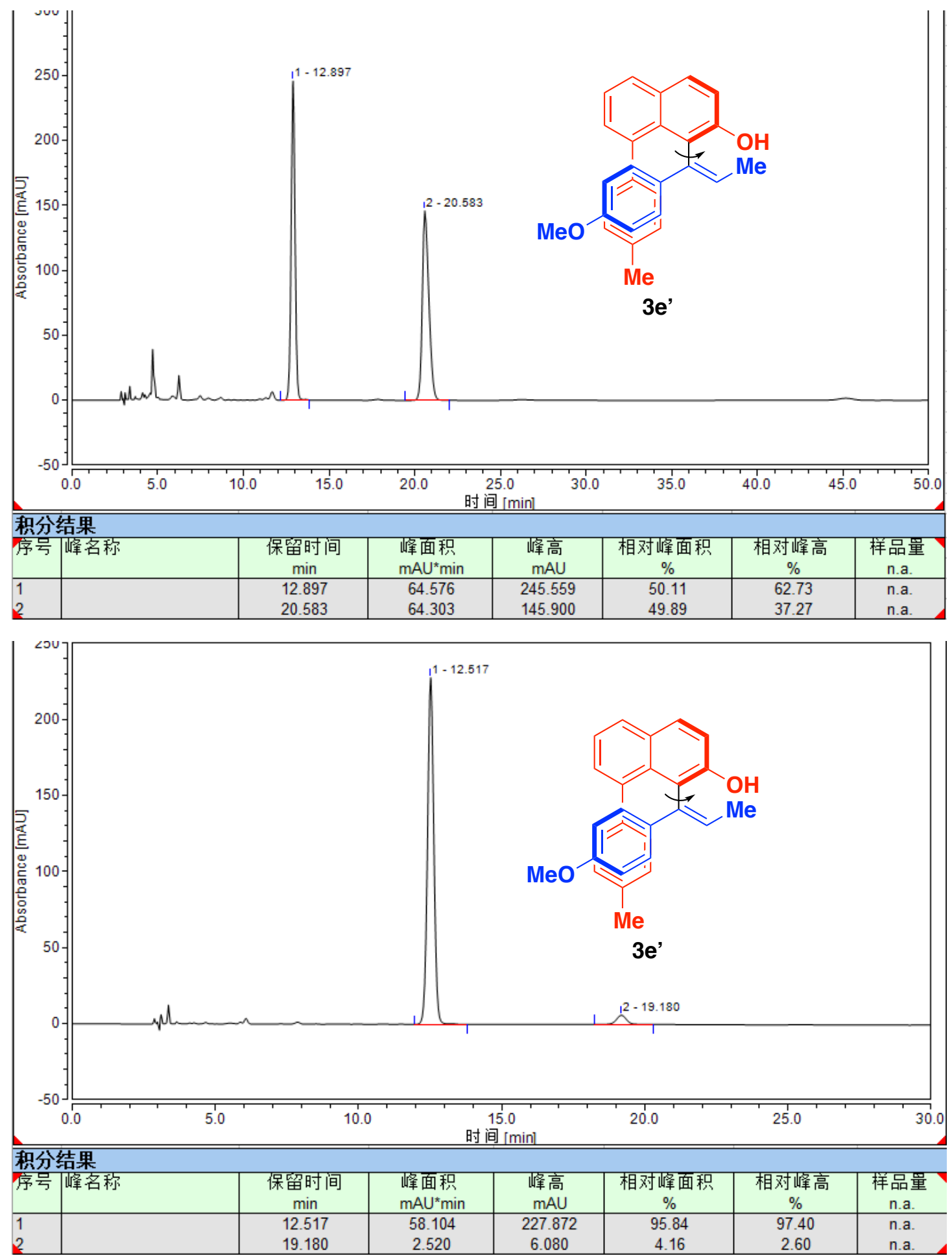

For comparison of Chinese and English of the HPLC data table.

\begin{tabular}{|l|l|l|l|l|l|l|l|}
\hline 序号 & $\begin{array}{l}\text { 峰名 } \\
\text { 称 }\end{array}$ & $\begin{array}{l}\text { 保留时间 } \\
(\mathrm{min})\end{array}$ & $\begin{array}{l}\text { 峰面积 } \\
(\mathrm{mAU} \text { *min) }\end{array}$ & $\begin{array}{l}\text { 峰高 } \\
(\mathrm{mAU})\end{array}$ & $\begin{array}{l}\text { 相对峰面 } \\
\text { 积 }(\%)\end{array}$ & $\begin{array}{l}\text { 相对峰高 } \\
(\%)\end{array}$ & $\begin{array}{l}\text { 样品量 } \\
\text { n.a. }\end{array}$ \\
\hline $\begin{array}{l}\text { Entr } \\
\mathrm{y}\end{array}$ & $\begin{array}{l}\text { Peak } \\
\text { name }\end{array}$ & $\begin{array}{l}\text { Retention } \\
\text { time (min) }\end{array}$ & $\begin{array}{l}\text { Peak area } \\
\left(\mathrm{mAU} \mathrm{U}^{*} \text { min }\right)\end{array}$ & $\begin{array}{l}\text { Peak } \\
\text { height } \\
(\mathrm{mAU})\end{array}$ & $\begin{array}{l}\text { Relative } \\
\text { peak area } \\
(\%)\end{array}$ & $\begin{array}{l}\text { Relative } \\
\text { Peak } \\
\text { height (\%) }\end{array}$ & $\begin{array}{l}\text { Sample } \\
\text { volume } \\
\text { n.a. }\end{array}$ \\
\hline
\end{tabular}



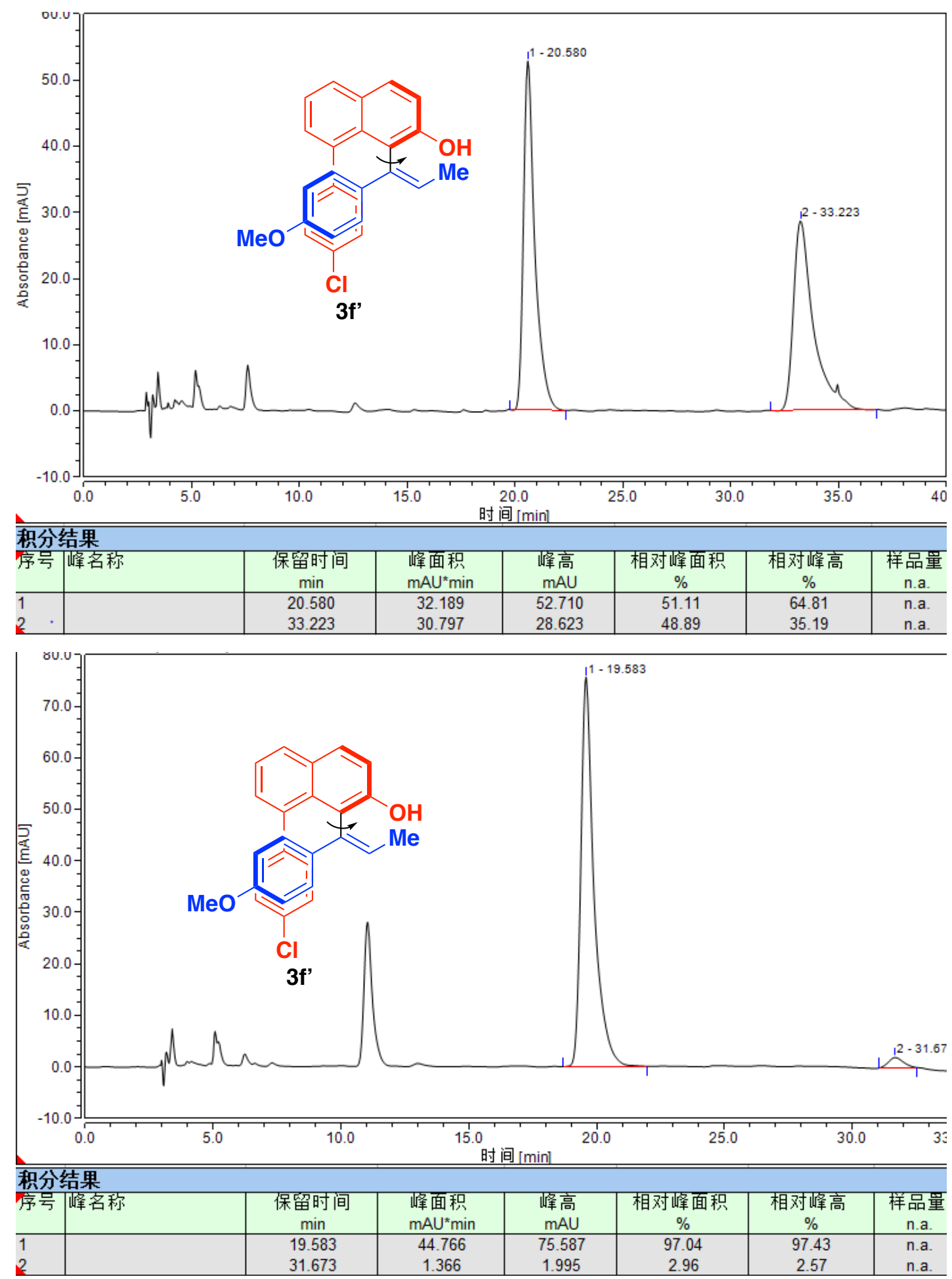

For comparison of Chinese and English of the HPLC data table.

\begin{tabular}{|l|l|l|l|l|l|l|l|}
\hline 序号 & $\begin{array}{l}\text { 峰名 } \\
\text { 称 }\end{array}$ & $\begin{array}{l}\text { 保留时间 } \\
(\mathrm{min})\end{array}$ & $\begin{array}{l}\text { 峰面积 } \\
(\mathrm{mAU} \text { *min) }\end{array}$ & $\begin{array}{l}\text { 峰高 } \\
(\mathrm{mAU})\end{array}$ & $\begin{array}{l}\text { 相对峰面 } \\
\text { 积 }(\%)\end{array}$ & $\begin{array}{l}\text { 相对峰高 } \\
(\%)\end{array}$ & $\begin{array}{l}\text { 样品量 } \\
\text { n.a. }\end{array}$ \\
\hline $\begin{array}{l}\text { Entr } \\
\mathrm{y}\end{array}$ & $\begin{array}{l}\text { Peak } \\
\text { name }\end{array}$ & $\begin{array}{l}\text { Retention } \\
\text { time (min) }\end{array}$ & $\begin{array}{l}\text { Peak area } \\
\left(\mathrm{mAU} \mathrm{U}^{*} \text { min }\right)\end{array}$ & $\begin{array}{l}\text { Peak } \\
\text { height } \\
(\mathrm{mAU})\end{array}$ & $\begin{array}{l}\text { Relative } \\
\text { peak area } \\
(\%)\end{array}$ & $\begin{array}{l}\text { Relative } \\
\text { Peak } \\
\text { height (\%) }\end{array}$ & $\begin{array}{l}\text { Sample } \\
\text { volume } \\
\text { n.a. }\end{array}$ \\
\hline
\end{tabular}



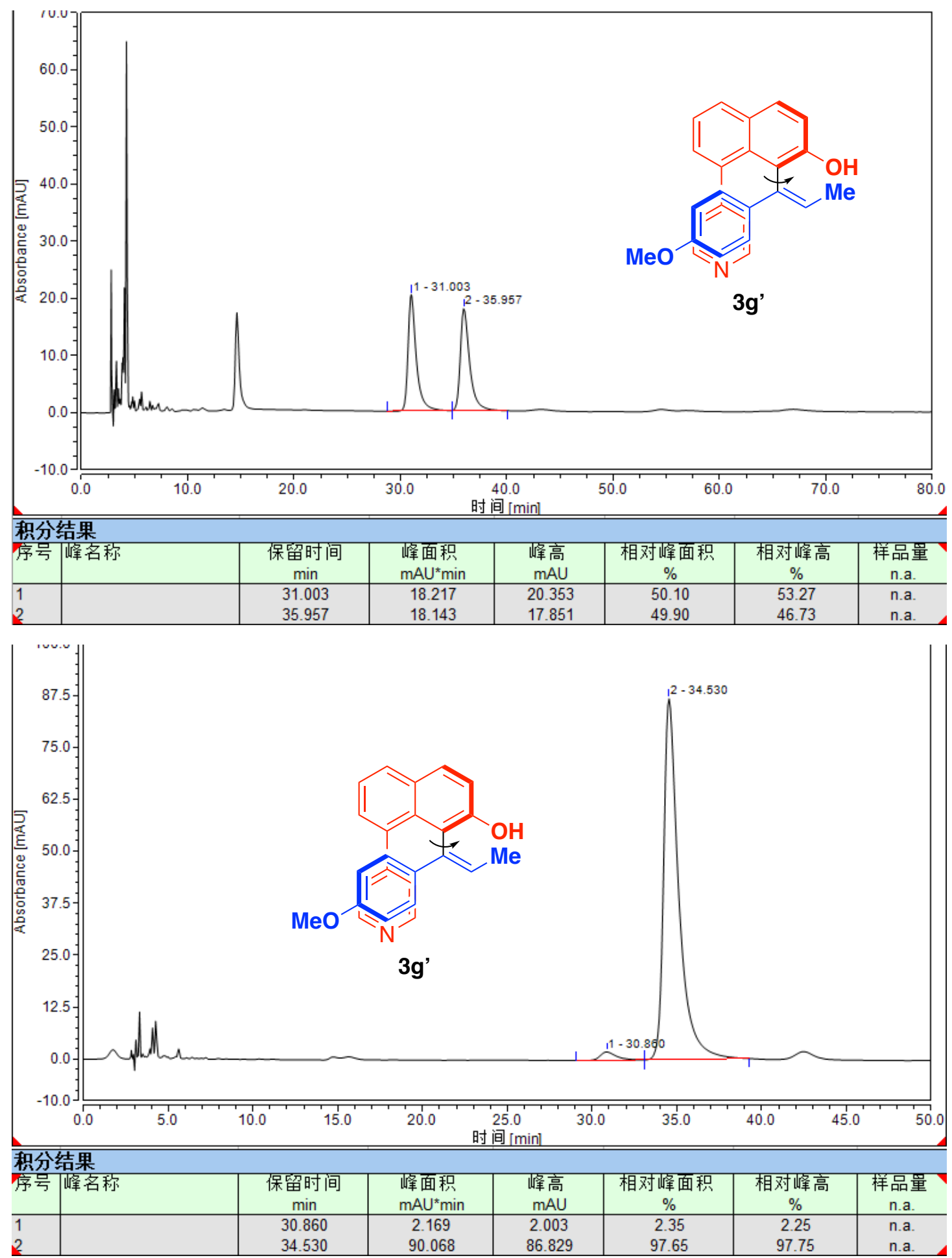

For comparison of Chinese and English of the HPLC data table.

\begin{tabular}{|l|l|l|l|l|l|l|l|}
\hline 序号 & $\begin{array}{l}\text { 峰名 } \\
\text { 称 }\end{array}$ & $\begin{array}{l}\text { 保留时间 } \\
(\mathrm{min})\end{array}$ & $\begin{array}{l}\text { 峰面积 } \\
(\mathrm{mAU} \text { *min) }\end{array}$ & $\begin{array}{l}\text { 峰高 } \\
(\mathrm{mAU})\end{array}$ & $\begin{array}{l}\text { 相对峰面 } \\
\text { 积 }(\%)\end{array}$ & $\begin{array}{l}\text { 相对峰高 } \\
(\%)\end{array}$ & $\begin{array}{l}\text { 样品量 } \\
\text { n.a. }\end{array}$ \\
\hline $\begin{array}{l}\text { Entr } \\
\mathrm{y}\end{array}$ & $\begin{array}{l}\text { Peak } \\
\text { name }\end{array}$ & $\begin{array}{l}\text { Retention } \\
\text { time (min) }\end{array}$ & $\begin{array}{l}\text { Peak area } \\
\left(\mathrm{mAU} \mathrm{U}^{*} \text { min }\right)\end{array}$ & $\begin{array}{l}\text { Peak } \\
\text { height } \\
(\mathrm{mAU})\end{array}$ & $\begin{array}{l}\text { Relative } \\
\text { peak area } \\
(\%)\end{array}$ & $\begin{array}{l}\text { Relative } \\
\text { Peak } \\
\text { height (\%) }\end{array}$ & $\begin{array}{l}\text { Sample } \\
\text { volume } \\
\text { n.a. }\end{array}$ \\
\hline
\end{tabular}



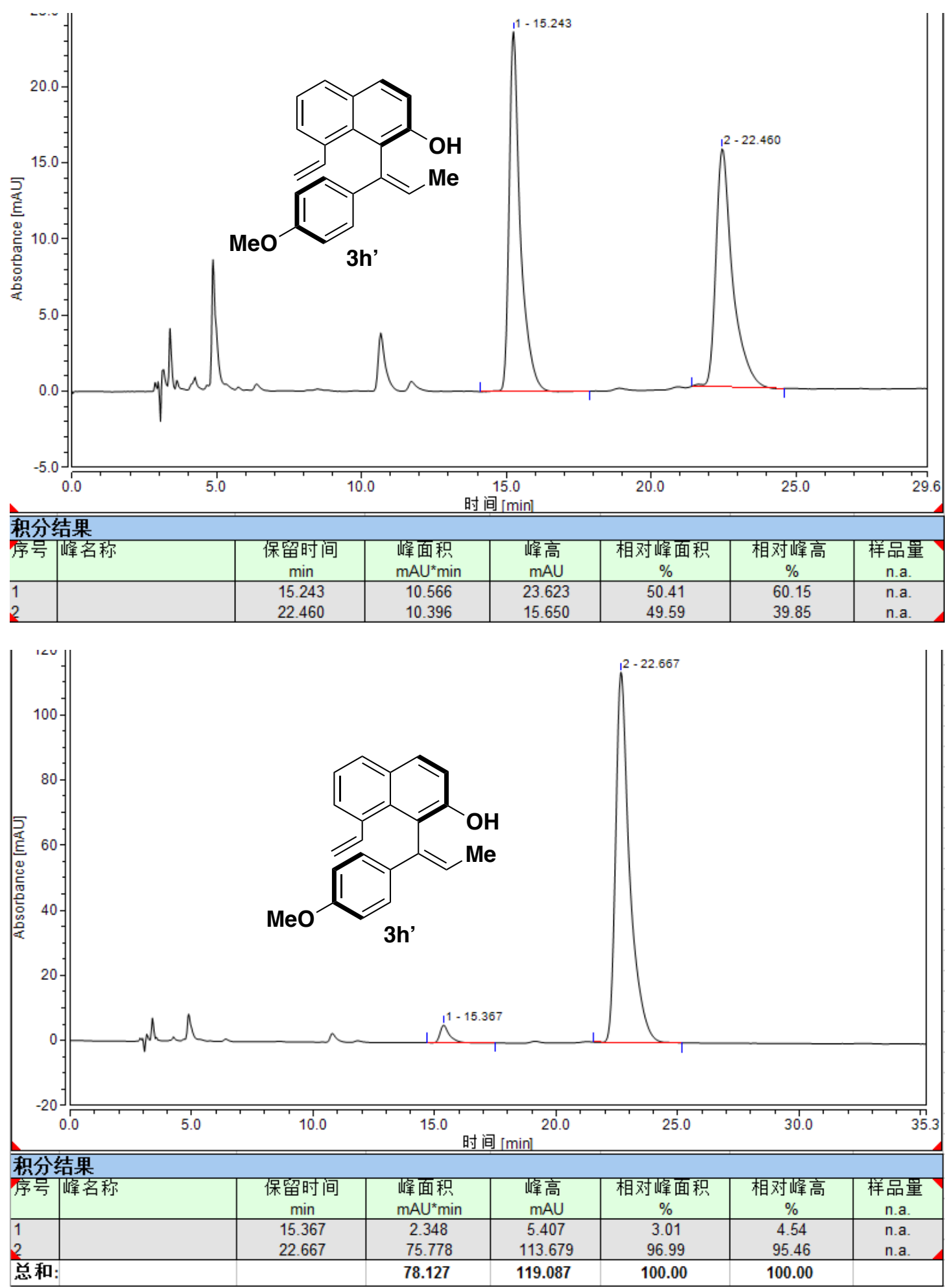

For comparison of Chinese and English of the HPLC data table.

\begin{tabular}{|c|c|c|c|c|c|c|c|}
\hline 序号 & $\begin{array}{l}\text { 峰名 } \\
\text { 称 }\end{array}$ & $\begin{array}{l}\text { 保留时间 } \\
\text { (min) }\end{array}$ & $\begin{array}{l}\text { 峰面积 } \\
\text { (mAU*min) }\end{array}$ & $\begin{array}{l}\text { 峰高 } \\
(\mathrm{mAU})\end{array}$ & $\begin{array}{l}\text { 相对峰面 } \\
\text { 积 }(\%)\end{array}$ & $\begin{array}{l}\text { 相对峰高 } \\
(\%)\end{array}$ & $\begin{array}{l}\text { 样品量 } \\
\text { n.a. }\end{array}$ \\
\hline $\begin{array}{l}\text { Entr } \\
\mathrm{y}\end{array}$ & $\begin{array}{l}\text { Peak } \\
\text { name }\end{array}$ & $\begin{array}{l}\text { Retention } \\
\text { time (min) }\end{array}$ & $\begin{array}{l}\text { Peak area } \\
\left(\mathrm{mAU}{ }^{*} \min \right)\end{array}$ & $\begin{array}{l}\text { Peak } \\
\text { height } \\
(\mathrm{mAU})\end{array}$ & $\begin{array}{l}\text { Relative } \\
\text { peak area } \\
(\%)\end{array}$ & $\begin{array}{l}\text { Relative } \\
\text { Peak } \\
\text { height (\%) }\end{array}$ & $\begin{array}{l}\text { Sample } \\
\text { volume } \\
\text { n.a. }\end{array}$ \\
\hline
\end{tabular}



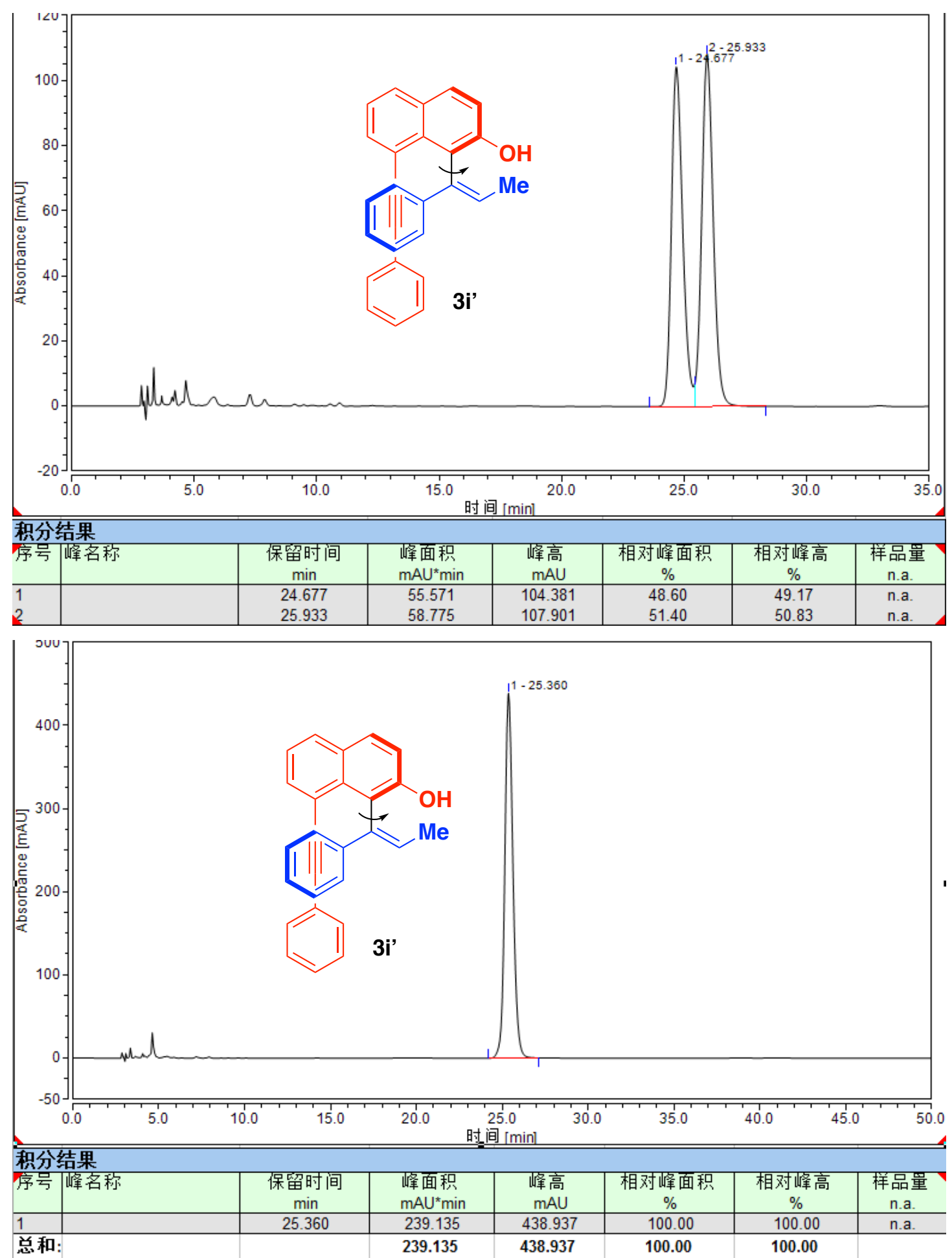

For comparison of Chinese and English of the HPLC data table.

\begin{tabular}{|l|l|l|l|l|l|l|l|}
\hline 序号 & $\begin{array}{l}\text { 峰名 } \\
\text { 称 }\end{array}$ & $\begin{array}{l}\text { 保留时间 } \\
(\mathrm{min})\end{array}$ & $\begin{array}{l}\text { 峰面积 } \\
(\mathrm{mAU} \text { *min) }\end{array}$ & $\begin{array}{l}\text { 峰高 } \\
(\mathrm{mAU})\end{array}$ & $\begin{array}{l}\text { 相对峰面 } \\
\text { 积 }(\%)\end{array}$ & $\begin{array}{l}\text { 相对峰高 } \\
(\%)\end{array}$ & $\begin{array}{l}\text { 样品量 } \\
\text { n.a. }\end{array}$ \\
\hline $\begin{array}{l}\text { Entr } \\
\mathrm{y}\end{array}$ & $\begin{array}{l}\text { Peak } \\
\text { name }\end{array}$ & $\begin{array}{l}\text { Retention } \\
\text { time (min) }\end{array}$ & $\begin{array}{l}\text { Peak area } \\
\left(\mathrm{mAU} \mathrm{U}^{*} \text { min }\right)\end{array}$ & $\begin{array}{l}\text { Peak } \\
\text { height } \\
(\mathrm{mAU})\end{array}$ & $\begin{array}{l}\text { Relative } \\
\text { peak area } \\
(\%)\end{array}$ & $\begin{array}{l}\text { Relative } \\
\text { Peak } \\
\text { height (\%) }\end{array}$ & $\begin{array}{l}\text { Sample } \\
\text { volume } \\
\text { n.a. }\end{array}$ \\
\hline
\end{tabular}



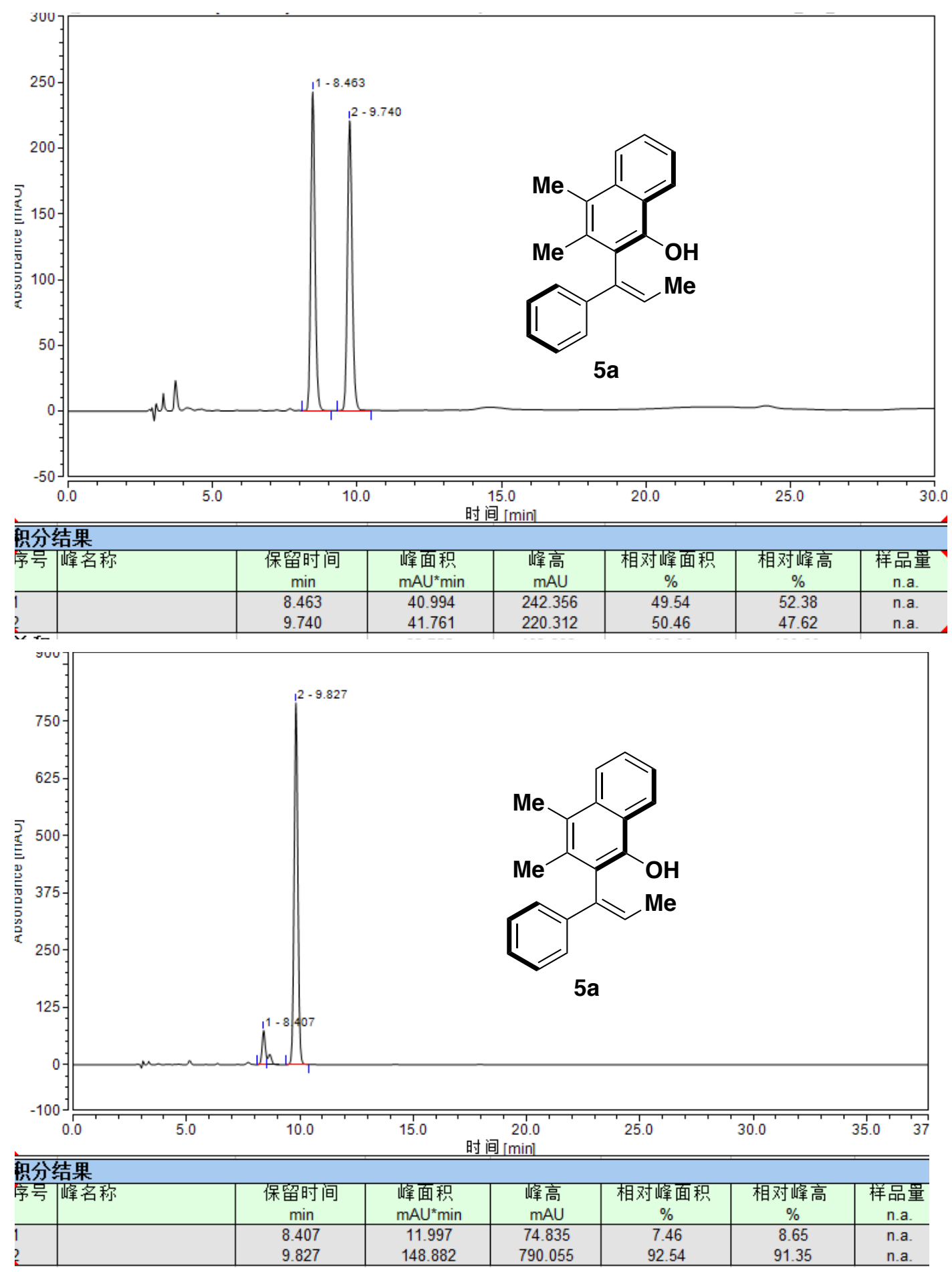

For comparison of Chinese and English of the HPLC data table.

\begin{tabular}{|c|c|c|c|c|c|c|c|}
\hline 序号 & $\begin{array}{l}\text { 峰名 } \\
\text { 称 }\end{array}$ & $\begin{array}{l}\text { 保留时间 } \\
\text { (min) }\end{array}$ & $\begin{array}{l}\text { 峰面积 } \\
\left(\mathrm{mAU}{ }^{*} \min \right)\end{array}$ & $\begin{array}{l}\text { 峰高 } \\
\text { (mAU) }\end{array}$ & $\begin{array}{l}\text { 相对峰面 } \\
\text { 积 (\%) }\end{array}$ & $\begin{array}{l}\text { 相对峰高 } \\
(\%)\end{array}$ & $\begin{array}{l}\text { 样品量 } \\
\text { n.a. }\end{array}$ \\
\hline $\begin{array}{l}\text { Entr } \\
\mathrm{y}\end{array}$ & $\begin{array}{l}\text { Peak } \\
\text { name }\end{array}$ & $\begin{array}{l}\text { Retention } \\
\text { time (min) }\end{array}$ & $\begin{array}{l}\text { Peak area } \\
\left(\mathrm{mAU}^{*} \min \right)\end{array}$ & $\begin{array}{l}\text { Peak } \\
\text { height } \\
(\mathrm{mAU})\end{array}$ & $\begin{array}{l}\text { Relative } \\
\text { peak area } \\
(\%)\end{array}$ & $\begin{array}{l}\text { Relative } \\
\text { Peak } \\
\text { height }(\%)\end{array}$ & $\begin{array}{l}\text { Sample } \\
\text { volume } \\
\text { n.a. }\end{array}$ \\
\hline
\end{tabular}



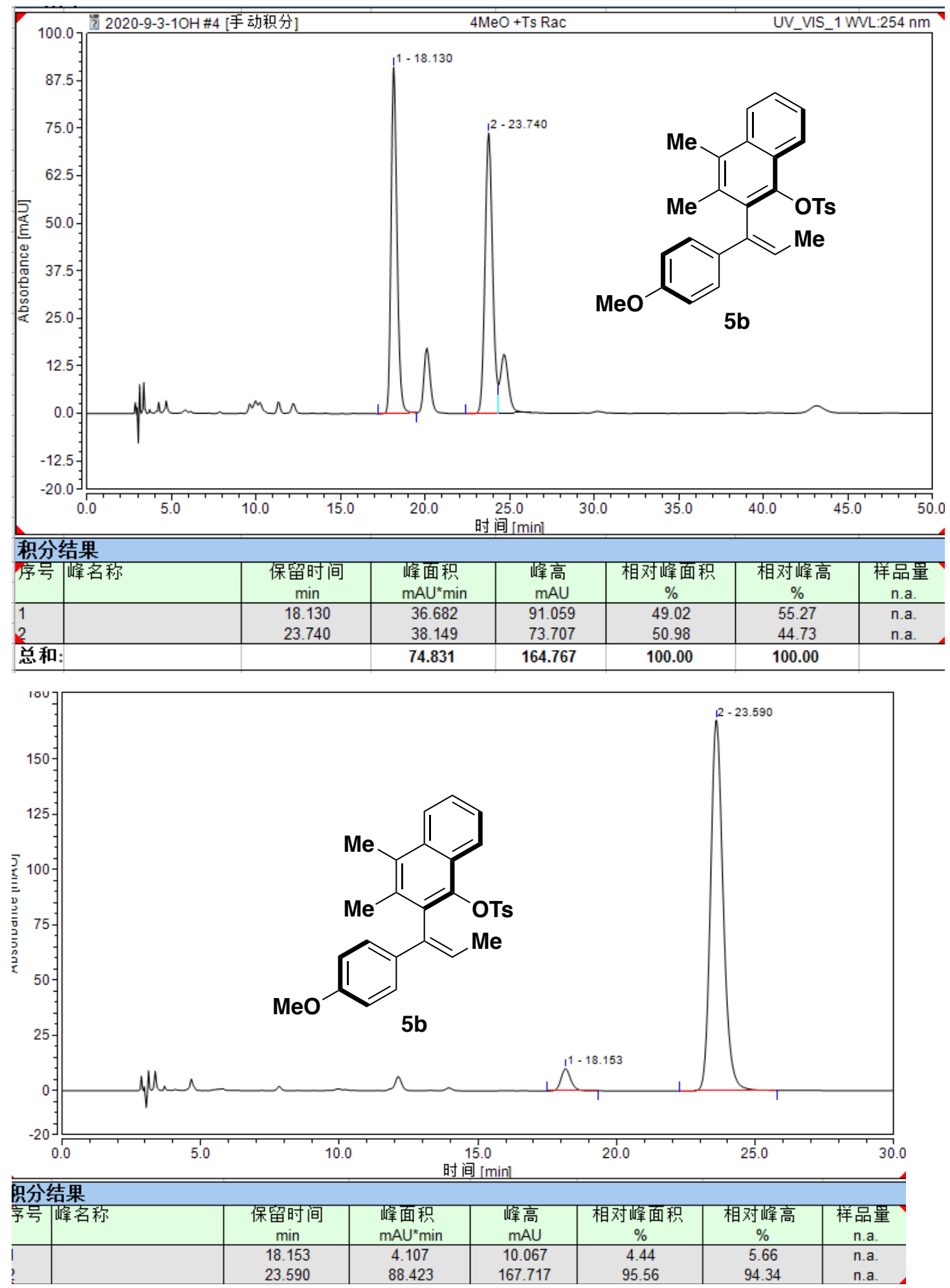

For comparison of Chinese and English of the HPLC data table.

\begin{tabular}{|l|l|l|l|l|l|l|l|}
\hline 序号 & $\begin{array}{l}\text { 峰名 } \\
\text { 称 }\end{array}$ & $\begin{array}{l}\text { 保留时间 } \\
(\mathrm{min})\end{array}$ & $\begin{array}{l}\text { 峰面积 } \\
(\mathrm{mAU} \text { *min) }\end{array}$ & $\begin{array}{l}\text { 峰高 } \\
(\mathrm{mAU})\end{array}$ & $\begin{array}{l}\text { 相对峰面 } \\
\text { 积 }(\%)\end{array}$ & $\begin{array}{l}\text { 相对峰高 } \\
(\%)\end{array}$ & $\begin{array}{l}\text { 样品量 } \\
\text { n.a. }\end{array}$ \\
\hline $\begin{array}{l}\text { Entr } \\
\mathrm{y}\end{array}$ & $\begin{array}{l}\text { Peak } \\
\text { name }\end{array}$ & $\begin{array}{l}\text { Retention } \\
\text { time (min) }\end{array}$ & $\begin{array}{l}\text { Peak area } \\
(\mathrm{mAU} * \mathrm{~min})\end{array}$ & $\begin{array}{l}\text { Peak } \\
\text { height } \\
(\mathrm{mAU})\end{array}$ & $\begin{array}{l}\text { Relative } \\
\text { peak area } \\
(\%)\end{array}$ & $\begin{array}{l}\text { Relative } \\
\text { Peak } \\
\text { height (\%) }\end{array}$ & $\begin{array}{l}\text { Sample } \\
\text { volume } \\
\text { n.a. }\end{array}$ \\
\hline
\end{tabular}




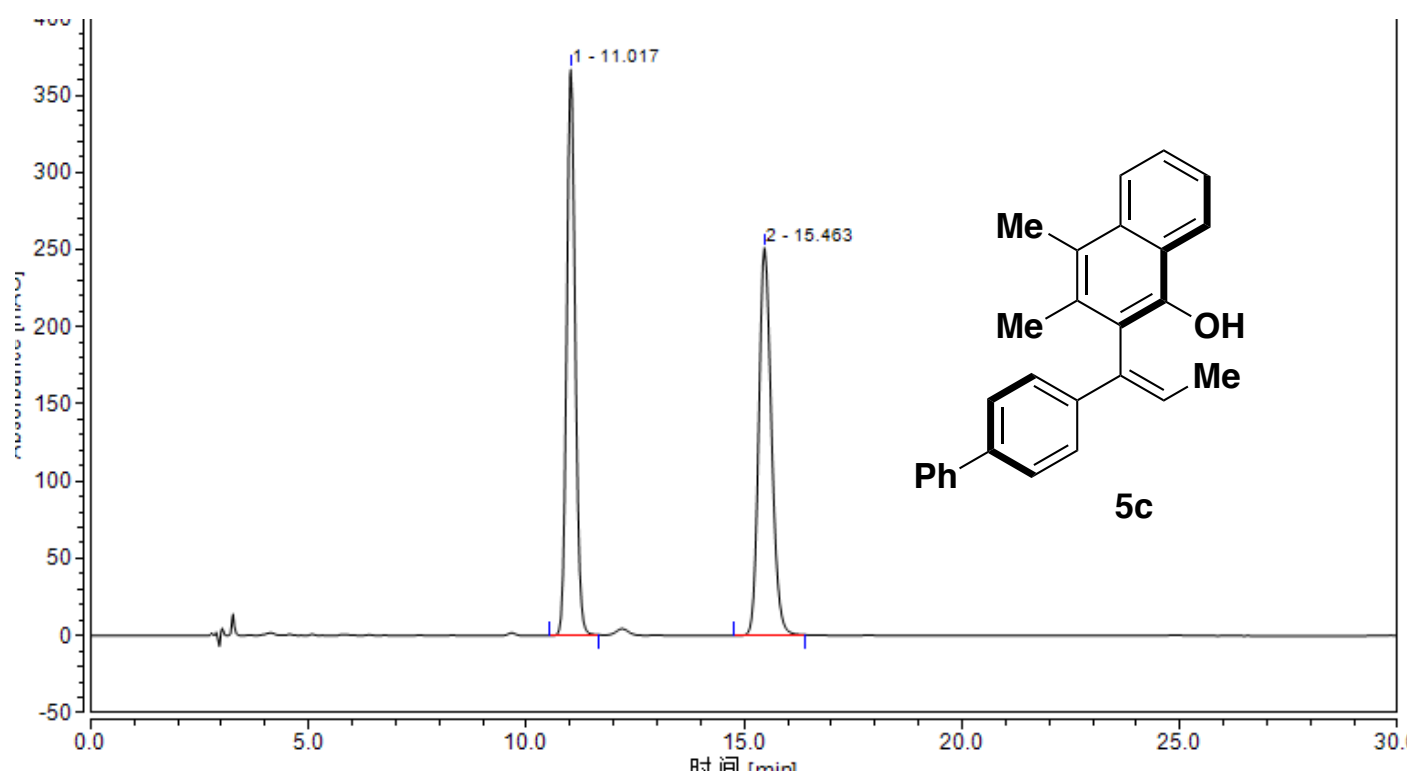

\begin{tabular}{|c|c|c|c|c|c|c|}
\hline \multicolumn{7}{|l|}{ 召分结果 } \\
\hline 予号峰名称 & $\begin{array}{c}\text { 保留时间 } \\
\text { min }\end{array}$ & $\begin{array}{l}\text { 峰面积 } \\
\mathrm{mAU}^{*} \text { min }\end{array}$ & $\begin{array}{c}\text { 峰高 } \\
\text { mAU }\end{array}$ & $\begin{array}{c}\text { 相对峰面积 } \\
\%\end{array}$ & $\begin{array}{c}\text { 相对峰高 } \\
\%\end{array}$ & $\begin{array}{c}\text { 样品量 } \\
\text { n.a. }\end{array}$ \\
\hline & 11.017 & 83.569 & 366.463 & 49.66 & 59.35 & n.a. \\
\hline & 15.463 & 84.701 & 250.963 & 50.34 & 40.65 & n.a. \\
\hline
\end{tabular}

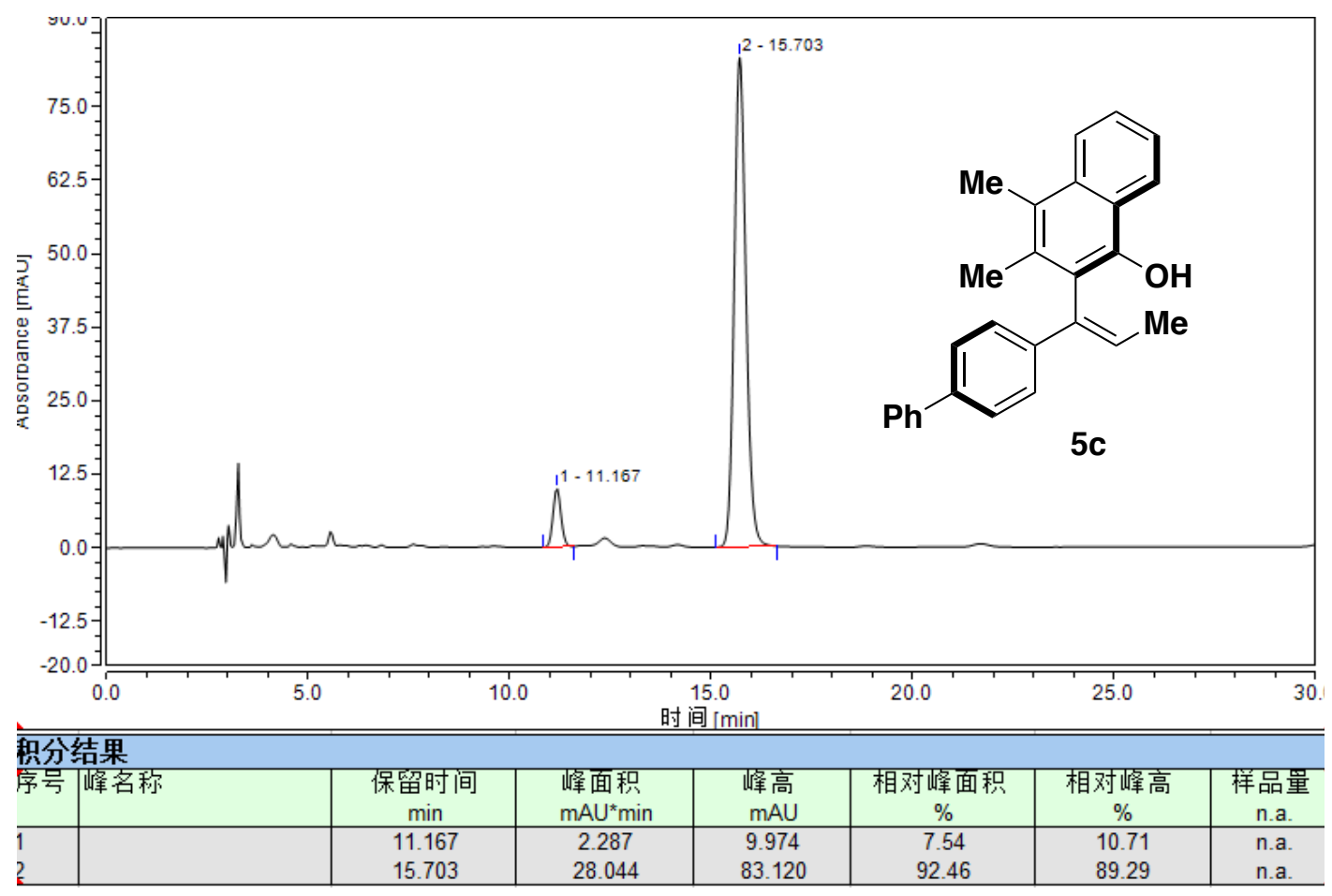

For comparison of Chinese and English of the HPLC data table.

\begin{tabular}{|l|l|l|l|l|l|l|l|}
\hline 序号 & $\begin{array}{l}\text { 峰名 } \\
\text { 称 }\end{array}$ & $\begin{array}{l}\text { 保留时间 } \\
(\mathrm{min})\end{array}$ & $\begin{array}{l}\text { 峰面积 } \\
(\mathrm{mAU} \text { *min) }\end{array}$ & $\begin{array}{l}\text { 峰高 } \\
(\mathrm{mAU})\end{array}$ & $\begin{array}{l}\text { 相对峰面 } \\
\text { 积 }(\%)\end{array}$ & $\begin{array}{l}\text { 相对峰高 } \\
(\%)\end{array}$ & $\begin{array}{l}\text { 样品量 } \\
\text { n.a. }\end{array}$ \\
\hline $\begin{array}{l}\text { Entr } \\
\mathrm{y}\end{array}$ & $\begin{array}{l}\text { Peak } \\
\text { name }\end{array}$ & $\begin{array}{l}\text { Retention } \\
\text { time (min) }\end{array}$ & $\begin{array}{l}\text { Peak area } \\
\left(\mathrm{mAU} \mathrm{U}^{*} \text { min }\right)\end{array}$ & $\begin{array}{l}\text { Peak } \\
\text { height } \\
(\mathrm{mAU})\end{array}$ & $\begin{array}{l}\text { Relative } \\
\text { peak area } \\
(\%)\end{array}$ & $\begin{array}{l}\text { Relative } \\
\text { Peak } \\
\text { height (\%) }\end{array}$ & $\begin{array}{l}\text { Sample } \\
\text { volume } \\
\text { n.a. }\end{array}$ \\
\hline
\end{tabular}




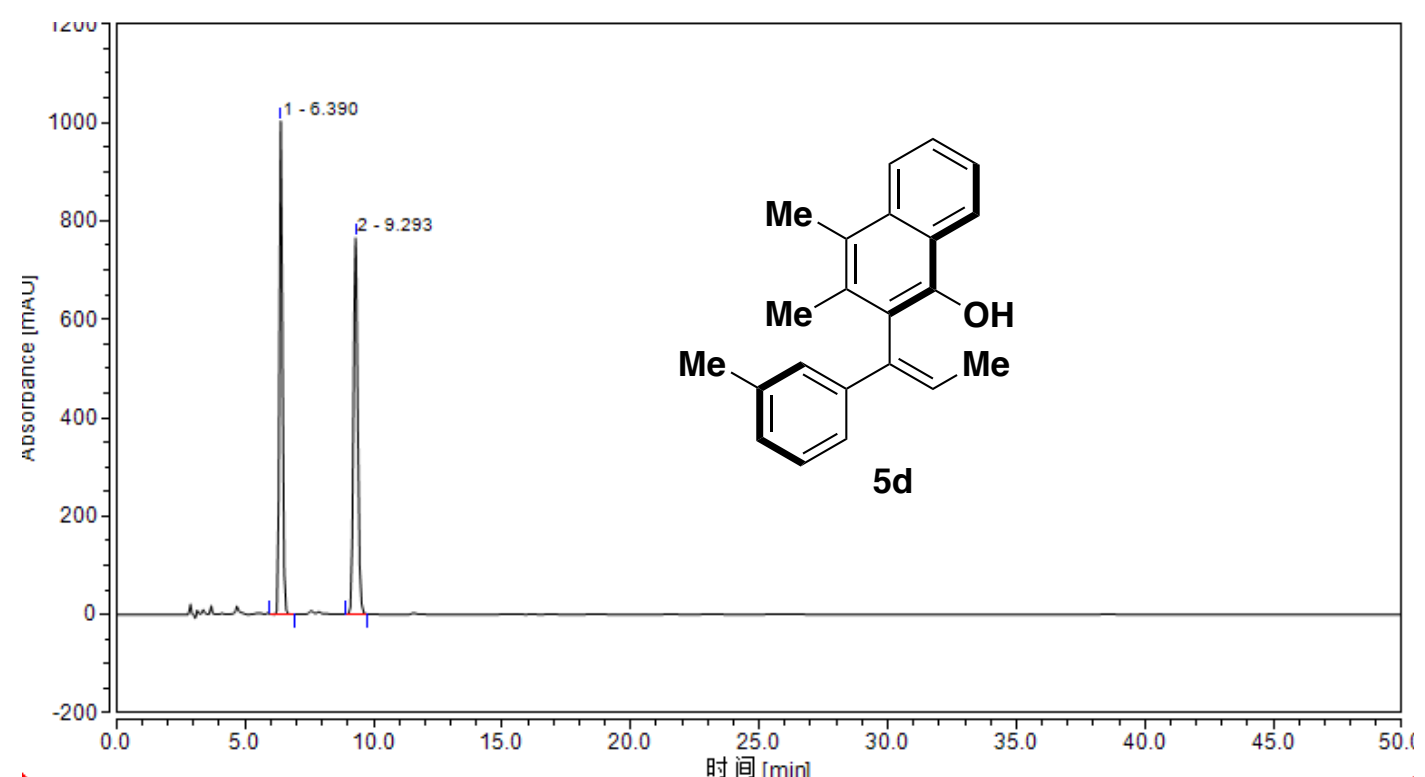

\begin{tabular}{|c|c|c|c|c|c|c|c|}
\hline \multicolumn{8}{|c|}{ 积分结果 } \\
\hline 序㕺 & 峰名称 & $\begin{array}{c}\text { 保留时间 } \\
\text { min }\end{array}$ & $\begin{array}{l}\text { 峰面积 } \\
m A U^{*} \text { min }\end{array}$ & $\begin{array}{l}\text { 峰高 } \\
\text { mAU }\end{array}$ & $\begin{array}{c}\text { 相对峰面积 } \\
\%\end{array}$ & $\begin{array}{c}\text { 相对峰高 } \\
\%\end{array}$ & $\begin{array}{c}\text { 样品黑 } \\
\text { n.a. }\end{array}$ \\
\hline ? & & $\begin{array}{l}6.390 \\
9.293 \\
\end{array}$ & $\begin{array}{l}140.476 \\
145.272 \\
\end{array}$ & $\begin{array}{l}1002.079 \\
764.410\end{array}$ & $\begin{array}{l}49.16 \\
50.84 \\
\end{array}$ & $\begin{array}{l}56.73 \\
43.27 \\
\end{array}$ & $\begin{array}{l}\text { n.a. } \\
\text { n.a. }\end{array}$ \\
\hline
\end{tabular}

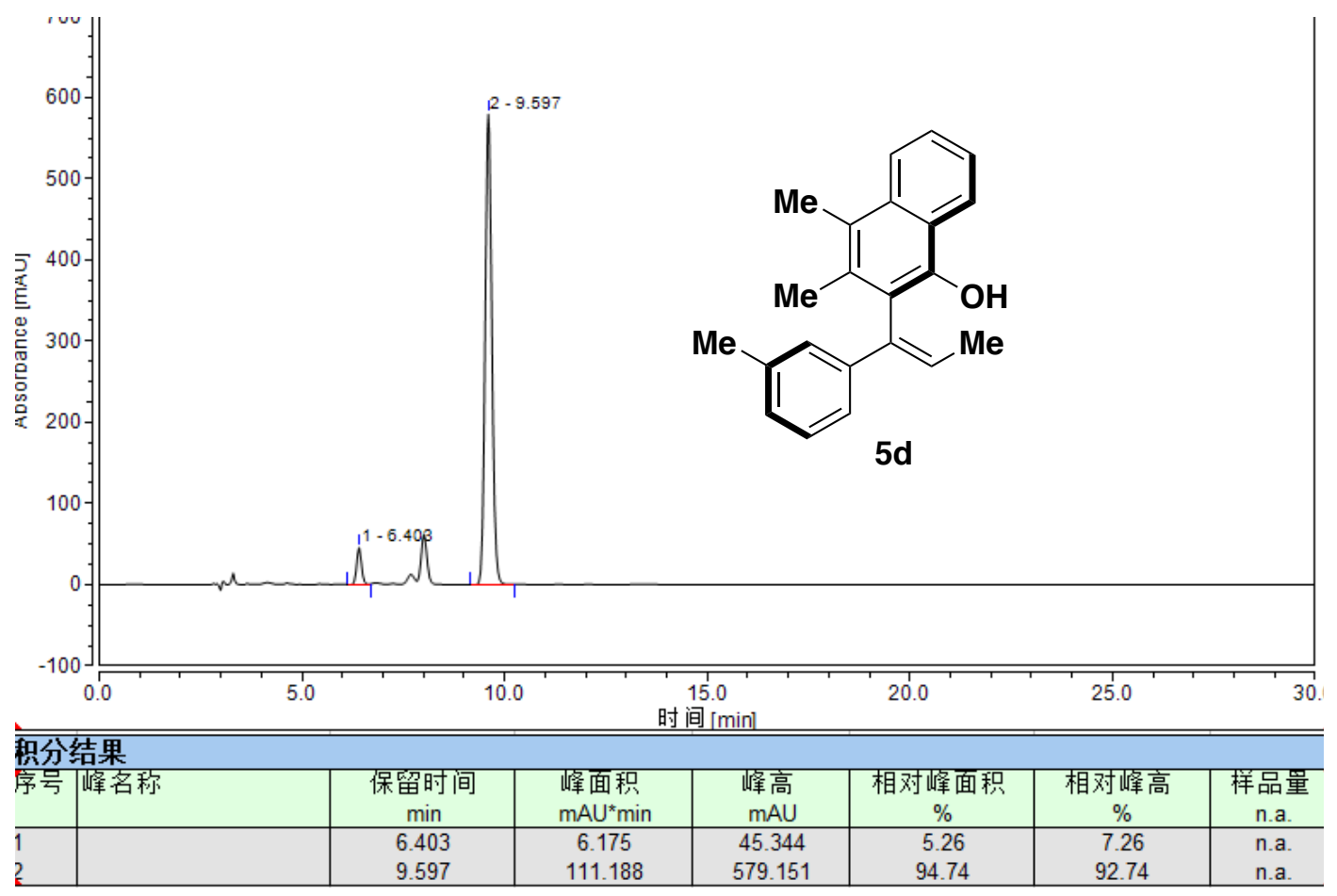

For comparison of Chinese and English of the HPLC data table.

\begin{tabular}{|c|c|c|c|c|c|c|c|}
\hline 序号 & $\begin{array}{l}\text { 峰名 } \\
\text { 称 }\end{array}$ & $\begin{array}{l}\text { 保留时间 } \\
\text { (min) }\end{array}$ & $\begin{array}{l}\text { 峰面积 } \\
\left(\mathrm{mAU} \mathrm{U}^{*} \mathrm{~min}\right)\end{array}$ & $\begin{array}{l}\text { 峰高 } \\
(\mathrm{mAU})\end{array}$ & $\begin{array}{l}\text { 相对峰面 } \\
\text { 积 }(\%)\end{array}$ & $\begin{array}{l}\text { 相对峰高 } \\
(\%)\end{array}$ & $\begin{array}{l}\text { 样品量 } \\
\text { n.a. }\end{array}$ \\
\hline $\begin{array}{l}\text { Entr } \\
\mathrm{y}\end{array}$ & $\begin{array}{l}\text { Peak } \\
\text { name }\end{array}$ & $\begin{array}{l}\text { Retention } \\
\text { time (min) }\end{array}$ & $\begin{array}{l}\text { Peak area } \\
\left(\mathrm{mAU} *^{*} \min \right)\end{array}$ & $\begin{array}{l}\text { Peak } \\
\text { height } \\
\text { (mAU) }\end{array}$ & $\begin{array}{l}\text { Relative } \\
\text { peak area } \\
(\%)\end{array}$ & $\begin{array}{l}\text { Relative } \\
\text { Peak } \\
\text { height }(\%)\end{array}$ & $\begin{array}{l}\text { Sample } \\
\text { volume } \\
\text { n.a. }\end{array}$ \\
\hline
\end{tabular}



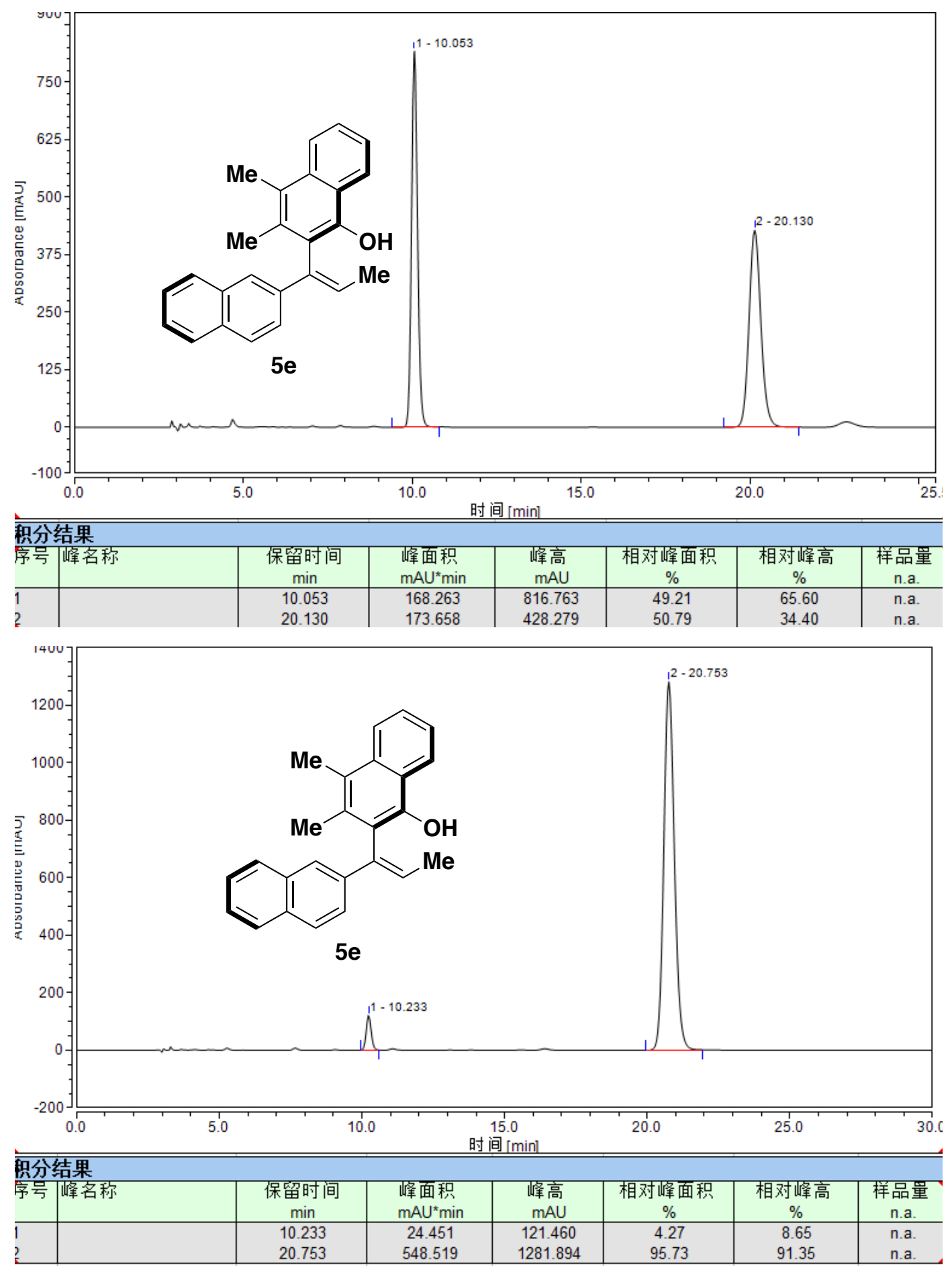

For comparison of Chinese and English of the HPLC data table.

\begin{tabular}{|c|c|c|c|c|c|c|c|}
\hline 序号 & $\begin{array}{l}\text { 峰名 } \\
\text { 称 }\end{array}$ & $\begin{array}{l}\text { 保留时间 } \\
\text { (min) }\end{array}$ & $\begin{array}{l}\text { 峰面积 } \\
\left(\mathrm{mAU}{ }^{*} \min \right)\end{array}$ & $\begin{array}{l}\text { 峰高 } \\
\text { (mAU) }\end{array}$ & $\begin{array}{l}\text { 相对峰面 } \\
\text { 积 (\%) }\end{array}$ & $\begin{array}{l}\text { 相对峰高 } \\
(\%)\end{array}$ & $\begin{array}{l}\text { 样品量 } \\
\text { n.a. }\end{array}$ \\
\hline $\begin{array}{l}\text { Entr } \\
\mathrm{y}\end{array}$ & $\begin{array}{l}\text { Peak } \\
\text { name }\end{array}$ & $\begin{array}{l}\text { Retention } \\
\text { time (min) }\end{array}$ & $\begin{array}{l}\text { Peak area } \\
\left(\mathrm{mAU}{ }^{*} \min \right)\end{array}$ & $\begin{array}{l}\text { Peak } \\
\text { height } \\
(\mathrm{mAU})\end{array}$ & $\begin{array}{l}\text { Relative } \\
\text { peak area } \\
(\%)\end{array}$ & $\begin{array}{l}\text { Relative } \\
\text { Peak } \\
\text { height (\%) }\end{array}$ & $\begin{array}{l}\text { Sample } \\
\text { volume } \\
\text { n.a. }\end{array}$ \\
\hline
\end{tabular}




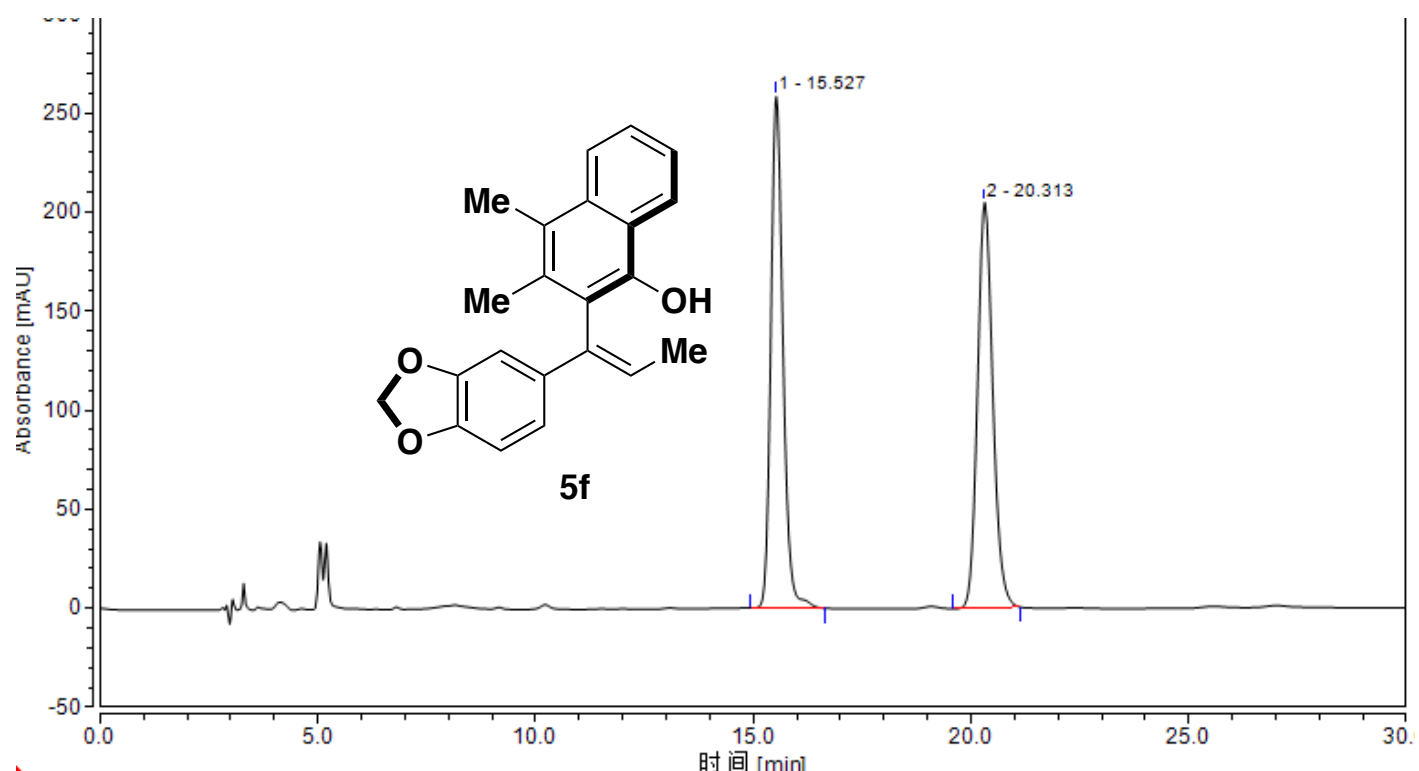

\begin{tabular}{|c|c|c|c|c|c|c|c|}
\hline \multicolumn{8}{|c|}{ 积分结果 } \\
\hline 序咢 & 峰名称 & $\begin{array}{c}\text { 保留时间 } \\
\text { min }\end{array}$ & $\begin{array}{l}\text { 峰面积 } \\
m A U^{*} \text { min }\end{array}$ & $\begin{array}{c}\text { 峰高 } \\
\text { mAU }\end{array}$ & $\begin{array}{c}\text { 相对峰面积 } \\
\%\end{array}$ & $\begin{array}{c}\text { 相对峰高 } \\
\%\end{array}$ & $\begin{array}{c}\text { 样品量 } \\
\text { n.a. }\end{array}$ \\
\hline ? & & $\begin{array}{l}15.527 \\
20.313\end{array}$ & $\begin{array}{l}83.069 \\
83.497\end{array}$ & $\begin{array}{l}258.281 \\
204.738\end{array}$ & $\begin{array}{l}49.87 \\
50.13\end{array}$ & $\begin{array}{l}55.78 \\
44.22\end{array}$ & $\begin{array}{l}\text { n.a. } \\
\text { n.a. }\end{array}$ \\
\hline
\end{tabular}

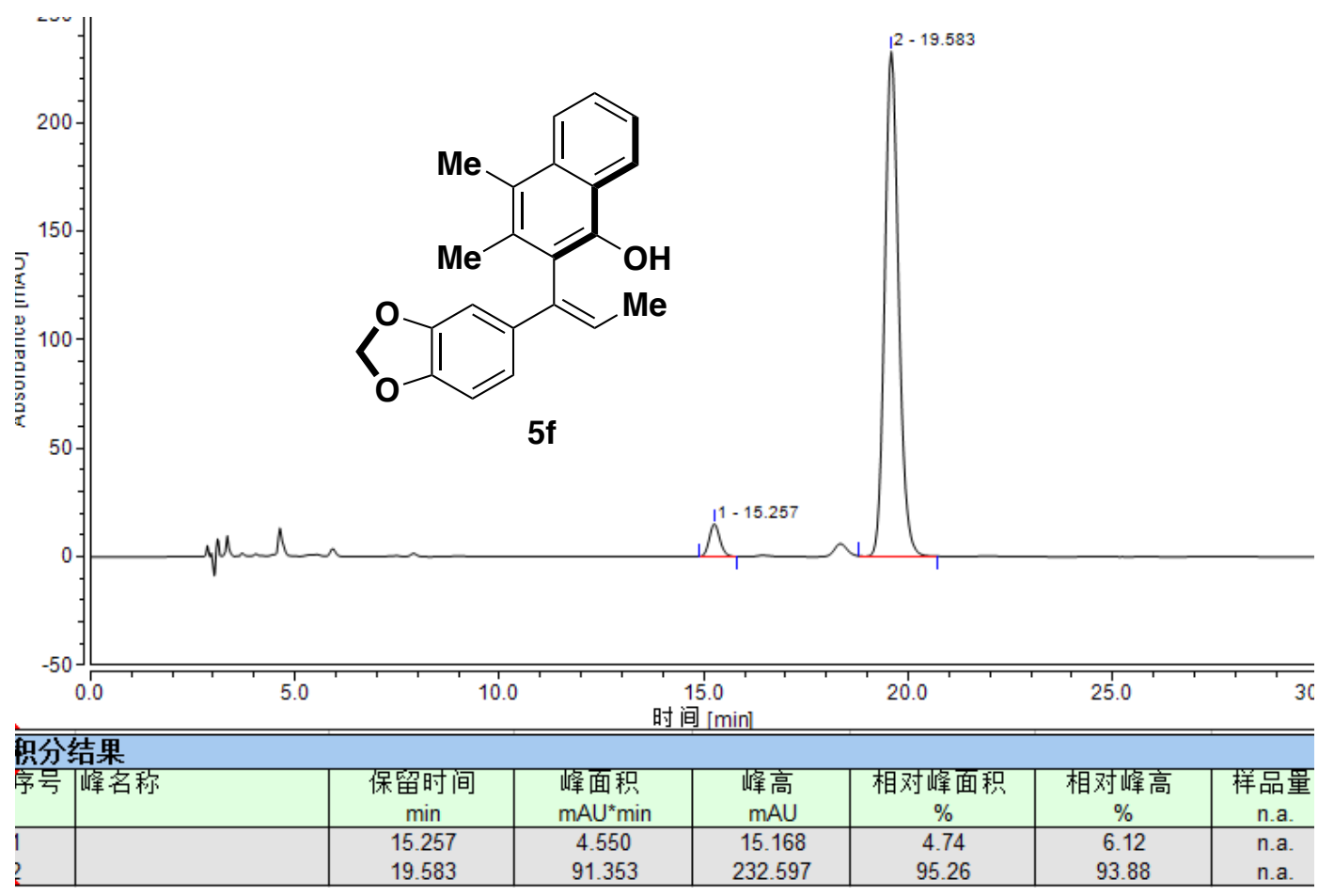

For comparison of Chinese and English of the HPLC data table.

\begin{tabular}{|c|c|c|c|c|c|c|c|}
\hline 序号 & $\begin{array}{l}\text { 峰名 } \\
\text { 称 }\end{array}$ & $\begin{array}{l}\text { 保留时间 } \\
\text { (min) }\end{array}$ & $\begin{array}{l}\text { 峰面积 } \\
\left(\mathrm{mAU}{ }^{*} \min \right)\end{array}$ & $\begin{array}{l}\begin{array}{l}\text { 峰高 } \\
(\mathrm{mAU})\end{array} \\
\end{array}$ & $\begin{array}{l}\text { 相对峰面 } \\
\text { 积 }(\%)\end{array}$ & $\begin{array}{l}\text { 相对峰高 } \\
(\%)\end{array}$ & $\begin{array}{l}\text { 样品量 } \\
\text { n.a. }\end{array}$ \\
\hline $\begin{array}{l}\text { Entr } \\
\mathrm{y}\end{array}$ & $\begin{array}{l}\text { Peak } \\
\text { name }\end{array}$ & $\begin{array}{l}\text { Retention } \\
\text { time (min) }\end{array}$ & $\begin{array}{l}\text { Peak area } \\
\left(\mathrm{mAU} *^{*} \min \right)\end{array}$ & $\begin{array}{l}\text { Peak } \\
\text { height } \\
(\mathrm{mAU})\end{array}$ & $\begin{array}{l}\text { Relative } \\
\text { peak area } \\
(\%)\end{array}$ & $\begin{array}{l}\text { Relative } \\
\text { Peak } \\
\text { height }(\%)\end{array}$ & $\begin{array}{l}\text { Sample } \\
\text { volume } \\
\text { n.a. }\end{array}$ \\
\hline
\end{tabular}




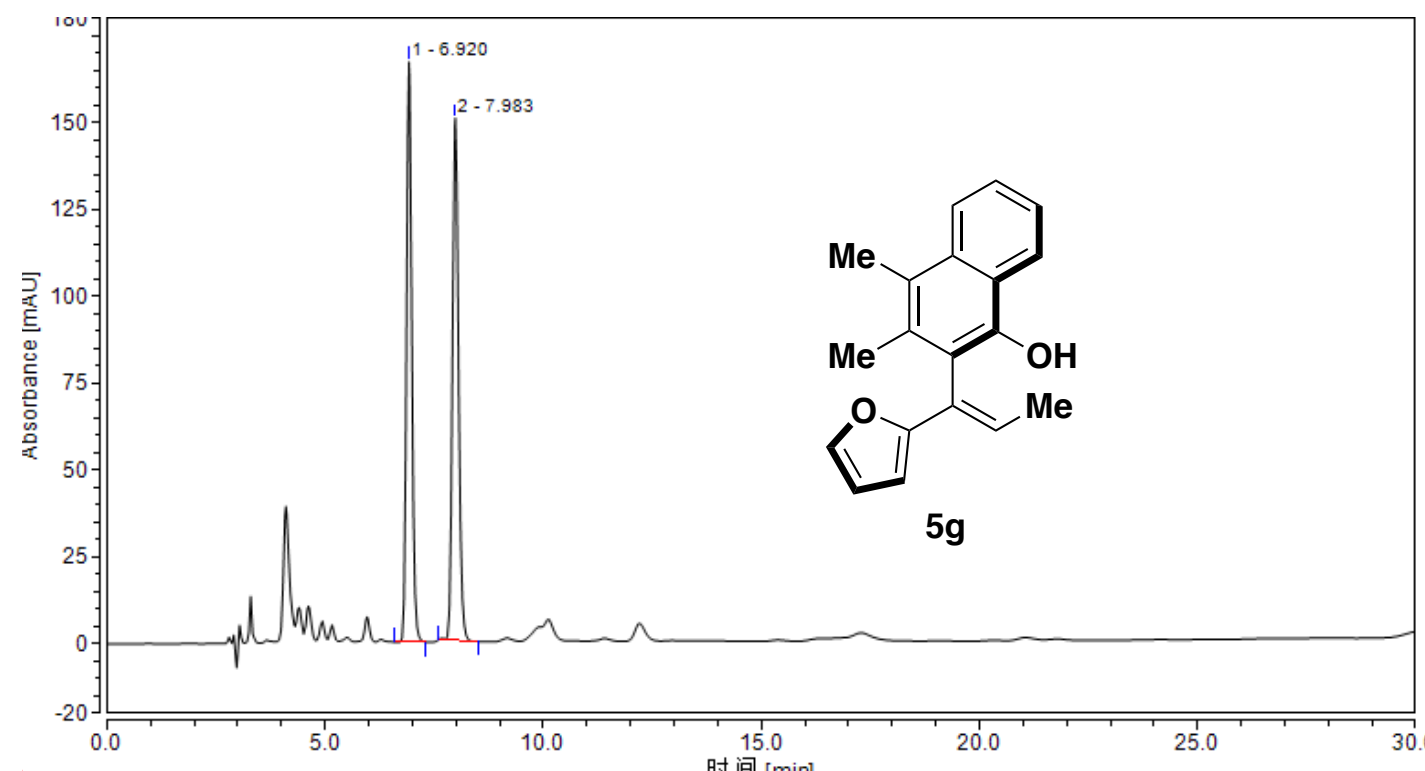

\begin{tabular}{|c|c|c|c|c|c|c|c|}
\hline \multicolumn{8}{|c|}{ 积分结果 } \\
\hline 序号 & 峰名称 & $\begin{array}{c}\text { 保留时间 } \\
\text { min }\end{array}$ & $\begin{array}{l}\text { 峰面积 } \\
m A U^{*} \min \end{array}$ & $\begin{array}{l}\text { 峰高 } \\
\text { mAU }\end{array}$ & $\begin{array}{c}\text { 相对峰面积 } \\
\%\end{array}$ & $\begin{array}{c}\text { 相对峰高 } \\
\%\end{array}$ & $\begin{array}{c}\text { 样品埋 } \\
\text { n.a. }\end{array}$ \\
\hline $\begin{array}{l}1 \\
?\end{array}$ & & $\begin{array}{l}6.920 \\
7.983 \\
\end{array}$ & $\begin{array}{l}23.391 \\
24.004\end{array}$ & $\begin{array}{l}166.924 \\
150.278\end{array}$ & $\begin{array}{l}49.35 \\
50.65 \\
\end{array}$ & $\begin{array}{l}52.62 \\
47.38 \\
\end{array}$ & $\begin{array}{l}\text { n.a. } \\
\text { n.a. }\end{array}$ \\
\hline
\end{tabular}

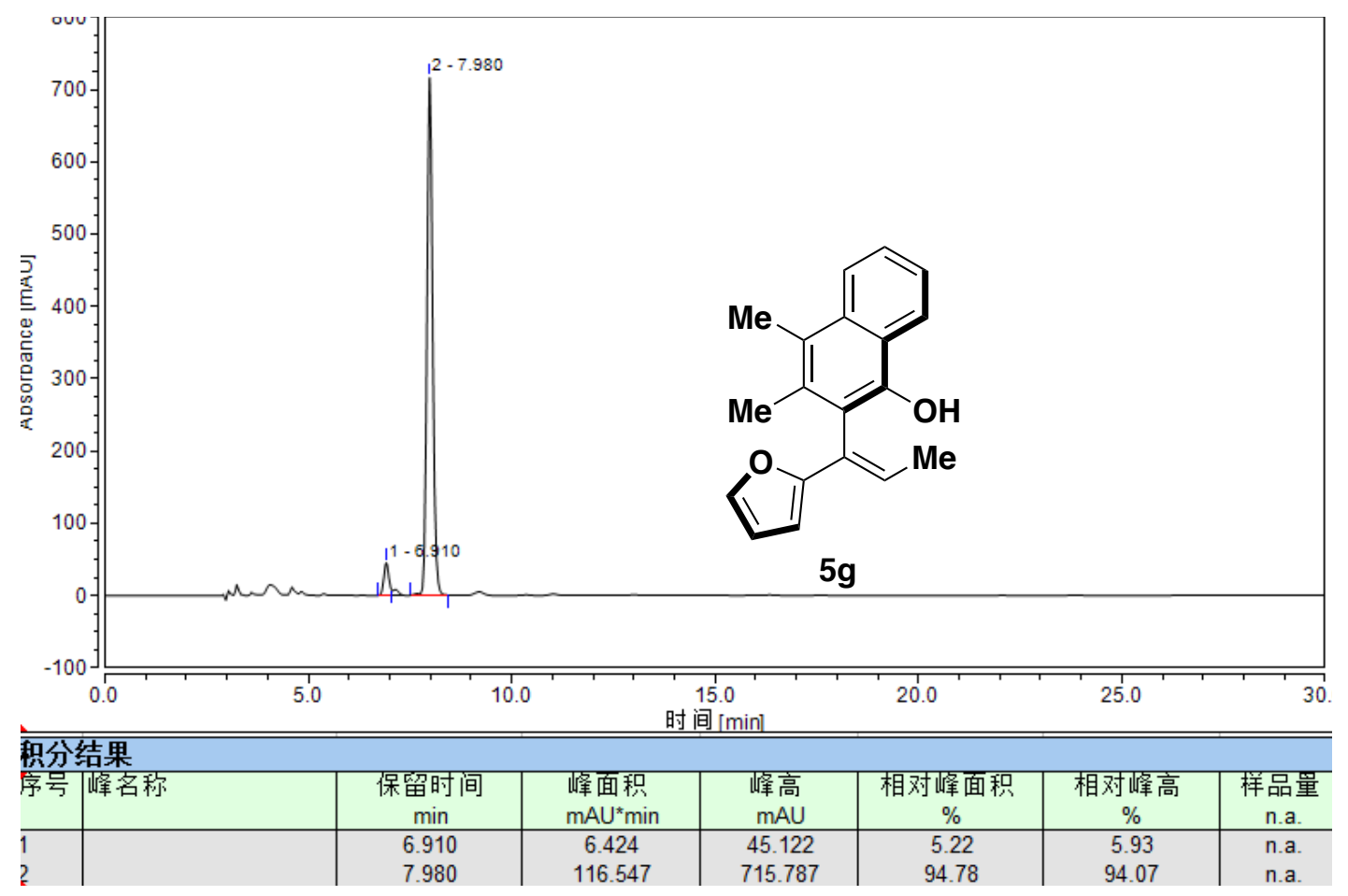

For comparison of Chinese and English of the HPLC data table.

\begin{tabular}{|c|c|c|c|c|c|c|c|}
\hline 序号 & $\begin{array}{l}\text { 峰名 } \\
\text { 称 }\end{array}$ & $\begin{array}{l}\text { 保留时间 } \\
\text { (min) }\end{array}$ & $\begin{array}{l}\text { 峰面积 } \\
\left(\mathrm{mAU} \mathrm{U}^{*} \mathrm{~min}\right)\end{array}$ & $\begin{array}{l}\text { 峰高 } \\
(\mathrm{mAU})\end{array}$ & $\begin{array}{l}\text { 相对峰面 } \\
\text { 积 }(\%)\end{array}$ & $\begin{array}{l}\text { 相对峰高 } \\
(\%)\end{array}$ & $\begin{array}{l}\text { 样品量 } \\
\text { n.a. }\end{array}$ \\
\hline $\begin{array}{l}\text { Entr } \\
\mathrm{y}\end{array}$ & $\begin{array}{l}\text { Peak } \\
\text { name }\end{array}$ & $\begin{array}{l}\text { Retention } \\
\text { time (min) }\end{array}$ & $\begin{array}{l}\text { Peak area } \\
\left(\mathrm{mAU} *^{*} \min \right)\end{array}$ & $\begin{array}{l}\text { Peak } \\
\text { height } \\
\text { (mAU) }\end{array}$ & $\begin{array}{l}\text { Relative } \\
\text { peak area } \\
(\%)\end{array}$ & $\begin{array}{l}\text { Relative } \\
\text { Peak } \\
\text { height }(\%)\end{array}$ & $\begin{array}{l}\text { Sample } \\
\text { volume } \\
\text { n.a. }\end{array}$ \\
\hline
\end{tabular}




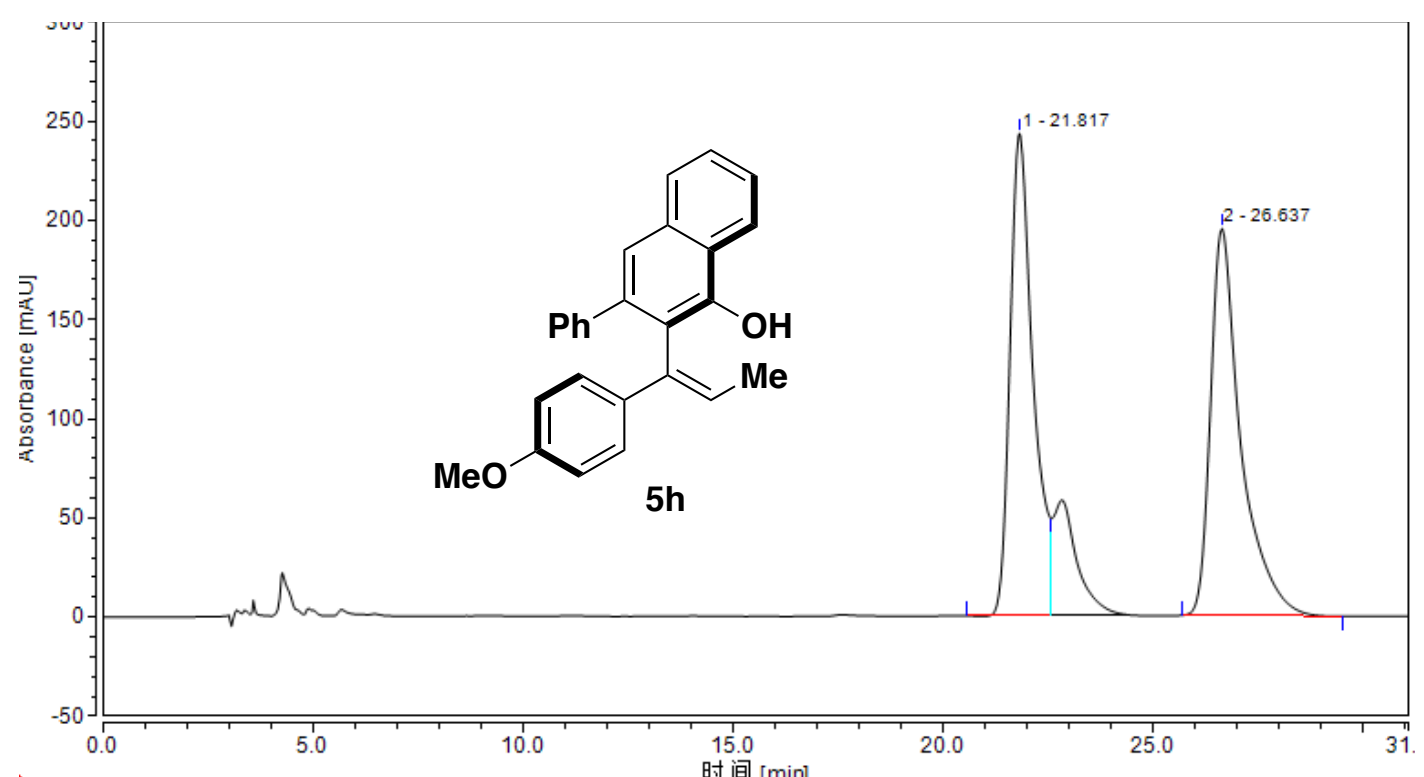

\begin{tabular}{|c|c|c|c|c|c|c|}
\hline \multicolumn{7}{|l|}{ 积分结果 } \\
\hline 序号峰名称 & $\begin{array}{c}\text { 保留时间 } \\
\text { min }\end{array}$ & $\begin{array}{l}\text { 峰面积 } \\
m U^{*} \text { min }\end{array}$ & $\begin{array}{c}\text { 峰高 } \\
\text { mAU }\end{array}$ & $\begin{array}{c}\text { 相对峰面积 } \\
\%\end{array}$ & $\begin{array}{c}\text { 相对峰高 } \\
\%\end{array}$ & $\begin{array}{c}\text { 样品量 } \\
\text { n.a. }\end{array}$ \\
\hline $\begin{array}{l}1 \\
?\end{array}$ & $\begin{array}{l}21.817 \\
26.637\end{array}$ & $\begin{array}{l}154.957 \\
163.064\end{array}$ & $\begin{array}{l}243.151 \\
195.286\end{array}$ & $\begin{array}{l}48.73 \\
51.27\end{array}$ & $\begin{array}{l}55.46 \\
44.54\end{array}$ & $\begin{array}{l}\text { n.a. } \\
\text { n.a. }\end{array}$ \\
\hline
\end{tabular}

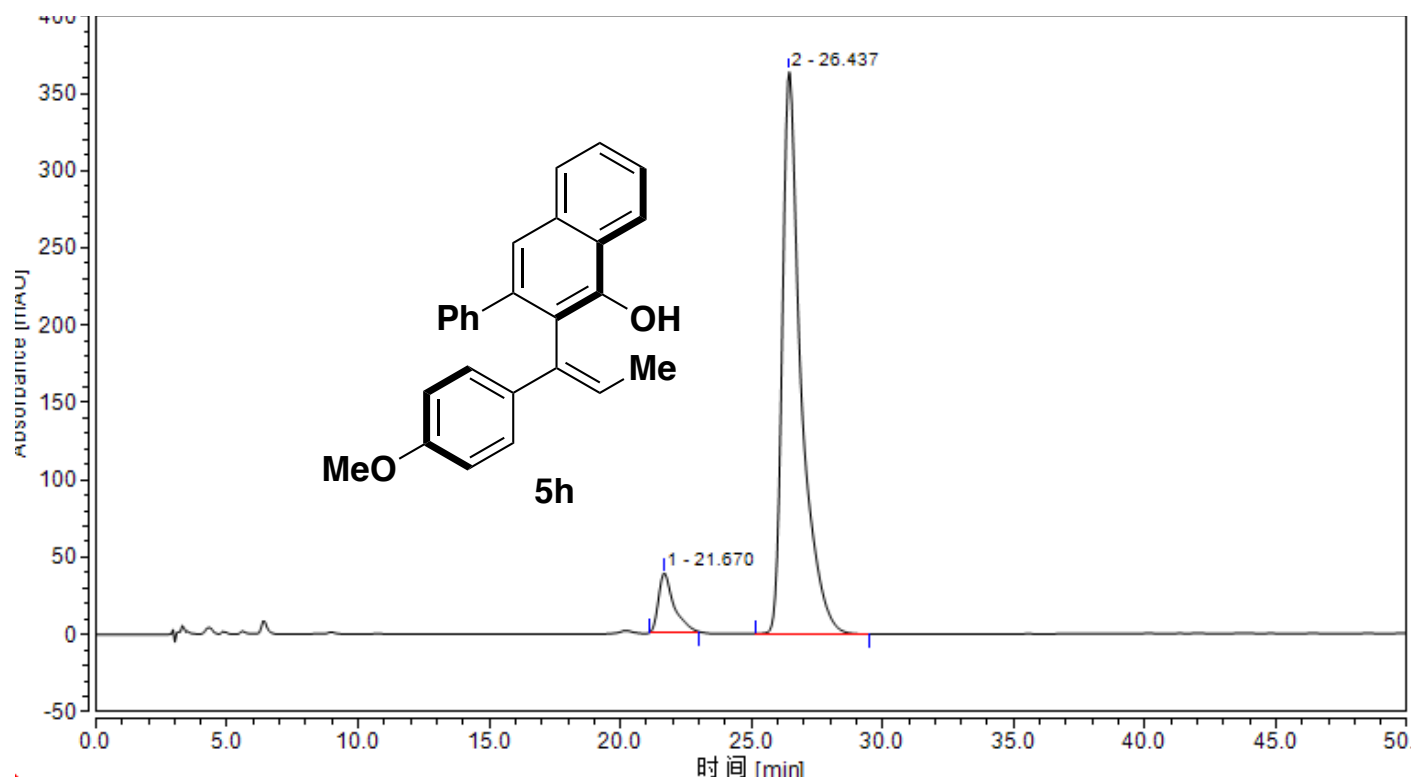

\begin{tabular}{|c|c|c|c|c|c|c|c|}
\hline \multicolumn{8}{|c|}{ 抧分结果 } \\
\hline 予号 & 峰名称 & $\begin{array}{c}\text { 保留时间 } \\
\text { min }\end{array}$ & $\begin{array}{l}\text { 峰面积 } \\
m U^{*} \text { min }\end{array}$ & $\begin{array}{l}\text { 峰高 } \\
\text { mAU }\end{array}$ & $\begin{array}{c}\text { 相对峰面积 } \\
\%\end{array}$ & $\begin{array}{c}\text { 相对峰高 } \\
\%\end{array}$ & $\begin{array}{c}\text { 样品黑 } \\
\text { n.a. }\end{array}$ \\
\hline ? & & $\begin{array}{l}21.670 \\
26.437\end{array}$ & $\begin{array}{c}26.336 \\
301.799\end{array}$ & $\begin{array}{c}38.573 \\
363.530\end{array}$ & $\begin{array}{c}8.03 \\
91.97\end{array}$ & $\begin{array}{c}9.59 \\
90.41\end{array}$ & $\begin{array}{l}\text { n.a. } \\
\text { n.a. }\end{array}$ \\
\hline
\end{tabular}

For comparison of Chinese and English of the HPLC data table.

\begin{tabular}{|l|l|l|l|l|l|l|l|}
\hline 序号 & $\begin{array}{l}\text { 峰名 } \\
\text { 称 }\end{array}$ & $\begin{array}{l}\text { 保留时间 } \\
(\mathrm{min})\end{array}$ & $\begin{array}{l}\text { 峰面积 } \\
(\mathrm{mAU} \text { *min) }\end{array}$ & $\begin{array}{l}\text { 峰高 } \\
(\mathrm{mAU})\end{array}$ & $\begin{array}{l}\text { 相对峰面 } \\
\text { 积 }(\%)\end{array}$ & $\begin{array}{l}\text { 相对峰高 } \\
(\%)\end{array}$ & $\begin{array}{l}\text { 样品量 } \\
\text { n.a. }\end{array}$ \\
\hline $\begin{array}{l}\text { Entr } \\
y\end{array}$ & $\begin{array}{l}\text { Peak } \\
\text { name }\end{array}$ & $\begin{array}{l}\text { Retention } \\
\text { time (min) }\end{array}$ & $\begin{array}{l}\text { Peak area } \\
\left(\mathrm{mAU} \mathrm{U}^{*} \text { min }\right)\end{array}$ & $\begin{array}{l}\text { Peak } \\
\text { height } \\
(\mathrm{mAU})\end{array}$ & $\begin{array}{l}\text { Relative } \\
\text { peak area } \\
(\%)\end{array}$ & $\begin{array}{l}\text { Relative } \\
\text { Peak } \\
\text { height (\%) }\end{array}$ & $\begin{array}{l}\text { Sample } \\
\text { volume } \\
\text { n.a. }\end{array}$ \\
\hline
\end{tabular}




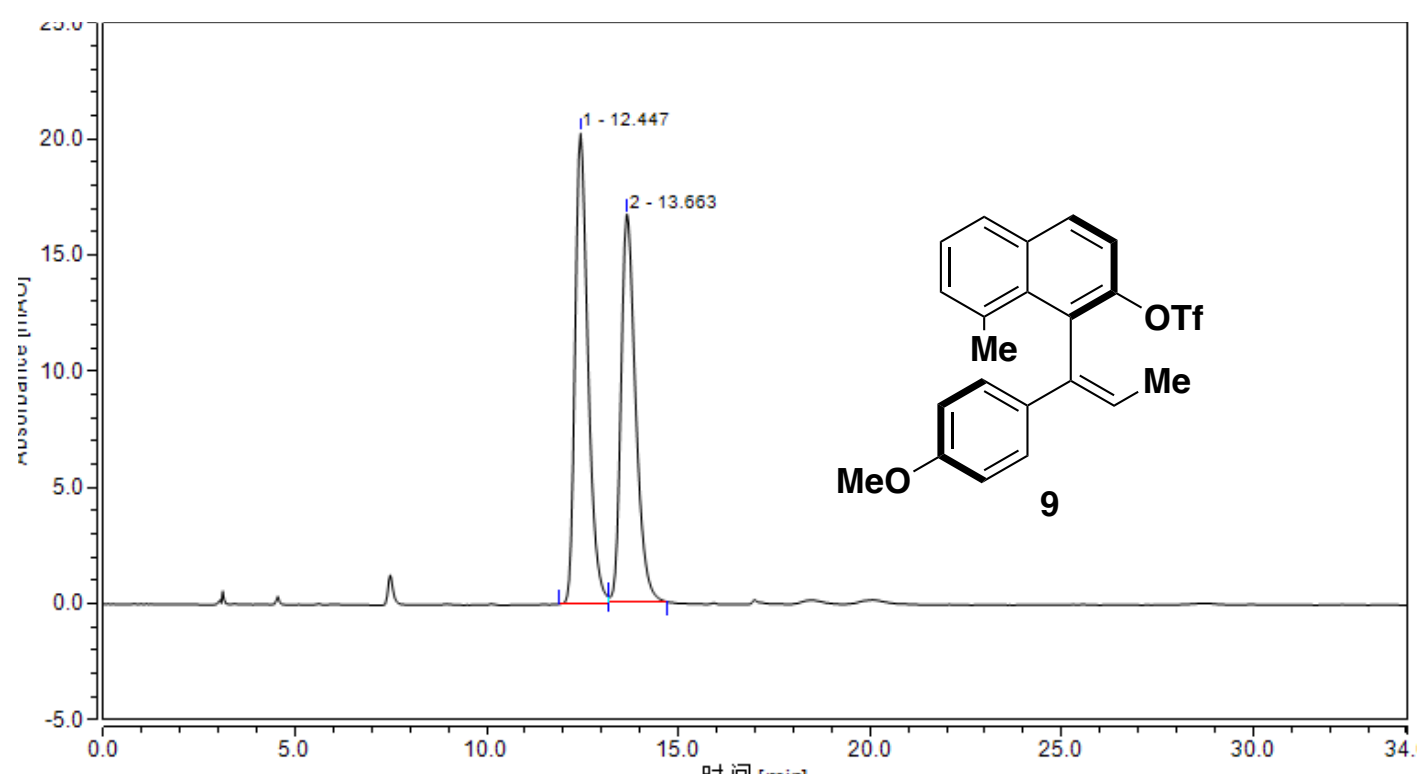

\begin{tabular}{|c|c|c|c|c|c|c|c|}
\hline \multicolumn{8}{|c|}{ 分分结果 } \\
\hline 予号 & 峰名称 & $\begin{array}{c}\text { 保留时间 } \\
\text { min }\end{array}$ & $\begin{array}{l}\text { 峰面积 } \\
m A U^{*} \text { min }\end{array}$ & $\begin{array}{c}\text { 峰高 } \\
\text { mAU }\end{array}$ & $\begin{array}{c}\text { 相对峰面积 } \\
\%\end{array}$ & $\begin{array}{c}\text { 相对峰高 } \\
\%\end{array}$ & $\begin{array}{c}\text { 样品裏 } \\
\text { n.a. }\end{array}$ \\
\hline ! & & $\begin{array}{l}12.447 \\
13.663 \\
\end{array}$ & $\begin{array}{l}7.899 \\
7.403 \\
\end{array}$ & $\begin{array}{l}20.238 \\
16.710 \\
\end{array}$ & $\begin{array}{l}51.62 \\
48.38 \\
\end{array}$ & $\begin{array}{l}54.77 \\
45.23 \\
\end{array}$ & $\begin{array}{l}\text { n.a. } \\
\text { n.a. }\end{array}$ \\
\hline
\end{tabular}
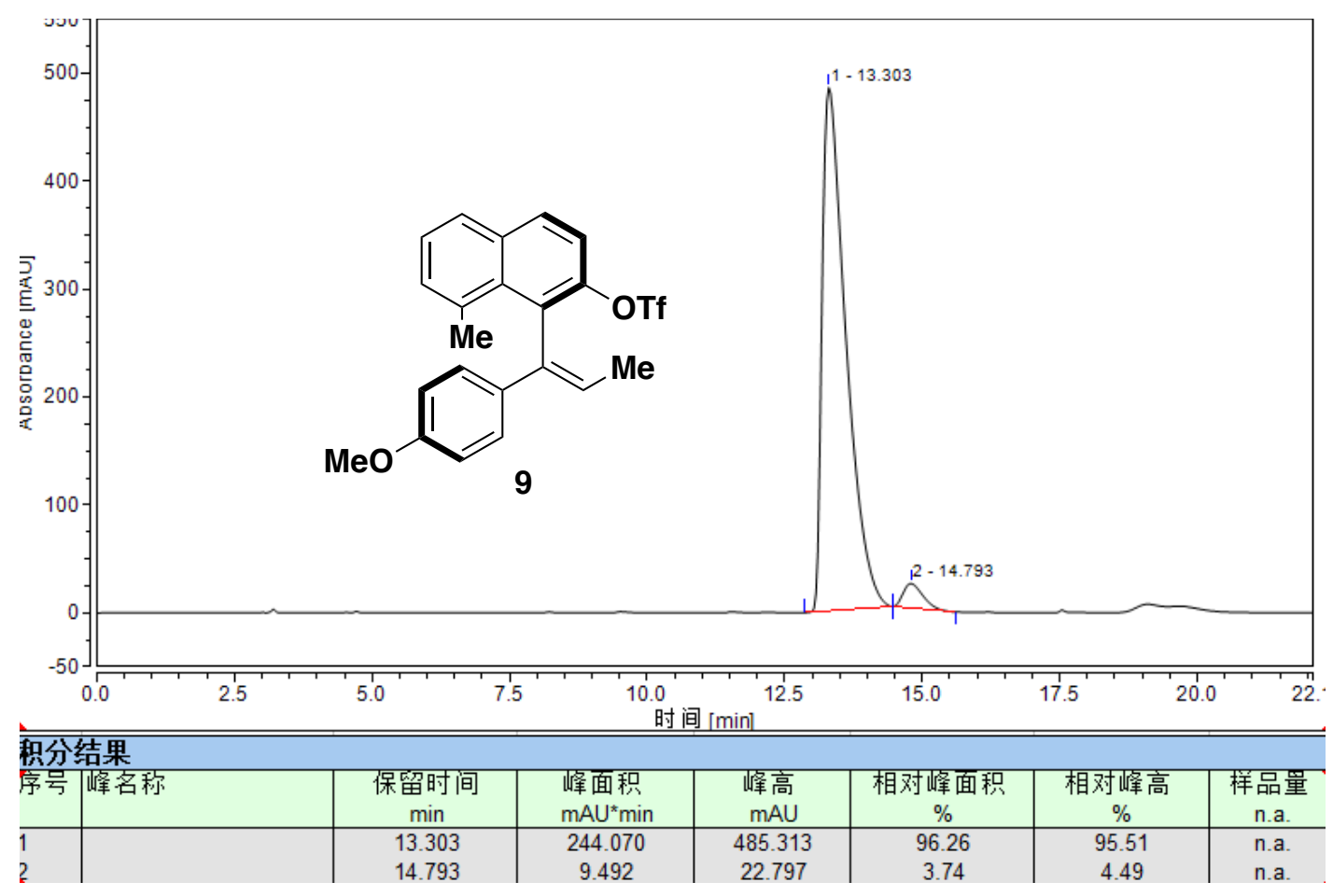

For comparison of Chinese and English of the HPLC data table.

\begin{tabular}{|c|c|c|c|c|c|c|c|}
\hline 序号 & $\begin{array}{l}\text { 峰名 } \\
\text { 称 }\end{array}$ & $\begin{array}{l}\text { 保留时间 } \\
\text { (min) }\end{array}$ & $\begin{array}{l}\text { 峰面积 } \\
\left(\mathrm{mAU} \mathrm{U}^{*} \mathrm{~min}\right)\end{array}$ & $\begin{array}{l}\text { 峰高 } \\
(\mathrm{mAU})\end{array}$ & $\begin{array}{l}\text { 相对峰面 } \\
\text { 积 }(\%)\end{array}$ & $\begin{array}{l}\text { 相对峰高 } \\
(\%)\end{array}$ & $\begin{array}{l}\text { 样品量 } \\
\text { n.a. }\end{array}$ \\
\hline $\begin{array}{l}\text { Entr } \\
\mathrm{y}\end{array}$ & $\begin{array}{l}\text { Peak } \\
\text { name }\end{array}$ & $\begin{array}{l}\text { Retention } \\
\text { time (min) }\end{array}$ & $\begin{array}{l}\text { Peak area } \\
\left(\mathrm{mAU} *^{*} \min \right)\end{array}$ & $\begin{array}{l}\text { Peak } \\
\text { height } \\
\text { (mAU) }\end{array}$ & $\begin{array}{l}\text { Relative } \\
\text { peak area } \\
(\%)\end{array}$ & $\begin{array}{l}\text { Relative } \\
\text { Peak } \\
\text { height }(\%)\end{array}$ & $\begin{array}{l}\text { Sample } \\
\text { volume } \\
\text { n.a. }\end{array}$ \\
\hline
\end{tabular}




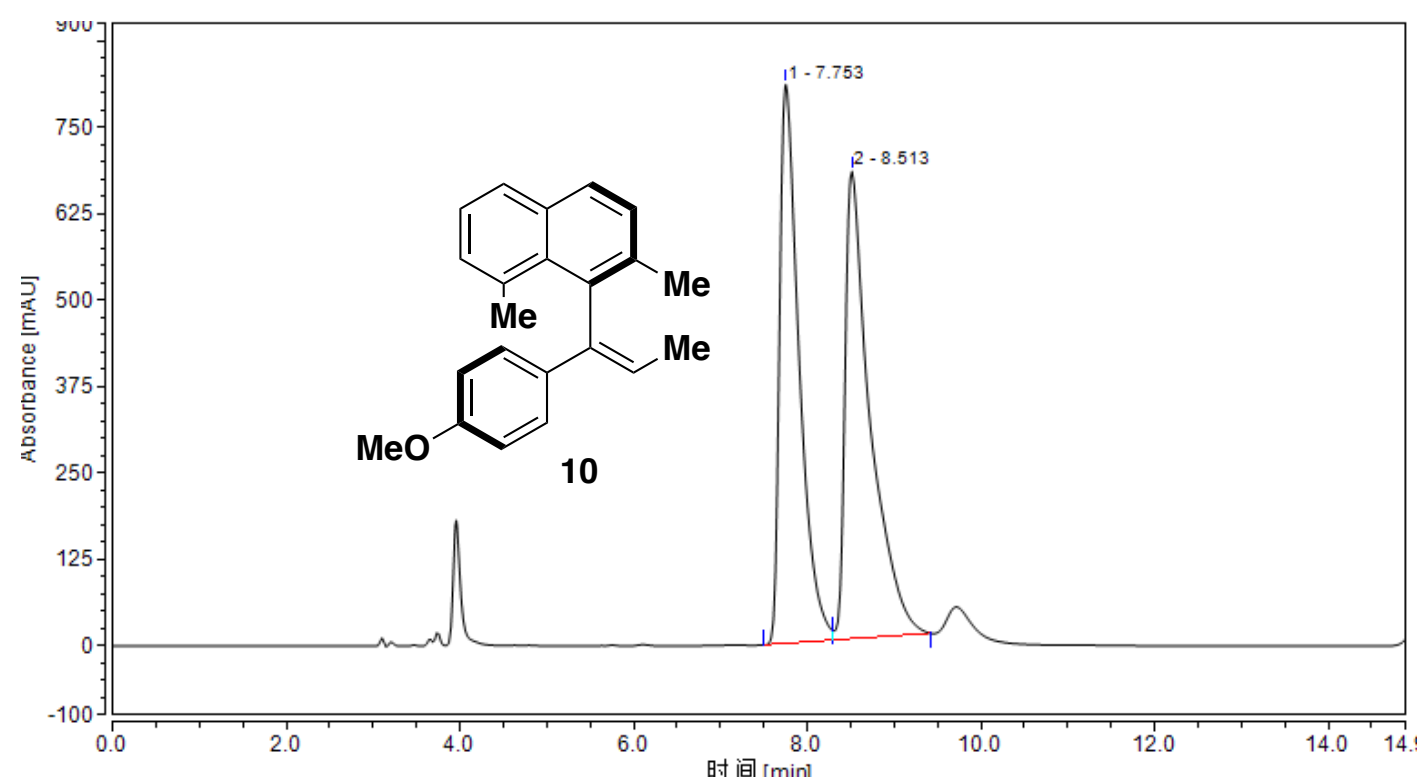

\begin{tabular}{|c|c|c|c|c|c|c|c|}
\hline \multicolumn{8}{|c|}{ 积分结果 } \\
\hline 序号 & 峰名称 & $\begin{array}{c}\text { 保留时间 } \\
\text { min }\end{array}$ & $\begin{array}{l}\text { 峰面积 } \\
m U^{*} \text { min }\end{array}$ & $\begin{array}{l}\text { 峰高 } \\
\text { mAU }\end{array}$ & $\begin{array}{c}\text { 相对峰面积 } \\
\%\end{array}$ & $\begin{array}{c}\text { 相对峰高 } \\
\%\end{array}$ & $\begin{array}{c}\text { 样品量 } \\
\text { n.a. }\end{array}$ \\
\hline $\begin{array}{l}1 \\
?\end{array}$ & & $\begin{array}{l}7.753 \\
8.513\end{array}$ & $\begin{array}{l}211.713 \\
226.653\end{array}$ & $\begin{array}{l}808.566 \\
676.893\end{array}$ & $\begin{array}{l}48.30 \\
51.70\end{array}$ & $\begin{array}{l}54.43 \\
45.57\end{array}$ & $\begin{array}{l}\text { n.a. } \\
\text { n.a. }\end{array}$ \\
\hline
\end{tabular}

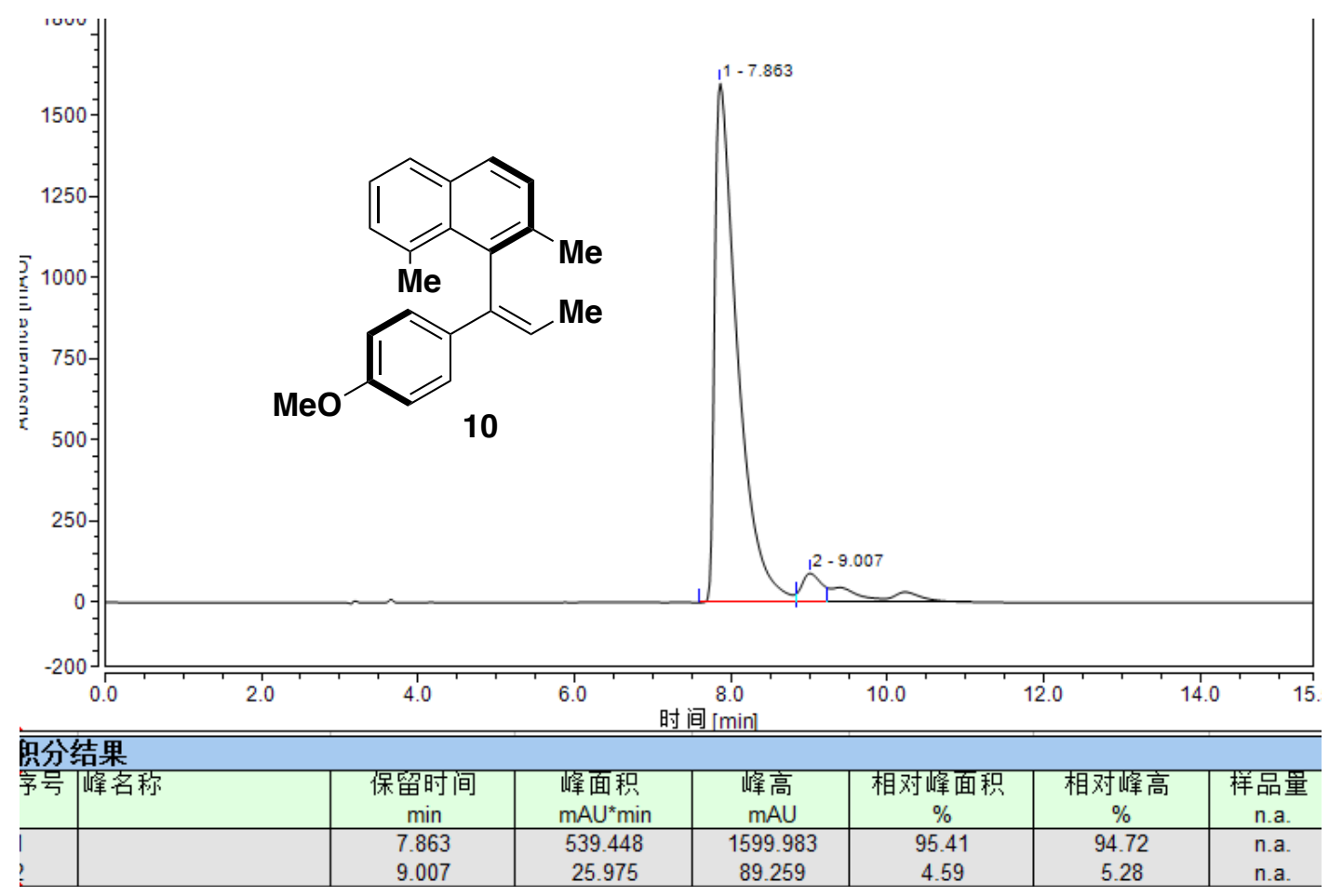

For comparison of Chinese and English of the HPLC data table.

\begin{tabular}{|c|c|c|c|c|c|c|c|}
\hline 序号 & $\begin{array}{l}\text { 峰名 } \\
\text { 称 }\end{array}$ & $\begin{array}{l}\text { 保留时间 } \\
\text { (min) }\end{array}$ & $\begin{array}{l}\text { 峰面积 } \\
\left(\mathrm{mAU} \mathrm{U}^{*} \mathrm{~min}\right)\end{array}$ & $\begin{array}{l}\text { 峰高 } \\
(\mathrm{mAU})\end{array}$ & $\begin{array}{l}\text { 相对峰面 } \\
\text { 积 }(\%)\end{array}$ & $\begin{array}{l}\text { 相对峰高 } \\
(\%)\end{array}$ & $\begin{array}{l}\text { 样品量 } \\
\text { n.a. }\end{array}$ \\
\hline $\begin{array}{l}\text { Entr } \\
\mathrm{y}\end{array}$ & $\begin{array}{l}\text { Peak } \\
\text { name }\end{array}$ & $\begin{array}{l}\text { Retention } \\
\text { time (min) }\end{array}$ & $\begin{array}{l}\text { Peak area } \\
\left(\mathrm{mAU} *^{*} \min \right)\end{array}$ & $\begin{array}{l}\text { Peak } \\
\text { height } \\
\text { (mAU) }\end{array}$ & $\begin{array}{l}\text { Relative } \\
\text { peak area } \\
(\%)\end{array}$ & $\begin{array}{l}\text { Relative } \\
\text { Peak } \\
\text { height }(\%)\end{array}$ & $\begin{array}{l}\text { Sample } \\
\text { volume } \\
\text { n.a. }\end{array}$ \\
\hline
\end{tabular}



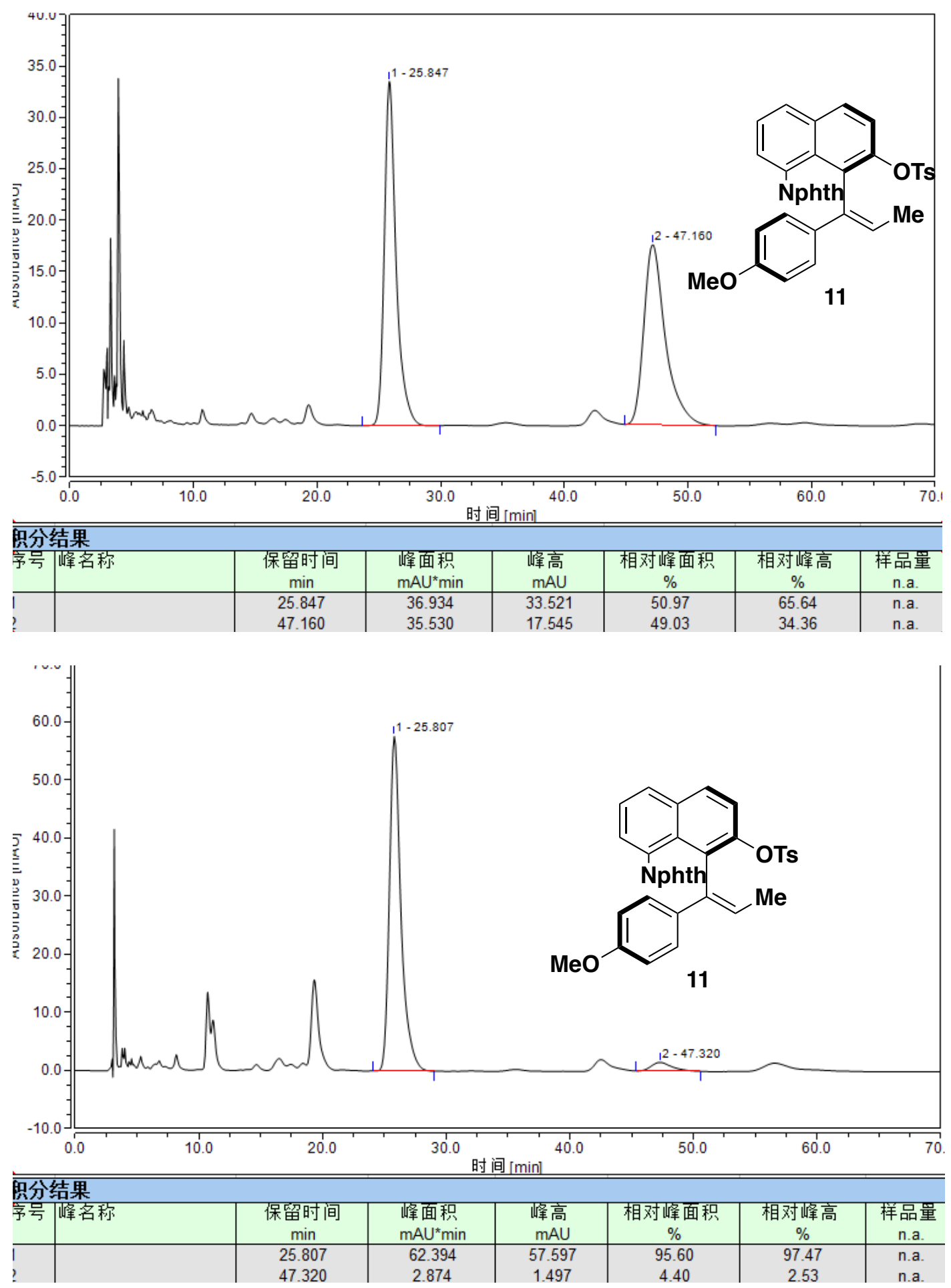

For comparison of Chinese and English of the HPLC data table.

\begin{tabular}{|l|l|l|l|l|l|l|l|}
\hline 序号 & $\begin{array}{l}\text { 峰名 } \\
\text { 称 }\end{array}$ & $\begin{array}{l}\text { 保留时间 } \\
(\mathrm{min})\end{array}$ & $\begin{array}{l}\text { 峰面积 } \\
(\mathrm{mAU} \text { *min) }\end{array}$ & $\begin{array}{l}\text { 峰高 } \\
(\mathrm{mAU})\end{array}$ & $\begin{array}{l}\text { 相对峰面 } \\
\text { 积 }(\%)\end{array}$ & $\begin{array}{l}\text { 相对峰高 } \\
(\%)\end{array}$ & $\begin{array}{l}\text { 样品量 } \\
\text { n.a. }\end{array}$ \\
\hline $\begin{array}{l}\text { Entr } \\
\mathrm{y}\end{array}$ & $\begin{array}{l}\text { Peak } \\
\text { name }\end{array}$ & $\begin{array}{l}\text { Retention } \\
\text { time (min) }\end{array}$ & $\begin{array}{l}\text { Peak area } \\
\left(\mathrm{mAU} \mathrm{U}^{*} \text { min }\right)\end{array}$ & $\begin{array}{l}\text { Peak } \\
\text { height } \\
(\mathrm{mAU})\end{array}$ & $\begin{array}{l}\text { Relative } \\
\text { peak area } \\
(\%)\end{array}$ & $\begin{array}{l}\text { Relative } \\
\text { Peak } \\
\text { height (\%) }\end{array}$ & $\begin{array}{l}\text { Sample } \\
\text { volume } \\
\text { n.a. }\end{array}$ \\
\hline
\end{tabular}



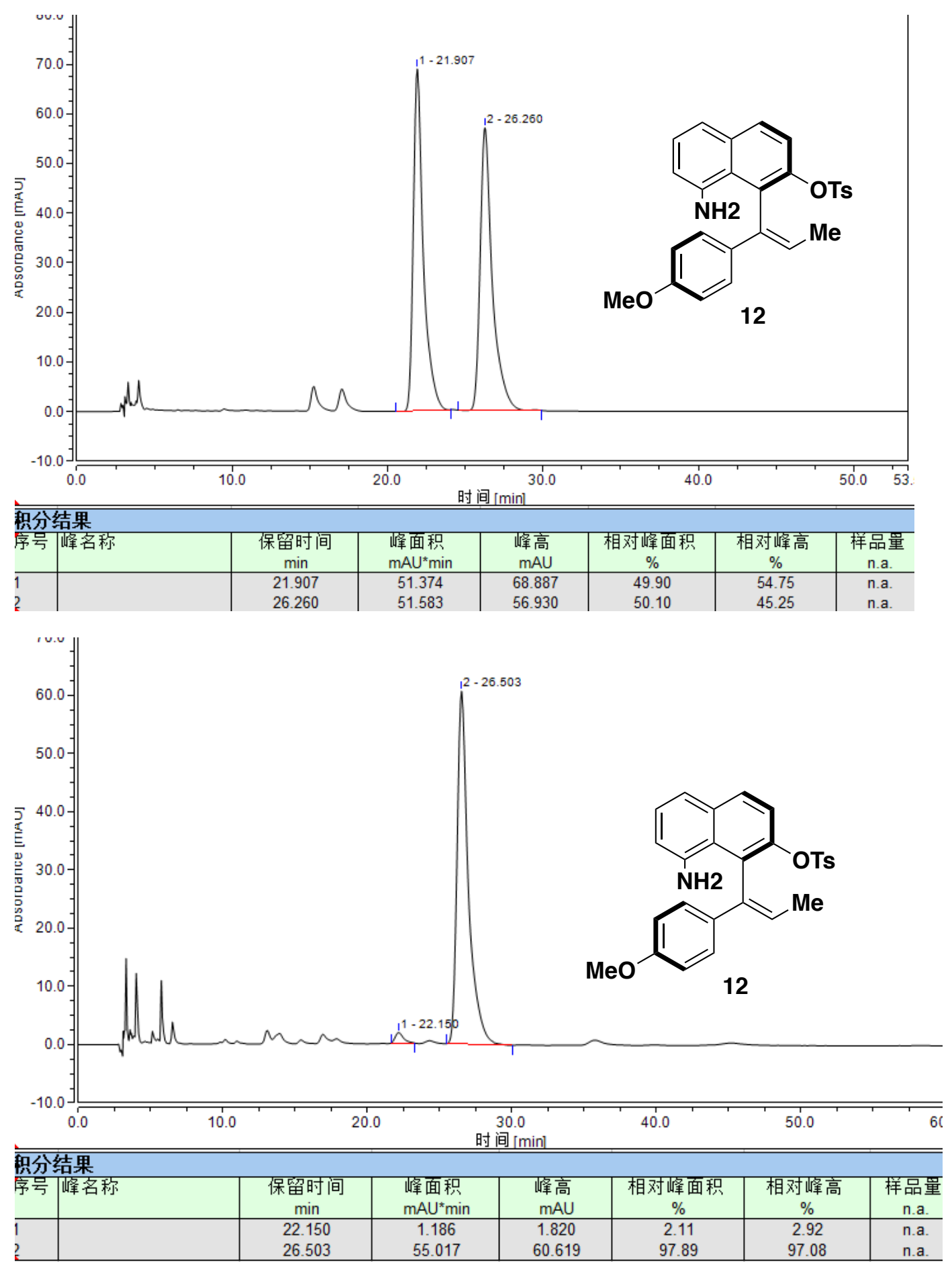

For comparison of Chinese and English of the HPLC data table.

\begin{tabular}{|c|c|c|c|c|c|c|c|}
\hline 序号 & $\begin{array}{l}\text { 峰名 } \\
\text { 称 }\end{array}$ & $\begin{array}{l}\text { 保留时间 } \\
\text { (min) }\end{array}$ & $\begin{array}{l}\text { 峰面积 } \\
\left(\mathrm{mAU}{ }^{*} \min \right)\end{array}$ & $\begin{array}{l}\text { 峰高 } \\
\text { (mAU) }\end{array}$ & $\begin{array}{l}\text { 相对峰面 } \\
\text { 积 (\%) }\end{array}$ & $\begin{array}{l}\text { 相对峰高 } \\
(\%)\end{array}$ & $\begin{array}{l}\text { 样品量 } \\
\text { n.a. }\end{array}$ \\
\hline $\begin{array}{l}\text { Entr } \\
\mathrm{y}\end{array}$ & $\begin{array}{l}\text { Peak } \\
\text { name }\end{array}$ & $\begin{array}{l}\text { Retention } \\
\text { time (min) }\end{array}$ & $\begin{array}{l}\text { Peak area } \\
\left(\mathrm{mAU}{ }^{*} \min \right)\end{array}$ & $\begin{array}{l}\text { Peak } \\
\text { height } \\
(\mathrm{mAU})\end{array}$ & $\begin{array}{l}\text { Relative } \\
\text { peak area } \\
(\%)\end{array}$ & $\begin{array}{l}\text { Relative } \\
\text { Peak } \\
\text { height (\%) }\end{array}$ & $\begin{array}{l}\text { Sample } \\
\text { volume } \\
\text { n.a. }\end{array}$ \\
\hline
\end{tabular}

